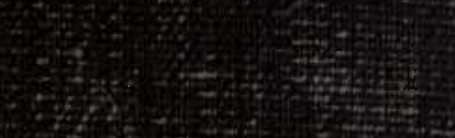

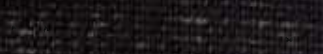

oct

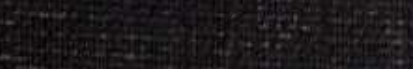

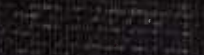

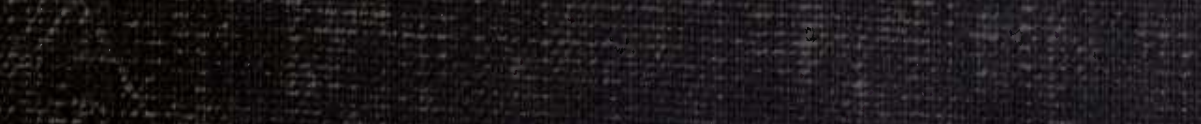
5.

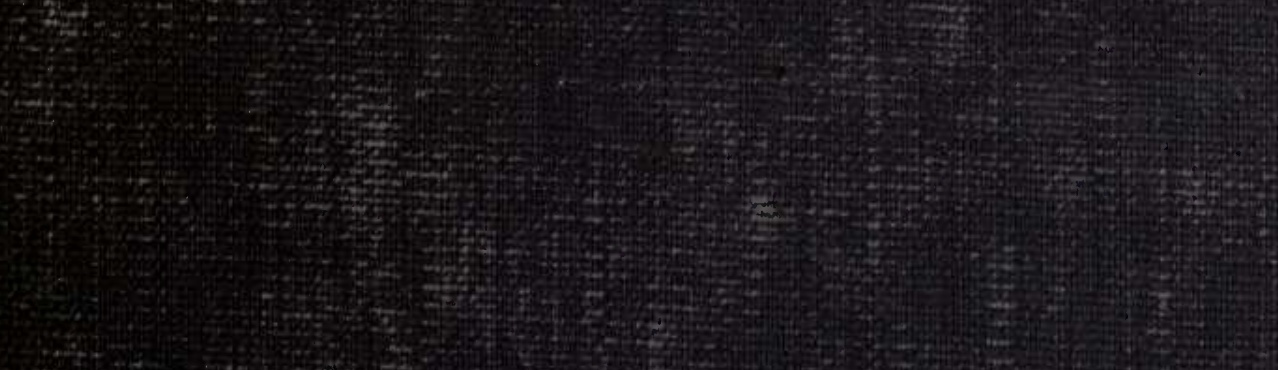

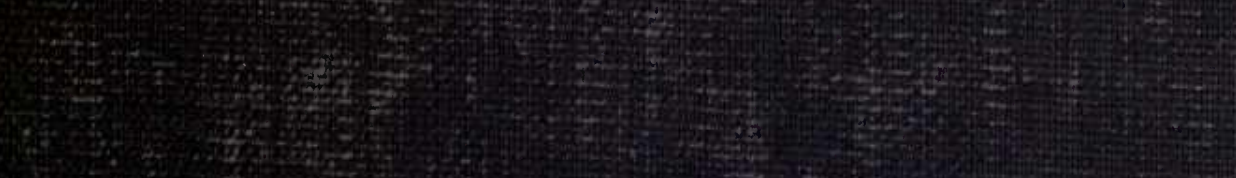

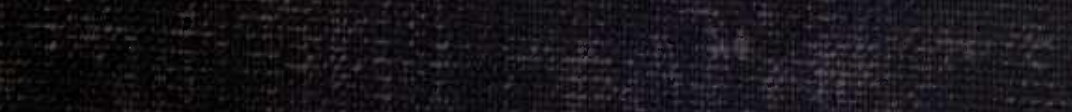

(1)

(1) 


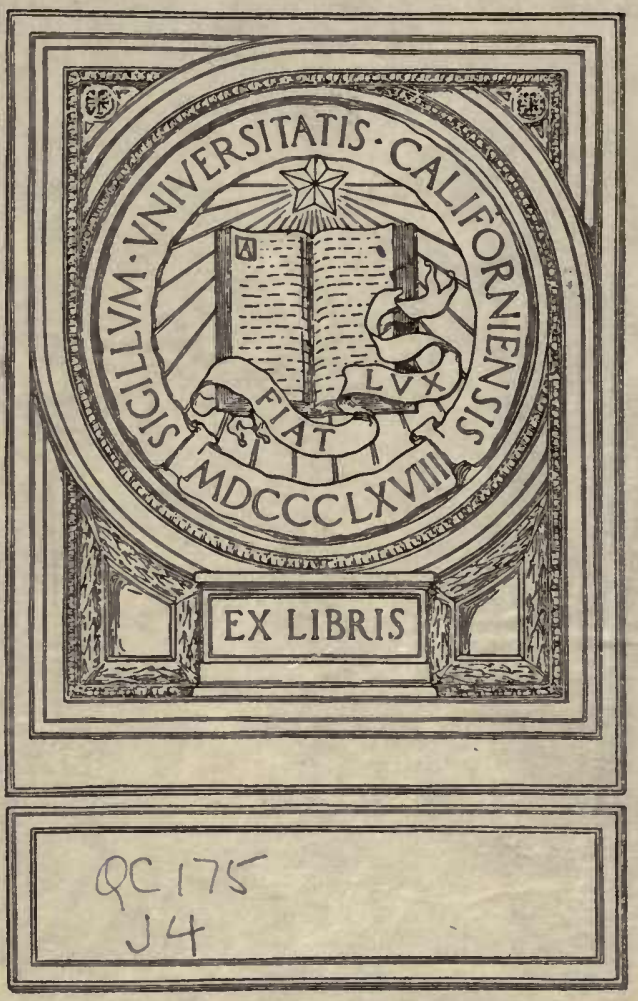



THE DYNAMICAL THEORY OF GASES 
Floñon: C. J. CLAY AND SONS, CAMBRIDGE UNIVERSITY PRESS WAREHOUSE, AVE MARIA LANE.

EFtassob: 50, WELLINGTON STREET.

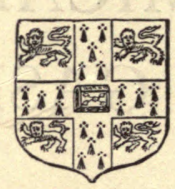

羊 eipุ̧ig: F. A. BROCKHAUS.

ferw ragork: THE MACMILLAN COMPANY.

Bombay añ Calcutta: MACMILLAN AND CO., LTD. 


\title{
THE DYNAMICAL THEORY OF GASES •
}

\author{
BY \\ J. H. JEANS, M.A., \\ FELLOW OF TRINITY COLLEGE, CAMBRIDGE.
}

Cambridge

at the University Press

I 904 


\section{XYOHHT UADIMAKXO}

\section{$2 \pi 2 A 0$}

$$
\text { Q } 31^{7^{7}}
$$

\section{Cambrioge:}

PRINTED BY J. AND C. F. CLAY,

AT THE UNIVERSITY PRESS.

$$
\because \because \quad \because 4 \vdots
$$




\section{PREFACE.}

TN writing the present book, my first aim has been to develop the Theory of Gases upon as exact a mathematical basis as possible. The need for a sure foundation and an exact treatment is, I think, sufficiently obvious. For some years the Kinetic Theory has been regarded by the great majority of physicists with ever-increasing distrust and suspicion. The origin of this attitude of mind is undoubtedly to be looked for in the fact that many results which have been deduced from the Theory are found to be in obvious opposition to experimental results. Deductions which have any relation at all to physical phenomena cannot of course be arrived at without definite physical assumptions, and any inconsistency between theoretical and experimental results must of necessity be traceable to imperfections in the physical assumptions on which the development of the theory is based. Unfortunately the assumptions frequently enter in so subtle a form that it is hardly possible to realise that a definite assumption has been made at all. Hence the imperative necessity for a strict logical treatment. I have hoped that some of the distrust which at present attaches itself to the Kinetic Theory, will be removed when the exact relation between premises and conclusions is pointed out.

One discrepancy between theory and experiment, that connected with the theorem of Equipartition of Energy and the consequent evaluation of the ratio of the specific heats of a gas, is of greater importance than all the others together. As regards this particular question, I have tried to emphasise the facts, (i) that the theorem of Equipartition is based upon a definite assumption, namely that there is no interaction between matter and ether, (ii) that wherever we look, in the most literal sense of the word, the evidence that the assumption is illegitimate thrusts itself upon us-every ray of light which reaches our eyes is evidence against the truth of the assumption-and (iii) that as soon as we abandon the assumption in question, theory and experiment harmonise as well as could be desired. 
An attempt at an exact treatment of the subject has unfortunately involved the introduction of a considerable quantity of mathematical analysis, but I have kept before me the desire to reduce the amount of analysis to the minimum consistent with efficiency, in the hope of making as much of the book as possible intelligible to readers who are not trained mathematicians.

I have also paid considerable attention to estimates of numerical values, to experimental results, and to the physical interpretation of the phenomena discussed, my ambition being to endow the subject with more life than would have been possible if it had been treated solely as a dynamical problem. As regards these parts of my work, I have been fortunate in receiving advice and criticism from Mr H. A. Wilson and Mr O. W. Richardson, Fellows of Trinity College. I therefore venture to hope that there are no serious inaccuracies in the more physical parts of the book. It will of course be understood that the book makes no claim to give anything like a full account of experimental results. Such results as are quoted are meant as illustrations only. The reader who wishes for a fuller account of experimental work is referred to Meyer's Kinetic Theory of Gases or Weinstein's Thermodynamik und Kinetik der Körper. From both these books, as also from Travers' Experimental Study of Gases, I have borrowed largely. In the more abstract parts of the subject I have made constant use of the writings of Maxwell, Rayleigh and Tait, of Boltzmann's Vorlesungen über Gastheorie, of the papers of the same author in the Wiener Sitzungsberichte, and of Kirchhoff's Vorlesungen über die Theorie der Wärme. To all these I express my indebtedness.

It remains only to record my thanks to all who have helped me with suggestions and criticisms, and to the staff of the University Press from whom I have received every possible assistance and courtesy during the printing of the book.

J. H. JEANS.

November, 1904. 


\section{CONTENTS.}

CHAP.

PAGE

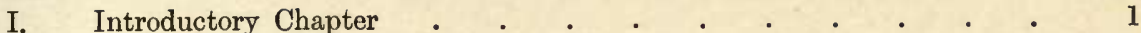

The Law of Distribution.

II. The Statistical Method . . . . . . . . . . 11

III. The Method of General Dynamics . . . . . . . 33

IV. Comparison between the two Methods . . . . . . . 53

V. Extension to Molecules of the most general conservative type . . 61

\section{Physical Properties.}

VI. Pressure, Density and Temperature . . . . . . . 105

VII. Mass Motion and Calorimetry . . . . . . . . 149

Theory of a Non-Conservative Gas.

VIII. General Dynamics of Non-conservative Systems . • . . 176

IX. The Transfer and Dissipation of Energy in a gas . . . . 197

$\mathrm{X}$. Calorimetry . • . • • • • • • . • . 210

Free Path Phenomena.

XI. Calculation of the Free Path . . . . . . . . . 229

XII. Viscosity . . . . . . . . . . . . . 242

XIII. Conduction of Heat . . . . . . . . . . 259

XIV. Diffusion . • . . . . . . . • . . . 268

XV. Maxwell's Theory of Viscosity, Conduction and Diffusion . . . 275

XVI. The Propagation of Sound . . . . . . . . 302

XVII. Planetary Atmospheres . . . . . . . . . 309

XVIII. Molecular Aggregation and Dissociation . • • . • 326

XIX. The Evidence of the Kinetic Theory as to the size of molecules - 339

\section{APPENDICES.}

A. Integrals involving exponentials . . . . . . . . 346

B. Tables for numerical calculations . . . . . . . 347

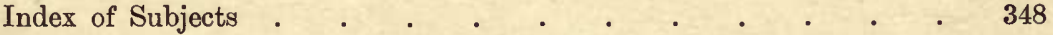

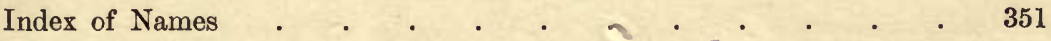




\section{ADDENDA AND CORRIGENDA.}

p. 110. \$126. Since writing this section, I have been interested to find that a precisely similar treatment had suggested itself to Maxwell in 1877, and was outlined in a letter to Garnett (see the Life of Maxwell by Campbell and Garnett, p. 570).

p. 161, last line, last expression. For $\frac{\nu_{b}}{2 h v} \operatorname{read} \frac{\nu b}{2 h v}$.

p. 170, last line of $\$ 199$. For unit mass read unit volume.

p. 219. Table of values of $C_{p}$. In last five values of $\frac{R}{J m}$, for $0 \cdot$ read $\cdot 0$. The corresponding values on p. 218 are correct.

p. 225, bottom of page. A step has been omitted, namely, a mention of the heat required to change the degree of dissociation when the temperature changes. The full argument is given in Chapter XVIII.

p. 267. Table of values of 9 . Some of the figures in the last column $\left(\frac{9}{\kappa C_{v}}\right)$ are inaccurate, the correct series being $1 \cdot 70,1 \cdot 77,1 \cdot 51,1 \cdot 76,1 \cdot 77,1 \cdot 61,1 \cdot 86,1 \cdot 50,1 \cdot 65$-mean : $1 \cdot 68$. 


\section{CHAPTER I.}

\section{INTRODUCTORY CHAPTER.}

\section{The Kinetic Theory conception of Matter.}

1. THE dynamical theory of gases, as developed in the present book, rests solely upon what is commonly termed the Kinetic Theory of matter.

The essential feature of the Kinetic Theory is that it interprets heat in matter as a manifestation of a motion of the molecules which compose the matter. It need hardly be said that this identification of heat and motion is only a hypothesis: it never has been, and from the nature of things never can be, proved. At the same time this hypothesis shews an ability to explain and even to predict natural phenomena, such that there can be little doubt that it rests upon a foundation of truth.

2. The Kinetic Theory assumes at the outset that all matter, whether solid, liquid or gaseous, is composed of a great number of small molecules, and that these molecules are similar to one another so long as they are molecules of the same chemical substance. In making this assumption we are again relying upon an unproved and unprovable hypothesis. This second hypothesis is of course not peculiar to the Kinetic Theory but is borrowed from the science of Chemistry. Chemistry further supposes molecules to be capable of division into smaller bodies, namely atoms, and modern investigations shew that these again may be capable of division into yet smaller bodies-ions, electrons, or corpuscles. The Kinetic Theory, however, is concerned primarily with molecules only, and it is hardly necessary, at any rate for the development of the pure Kinetic Theory, to discuss any hypotheses or speculations as to the structure or divisibility of molecules.

Having once postulated this divisibility of matter into molecules, our every-day experience gives a certain amount of guidance as to the properties which are to be attributed to molecules. For instance, we know that two bodies cannot occupy the same space; any attempt to compel them to do 
so brings into play a system of repulsive forces tending to keep the two bodies apart, and this system of forces can only be interpreted as the aggregate of the forces from individual molecules. It follows that molecules exert forces on one another, and that these forces are, in general, repulsive when the molecules are sufficiently close to one another. On the other hand, the phenomenon of cohesion shews that the force between two molecules may, under certain conditions, be one of attraction.

\section{The Three States of Matter.}

3. The fact that a solid body, when in its natural state, resists both compression and dilatation, indicates that the force between molecules changes from one of repulsion at small distances to one of attraction at greater distances. This change from a repulsive to an attractive force suggests a position of stable equilibrium in which a pair of molecules can rest in proximity to one another. If we imagine a great number of molecules placed in proximity and at rest in an equilibrium configuration, we have on the Kinetic Theory conception of matter, a mass of matter in the solid state, and, as there is no motion, this matter must be supposed, in accordance with the fundamental hypothesis of the theory, to be entirely devoid of heat.

The molecules of which the substance is formed will be capable of vibration about their positions of equilibrium, and when these vibrations occur, we say that the body possesses heat. As the vibrations become more vigorous we say that the temperature of the body increases.

For example, we may imagine the vibratory motion of the molecules to be set up in the first instance by rubbing the surface of the body against a surface of a similar body: here we have a case of heat generated by friction. The act of rubbing will consist firstly in placing the surfaces of the two bodies so near to one another that the molecules near the surface of one exert a perceptible action on the molecules near the surface of the other, and then in moving the surfaces over one another so as to disturb these surface molecules from their positions of equilibrium. At first the motion will be confined to the neighbourhood of the parts actually rubbed, but the motion of these parts will gradually induce motion in the adjoining regions, until ultimately the motion will have spread over the whole mass. In this latter phenomenon we have an instance of conduction of heat.

As a second example, we may imagine two masses, both devoid of internal motion, to impinge one upon the other. The impact will at first cause systems of waves to be set up in the masses, but after a sufficient time the wave character of the motion will have become obliterated, whilst 
motion of some kind must persist in order to account for the energy of the original motion. This original motion will, in fact, have become replaced by a small vibratory motion of the molecules about their positions of equilibrium-according to the Kinetic Theory, by heat-motion.

4. If the body under consideration continues to acquire heat in any way-if, that is, the energy of vibration is caused continually to increasea stage will in time be reached in which some of the molecules are possessed of so much kinetic energy that the forces from the other molecules no longer suffice to hold them in position: they will, to borrow an astronomical term, escape from their orbits. When the body has reached a state such that this is true of a great number of molecules, it is clear that the application of even a small force, provided it is continued for a sufficient length of time, will, by taking advantage time after time, as opportunity occurs, of the weakness of the forces tending to retain individual molecules, cause the mass to change its shape. When this stage is reached, the body has assumed a plastic or liquid state.

When a molecule of a liquid escapes from its orbit it will in general wander about amongst the other molecules until it falls into a new orbit. If, however, it was initially near to the surface of the liquid, it may be possible for it to escape altogether from the attraction of the other molecules, just as it is possible for a projectile, if projected from the earth's surface with sufficient velocity, to escape from the earth altogether. When this happens the molecule will leave the liquid, so that the mass and volume of liquid will continually diminish owing to the loss of such molecules. Here we have the Kinetic Theory interpretation of the process of evaporation, the vapour being formed by the escaped molecules.

If the liquid is contained in a closed vessel the escaping molecules will impinge on the side of the vessel, and after a certain number of impacts, may fall back again into the liquid. When a state is reached in which the number of molecules which fall back in this way is exactly balanced by the number which escape, we have, according to the Kinetic Theory, a liquid in equilibrium with its own vapour.

5. If we suppose the whole of the liquid transformed into vapour in this way, we have the Kinetic Theory conception of a gas. The molecules can no longer be said to describe orbits, but describe rectilinear paths with uniform velocity except when they encounter other molecules or the walls of the containing vessel. It is clear that this view of the nature of a gas will sufficiently explain the property which a gas possesses of spreading throughout the whole of any closed space in which it is placed. It is not necessary to suppose, as was at one time done, that this expansive property of a gas is the result of repulsive forces between the molecules. 
Of the three states of matter which have just been sketched, the mathematical development of the Kinetic Theory is at present concerned only with the last. A properly developed Kinetic Theory of liquids and solids is still wanting, and throughout the remainder of this book we shall be content to discuss matter in its gaseous state.

\section{Mechanical Illustration of the Kinetic Theory of Gases.}

6. It is important to form as clear an idea as possible of the conception of a gas which has just been reached. Now very little is known as to the structure or shape of actual molecules, or of the way in which they act upon one another. Since, however, it is advisable to have as concrete a representation as possible before the mind, we may for the present (following the usual procedure in the development of the Kinetic Theory) agree to associate the idea of a molecule with that of a spherical body of great elasticity and rigidity - to make the picture quite definite, let us say a billiard-ball. The justification for this procedure lies in its success: it will be found that the behaviour of a gas in which the molecules are complex structures of the most general kind, can, to a large extent, be predicted from the behaviour of the much simpler ideal gas of which the-molecules are of the simple type just described. In fact one of the most striking features of the Kinetic Theory is the way in which we are enabled to predict the behaviour of a gas as a whole while remaining in almost complete ignorance of the behaviour and properties of the molecules of which it is composed.

7. Before proceeding to exact mathematical analysis, it may be profitable roughly to indicate the questions which are to be examined, and at the same time to forecast some of the results which will be obtained. This can well be done by taking a mechanical illustration in two dimensions.

Let us represent the molecules of the gas by billiard-balls, and the vessel in which the gas is enclosed by a large billiard-table. The walls of the vessel are of course represented by the cushions of the table, and if the vessel represented is to be a closed one, there must be no pockets. The materials must be of such good quality that a ball once set in motion will collide thousands of times with the cushions before it is brought to rest by the friction and various other passive forces which tend to destroy its motion. Most of the properties of gases can be illustrated with this imaginary apparatus.

If we take a very large number of balls and start them at random on the table with random velocities, the resulting state of motion will give a representation of the supposed state of the gas. Every ball will continually be colliding both with the other balls and with the cushions. The velocities of the balls will be of the most varying kinds : at one instant a ball may be 
brought absolutely to rest, while at another instant, as the result of a succession of favourable collisions, it may possess a velocity far in excess of the average velocity of the other balls. (One of the problems we shall have to solve will be to find how the velocities of the various balls are distributed about the mean velocity.) We shall find that whatever the way in which the velocities are grouped at the outset, they will tend, after a sufficient number of collisions, to group themselves according to the so-called law of trial and error-the law which governs the grouping in position of shots fired at a target.

If the cushions of the table were not fixed in position, they would be driven back by the continued impacts of the balls. The force exerted on the cushions by the balls colliding with them accordingly represents the pressure exerted on the walls of the containing vessel by the gas. Let us imagine a moveable barrier placed initially against one of the cushions, and capable of motion parallel to this cushion. Moving this barrier forward is equivalent to decreasing the volume of the gas. If the barrier is moved forwards while the motion of the billiard-balls is in progress, the impacts both on the moveable barrier and on the three fixed cushions will of course become more frequent: here we have a representation of an increase of pressure accompanying a diminution of volume of a gas. (We shall have to discuss how far the law connecting the pressure and density of a gas, constituted in the way imagined by the Kinetic Theory, is in agreement with that found by experiment for an actual gas.)

Let us imagine the barrier on our supposed billiard-table to be moved half-way up the table. Let us suppose that the part of the table in front of the barrier is occupied by white balls moving on the average with a large velocity, while the part behind it is similarly occupied by red balls moving on the average with a much smaller velocity. Here we may imagine that we have divided our vessel into two separate chambers; the one is occupied by a gas of one kind at a high temperature, the other by a gas of a different kind at a lower temperature. Returning to the billiard-table, let the barrier suddenly be removed. The white balls will immediately invade the part which was formerly occupied only by red balls, and vice-versa. Also the rapidly moving white balls will be continually losing energy by collision with the slower red balls, and the red of course gaining energy through impact with the white. After the motion has been in progress for a sufficient time the white and red balls will be equally distributed over the whole of the table, and the average velocities of the balls of the two colours will be the same. (Here we have simple illustrations of the diffusion of gases, and of equalisation of temperature.) The actual problem to be solved is, however, obviously more complex than that suggested by this analogy, for in nature the molecules of different gases differ by something more than mere colour. 
There is one further point to be mentioned. However elastic the billiardballs and table may be, the motion cannot continue indefinitely. In time the energy of this motion will be frittered away, partly by the vibrations set up at collisions and partly, perhaps, by frictional forces. What, then, does this represent in the gas, and how is it that a gas, if constituted as we have supposed, does not in a short time lose the translational motion of its molecules, and replace it by a vibrational motion internal to the molecules? An answer, as will appear later, is supplied by the extreme "elasticity" of the molecules, this elasticity being put in evidence by the high frequency of the light-vibrations emitted by the molecules. This frittering away of the translational motion is in fact continually taking place in a gas, but the process is so slow that, as we shall see, gases may exist for millions of years without their stock of energy being replenished, before the energy of the translational motion is appreciably diminished.

\section{Numerical Values.}

8. The foregoing rough sketch will, it is hoped, have given some idea of the nature of the problems to be attacked. As a conclusion to this preliminary chapter, it may be useful to give some approximate numerical values, as an indication of the magnitude of the quantities with which we shall be dealing.

Number of molecules per cubic centimetre. The number of molecules in a cubic centimetre of gas at normal temperature and pressure is, in accordance with Avogadro's law ( $\$ 113$ infra), independent of the chemical composition of the gas. Probably the most accurate values obtained experimentally for this number are those obtained by electrical methods which are quite independent of the Kinetic Theory*. Professor J. J. Thomsont finds for this number, referred to normal temperature and pressure, the value $3.6 \times 10^{19}, \mathrm{Mr} \mathrm{H}$. A. Wilson + finds the value $4.0 \times 10^{19}$, whilst from some earlier experiments of Professor Townsend $\S$ it is possible to deduce the value $4 \cdot 1 \times 10^{10}$. We may, therefore, conclude that the value of this number, certainly as regards order of magnitude, and possibly to within an error of a few per cent., is equal to about $4 \times 10^{30} \|$. Since the mass of the molecule must be supposed to remain constant thrughout all changes of density, temperature and volume of the gas, the number of molecules per cubic centimetre will obviously be directly proportional to the density of the gas.

* A review of these methods is given by Prof. J. S. Townsend, Phil. Mag. vII. p. 278.

+ Phil. Mag. v. p. 335.

$\S$ Proc. Camb. Phil. Soc. 1x. Part 5.

|| A discussion of earlier attempts to estimate this number is given by Lord Kelvin, Baltimore $\ddagger$ Phil. Mag. v. p. 441. Lectures, Lecture xvir., or Phil. MIag. 1v. pp. 177 and 281.

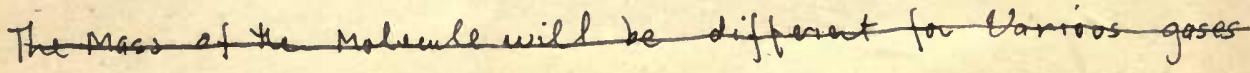

.

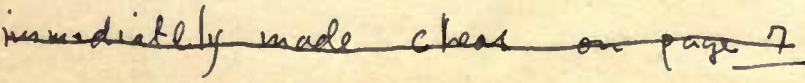


Mass of a molecule. The mass of a cubic centimetre of oxygen under normal conditions* is 001429 gms., so that from the number just given it follows that the mass of a molecule of oxygen is, to the same degree of accuracy as before, equal to $3.5 \times 10^{-23} \mathrm{gms}$. The masses of other molecules are of course in proportion to their chemical molecular-weights. That of hydrogen, for instance, is about $2.2 \times 10^{-24} \mathrm{gms}$.

Velocity. The velocity of the molecules is known with great accuracy. For air at a temperature of $15^{\circ} \mathrm{C}$. the average velocity is about 459 metres per second. For hydrogen at $0^{\circ} \mathrm{C}$. it is about 1694 metres per second. As regards velocities under other conditions it may be said that, roughly, the mean velocity of a molecule of molecular weight $m$, at a temperature of $\theta$ degrees Centigrade, is proportional to $\sqrt{273+\theta}$, and is inversely proportional to $\sqrt{m}$. It is approximately independent of the density. A table of velocities will be given later.

If all the molecules were moving with a velocity equal to the average velocity, the total distance described by the $4 \times 10^{19}$ molecules in a c.c. of hydrogen at $0^{\circ} \mathrm{C}$. would be about $677 \times 10^{17}$ kilometres per second. The actual distance, after allowing for the variation in the velocities of the molecules, is, as will be seen without trouble, exactly equal to this.

Size. It is a matter of some difficulty to determine or even to define the size of a molecule. The trouble arises primarily from our ignorance of the shape and other properties of the molecule. If the molecules were known to be elastic spheres the question would be simple enough, and the size of the molecule would be measured by the diameter of the sphere. If, however, the molecules are assumed as a first approximation to be elastic spheres, experiment leads to discordant results for the diameters of these spheres, shewing that the original assumption is unjustifiable. The divergencies arise not only from the fact that the shape of the molecules is not spherical, but also from the fact that the molecules are surrounded by fields of force, and in most experiments it is the extension of this field of force, rather than that of the molecules themselves, with which we are concerned.

If, however, we agree to regard the molecules as roughly represented by elastic spheres, it is found that these spheres must be supposed in the case of hydrogen to have a radius of about $10^{-8} \mathrm{cms}$. The size ought strictly to be different for different molecules, and more exact figures will be given later, but as the difference in size is hardly more than comparable with the error introduced by the supposition that the molecules are elastic spheres, these differences need not be discussed here. Regarding the molecule of hydrogen as a sphere of radius $10^{-8} \mathrm{cms}$. the number of collisions per cubic centimetre of hydrogen at $0^{\circ} \mathrm{C}$. is found to be about $24 \times 10^{29}$ per second.

* Travers, Experimental Study of Gases, p. 133. 
Free Paths. Each collision is the termination of two free paths, hence the number of free paths described in the gas just considered is about $4.8 \times 10^{20}$ per second. It has already been said that the total distance described-i.e., the aggregate of these free paths-is $677 \times 10^{22} \mathrm{cms}$. Hence on division we see that the mean length of these free paths is $1.4 \times 10^{-5} \mathrm{cms}$.

It is obvious that the mean free path, being a pure length, will depend only on the diameter of the molecules, and on the number of molecules per cubic centimetre; it will not depend on the velocities of motion of the molecules. Thus the values we have obtained for the mean free path are approximately true for all gases so long as the molecules are supposed uniformly to be spheres of radius $10^{-8} \mathrm{cms}$. The free path is, however, inversely proportional to the number of molecules per cubic centimetre of gas. For instance in a vacuum tube in which the pressure is that of half a millimetre of mercury, the density of gas is only $1: 1520$ of the normal - density, and therefore the free path is roughly equal to a quarter of a millimetre.

It appears from these figures that the mean free path of a molecule is about 700 times its diameter in a gas at normal pressure, and is over a million times its diameter when the pressure is reduced to half a millimetre of mercury. There is therefore every justification for assuming, as a first approximation, that the linear dimensions of molecules are small in comparison with their free paths.

Comparing the values obtained for the free path with the values previously given for the velocity of motion, we find that the mean time of describing a free path ranges from about $3 \times 10^{-10}$ seconds in the case of air under normal conditions, to about $1.3 \times 10^{-7}$ seconds in the case of hydrogen at a pressure equal to that of half a millimetre of mercury.

The principal lesson to be learned from the foregoing figures is that the mechanism of the Kinetic Theory is extremely "fine-grained" when measured by ordinary standards. Molecules are, in fact, not infinitely small, and neither is their motion infinitely rapid, but the units of space and time appropriate for the measurement of the motion of individual molecules are so small in comparison with even the smallest quantities which we can measure experimentally that the phenomena exhibited by a gas constituted in the way described will be indistinguishable, so far as experiment and human observation go, from those of a continuous medium. It is for this reason that the hypothesis upon which the Kinetic Theory rests is, and probably will always remain, an unproved hypothesis. 


\section{Historical Note.}

9. The rise of the Kinetic Theory was of a gradual nature, and it is difficult to mention any time at which the theory may be said to have arisen, or any single name to whom honour of its establishment is due. Three stages in its development may be traced. There is first the stage of speculative opinion, unsupported by scientific evidence. Given that a great number of thinkers are speculating as to the structure of matter, it is only in accordance with the laws of probability that some of them should arrive fairly near to the truth. An opinion which turns out ultimately to be near the truth remains, however, of no greater value to the advancement of science, than a more erroneous opinion, until scientific reasons can be given for supposing the former to be more accurate than the latter. When this point is reached the theory may be said to have entered upon the second stage of its development; the true and false opinions are still equally in the field, but the former is supplied with weapons for defeating the latter. In the third stage there is general agreement as to the main foundations of the theory and their truth, and labour is devoted no longer to defeating adverse opinion, but to the elaboration of the detail of the theory, and to attempts to extend its boundaries.

In its earliest stage the growth of the Kinetic Theory is hardly distinguishable from that of the atomic theory. The view that matter was to be regarded as an aggregation of hard, indivisible and similar parts was upheld by Lucretius, who appears to have taken his opinions from Democritus and Epicurus, who again had been guided by Leucippus. This theory was revived by Gassendi in the middle of the seventeenth century*. Apparently Gassendi was the first to suspect that the motion alone of the atoms was sufficient to account for a number of phenomena, without the introduction of adventitious hypotheses to account separately for these phenomena. Lasswitz† describes Gassendi's work as follows: "Following Democritus and Epicurus, Gassendi in the seventeenth century re-established and elaborated an atomic theory based upon the assumption that all material phenomena can be referred to the indestructible motion of atoms and can therefore be described as "kinetic." Gassendi's atoms are devoid of all qualities except absolute rigidity; they are similar in substance, but different in size and form, and move in all directions through empty space. On this basis Gassendi explains a number of physical processes, in particular the three states of matter and the transitions from one to another, in a way very little different from that of the modern kinetic theory." It is obvious, then, that with Gassendi the theory is entering upon the second stage of its existence.

* Syntagma Philosophicum, 1658, Lugduni.

+ "Der Verfall der kinetischen Atomistik im 17 Jahrhundert," Pogg. Ann. curr. p. 373 (1874). 
Twenty years later ideas of the same nature seem to have occurred independently to Hooke, the recognition of whose work in the foundation of the Kinetic Theory is due to Professor Tait*.

The next advance in the theory is due to Daniel Bernoullit, who frequently is credited with having been the first to make the discoveries of Gassendi and Hooke. In his Hydrodynamica, published in 1738, he points out that the elasticity of a gas may be regarded as due to the impacts of particles on the boundary. He deduces Boyle's law for the relation between pressure and volume, and attempts to find a general relation between pressure and volume when the finite size of the molecules, supposed absolutely hard and spherical, is taken into account.

After Bernoulli, there is little to record for almost a century. Then we find that in rapid succession Herapath + (1821), Waterston (1845), Joule $\S$ (1848), Krönig\|(1856), and Clausius (1857) take up the subject. Waterston attempted to a scientific mathematical theory of the subject; but his paper, which was presented to the Royal Society in 1845 , contained certain inaccuracies, and was for this reason not published in the Philosophical Transactions until 1892 , when Lord Rayleigh had it published on account of its historical interest. Clausius, in his first paper**, calculates accurately the relation between temperature, pressure and volume, and also the value of the ratio of the two specific heats for a gas in which the energy of the molecules is wholly one of translation. In 1859, Clerk Maxwell was added to the number of contributors to the theory, reading a paper on the subject before the British Association at Aberdeen $+\dagger$. It has been suggested that Maxwell was first led to take an interest in the subject by his investigations on the motion of Saturn's rings, which gained for him the Adams Prize in 1857+甘. In the hands of Clausius and Maxwell the theory developed with great rapidity, so that to write the history of the subject from this time, would be hardly less than to give an account of the theory in its present form. Among the more prominent contributors to the theory since the time of Clausius and Maxwell may be mentioned Boltzmanu, Kirchhoff, Van der Waals and Lorentz on the continent, and in England Tait, Lord Rayleigh and Burbury.

* "Hooke's Anticipation of the Kinetic Theory." Proc. Edin. Roy. Soc., March 16, 1885. Tait's Collected Works, ir. p. 122.

+ Daniel Bernoulli, Hydrodynamica. Argentoria, 1738. Sectio decima, "De affectionibus atque motibus fluidorum elasticorum, praecipue autem aeris."

† Annals of Philosophy, [2], I. p. 273.

$\S$ British Association Report, 1848, Part Ir. p. 21 ; Memoirs of the Manchester Literary and Philosophical Society, [2], Ix. p. 107.

\| Poggendorff's Annalen.

T Phil. Trans. cLxxxir. p. 1.

** "Ueber die Art der Bewegung welche wir Wärme nennen," Pogg. Annalen, c. p. 353.

†† Phil. Mag. Jan. and July, 1860. Collected Works, 1. p. 377.

$¥+$ See W. D. Niven, preface to Maxwell's Collected Works, p. xv. 


\section{CHAPTER II.}

\section{THE LAW OF DISTRIBUTION OF VELOCITIES.}

\section{The Statistical Method.}

10. THE mathematical difficulties of the subject commence when we attempt to discuss the law according to which the velocities of the molecules are grouped about their mean value.) We are of course at liberty to consider an imaginary gas in which the velocities are grouped at the outset according to any law we please, but in general every collision which occurs will tend to change this law. The problem before us is to investigate whether there is any law which remains, on the whole, unchanged by collisions; and if so whether the velocities of the molecules ' of a gas, starting from some arbitrarily chosen law, will tend after a sufficient time, to obey some definite law which is independent of the particular law from which the gas started.

There are two totally distinct methods of attacking these problems, and these are given in this chapter and the next, the relation between them being discussed in Chapter IV. The present chapter contains the classical method of which the development is due mainly to Clerk Maxwell and Boltzmann (see $§ 60$ below).

\section{The definition of Density.}

11. There is no difficulty in defining the density of a continuous substance. If we take a small volume $v$, enclosing a given point $P$, and denote by $m$ the mass of matter contained within this volume, then the assumption of continuity ensures that as the volume $v$ shrinks until it is of infinitesimal size, while still enclosing the point $P$, then the ratio $m / v$ will approach a definite limit $\rho$, and we define the density at the point $P$ as being the value of the limit $\rho$.

Again, when, as in the Kinetic Theory, the matter is composed of discrete molecules, there is no difficulty in defining density if the matter is homogeneous and if also it can be supposed that there is an infinitely great 
quantity of it. In this case, we take a volume $V$ and suppose $M$ to be the mass enclosed within it. The homogeneity of the matter now ensures that as $V$ is jucreased indefinitely, the ratio $M / V$ will approsimate to a definite limit $\rho$, and, as before, we define the density of the matter to be the value of the limit $\rho$.

The gas of the Kinetic Theory will, in general, be neither continuons nor homogeneous. It will therefore be impossible to frame a general definition upon the model of the two foregoing definitions, since to do this we should have to suppose the element of rolume to become infinitely great and infinitely small at the same time. But with reference to the actual conditions of nature this objection is not serious. We can find an element of volume which may, without appreciable error, be supposed to be infinity great in comparison with the distance between neighbouring molecules, and at the same time infinitely sraall compared with the scale of variation of density of the gas. For instance, the density of a gas may generally be supposed homogeneous throughout a cube of edge equal to one millimetre, while such a cube is large compared with the scale of molecular structure, containing, as has already been mentioned, about $4 \times 10^{15}$ molecules in the case of a gas under normal conditions of pressure and temperature.

The ratio of the mass contained in an element of this kind, to the volume of the element, will give the mass-density of the gas. If we substitute "number of molecules whose centre is contained in" for "mass contained in," the definition gires the molecular-density of the gas. We shall find it convenient to denote the mass-density by $p$ and the moleculardensity by $v$. If $m$ is the mass of each molecule, we have

$$
\rho=m \nu
$$

It will be seen that this definition of density is not logically perfect, but it will be admitted that it is adequate for practical use. The difficulty of obtaining a logically perfect definition has been discussed by Burbary *. A similar difficulty is of common occurrence in statistical work: consider, for instance, the statement "the density of population in parts of London is as high as 105 per acre."

12. If $\Omega$ is a volume throughout which the density is sensibly constant, the number of molecules of which the centres are contained within this volume would, if the foregoing definitions were logically perfect, be $\Omega v$. As the definitions are not perfect, we must examine within what limits the statement is true, that the number of molecules is $\Omega v$. It is certainly not literally true, for neither $\Omega$ nor will in general be integers, while

\section{* S. H. Barturg, Kingtic Theory of Guskes, p- 3.}


the number of molecules whose oentres are contained in the rolume $\Omega$ must necessarily be integral. In the language of the theory of probability the statement may be iaken to mean that the "expectation " of the nusiber of molecules in the region in question is $\Omega v$. Any appeal to the theory of probability iroplies that a oertain amount of knowledge is given, while we remain in ignoranoe of the remaining facts. In this particular case, what is hoown is that the molecular density throughout the region $\Omega$ is $\nu$; what is not koown is the position of the indiridual molecules of the gas.

With this understandiog it will be pernissible to say that the number of molecules in an element of volume dxdyds selected et random is rdxdyds. What is meant is that the probability of finding the centre of a molecule inside this element of volume is $y d x d y d z$.

\section{The definition of the Law of Dituribution of Telocities.}

13. The difficulties of the last two sections necur when we atternpt to define the law of distribution of velocities. 'In fact at present we may cossider that a molecale possesses six coondinates-the ocordinates in space of its centre of gravity which we denote by $x, y, z$, and the corresponding velosity conopsoents, which we shall denote by $r, \boldsymbol{r}, \boldsymbol{s}$, In the last two sectiots we were rirtally discussing the law of grouping of the coondinates $x, y=x$; we now have to discuss the law of grouping of the velocities

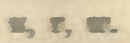

Lat es take some fixed imaginary point as origin, and draw from this point a eystem of lines to represent in magnitude and direction the relocities of the different molecules of the gas Peferred to orthogonal ares the owndinates of the extremity of any line will be $\mathrm{s}, \mathrm{E}, \mathrm{w}$, the components of relhuity of the oumespowding molecule A discussion of the law of distribution of relocities is exactly equivaleat to a discussion of the law of density of these points :

Sabject to the limitations already mentioned, we can define the density of these points in the masoer already explained. If we may denote this density by $;$, then, on our furwer understaoding, we can say that the number

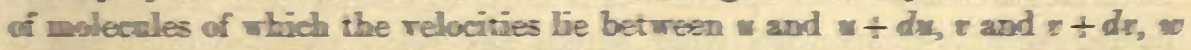

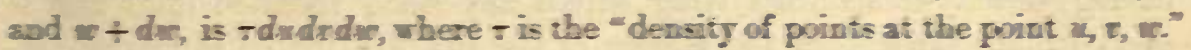
We shall find it convewient to replace - by $\mathbb{N} f$, where $\mathbb{N}$ is the total nutwbor of molecales of which the relocities bave boen represented. When it is mecessary to spocify the puint $\mathrm{n}, \mathrm{r}, \mathrm{n}$ at which $f$ is measured, we shall write $f(s, r, v)$ instead of $f$.

11 Te therefore see that the probability that the velocity of a molecule selected at randow sball have componeats lying between $a$ and $a \div d m, \varepsilon$ and $v+d r, v$ and $w+d s$ is $f(n, v, v) d u d r d s$. To avoid the continual repetition of 
these limits, let us agree to say that a molecule of which the components lie between $u$ and $u+d u, v$ and $v+d v, w$ and $w+d w$ is a molecule of class A.

The total number of molecules of class $A$ is of course

$$
N f(u, v, w) d u d v d w .
$$

In accordance with the definition of $\S 12$, we can say that the number of molecules belonging to class $\mathrm{A}$ which are found within the element of volume $d x d y d z$ selected at random is

$$
\nu f(u, v, w) d u d v d w d x d y d z
$$

Interpreted literally this statement is unintelligible for $d u d v d w d x d y d z$ is a small quantity of the sixth order; interpreted in the sense already explained, no exception can be taken either to its intelligibility or truth.

\section{The assumption of Molecular Chaos.}

15. Let us imagine that instead of the element $d x d y d z$ having been selected at random, we had supposed it to be an element in the immediate neighbourhood of a second molecule of which the components of velocity were known to lie between $u^{\prime}$ and $u^{\prime}+d u^{\prime}, v^{\prime}$ and $v^{\prime}+d v^{\prime}, w^{\prime}$ and $w^{\prime}+d w^{\prime}$, let us say a molecule of class $B$. We are no longer justified in saying that the probability of finding a molecule belonging to class $\mathrm{A}$ inside this element is given by expression (2). If all the molecules of class A were distributed at random, and then those of class B were independently distributed at random, the statement would be true enough. But if the gas is moving in accordance with the dynamical conditions of nature, it is quite conceivable that, for instance, molecules possessing nearly equal velocities tend to flock together. If this were so the probability we are discussing would be greater than that given by expression (2) when the velocities of the two molecules of classes A and B were nearly equal; in general, it would depend on $u^{\prime}, v^{\prime}, w^{\prime}$, as well as on $u, v, w$.

In the case which is discussed in the present chapter-that in which the molecules are hard elastic spheres-it is usual to assume that the molecules having velocity-components lying within any small specified limit are, at every instant throughout the motion of the gas, distributed at random, independently of the positions or velocities of the other molecules, provided only that two molecules do not occupy the same space. The legitimacy of this assumption is not self-evident. Indeed, nothing but a discussion of the dynamical equations which determine the motion of the molecules can decide whether the assumption is true or not. Such a discussion will be given in the next chapter; for the present we shall be content to make the assumption, without discussing its validity. 


\section{The changes produced by Collisions when the Molecules are Elastic Spheres.}

16. The state of a gas is fully known from the statistical point of view, when the density and the law of distribution of velocities at every point of the gas are known. The main problem of this chapter, which we now proceed to attack, is to search for a steady state: i.e., a state in which the density and law of distribution of velocities remain the same at every point of the gas throughout all time.

We begin by discussing the simplest case. Not only are the molecules supposed to be hard rigid spheres, but we suppose that the external physical conditions are the same at every point of space, and that the gas fills infinite space. The latter assumption is a temporary one, which enables us to consider separately the elements of the problem which are introduced by the presence of a containing vessel.

Under the conditions now postulated, we may clearly begin by assuming the gas to have the same molecular density $\nu$ and the same law of distribution of velocities $f$ at every point of space. Since there is nothing to distinguish the different regions in space, this uniformity in space will obviously be maintained throughout all time, but the actual form of the function $f$ will change with the time.

17. The first problem is to find an expression for the change in the number of molecules belonging to class A (defined on p. 14) which occurs during an interval of time $d t$. Since the motion of the molecules is one of uniform velocity except when collisions take place, it appears that molecules can only enter or leave class A through the occurrence of collisions. We begin by considering molecules which leave class $\mathrm{A}$ through collisions.

Let us consider a special kind of collision which we shall call a collision of class $\alpha$. This is to be defined as a collision in which the three following conditions are satisfied.

(i) One of the two colliding molecules is to be a molecule of class A.

(ii) The second colliding molecule is to be of class $B$ (defined on p. 14).

(iii) The direction of the line joining the centre of the former molecule to that of the latter at the moment of impact is to be such that a line drawn parallel to it from the centre of a fixed sphere of unit radius to the surface of this sphere meets the surface inside a small element of area $d \omega$, this element being such that the direction-cosines of a line drawn to its centre from the centre of the sphere are $l, m, n$. 
The number of molecules of class $\mathrm{A}$ is $\nu f(u, v, w) d u d v d w$ per unit volume, and each of these is capable of taking part in a collision of class $\alpha$. Let $\sigma$ be the diameter of a molecule, and imagine a sphere of radius $\sigma$ drawn round each molecule and concentric with it. As the molecule moves, the sphere is to move so as to remain concentric, but is not to rotate with the molecule. If a collision of class $\alpha$ occurs, the centre of the second molecule-that of class B-must lie on this sphere at the moment of impact, and further, since condition (iii) is to be satisfied, must lie within a small element of surface of area $\sigma^{2} d \omega$. In figure 1 , the sphere of radius $\sigma$ is drawn thick. The other spheres represent the two molecules just before and at the instant of collision.

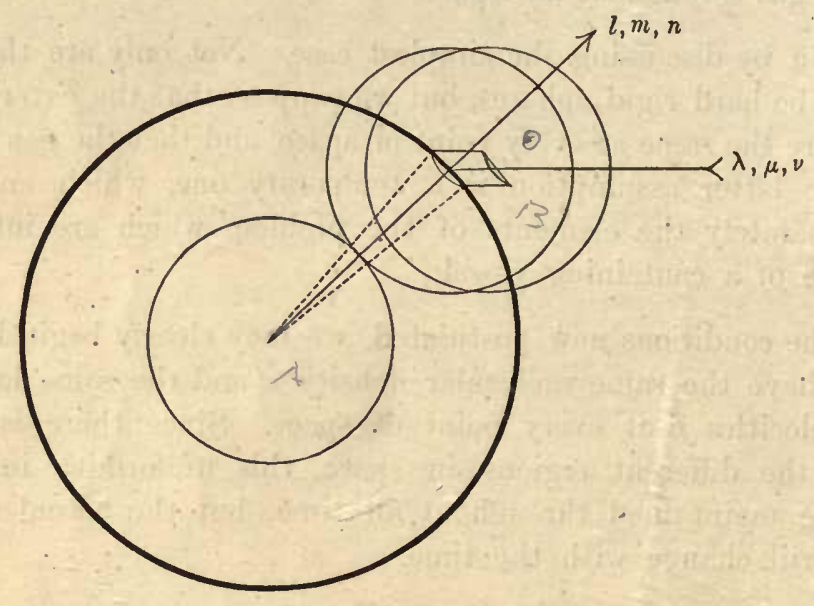

FIG. 1.

Supposing that a collision of class $\alpha$ takes place, we see that before collision the second molecule must have been moving relatively to the first with a velocity of which the components, except for infinitesimally small quantities, were $u^{\prime}-u, v^{\prime}-v, w^{\prime}-w$; let us say a velocity $V$ in a direction $\lambda, \mu, \nu$. Hence at an infinitesimally small time $\delta t$ before collision, the centre of the second molecule must bave been upon a small element of area $\sigma^{2} d \omega$ obtained by moving the element $\sigma^{2} d \omega$ from an initial position upon the surface of the sphere through a distance $V \delta t$ in a direction $-\lambda,-\mu,-\nu$. If therefore, a collision is to take place within an interval $d t$ the centre of the second molecule must, at the beginning of this interval, have been inside the cylinder which is described by moving the original element through a distance $V d t$. in this same direction.

The volume of this cylinder is equal to its base multiplied by its height. The former is $\sigma^{2} d \omega$, the latter is $V d t \cos \theta$, where $\theta$ is the angle between the axis of the cylinder and a perpendicular to the base. The direction cosines of the axis are $-\lambda,-\mu,-\nu$, those of the perpendicular to the base are of course $l, m, n$, so that

$$
\cos \theta=-(l \lambda+m \mu+n \nu)
$$


The volume of the cylinder is therefore $V \sigma^{2} \cos \theta d \omega d t$, so that for any single molecule of class $\mathrm{A}$, the probability that the centre of a molecule of class $\mathrm{B}$ shall lie within this cylinder at the beginning of the interval $d t$ is, in accordance with $§ 15$,

$$
\nu f\left(u^{\prime}, v^{\prime}, w^{\prime}\right) d u^{\prime} d v^{\prime} d w^{\prime} V \sigma^{2} \cos \theta d \omega d t .
$$

This, then, is the probability for each molecule of class A that a collision of class $\alpha$ shall occur during the interval $d t$. The number of molecules of class A is $\nu f(u, v, w) d u d v d w$ per unit volume, so that the "expectation" of the total number of collisions of class $\alpha$ which occur in time $d t$ per unit volume will be

$$
\nu^{2} f(u, v, w) f\left(u^{\prime}, v^{\prime}, w^{\prime}\right) V \sigma^{2} \cos \theta d u d v d w d u^{\prime} d v^{\prime} d w^{\prime} d \omega d t .
$$

18. We now consider a second type of collision, class $\beta$. This is to be a type of collision through which a molecule enters into class $A$, and is to be defined as a collision in which the three following conditions are satisfied:

(i) After the collision, one of the molecules is to be of class $\mathrm{A}$.

(ii) After the collision the second molecule is to be of class $\mathrm{B}$.

(iii) The direction of the line of centres at impact is to satisfy the same condition as for a collision of class $\alpha$ (p. 15).

The velocities before the collision can be found without trouble. For the relative velocity can be divided into two parts-the one in the common tangent plane through the point of contact of the spheres, and the other along the line of centres. Of these, the former remains unaltered by the collision, while the latter is reversed in direction, but remains unaltered in magnitude. Now the normal relative velocity after impact must, in virtue of the three conditions satisfied, be the same as for a collision of class a before impact. It must, therefore, be $V \cos \theta$. We shall denote $V \cos \theta$ by $W$, and therefore have, by equation (3),

$$
\begin{aligned}
V & =-V(l \lambda+m \mu+n \nu) \\
& =l\left(u-u^{\prime}\right)+m\left(v-v^{\prime}\right)+n\left(w-w^{\prime}\right) .
\end{aligned}
$$

Let $\bar{u}, \bar{v}, \bar{w}$ and $\bar{u}^{\prime}, \bar{v}^{\prime}, \bar{w}^{\prime}$ be the components of the velocities of two molecules such that after a collision along a line of centres having direction cosines $l, m, n$ the velocities are $u, v, w$ and $u^{\prime}, v^{\prime}, w^{\prime}$, then by what hust been said we must have.

$$
\begin{aligned}
& \bar{u}=u-l W=u-\left\{l^{2}\left(u-u^{\prime}\right)+\operatorname{lm}\left(v-v^{\prime}\right)+\ln \left(w-w^{\prime}\right)\right\} \\
& \bar{u}^{\prime}=u^{\prime}+l W=u^{\prime}-\left\{l^{2}\left(u^{\prime}-u\right)+\operatorname{lm}\left(v^{\prime}-v\right)+\ln \left(w^{\prime}-w\right)\right\}
\end{aligned}
$$

The number of collisions per unit volume, such that before collision the components of the velocities lie between $\bar{u}$ and $\bar{u}+d \bar{u}$, etc., and $\bar{u}^{\prime}$ and 
$\bar{u}^{\prime}+d \bar{u}^{\prime}$, etc., and such that the third condition of $p .15$ is satisfied by the line of centres at impact is, by comparison with expression (4), seen to be

$$
\nu^{2} f(\bar{u}, \bar{v}, \bar{w}) f\left(\bar{u}^{\prime}, \bar{v}^{\prime}, \bar{w}^{\prime}\right) V \sigma^{2} \cos \theta d \bar{u} d \bar{v} d \bar{w} d \bar{u}^{\prime} d \bar{v}^{\prime} d \bar{w}^{\prime} d \omega d t .
$$

19. These collisions will all belong to class $\beta$, provided that the limits determined by $d \bar{u}, d \bar{v}$, etc., are such that the values of $u, v$ given by equations (5) and (6) lie within the appropriate limits $u$ and $u+d u, v$ and $v+d v$, etc. To obtain the whole number of collisions of class $\beta$ we must integrate expression ( 7 ) over all values of $\bar{u}, \bar{v}$, etc., such that the values of $u, v$, etc., lie within these limits.

To do this we need only consider the ratio of the two products of differentials $d u d v d w d u^{\prime} d v^{\prime} d w^{\prime}$ and $d \bar{u} d \bar{v} d \bar{w} d \bar{u}^{\prime} d \bar{v}^{\prime} d \bar{w}^{\prime}$. We use Jacobi's theorem that

$$
d \bar{u} d \bar{v} d \bar{w} d \bar{u}^{\prime} d \bar{v}^{\prime} d \bar{w}^{\prime}=\Delta d u d v d w d u^{\prime} d v^{\prime} d w^{\prime}
$$

where $\Delta$ denotes the determinant

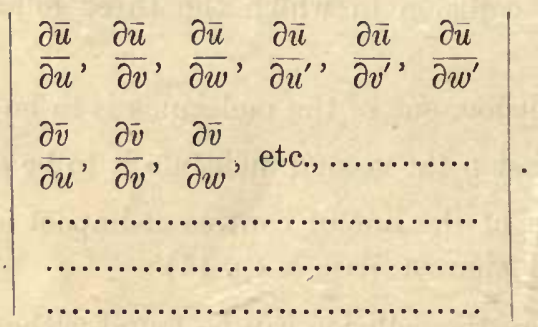

Using the values given by equations (5) and (6) we find without trouble that $\Delta=1$. This may in fact be seen without actual calculation. For since equations (5) and (6) are linear as regards the velocities, the value of the above determinant cannot depend on the velocities. Also since the relation between the velocities before and after collision is, on account of the reversibility of the motion, a reciprocal relation, it is clear from equation (8) that the only possible values for $\Delta$ are \pm 1 , of which the negative value may for obvious reasons be rejected.

Hence equation (8) becomes

$d \bar{u} d \bar{v} d \bar{w} d \bar{u}^{\prime} d \bar{v}^{\prime} d \bar{w}^{\prime}=d u d v d w d u^{\prime} d v^{\prime} d w^{\prime}$

and expression (7) may be written in the form

$$
\nu^{2} f(\bar{u}, \bar{v}, \bar{w}) f\left(\bar{u}^{\prime}, \bar{v}^{\prime}, \bar{w}^{\prime}\right) V \sigma^{2} \cos \theta d u d v d w d u^{\prime} d v^{\prime} d w^{\prime} d \omega d t \ldots . .(10) \text {. }
$$

If this number of collisions is exactly to include all of class $\beta$, the values of $d u, d v, d w, d u^{\prime}, d v^{\prime}, d w^{\prime}$ must be those which occur in the specification of a collision of class $\alpha$ (p. 15) and therefore those which occur in expression (4).

20. Suppose that expression (4) is summed over all possible classes of collisions which can occur to a molecule of class A. Or, what is the same thing, suppose that expression. (4) is integrated over all possible values of 
$u^{\prime}, v^{\prime}, w^{\prime}$ and over all elements of spherical surface $d \omega$ for which a collision is possible. Obviously the quantity obtained in this way will represent the total number of molecules of class $\mathrm{A}$ which enter into collision in the interval $d t^{*}$. So also expression (10) integrated through the same range of values gives the total number of molecules of class A which emerge from collision during the same interval.

The net gain to class $\mathbf{A}$ in the interval $d t$ is therefore the difference of these two integrals, and this is

$$
\nu^{2} d u d v d w d t \iiint \iint\left(\bar{f} \bar{f}^{\prime}-f f^{\prime}\right) V \sigma^{2} \cos \theta d u^{\prime} d v^{\prime} d w^{\prime} d \omega
$$

in which $f, f^{\prime}, \bar{f}, \bar{f}^{\prime}$ are written for $f(u, v, w), f\left(u^{\prime}, v^{\prime}, w^{\prime}\right), f(\bar{u}, \bar{v}, \bar{w})$ and $f\left(\bar{u}^{\prime}, \bar{v}^{\prime}, \bar{w}^{\prime}\right)$ respectively.

21. The number of molecules which belong to class $\mathrm{A}$ at the beginning of the interval $d t$ is known to have been $\nu f d u d v d w$ per unit volume, whilst the number at the end of this interval may be supposed to be

$$
\nu\left(f+\frac{\partial f}{\partial t} d t\right) d u d v d w
$$

The gain to class $\mathrm{A}$ is therefore

$$
\nu \frac{\partial f}{\partial t} d u d v d w d t
$$

Equating this quantity to that given by expression (11), we obtain the equation

$$
\frac{\partial f}{\partial t}=\nu \iiint \iint\left(\bar{f} \bar{f}^{\prime}-f f^{\prime}\right) V \sigma^{2} \cos \theta d u^{\prime} d v^{\prime} d w^{\prime} d \omega
$$

The condition for a steady state is that $\partial f / \partial t$ shall vanish for all values of $u, v$ and $w$. No progress can however be made by equating the right-hand side of equation (12) to zero: the problem of determining the steady state has to be attacked in a different manner.

\section{The H-theorem.}

22. Consider the quantity $H$ defined by

$$
H=\iiint f \log f d u d v d w
$$

in which the integration is to extend over all possible values of $u, v, w$, so that $H$ is a pure quantity and not a function of $u, v, w$. This quantity depends solely upon the law of distribution of velocities and therefore remains un-

* Not the total number of collisions in which a molecule initially of class A is involved, since collisions for which both molecules are of class A are counted twice. 
changed so long as this law remains unchanged. Hence a necessary condition for a steady state is given by $d H / d t=0$. We proceed to evaluate $d H / d t$ in the general case.

After an interval $d t$ the value of $f \log f$ corresponding to any specified values of $u, v, w$ will of course have changed into

$$
f \log f+\frac{\partial}{\partial t}(f \log f) d t
$$

or, what is the same thing, into

$$
f \log f+(1+\log f) \frac{\partial f}{\partial t} d t
$$

Hence the increase in $H$, which may be written $\frac{d H}{d t} d t$, will be given by

$$
\frac{d H}{d t} d t=\left\{\iiint(1+\log f) \frac{\partial f}{\partial t} d u d v d w\right\} d t
$$

and if we substitute the value of $\partial f / \partial t$ from equation (12), this becomes $\frac{d H}{d t}=\nu \iiint \iiint \iint(1+\log f)\left(\bar{f} \bar{f}^{\prime}-f f^{\prime}\right) V \sigma^{2} \cos \theta d u d v d w d u^{\prime} d v^{\prime} d w^{\prime} d \omega \ldots$

23. Equation (13) regards $H$ as the sum of a number of contributions, one from each class of molecule, and in this equation class $\mathrm{A}$ is taken as the typical class. If we had chosen class B as the typical class, we might have written $H$ in the form

$$
H=\iiint f^{\prime} \log f^{\prime} d u^{\prime} d v^{\prime} d w^{\prime}
$$

and the increase in $H$, instead of being given by equation (14), would then have been given by

$$
\frac{d H}{d t}=\iiint\left(1+\log f^{\prime}\right) \frac{\partial f^{\prime}}{\partial t} d u^{\prime} d v^{\prime} d w^{\prime}
$$

To evaluate the right-hand member of this equation we need to know the value of $\partial f^{\prime} / \partial t$. Now equation (12) regards the change in $f$ as the sum of a number of contributions, one from every class of collision in which either of the molecules either before or after impact is of class $\mathrm{A}$, and the typical classes of collision are taken to be classes $\alpha$ and $\beta$. In a similar way we can express $\partial f^{\prime} \partial t$ as the sum of a number of contributions, one from every class of collision in which either of the molecules either before or after impact is of class B. The typical classes of collision may again be taken to be classes $\alpha$ and $\beta$, and if this is done we obtain for $\partial f^{\prime} / \partial t$ an expression similar to that given for $\partial f / \partial t$ in equation (12), except that accented symbols replace unaccented, and vice-versâ. In fact molecules of classes A and B exchange rôles. 
If we now substitute this value for $\partial f^{\prime} / \partial t$ in equation (17) we obtain (cf. equation 15)

$$
\frac{d H}{d t}=\nu \iiint \iiint \int\left(1+\log f^{\prime}\right)\left(\bar{f} \bar{f}^{\prime}-f f^{\prime}\right) V \sigma^{2} \cos \theta d u d v d w d u^{\prime} d v^{\prime} d w^{\prime} d \omega,
$$

an equation which is of course the same as (15) except that accented and unaccented symbols are interchanged. If we add together the two values for $d H / d t$ which have been obtained, we have

$\frac{d H}{d t}=\frac{1}{2} \nu \iiint \iiint \iint\left(2+\log f f^{\prime}\right)\left(\bar{f} \bar{f}^{\prime}-f f^{\prime}\right) V \sigma^{2} \cos \theta d u d v d w d u^{\prime} d v^{\prime} d w^{\prime} d \omega \ldots$

This equation expresses $d H / d t$ as the sum of a number of contributions, one from every possible class of collision. The typical class is taken to be class $\alpha$, in which

become changed into

$$
u, v, w, u^{\prime}, v^{\prime}, w^{\prime}
$$$$
\bar{u}, \bar{v}, \bar{w}, \bar{u}^{\prime}, \bar{v}^{\prime}, \bar{w}^{\prime} .
$$

If we use the same equation, but take as the typical collision one of class $\beta$, in which

become changed into

$$
\bar{u}, \bar{v}, \bar{w}, \bar{u}^{\prime}, \bar{v}^{\prime}, \bar{w}^{\prime},
$$

$$
u, v, w, u^{\prime}, v^{\prime}, w^{\prime}
$$

we obtain, as a still different form for $d H / d t$,

$\frac{d H}{d t}=\frac{1}{2} \nu \iiint \iiint \iint\left(2+\log \bar{f} \bar{f}^{\prime}\right)\left(f f^{\prime}-\bar{f} \bar{f}^{\prime}\right) \nabla \sigma^{2} \cos \theta d \bar{u} d \bar{v} d \bar{w} d \bar{u}^{\prime} d \bar{v}^{\prime} d \bar{w}^{\prime} d \omega \ldots$

Equation (9) enables us to replace the product of the first six differentials on the right-hand of this equation by $d u d v d w d u^{\prime} d v^{\prime} d w^{\prime}$ and if we add this modified value of $d H / d t$ to that given by equation (18) we obtain $\frac{d H}{d t}=\frac{1}{4} \nu \iiint \iiint \iint\left(\log f f^{\prime}-\log \bar{f} \bar{f}^{\prime}\right)\left(\bar{f} \bar{f}^{\prime}-f f^{\prime}\right) V \sigma^{2} \cos \theta d u d v d w d u^{\prime} d v^{\prime} d w^{\prime} d \omega$ (20).

Now $\left(\log f f^{\prime}-\log \bar{f} \bar{f}^{\prime}\right)$ is positive or negative according as $f f^{\prime}$ is greater or is less than $\bar{f} \bar{f}^{\prime}$ and is therefore always of the sign opposite to that of $\bar{f} \bar{f}^{\prime}-f f$. Hence the product

$$
\left(\log f f^{\prime}-\log \bar{f} \bar{f}^{\prime}\right)\left(\bar{f} \bar{f}^{\prime}-f f^{\prime}\right),
$$

if not zero, is necessarily negative. Since $V \cos \theta$, the relative velocity along the line of centres before impact, is necessarily positive for every type of collision, it follows that the integrand of equation (20) is always either negative or zero. Hence equation (20) shews that $d H / d t$ is either negative or zero. 


\section{The Solution for a Steady State.}

24. In order that the gas may be in a steady state, it is necessary, as has been already said, that $d H / d t$ shall be zero. Now equation (20), as we have seen, expresses $d H / d t$ as the sum of a number of contributions, one from every type of collision, and each contribution is either negative or zero. Hence for $d H / d t$ to be zero, each contribution separately must be zero. In other words we must have

for every type of collision.

$$
f f^{\prime}=\bar{f} \bar{f}^{\prime}
$$

'This condition has been seen to be necessary for a steady state. Equation (12) shews that it is also sufficient, for if it is satisfied then $\partial f / \partial t=0$ for every value of $u, v$ and $w$. The problem of determining the steady state is therefore reduced to the problem of obtaining the solution of equation (21). We shall find it convenient to take logarithms of both sides, and write the equation in the form

$$
\log f+\log f^{\prime}=\log \bar{f}+\log \bar{f}^{\prime}
$$

25. Let $\chi$ be a function of the velocities $u, v, w$, such that when two molecules collide, the sum of the $\chi$ 's appropriate to the two molecules before impact is equal to the sum of the two $\chi$ 's after impact. Since $\chi$ is, by hypothesis, a function only of $u, v, w$, the value of $\chi$ will remain the same for eyery molecule except when it is altered by collision. We may therefore say that $\chi$ is defined as being capable of exchange between molecules at a collision, but is indestructible; $\Sigma \chi$ remains the same throughout the motion, where $\Sigma$ denotes summation which extends over all the molecules of the gas.

It is clear that a particular solution of equation (22) is

$$
\log f=\chi
$$

Further it will be seen that the difference between $\chi$ and the most general solution of (22) is such as to satisfy the conditions postulated for $\chi$. Let $\chi_{1}, \chi_{2}, \chi_{3} \ldots$ be independent quantities, each satisfying these conditions, and let it be supposed that there are no other such quantities, then the most general solution of (22) must be

$$
\log f=\alpha_{1} \chi_{1}+\alpha_{2} \chi_{2}+\alpha_{3} \chi_{3}+\ldots
$$

in which $\alpha_{1}, \alpha_{2}, \alpha_{3} \ldots$ are independent and, so far, arbitrary constants.

From the dynamics of a collision we know that there are four quantities which satisfy the condition in question: namely, the energy and the three components of linear momentum. These give four forms for $\chi$ : a fifth is obtained by taking $\chi$ equal to a constant, and it is obvious that there can be no others. For if there were any additional form possible for $\chi$, there would be five equations giving $\bar{u}, \bar{v}, \bar{w}, \bar{u}^{\prime}, \bar{v}^{\prime}, \bar{w}^{\prime}$ in terms of $u, v, w, u^{\prime}, v^{\prime}, w^{\prime}$, so that $\bar{u}, \bar{v}, \bar{w}, \bar{u}^{\prime}, \bar{v}^{\prime}, \bar{w}^{\prime}$ would be determined except for one unknown. There must however be two unknowns, as the direction of the line of centres is unknown. 
The general solution of equation (22) is therefore seen to be

$$
\log f=\alpha_{1}^{-1} m\left(u^{2}+v^{2}+w^{2}\right)+\alpha_{2} m u+\alpha_{3} m v+\alpha_{4} m w+\alpha_{5}
$$

The constants $\alpha_{2}, \alpha_{3}, \alpha_{4}, \alpha_{5}$ may be replaced by new ones and the solution written in the form

$$
\log f=\alpha_{1} m\left[\left(u-u_{0}\right)^{2}+\left(v-v_{0}\right)^{2}+\left(w-w_{0}\right)^{2}\right]+\alpha_{6},
$$

or, if we still further change the constants,

$$
f=A e^{-h m\left[\left(u-u_{0}\right)^{2}+\left(v-v_{0}\right)^{2}+\left(w-w_{0}\right)^{2}\right]} .
$$

in which $A, h, u_{0} ; v_{0}, w_{0}$ are new arbitrary constants.

26. By giving different values to these five constants we obtain all the steady states which are possible for a gas. The different values of the constants depend upon the different values of $\Sigma \chi_{1}, \Sigma \chi_{2}, \Sigma \chi_{3}, \Sigma \chi_{4}, \Sigma \chi_{5}$, i.e., upon the total energy, momentum and mass of the gas. We proceed to determine the relations between these constants and the corresponding physical quantities.

The value per unit volume of any quantity $\chi$ summed over all the molecules is given by

$$
\Sigma \chi=\nu \iiint \chi A e^{-h m\left[\left(u-u_{0}\right)^{2}+\left(v-v_{0}\right)^{2}+\left(w-w_{0}\right)^{2}\right]} d u d v d w
$$

If we write

$$
\begin{aligned}
& u-u_{0}=\mathrm{U}, \\
& v-v_{0}=\mathrm{V}, \\
& w-w_{0}=\mathrm{W},
\end{aligned}
$$

this becomes transformed into

$$
\Sigma \chi=\nu \iiint \chi A e^{-h m\left(\mathbf{u}^{2}+\mathrm{v}^{2}+\mathrm{w}^{2}\right)} d \mathbf{u} d \mathbf{v} d \mathrm{w}
$$

and if we further transform variables according to the scheme

the equation becomes

$$
\left.\begin{array}{l}
\mathrm{U}=c \sin \theta \cos \phi \\
\mathrm{V}=c \sin \theta \sin \phi \\
\mathrm{W}=c \cos \theta
\end{array}\right\}
$$

$$
\Sigma \chi=\nu \iiint \chi A e^{-h m c^{2}} c^{2} \sin \theta d \theta d \phi d c
$$

If we take $\chi=1, \Sigma \chi$ is the number of molecules per unit volume, and is therefore equal to $\nu$. Equation (29) accordingly becomes

$$
1=4 \pi A \int_{0}^{\infty} e^{-h m c^{2} c^{2}} d c
$$

and since the value of the integral is known to be $\frac{1}{4} \sqrt{\frac{\pi}{h^{3} m^{3}}}$ this gives the relation

$$
A=\sqrt{\frac{h^{3} m^{3}}{\pi^{3}}}
$$


Next put $\chi=u$ in equation (27). We obtain

$$
\Sigma \mathrm{U}=\nu A \int_{-\infty}^{+\infty} e^{-h m \mathrm{u}^{2} \mathrm{u}} d \mathbf{u} \int_{-\infty}^{+\infty} e^{-h m \mathrm{v}^{2}} d \mathrm{v} \int_{-\infty}^{+\infty} e^{-h m \mathrm{w}^{2}} d \mathrm{~W},
$$

and the right-hand vanishes, since the value of the first integral is zero. Hence $\Sigma U=0$, or, what is the same thing,

$$
\Sigma u=\Sigma u_{0}=\nu u_{0} .
$$

Thus $u_{0}$ is the mean value of $u$, and is therefore the $x$-component of the meanvelocity of the gas. Similarly $v_{0}, w_{0}$ are the $y$ and $z$ components of this velocity.

Lastly, let us put $\chi=u^{2}+v^{2}+w^{2}$. If we substitute this value in equation (29) we obtain

$$
\Sigma\left(\mathrm{U}^{2}+\mathrm{V}^{2}+\mathrm{W}^{2}\right)=4 \pi \nu A \int_{0}^{\infty} e^{-h m c^{2}} c^{4} d c .
$$

The value of the integral on the right-hand is known to be $\frac{3}{8} \sqrt{\frac{\pi}{h^{5} m^{5}}}$, and this leads to

$$
\Sigma\left(U^{2}+v^{2}+W^{2}\right)=\frac{3}{2} A \sqrt{\frac{\pi^{3}}{h^{5} m^{5}} \nu,}
$$

or, if we substitute the value of $A$ from equation (30),

$$
\Sigma\left(u^{2}+v^{2}+w^{2}\right)=\frac{3}{2 h m} \nu
$$

The kinetic energy per unit volume of the gas is $\Sigma \frac{1}{2} m\left(u^{2}+v^{2}+w^{2}\right)$, and since $\Sigma u=\Sigma v=\Sigma w=0$, we have

$$
\begin{aligned}
& \Sigma \frac{1}{2} m\left(u^{2}+v^{2}+w^{2}\right)=\frac{1}{2} m \Sigma\left(\left(\mathrm{U}+u_{0}\right)^{2}+\left(\mathrm{V}+v_{0}\right)^{2}+\left(\mathrm{W}+w_{0}\right)^{2}\right) \\
& =\frac{1}{2} m \Sigma\left(\mathrm{U}^{2}+\mathrm{V}^{2}+\mathrm{W}^{2}+u_{0}{ }^{2}+v_{0}{ }^{2}+w_{0}{ }^{2}\right) \\
& =\frac{1}{2} m \nu\left(\frac{3}{2 h m}+u_{0}^{2}+v_{0}^{2}+w_{0}^{2}\right) \\
& =\frac{3}{4 h} \nu+\frac{1}{2} \rho\left(u_{0}{ }^{2}+v_{0}{ }^{2}+w_{0}{ }^{2}\right) \ldots . .
\end{aligned}
$$

where $\rho$ is the mass density of the gas, given by equation (1).

We have now determined five relations between the unknown constants and the density, kinetic energy and momenta of the gas. It appears that for given density, kinetic energy and momenta the values of the constants are unique, $h$ being determined by equation (32), $u_{0}, v_{0}, w_{0}$ by the momenta, and $A$ by equation (30). Hence there is only one steady state possible for given values of the density, energy and momenta. 


\section{Gas in a closed vessel.}

27. This completes the determination of the steady states of an infinite mass of gas. We have next to consider the modifications introduced when the gas is confined in a closed vessel. Supposing the walls of this vessel to be absolutely rigid and elastic, we shall shew that the law of distribution already found in equation (25), namely

$$
f=A e^{-h m\left[\left(u-u_{0}\right)^{2}+\left(v-v_{0}\right)^{2}+\left(w-w_{0}\right)^{2}\right]} .
$$

will still represent a steady state, independently of the shape of the containing vessel, provided that this vessel is moving with a velocity $u_{0}, v_{0}, w_{0}$.

To prove this we consider the collisions of molecules with a single small element of the wall of the containing vessel. Let this element be of area $d \sigma$ and let the direction-cosines of a line drawn perpendicular to it be $l, m, n$. Consider the class of collisions such that the components of velocity of the colliding molecule before impact lie between

$$
u \text { and } u+d u, v \text { and } v+d v, w \text { and } w+d w
$$

As before, let us call all such molecules, molecules of class A. Let us, as on a former occasion ( $\$ 13)$, take a fixed point $O$ as origin and represent the velocities of the different molecules in magnitude and direction, by a system of lines drawn from this point. All the molecules of class A will be represented by lines having their representative points inside a certain small rectangular parallelepiped - the rectangular parallelepiped of which the orthogonal coordinates lie within the limits (34).

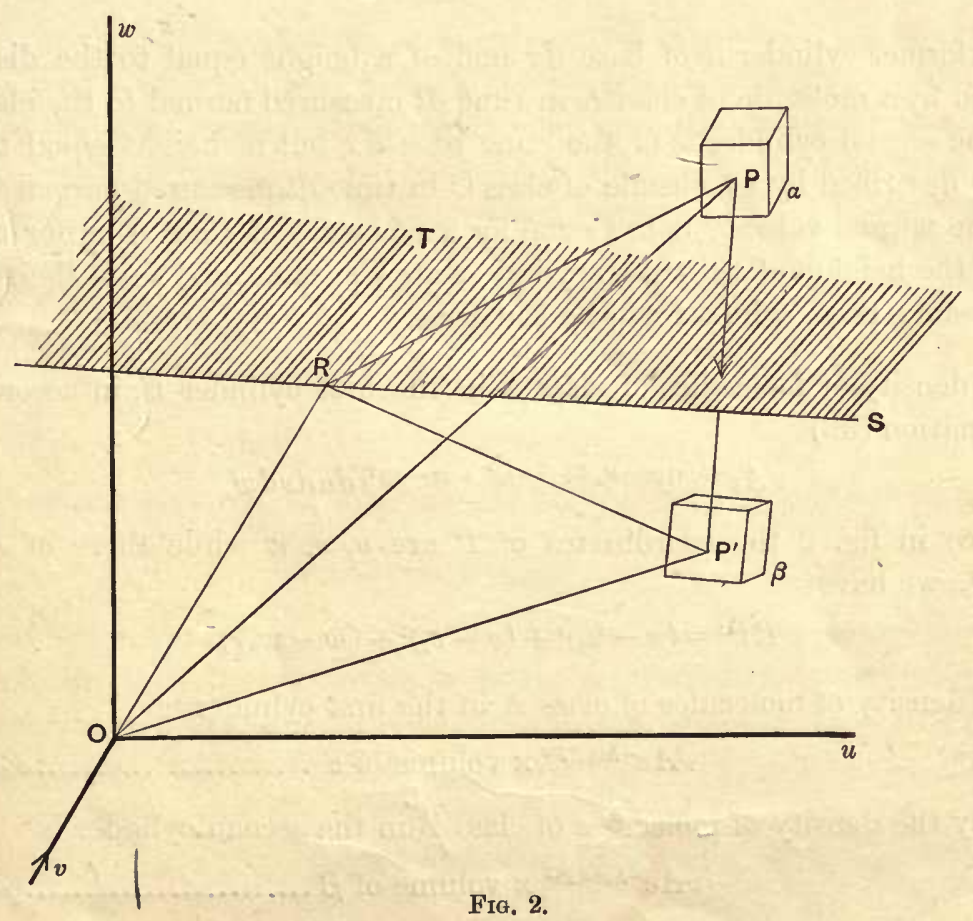


Let $P$ be any one of these points, so that $O P$ represents the velocity of the corresponding molecule. Let $O R$ represent the velocity $u_{0}, v_{0}, w_{0}$ of the vessel, then $R P$ will represent the velocity of the molecule relatively to the vessel. After collision with the element $d \sigma$, the normal component of this velocity will be reversed, while the tangential component will persist unaltered. Hence if $T R S$ is a plane through $R$ parallel to the element $d \sigma$, the relative velocity after impact is $R P^{\prime}$ where $R P^{\prime}$ is the image of $P$ in the plane $T R S$.

The small parallelepiped in which $P$ must lie if the corresponding molecule is to belong to class $\mathrm{A}$ will have as its image in the plane TRS a second parallelepiped which is obviously of the same volume as the former. Let us denote the two parallelepipeds by $\alpha, \beta$, and when the velocity of a molecule is such that the line representing it has its end within $\beta$, let us say that the molecule is of class C. Then we have seen that a molecule of class A is changed by collision into a molecule of class $\mathrm{C}$, and from symmetry it is obvious that the converse is true.

The number of molecules of class $\mathrm{A}$ which collide with the element $d \sigma$ in time $d t$ is equal to the number of molecules of class A which lie within a certain cylinder at the beginning of the interval $d t$. Similarly the number of molecules of class $\mathrm{C}$ which collide with $d \sigma$ in the same time $d t$ is equal to the number of molecules of class $\mathrm{C}$ which lie within a second cylinder at the same instant.

The former cylinder is of base $d \sigma$ and of a height equal to the distance described by a molecule of class $\mathrm{A}$ in time $d t$ measured normal to the element $d \sigma$. The second cylinder is of the same base $d \sigma$, but of height equal to the distance described by a molecule of class $\mathrm{C}$ in time $d t$ measured normal to $d \sigma$. Since the normal velocity is the same for a molecule of class $A$ as for one of class $\mathrm{C}$, the heights of these two cylinders are the same, and since their bases are the same, their volumes will be the same.

The density of molecules of class $A$ in the first cylinder is, in accordance with equation (33)

$$
\nu A e^{-h m\left[\left(u-u_{0}\right)^{2}+\left(v-v_{0}\right)^{2}+\left(w-w_{0}\right)^{2}\right]} d u d v d w,
$$

and since in fig. 2 the coordinates of $P$ are $u, v, w$ while those of $R$ are $u_{0}, v_{0}, w_{0}$, we have

$$
R P^{2}=\left(u-u_{0}\right)^{2}+\left(v-v_{0}\right)^{2}+\left(w-w_{0}\right)^{2}
$$

and the density of molecules of class $\mathbf{A}$ in the first cylinder is

$$
\nu A e^{-h m R P^{2}} \times \text { volume of } \alpha
$$

Similarly the density of molecules of class $\not B^{C}$ in the second cylinder is

$$
\nu A e^{-h m R P^{\prime 2}} \times \text { volume of } \beta
$$


Now $R P=R P^{\prime}$, and the volume of $\alpha$ has been seen to be equal to the volume of $\beta$. Hence the two densities (35) and (36) are equal. Since the two cylinders have also been shewn to be equal it follows that the number of molecules of class $\mathrm{A}$ which collide with the element $d \sigma$ in the interval $d t$ is equal to the number of molecules of class $\mathrm{C}$ which do the same thing. Each of the former molecules is changed by collision from a molecule of class A to one of class $\mathrm{C}$, and each of the latter from a molecule of class $\mathrm{C}$ to one of class A. Hence the number of molecules of class A remains unaltered by collisions with the element $d \sigma$. The same is of course true of every other class of molecule, and of every other element of the surface of the containing vessel, whence we see that the whole law of distribution is unaltered by the presence of the walls, or, in other words, that the law of distribution (33) represents a steady state.

It now appears, however, that there are only the two constants $A$ and $h$ at our disposal in the case of a gas enclosed in a vessel which is either at rest or moving with a known velocity $u_{0}, v_{0}, w_{0}$, and these two constants are of course connected by the relation (30). By varying these constants we are enabled to assign to our gas different values of the total energy, or, speaking physically, different temperatures. Similarly by varying $\nu$ we are enabled to assign different densities to the gas.

\section{Mass Motion and Molecular Motion.}

28. We have seen that the most general "steady state" possible consists of a motion compounded of a mass-motion and a molecular-motion. The mass-motion has velocity components $u_{0}, v_{0}, w_{0}$, the molecular-motion has velocity components $u-u_{0}, v-v_{0}, w-w_{0}$, which we have denoted (p. 23) by $u, v, w$. The number of molecules having molecular velocities lying between $u$ and $u+d u, v$ and $v+d v, w$ and $w+d w$ is the number of molecules having actual resultant velocities lying between $u$ and $u+d u$ etc., ete., and this by equation (25)

$$
\begin{aligned}
& =A e^{-h m\left[\left(u-u_{0}\right)^{2}+\left(v-v_{0}\right)^{2}+\left(w-w_{0}\right)^{2}\right]} d u d v d \mathrm{w} \\
& =A e^{-h m\left(\mathrm{U}^{2}+\mathrm{v}^{2}+\mathrm{w}^{2}\right)} d \mathrm{u} d \mathrm{v} d \mathrm{w} .
\end{aligned}
$$

Hence we may suppose the molecular velocities distributed according to the law

$$
A e^{-h m\left(\mathbf{u}^{2}+\mathrm{v}^{2}+\mathrm{w}^{2}\right)} d \mathbf{u} d \mathbf{v} d \mathbf{w}
$$

If we adopt the scheme of transformation (28) we may replace the velocity of which the components are $\mathrm{u}, \mathrm{v}, \mathrm{w}$ by a velocity of magnitude $c$, in a direction which makes an angle $\theta$ with the axis of $z$, and such that a plane through this direction and the axis of $z$ makes an angle $\phi$ with the axis of $x$. The law of distribution (37) now becomes

$$
A e^{-h m c^{2}} c^{2} \sin \theta d \theta d \phi d c
$$


This shews that the velocities of molecular motion are distributed equally in all directions in space. The law of distribution of the magnitudes of these velocities independently of their directions in space is found, upon integration of expression (38) with respect to $\theta$ and $\phi$, to be

$$
4 \pi A e^{-h m c^{2}} c^{2} d c
$$

The law indicated by expression (37) can also be written in the form

$$
A\left[e^{-h m \mathrm{u}^{2}} d \mathrm{u}\right]\left[e^{-h m \mathrm{v}^{2}} d \mathrm{v}\right]\left[e^{-h m \mathrm{w}^{2}} d \mathrm{w}\right]
$$

shewing that the distributions of $u, v, w$ are independent.

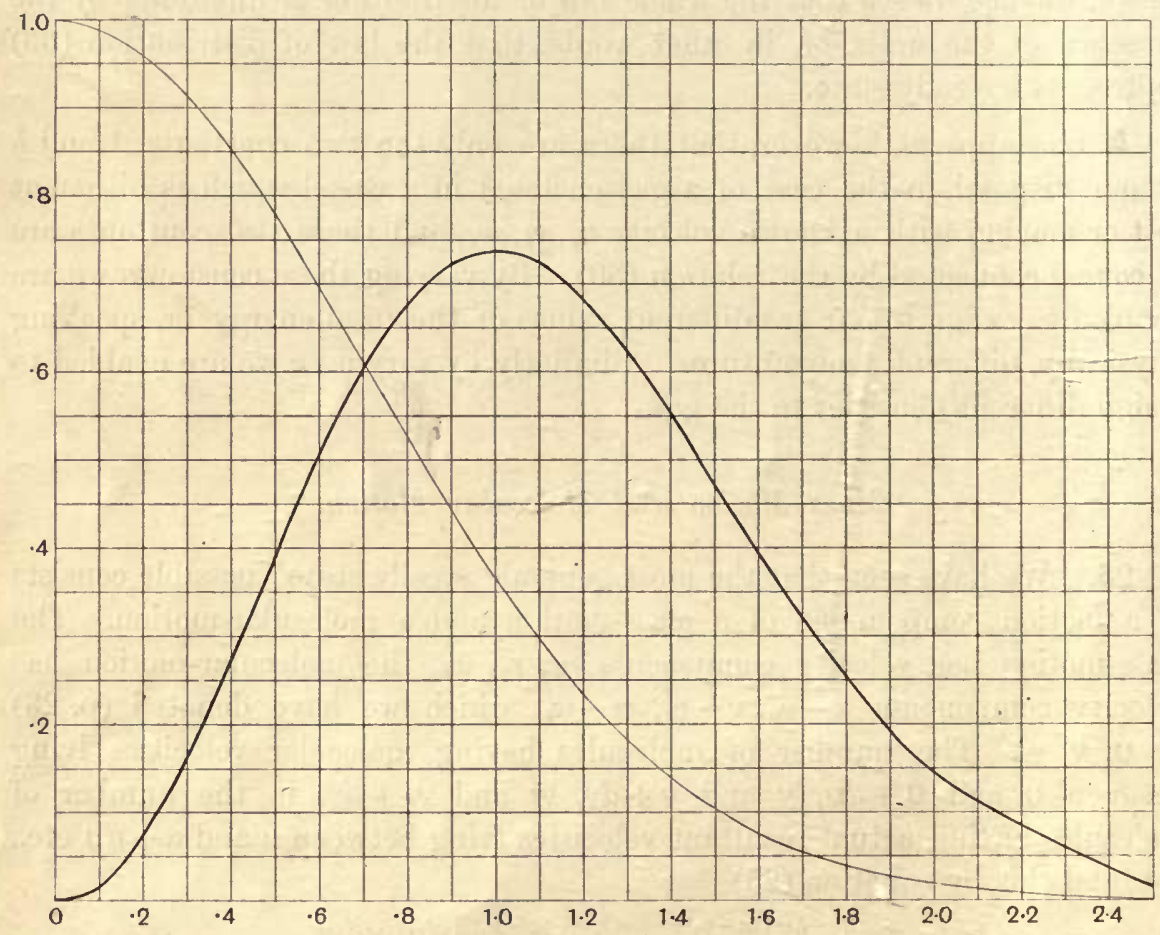

FIa. 3.

In fig. 3 , the thick line is the curve

$$
y=2 x^{2} e^{-x^{2}}
$$

while the thin line is the curve

$$
y=e^{-x^{2}}
$$

The factor 2 is introduced in the former equation in order that the two curves may have the same area, namely $\frac{1}{2} \sqrt{ } \pi$. The former curve shews the grouping of the magnitudes of the velocities independently of their directions in space, the latter that of the magnitudes of a single component. 
29. Equation (32) expresses that the total energy of a gas may be regarded as the sum of the energies of a mass-motion and a molecular motion. In the language of the older physics, one would say that the total energy was partly kinetic and partly thermal. In the language of the Kinetic Theory, both energies are equally kinetic.

Let us suppose that the containing vessel, which has so far been moving with a velocity of which the components are $u_{0}, v_{0}, w_{0}$, is suddenly brought to a standstill. This will of course destroy the steady state of the gas, but after a sufficient time, the gas will assume a new and different steady state. The mass-velocity of this steady state will obviously be nil, and the energy wholly molecular. The individual molecules have not been acted upon by any external forces except in their impacts with the containing vessel, and these leave their energy unchanged. The new molecular energy is therefore equal to the former total energy. These data enable us to determine the new steady state. In the language of the older physics, one would say that by suddenly stopping the forward motion of the gas the kinetic energy of this motion had been transformed into heat. In the language of the Kinetic Theory, we say that the total kinetic energy has been redistributed, so as now to be wholly molecular.

An interesting region of thought, although one outside the domain of pure Kinetic Theory, is opened up by the consideration of the processes by which this new steady state is arrived at. To examine the simplest case, let us suppose the gas to be contained in a cubical box, and to have been moving originally in a direction perpendicular to one of the sides. The hydrodynamical theory of sound is capable of tracing the motion of the gas throughout all time, subject of course to the assumptions on which the theory is based. The solution obtained to the problem from the hydrodynamical standpoint is that the original motion of the gas is perpetuated in the form of plane waves of sound in the gas, the wave fronts all being perpendicular to the original direction of motion. This solution is obviously very different from that arrived at by the Kinetic Theory. For instance, the solution of hydrodynamics indicates that the original direction of motion remains differentiated from other directions in space through all time, whereas the solution of the Kinetic Theory indicates that a state is soon attained in which there is no differentiation between directions in space.

The explanation of the divergence of the two solutions is naturally to be looked for in the differences of the assumptions made. The conception of the perfect non-viscous fluid postulated by hydrodynamics is an abstract ideal which is logically inconsistent with the molecular constitution of matter postulated by the Kinetic Theory. Indeed we shall in a later part of the book be able to shew that the actual viscosity of gases is simply and fully accounted for by their molecular structure. If we introduce viscosity terms 
into the hydrodynamical equations, these equations will lead to a solution in which the ultimate state is one in which there is no mass motion in the gas. On the hydrodynamical view, the energy of the original motion has been "dissipated" by viscosity. On the Kinetic Theory view, this energy has been converted into molecular motion. In fact the Kinetic Theory enables us to trace as molecular motion, energy which other theories are content to regard as lost.

\section{Number of Collisions, Mean Free Path, etc.}

30. We shall now use the results which have been obtained, to calculate the total number of collisions per unit volume of gas. Since the number of collisions is not affected by the mass-motion of the gas, we shall take this mass-motion to be zero.

In expression (4) we found the number of collisions of class a occurring per unit time to be

$$
\nu^{2} f(u, v, w) f\left(u^{\prime}, v^{\prime}, w^{\prime}\right) V \sigma^{2} \cos \theta d u d v d w d u^{\prime} d v^{\prime} d w^{\prime} d \omega
$$

and the problem of determining the total number of collisions amounts to integrating this expression over all values of the variables when $f(u, v, w)$ has the form appropriate to the steady state, i.e., when

$$
f(u, v, w)=\sqrt{\frac{h^{3} m^{3}}{\pi^{3}}} e^{-h m c^{2}}
$$

In expression (41), $V$ is the relative velocity and $\theta$ is the angle between this velocity and that of the line of centres. If $\phi$ is the azimuth of the line of centres referred to any definite plane through the direction of the relative velocity, we may, in expression (41), replace $d \omega$ by $\sin \theta d \theta d \phi$. Since collisions can occur for all values of $\phi$ and for all values of $\theta$ from 0 to $\pi / 2$, we must integrate expression (41) from $\phi=0$ to $\phi=2 \pi$ and from $\theta=0$ to $\theta=\pi / 2$. Performing the integrations, and substituting for $f(u, v, w)$ from equation (42), we obtain

$$
\pi \nu^{2}\left(\frac{h^{3} m^{3}}{\pi^{3}} e^{-h m\left(c^{2}+c^{\prime 2}\right)}\right) V \sigma^{2} d u d v d w d u^{\prime} d v^{\prime} d w^{\prime}
$$

as the total number of collisions in which the molecules before collision have velocities lying within the usual limits $d u d v d w d u^{\prime} d v^{\prime} d w^{\prime}$.

Let us now suppose that the variables are transformed to new variables, given by

$$
\mathbf{u}=\frac{1}{2}\left(u+u^{\prime}\right), \text { etc., } \alpha=u^{\prime}-u \text {, etc., }
$$

so that $\mathbf{u}, \mathbf{v}, \mathbf{w}$ are the components of the velocity of the centre of gravity of the two molecules, and $\alpha, \beta, \gamma$ are the components of the velocity of the second molecule relatively to the first. We have

$$
\frac{\partial(\mathbf{u}, \alpha)}{\partial\left(u, u^{\prime}\right)}=\left|\begin{array}{cc}
\frac{1}{2}, & \frac{1}{2} \\
-1, & 1
\end{array}\right|=1
$$


Hence from expression (43) the number of collisions for which the new variables lie within a range $d \mathbf{u} d \mathbf{v} d \mathbf{w} d \alpha d \beta d \gamma$ is

$$
\pi \nu^{2}\left(\frac{h^{3} m^{3}}{\pi^{3}} e^{-h m\left(c^{2}+c^{\prime 2}\right)}\right) V \sigma^{2} d \mathbf{u} d \mathbf{v} d \mathbf{w} d \alpha d \beta d \gamma
$$

in which we have

$$
\begin{gathered}
V^{2}=\left(u^{\prime}-u\right)^{2}+\left(v^{\prime}-v\right)^{2}+\left(w^{\prime}-w\right)^{2}=\alpha^{2}+\beta^{2}+\gamma^{2}, \\
c^{2}+c^{\prime 2}=u^{2}+u^{\prime 2}+v^{2}+v^{\prime 2}+w^{2}+w^{\prime 2}=2\left(\mathbf{u}^{2}+\mathbf{v}^{2}+\mathbf{w}^{2}\right)+\frac{1}{2}\left(\alpha^{2}+\beta^{2}+\gamma^{2}\right),
\end{gathered}
$$

or, if we write $\mathbf{u}^{2}+\mathbf{v}^{2}+\mathbf{w}^{2}=\mathbf{c}^{2}$,

$$
c^{2}+c^{\prime 2}=2 c^{2}+\frac{1}{2} V^{2}
$$

Let us again transform variables according to the schemes

$$
\begin{array}{ll}
\mathbf{u}=\mathbf{c} \sin \theta \cos \phi, & \alpha=V \sin \psi \cos \chi, \\
\mathbf{v}=\mathbf{c} \sin \theta \sin \phi, & \beta=V \sin \psi \sin \dot{\chi}, \\
\mathbf{w}=\mathbf{c} \cos \theta, & \gamma=V \cos \psi .
\end{array}
$$

In order that $\mathbf{u}, \mathbf{v}, \mathbf{w}$ may have all possible values, $\theta$ must range from 0 to $\pi, \phi$ from 0 to $2 \pi$, and $\mathrm{c}$ from 0 to $\infty$. If, however, we give a similar range to the new variables in the second scheme of transformation, we shall be counting each collision twice over. For a collision in which $\alpha, \beta, \gamma$ have given values can, by merely changing the rôles of the two molecules, be regarded as a new collision in which the signs of $\alpha, \beta, \gamma$ are altered. This source of error can be eliminated by limiting the integration with respect to $\psi$ from 0 to $\pi / 2$, instead of from 0 to $\pi$. Hence we obtain, for the number for which $\mathbf{c}$ lies between $\mathbf{c}$ and $\mathbf{c}+d \mathbf{c}$, while $V$ lies between $V$ and $V+d V$,

$$
8 h^{3} m^{3} \nu^{2} \sigma^{2} e^{-h m\left(2 \mathbf{c}^{2}+\frac{1}{2} V^{2}\right)} \mathbf{c}^{2} V^{3} d \mathbf{c} d V \text {. }
$$

Integrating with respect to $\mathbf{c}$ from 0 to $\infty$, the number of collisions for which $V$ lies between $V$ and $V+d V$ is

or

$$
\begin{gathered}
8 h^{3} m^{3} \nu^{2} \sigma^{2}\left[\int_{0}^{\infty} e^{-2 h m c^{2}} \mathbf{c}^{2} d \mathbf{c}\right] e^{-\frac{1}{2} h m V^{2}} V^{3} d V, \\
\nu^{2} \sigma^{2} \sqrt{\frac{\pi h^{3} m^{3}}{2}} e^{-\frac{1}{2} h m V^{2}} V^{3} d V \ldots \ldots \ldots
\end{gathered}
$$

a result which will be required later.

If we finally integrate this from $V=0$ to $V=\infty$, we obtain for the total number of collisions

$$
\nu^{2} \sigma^{2} \sqrt{\overline{2 \pi}}
$$

There are $\nu$ molecules per unit volume, and each collision terminates two free paths. Hence the $\nu$ molecules describe

$$
2 \nu^{2} \sigma^{2} \sqrt{\frac{2 \pi}{h m}}
$$

free paths per unit time. 
The average duration of a free path is accordingly

$$
\frac{1}{2 \nu \sigma^{2}} \sqrt{\frac{h m}{2 \pi}}
$$

The distance described per unit time by the $\nu$ molecules occupying unit volume

$$
\begin{aligned}
& =\nu \iint_{-\infty}^{+\infty} \int_{\frac{h^{3} m^{3}}{\pi^{3}}} e^{-h m c^{2}} c d u d v d w \\
& =4 \pi \nu \sqrt{\frac{h^{3} m^{3}}{\pi^{3}}} \int_{0}^{\infty} e^{-h m c^{2} c^{3} d c} \\
& =\frac{2 \nu}{\sqrt{\pi h m}} .
\end{aligned}
$$

This distance is the aggregate of all the free paths, of which the number is given by expression (47). By division we find as the length of the mean free path

$$
\frac{1}{\sqrt{2} \pi \nu \sigma^{2}}=\frac{\cdot 7071 . .}{\pi \nu \sigma^{2}}
$$

If the average is taken in any other way, the result is of course different. We might for instance average over all the free paths which are being deseribed at a particular instant of time.

Tait* takes a particular instant of time, and defines the mean free path as the average of the distances described by each molecule between this instant and the instant of its next collision. He calculates as the value of the mean free path defined in this way,

$$
\frac{677 . .}{\pi \nu \sigma^{2}}
$$

the factor $677 \ldots$ arising from an integral of which the value cannot be calculated in finite terms. We shall return later to the actual calculations by which this result is obtained.

From the results obtained in this section the numerical values given in $\S 8$ can be ealeulated without trouble.

\section{Apparent Irreversibility of Motion.}

31. When a gas is not in a steady state, it follows from $\S 23$ (p. 21), that $d H / d t$ must be negative. Former writers have interpreted this to mean that $H$ will continually decrease, until it reaches a minimum value, and will then retain that value for ever after. A motion of this kind would, however, be dynamically irreversible, and therefore inconsistent with the dynamical equations of motion from which it ought to have been deduced. As will appear from the next chapter, the truth is that we have at this point reached the limit within which the assumption of molecular ehaos leads to accurate results. The motion is, in point of fact, strictly reversible, and the apparent irreversibility is merely an illusion introduced by the imperfections of the statistical method.

* Royal Soc. Edin. Trans. xxxıı., p. 74 (1886). 


\section{CHAPTER III.}

\section{THE LAW OF DISTRIBUTION (CONTINUED).}

\section{The Method of General Dynamics.}

\section{The Conception of a Generalised Space.}

32. In the last chapter it was twice found convenient to represent the three velocity coordinates $u, v, w$ of a molecule, by a point in space of which the coordinates referred to three rectangular axes were $u, v, w$. The principle involved is a useful one, capable of almost indefinite extension, and will be largely used both in the present chapter and elsewhere in the book.

The space of nature possesses three dimensions, but just as it is open for us to represent any two coordinates in an imaginary space of only two dimensions, so in the same way we may represent any four coordinates in an imaginary space of four dimensions. Similarly if a dynamical system is specified by $n$ coordinates, we can represent these coordinates in a space of $n$ dimensions, and the various points in this space will correspond to the various configurations of the dynamical system.

In the present chapter, we attempt to find the law of distribution of velocities by a method which consists essentially in regarding the whole gas as a single dynamical system, and in representing its coordinates in a single imaginary space of the appropriate number of dimensions.

Let us suppose that the gas consists of a great number $N$ of exactly similar molecules, enclosed in a vessel of volume $\Omega$. At the outset we shall suppose these molecules to be elastic spheres of the kind already described. Each molecule will possess six coordinates, the three positional coordinates of its centre referred to three fixed rectangular axes in the containing vessel, and the three components of the velocity of its centre resolved parallel to these three axes. We shall denote the separate molecules by the letters $A, B, C$, etc., and the six coordinates of molecule $A$ will be denoted by $x_{a}, y_{a}, z_{a}, u_{a}, v_{a}, w_{a}$. The whole gas may accordingly be regarded as a single dynamical system possessing $6 \mathrm{~N}$ coordinates, namely,

$$
x_{a}, y_{a}, z_{a}, u_{a}, v_{a}, w_{a}, x_{b}, y_{b}, z_{b}, u_{b}, v_{b}, w_{b}, x_{c} \ldots \text { etc........(51). }
$$

J. 
We can suppose this dynamical system represented in a generalised space of $6 \mathrm{~N}$ dimensions. The configuration of the system in which the coordinates are those given by (51) will be represented by a single point in this space, namely the point of which the coordinates referred to $6 \mathrm{~N}$ rectangular axes are those given by (51).

33. In this way every possible configuration is represented by a point, but it does not follow that every point represents a possible configuration. For instance if as before the diameter of each molecule is $\sigma$, then a configuration in which

$$
\left(x_{a}-x_{b}\right)^{2}+\left(y_{a}-y_{b}\right)^{2}+\left(z_{a}-z_{b}\right)^{2}<\sigma^{2}
$$

is physically impossible because it represents a configuration in which the centres of molecules $A$ and $B$ are separated by a distance less than $\sigma-a$ configuration, therefore, in which parts of these two molecules occupy the same space. We must therefore suppose the region in which the inequality (52) is satisfied to be excluded from our generalised space. If we shut off all such regions, found by substituting for $a, b$ in (52) the suffixes corresponding to all possible pairs of molecules, we see that every point in the space which is left will represent a system which is physically possible in so far that no two molecules overlap.

There is still the boundary to be considered. For a configuration to be physically possible it is necessary that the centre of each molecule shall be at a normal distance from the boundary which is greater than the radius of the molecule. Thus all parts of the space must be excluded which do not satisfy equations of the form

$$
\left.\begin{array}{l}
\phi\left(x_{a}, y_{a}, z_{a}\right)>\frac{1}{2} \sigma \ldots \text { etc. } \\
\phi\left(x_{b}, y_{b}, z_{b}\right)>\frac{1}{2} \sigma \ldots \text { etc. }
\end{array}\right\}
$$

where $\phi(x, y, z)$ is the shortest normal distance from $x, y, z$ to the surface of the enclosing vessel.

34. If we exclude all the regions just indicated, it is clear that any point in the space which remains will represent a configuration of the system of molecules which is physically possible. In the course of the motion of the gas, this configuration will give place to other configurations, and by tracing out this series of configurations in the generalised space, we should obtain a "path" indicating the motion of the gas. By starting from a great number of points, and tracing the motion backwards as well as forwards, the whole space can be mapped out into paths in this way. Since the motion of the gas is completely determined when all the coordinates (51) are known, it follows that through any point there is one and only one path; two paths can never intersect. Also, of course, the paths are fixed in the generalised space; the motion of a gas, starting from given values of velocity and position coordinates, is always the same. These paths are identical with the "trajectories" of abstract dynamics. 


\section{The Motion in the Generalised Space.}

35. The general nature of these paths can be seen without trouble. A collision either of two molecules, or of a molecule and the boundary, occurs when, and only when, a path meets one of the surfaces of the regions excluded in $\S 33$. Now between collisions every molecule moves with uniform velocity in a straight line. Thus if at time $t=0$, the coordinates of a system are

$$
x_{a}^{\prime}, y_{a}^{\prime}, z_{a}^{\prime}, u_{a}^{\prime}, v_{a}^{\prime}, w_{a}^{\prime}, x_{b}^{\prime}, y_{b}^{\prime}, z_{b}^{\prime}, u_{b}^{\prime}, v_{b}^{\prime}, w_{b}^{\prime}
$$

the coordinates at time $t$, assuming that no collision has taken place in the interval, will be given by

$$
\begin{array}{ll}
x_{a}=x_{a}^{\prime}+u_{a}^{\prime} t, & y_{a}=y_{a}^{\prime}+v_{a}^{\prime} t, \text { etc. } \\
u_{a}=u_{a}^{\prime}, & v_{a}=v_{a}^{\prime}, \text { etc. }
\end{array}
$$

To find the equations of the path described by the representative point in the generalised space we eliminate $t$, and so obtain

$$
\left.\begin{array}{r}
\frac{x_{a}-x_{a}^{\prime}}{u_{a}^{\prime}}=\frac{y_{a}-y_{a}^{\prime}}{v_{a}^{\prime}}=\ldots . . \text { ete. } \\
u_{a}=u_{a}^{\prime}, v_{a}=v_{a}^{\prime}, w_{a}=w_{a}^{\prime} \ldots \text { etc. }
\end{array}\right\}
$$

and since these equations are linear they are of course the equations of a straight line. We therefore see that the paths in the generalised space are rectilinear except when they meet the excluded regions. Along the rectilinear parts of any paths, all the coordinates $u_{a}, v_{a}, w_{a}, u_{b} \ldots$ etc., maintain constant values, and any series of paths for which these constant values are the same are all parallel. When a representative point, moving along one of these paths, meets a boundary of the excluded space-corresponding to a collision-it must be supposed to move along this boundary until it reaches the point of which the eoordinates are those of the system after eollision, and then to start from here and describe the new rectilinear path through this point.

36. Now in the gas of the Kinetic Theory, we do not know anything as to the coordinates of the individual molecules of the gas: the problem we have to attack is virtually that of finding as much as we can about the behaviour of a dynamical system, without knowing on which of the paths in our generalised space its representative point is moving.

Our method is therefore to start an infinite number of systems, each system being a complete gas of the kind already specified, so as to have systems starting from every eoneeivable configuration, and moving over every path; and to investigate, as far as possible, the motion of this series of systems, in the hope of finding features common to all. Or, what eomes to exactly the same thing, we shall imagine our generalised space filled 
with a continuous fluid, we shall suppose the different points in this fluid to move, as directed by the dynamical equations of the gas, along the paths in our generalised space as stream-lines, and we shall then examine the motion of this fluid.

It is obvious that the initial distribution of density of this fluid may be chosen quite arbitrarily. We therefore choose that the initial distribution shall be homogeneous. The advantage of this choice is that the fluid remains homogeneous throughout its subsequent motion. This result follows from a general theorem which will be proved later $(\$ 72)$, but we now proceed to give a separate proof for the special case at present under consideration.

37. It has been seen that throughout the motion which takes place between two collisions, all the velocity coordinates $u_{a}, v_{a}, w_{a}, u_{b} \ldots$ etc., remain constant for any single path.

Consider a series of systems starting simultaneously with the same values of these velocity coordinates, but having positional coordinates lying between

$$
\begin{aligned}
& x_{a} \text { and } x_{a}+d x_{a}, y_{a} \text { and } y_{a}+d y_{a}, z_{a} \text { and } z_{a}+d z_{a} \text {, } \\
& x_{b} \text { and } x_{b}+d x_{b}, \ldots \text { etc. }
\end{aligned}
$$

Let these systems move for a time $d t$, and let it be supposed that no collision occurs during this interval, then it is clear that at the end of the interval the various positional coordinates will have values lying between

$$
x_{a}+u_{a} d t \text { and } x_{a}+u_{a} d t+d x_{a} \ldots \text { etc., etc., }
$$

while the velocity coordinates of course remain unaltered.

Hence the element of generalised space occupied by these systems remains unaltered in shape, size and orientation, but has in the course of the time $d t$ moved parallel to itself a distance $u_{a} d t$ parallel to the axis of $x_{u}, v_{a} d t$ parallel to the axis of $y_{a}$, etc. It follows that the density of the fluid with which the element of generalised space may be supposed to have been filled, has remained constant through this rectilinear motion.

Just as rectilinear motion leaves the velocity coordinates unchanged while altering the positional coordinates, so a collision leaves the positional coordinates unchanged while altering the velocity coordinates. There are two types of collisions to be discussed-collisions between molecules and the boundary, and collisions between pairs of molecules.

As a specimen of the former, consider a collision between molecule $A$ and the boundary: this leaves all the coordinates unchanged except $u_{a}, v_{a}, w_{a}$. Considering a series of systems in which before collision all the coordinates except $u_{a}, v_{a}, w_{a}$ have the same values for each member of the system, whilst $u_{a}, v_{a}, w_{a}$ lie between $u_{a}$ and $u_{a}+d u_{a}, v_{a}$ and $v_{a}+d v_{a}, w_{a}$ and $w_{a}+d w_{a}$, we see that after collision all the coordinates will remain unaltered except $u_{a}, v_{a}, w_{a}$, while these will lie within a new set of limits. Now in fig. 2 
(p. 25) we may suppose the former limits represented by the parallelepiped $\alpha$, in which case the latter set will be represented by the parallelepiped $\beta$. These parallelepipeds have been shewn to be equal in size although in the present case the orientations are not the same. This proves that the size of the clement of volume of generalised space occupied by the series of systems now under consideration is unaltered by a collision of molecule $A$ with the boundary, and hence that the density of the fluid may be supposed to remain unaltered.

The case of a collision between a pair of molecules may be treated in the same way. If the molecules are $A$ and $B$, all the coordinates remain unaltered except $u_{a}, v_{a}, w_{a}, u_{b}, v_{b}, w_{b}$, and the result follows at once from equation (9) if we change the notation so as to replace $u, v, w$ by $u_{a}, v_{a}, w_{a}$ and $u^{\prime}, v^{\prime}, w^{\prime}$ by $u_{b}, v_{b}, w_{b}$.

Hence, examining the motion of any small element of fluid in our generalised space, we have proved that the density of this element remains unchanged by steady motion and by collisions, i.e., remains unchanged throughout the whole motion of the gas. It follows that if the whole generalised space is filled with fluid initially homogeneous, then this fluid will remain homogeneous throughout the entire motion.

38. It has already been remarked that the stream-lines along which the fluid moves are permanently fixed in the generalised space. This fact, combined with the result just proved, shews that the motion of the fluid we are discussing is a "steady-motion" in the hydrodynamical sense.

One further feature of this motion must be noticed. If we denote the total kinetic energy of any system by $E$, so that

$$
2 E=m\left(u_{a}^{2}+v_{a}^{2}+w_{a}^{2}+u_{b}{ }^{2}+\ldots\right)
$$

it is clear that $E$ remains constant throughout the whole length of any stream-line. When $E$ is a constant, equation (57), regarded as a relation between the Cartesian coordinates of a point in the generalised space, expresses that the point lies on a certain locus (of dimensions $6 N-1$ ) in this space. It follows, then, that the motion of any element of the fluid is confined to that member of the family of loci $E=$ constant, in which it started.

To obtain some idea of the disposition of this family of loci in our generalised space, we notice that $2 E / m$ is the square of the perpendicular distance from

$$
\text { - } u_{a}=v_{a}=w_{a}=u_{b}=\ldots=0 \text {, }
$$

or, what is the same thing, from $E=0$. Hence the loci enclose one another, being in fact a system of tubular surfaces of which the cross-sections are spherical loci of $3 N$ dimensions. The tubes do not extend to infinity along their length. For we pass along a generator of a tube by varying $x_{a}, y_{a}, z_{a}, x_{b} \ldots$ etc., and none of these coordinates can become infinite, 
because each molecule of the gas is supposed to be contained in a finite closed vessel. The surfaces $E=$ constant are therefore finite closed surfaces in the generalised space, the surface $E=\infty$ alone being, in the limit, infinite and enclosing all the others.

Hence the motion of the fluid in the generalised space is one of circulation in closed surfaces, and, in particular, there is no motion of the fluid across the boundary at infinity.

Similarly, if there were any other quantities $\chi_{1}, \chi_{2} \ldots$, functions of the coordinates in the generalised space, which remained constant throughout the motion of the gas, then the motion of the fluid in the generalised space would be confined to the loci

$$
\chi_{1}=\text { constant }, \quad \chi_{2}=\text { constant, etc. }
$$

The only quantities of which we know, other than the energy, which remain constant over a collision between any two molecules, are the three components of linear momentum, the three moments of angular momentum and the number of molecules in the gas; of these the components of momentum both linear and angular are in general changed by a collision between a molecule and the boundary, and the number of molecules in the gas is not a function of the coordinates in the generalised space. Thus in general the energy is the only quantity of which we know, satisfying the conditions in question.

An exception to this may occur if the vessel containing the gas is a figure of revolution, having its interior surface perfectly smooth. For then there is always a component of momentum which is not changed by a collision between a molecule and the boundary; namely, that parallel to a tangent to the containing vessel at the point at which the collision takes place. In this case, then, the moment of momentum of the whole gas about the axis of figure of the containing vessel remains constant throughout the motion. It will, however, be convenient to defer the consideration of special cases of this type until Chapter V.

\section{The Partition of the Generalised Space-Positional Coordinates.}

39. We have supposed the volume of the containing vessel to be $\Omega$. Let us suppose the vessel divided up in a number $n$ of small "cells" each of the same volume $\omega$, so that $n \omega=\Omega$. These cells will be referred to as cell 1 , cell $2, \ldots$, respectively. The different possible configurations of the gas are now to be classified according to the number of molecules of which the centres fall within the different cells. As a typical class, we consider a class such that $a_{1}$ molecules have their centres in cell $1, a_{2}$ in cell 2 , and so on. Let this class be referred to as class A. We have to examine what proportion of the whole of the generalised space represents systems of class A. 
Let us, for the present, suppose that the radius of the molecules is vanishingly small, so that those parts of the generalised space excluded by the conditions of $\S 33$ may be neglected. Then the representative points of systems which are such that the centre of the molecule $A$ lies within a single specified cell,-i.e., is restricted to a range $\omega$ out of the whole volume $\Omega$ of the containing vessel-will clearly occupy a fraction $\omega / \Omega$ of the whole of the generalised space. Since $n \omega=\Omega$, this may be written $n^{-1}$. If two molecules $A$ and $B$ both lie within specified cells, the representative points occupy a fraction $n^{-2}$ of the whole, and so on. Thus if each of the $N$ molecules lies within a specified cell, the representative points will occupy a fraction $n^{-N}$ of the whole of the generalised space.

Now the number of different ways in which the $N$ molecules can be assigned to the $n$ different cells, so that the system shall belong to class $\mathrm{A}$, defined as above, is

$$
\frac{N !}{a_{1} ! a_{2} ! a_{3} ! \ldots a_{n} !}
$$

where, since the total number of molecules is $N$, we have of course

$$
a_{1}+a_{2}+a_{3}+\ldots+a_{n}=N \text {. }
$$

Hence it follows that the representative points of systems of class $\mathbf{A}$ will occupy a fraction

$$
\frac{N !}{a_{1} ! a_{2} ! a_{3} ! \ldots a_{n} !} n^{-N}
$$

of the whole of the generalised space.

40. We have already supposed $N$ to be great; we now suppose that $a_{1}, a_{2}, a_{3} \ldots a_{n}$ separately are very great. This enables us to express (60) in a simpler form, by using the well-known theorem of Stirling,

$$
\mathrm{L}_{p=\infty}^{\mathrm{t}} p !=\sqrt{2 p \pi}\left(\frac{p}{e}\right)^{p}
$$

If we take logarithms of both sides, this becomes

$$
\underset{p=\infty}{\mathrm{L}^{\mathrm{t}}} \log p !=\frac{1}{2} \log 2 \pi+\left(p+\frac{1}{2}\right) \log p-p
$$

Denoting expression (60) by $\theta_{a}$, we have

$$
\log \theta_{a}=\log N !-\sum_{s=1}^{s=n} \log a_{s} !-N \log n,
$$

and in the limit, when $a_{1}, a_{2} \ldots a_{n}, N$ are all infinite, this may, in virtue of equation (62), be replaced by

$$
\begin{aligned}
\log \theta_{a}=\frac{1}{2} \log 2 \pi+\left(N+\frac{1}{2}\right) & \log N-\dot{N} \\
& -\sum_{s=1}^{s=n}\left\{\frac{1}{2} \log 2 \pi+\left(a_{s}+\frac{1}{2}\right) \dot{\log } a_{s}-a_{s}\right\}-N \dot{\log n} .
\end{aligned}
$$


Now $\Sigma a_{8}=N$, so that $\Sigma\left(a_{8}+\frac{1}{2}\right)=N+\frac{1}{2} n$, and hence it will be found that the foregoing equation may be transformed into

$$
\log \theta_{a}=\frac{n}{2} \log n-\frac{n-1}{2} \log 2 \pi N-\sum_{s=1}^{s=n}\left(a_{s}+\frac{1}{2}\right) \log \frac{n a_{8}}{N}
$$

It will be convenient to write

$$
K_{a}=\frac{1}{N} \sum_{s=1}^{s=n}\left(a_{8}+\frac{1}{2}\right) \log \frac{n a_{s}}{N}
$$

so that $\theta_{a}$, the fraction of the geueralised space which represents systems of class $A$, is given by

$$
\theta_{a}=\frac{n^{\frac{3}{2} n}}{(2 \pi N)^{\frac{n-1}{2}}} e^{-N K a}
$$

41. It is now necessary to represent the various possible classes of systems in a new generalised space. Let us imagine a space of $n$ dimensions, in which the position of a point is specified by $n$ rectangular coordinates $x_{1}, x_{2} \ldots x_{n}$. Then we may suppose a system of class $\mathrm{A}$ represented in this space by the point

$$
x_{1}=a_{1}, x_{2}=a_{2}, \ldots \ldots ., x_{n}=a_{n}
$$

Not every point in this generalised space will represent a possible class of system, for we are supposing $a_{1}, a_{2} \ldots$ to be integers, and their sum to be $N$. The different possible classes will be represented by those points for which $x_{1}, x_{2} \ldots x_{n}$ have integral values such that

$$
x_{1}+x_{2}+\ldots+x_{n}=N \text {. }
$$

Just as in two-dimensional space a linear equation between the coordinates represents a line (of one dimension), or as in three-dimensional space a linear relation between the coordinates represents a plane (of two dimensions), so in the present $n$-dimensional space, the linear relation (67) represents a region of $(n-1)$ dimensions. All values of the coordinates which satisfy (67) will represent points in this $(n-1)$-dimensional region, and therefore all possible classes of systems may be represented in this region. The representation is still limited to points for which the $x^{\prime}$ s have integral values, and these points are perhaps most easily thought of as the intersections of systems of parallel straight lines.

If we now place a mass $\theta_{a}$ (cf. equation 65) at the point representing class A, and a similar mass for every other class, then the various possible classes will be represented both in nature and magnitude in this new generalised space.

42. Consider the function $K$, a continuous function of position in this new generalised space, defined by

$$
K=\frac{1}{N} \sum_{s=1}^{s=n}\left(x_{8}+\frac{1}{2}\right) \log \frac{n x_{8}}{N}
$$


Comparison with equation (64) shews that $K$ becomes equal to $K_{a}$ at the point representing class $\mathrm{A}$, equal to $K_{b}$ at the point representing class $\mathrm{B}$, and so on. We have now to examine the way in which values of $K$ are distributed in the new generalised space, and shall find that there is a single point at which $K$ has a minimum value $K=0$, and that at all other points $K$ is positive.

43. To find maximum or minimum values of $K$, if such exist, we proceed to the variation of equation (68). The equation expressing this variation is

$$
\delta K=\frac{1}{N} \sum_{s=1}^{s=n}\left(\log x_{8}+1+\frac{1}{2 x_{8}}\right) \delta x_{8} \ldots \frac{1}{N} \sum \log \frac{n}{N} \delta x_{s} \ldots . . . .(69)
$$

Since, however, $x_{1}, x_{2} \ldots$ cannot vary independently, but are subject to equation (67), their variations will be connected by the equation obtained by the variation of (67), namely

$$
\sum_{s=1}^{s=n} \delta x_{s}=0
$$

Following the usual method, we combine equations (69) and (70) in the single equation

$$
\delta K=\frac{1}{N} \sum_{s=1}^{s=n}\left(\log x_{s}+1+\frac{1}{2 x_{s}}+\lambda\right) \delta x_{s}
$$

where $\lambda$ is an undetermined multiplier. The condition that $\delta K$ shall vanish is now given by the elimination of $\lambda$ from the system of equations

$$
\log x_{8}+1+\frac{1}{2 x_{8}}+\lambda=0 \ldots(s=1,2, \ldots n) .
$$

The result of this elimination is

$$
x_{1}=x_{2}=\ldots=x_{n},
$$

and if we combine this with equation (67) we find that $\delta K$ vanishes, subject to equation (67), when

$$
x_{1}=x_{2}=\ldots=\frac{N}{n}
$$

There is therefore one and only one point at which $K$ has a stationary value.

44. The next step is to expand $K$ in the neighbourhood of the point given by equations (72) at which $\delta K=0$. A point adjacent to this point may be taken to be

$$
x_{1}=\frac{N}{n}+\epsilon_{1}, x_{2}=\frac{N}{n}+\epsilon_{2}, \ldots
$$

where $\epsilon_{1}, \epsilon_{2} \ldots$ are small, and where, since equation (67) must still be satisfied, $\epsilon_{1}, \epsilon_{2} \ldots$ are connected by the relation

$$
\epsilon_{1}+\epsilon_{2}+\ldots+\epsilon_{n}=0
$$


At this point, by equation (68), the value of $K$ is

$$
\begin{aligned}
K & =\frac{1}{N} \sum_{s=1}^{s=n}\left(\frac{N}{n}+\epsilon_{s}+\frac{1}{2}\right) \log \left(1+\frac{n \epsilon_{s}}{N}\right) \\
& =\frac{1}{N} \sum_{s=1}^{s=n}\left(\frac{N}{n}+\epsilon_{s}+\frac{1}{2}\right)\left(\frac{n \epsilon_{s}}{N}-\frac{1}{2}\left(\frac{n}{N}\right)^{2} \epsilon_{s}{ }^{2}+\frac{1}{3}\left(\frac{n}{N}\right)^{3} \epsilon_{s}{ }^{3}+\ldots\right) .
\end{aligned}
$$

Expanded in powers of $\epsilon_{1}, \epsilon_{2}, \ldots$ this becomes

$$
K=\frac{1}{2} \frac{\dot{n}}{N^{2}}\left(1-\frac{1}{2} \frac{n}{N}\right) \sum_{s=1}^{s=n} \epsilon_{8}{ }^{2}-\frac{1}{6} \frac{n^{2}}{N^{3}}\left(1-\frac{n}{N}\right) \sum_{s=1}^{s=n} \epsilon_{8}{ }^{3}+\ldots,
$$

or, since $N$ and $\frac{N}{n}$ are supposed to be infinite,

$$
K=\frac{1}{2} \frac{n}{N^{2}} \sum_{s=1}^{s=n} \epsilon_{s}{ }^{2}-\frac{1}{6} \frac{n^{2}}{N^{3}} \sum_{s=1}^{s=n} \epsilon_{s}{ }^{3}+\ldots
$$

For small values of $\epsilon_{1}, \epsilon_{2} \ldots$ the right-hand member of this equation reduces to its first term, which is positive for all values of the $\epsilon$ 's. It follows that except at the point (72) the value of $K$ is always greater than the value which it has at this point, and therefore that this value is a true minimum value. Moreover, since the point in question is the only point at which $\delta K=0$, it follows that there are no other maxima or minima for $K$ and therefore that $K$ is positive at all points except the point given by (72), at which it vanishes.

45. On the right-hand of equation (75) the second term stands to the first in the ratio

$$
\frac{1}{3} \frac{n}{N} \frac{\sum \epsilon^{3}}{\sum \epsilon^{2}}
$$

and the condition that this shall vanish is that the $\epsilon$ 's shall vanish in comparison with $N$-a condition which of course admits of the $\epsilon$ 's being finite or even infinite. If the condition is satisfied, equation (75) may be replaced by

$$
\epsilon_{1}^{2}+\epsilon_{2}^{2}+\ldots+\epsilon_{n}{ }^{2}=\frac{2 N^{2} K}{n}
$$

If we substitute the values of $\epsilon_{1}, \epsilon_{2} \ldots$ from equations (73) this becomes

$$
\left(\frac{N}{n}-x_{1}\right)^{2}+\left(\frac{N}{n}-x_{2}\right)^{2}+\ldots=\frac{2 N^{2} K}{n}
$$

so that $K$ is proportional to the square of the distance from the point at which $K=0$.

If $K$ is kept eonstant, equation (78) expresses that in the generalised space the distance from the fixed point $\frac{N}{n}, \frac{N}{n} \ldots$ to the variable point $x_{1}, x_{2} \ldots$

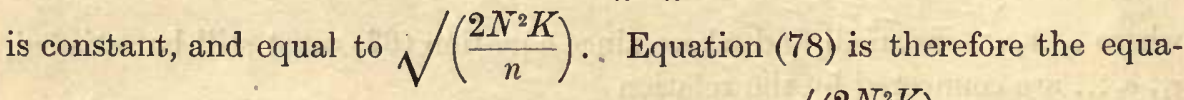
tion of a sphere of $n$ dimensions, its radius being $\sqrt{ }\left(\frac{2 N^{2} K}{n}\right)$. The centre of 
this sphere satisfies equation (67), so that points of which the coordinates satisfy equations (67) and (78) simultaneously lie on a sphere of $(n-1)$ dimensions and of radius equal to that of the former sphere.

We see then that the region of the generalised space in which equation (67) is satisfied and in which $K<K_{0}$, is a sphere of $(n-1)$ dimensions, and of radius $\sqrt{ }\left(\frac{2 N^{2} K_{0}}{n}\right)$, provided that the $\epsilon$ 's are finite everywhere inside this sphere.

46. In space of $s$ dimensions $\left(\xi_{1}, \xi_{2} \ldots \xi_{8}\right)$ the volume of a sphere of radius $R$ is

$$
\iiint \ldots d \xi_{1} d \xi_{2} \ldots d \xi_{8}
$$

where the integration extends over all values of the variables which are such that

$$
\xi_{1}{ }^{2}+\xi_{2}^{2}+\ldots+\xi_{8}{ }^{2} \leq R^{2}
$$

The multiple integral (79) is of the type associated with the name of Dirichlet, and its value is known to be*

$$
\frac{\pi^{\frac{s}{2}}}{\Gamma\left(1+\frac{s}{2}\right)} R^{s}
$$

47. It follows that the volume of our $(n-1)$-dimensional sphere of

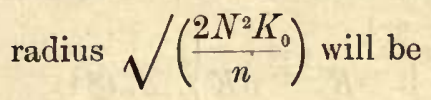

$$
\frac{\pi^{\frac{n-1}{2}}}{\Gamma\left(\frac{n+1}{2}\right)}\left(\frac{2 N^{2} K_{0}}{n}\right)^{\frac{n-1}{2}}
$$

By differentiation with respect to $K_{0}$, it follows that the volume for which $K$ lies between $K_{0}$ and $K_{0}+d K$ is

$$
\frac{\pi^{\frac{n-1}{2}}}{\Gamma\left(\frac{n+1}{2}\right)} \frac{n-1}{2}\left(\frac{2 N^{2}}{n}\right)^{\frac{n-1}{2}} K_{0}^{\frac{n-3}{2}} d K
$$

and in virtue of the general property of the Gamma-function which is expressed by the equation

$$
\Gamma(p+1) \stackrel{\star}{=} \Gamma(p),
$$

this may be written in the simpler form

$$
\frac{\pi^{\frac{n-1}{2}}}{\Gamma\left(\frac{n-1}{2}\right)}\left(\frac{2 N^{2}}{n}\right)^{\frac{n-1}{2}} K_{0}^{\frac{n-3}{2}} d K
$$

* Williamson, Integral Calculus, p. 320. 
48. We must now determine the number of mass-centres-i.e., points at which all the $x$ 's have integral values-which occur within this region. The density of distribution of these centres is of course constant throughout the $(n-1)$-dimensional space in which equation (67) is satisfied. If a system of lines is drawn through these centres parallel to one of the axes of coordinates, these lines will form a network of parallel lines at unit distance apart; their intersections with any orthogonal $(n-1)$-dimensional region will therefore be at the rate of unity per unit volume of this latter region. The region specified by equation (67) is not orthogonal to this system of lines, but since it has direction cosines

$$
n^{-\frac{1}{2}}, n^{-\frac{1}{2}}, n^{-\frac{1}{2}} \ldots
$$

it will make an angle $\cos ^{-1}\left(n^{-\frac{1}{2}}\right)$ with any orthogonal region. Hence the number of intersections of the system of lines with the region specified by equation (67) is $n^{-\frac{1}{2}}$ per unit volume.

The number of mass-centres in this region for which $K$ lies between $K_{0}$ and $K_{0}+d K$ is accordingly equal to expression (82) multiplied by $n^{-\frac{1}{3}}$. In accordance with equation (65) we place at each of these centres a mass

$$
\frac{n^{\frac{1 n}{n}}}{(2 \pi N)^{\frac{n-1}{2}} e^{-N K_{0}}}
$$

and we therefore have, for the total mass in the region in which $K$ lies between $K_{0}$ and $K_{0}+d K$,

$$
n^{-\frac{1}{2}}\left\{\frac{n^{\frac{1}{3} n}}{(2 \pi N)^{\frac{n-1}{2}}} e^{-N K_{0}}\right\}\left\{\frac{\pi^{\frac{n-1}{2}}}{\Gamma\left(\frac{n-1}{2}\right)}\left(\frac{2 N^{2}}{n}\right)^{\frac{n-1}{2}} K_{0}^{\frac{n-3}{2}} d K\right\} \ldots \ldots
$$

After simplification this expression reduces to

$$
\frac{N^{\frac{n-1}{2}}}{\Gamma\left(\frac{n-1}{2}\right)} e^{-N K_{0}} K_{0}^{\frac{n-3}{2}} d K
$$

49. Remembering the significance of the masses placed in the new generalised space, it appears that in the original generalised space of $6 \mathrm{~N}$ dimensions, that fraction of the whole space which represents systems for which $K$ lies between $K_{0}$ and $K_{0}+d K$ is given by expression (85). By integration, the fraction for which $K$ lies between $K_{1}$ and $K_{2}$ is given by

$$
\frac{N^{\frac{n-1}{2}}}{\Gamma\left(\frac{n-1}{2}\right)} \int_{K=K_{1}}^{K=K_{2}} e^{-N K} K^{\frac{n-8}{2}} d K
$$

This result, it must be remembered, has been obtained on the assumption that the $\epsilon^{\prime}$ s are small compared with $N$ and is therefore, by equation (77), 
- only true provided $K_{1}$ and $K_{2}$ are both small. This condition, however, does not require that $N K$ shall be sinall, or even finite.

The general identity

$$
N^{\frac{n-1}{2}} \int_{K=0}^{K=\infty} e^{-N K} K^{\frac{n-3}{2}} d K=\Gamma\left(\frac{n-1}{2}\right)
$$

holds for all values of $N$. When, in the limit, $N$ is made infinite, the expression on the right-hand will of course remain finite, and the whole value of the integral on the left-hand arises from the contributions made to this integral by the infinitesimal range of values of $K$ which is in the immediate neighbourhood of $K=0$.

It follows that expression (86) vanishes unless $K_{1}=0$, and that it is equal to unity when $K_{1}=0$ and $K_{2}$ is not equal to zero.

50. Hence it follows that all except an infinitesimal fraction of the original generalised space represents systems for which $K$ has its minimum value, or for which its value is only infinitesimally different from this.

51. Transforming expression (86) by the use of equation (87) we see that the fraction of the generalised space which represents systems for which $K$ is less than $K_{2}$ is

$$
\frac{\int_{K=0}^{K=K_{2}} e^{-N K} K^{\frac{n-3}{2}} d K}{\int_{K=0}^{K=\infty} e^{-N K} K^{\frac{n-3}{2}} d K},
$$

and hence that the fraction which represents systems for which $K$ is greater than $K_{2}$ is

$$
\frac{\int_{K=K_{2}}^{K=\infty} e^{-N K} K^{\frac{n-3}{2}} d K}{\int_{K=0}^{K=\infty} e^{-N K} \cdot K^{\frac{n-3}{2}} d K}
$$

As in the former case, the whole value of this fraction arises from a contribution made to the integral in the numerator by a small range of values for $K$ in the immediate neighbourhood of the lower limit.

When $K_{2}$ is not small, expression (88) will require correction. The surfaces $K=$ constant are no longer spheres as in $\S 45$, and the consequence of this is that every integrand in the numerator of (88) requires to be multiplied by a correcting factor. This factor, however, is always finite and is independent of $N$. Hence it follows that the result stated in the last paragraph is true independently of the smallness of $K_{2}$.

52. It therefore appears that of the systems for which $K \geqslant K_{1}$, where $K_{1}$ is some finite positive quantity, all except an infinitesimal fraction have values of $K$ which are either equal to $K_{1}$ or differ from $K_{1}$ only by an infinitesimal quantity. 


\section{Physical Interpretation.}

53. In $\S 11$ we discussed the conditions under which it is possible logically to define the "density at a point" in a gas, in such a way that the density is a definite continuous function of the position of the point at which it is estimated. It was there found to be necessary for us to be able to divide the gas into elements of volume, "which may, without appreciable error, be supposed to be infinitely great in comparison with the distances between neighbouring molecules, and at the same time infinitely small compared with the scale of variation of the gas." We shall now see that when these conditions are satisfied the quantity $K$ admits of a physical interpretation which is independent of the way in which the volume of the gas is divided up into cells.

In assuming the quantities $a_{1}, a_{2} \ldots a_{n}$ to be infinite, we have already supposed the first of the above mentioned conditions to be satisfied by the cells. If we assume the second also to be satisfied, we can speak of the density of the gas at a point, and this density may, without appreciable error, be taken to be constant throughout any single cell. In general let $\nu$ denote the molecular density, let $\nu_{s}$ denote the mean molecular density in the $s$ th cell, and $\nu_{0}$ that in the whole gas. Then, obviously

and therefore

$$
\nu_{s}=\frac{a_{s}}{\omega}=\frac{n a_{s}}{\Omega}, \quad \nu_{0}=\frac{N}{\Omega},
$$

$$
\frac{n a_{8}}{N}=\frac{\nu_{8}}{\nu_{0}}
$$

Also $a_{s}$, the number of molecules in the sth cell, may be taken equal to $\nu_{s}$ multiplied by the volume of the cell, so that

$$
a_{s}=\iiint \nu d x d y d z
$$

where the integral extends throughout the $s$ th cell.

The value of $K_{a}$ is given by equation (64), in which $\left(a_{s}+\frac{1}{2}\right)$ may be replaced by $a_{s}$ and therefore by expression (90). If we further use equation (89), we find that (64) can be replaced by

$$
K=\frac{1}{N} \iiint \nu \log \left(\frac{\nu}{\nu_{0}}\right) d x d y d z
$$

in which the integration now extends throughout the whole volume of the gas. The equation may also be put in the form

$$
K=\frac{1}{\Omega} \iiint \frac{\nu}{\nu_{0}} \log \frac{\nu}{\nu_{0}} d x d y d z
$$

expressing that $K$ is the mean value of $\left(\nu / \nu_{0}\right) \log \left(\nu / \nu_{0}\right)$ throughout the gas. The results of $\$ 50$ and 52 are, however, only concerned with the minimum value of $K$, and comparisons between two different values of $K$. It is there- 
fore clear that these results will not be affected by constant multipliers or additive constants in the definition of $K$. Hence $K$ might equally well and more simply, have been defined by

$$
K=\iiint \nu \log \nu d x d y d z
$$

54. In whatever form $K$ is defined, the minimum value of $K$ is given by equations (72), and this is equivalent to

$$
\nu=\text { constant. }
$$

Thus the result of $\S 50$ states that in the original generalised space, all except an infinitesimal fraction of the whole space represents systems in which $\nu$ is constant throughout the gas. This is the result which we might $\grave{a}$ priori have expected. For selecting a point at random in the generalised space is equivalent to assigning positions to the molecules at random, and we should in this case naturally expect the resulting gas to be of uniform density.

When the gas is nearly, but not quite, of uniform density, let the density at any point be denoted by $\nu=\nu_{0}+\delta \nu$, then equation (92) becomes

$$
\begin{aligned}
K & =\frac{1}{\Omega} \iiint\left(1+\frac{\delta \nu}{\nu_{0}}\right) \log \left(1+\frac{\delta \nu}{\nu_{0}}\right) d x d y d z \\
& =\frac{1}{2 \Omega} \iiint\left(\frac{\delta \nu}{\nu_{0}}\right)^{2} d x d y d z .
\end{aligned}
$$

Hence $K$ is proportional to the mean value of $(\delta \nu)^{2}$ averaged through the gas, and we see that $K$ is a quantity which might naturally be taken to measure divergence from homogeneity.

\section{The Partition of the Generalised Space-Velocity Coordinates.}

55. We can investigate the partition of the velocity coordinates in a way exactly similar to that in which we have investigated the partition of the positional coordinates. The density $\nu$ must be replaced by the density $\tau$ defined as in $\S 13$. If we introduce a quantity $H$ defined by

$$
H=\iiint \tau \log \tau d u d v d w
$$

we can prove, exactly as before, that for all except an infinitesimal fraction of the whole series of systems represented in the generalised space, the quantity $H$ only differs infinitesimally from its minimum value. Since we are not tied down as regards additive or multiplicative constants in the definition of this function, we may replace equation (95) by

$$
H=\iiint f \log f d u d v d w
$$

where $f$ is the $f$ of $\S 13$, so that $H$ is now identical with the $H$ of the preceding chapter, defined by equation (13). 
There is, however, a feature about the $u, v, w$ coordinates which did not occur in the case of $x, y, z$ coordinates. Throughout the motion of a gas the energy remains constant, or, as in $\S 38$, the trajectories are confined to single members of the family of loci $E=$ constant. From this it results that we are not interested in the partition of the velocity coordinates throughout the whole of the generalised space, but only throughout the region which corresponds to a single definite value of $E$. Hence in finding the minimum value of $H$, we suppose $f$ subject not only to

$$
\iiint f d u d v d w=1
$$

the equation corresponding to the former equation (67), indicating that the number of molecules in the gas is equal to $N$, but also subject to

$$
\iiint \frac{1}{2} m\left(u^{2}+v^{2}+w^{2}\right) f d u d v d w=E
$$

expressing that the kinetic energy of the system is equal to $E$.

The equation of variation of $H$, subject to equations (97) and (98), is seen to be

$$
\delta H=\iiint\left\{1+\log f+\lambda+\frac{1}{2} \mu m\left(u^{2}+v^{2}+w^{2}\right)\right\} \delta f d u d v d w \ldots \ldots(99),
$$

in which $\lambda, \mu$ are undetermined multipliers. The minimum value of $H$ is accordingly given by

$$
1+\log f+\lambda+\frac{1}{2} \mu m\left(u^{2}+v^{2}+w^{2}\right)=0
$$

leading, if we change the constants, to the solution

$$
f=A e^{-h m\left(u^{2}+v^{2}+v^{2}\right)}
$$

56. It could now be shewn, exactly as in the former case, that throughout all but an infinitesimal fraction of the whole of that part of the generalised space in which the energy of the corresponding system is $E$, the law of distribution of velocities is that given by equation (101). It does not seem necessary to reproduce the details of this proof; the mathematician will be able to construct them for himself, while the physicist will probably not wish to be detained over them.

The law of distribution of velocities expressed by equation (101) is a special case of the general law found for the "steady state" in the last chapter. We are limited to this special case because we have, at the outset, supposed our containing vessel to be fixed in space. If, on the contrary, the vessel is moving in space with a velocity of components $u_{0}, v_{0}, w_{0}$ the analysis of this chapter can be made to apply by supposing all coordinates referred to moving axes, moving with a velocity of components $u_{0}, v_{0}, w_{0}$. In this case equation (91) expresses the law of distribution of velocities relative to these moving axes. The law of distribution of absolute velocities in space is therefore

$$
f=A e^{-h m\left[\left(u-u_{0}\right)^{2}+\left(v-v_{0}\right)^{2}+\left(w-w_{0}\right)^{2}\right]}
$$

which is the general law for the steady state given by equation (25). 
As in the case of the positional coordinates the law of distribution of velocities could have been predicted from considerations of probability. For selecting a point at random in the region corresponding to systems of energy $E$ is equivalent to assigning velocity coordinates $u, v, w$ to the molecules at random, subject only to the condition that their squares shall be distributed about a certain mean-value. It is therefore natural to find that the distribution of velocities should be in accordance with the law of trial and error.

This law, it will have already been noticed, gives $f=0$ when $u, v$ or $w$ is infinite. There is therefore the $\dot{a}$ posteriori objection to the analysis by which it has been obtained, that if we divide all possible velocities into "cells" in the manner of $\S 39$, the number of molecules in some of these cells cannot legitimately be treated as infinitely great. The difficulty is best met by taking a definite velocity $V$ such that those molecules of which the velocities do not satisfy the inequalities

$$
u<V, \quad v<V, \quad w<V,
$$

form an infinitesimal fraction of the whole. If the velocities which satisfy these inequalities can be partitioned into cells in the manner of $\S 39$, so as to satisfy the condition that the number in each cell is very great, then there is no further difficulty, and equation (102) will give the law of distribution of velocities which are less than $V$. The law now has no meaning for velocities greater than $V$. It is obvious, for instance, that the law expressed by equation (102) does not impose any upper limit whatever on the possible values of $u, v$ and $w$ for a single molecule, whereas in point of fact such a limit is definitely imposed by the energy equation.

\section{Molecules of finite size.}

57. It is obvious that it is in no way material to the analysis of $\$ 55$ and 56 , from which the law of distribution of velocities is found, whether the regions mentioned in $\S 33$ are excluded from the generalised space or not. For the exclusion of these regions affects the velocity coordinates equally throughout. Thus the law of distribution of velocities is the same whether the spheres are of finite size or are infinitesimal. It remains the same right up to the extreme limiting case in which the spheres are packed so tightly in the containing vessel that they cannot move.

\section{The Normal State.}

58. In the last chapter it was found, with the help of the unwarranted assumption of molecular chaos $(\S 15)$, that the law of distribution expressed by equation (102) represented a "steady state" for the gas. In the present chapter it has been shewn, without making any use of this assumption, that, 
except for an infinitesimal probability of error, a system selected at random from the generalised space will be in the state specified by equation (102). It will be convenient to refer to this state as the "Normal State." And when a result is certain except for an infinitesimal probability of error, it will be convenient to speak of the result as "infinitely probable."

If, therefore, a system is selected at random, it is infinitely probable that it will be in the "Normal State." Suppose that a system is selected at random, and then allowed to move under its natural motion for a time $t$, what do we know now as to its probable state after a time $t$ ? The answer is provided by the theorem proved in $\S 37$. The motion of all possible systems is represented in our generalised space by the motion of the supposed fluid. Instead of selecting a system at random and allowing it to move for time $t$, we may allow the whole fluid in the generalised space to move for a time $t$, and select a system at random after the motion has proceeded for a time $t$. The theorem of $\S 37^{\circ}$ proved the motion of the fluid in the generalised space to be a "steady motion." Hence selecting a system after time $t$ is the same thing as selecting a system at time 0 , and it is infinitely probable that the system thus selected will be in a normal state.

59. This completes our information abont the motion of the gas. At any instant it is infinitely probable that it is in a normal state. In the course of the motion departures from the normal state will occur, but it is infinitely probable that these will only occupy an infinitesimal fraction of the time occupied by the whole motion.

There is in theory a possibility of a gas continuing throughout its motion in a state different from the normal. Suppose for instance that the containing vessel is cubical, and that the molecules are started so that all move perpendicular to one edge along a system of parallel lines, no two of which are at a less distance than the diameter of a molecule. Then it is obvious that the molecules will not leave the lines on which they start, and will not change their velocities. In this case any law of velocities $f(u, 0,0)$ will be permanent, where $u$ is the velocity in the direction of the parallel lines.

The analysis of this chapter breaks down, it will be seen, because the supposition made in $\S 38$ is no longer true, that there are no functions of the coordinates in the generalised space except the energy which remain constant through the motion. For obviously we have $u_{a}^{2}=$ constant, $u_{b}^{2}=$ constant, etc., and $v_{a}=w_{a}=0, v_{b}=w_{b}=0$, etc.

Our results shew that it is infinitely probable that a system selected at random will not be of this special type. The connection between the trajectories of such systems and the "periodic orbits" of abstract dynamics is interesting, but cannot be discussed here. We shall return to the discussion of cases in which there are constants other than the energy in Chapter V. 


\section{Historical Note.}

60. The law of distribution of velocities which has been found both in this chapter and the preceding one was discovered by Maxwell, and is generally associated with his name. It first appears in the paper already referred to $(\$ 9)$, communicated to the British Association in 1859. The original proof is now universally admitted to be unsatisfactory, but is of interest from its historical importance. Except for a slight change of notation, the form in which it was given is as follows*.

"Let $N$ be the whole number of particles. Let $u, v, w$ be the components of the velocity of each particle in three rectangular directions, and let the number of particles for which $u$ lies between $u$ and $u+d u$ be $N f(u) d u$, where $f(u)$ is a function of $u$ to be determined.

"The number of particles for which $v$ lies between $v$ and $v+d v$ will be $N f(v) d v$, and the number for which $w$ lies between $w$ and $w+d w$ will be $N f(w) d w$, where $f$ always stands for the same function.

"Now the existence of the velocity $u$ does not in any way affect that of the velocities $v$ or $w$, since these are all at right angles to each other and independent, so that the number of particles whose velocity lies between $u$ and $u+d u$, and also between $v$ and $v+d v$ and also between $w$ and $w+d w$ is

$$
N f(u) f(v) f(w) d u d v d w .
$$

If we suppose the $N$ particles to start from the origin at the same instant, then this will be the number in the element of volume $d u d v d w$ after unit of time, and the number referred to unit of volume will be

$$
N f(u) f(v) f(w) \text {. }
$$

"But the directions of the coordinates are perfectly arbitrary, and therefore this number must depend on the distance from the origin alone, that is

$$
f(u) f(v) f(w)=\phi\left(u^{2}+v^{2}+w^{2}\right)
$$

Solving this functional equation, we find

$$
f(u)=C e^{A u^{2}}, \quad \phi\left(u^{2}+v^{2}+w^{2}\right)=C^{3} e^{A\left(u^{2}+v^{2}+w^{2}\right)}, "
$$

This proof must be admitted to be unsatisfactory, because it assumes the three velocity components to be independent. The velocities do not, however, enter independently into the dynamical equations of collisions between molecules, so that until the contrary has been proved, we should expect to find correlation between these velocities.

On account of this defect, Maxwell attempted a second proof $\uparrow$, which after emendations by Boltzmann + and Lorentz§ assumes the form given

* J. C. Maxwell, Collected Works, I. p. 380.

+ Collected Works, Ir. p. 43.

† Wiener Sitzungsber., LvIII. p. 517 (1868), Lxvi. p. 275 (1872), xcv. p. 153 (1887), Vorlesungen über Gastheorie, 1. p. 15.

$\S$ Wiener Sitzungsber., xcV. p. 115 (1887). 
in Chapter II. It is however very doubtful whether this proof can claim any superiority on grounds of logical consistency or completeness over Maxwell's original proof. The later proof finds it necessary to assume that there is no correlation between the velocity and space coordinates, while the earlier proof merely assumed that there was no correlation between the separate velocity components inter se. In each case the dynamical conditions equally suggest correlation, until the contrary has been proved, and it would be hard to give reasons why one assumption of no correlation is more justifiable than the other. It should be mentioned that Burbury* has always been of opinion that the later proof of Maxwell is not only logically unsound, but leads to an inaccurate result. He maintains that correlation actually takes place, except in the limiting case of an infinitely rare gas. This view, however, is not borne out by the analysis of the present and of the succeeding chapter. (Cf. $§ 66$, infra.)

A second class of proof of the law is represented by the proof which has been given in this chapter. In this class of proof the aim is to deduce a law from general dynamical considerations. As important examples of this class of proof may be mentioned a proof due to Kirchhoff, given in his lecturest, and one due to Meyer and Pirogoff, given in Meyer's Kinetic Theory of Gasesł. Both these proofs depend upon a use of the calculus of probabilities which cannot be justified. The proof given in this chapter is my own§: it also has been criticised by Burbury $\uparrow$, but I cannot persuade myself that his criticisms as to the validity of the proof are well founded $\|$.

* S. H. Burbury, The Kinetic Theory of Gases, Cambridge, 1899.

+ Kirchhoff, Vorlesungen über die Theorie der Wärme, p. 142.

¥ Meyer, Kinetic Theory of Gases, Eng. Trans. by Baynes, p. 370.

§ Phil. Mag. v. p. 597.

II Phil. Mag. vr. p. 529, vir. p. 467.

|| Phil. Mag. vi. p. 720, vir. p. 468. 


\section{CHAPTER IV.}

\section{THE LAW OF DISTRIBUTION (CONTINUED).}

\section{Comparison between the Methods of the two preceding Chapters.}

61. THE problem of the present chapter will be to consider the relation between the methods of procedure adopted in Chapters II. and III.

The discussion of Chapter II. was based upon certain questions of probability, and an answer to these questions was made possible and was obtained by the help of the assumption of molecular chaos enunciated in $\S 15$.

The discussion of Chapter III. also rested, although in a different sense, upon the theory of probability. The generalised space filled with fluid supplied a basis for the calculation of probabilities, and as the motion of the fluid was proved to be steady-motion, it followed that this basis was independent of the time. For the present, we continue to take this generalised space as the basis of probability calculations. The question "What is the probability that a system satisfies condition $p$ ?" will be taken to mean: "For what proportion of the generalised space is condition $p$ satisfied?" The further question: "Given that a system satisfies condition $p$, what is the probability that it also satisfies condition $q$ ?" will be interpreted to mean : "A point is selected at random from all those parts of the generalised space in which condition $p$ is satisfied: what is the probability that at this point condition $q$ also is satisfied?" And if $v_{p}$ is the total volume of that part of the space in which condition $p$ is satisfied, and $v_{p q}$ that of the space in which condition $q$ also is satisfied, the value of the probability required is of course the ratio

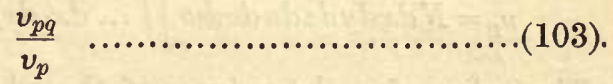

If $v$ is the whole volume of this generalised space (or of any part of it to which we choose to limit our consideration) the chance that condition $q$ shall be satisfied independently of condition $p$ is

$$
\frac{v_{q}}{v}
$$


Hence the condition that the probabilities of $p$ and $q$ being satisfied may be regarded as "independent" is that expressions (103) and (104) shall be equal, or, written symmetrically, that

$$
\frac{v_{p q}}{v}=\frac{v_{p}}{v} \frac{v_{q}}{v}
$$

\section{Analysis of the Assumption of Molecular Chaos.}

62. The assumption of molecular chaos was tantamount to an assumption that two probabilities might be regarded as independent. Equation (10j) accordingly enables us to test whether this assumption is legitimate or not relatively to our present basis of probability-namely, the generalised space filled with homogeneous fluid.

To do this, let us define condition $p$ as the condition that a molecule of class A shall be found in the element $d x d y d z$ of the gas-in other words, that one of the $N$ molecules shall have coordinates lying between the limits,

$$
\left.\begin{array}{l}
x \text { and } x+d x, y \text { and } y+d y, \quad z \text { and } z+d z \\
u \text { and } u+d u, v \text { and } v+d v, w \text { and } w+d w
\end{array}\right\}
$$

For certain systems this condition is satisfied by molecule $\mathrm{A}$, and these systems are represented in the generalised space by that region for which $x_{a}$ lies between $x$ and $x+d x$, and for which similar conditions are satisfied by $y_{a}, z_{a}, u_{a}, v_{a}, w_{a}$. This region supplies to $v_{p}$ a contribution of amount

$$
\iiint \ldots d x_{a} d x_{b} d x_{c} \ldots d u_{a} d u_{b} d u_{c} \ldots
$$

where the integration extends over all values of the variables which are not excluded by $\S 33$, except in the case of $x_{a}, y_{a}, z_{a}, u_{a}, v_{a}, w_{a}$, for which the limits are those given by (106). The integral may be written in the form

$$
d x d y d z d u d v d w \iint \ldots d x_{b} d x_{c} \ldots \int_{-\infty}^{+\infty} d u_{b} \int_{-\infty}^{+\infty} d u_{c} \ldots \quad \ldots(108)
$$

For other systems, condition $p$ is satisfied by molecule $\mathrm{B}$, and these systems again supply a contribution of amount equal to the above. Each of the $N$ molecules contributes in this way to $v_{p}$ an amount equal to that given by expression (108), so that the total value of $v_{p}$ is

$$
v_{p}=N d x d y d z d u d v d w \iint \ldots d x_{b} d x_{c} \ldots \int_{-\infty}^{+\infty} d u_{b} \int_{-\infty}^{+\infty} d u_{c} \ldots \ldots(109) .
$$

The value of $v$, the volume of the whole space, is given by (107), if the integrals are taken through all values of all the variables except those values excluded by $\S 33$. This integral may of course be written in the form

$$
v=\iiint \ldots d x_{a} d x_{b} d x_{c} \ldots \int_{-\infty}^{+\infty} d u_{a} \int_{-\infty}^{+\infty} d u_{b} \ldots
$$


and from equations (109) and (110) we now find

$$
\frac{v_{p}}{v}=N \frac{d x d y d z \iint \ldots d x_{b} d x_{c} \ldots}{\iiint d x_{a} d x_{b} d x_{c} \ldots} \frac{d u d v d w}{\int_{-\infty}^{+\infty} d u \int_{-\infty}^{+\infty} d v \int_{-\infty}^{+\infty} d w} \ldots . .(111)
$$

This vanishes through the last factor, for the obvious reason that when the molecules are equally likely to have all velocities, the probability is infinitely against a single molecule belonging to any specified class.

63. Let us now suppose that the velocities of the individual molecules are given, and let us calculate the probability in this case that condition $p$ is satisfied. Let us suppose that the velocities of molecule A are known to lie within the limits

$$
u_{a} \text { and } u_{a}+\delta u_{a}, v_{a} \text { and } v_{a}+\delta v_{a}, w_{a} \text { and } w_{a}+\delta w_{a} \ldots \ldots .(112),
$$

and that we have similar knowledge for the other molecules. The value of $v$, the whole space representing systems for which the molecules have the given velocities, is given by equation (110) if the integration is from $u_{a}$ to $u_{a}+\delta u_{a}$ for $u_{a}$ instead of from $-\infty$ to $+\infty$, and similarly for the other velocities.

Thus we have as the new value of $v$,

$$
v=\delta u_{a} \delta v_{a} \ldots \iiint \ldots d x_{a} d x_{b} d x_{c} \ldots
$$

As before, the systems for which condition $p$ is satisfied by molecule A, are represented by those parts of the space $v$ for which $x_{a}$ lies between $x$ and $x+d x$, and similar conditions are satisfied by $y_{a}, z_{a}, u_{a}, v_{a}, w_{a}$. We shall suppose, as we legitimately may, that the $\delta u_{a}, \delta v_{a}, \delta w_{a}$ of the limits (112) are infinitesimal in comparison with $d u d v d w$. Then provided that the range for $u_{a}$ given by (112) lies within the range $u$ and $u+d u$, and that similar conditions are satisfied by $v_{a}, w_{a}$, the contribution to $v_{p}$ corresponding to molecule $A$ is given by the right-hand of (113) provided the integration with respect to $x_{a}$ extends only from $x$ to $x+d x$, and similarly for $y_{a}, z_{a}$. Thus if the velocities of molecule $A$ lie within the specified ranges, there is a contribution from molecule $\mathrm{A}$ to $v_{p}$ of amount

$$
d x d y d z \delta u_{a} \delta v_{a} \ldots \iiint \int \ldots d x_{b} d x_{c} \ldots d y_{b} d y_{c} \ldots
$$

If the velocities given by the limits (112) do not lie within this range $d u d v d w$ the contribution is nil. The number of molecules of which the velocities satisfy the condition of lying within this range-in other words, the number of molecules capable of taking the rôle of molecule $\mathrm{A}$ in expression . (114) - may be taken to be

$$
N f(u, v, w) d u d v d w
$$


The product of expressions (114) and (115) gives $v_{p}$. From this and equation (113) we get

$\frac{v_{p}}{v}=N f(u, v, w) d u d v d w d x d y d z \frac{\iiint \int \ldots d x_{b} d x_{c} \ldots d y_{b} d y_{c} \ldots}{\iiint \iiint \ldots d x_{a} d x_{b} d x_{c} \ldots d y_{a} d y_{b} d y_{c}}$

64. Let us define condition $q$ as the condition that there shall be a molecule having its coordinates within the limits $x^{\prime}$ and $x^{\prime}+d x^{\prime}$, etc. The volume $v_{p q}$ for which conditions $p$ and $q$ are both satisfied will consist of contributions from different pairs of molecules. In expression (116) we suppose molecule A to satisfy condition $p$. If molecule $\mathrm{B}$ satisfies condition $q$ the corresponding contribution to $v_{p q} / v$ can be obtained from the right-hand of (116) by limiting the integration in the numerator to the range from $x^{\prime}$ to $x^{\prime}+d x^{\prime}$ as regards $x_{b}$, and to similar ranges as regards $y_{b}, z_{b}$. The number of molecules capable of taking the rôle of $\mathrm{B}$ is

$$
N f\left(u^{\prime}, v^{\prime}, w^{\prime}\right) d u^{\prime} d v^{\prime} d w^{\prime}
$$

Hence we obtain as the value of $v_{p q} / v$ $\frac{v_{p q}}{v}=N^{2} f(u, v, w) f\left(u^{\prime}, v^{\prime}, w^{\prime}\right) d u d v d w d u^{\prime} d v^{\prime} d w^{\prime} d x d y d z d x^{\prime} d y^{\prime} d z^{\prime}$

$$
\times \frac{\iint \ldots d x_{c} \ldots d y_{c} \ldots}{\iiint \iint \ldots d x_{a} d x_{b} d x_{c} \ldots d y_{a} d y_{b} d y_{c}} \ldots \ldots
$$

The integration extends throughout all the values of the variables except such as are excluded by the conditions of $\S 33$. In applying these conditions to the numerator, we must replace $x_{a}, y_{a}, z_{a}$ by $x, y, z$ and $x_{b}, y_{b}, z_{b}$ by $x^{\prime}, y^{\prime}, z^{\prime}$. We therefore find, as we ought, that $v_{p q}$ vanishes when the points $x, y, z$ and $x^{\prime}, y^{\prime}, z^{\prime}$ are at a shorter distance than $\sigma$, or when either of them is at a distance from the boundary less than $\frac{1}{2} \sigma$. We also see that $v_{p q} / v$ is not equal to the product of $v_{p} / v$ and $v_{q} / v$, so that the fulfilment of conditions $p$ and $q$ cannot be treated as independent events.

65. In the special case in which the radii of the molecules are vanishingly small, those parts of the generalised space which are excluded in $\S 33$ may be neglected. In the integrals of equations (116) and (117) the integrations with respect to the variables with different suffixes now become independent. We may for instance write

$$
\iiint \iiint_{0} d x_{a} d x_{b} d x_{c} \ldots d y_{a} d y_{b} d y_{c} \ldots=\iiint d x_{a} d y_{a} d z_{a} \iiint d x_{b} d y_{b} d z_{b} \ldots=\Omega^{N}
$$

The other integrals can be simplified in a similar manner, and we obtain

$$
\frac{v_{p}}{v}=\frac{N}{\Omega} f(u, v, w) d u d v d w d x d y d z
$$

$\frac{v_{p q}}{v}=\left(\frac{\bar{N}}{\Omega}\right)^{2} f(u, v, w) f\left(u^{\prime}, v^{\prime}, w^{\prime}\right) d u d v d w d u^{\prime} d v^{\prime} d w^{\prime} d x d y d z d x^{\prime} d y^{\prime} d z^{\prime} \ldots$ 
Writing $\nu$ for $N / \Omega$ we see that the right-hand of equation (118) becomes identical with our former expression (2) (p. 14). In other words, in the case in which the density is constant throughout the gas, the probability that condition $p$ shall be satisfied for a system selected at random, is equal to the probability calculated in $\S 14$.

From equations (118) and (119) we have the important result

$$
\frac{v_{p q}}{v}=\frac{v_{p}}{v} \frac{v_{q}}{v}
$$

Thus the fulfilment of conditions $\alpha$ and $\beta$ are now independent events. In other words, we have proved that, relatively to our present basis of probability, the assumption of molecular chaos enunciated in $\$ 15$ is justifiable in the case in which the radii of the molecules are vanishingly small.

To justify the way in which this assumption was used in Chapter II. we must go somewhat further. In expression (4) we found a value which we supposed to be equal to the number of collisions of a certain class $\alpha$. The actual value of this expression was, however, equal to

(the number of possible collisions of class $\alpha$ )

$\times$ (the probability of each collision happening).

This quantity therefore expresses the probable number of collisions, the number which actually occur averaged over a large number of cases, or the "expectation" of collisions, but does not necessarily express the actual number in any particular case. Looked at from another point of view, however, the quantity expressed

$$
\begin{aligned}
& =(\text { the sum of a number of small elements of volume }) \\
& \qquad \times \text { (the density of molecules of class B)........(121). }
\end{aligned}
$$

Now there was nothing in analysis of Chapter III. to compel us to take the "cells" to be continuous in space. We may accordingly regard the first factor in expression (121) as one of these cells. We now see in accordance with the results of Chapter III. that expression (121) not only gives the "expectation" of molecules of class B in these elements of volume, but that it is infinitely probable that it gives the actual number, to within an infinitesimal fraction of the whole. It follows that the number of collisions of a given type found in Chapter III. not only gives the most probable number of collisions, but that it is infinitely probable that it gives the true number, to within an infinitesimal fraction of itself.

66. When the molecules are not vanishingly small, let us denote the integral

$$
\iiint \int \ldots d x_{b} d x_{c} \ldots d y_{b} d y_{c} \ldots
$$

taken over all values of the variables which are not excluded by $\S 33$, by $I(b, c \ldots)$. The value of the integral can only depend on $x_{a}, y_{a}, z_{a}$, and 
since we have supposed the molecule $\mathrm{A}$ to be at the point $x, y, z$, we may say that $I(b, c, \ldots)$ is a function of $x, y, z$ only. Equations (117) and (118) now become

$$
\begin{aligned}
\frac{v_{p}}{v} & \left.=N f(u, v, w) d u d v d w d x d y d z \frac{I(b, c, d \ldots)}{I(a, b, c, d \ldots)} \ldots \ldots \ldots \ldots \ldots \ldots \ldots . . . . . . . . . . .122\right) \\
\frac{v_{p q}}{v} & =N^{2} f(u, v, w) f\left(u^{\prime}, v^{\prime}, w^{\prime}\right) d u d v d w d x d y d z d u^{\prime} d v^{\prime} d w^{\prime} d x^{\prime} d y^{\prime} d z^{\prime} \frac{I(c, d \ldots)}{I(a, b, c, d \ldots)}
\end{aligned}
$$

From equation (122) we see that the density of molecules of class A at $x, y, z$ may no longer be taken to be

$$
\nu f(u, v, w) d u d v d w
$$

but must be taken to be

where

$$
\begin{aligned}
& \nu_{1} f(u, v, w) d u d v d w \\
& \nu_{1}=N \frac{I(b, c \ldots)}{I(a, b, c \ldots)} \ldots
\end{aligned}
$$

a quantity which reduces to $\frac{N}{\Omega}$, and therefore to $\nu$, when the molecules are infinitely small. In general $\nu_{1}$ is a function of $x, y, z$ but it is not a function of $u, v, w$. We may conveniently refer to $\nu_{1}$ as the "effective molecular density" at the point $x, y, z$. When we require to specify the point $x, y, z$ at which $\nu_{1}$ is estimated, we shall replace $\nu_{1}$ by $\nu_{x, y, z}$.

The "expectation" of the number of molecules in the whole vessel is equal to the total number of molecules actually present in the vessel, so that

$$
\iint \nu_{x, y, z} d x d y d z=\nu \Omega
$$

Thus $\nu$ is the mean value of $\nu_{x, y, z}$ averaged throughout the vessel. We shall see later the importance of the distinction between $\nu_{x, y, z}$ and $\nu$.

From equations (122) and (123) we obtain

$$
\frac{v_{p}}{v} \frac{v_{q}}{v}=\frac{v_{p q}}{v} \frac{I(b, c, d \ldots) I(a, c, d \ldots)}{I(c, d \ldots) I(a, b, c, d \ldots)} .
$$

Hence given that the molecule $\mathrm{A}$ is in position at $x, y, z$ the probability that a second molecule $\mathrm{B}$ has a position at $x^{\prime}, y^{\prime}, z^{\prime}$ is not that which would be given by the assumption of molecular chaos, but is equal to this value multiplied by

$$
\frac{I(c, d \ldots) I(a, b, c, d \ldots)}{I(b, c, d \ldots) I(a, c, d \ldots)}
$$

a function which is symmetrical as regards the $x, y, z$ coordinates of $\mathrm{A}$ and. $\mathrm{B}$, and which is independent of the velocities of the molecules $A$ and $B$. 


\section{Analysis of the H-theorem.}

67. The assumption of molecular chaos (corrected, if necessary, in accordance with $\S 66$ ) will therefore give correct results, provided it is interpreted with reference to the basis of probability supplied by our generalised space, and provided it is understood that it gives probable, and not certain, results. If we wish to obtain strictly accurate results the quantities calculated from it must not be regarded as applying to a single system, but must be supposed to be averaged over all the systems in the generalised space which satisfy certain conditions. For instance, the value of $\frac{\partial f}{\partial t}$ given by equation (12) is merely the value of $\frac{\partial f}{\partial t}$ averaged throughout all those parts of the space for which the system has a given $f$. So also we must interpret the value of $\frac{\partial H}{\partial t}$ given by equation (20) as an average value for $\frac{\partial H}{\partial t}$, taken over all systems in our space which have a given value for $H$.

68. We now come to what is, at first sight, a paradox. Let us suppose that $f$ is an even function of $u, v, w$ different from the normal function $A e^{-h m\left(u^{2}+v^{2}+w^{2}\right)}$. Then from Chapter II. $(\S 23)$ it follows that $d H / d t$ is negative, $d H / d t$ indicating, as we have just seen, the value of $d H / d t$ averaged over all the systems in our space which have this given $f$. Now these systems may be divided up into pairs. Corresponding to any system there will be a second system of which the positional coordinates will be the same as those of the first system and of which the velocity coordinates will be the same in magnitude but opposite in sign. Since $f$ is an even function of the velocity coordinates, the value of $f$ will be the same for each of these two systems and both systems are equally to be included in the average of $d H / d t$. But the motion of the first system is exactly the reverse of that of the second system. It would therefore appear as though the values of $d H / d t$ must be equal and opposite for the two systems, so that the average of $d H / d t$ for these two must be zero. Since the whole of the systems corresponding to a given $f$ fall into pairs of this type, it might be inferred that the average value of $d H / d t$ must be zero.

69. The explanation of the apparent paradox will be found to be contained in the result of $\S 52$, if we substitute $H$ for $K$. By a proof similar to that which led to the result in the case of the function $K$, we can prove the result for the function $H$. We therefore see that, of the systems for which $H$ has a value greater than some value $H_{1}$ other than the minimum value for $H$, all except an infinitesimal fraction have a value for $H$ which only differs infinitesimally from $H_{1}$. If, therefore, we select at random a point at which the value of $H$ is $H_{1}$, it is infinitely probable that $H$ will 
decrease as we recede from this point in either direction along the trajectory through the point. In other words, it is infinitely probable that the value $H=H_{1}$ is a maximum value of $H$ for the trajectory through the point.

70. It may, perhaps, still be thought paradoxical that $d H / d t$ is not zero at each of these maxima. The explanation is that the variation of $H$ is not governed by the laws of the differential calculus, since this variation is not, strictly speaking, continuous. The value of $H$ is constant between collisions of the molecules, and changes abruptly at every collision. When the number of molecules in the gas is infinite, the interval between successive collisions will become infinitely small, but in general the variation in $H$ will not be continuous. For obviously the differential coefficients of $H$ vanish between collisions and become infinite at every collision, so that $H$, regarded as a function of $t$ as we follow any trajectory, will be a function of the well-known type which possesses an infinite number of maxima and minima within a finite range of the variable. We can, however, "smooth out" the curve obtained for $H$ as a function of $t$ and in this way obtain the function $H$ as a continuous function of the time. This is the $H$ contemplated by the analysis of Chapter II. But now we can also see that there is no reason to suppose that $d H / d t$ will vanish when $H$ attains a maximum value, but that on the contrary $H$ will in general change sign abruptly at such a point. It is therefore clear that, averaged over all systems which have a given $f$, $d H / d t$ will be negative except when $f$ is the law of distribution for the normal state, in which case it is zero. This result is now in agreement with that of Chapter II. 


\section{CHAPTER V.}

THE LAW OF DISTRIBUTION“ (CONTINUED).

\section{Extension to Molecules of the most general Conservative Type.}

71. In the present chapter we abandon the supposition that the molecules are hard and smooth elastic spheres, and try to develop a theory for a gas of which the molecules are conservative systems of the most general type. The assumption that the molecules form conservative systems finds no justification in nature, but the analysis of this chapter is a necessary preliminary to the discussion of the more general case, in which the dissipation of energy is taken into account.

We shall no longer suppose the molecules of the gas to be all exactly similar, but shall suppose that the gas consists of a mixture of a finite number of definite types of molecules. Also we shall no longer suppose that forces only come into play between two molecules when they are in actual contact, but shall suppose that the forces may act between the molecules at all distances. These forces may be of the most general type consistent with the conservation of energy, subject only to an assumption which is justified by experiment, and which will be explained later. We may still find it convenient to speak of a "collision" between a pair of molecules, but a "collision" is not, as before, a clearly defined occurrence. When the forces between a pair of molecules become very great, as they necessarily will when the centres of the molecules approach sufficiently near to one another, we may, if we please, say that the molecules are in collision with one another, but this statement rests upon the assumption of a purely arbitrary standard for the magnitude to which the forces must attain before we regard their action as constituting a collision. If we assign a high value to this quantity, a collision may, in general, be expected to change somewhat abruptly the paths described by both molecules, but we shall not be justified in assuming that the path between collisions is rectilinear. If on the other hand we assign to it a low value, the average change in the paths of the molecules will be much less, but then possibly it will not be legitimate to assume, even as a first approximation, that a molecule is not engaged in more than one collision at once. 
We shall suppose that in addition to these intermolecular forces, the whole gas is under the influence of a permanent external field of force. No assumption will be made about this field of force, except that the potential energy of a molecule in the field is a single-valued function of the coordinates of the molecule, this assumption being required to ensure conservation of energy.

\section{Liouville's Theorem.}

72. Let a conservative dynamical system of the most general kind be specified by $n$ coordinates $q_{1}, q_{2} \ldots q_{n}$. Let the corresponding momenta be denoted by $p_{1}, p_{2} \ldots p_{n}$, these being given by

$$
p_{8}=\frac{\partial E}{\partial \dot{q}_{8}}, \text { etc. }
$$

where $E$ is the energy of the system expressed as a function of the $q$ 's and $\dot{q}$ 's. We can represent this dynamical system in a space of $2 n$ dimensions corresponding to $2 n$ variable coordinates

$$
p_{1}, p_{2} \ldots p_{n}, q_{1}, q_{2} \ldots q_{n}
$$

The equations of motion of the system expressed in the Hamiltonian form are of the type

$$
\begin{aligned}
& \frac{\partial p_{8}}{\partial t}=-\frac{\partial E}{\partial q_{8}} \\
& \frac{\partial q_{8}}{\partial t}=\frac{\partial E}{\partial p_{8}}
\end{aligned}
$$

These, therefore, are the equations of the trajectories in this generalised space. Let us suppose the space filled with fluid, initially of density $\rho$. Let $D \rho / D t$ be the rate of increase of $\rho$ as we follow any element of fluid in its motion. In three dimensions, the hydrodynamical equation of continuity can, with the usual notation, be put in the form

$$
\frac{D \rho}{D t}+\rho\left(\frac{\partial u}{\partial x}+\frac{\partial v}{\partial y}+\frac{\partial w}{\partial z}\right)=0
$$

In our present $2 n$-dimensional case the last bracket must be replaced by

$$
\Sigma \frac{\partial \dot{\xi}}{\partial \xi}
$$

where $\xi$ is any one of the $2 n$ coordinates (127), and the summation extends to all. This again may be replaced by

$$
\sum_{s=1}^{s=n}\left(\frac{\partial \dot{p}_{8}}{\partial p_{8}}+\frac{\partial \dot{q}_{8}}{\partial q_{8}}\right)
$$

and the general equation of continuity for our present space is therefore

$$
\frac{D \rho}{D t}+\rho \sum_{s=1}^{s=n}\left(\frac{\partial \dot{p}_{8}}{\partial p_{8}}+\frac{\partial \dot{q}_{s}}{\partial q_{s}}\right)=0
$$


If the fluid is to represent the motion of the system, the equations of motion of the fluid must be equations (128) and (129). From these we get, by differentiating with respect to $p_{s}, q_{8}$ and adding,

$$
\frac{\partial \dot{p}_{8}}{\partial p_{s}}+\frac{\partial \dot{q}_{8}}{\partial q_{s}}=0
$$

whence equation (130) takes the form

$$
\frac{D \rho}{D t}=0
$$

In other words, $\rho$ remains constant throughout the whole motion of any element, so that if the fluid is initially homogeneous it will always remain so. This is the general theorem of which the result proved in $\S 37$ was a particular case. We now see that the result there proved is true for a generalised space representing any conservative dynamical system, provided the coordinates in this space are the generalised coordinates of this system in the sense required for the Hamiltonian equations (128) and (129).

\section{Application to a Gas.}

73. We now suppose our system, as before, to be the gas contained in a vessel. We shall suppose this gas to consist of

$N_{\alpha}$ molecules of type $\alpha$;

$N_{\beta}$ molecules of type $\beta$, and so on.

A molecule of type $\alpha$ will be specified, let us suppose, by $n_{\alpha}$ positional coordinates, and the corresponding $n_{a}$ momenta $-2 n_{a}$ coordinates in all. The whole system will therefore be specified by

$$
\Sigma N_{a} n_{a} \equiv N_{a} n_{a}+N_{\beta} n_{\beta}+\ldots
$$

positional coordinates and an equal number of momenta.

Let $E_{a}, E_{b}$ denote the energies of molecules $\mathrm{A}, \mathrm{B}, \ldots$ when free from intermolecular forces, but when in position in the permanent field of force. Then $E_{a}$, for instance, is a function of the $2 n_{a}$ coordinates of position and momentum which belong to molecule $\mathrm{A}$, and if $q_{8}, p_{8}$ are a pair of corresponding coordinates of position and momentum belonging to this molecule, we have

$$
p_{s}=\frac{\partial E_{a}}{\partial \dot{q}_{s}}
$$

The total energy of the gas is

$$
\mathbb{E}=E_{a}+E_{b}+\ldots+\Phi .
$$

where $\Phi$ is the total potential energy arising from the intermolecular forces. This quantity $\Phi$ is a function solely of the positional coordinates of the various molecules: it does not depend on velocities. Hence in the righthand member of equation (133) none of the terms will depend on $\dot{q}_{s}$ except 
$E_{a}$, the energy of the molecule A to which the coordinate $q_{8}$ belongs; and hence we have

$$
\frac{\partial \mathfrak{E}}{\partial \dot{q}_{8}}=\frac{\partial E_{a}}{\partial \dot{q}_{8}} .
$$

Equation (132) may now be written in the form

$$
p_{8}=\frac{\partial \dot{\xi}}{\partial \dot{q}_{s}}
$$

and from this it follows that the coordinates we have specified form pairs of true coordinates of position and momenta for the whole system of molecules of which the energy is $\mathbb{E}$.

If, therefore, we construct a generalised space of $2 \Sigma N_{a} n_{a}$ coordinates, to represent all the coordinates of position and momenta of all the molecules, and if we fill this space with fluid, initially homogeneous, the motion of which is to represent the motion of the gas, then this fluid will, by the theorem proved in the last section, remain homogeneous through all time.

74. We now turn to the assumption which has to be made about the intermolecular forces.

If a gas is compressed into a vessel, and is then allowed to stream out into a vacuum, the total energy of the gas, given by equation (133), must remain unaltered by the process.

Now as we shall see later, a measure of the quantity $E_{a}+E_{b}+\ldots$, or what is the same thing, of $\mathbb{E}-\Phi$ is supplied by the temperature of the gas. Joule and Kelvin have found experimentally that the change in the temperature of a gas, consequent on allowing it to stream into a vacuum, is extremely small. The inference is that the change in the quantity $\Phi$ is extremely small. When the gas is very rare, it is obvious that $\Phi$ must be very small, and hence we conclude that for a gas $\Phi$ is in general very small, at any rate within the limits covered by the experiments just quoted.

The quantity $\Phi$ is of course the potential energy of the forces of cohesion of the gas. Thus Joule and Kelvin's result may be stated by saying that the cohesion in gases is extremely small. And from this we infer that equation (133) may, without appreciable error, be written in the form

$$
\mathfrak{E}=E_{a}+E_{b}+\ldots
$$

Stated in words this equation expresses that the energy of a gas may be assumed equal to the sum of the energies of its molecules.

75. From this point the treatment is exactly similar to that of Chapter III.

Let a molecule of type $\alpha$ be specified by the $2 n_{a}$ coordinates

$$
\xi_{1}, \xi_{2} \ldots \xi_{2 n_{a}}
$$


and let the number of molecules for which $\xi_{1}$ lies between $\xi_{1}$ and $\xi_{1}+d \xi_{1}$, etc., be

$$
N_{a} f_{a}\left(\xi_{1}, \xi_{2} \ldots\right) d \xi_{1} d \xi_{2} \ldots
$$

Then, if $\mathfrak{S}$ is defined by

$$
\mathfrak{H}=\Sigma_{a} N_{a} \iint \ldots f_{a} \log f_{a} d \xi_{1} d \xi_{2} \ldots
$$

where the summation extends over all types of molecules, it can be shewn, precisely as before, that for all except an infinitesimal fraction of that part of the space for which $\mathbb{E}$ has a given value, $\mathfrak{S}$ has the minimum value which is consistent with this value of $(5$.

Let $E_{a}$ be the energy of a molecule of type $\alpha$ specified as a function of the $2 n_{a}$ coordinates (136). Then equation (135) may be written in the form

$$
\xi=\sum_{a} N_{a} \iint \ldots E_{a} f_{a} d \xi_{1} d \xi_{2} \ldots
$$

in which the summation extends over all the types of molecules. In addition to the functions $f_{a}, f_{\beta} \ldots$ being subject to the constraint expressed by equation (139), they are subject also to separate constraints expressing that the number of molecules of each type remains constant. The equation expressing this constraint in the case of $f_{a}$ is

$$
\iint \ldots N_{a} f_{a} d \xi_{1} d \xi_{2} \ldots=N_{a}
$$

The equations resulting from the variation of equations (138), (139) and (140), keeping $\&$ and $N_{a}, N_{\beta} \ldots$ constant, are

$$
\begin{aligned}
\delta \mathfrak{S} & =\sum_{a} N_{a} \iint \ldots\left(1+\log f_{a}\right) \delta f_{a} d \xi_{1} d \xi_{2} \ldots \\
0 & =\sum_{a} N_{a} \iint \ldots E_{a} \delta f_{a} d \xi_{1} d \xi_{2} \ldots \ldots \ldots . \\
0 & =N_{a} \iint \ldots \delta f_{a} d \xi_{1} d \xi_{2} \ldots \ldots \ldots \ldots \ldots . .
\end{aligned}
$$

The most general variation possible, subject to the constancy of $(5$, $N_{a}, N_{\beta} \ldots$, is therefore given by the equation

$$
\delta \mathfrak{S}=\sum_{a} N_{a} \iint \ldots\left(1+\log f_{a}+\lambda E_{a}+\mu_{a}\right) \delta f_{a} d \xi_{1} d \xi_{2} \ldots \ldots(144),
$$

where $\lambda, \mu_{a}, \mu_{\beta} \ldots$ are undetermined multipliers.

The minimum value for $\mathfrak{H}$ is accordingly given by the system of equations

$$
\left.\begin{array}{l}
1+\log f_{a}+\lambda E_{\alpha}+\mu_{\alpha}=0, \\
1+\log f_{\beta}+\lambda E_{\beta}+\mu_{\beta}=0, \text { etc. }
\end{array}\right\}
$$

Changing the constants, the solution of these equations is given by

$$
\left.\begin{array}{l}
f_{a}=A_{\alpha} e^{-2 h E_{a}}, \\
f_{\beta}=A_{\beta} e^{-2 h E_{\beta}, \text { etc. }}
\end{array}\right\}
$$

J. 
The constants $A_{\alpha}, A_{\beta} \ldots$ are to be determined from equations of the type (140). The constant $h$ depends on the total energy, and must be determined from equation (139).

76. We have therefore proved that for a gas about which nothing is known except the total energy, it is infinitely probable that the law of distribution of coordinates will be that expressed by equations (146). But in the present case the function $(\xi$ does not necessarily maintain the position which it has been supposed up to now to hold, of being the only function of the coordinates in the generalised space which remains constant throughout the motion of the gas. In arriving at equations (146) it has in no way been assumed that $\&$ does hold this position, but if $(\xi$ does not hold this position the importance of equations (146) is diminished, in that the information then supplied is not precisely that which is of most importance.

Let us suppose that there are other quantities $\chi, \psi \ldots$ functions of the coordinates of the generalised space, which have the property, in common with $\mathbb{E}$, of remaining constant throughout every possible motion of the gas. Then if we know the values of the quantities $\chi, \psi \ldots$ as well as of $\mathbb{E}$, the information we require is the most probable law of distribution consistent with these values of $\chi, \psi \ldots$ and $\xi$. This information is not supplied by equations (146), for the law of distribution implied in these equations may be inconsistent with these values of $\chi, \psi \ldots$, and even if it is not, it is by no means obvious that this law remains the most probable after the limitations have been imposed on the values of $\chi, \psi \ldots$.

If we know the values of $\chi, \psi \ldots$ in practice, these values must be deducible from the mass-properties of the gas, without reference to its molecular properties. And consistent with the mass-equilibrium of the gas, there are only six quantities other than the energy, which can be measured from the mass-properties of the gas, these being the three components of linear momentum and the three components of angular momentum. These quantities are of course obtained by summation over all the molecules. They remain constant, except in special cases, only when the gas has no boundary, or when the boundary is either of a special shape, as in $\S 38$, or moves or rotates with a given velocity.

If some or all of these quantities remain constant throughout the motion, the variation of $\mathfrak{S}$ will be subject not only to equations (141), (142), (143), but also to some or all of the following equations

$$
0=\sum_{\alpha} N_{\alpha} \iint \ldots m_{\alpha} u \delta f_{\alpha} d \xi_{1} d \xi_{2} \ldots
$$

and two similar equations for $v$ and $w$,

$$
0=\sum_{\alpha} N_{\alpha} \iint \ldots m_{\alpha}(y w-z v) \delta f_{a} d \xi_{1} d \xi_{2} \ldots
$$


and two similar equations with $z u-x w$ and $x v-y u$ respectively instead of $y w-z v$.

Instead of being given by equations (145) the minimum value of $\mathfrak{S}$ is now given by a system of equations of the form

$$
\left.\begin{array}{l}
1+\log f_{a}+\lambda E_{a}+\mu_{a}+\Sigma p_{1} m_{a} u+\Sigma q_{1} m_{a}(y w-z u)=0 \\
1+\log f_{\beta}+\lambda E_{\beta}+\mu_{\beta}+\Sigma p_{1} m_{\beta} u+\Sigma q_{1} m_{\beta}(y w-z u)=0
\end{array}\right\}
$$

in which $p_{1}, p_{2}, p_{3}, q_{1}, q_{2}, q_{3}$ are new undetermined multipliers derived from the six equations of which (147) and (148) are typical. In the case in which any of the six momenta are not known to be constant, the corresponding multipliers must be put equal to zero in equations (149).

The solution (146) must now be replaced by the more general solution

$$
\left.\begin{array}{l}
f_{\alpha}=A_{\alpha} e^{-2 h E_{\alpha}-m_{\alpha} \Sigma\left\{p_{1} u+q_{1}(y w-z v)\right\}}, \\
f_{\beta}=A_{\beta} e^{-2 h E_{\beta}-m_{\beta} \Sigma\left\{p_{1} u+q_{1}(y w-z v)\right\}}, \text { etc. }
\end{array}\right\}
$$

in which the unknown quantities $p_{1}, p_{2}, p_{3}, q_{1}, q_{2}, q_{3}$ are to be deduced from the corresponding momenta when these remain constant, and put equal to zero when they do not remain constant. Obviously the solution expressed by equation (146) is a special case of that expressed by (150).

This generalisation of equations (146) is, however, of abstract interest only. In future we shall suppose the law of distribution to be expressed by equations (146), assuming in so doing that the momenta are zero, so that the gas as a whole has neither translational nor rotational motion.

\section{The Partition of Energy.}

77. Of the $2 n_{a}$ coordinates (136) of which $E_{a}$ is a function, let $\xi_{1}, \xi_{2} \ldots \xi_{n}$ be positional coordinates, and $\xi_{n+1} \ldots \xi_{2 n}$ be momenta, the suffix $\alpha$ now being omitted, as we are only going to consider one type of molecule. In general $E_{a}$ will consist of two parts, the kinetic energy $L$ which will be a quadratic function of $\xi_{n+1} \ldots \xi_{2 n}$ with coefficients involving $\xi_{1} \ldots \xi_{n}$, and secondly the potential energy $V$ which will depend only on $\xi_{1} \ldots \xi_{n}$. We therefore write

$$
E_{a}=L+V \text {, }
$$

where

$$
2 L=a_{11} \xi_{n+1}^{2}+2 a_{12} \xi_{n+1} \xi_{n+2}+
$$

It is known that by a linear substitution of the form

$$
\left.\begin{array}{l}
\xi_{n+1}=b_{11} \eta_{1}+b_{12} \eta_{2}+\ldots \\
\xi_{n+2}=b_{21} \eta_{1}+b_{22} \eta_{2}+\ldots, \text { etc. }
\end{array}\right\}
$$

the function $L$ may be reduced to a sum of squares of the form

$$
2 L=c_{1} \eta_{1}^{2}+c_{2} \eta_{2}^{2}+\ldots+c_{n} \eta_{n}^{2} \text {. }
$$


The quantities $\eta_{1}, \eta_{2} \ldots$ will be referred to as momentoids, and the form of equation (153) shews that the kinetic energy of the molecule may be regarded as the sum of contributions from various momentoids.

There are an infinite number of possible substitutions of the kind required, for if in (152) we replace $b_{11}, b_{12} \ldots$ by $K b_{11}, K b_{12} \ldots$, and $\eta_{1}, \eta_{2} \ldots$ by $\eta_{1} / K, \eta_{2} / K \ldots$, the substitution is still a possible one and reduces $2 L$ to a sum of squares as before. The modulus of transformation for this new substitution is the Jacobian

$$
\frac{\partial\left(\xi_{n+1}, \xi_{n+2} \ldots\right)}{\partial\left(\frac{\eta_{1}}{K}, \frac{\eta_{2}}{K} \ldots\right)}=K^{n}\left|\begin{array}{c}
b_{11}, b_{12} \ldots \\
b_{21}, b_{22} \ldots \\
\ldots \ldots \ldots \ldots . . . \\
\ldots \ldots \ldots . .
\end{array}\right|,
$$

and is therefore $K^{n}$ times the modulus of the original substitution. By a suitable choice of $K$, the right-hand member of the foregoing equation can always be made equal to unity. We shall suppose such a value of $K$ to have already been absorbed in the quantities which occur in the transformation (152), so that

$$
\frac{\partial\left(\xi_{n+1}, \xi_{n+2} \ldots\right)}{\partial\left(\eta_{1}, \eta_{2} \ldots\right)}=1
$$

If we now transform variables according to scheme (152), so that the new variables are

$$
\xi_{1}, \xi_{2} \ldots \xi_{n}, \eta_{1}, \eta_{2} \ldots \eta_{n} \ldots \ldots \ldots \ldots \ldots \ldots \ldots \text { (155), }
$$

we see from expression (137) that the number of molecules for which $\xi_{1}$ lies between $\xi_{1}$ and $\xi_{1}+d \xi_{1}, \eta_{1}$ between $\eta_{1}$ and $\eta_{1}+d \eta_{1}$, etc., will be

$$
N_{a} f_{a}\left(\xi_{1}, \xi_{2} \ldots \xi_{n}, \eta_{1} \ldots \eta_{n}\right) d \xi_{1} d \xi_{2} \ldots d \xi_{n} \frac{\partial\left(\xi_{n+1}, \xi_{n+2} \ldots\right)}{\partial\left(\eta_{1}, \eta_{2} \ldots\right)} d \eta_{1} \ldots d \eta_{n},
$$

and this, by equation (154), may be written

$$
N_{a} f_{\alpha}\left(\xi_{1}, \xi_{2} \ldots \xi_{n}, \eta_{1} \ldots \eta_{n}\right) d \xi_{1} d \xi_{2} \ldots d \xi_{n} d \eta_{1} \ldots d \eta_{n}
$$

while from equation (146) we have

$$
f_{\alpha}=A_{a} e^{-2 h E_{a}}=A_{a} e^{-2 h V-h\left(c_{1} \eta_{1}^{2}+\ldots+c_{n} \eta_{n}^{2}\right)}
$$

If, then, we denote by $\overline{c_{1} \eta_{1}{ }^{2}}$ the value of $c_{1} \eta_{1}{ }^{2}$ averaged over all molecules of type $\alpha$, we shall have

$$
\overline{c_{1} \eta_{1}^{2}}=\frac{\iiint \int \ldots e^{-2 h V-h\left(c_{1} \eta_{1}{ }^{2}+\ldots+c_{n} \eta_{n}{ }^{2}\right.} c_{1} \eta_{1}{ }^{2} d \xi_{1} \ldots d \xi_{n} d \eta_{1} \ldots d \eta_{n}}{\iiint \int \ldots e^{-2 h V-h\left(c_{1} \eta_{1}{ }^{2}+\ldots+c_{n} \eta_{n}{ }^{2}\right)} d \xi_{1} \ldots d \xi_{n} d \eta_{1} \ldots d \eta_{n}} \ldots \ldots
$$

The integrals in both numerator and denominator are of the type

$$
\iiint \int \ldots I e^{-2 h V-h\left(c_{2} \eta_{2}^{2}+\ldots+c_{n} \eta_{n}{ }^{2}\right)} d \xi_{1} \ldots d \xi_{n} d \eta_{2} \ldots d \eta_{n}
$$


where in the numerator we have as the value of $I$,

$$
I_{n} \equiv \int_{-\infty}^{+\infty} e^{-h e \eta_{1}{ }^{2}} c_{1} \eta_{1}^{2} d \eta_{1}
$$

and in the denominator we bave as the value of $I$,

$$
I_{d} \equiv \int_{-\infty}^{+\infty} e^{-h c_{1} \eta_{1}^{2}} d \eta_{1}
$$

Since $c_{1}$ does not involve $\eta_{1}$, we have

$$
\begin{aligned}
& I_{n}=\frac{1}{\sqrt{h^{3} c_{1}}} \int_{-\infty}^{+\infty} e^{-x^{2}} x^{2} d x=\frac{1}{2} \sqrt{\frac{\pi}{h^{3} c_{1}}}, \\
& I_{d}=\frac{1}{\sqrt{h c_{1}}} \int_{-\infty}^{+\infty} e^{-x^{2}} d x=\sqrt{\frac{\pi}{h c_{1}}} .
\end{aligned}
$$

Substituting these values in the numerator and denominator of equation $(158)$ we find that the whole equation reduces to

$$
\overline{c_{1} \eta_{1}^{2}}=\frac{1}{2 h}
$$

This equation shews that the mean energy of each-momentoid is equal to $\frac{1}{4 h}$, a quantity which is independent not only of the particular momentoid chosen, but also of the type of molecule to which it belongs.

For any type of molecule, three of the momentoids may always be taken to correspond to the three components of the velocity of the centre of gravity. Taking these to be the last three momentoids, we have

$$
E_{a}=V+\frac{1}{2}\left[m_{a}\left(u^{2}+v^{2}+w^{2}\right)+c_{1} \eta_{1}{ }^{2}+c_{2} \eta_{2}{ }^{2}+\ldots+c_{n-3} \eta_{n-3}{ }^{2}\right] \ldots(160),
$$

and our result may be expressed in the form

$$
\overline{m_{a} u^{2}}=\overline{m_{a} v^{2}}=\overline{m_{a} w^{2}}=\overline{c_{1} \eta_{1}^{2}}=\ldots=\overline{m_{\beta} u^{2}}=\overline{m_{\beta} v^{2}}=\ldots=\frac{1}{2 h} \ldots
$$

These equations express the law of Equipartition of Kinetic Energy, a law of which the importance will appear later.

\section{Law of Distribution of Velocities and Density.}

78. From expression (156) it follows that if $\eta_{n-2}, \eta_{n-1}$ and $\eta_{n}$ are the momentoids of translational energy, the number of molecules of type $\alpha$ for which $u, v, w$ lie within a small range $d u d v d w$ is

$$
\begin{aligned}
& N_{a} d u d v d w \iiint \ldots f_{a}\left(\xi_{1}, \xi_{2} \ldots \xi_{n}, \eta_{1}, \eta_{2} \ldots \eta_{n}\right) \\
& \qquad \frac{\partial\left(\eta_{n-2}, \eta_{n-1}, \eta_{n}\right)}{\partial(u, v, w)} d \xi_{1} d \xi_{2} \ldots d \xi_{n} d \eta_{1} \ldots d \eta_{n-3} \ldots \text { ( }
\end{aligned}
$$

In the present case,

$$
f_{a}\left(\xi_{1} \ldots \xi_{n}, \eta_{1} \ldots \eta_{n}\right)=e^{-h m_{a}\left(u^{2}+v^{2}+w^{2}\right)} A_{a} e^{-2 h V-h\left(c_{1} \eta_{1}{ }^{2}+\ldots+c_{n-3} \eta_{n-3} \eta^{2}\right)},
$$


so that expression (162) can be written in the form

$$
B_{a} e^{-h m_{a}\left(u^{2}+v^{2}+w^{2}\right)} d u d v d w \text {. }
$$

where $B_{a}$ is independent of $u, v$ and $w$. It follows that the law of distribution of translational velocities is of the form

$$
f_{a}(u, v, w)=C_{a} e^{-h m_{a}\left(u^{2}+v^{2}+w^{2}\right)}
$$

In other words, the law of distribution of translational velocities is the same as if the molecules were the smooth rigid spheres of Chapters II. and III.

79. In the only case of any importance, the potential energy can be written in the form

$$
V=V_{i}+\chi
$$

where $V_{i}$ is the potential energy of the molecule under its internal forces, and $\chi$ is its potential in the external field of force and depends only on $x, y, z$, the coordinates of its centre of gravity. This covers the case of a gas acted upon by a gravitational field of force-for instance, the atmosphere. In this case we have

$$
E_{\alpha}=V_{i}+\chi+L
$$

and $\chi$ is the only part which involves $x, y, z$.

By an argument similar to that of the last section, it will be seen that the number of molecules for which $x$ lies between $x$ and $x+d x$, and similar conditions are satisfied by $y, z$, is of the form

$$
D_{a} e^{-2 h x} d x d y d z \text {. }
$$

In other words, if $\nu_{\alpha}$ is the molecular density of gas of type $\alpha$ at the point $x, y, z$ we have

$$
\nu_{\alpha}=D_{\alpha} e^{-2 h x}
$$

Also it appears that the number of molecules of type $\alpha$ which occur per unit volume at $x, y, z$ and of which the coordinates lie within the usual range of limits $d \xi_{1} \ldots d \xi_{n-3} d \eta_{1} \ldots d \eta_{n}$ is proportional to

$$
\nu_{a} e^{-2 h\left(V_{i}+L\right)} d \xi_{1} \ldots d \xi_{n-3} d \eta_{1} \ldots d \eta_{n}
$$

This shews that the field of external force does not affect the law of distribution of the coordinates of the molecule, other than $x, y, z$, or the law of distribution of velocities, but that the distribution of density alone is affected.

The result expressed by equation (165) is simply a more general case of that expressed by equation (94), namely

$$
\nu=\text { constant, }
$$

in the case of hard spherical molecules. The quantity $\nu$ is the moleculardensity defined as in $\S 11$, being in fact the true density averaged through a "cell" which is sufficiently large to contain a very great number of molecules. 


\section{Rotating Gas.}

80. An interesting example of the treatment of a permanent field of force is supplied by the case of a gas and its containing vessel rotating together with an angular velocity $\omega$ about the axis of $z$. We can reduce the problem to a statical one by supposing the gas acted on by a field of force of potential $\frac{1}{2} \omega^{2}\left(x^{2}+y^{2}\right)$, and therefore have as the law of distribution (equation 146)

$$
f=A e^{-2 h E+h m \omega^{2}\left(x^{2}+y^{2}\right)}
$$

where $E$ stands for $\frac{1}{2} m\left(u^{2}+v^{2}+w^{2}\right)+\ldots$ and $u, v, w$ are the velocities referred to axes fixed in the containing vessel. To transform these velocities to axes fixed in space, we must write $u-\omega y$ for $u$ and $v+\omega x$ for $v$. The value of $E$, referred to fixed axes, must therefore be taken to be

$$
E=E_{0}+m \omega(x v-y u)+\frac{1}{2} m \omega^{2}\left(x^{2}+y^{2}\right),
$$

where $E_{0}$ is formally the same as $E$, but the velocities are now referred to fixed axes. Equation (167) now becomes

$$
f=A e^{-2 h\left(E_{0}-m \omega(x v-y u)\right)}
$$

giving the law of distribution of velocities referred to fixed axes. This equation is, as it ought to be, a special case of equation (150) in which the same law of distribution was found by another method.

\section{Calculation of Effective Density.}

81. If we wish to calculate the probability of finding the centre of a molecule within a specified small element of volume of dimensions comparable with the size of a single molecule, we must proceed as was done in the case of hard spherical molecules in Chapter IV. This probability was there calculated from an "effective-density" which became identical with the true density when the molecules were infinitely small, but which in general depended on the size of the molecules.

In the former instance the size of the molecules entered into our calculations by excluding a certain region of the generalised space. In the present case the size of the molecules is not a very definite conception, any more than is a collision between molecules (cf. $\S 71$ ), but there is; as we shall now see, no want of definiteness as to the regions of the generalised space which are to be excluded.

In equation (133) we expressed the total energy of the gas in the form

$$
\tilde{E}=E_{a}+E_{b}+\ldots+\Phi
$$

where $E_{a}, E_{b}, \ldots$ are the energies of the separate molecules, and $\Phi$ is the total potential energy of the intermolecular forces, and is therefore a function of the positional coordinates only. In the case of the elastic spheres, $\Phi$ 
vanishes so long as no two molecules or no molecule and the boundary overlap, and becomes infinite if this takes place. Or, what is the same thing, $\Phi$ vanishes throughout all those parts of the generalised space which were retained in $\S 33$, and becomes infinite throughout those parts which were excluded. The parts which were excluded might have been supposed to be determined either by the condition that $\Phi$ should be greater than zero, or that $\Phi$ should be infinite.

In the present instance we shall suppose all regions excluded for which

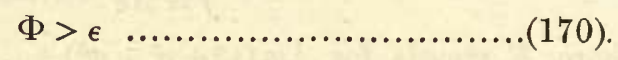

If $\epsilon$ is small in comparison with $(\xi$, equation (169) may, without perceptible error, be replaced by the equation

$$
\text { E }=E_{a}+E_{b}+\ldots
$$

as has been done throughout this Chapter.

If $\epsilon$ is so great that a system is only inside the region defined by the inequality (170) throughout an infinitesimal fraction of its whole path, then the results obtained in this Chapter (which in strictness apply only to a gas in the non-excluded region) may be supposed to apply to a gas throughout its whole path.

In virtue of the assumption which it was announced that we were going to make in $\S 74$, and for which experimental justification was given, it is possible to choose $\epsilon$ so that these two conditions shall be satisfied simultaneously. We therefore agree that the region in which $\Phi>\epsilon$ is to be excluded from the generalised space, where $\epsilon$ is chosen in this way.

The effective molecular density can now be determined exactly as in Chapter IV. We are, however, ignorant of the particular nature of the intermolecular forces which occur in nature, and it would hardly be profitable to try to determine the effective molecular density in general terms. It will perhaps be sufficient to remark that it is a function not only of $x, y, z$ the coordinates of the point in space, but also of the type of molecule which we are supposing to be found there, and also of the internal positional coordinates of this molecule, if we assume the intermolecular forces to depend on these coordinates.

\section{Sphere of Molecular Action.}

82. In this connection, the conception of a "sphere of molecular action" is sometimes useful. We assume that a sphere of diameter $\sigma$ can be drawn about the centre of gravity of every molecule, such that

(i) the intermolecular forces between two molecules are inappreciable, so long as the two "spheres of molecular action" do not intersect, 
(ii) when two "spheres of molecular action" intersect, the time which elapses before they again separate is inappreciable in comparison with the average time between two consecutive collisions of the same molecule, a collision now being supposed to take place whenever two spheres intersect.

It will be noticed that although the quantity $\sigma$ is at our disposal, yet we have to assume it to satisfy two distinct conditions, and these will not, in general, be satisfied by the same values of $\sigma$. There is no experimental justification for assuming that in the case of an actual gas they can be satisfied simultaneously: the sole merit of the conception of a "sphere of molecular action," is that it introduces a certain definiteness into a class of problems of which the treatment otherwise is often impracticable, and that at the same time it leads to results which, although not strictly accurate, often give a very fair approximation to what is found in nature.

If we decide provisionally to admit this conception, it is clear that we may exclude from the generalised space all regions in which two spheres intersect, or of course in which one sphere intersects the boundary, and may suppose that when a collision occurs, the representative point in the generalised space moves along the boundary of the excluded region, exactly as in $\S 35$. In fact we may, in calculating the "effective molecular density" regard the molecules as the rigid spheres of Chapter IV., the diameter of the sphere of molecular action now being identical with the diameter of the actual molecule.

At the same time it is important to notice that the assumption of a sphere of molecular action leaves very much more freedom for the structure of the molecule than does the assumption that the molecules are elastic spheres. For after assuming the existence of a sphere of molecular action, it is still open for the molecule to have any shape we please: all that is required is that it shall not protrude outside its sphere of action. Also its interaction with a second molecule may be of any nature we please: all that is required is that the interaction between two molecules shall be negligible so long as their spheres do not overlap. Lastly, consistent with the assumption of a sphere of molecular action, a molecule may have an internal structure as complex as we please.

\section{General Intermolecular Forces.}

83. Before leaving the questions of the present Chapter, let us examine the problem of determining the law of distribution of velocities and coordinates in a gas in which the assumption of the smallness of the intermolecular forces is not justifiable. Let us say that an encounter between two molecules takes place as soon as the potential energy of the inter- 
molecular forces between them becomes appreciable, and let us suppose encounters to be possible in which more than two molecules are engaged at once.

Instead of being written in the form (133) the total energy of the gas may be put in the form

$$
\tilde{E}=E_{a}+E_{b}+\ldots+W_{a b}+\ldots+W_{c d e}+\ldots
$$

in which $W_{a b}$ is the potential energy of the intermolecular forces between the molecules $A$ and $B$, supposed engaged in an encounter in which no other molecules are engaged, $W_{\text {cde }}$ is the potential energy of the intermolecular forces between the three molecules $C, D$, and $E$, supposed engaged in an encounter in which no other molecules are engaged, and so on. This equation only differs from equation (133) in that the intermolecular energy $\Phi$ is expressed as the sum of the intermolecular energies of the various encounters.

Let us now regard the molecules $A$ and $B$ as forming a single dynamical system, which we shall refer to as a "double-molecule." Let the molecules $C, D$, and $E$ form a single dynamical system-a "triple-molecule"; and so on. The energy $E_{a b}$ of the double molecule $A B$ may be supposed to be given by

$$
E_{a b}=E_{a}+E_{b}+W_{a b}
$$

that of the triple molecule $C D E$ by

$$
E_{\text {cde }}=E_{c}+E_{d}+E_{e}+W_{\text {cde }}
$$

and so on. The whole energy of the gas at any single instant is now given by

$$
\leftleftarrows=\Sigma_{1} E+\Sigma_{2} E+\Sigma_{3} E+\ldots
$$

where $\Sigma_{1} E$ is the sum of the energies of all the single molecules, i.e., molecules which are not engaged in encounter at all, $\Sigma_{2} E$ is the sum of the energies of all the double molecules, and so on. The energy has now been expressed as the sum of the energies of a number of molecules of different kinds, regarding multiple molecules merely as new species of molecules. To this extent the problem is the same as the problem already dealt with in $\$ 75$. There is, however, an important difference. In the former case a molecule belonged to the same type for ever: in the present case single molecules are always combining into multiple molecules, and vice versâ. In fact we cannot assume that the total number of molecules of each, or even of all, kinds is constant.

All the constituent molecules $A, B, \ldots$ of the gas must be represented separately in the generalised space, as before. A double molecule $A B$ comes into existence when certain relations are satisfied between the coordinates of $A$ and those of $B$. We shall find it convenient to make a slight alteration 
in the form in which the law of distribution is expressed. We shall suppose that the number of single molecules of type $\alpha$ for which the $2 n_{\alpha}$ coordinates

$$
\xi_{1}, \xi_{2} \ldots \xi_{2 n_{\alpha}}
$$

lie within the range $d \xi_{1} d \xi_{2} \ldots d \xi_{2 n_{\alpha}}$ is

$$
\phi_{a}\left(\xi_{1}, \xi_{2} \ldots\right) d \xi_{1} d \xi_{2} \ldots
$$

so that by comparison with (137) we see that $\phi_{a}$ replaces the old $N_{a} f_{a}$. The point of this change is to absorb the variable quantity $N_{a}$ into the variable function $f_{\alpha}$. In the former case, $N_{\alpha}$ was constant and this absorption was unnecessary : in the present case it reduces the apparent number of variables from two to one.

A double molecule of type $\alpha \beta$ will be a molecule composed of a molecule of type $\alpha$ and a molecule of type $\beta$ in encounter. The coordinates specifying such a double molecule will be the coordinates,

$$
\xi_{1}, \xi_{2} \ldots \xi_{2 n_{\alpha}}, \xi_{1}^{\prime}, \xi_{2}^{\prime} \ldots \xi_{2 n_{\beta}}^{\prime}
$$

of its components, and the number of double molecules for which these coordinates lie within the usual range will be taken to be

$$
\phi_{a \beta}\left(\xi_{1}, \xi_{2}, \ldots \xi_{1}^{\prime}, \xi_{2}^{\prime} \ldots\right) d \xi_{1} d \xi_{2} \ldots d \xi_{1}^{\prime} d \xi_{2}^{\prime} \ldots
$$

Let $E_{\alpha}$ be the energy of a single molecule of type $\alpha$, expressed as a function of the coordinates (173), $E_{a \beta}$ the energy of a double molecule of type $\alpha \beta$ expressed as a function of the coordinates (17う), and so on. Then the total energy as expressed by equation (172) may be put in the form

$$
\xi=\int \phi_{a} E_{a}+\int \dot{\phi}_{\beta} E_{\beta}+\ldots+\int \dot{\phi}_{\alpha \beta} E_{\alpha \beta}+\ldots
$$

in which the differentials are omitted to save printing, and the single thick integral denotes integration over all values of these differentials.

There will be a constituent molecule of type $\alpha$, (i) in each single molecule of type ( $\alpha$ ), (ii) in each double molecule of the types $\alpha \beta, \alpha \gamma \ldots$, and (iii) there will be two such molecules in each double molecule of type $\alpha \alpha$; and so on for more complex molecules. Let $N_{\alpha}, N_{\beta}, N_{\alpha \beta} \ldots$ be the number of molecules of types $\alpha, \beta, \alpha \beta \ldots$, and let $\mathfrak{N}_{\alpha}, \Re_{\beta}, \ldots$ be the numbers of the permanent constituent molecules of types $\alpha, \beta \ldots$. Then we have

$$
\Re_{a}=N_{a}+2 N_{\alpha a}+N_{a \beta}+N_{\alpha \gamma}+\ldots
$$

and since we have equations of the form

this may be written

$$
N_{\alpha}=\int \phi_{\alpha}, \quad N_{a \beta}=\int \phi_{a \beta}
$$

$$
\Re_{\alpha}=\int \phi_{a}+2 \int \phi_{\alpha \alpha}+\int \phi_{\alpha \beta}+\int \phi_{\alpha \gamma}+\ldots
$$

There are similar equations for each of the types $\beta, \gamma \ldots$. 
The analysis of $\$ 72$ holds for the most general field of intermolecular force. Hence it appears that in the present case, as before, the density of fluid in the generalised space may be regarded as constant throughout all time, and therefore we need only discuss the ratio of the volumes of this space which are occupied by the different possible classes of systems.

84. Let us agree to adopt the artifice explained in $\S 56$, to limit the variation of the coordinates of the various types of molecules to a finite range. Let us divide up the possible range of coordinates for a single molecule of type $\alpha$ into $n_{a}$ equal "cells," the possible range for a single molecule of type $\beta$ into $n_{\beta}$ equal cells, and so on. The range for a compound molecule of type $\alpha \beta$ will then be $n_{\alpha} n_{\beta}$ cells, if for the moment we regard any combination of an $\alpha$ molecule with a $\beta$ molecule as a compound of the $\alpha \beta$ type. If we only regard these as forming a double molecule when the intermolecular force exceeds a certain amount, then it follows that double molecules can only occur in certain of these $n_{a} n_{\beta}$ cells, and not in all-it does not at present matter in how many.

Let us now consider a special class of system-class A-in which there are

$$
\alpha_{1}, \alpha_{2} \ldots \text { single molecules of type } \alpha \text { in the respective } n_{a} \text { cells, }
$$

$$
\begin{aligned}
& \beta_{1}, \beta_{2} \ldots \text { " " " } \beta \text { " " } \quad n_{\beta} \text { " }
\end{aligned}
$$

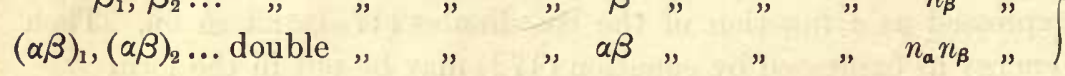

and so on.

Each arrangement of molecules which form a system of class $\mathrm{A}$ will be represented in an element of the generalised space which forms a fraction

$$
\frac{1}{n_{a} N_{a} n_{\beta} N_{\beta} \ldots\left(n_{a}^{2}\right)^{N_{a \alpha}}\left(n_{a} n_{\beta}\right)^{N_{a \beta} \ldots}}
$$

of the whole. Using equations (178) this becomes

$$
\frac{1}{n_{a} \Re_{a} n_{\beta} \Re_{\beta} \ldots}
$$

Now the number of ways of distributing the rôles of the various constituent molecules so that conditions (181) are satisfied is

$$
\frac{\mathfrak{R}_{\alpha} ! \mathfrak{N}_{\beta} ! \ldots}{\alpha_{1} ! \alpha_{2} ! \ldots \beta_{1} ! \beta_{2} ! \ldots(\alpha \beta)_{1} !(\alpha \beta)_{2} ! \ldots}
$$

Here the factor $\mathfrak{N}_{\alpha}$ ! is the number of ways in which the $\mathfrak{R}_{a}$ permanent constituent molecules of type $\alpha$ can be permuted inter se, $\alpha_{1} !$ is the number of ways in which the molecules in the first of the $n_{a}$ cells can be permuted inter se, and so on. Expression (183), then, gives the number of elements which represent systems of class A. Multiplying expressions (183) and (182) 
together, we find that the fraction of the whole generalised space which is occupied by systems of class $\mathrm{A}$ is

$$
\theta_{\alpha}=\frac{1}{n_{a} \mathfrak{R}_{a} n_{\beta} \mathfrak{l l}_{\beta} \ldots} \frac{\mathfrak{R}_{a} ! \mathfrak{N}_{\beta} ! \ldots}{\alpha_{1} ! \alpha_{2} ! \ldots}
$$

85. The value of $\theta_{a}$ just found is a generalised form of that given by expression (60). If we proceed as in $\S 40$, using Stirling's Theorem [equation $(61)]$ in the form

$$
\underset{p=\infty}{\mathrm{Lt}} \log p !=\frac{1}{2} \log (2 \pi e)+\left(p+\frac{1}{2}\right) \log \frac{p}{e},
$$

we obtain $\log \theta_{a}=C-\sum_{n_{a}}\left(\alpha_{8}+\frac{1}{2}\right) \log \frac{\alpha_{8}}{e}-\sum_{n_{\beta}}\left(\beta_{8}+\frac{1}{2}\right) \log \frac{\beta_{8}}{e}-\ldots$

$$
-\sum_{n_{a} n_{\beta}}\left((\alpha \beta)_{8}+\frac{1}{2}\right) \log \frac{(\alpha \beta)_{8}}{e}-\ldots
$$

where $C$ is a constant depending on the constants $\mathfrak{N}_{a}, \mathfrak{N}_{\beta} \ldots n_{a}, n_{\beta} \ldots$. From this equation it follows that the normal state is obtained by making $\mathfrak{\mathfrak { g }}$ a minimum, where

$$
\mathfrak{S}=\int \dot{\phi}_{a} \log \frac{\phi_{a}}{e}+\int \dot{\phi}_{\beta} \log \frac{\phi_{\beta}}{e}+\ldots+\int \dot{\phi}_{\alpha \beta} \log \frac{\phi_{\alpha \beta}}{e}+\ldots \ldots \ldots
$$

The variation of $\mathfrak{S}$ is subject to the energy equation (177) and to equations of the type (180) expressing the permanency of the separate types of permanent molecules. If we vary equation (185) and add the variation of equation (177) multiplied by an undetermined multiplier $\lambda$, and that of the equations of the type (180) multiplied by $\mu_{a}, \mu_{\beta} \ldots$ we obtain

$$
\begin{aligned}
\delta \mathfrak{Y}= & \left.\int \log \phi_{a}+\lambda E_{a}+\mu_{\alpha}\right) \delta \phi_{a}+\ldots \\
& +\int\left(\log \phi_{a \beta}+\lambda E_{\alpha \beta}+\mu_{a}+\mu_{\beta}\right) \delta \phi_{a \beta}+\ldots \\
& +\int\left(\log \phi_{\alpha a}+\lambda E_{\alpha a}+2 \mu_{\alpha}\right) \delta \phi_{\alpha a}+\ldots
\end{aligned}
$$

and the condition that $\mathfrak{F}$ shall be a minimum is given by the systems of equations

$$
\begin{gathered}
\log \phi_{a}+\lambda E_{a}+\mu_{a}=0, \text { etc., } \\
\log \phi_{a \beta}+\lambda E_{a \beta}+\mu_{a}+\mu_{\beta}=0, \text { etc., } \\
\log \phi_{a a}+\lambda E_{a a}+2 \mu_{a}=0, \text { etc., } \\
\text { etc. }
\end{gathered}
$$

Changing the constants $\lambda, \mu_{\alpha}, \mu_{\beta} \ldots$, and substituting for $E_{\alpha \beta}, E_{\alpha a} \ldots$ these equations lead at once to the equations

$$
\left.\begin{array}{rl}
\phi_{a} & =A e^{-2 h E_{\alpha}} \\
\phi_{\beta} & =B e^{-2 h E_{\beta}} \\
\phi_{\alpha \beta} & =A B e^{-2 h\left(E_{a}+E_{\beta}+W_{\alpha \beta}\right)} \\
\phi_{\alpha a} & =A^{2} e^{-2 h\left(E_{\alpha}+E_{a}^{\prime}+W_{\alpha a}\right)}
\end{array}\right\}
$$

etc. 
In general, for the multiple molecule $\alpha \beta \gamma \ldots$ we have

$$
\phi_{a \beta \gamma \ldots}=A B C \ldots e^{-2 h\left(E_{\alpha}+E_{\beta}+E_{\gamma}+\ldots+W_{\alpha \beta \gamma} \ldots\right)}
$$

86. From the well-known formula in attractions

$$
W=\frac{1}{2} \iiint \rho V d x d y d z
$$

where $\rho, V$ are density and potential at the point $x, y, z$, it follows that we can write $W_{a \beta \gamma . . .}$ in the form

$$
W_{\alpha \beta \gamma \ldots}=\frac{1}{2}\left(\chi_{a}+\chi_{\beta}+\chi_{\gamma}+\ldots\right),
$$

where $\chi_{a}$ is the potential of the molecule of type $\alpha$ in the field of intermolecular forces arising from the other molecules, and so on. Hence in equation (187) we may write

where

$$
\begin{aligned}
\phi_{a \beta \gamma \ldots} & =\psi_{a} \psi_{\beta} \psi_{\gamma} \ldots \\
\psi_{a} & =A e^{-2 h E_{\alpha}-h \chi_{a}}, \text { etc. }
\end{aligned}
$$

We may therefore regard the probability of a combination of molecules having any specified coordinates, as the product of the probabilities of the constituent molecules having the appropriate coordinates, if we take the probability of a molecule of type $\alpha$ having its coordinates within the usual range $d \xi_{1} d \xi_{2} \ldots$ to be

$$
A e^{-2 h E_{\alpha}-h x_{\alpha}} d \xi_{1} d \xi_{2} \ldots
$$

Since the quantity $\chi_{o}$ does not involve the velocity coordinates it is clear that the analysis of $\S 77$ can be made to apply to this case, and hence that the result expressed by equation (161) is true, even when intermolecular forces are taken into account. Thus we see that the law of distribution of velocity-coordinates is unaltered by the presence of intermolecular forces, and that the law of equipartition of kinetic energy remains valid independently of the existence of such forces.

87. Before leaving the subject we must, notice the similarity between the effects of an intermolecular and an external field of force. If $\chi_{a}$ instead of being the potential of a molecule type $\alpha$ in an intermolecular field of force, had been the potential in a permanent external field of force, then the law of distribution of molecules of type a would, by $\S 75$, be exactly the same as that expressed by (190), except that $\chi_{\alpha}$ would have been replaced by $2 \chi_{\alpha}$. 


\section{An alternative treatment of the Partition of Energy.}

88. The doctrine of the equipartition of energy in a system of molecules of varying masses was discovered and enunciated by Waterston* in 1821, in the paper which has already been referred to. He states the doctrine in the following form: "In mixed media, the mean square molecular velocity is inversely proportional to the specific weight of the molecule. This is the law of the equilibrium of vis-viva." Lord Rayleigh, in a footnote, says "This is the first statement of a very important theorem. The demonstration, however,...can hardly be defended." Exactly the same theorem was brought forward independently by Maxwell in 1859, in the British Association paper already referred to $\dagger$. He states the proposition: "Two systems of molecules move in the same vessel; to prove that the mean vis-viva of each particle will become the same in the two systems." The question was again brought into prominence by the publication of a paper by Boltzmann in 1861+. In 1879 Maxwell also published a paper on equipartition in which he regarded the whole question from a somewhat different standpoint§. In what follows we shall treat the question from Maxwell's point of view, the only difference being that the mathematical analysis can be put much more concisely by the help of the conception of a generalised space.

89. Let us, as before, represent a general dynamical system having $n$ degrees of freedom in a space of $2 n$ dimensions. We shall now find it convenient to take as variables

$$
q_{1}, q_{2} \ldots q_{n}, \quad \eta_{1}, \eta_{2} \ldots \eta_{n} \ldots \ldots \ldots \ldots \ldots \ldots \ldots(191),
$$

where $q_{1}, q_{2} \ldots q_{n}$ are generalised coordinates of the system, and $\eta_{1}, \eta_{2} \ldots \eta_{n}$ are the momentoids of $\S 77$. Since

$$
\frac{\partial\left(\eta_{1}, \eta_{2} \ldots \eta_{n}\right)}{\partial\left(p_{1}, p_{2} \ldots p_{n}\right)}=1
$$

it is clear that any assemblage of systems will be represented in the present space by fluid of the same density as that by which it was represented in the former space. If, therefore, the fluid in the present space is taken to be initially homogeneous, it will remain homogeneous throughout all time\|.

* Phil. Trans. clxxxin. p. 1.

+ "Illustrations of the Dynamical Theory of Gases," Phil. Mag. Jan. and July, 1860. Collected Works, I. p. 378.

‡ "Studien über das Gleichgewicht der lebendigen Kraft zwischen bewegten materiellen Punkten," Sitzungsber, der K. Akad. Wien, Lvir.

$\S$ "On Boltzmann's Theorem on the average distribution of energy in a system of material points," Camb. Phil. Trans. xir. Collected Works, I1. p. 713.

\| This treatment seems to obviate, in a simple manner, a criticism which has often been urged against Maxwell's original proof. Maxwell takes coordinates in which the kinetic energy is already expressed as the sum of squares, and assumes these to form true Lagrangian co- 
90. We may express the energy $E$ of the system in the forms

$$
\begin{aligned}
E & =V+L \\
& =V+\frac{1}{2}\left(c_{1} \eta_{1}{ }^{2}+c_{2} \eta_{2}{ }^{2}+\ldots+c_{n} \eta_{n}{ }^{2}\right)
\end{aligned}
$$

where $V$ is the potential energy, a function of the $q$ 's only, and $c_{1}, c_{2} \ldots$ are also functions of the $q$ 's only.

The volume of the generalised space for which $q_{1}, q_{2} \ldots q_{n}$ lie within specified ranges $d q_{1}, d q_{2} \ldots d q_{n}$, while $\eta_{1}, \eta_{2} \ldots \eta_{n}$ have all values such that $E<E_{0}$ is given by

$$
d q_{1} d q_{2} \ldots d q_{n} \iiint \ldots d \eta_{1}, d \eta_{2} \ldots d \eta_{n}
$$

where $\eta_{1}, \eta_{2} \ldots \eta_{n}$ have all values subject to

$$
c_{1} \eta_{1}{ }^{2}+c_{2} \eta_{2}{ }^{2}+\ldots+c_{n} \eta_{n}{ }^{2}<2\left(E_{0}-V\right)
$$

The integral is a Dirichlet Integral* of which the value is known to be

$$
\frac{\pi^{\frac{n}{2}}}{\Gamma\left(1+\frac{n}{2}\right)}\left(c_{1} c_{2} \ldots c_{n}\right)^{-\frac{1}{2}}\left(2 E_{0}-2 V\right)^{\frac{n}{2}}
$$

Differentiating with respect to $E_{0}$, we find that the volume representing systems for which $q_{1}, q_{2} \ldots q_{n}$ lie within the same range as before, while $E$ lies between $E_{0}$ and $E_{0}+d E$, is

$$
2 d q_{1} d q_{2} \ldots d q_{n} d E \frac{\pi^{\frac{n}{2}}}{\Gamma\left(\frac{n}{2}\right)}\left(c_{1} c_{2} \ldots c_{n}\right)^{-1}\left(2 E_{0}-2 V\right)^{\frac{n}{2}-1}
$$

If we introduce a new condition that $\eta_{n}$ is to lie between $\eta_{n}$ and $\eta_{n}+d \eta_{n}$, the alterations necessary to transform expression (198) to suit the new conditions will consist in writing $n-1$ for $n, 2 E_{0}-2 V-c_{n} \eta_{n}{ }^{2}$ for $2 E_{0}-2 V$ and introducing the new differential $d \eta_{n}$. Making these alterations the expression becomes

$$
2 d q_{1} d q_{2} \ldots d q_{n} d E d \eta_{n} \frac{\pi^{\frac{n-1}{2}}}{\Gamma\left(\frac{n-1}{2}\right)}\left(c_{1} c_{2} \ldots c_{n-1}\right)^{-\frac{1}{2}}\left(2 E_{0}-2 V-c_{n} \eta_{n}{ }^{2}\right)^{\frac{n-3}{2}} \ldots(199)
$$

ordinates. Unfortunately it is not always possible to find coordinates satisfying these conditions. To take the simplest case, the kinetic energy of rotation of a rigid body can be expressed as a sum of squares in many ways, but in no case are the coordinates true Lagrangian coordinates. If, for instance, we write

$$
2 L=A \omega_{1}^{2}+B \omega_{2}^{2}+C \omega_{3}^{2},
$$

we know that $\int \omega_{1} d t$, etc., are not true Lagrangian coordinates.

* Williamson, Integral Calculus, p. 320. 
The ratio of this expression to (198), on replacing $\sqrt{\pi}$ by $\Gamma\left(\frac{1}{2}\right)$, is found to be

$$
\frac{\Gamma\left(\frac{n}{2}\right)}{\Gamma\left(\frac{n-1}{2}\right) \Gamma\left(\frac{1}{2}\right)} \frac{\left(2 E_{0}-2 V-c_{n} \eta_{n}^{2}\right)^{\frac{n-3}{2}}}{\left(2 E_{0}-2 V\right)^{\frac{n-2}{2}}} c_{n}^{\frac{1}{2}} d \eta_{n}
$$

This is the fraction for which $\eta_{n}$ lies between $\eta_{n}$ and $\eta_{n}+d \eta_{n}$, of all the systems for which $q_{1}, q_{2} \ldots q_{n}, E$ lie within the specified small ranges.

Let us write

$$
K_{n}=\frac{1}{2} c_{n} \eta_{n}^{2}
$$

so that $K_{n}$ is the kinetic energy corresponding to the momentoid $\eta_{n}$; then

$$
c_{n}^{\frac{1}{2}} d \eta_{n}=\frac{d K_{n}}{\sqrt{2 K_{n}}},
$$

and therefore expression (200) becomes

$$
\frac{\Gamma\left(\frac{n}{2}\right)}{\Gamma\left(\frac{n-1}{2}\right) \Gamma\left(\frac{1}{2}\right)} \frac{\left(E_{0}-V-K_{n}\right)^{\frac{n-3}{2}}}{\left(E_{0}-V\right)^{\frac{n-2}{2}}} \frac{d K_{n}}{\sqrt{K_{n}}}
$$

The mean value of $K_{n}$ averaged over all the systems for which $q_{1}, q_{2} \ldots q_{n}$ and $E$ lie within the specified ranges, say $\bar{K}_{n}$, is therefore

$$
\begin{aligned}
\bar{K}_{n} & =\frac{\Gamma\left(\frac{n}{2}\right)}{\Gamma\left(\frac{n-1}{2}\right) \Gamma\left(\frac{1}{2}\right)} \int_{K_{n}=0}^{K_{n}=E_{0}-V} \frac{\left(E_{0}-V-K_{n}\right)^{\frac{n-3}{2}}}{\left(E_{0}-V\right)^{\frac{n-2}{2}}} K_{n}^{\frac{1}{2}} d K_{n} \\
& =\frac{\Gamma\left(\frac{n}{2}\right)}{\Gamma\left(\frac{n-1}{2}\right) \Gamma\left(\frac{1}{2}\right)} B\left(\frac{n-1}{2}, \frac{3}{2}\right)\left(E_{0}-V\right) \\
& =\frac{E_{0}-V}{n} *
\end{aligned}
$$

so that from symmetry

$$
\bar{K}_{1}=\bar{K}_{2}=\ldots=\bar{K}_{1}
$$

In words, this result states that, averaged through all those parts of the generalised space in which $q_{1}, q_{2} \ldots q_{n}$ and $E$ have specified value, the energies of the various momentoids are equal. By addition, it follows that, averaged through all parts of the generalised space for which $E$ has a given value, the energies of the various momentoids are equal. 
91. The result just reached is Maxwell's main result. If we wish it to apply to the motion of dynamical systems, we must suppose an assemblage of systems started with energies intermediate between the narrow limits $E_{0}$ and $E_{0}+d E$, in such a way that their density in the generalised space is uniform, i.e., so that all values of the coordinates and momenta which are consistent with the energy lying within the specified limits, are equally probable. The separate systems have of course no interaction one with another. It then follows that initially and throughout all time the mean energies of the various momentoids are equal.

By addition over all possible values of the energy, we can arrive at the result that for an assemblage of systems having all possible values for the coordinates and momenta, provided only they are started so that the initial density in the generalised space is uniform, the mean energies of the various momentoids are equal.

We can, however, obtain a result more general than this. The motion in the generalised space is confined to the loci $E=$ constant, so that if we take an initial distribution of density $\left(\rho_{1}\right)$ in the generalised space such that

$$
\rho_{1}=\phi(E)
$$

where $\phi(E)$ is any function of the energy, then this distribution is a permanent distribution, i.e., equation (203) is satisfied through all time. And by addition of the result obtained in the last section, it follows that in this assemblage the mean values of the energies of the various momentoids are equal.

\section{Continuity of path.}

92. Attempts have been made to extend this result to the time-average of the energies of the various momentoids of a single system. It is obvious that this cannot be done without further assumption of some kind. For instance it may be that the path of the single system is entirely confined to a certain definite region of the energy-surface on which it is moving, and in this case it would obviously be fallacious to calculate the time-average by integrating over the whole surface. The assumption which is usually made, in order to make the extension to a-time-average possible, is that generally known as the assumption of continuity of path. It is "that the system, if left to itself, will, sooner or later, pass through every phase which is consistent with the conservation of energy"*. Lord Rayleigh $\dagger$ points out that " if we take it quite literally, the assumption is of a severely restrictive character; for it asserts that the systems, starting from any phase, will traverse every other phase (consistent with the energy condition) before

* Maxwell, Collected Works, Ir. p. 714.

† "The law of partition of Kinetic Energy," Phil. Mag. [5] xuIx. p. 111, 1900. 
returning to the initial phase. As soon as the initial phase is recovered, a cycle is established and no new phases can be reached, however long the motion may continue."

It is, however, pretty clear that the assumption cannot be justified, if taken quite literally. It is known that in connection with every dynamical problem, there are an infinite number of re-entrant paths-the "periodic orbits" of astronomy - so that obviously a system on one of these paths will never reach the phases outside the one particular path, while a system not on one of these paths can never reach the phases represented by points on them.

This objection might be met by arguing that the re-entrant paths only form an infinitesimal fraction of the whole, and that it is quite conceivable that all the phases outside these re-entrant paths form a single path. If this were so, it would be immaterial, for a system on this single path, whether we averaged over the whole energy surface, or only over the path. This defence, at any rate at first sight, does not seem very plausible. It requires us to suppose that the paths are all re-entrant, but that one of them is infinitely longer than all the others added together. It must also be noticed that there are dynamical systems in which all the paths are re-entrant and of finite length, as for example occurs in the case instanced by Lord Rayleigh (l.c. ante) of a particle describing an orbit about a centre of force, the law of force being $\mu r$.

93. An escape is perhaps made possible by assuming that the system does not continually traverse a single path undisturbed, but that by the agency of external forces, it is at times removed from one path to another. If the action of these external agencies is sufficiently fortuitous it may be that it is legitimate to suppose that the system passes through all phases on the energy surface. A warning must, however, be entered as to the nature of the agencies which may be regarded as fortuitous. The essential elements of the question may all be represented by the simple case of a billiard ball moving on a smooth billiard table. Here the impacts of the ball on the cushions are not fortuitous. In fact the cushions may be replaced by a field of repulsive force which becomes infinite at the cushions and vanishes elsewhere, and the motion of the ball is now undisturbed motion in this field of force. Again, if the system consists of two billiard balls moving upon the same table, the collisions between them cannot be regarded as fortuitous, because the impulsive forces between them at collisions can be treated as a special case of a continuous system of forces acting between them. Obviously the same consideration covers the case of a gas of the most general kind moving undisturbed by external agencies, in a closed vessel of any kind. 


\section{Extension to time-averages.}

94. Even after agreeing to shelve these difficulties connected with "Continuity of path," there is considerable disagreement among authorities as to the legitimacy of the extension to time-averages.

Lord Rayleigh writes as follows*:

"The extension to time-averages, the aspect under which Lord Kelvin has always considered the problem, is important, the more as some authors appear to doubt the possibility of such extension. Thus Professor Bryant, speaking of Maxwell's assumption, writes:- "To discover, if possible, a "general class of dynamical systems satisfying the assumption, would form " an interesting subject for future investigation. It is, however, doubtful "how far Maxwell's law would be applicable to the time-averages of the " energies in any such system. We shall see, in what follows, that the law of "permanent distribution of a very large number of systems is in many cases "not unique. Where there is more than one possible distribution it would "be difficult to draw any inference with regard to the average distribution "(taken with respect to the time) for one system."

"The extension to time-averages appears to me" (we are still quoting Lord Rayleigh) “to require nothing more than Maxwell's assumption, without which the law of distribution itself is only an artificial arrangement, sufficient indeed, but not necessary for steadiness. We shall still speak of the particle moving in two dimensions, though the argument is general. It has been shewn that at any moment the $u$-energy and the $v$-energy of the groups of particlest is the same; and it is evident that the equality subsists if we integrate over any period of time. But if this period be sufficiently prolonged, and if Maxwell's assumption be applicable, it makes no difference whether we contemplate the whole group of particles or limit ourselves to a single member of it. It follows that for a single particle the time-averages of $u^{2}$ and $v^{2}$ are equal, provided the averages are taken over a sufficient length of time.

"On the other hand, if in any case Maxwell's assumption be untrue, not only is the special distribution unnecessary for steadiness, but even if it be artificially arranged, the law of equal time-averages does not follow as a consequence."

In the case in which Maxivell's assumption is true, Lord Rayleigh's argument certainly seems (to the present author at least) to be incontrovertible, and it applies, as he states, to the most general system. The

* Phil. Mag. [5] xurx. p. 108.

+ British Association Report, 1894, §11.

‡ Lord Rayleigh's "group of particles" is the same as our assemblage of systems represented in the generalised space. 
assemblage of systems represented in a generalised space will all pass over the same paths if allowed sufficient time, and hence the time-averages will be the same for every system. Since the time-averages summed over all systems are equal, it follows that the time-averages for each individual system are equal.

If, however, Maxwell's assumption is untrue-and it must be borne in mind that no single system has yet been discovered in which it is not untrue-there seems to be nothing to be said in justification of deducing the equality of the time-averages from the theorem of $\S 90$. The main point to be noticed is that if the systems are subject to fortuitous disturbances, there is no reason for supposing that a homogeneous distribution of density in the generalised space (or, more generally, a distribution satisfying equation (203)) will be permanent, or conversely that the permanent state will satisfy the condition expressed by equation (203). And if this is not so, the attempted extension to time-averages fails entirely.

95. It may nevertheless be true that for fortuitous disturbances of a special type the distribution expressed by equation (203) remains permanent, and it may also be that the converse is true, and that the only permanent distribution is that represented by equation (203). I have thought it worth while attempting to shew that this is actually the case with a gas.

Before attacking this question, one further observation must be made. If our gas consists of an infinite number of molecules, we can select one single molecule from the rest, and regard the remainder of the molecules as the dynamical system, while the single molecule plays the part of the fortuitous disturbing agency. The disturbances are not fortuitous in the true sense, but since the single molecule collides only with an infinite number of different molecules in turn, it might be legitimate to regard its action as fortuitous. And again the energy of the system is not constant when the single molecule has been removed from consideration, but it might be legitimate to neglect the deviations of energy which are infinitesimal in comparison with the whole. In the following sections, however, the argument is not based on the somewhat doubtful foundations just sketched out: it rests on the assumption of molecular chaos.

\section{Application to a gas.}

96. We shall suppose a gas to be composed of a number of exactly similar dynamical systems - the molecules. We suppose that each molecule is surrounded by a sphere of molecular action of diameter $\sigma$, these spheres, as in $\$ 82$, being of such a size that two molecules exert no action upon one another except when their spheres intersect. 
When the spheres of two or more molecules intersect, an "encounter" is said to take place, lasting until the spheres again become clear of one another. The individual molecules are now to be the systems under discussion, and the "encounters" are to play the part of the fortuitous agencies.which disturb their motion. Each molecule is to have $n$ degrees of freedom, in addition to the three degrees of freedom represented by the motion of its centre of gravity in space, and the possible states of a single molecule are to be represented in a space of $2 n+3$ dimensions, of which $2 n$ represent the internal coordinates and momenta of the molecule, and the remaining three represent the velocities of the centre of gravity.

\section{Binary Encounters.}

97. We shall begin by considering binary encounters only. That is to say, we work on the hypothesis that the event of a sphere being intersected by two other spheres simultaneously is so rare that it may be neglected. In this case the spheres become identical with the "spheres of molecular action" of $\S 82$.

We treat this case as follows. As soon as an encounter begins between two molecules their existence as single molecules is supposed to be abruptly terminated, and their representative points are removed from our generalised space of $2 n+3$ dimensions. During the progress of the encounter the two molecules together will be supposed to form a new dynamical system-a double molecule. This system will be specified by $4 n+9$ independent coordinates, $2 n$ for the internal coordinates of each constituent molecule, six for the velocity and position of the centre of gravity of one molecule relatively to that of the other, and three for the velocity of the centre of gravity of the whole system in space. Hence any such system can be represented by a point in a space of $4 n+9$ dimensions. We shall not, however, require the whole of this $4 n+9$ dimensional space. If $x, y, z, x^{\prime}, y^{\prime}, z^{\prime}$ are the coordinates of the centres of the two molecules, the condition that an encounter is beginning or ending is

$$
\left(x-x^{\prime}\right)^{2}+\left(y-y^{\prime}\right)^{2}+\left(z-z^{\prime}\right)^{2}=\sigma^{2} \ldots \ldots \ldots \ldots \ldots \ldots(204) .
$$

In the $4 n+9$ dimensional space this equation will be the equation of a certain "surface" $S$ (of dimensions $4 n+8$ ), and the representative points of all double molecules will be inside $S$. We shall find it convenient to denote each double molecule by two representative points, since the rôles of first and second molecule can be allotted in two different ways.

Let $\rho_{2}$ be the density of representative points in any small element of volume in this new space, and $\rho_{1}$ the density in the original space of $2 n+3$ 
dimensions. Then the necessary and sufficient conditions for a steady state are

$$
\begin{aligned}
& \frac{d \rho_{2}}{d t}=0 \\
& \frac{d \rho_{1}}{d t}=0
\end{aligned}
$$

in the latter of which the change in $\rho_{1}$ includes that caused by the formation and dissolution of double molecules.

98. To determine the relation between $\rho_{1}$ and $\rho_{2}$, we make the assumption of $\S 15$, namely, that the gas is in a state of molecular chaos. Having made this assumption we proceed to calculate the number of encounters of a given kind which occur in an interval $d t$. If $\xi_{1}, \xi_{2} \ldots \xi_{2 n}$ are the internal coordinates of a molecule, the number of molecules per unit volume for which $\xi_{1}, \xi_{2} \ldots \xi_{2 n}, u, v, w$ lie within a range

$$
d \xi_{1} d \xi_{2} \ldots d \xi_{2 n} d u d v d w
$$

will be

$$
\rho_{1} d \xi_{1} d \xi_{2} \ldots d \xi_{2 n} d u d v d w \text {. }
$$

Hence, as in expression (4), the number of collisions in time $d t$ for which the coordinates of the first molecule lie within a range (207), and those of the second within a similar range in which the variables are accented, while the line joining their centres meets a unit sphere in a given element of surface $d \omega$, will be

$$
\rho_{1} \rho_{1}^{\prime} V \sigma^{2} \cos \theta d \xi_{1} d \xi_{2} \ldots d \xi_{2 n} d \xi_{1}^{\prime} d \xi_{2}^{\prime} \ldots d \xi_{2 n}^{\prime} d u d v d w d u^{\prime} d v^{\prime} d w^{\prime} d \omega d t \ldots \text { (208). }
$$

This number of collisions must however be equal to the number of double molecules which cross a certain element of the surface $S$ in the $4 n+9$ dimensional space in time $d t$, and this number will be

$$
\rho_{2} \frac{\partial \epsilon}{\partial t} d S d t
$$

where $d S$ is the element of the surface $S$ representing collisions of the type in question, and $\frac{\partial \epsilon}{\partial t}$ is the velocity in this space at the element of surface $d S$ measured inward along the normal. The equation of the surface $S$ being equation (204), we may clearly suppose the normal to be the shortest distance from $d S$ to

$$
x-x^{\prime}=0, y-y^{\prime}=0, z-z^{\prime}=0,
$$

and therefore write

Thus

$$
\epsilon^{2}=\left(x-x^{\prime}\right)^{2}+\left(y-y^{\prime}\right)^{2}+\left(z-z^{\prime}\right)^{2} .
$$

$$
\begin{aligned}
\frac{\partial \epsilon}{\partial t} & =\frac{x-x^{\prime}}{\epsilon} \frac{\partial}{\partial t}\left(x-x^{\prime}\right)+\ldots \\
& =\lambda\left(u-u^{\prime}\right)+\ldots \\
& =V \cos \theta \ldots \ldots \ldots \ldots \ldots
\end{aligned}
$$


where $\lambda, \mu, \nu$, as in Chapter II., are the direction-cosines of the line of centres. The value of $d S$ corresponding to collisions of the type specified will clearly be

$$
d S=d \xi_{1} \ldots d \xi_{2 n} d u d v d w d \xi_{1}^{\prime} \ldots d \xi_{2 n}^{\prime} d u^{\prime} d v^{\prime} d w^{\prime} \sigma^{2} d \omega
$$

If we substitute the value for $\frac{\partial \epsilon}{\partial t}$ from equation (210) and for $d S$ from equation (211) into expression (209), and equate the value so obtained to expression (208), we obtain, after dividing through by common multipliers,

$$
\rho_{1} \rho_{1}^{\prime}=\rho_{2}
$$

This equation may be regarded as giving the density $\rho_{2}$ at all points on the surface $S$ in the $4 n+8$ dimensional space, in terms of the densities at points in the $2 n+3$ dimensional space.

99. Since the systems represented in the $4 n+9$ dimensional space are not acted upon by any external forces, we have, as in $\$ 72$ (equation (131))

$$
\frac{D \rho_{2}}{D t}=0
$$

where $D / D t$ denotes differentiation with respect to the time as we follow the fluid in its motion. We may however write

$$
\frac{D \rho_{2}}{D t}=\frac{d \rho_{2}}{d t}+\frac{\partial s}{\partial t} \frac{d \rho_{2}}{d s}
$$

where $d \rho_{2} / d t$ denotes the rate of increase at a fixed point, $\partial s / \partial t$ is the velocity along a stream line, and $d \rho_{2} / d s$ is the increase of $\rho_{2}$ per unit length along the stream line. If we combine equation (213) with the condition for a steady state (equation (205)), equation (214) reduces to

$$
\frac{d \rho_{2}}{d s}=0
$$

so that $\rho_{2}$ must be constant along every stream line.

Let $\rho_{1}, \rho_{1}^{\prime}$ be the densities in the $2 n+3$ dimensional space, at points occupied by the representative points of the two component molecules at the formation of a double molecule, and let $\bar{\rho}_{1}, \bar{\rho}_{1}^{\prime}$ be the densities at the points representative of the same two molecules at the dissolution of the double molecule. Then by equation (212) $\rho_{1} \rho_{1}^{\prime}$ and $\bar{\rho}_{1} \bar{\rho}_{1}^{\prime}$ are the two values of $\rho_{2}$ at the two ends of a single stream line in the $4 n+9$ dimensional space, and, therefore, by equation (215),

$$
\rho_{1} \rho_{1}^{\prime}=\bar{\rho}_{1} \bar{\rho}_{1}^{\prime}
$$

the same result, it will be noticed, as is obtained by the $H$-theorem of Chapter II. (cf. equation (21)).

Since the motion is dynamically reversible we may equally well take $\bar{\rho}_{1}, \bar{\rho}_{1}^{\prime}$ to be the densities at formation, then $\rho_{1}, \rho_{1}^{\prime}$ will be the densities at dissolution, and the same result holds. 
From this it follows that in equation (206) the decrease in $\rho_{1}$ caused by the formation of double molecules of any specified kind is exactly counterbalanced by the increase caused by the dissolution of double molecules of the same kind. Hence in equation (206), $d \rho_{1} / d t$ may be taken to be the change in $\rho_{1}$ caused solely by the continuous motion of the fluid, and may be treated as $\rho_{2}$ has been treated, leading to the result that $\rho_{1}$ must be constant along every stream line.

100. We have found, therefore, that the conditions for steady motion, on the hypothesis of binary encounters, may be expressed as follows:

(a) Throughout the $2 n+3$ dimensional space, $\rho_{1}$ must be constant along every stream line.

( $\beta$ ) Throughout the $4 n+9$ dimensional space, $\rho_{2}$ must be constant along every stream line.

( $\gamma$ ) At every point on the boundary of the $4 n+9$ dimensional space we must have

$$
\rho_{2}=\rho_{1} \rho_{1}^{\prime} .
$$

To these may be added a fourth condition:

( $\delta)$ At every point on the boundary of the $2 n+3$ dimensional space (i.e. at infinity) the flow across the boundary must be nil, or what is the same thing, we must have

$$
\rho_{1}=0,
$$

this last condition securing that steadiness is maintained without a supply of new systems from infinity.

These conditions are necessary and sufficient for. steady motion.

\section{Ternary and Higher Encounters.}

101. By a simple extension of the method already explained, the possibility of encounters of ternary and higher orders may be considered. For instance, to take ternary encounters into account we imagine systems of triple molecules, these being represented in a suitable space, in which the number of dimensions will be $6 n+15$, namely $2 n+6$ for each constituent molecule, less three for the position of the centre of gravity of the whole system. The density in this space being $\rho_{3}$, we have as conditions additional to those just given :

(є) Throughout the $6 n+15$ dimensional space, $\rho_{3}$ must be constant along every stream line.

(५) At every point on the boundary of the $6 n+15$ dimensional space we must have

$$
\rho_{3}=\rho_{2} \rho_{1} .
$$


102. Encounters of higher orders may be similarly treated. If $\rho_{K}$ is used to denote the density in the space of $2 K n+6 K-3$ dimensions, in which $K$-ple molecules are represented, the complete system of conditions for steady motion is

(i) Along every stream line in the $2 K n+6 K-3$ dimensional space

$$
\rho_{K}=\text { constant }
$$

(ii) At every point on the boundary of this space

$$
\rho_{K}=\rho_{a} \rho_{b}
$$

in which $\rho_{a}, \rho_{b}$ refer to the two systems of molecules of orders $a, b$, of which the encounter results in the particular system of order $K$ which is represented at the point in question (we therefore have always $a+b=K$ ).

If encounters of all orders are to be taken into account these conditions must be satisfied for all values of $K$ from $K=1$ to $K=\infty$. In the case of $K=1$, equation (218) must be interpreted so as to become identical with the condition $(\delta)$ of $\$ 100$.

It will be noticed that if these conditions are satisfied for all values up to $K=\infty$ no hypothesis need be made as to the smallness of the radius of molecular action in comparison with the free path. The only assumption now made is that the gas is in a state of molecular chaos.

\section{Solution of Equations.}

103. As before, let $\chi$ be a quantity, a function of the coordinates of a molecule or system of molecules, such that throughout the undisturbed motion of the molecule or system, $\chi$ maintains a constant value, and such that when two molecules or systems combine to form a new system, the $\chi$ of the new system is equal to the sum of the $\chi$ 's of the component systems. Speaking loosely we may say that $\chi$ is defined as being capable of exchange between molecules at a collision, but is indestructible.

Then a solution of equations (217) and (218) will be seen to be

$$
\log \rho_{K}=\Sigma_{K} \chi \ldots(K=1,2, \ldots \infty)
$$

where $\Sigma_{K} \chi$ is the value of $\chi$ for a $K$-ple molecule, being by definition equal to the sum of the $\chi$ 's of the $K$ constituent molecules. If $\chi_{1}, \chi_{2} \ldots$ are all the possible values of $\chi$, the most general solution is

$$
\log \rho_{K}=\sum_{K}\left(A_{1} \chi_{1}+A_{2} \chi_{2}+\ldots\right)
$$

As regards the number and meaning of the $\chi$ 's the question stands as in $\S 76$; and for the reasons there given we may, in the case of a gas which has no mass-motion, reject all except

$$
\begin{aligned}
& \chi_{1}=1, \\
& \chi_{2}=E, \text { the energy of a molecule. }
\end{aligned}
$$


Hence the solution becomes

$$
\begin{aligned}
& \rho_{1}=A e^{-2 h E}, \\
& \rho_{2}=A^{2} e^{-2 h\left(E+E^{\prime}+W\right)} \text {, } \\
& \rho_{K}=A^{K} e^{-2 h\left(\sum_{E} E+W\right)}
\end{aligned}
$$

where $E, E^{\prime} \ldots$ are the energies of the separate molecules, and $W$ is the potential energy of the intermolecular forces acting between molecules, this not now being included in $E$ and $E^{\prime}$.

This solution agrees with that of $\S 85$.

104. We have therefore seen that Maxwell's treatment of equipartition can be made to lead to the right result if we make the assumption of molecular chaos, or, more accurately, if we assume that the number of collisions of a given kind is that given by expression (208). It is infinitely probable, but not certain, that this expression will be accurate, so that it is infinitely probable, but not certain, that (221) will be the solution in a steady state. This is exactly the result arrived at before.

It is of interest to notice that it could have been predicted $\dot{a}$ priori, that it would be necessary to supplement Maxwell's treatment by some assumption of this kind.

This, as we shall now see, follows from the fact that the problem is a "statistical" problem, and not a dynamical problem of the ordinary type. A dynamical problem may, in accordance with accepted usage, be said to be one of statistical mechanics when the data and objects of inquiry are not the actual values of the various coordinates, but the law of distribution of these coordinates. Since the data of a problem in statistical mechanics do not completely specify the dynamical coordinates of the system, we are, in a problem of statistical mechanics, discussing an infinite number of different systems at once, and without differentiation inter se. The motion of these systems will naturally diverge in the course of time. It may be that after the motion a single statistical specification can be given which covers all except an infinitesimal fraction of the systems. In this case a solution may be said to have been found to the problem. It cannot be that a solution can be obtained which covers all the systems, the reason for this being that, even after the initial system has been fully specified statistically, there are still an infinite number of undetermined variables; and, by giving suitable values to these, we can obtain any chosen infinite number of relations between the coordinates of the final result, and can therefore cause this final result to disagree with any single statistical specification. It is therefore clear that a statistical problem must always have an element of uncertainty in its final solution, although in virtue of the infinite number of the variables, this 
uncertainty may be represented by an infinitesimally small probability of error: it may, in the terminology previously used, be "infinitely probable" that the result is true.

We have found that the assumption of molecular chaos, on the other hand, leads to a definite certain result, and not merely to one which is infinitely probable. Incidentally, this circumstance enables us to trace out some of the inner significance of this assumption. We see at once that the assumption rests on the supposition that the systems with which it deals have at every instant a definite statistical specification. It therefore just excludes those systems, an infinitesimal fraction of the whole, which wander away from the statistical specification obeyed by the majority. In other words, it implies that any system under discussion has the statistical specification of the majority, and therefore naturally leads to a certain result instead of leading merely to one of infinite probability.

As regards Maxwell's treatment of equipartition, enough has perhaps been said to shew that in dealing statistically with a gas, we can never arrive at absolute certainty: it is therefore impossible to reach any definite result unless a loophole of escape from absolute certainty has been introduced into the premises on which we work.

\section{Mechanical Illustration of Equipartition.}

105. It may be useful to illustrate the abstract results which have been obtained in this chapter by a concrete mechanical example.

Let us again suppose, as in Chapter II., that the molecules of a gas are hard, rigid spheres; but, in order to get more than the three degrees of freedom represented by the motion of translation of a molecule, let us also suppose that the centre of gravity of these spheres does not coincide with their geometrical centre, so that rotations are set up by collisions.

Each molecule possesses an axis of symmetry, namely, the line joining the centre of gravity to the centre of figure. Let us take any two other axes, fixed in the molecule in the plane perpendicular to the axis of symmetry, and let us denote the components of angular velocity about these two axes by $\varpi_{1}, \varpi_{2}$, and that about the axis of symmetry by $\varpi_{3}$. If the corresponding radii of gyration are $k, k, k^{\prime}$, the kinetic energy $L$ will be given by

$$
2 L=m\left(u^{2}+v^{2}+w^{2}\right)+m k^{2}\left(\varpi_{1}^{2}+\varpi_{2}^{2}\right)+m k^{\prime 2} \varpi_{3}^{2} .
$$

It is clear at the outset that the velocity $\varpi_{3}$ is peculiar in that its value cannot be changed by collisions. It follows, then, that the system, as at present specified, does not satisfy Maxwell's condition of continuity of path. Or, again, there are other constants besides the energy, namely, the $\varpi_{8}$ coordinates of the various molecules, which remain constant throughout the 
whole motion, just as, in the example of $\$ 59$, the values of $u^{2}$ for the various molecules remained constant. It is, then, clear that the general result obtained in the present chapter will not apply to the present system, without some modification being made.

106. The most obvious modification to make is to ignore the rotation $\varpi_{3}$ altogether, just as, in finding the law of distribution for a system of symmetrical spheres in Chapter II., we ignored all the three rotations. In either case the motion is the same as if the ignored rotations were non-existent.

Let us, then, suppose the kinetic energy given by

$$
2 L=m\left(u^{2}+v^{2}+w^{2}\right)+m k^{2}\left(\varpi_{1}^{2}+\varpi_{2}^{2}\right) .
$$

There are now 'five degrees of freedom, and the separate terms on the righthand of the above equation may be regarded as contributions from separate momentoids, in the sense required in $\$ 77$. The analysis of this chapter accordingly shews that in the steady state we must have

$$
\overline{m u^{2}}=\overline{m v^{2}}=\overline{m w^{2}}=\overline{m k^{2} \varpi_{1}^{2}}=\overline{m k^{2} \varpi_{2}^{2}}
$$

We can, however, investigate the steady state by considering the effect of individual collisions, somewhat after the method of Chapter II. It will be of interest to do this, and so to verify the result expressed by equation (222). The main importance of the problem, however, is that we shall obtain information, which will subsequently be found useful, as to the rate at which the gas, if disturbed from the steady state, returns to that state. This information cannot be obtained by the general methods which have been used to determine the steady state.

\section{The Transfer of Energy in a system of Loaded Spheres.}

107. Let us suppose the distance of the centre of gravity from the geometrical centre to be $r$ in each molecule, $r$ being small in comparison with $\sigma$, the diameter of the molecule. In the final result it is obvious that only even powers of $r$ can occur, for we can replace $r$ by $-r$ without altering the nature of the gas. The solution of the whole problem when $r=0$ is known, for the problem then reduces to that of the symmetrical spheres of Chapter II. For the present purpose we shall be content to find the solution as far as $r^{2}$ only, neglecting $r^{4}, r^{6}$, etc.

In addition to the rectangular axes $x, y, z$ fixed in space, let us suppose there to be a system of rectangular axes $\xi, \eta, \zeta$ fixed in each molecule, having the centre of gravity for origin, and coinciding with the three axes of rotation already specified. The coordinates of the geometrical centre will then be $0,0, r$, and the moments of inertia about the axes $\xi, \eta$, $\zeta$ will be $m k^{2}$, $m k^{2}, m k^{\prime 2}$ respectively. 
Let us suppose that for any single sphere the directions of the axes $\xi, \eta, \zeta$ are connected with those of the fixed axes $x, y, z$ by the following scheme of direction-cosines :

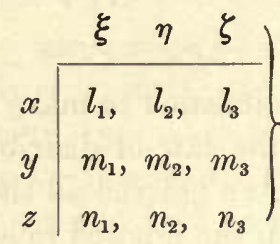

The condition of the sphere at any instant may be regarded as determined by $u, v, w$, the velocities of the centre of gravity parallel to $x, y, z ; \varpi_{1}, \varpi_{2}$, the angular velocities of rotation about the axes $\xi, \eta$; and the nine directioncosines of scheme (223) of which three only are independent.

There are therefore eight independent variables necessary to determine the condition of a sphere. In discussing a collision between two spheres, it is necessary to know not only the conditions of the two spheres, but also $\lambda, \mu, \nu$, the direction-cosines of the line of centres at impact referred to the fixed axes $x, y, z$. This introduces two more variables, so that a collision requires eighteen independent variables for its complete specification.

108. Let us examine first the changes in the eight variables of a sphere, which are produced by an impulse $I$ acting along the radius of which the direction-cosines are $\lambda, \mu, \nu$.

Referred to $\xi, \eta$, $\zeta$ axes, the point of application of this impulse may be taken to be $0,0, r$. The components of the impulse will be

$$
\left(l_{1} \lambda+m_{1} \mu+n_{1} \nu\right) I \text {, etc. }
$$

and the components of the resulting couple

$$
-r\left(l_{2} \lambda+m_{2} \mu+n_{2} \nu\right) I, \quad r\left(l_{1} \lambda+m_{1} \mu+n_{1} \nu\right) I, 0 .
$$

Hence, if variables after the impulse are distinguished by a horizontal bar, the new velocities of translation will be given by

$$
\bar{u}=u+\frac{I \lambda}{m}, \text { etc. }
$$

and those of rotation by

$$
\left.\begin{array}{c}
\bar{\varpi}_{1}=\varpi_{1}-\frac{r\left(l_{2} \lambda+m_{2} \mu+n_{2} \nu\right)}{m k^{2}} I \\
\bar{\varpi}_{2}=\varpi_{2}+\frac{r\left(l_{1} \lambda+m_{1} \mu+n_{1} \nu\right)}{m k^{2}} I
\end{array}\right\}
$$

Let us next regard this impulse as arising from a second sphere, of which the condition is determined by accented variables $u^{\prime}, v^{\prime}, w^{\prime} \ldots$, the line of centres at impact having direction-cosines $\lambda, \mu, \nu$. 
Before impact the components parallel to $x, y, z$ of the velocity of the centre of the first sphere are

$$
\begin{aligned}
& u+r\left(l_{1} \varpi_{2}-l_{2} \varpi_{1}\right), \\
& v+r\left(m_{1} \varpi_{2}-m_{2} \varpi_{1}\right), \\
& w+r\left(n_{1} \varpi_{2}-n_{2} \varpi_{1}\right) .
\end{aligned}
$$

Hence if $\alpha, \beta, \gamma$ denote the components of the velocity of the centre of the second sphere, relatively to that of the first, we have

$$
\alpha=u^{\prime}-u+r\left(l_{1}^{\prime} \varpi_{2}^{\prime}-l_{2}^{\prime} \varpi_{1}^{\prime}-l_{1} \varpi_{2}+l_{2} \varpi_{1}\right) \text {, etc. }
$$

The components of relative velocity after impact can be deduced by writing $\bar{u}, \bar{u}^{\prime} \ldots$ in place of $u, u^{\prime} \ldots$ The quantities $\bar{u} \ldots$ are given by equations (224), (225); the quantities $\bar{u}^{\prime} \ldots$ are given by similar equations, except that $I$ must be replaced by $-I$, since the impulse on the second sphere in the direction $\lambda, \mu, \nu$ is $-I$. Substituting this value, and comparing with equation (226), we obtain

$\bar{\alpha}=\alpha-\frac{2 I \lambda}{m}-\frac{r^{2} I}{m k^{2}}\left\{l_{1}\left(l_{1} \lambda+m_{1} \mu+n_{1} \nu\right)+l_{2}\left(l_{2} \lambda+m_{2} \mu+\bar{n}_{2} \nu\right)\right.$

$$
\left.+l_{1}^{\prime}\left(l_{1}^{\prime} \lambda+m_{1}^{\prime} \mu+n_{1}^{\prime} \nu\right)+l_{2}^{\prime}\left(l_{2}^{\prime} \lambda+m_{2}^{\prime} \mu+n_{2}^{\prime} \nu\right)\right\},
$$

and there are similar equations for $\bar{\beta}, \bar{\gamma}$.

Multiplying these three equations by $\lambda, \mu, \nu$ and adding, we obtain

$$
\lambda \bar{\alpha}+\mu \bar{\beta}+\nu \bar{\gamma}=\lambda \alpha+\mu \beta+\nu \gamma-\frac{2 I}{m}\left(1+A r^{2}\right) .
$$

where $A=\frac{1}{2 k^{2}}\left\{\left(l_{1} \lambda+m_{1} \mu+n_{1} \nu\right)^{2}+\left(l_{2} \lambda+m_{2} \mu+n_{2} \nu\right)^{2}\right.$

$$
\left.+\left(l_{1}^{\prime} \lambda+m_{1}^{\prime} \mu+n_{1}^{\prime} \nu\right)^{2}+\left(l_{2}^{\prime} \lambda+\overrightarrow{m_{2}} \mu+n_{2}^{\prime} \nu\right)^{2}\right\}
$$

At the moment of greatest compression, the components of relative velocity will be $\frac{1}{2}(\alpha+\bar{\alpha}), \frac{1}{2}(\beta+\bar{\beta}), \frac{1}{2}(\gamma+\bar{\gamma})$, and therefore the relative velocity along the line of centres will be

$$
\frac{1}{2}(\lambda \alpha+\mu \beta+\nu \gamma+\lambda \bar{\alpha}+\mu \bar{\beta}+\nu \bar{\gamma}) .
$$

This vanishes, and therefore, by equation (227),

$$
\begin{aligned}
\lambda \alpha+\mu \beta+\nu \gamma & =-(\lambda \bar{\alpha}+\mu \bar{\beta}+\nu \bar{\gamma}) \\
& =\left(1+A r^{2}\right) \frac{I}{m} .
\end{aligned}
$$

Hence, since we are neglecting $r^{4}$,

$$
\frac{I}{m}=\left(1-A r^{2}\right)(\lambda \alpha+\mu \beta+\nu \gamma)
$$

Substituting this value for $I$ in equations (224) and (225), we obtain the velocities of the first sphere after impact. 
109. Let us now pass to the consideration of impacts in which the two colliding spheres are in given conditions, but the direction-cosines $\lambda, \mu, \nu$ may have any values which are consistent with a collision.

The path described by the centre of the second molecule before collision relatively to that of the first is, since the spheres are loaded, not a straight line; but since the relative velocity $\alpha, \beta, \gamma$ is the same for all the impacts now being considered, this path is always the same as regards shape and direction, being in fact a curtate or prolate cycloid according to the magnitudes of the velocities.

Imagine the geometrical centre of the first molecule reduced to rest and surrounded by a sphere of radius $\sigma$. Let the centre of the second molecule describe its path relatively to the first molecule, then, whenever an impact occurs this centre must lie on the sphere of radius $\sigma$.

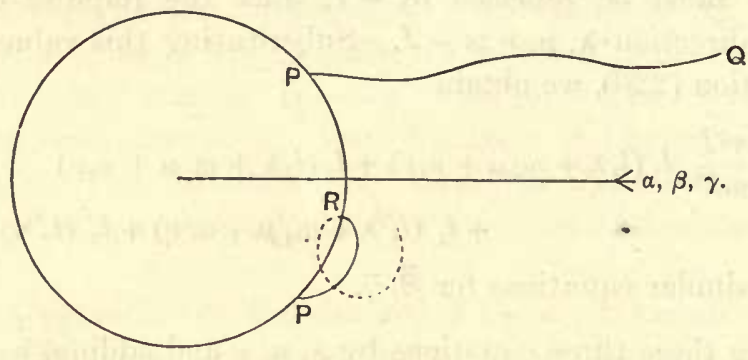

FIG. 4.

Let us start from any point $P$ on this sphere and trace the relative path backwards. This path, being a curved line, either may or may not intersect the sphere again in some point $R$.

In the former case an impact at $P$ will only be possible if an impact has occurred while the centre of the second sphere was at some point in $P R$. In the latter case there is no limitation of this kind, so that the probability of an impact occurring on any small region surrounding $P$ is simply proportional to the projection of this area on a plane perpendicular to the direction of the relative velocity at impact.

Owing to the smallness of $r$, the relative path will, in general, be only slightly curved. If the maximum curvature of this path is less than that of the sphere of radius $\sigma$, then obviously so long as the angle between the normal at $P$ and the relative velocity is acute, the relative path will not intersect the sphere except at $P$.

110. For the present we shall assume the velocities of the two molecules to be such that this latter condition is satisfied. In this case the proportion to the total number of collisions of those for which the angle between the directions of the relative velocity and line of centres lies between $\psi$ and $\psi+d \psi$, is easily seen to be $2 \sin \psi \cos \psi d \psi$, the limits for $\psi$ being 0 and $\frac{1}{2} \pi$. 
By $[q]$ we shall denote the average value of any quantity $q$ taken over all collisions in which the two spheres are, before impact, in certain specified conditions.

By squaring and adding equations similar to (224),

$$
\overline{u^{2}}+\overline{v^{2}}+\overline{w^{2}}=u^{2}+v^{2}+w^{2}+\frac{2 I}{m}(\lambda u+\mu v+\nu w)+\frac{I^{2}}{m^{2}}
$$

Before averaging this equation, we must calculate

$$
\left[\frac{I^{2}}{m^{2}}\right] \text { and }\left[\frac{I}{m}(\lambda u+\mu v+\nu w)\right] \text {. }
$$

If $V$ is the relative velocity,

$$
\lambda \alpha+\mu \beta+\nu \gamma=V \cos \psi
$$

hence by equation (229), we have as far as $r^{2}$,

$$
\frac{I^{2}}{m^{2}}=V^{2} \cos ^{2} \psi-2 r^{2} A V^{2} \cos ^{2} \psi
$$

Now

$$
\left[V^{2} \cos ^{2} \psi\right]=V^{2} \int_{0}^{\frac{1}{3} \pi} 2 \sin \psi \cos ^{3} \psi d \psi=\frac{1}{2} V^{2}
$$

Hence

$$
\left[\frac{I^{2}}{m^{2}}\right]=\frac{1}{2} V^{2}-2 r^{2}\left[A V^{2} \cos ^{2} \psi\right] \text {. }
$$

Again, from equation (229),

$$
\begin{aligned}
{\left[\frac{I}{m}(\lambda u+\mu v+\nu w)\right]=\Sigma\left[\lambda^{2}\right] \alpha u+\Sigma[\mu \nu](\beta w+\gamma v) } & \\
& -r^{2}\left\{\Sigma\left[A \lambda^{2}\right] \alpha u+\Sigma[A \mu \nu](\beta w+\gamma v)\right\}
\end{aligned}
$$

111. If we take the direction of the relative velocity for pole, and denote the coordinates of a point on a unit sphere, referred to this pole, by $\Theta, \Phi$, then the proportion of cases in which the direction $(\lambda, \mu, \nu)$ meets this unit sphere within limits $d \Theta d \Phi$ is $\frac{1}{\pi} \sin \Theta \cos \Theta d \Theta d \Phi$. Hence

$$
\left[\lambda^{2}\right]=\frac{1}{\pi} \int_{0}^{\frac{1}{2} \pi} \int_{0}^{2 \pi} \lambda^{2} \sin \Theta \cos \Theta d \Theta d \Phi
$$

If we take the coordinates of the axis of $x$ to be $(\theta, \phi)$, we have

$$
\lambda=\cos \Theta \cos \theta-\sin \Theta \sin \theta \cos (\Phi-\phi)
$$

Substituting this value for $\lambda$ in equation (232) and integrating, we obtain

$$
\begin{aligned}
{\left[\lambda^{2}\right] } & =\frac{1}{2} \cos ^{2} \theta+\frac{1}{4} \sin ^{2} \theta \\
& =\frac{1}{4}\left(1+\cos ^{2} \theta\right) \\
& =\frac{1}{4}\left(1+\frac{\alpha^{2}}{V^{2}}\right) \ldots \ldots
\end{aligned}
$$

and similar equations give $\left[\mu^{2}\right],\left[\nu^{2}\right]$. 
If we suppose the axis of $y$ to be $\left(\theta^{\prime}, \phi^{\prime}\right)$, then

$$
\mu=\cos \Theta \cos \theta^{\prime}-\sin \Theta \sin \theta^{\prime} \cos \left(\Phi-\phi^{\prime}\right),
$$

and multiplying this by the respective sides of equation (233) and integrating, we obtain

$$
[\lambda \mu]=\frac{1}{4} \cos \theta \cos \theta^{\prime}=\frac{\alpha \beta}{4 V^{2}}
$$

and similar equations give $[\mu \nu],[\nu \lambda]$.

112. Substituting these values in equation (231),

$$
\left[\frac{I}{m}(\lambda u+\mu v+\nu w)\right]=\frac{1}{4} \Sigma \alpha u+\frac{1}{4} \Sigma \frac{\alpha^{3} u}{V^{2}}+\frac{1}{4} \Sigma \frac{\beta \gamma(\beta w+\gamma v)}{V^{2}}-r^{2} D,
$$

where

$$
D=\Sigma\left[A \lambda^{2}\right] \alpha u+\Sigma[A \mu \nu](\beta w+\gamma v)
$$

Or, simplifying,

$$
\left[\frac{I}{m}(\lambda u+\mu v+\nu w)\right]=\frac{1}{2}(\alpha u+\beta v+\gamma w)-r^{2} D,
$$

and hence from equation (230),

$$
\begin{array}{r}
{\left[\overline{u^{2}}+\overline{v^{2}}+\overline{w^{2}}\right]=u^{2}+v^{2}+w^{2}+a u+\beta v+\gamma w+\frac{1}{2} V^{2}} \\
-2 r^{2}\left(D+\left[A V^{2} \cos ^{2} \psi\right]\right)
\end{array}
$$

On the right-hand side the upper line

$$
\begin{aligned}
& =\frac{1}{2}\left\{u^{2}+v^{2}+w^{2}+(u+\alpha)^{2}+(v+\beta)^{2}+(w+\gamma)^{2}\right\} \\
& =\frac{1}{2} \Sigma u^{2}+\frac{1}{2} \Sigma\left\{u^{\prime}+r\left(l_{1}^{\prime} \varpi_{2}^{\prime}-l_{2}^{\prime} \varpi_{1}^{\prime}-l_{1} \varpi_{2}+l_{2} \varpi_{1}\right)^{2}\right\},
\end{aligned}
$$

by equation (226),

$$
\begin{aligned}
=\frac{1}{2} \Sigma\left(u^{2}+u^{\prime 2}\right)+ & r \Sigma u^{\prime}\left(l_{1}^{\prime} \varpi_{2}^{\prime}-l_{2}^{\prime} \varpi_{1}{ }^{\prime}-l_{1} \varpi_{2}+l_{2} \varpi_{1}\right) \\
& +\frac{1}{2} r^{2}\left(\varpi_{1}{ }^{2}+\varpi_{2}{ }^{2}+\varpi_{1}{ }^{2}+\varpi_{2}{ }^{2}\right) \\
& -r^{2} \Sigma\left(l_{1} l_{2} \varpi_{1} \varpi_{2}-l_{1} l_{2}^{\prime} \varpi_{1}{ }^{\prime} \varpi_{2}+\ldots\right) .
\end{aligned}
$$

If therefore we write

$$
\begin{aligned}
u^{2}+v^{2}+w^{2} & =c^{2}, \\
\overline{u^{2}}+\overline{v^{2}}+\overline{w^{2}} & =\overline{c^{2},} \\
\varpi_{1}^{2}+\varpi_{2}^{2} & =\varpi^{2}, \\
\overline{\varpi_{1}}+\overline{\varpi_{2}} & =\overline{\varpi^{2}},
\end{aligned}
$$

and adopt a similar notation in accented symbols, equation (237) may be written

$$
\begin{aligned}
{\left[\overline{c^{2}}\right]=\frac{1}{2}\left(c^{2}+c^{\prime 2}\right) } & +r \Sigma u^{\prime}\left(l_{1}^{\prime} \varpi_{2}^{\prime}-\ldots\right)+\frac{1}{2} r^{2}\left(\varpi^{2}+\varpi^{\prime 2}\right) \\
& -r^{2} \Sigma\left(l_{1} l_{2} \varpi_{1} \varpi_{2}-\ldots\right)-2 r^{2}\left(D+\left[A V^{2} \cos ^{2} \psi\right]\right)
\end{aligned}
$$

113. We must now carry the process of averaging still further, so as to apply to all possible positions of the axes of the colliding spheres; that is to say, we must average over all collisions determined by given values of the ten velocities

$$
u, v, w, u^{\prime}, v^{\prime}, w^{\prime}, \varpi_{1}, \varpi_{2}, \varpi_{1}^{\prime}, \varpi_{2}^{\prime}
$$


Let the result of averaging in this manner be denoted by $\{q\}$.

When we require to average any quantity $p$ over all possible positions of the axes of the first sphere, all positions being regarded as equally probable, we shall denote the result by $\int p d \omega$, and the same quantity averaged over all positions of the axes of the second sphere will be denoted by $\int p d \omega^{\prime}$. The probability of an impact occurring for any given positions of the axes is, however, proportional to $V$, and this depends on the positions of the axes. Thus, averaging over all collisions for which the coordinates (239) have given values, we shall have

$$
\{q\}=\frac{\iint q V d \omega d \omega^{\prime}}{\iint V d \omega d \omega^{\prime}}
$$

To calculate $\left\{\overline{c^{2}}\right\}$ from equation (238), we must first evaluate $\left\{l_{1}\right\} \ldots$, $\left\{l_{1} l_{1}^{\prime}\right\} \ldots,\{D\}$, and $\left\{A V^{2} \cos ^{2} \phi\right\}$. Since, however, we are neglecting $r^{3}$, we require $\left\{l_{1}\right\} \ldots$ only as far as terms in $r$, and $\left\{l_{1} l_{1}^{\prime}\right\} \ldots$ and the remaining averages only when $r=0$.

From equation (226)

$$
V^{2}=\Sigma\left\{u^{\prime}-u+r\left(l_{1}^{\prime} \varpi_{2}^{\prime}-l_{2}^{\prime} \varpi_{1}^{\prime}-l_{1} \varpi_{2}+l_{2} \varpi_{1}\right)\right\}^{2},
$$

so that if we write

$$
U^{2}=\left(u^{\prime}-u\right)^{2}+\left(v^{\prime}-v\right)^{2}+\left(w^{\prime}-w\right)^{2},
$$

so that $U$ is the relative velocity of the centres of gravity, we have, as far as terms in $r$,

$$
V=U+\frac{r}{U} \Sigma\left(u^{\prime}-u\right)\left(l_{1}^{\prime} \varpi_{2}^{\prime}-l_{2}^{\prime} \varpi_{1}^{\prime}-l_{1} \varpi_{2}+l_{2} \varpi_{1}\right) .
$$

Now obviously $\iint p d \omega d \omega^{\prime}=0$, when $p$ has any of the values

$$
l_{1}, l_{2} \ldots, l_{1}^{\prime}, l_{2}^{\prime} \ldots, l_{1} l_{1}^{\prime} \ldots \text {, etc., }
$$

so that $\iint V d \omega d \omega^{\prime}=U$ as far as terms in $r$, and hence, from formula (240),

$$
\left\{l_{1}^{\prime}\right\}=\frac{\iint\left(\frac{r}{U} l_{1}^{\prime 2} \varpi_{2}^{\prime}\left(u^{\prime}-u\right)\right) d \omega d \omega^{\prime}}{U},
$$

and since obviously $\iint l_{1}^{2} d \omega d \omega^{\prime}=\frac{1}{3}$, this becomes

$$
\left\{l_{1}^{\prime}\right\}=\frac{r \varpi_{2}^{\prime}\left(u^{\prime}-u\right)}{3 U^{2}} .
$$

With the help of this and similar other expressions, we have as the averaged value of one term in equation (238)

$$
r \Sigma\left\{u^{\prime}\left(l_{1}^{\prime} \varpi_{2}^{\prime}-\ldots\right)\right\}=\frac{r^{2}\left(\varpi^{2}+\varpi^{\prime 2}\right)}{3 U^{2}} \Sigma u^{\prime}\left(u^{\prime}-u\right) .
$$


The remaining averages are required only when $r=0$. We may therefore write $U$ for $V$, and $u^{\prime}-u$ for $\alpha$, etc.

It is obvious at once that when $r=0,\left\{l_{1} l_{1}^{\prime}\right\}=0$, and hence that as the averaged value of a further term in equation (238) we have

$$
\left\{r^{2} \Sigma\left(l_{1} l_{2} \varpi_{1} \varpi_{2}-\ldots\right)\right\}=0 \text {. }
$$

As regards the remaining terms, on putting $r=0$ we obtain

$$
\{D\}=\Sigma\left\{\left[A \lambda^{2}\right] \alpha u\right\}+\Sigma\{[A \mu \nu](\beta v+\gamma v)\}
$$

by equation (236); and since the various systems of quantities may now be supposed to vary independently

$$
\begin{aligned}
\left\{\left[A \lambda^{2}\right] \alpha u\right\} & =\{A\}\left\{\lambda^{2}\right\}\left(u^{\prime}-u\right) u \\
& =\frac{1}{4}\left(1+\frac{\left(u^{\prime}-u\right)^{2}}{U^{2}}\right)\left(u^{\prime}-u\right) u\{A\},
\end{aligned}
$$

by equation (234), and

$$
\{[A \mu \nu](\beta w+\gamma v)\}=\left\{\left(v-v^{\prime}\right) w+\left(w^{\prime}-w\right) v\right\} \frac{\left(v^{\prime}-v\right)\left(w^{\prime}-w\right)}{4 U^{2}}\{A\}, \text { etc., etc., }
$$

and from equation (228)

$$
\{A\}=\frac{1}{2 k^{2}}\left(\frac{1}{3}+\frac{1}{3}+\frac{1}{3}+\frac{1}{3}\right)=\frac{2}{3 k^{2}} .
$$

Substituting these values in equation (241), we find that

$$
\{D\}=\frac{1}{3 k^{2}} \Sigma u\left(u^{\prime}-u\right),
$$

and in a similar way we have, for the remaining term in equation (238),

$$
\begin{aligned}
\left\{A V^{2} \cos ^{2} \psi\right\} & =\{A\} V^{2}\left\{\cos ^{2} \psi\right\} \\
& =\frac{2}{3 k^{2}} V^{2} \int_{0}^{\frac{\pi}{2}}\left(\cos ^{2} \psi\right) 2 \sin \psi \cos \psi d \psi, \\
\text { as in } \$ 110, \quad & =\frac{V^{2}}{3 k^{2}} \\
& =\frac{U^{2}}{3 k^{2}},
\end{aligned}
$$

since we are now neglecting $r$ altogether.

Hence, averaging equation (238), we obtain on substituting the averages which have been found for the separate terms,

$$
\begin{aligned}
\left\{\overline{c^{2}}\right\}=\frac{1}{2}\left(c^{2}+c^{\prime 2}\right)+\frac{1}{2} r^{2}\left(\varpi^{2}+\varpi^{\prime 2}\right) & +\frac{r^{2}\left(\varpi^{2}+\varpi^{\prime 2}\right)}{3 U^{2}} \Sigma u^{\prime}\left(u^{\prime}-u\right) \\
& -\frac{2 r^{2}}{3 k^{2}}\left(\Sigma u\left(u^{\prime}-u\right)+U^{2}\right) .
\end{aligned}
$$


There is an exactly similar equation for $\left\{c^{\overline{2}}\right\}$, and by addition of these two equations we obtain, after simplification

$$
\left\{\overline{c^{2}}+\overline{c^{2}}\right\}=c^{2}+c^{\prime 2}+\frac{4}{3} r^{2}\left(\varpi^{2}+\varpi^{\prime 2}\right)-\frac{2 r^{2}}{3 k^{2}} U^{2} .
$$

If we use the symbol $\Delta$ to denote the increase caused by collision of the quantity to which it is applied, the equation just obtained may be written in the form

$$
\left\{\Delta\left(c^{2}+c^{\prime 2}\right)\right\}=r^{2}\left\{\frac{4}{3}\left(\varpi^{2}+\varpi^{\prime 2}\right)-\frac{2 V^{2}}{3 k^{2}}\right\}
$$

in which $U$ has again been replaced by $V$.

114. So long as we remain in ignorance of the law of distribution of coordinates, it is impossible to carry any further the process of direct averaging. We may, however, proceed as follows.

An expression has just been found for the mean change of translational energy produced by collisions in which the molecules have given velocities. A summation extending over all collisions which occur during a short interval of time $d t$ will lead to an expression for the total change in the translational energy of the gas during that period. From the form of equation (242) it is clear that this expression will contain $r^{2}$ as a factor. But as we are neglecting powers of $r$ of a degree higher than the second, we may put $r=0$ in all terms multiplied by $r^{2}$. It follows that to find $\Sigma \Delta\left(c^{2}+c^{\prime 2}\right)$ as far as $r^{2}$, we need only calculate $\Sigma\left(\varpi^{2}+\varpi^{\prime 2}\right)$ and $\Sigma U^{2}$ on the assumption that $r=0$, the summation extending in each case to all the collisions which occur in the time $d t$.

This neglects a correction which is required by the considerations mentioned in $\S 109$, a correction which would consist in adding to the expression for $\Sigma \Delta\left(c^{2}+c^{\prime 2}\right)$, a quantity equal to the sum of all the separate corrections to be applied to the right-hand member of equation (242) in cases in which this equation fails. Now each separate correction will clearly contain $r^{2}$ as a factor, and on averaging this must be further multiplied by a factor proportional to the number of cases in which the correction is required; i.e. by a factor which vanishes when $r=0$. The correction to the final result is therefore of a higher order of small quantities than $r^{2}$, and may therefore be neglected.

For a similar reason we may, in calculating $\Sigma\left(\varpi^{2}+\varpi^{\prime 2}\right)$ and $\Sigma U^{2}$, assume the law of distribution of velocities to be that which would obtain in the case of $r=0$. We therefore assume the number of molecules per unit volume for which $c$ and $\varpi$ lie within ranges $d c, d \varpi$ to be

$$
4 \pi \nu A c^{2} e^{-h m c^{2}} f(\varpi) d c d \varpi
$$


It is not necessary to make any assumptions as to the form of $f(\varpi)$, but we shall suppose that $\int_{0}^{\infty} f(\varpi) d \varpi=1$, any constant multiplier being absorbed into the constant $A$, so that as in equation (30),

$$
A=\sqrt{\frac{h^{3} m^{3}}{\pi^{3}}}
$$

'The number of collisions per unit time per unit volume for which $V$ lies between $V$ and $V+d V$, is from expression (45),

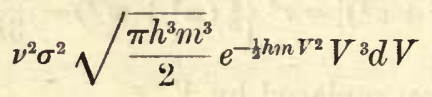

Hence the mean value of $V^{2}$ averaged over all collisions is

$$
\frac{\int_{0}^{\infty} e^{-\frac{1}{2} h m V^{2}} V^{5} d V}{\int_{0}^{\infty} e^{-\frac{1}{2} h m V^{2} V^{3} d V}}=\frac{4}{h m}=\frac{8}{3} c^{2}
$$

where $\overline{c^{2}}$ is now the mean value of $c^{2}$ averaged over all the molecules of the gas (cf. equation (161)).

The mean value of $\varpi^{2}+\varpi^{\prime 2}$ averaged over all collisions is clearly $2 \varpi^{2}$, where $\overline{\varpi^{2}}$ is the average value of $\varpi^{2}$ taken over all the molecules of the gas.

Substituting these values for $\left\{\varpi^{2}+\varpi^{\prime 2}\right\}$ and $\left\{V^{2}\right\}$ in equation (242), we obtain

$$
\left\{\Delta\left(c^{2}+c^{\prime 2}\right)\right\}=r^{2}\left(\frac{8}{3} \overline{\sigma^{2}}-\frac{16 \overline{c^{2}}}{9 k^{2}}\right)
$$

Let us write

$$
\begin{aligned}
& \mathbf{H}=\frac{1}{2} m k^{2} \overline{\varpi^{2},} \\
& \mathbf{K}=\frac{1}{2} m \overline{c^{2},}
\end{aligned}
$$

so that $K$ is the mean energy of translation, and $H$ is the mean energy of rotation, excluding rotation about the axis of symmetry. Then equation (246) can be written

$$
\left\{\Delta\left(c^{2}+c^{\prime 2}\right)\right\}=\frac{16 r^{2}}{3 m k^{2}}\left(\mathrm{H}-\frac{2}{3} \mathrm{~K}\right)
$$

115. There are $\nu$ molecules per unit volume, and, also per unit volume there are, by formula (46)

$$
\nu^{2} \sigma^{2} \sqrt{\frac{2 \pi}{h m}}
$$

collisions per unit time. Hence summing equation (247) over all the collisions which occur in time $d t$, we find that the change in the sum of the values of $c^{2}$ for all the $\nu$ molecules, which is produced by collisions in time $d t$, is

$$
\frac{16 r^{2}}{3 m k^{2}}\left(\mathrm{H}-\frac{2}{3} \mathrm{~K}\right) \nu^{2} \sigma^{2} \sqrt{\frac{2 \pi}{h m}} d t
$$


The sum of the values of $c^{2}$ is, however,

so that the change in time $d t$ is

$$
\nu \overline{c^{2}}, \text { or } \frac{2 v}{m} \mathrm{k},
$$

$$
\frac{2 \nu}{m} \frac{d \mathrm{\kappa}}{d t} d t
$$

Equating expressions (248) and (249),

$$
\begin{aligned}
\frac{d K}{d t} & =\frac{8 r^{2} \sigma^{2} \nu}{3 k k^{2}} \sqrt{\frac{2 \pi}{h m}}\left(\mathrm{H}-\frac{2}{3} \mathrm{~K}\right) \\
& =\frac{16 r^{2} \sigma^{2} \nu}{3 k^{2}} \sqrt{\left(\frac{2 \pi}{3 m} \mathrm{~K}\right)}\left(\mathrm{H}-\frac{2}{3} \mathrm{~K}\right)
\end{aligned}
$$

Or if, for the sake of brevity, we write

$$
\beta=\frac{16 r^{2} \sigma^{2}}{3 k^{2}} \sqrt{\frac{2 \pi}{3 m}}
$$

so that $\beta$ is a constant depending only on the structure of the molecules,

$$
\frac{d \mathrm{~K}}{d t}=\beta \nu \sqrt{\mathrm{K}}\left(\mathrm{H}-\frac{2}{3} \mathrm{~K}\right)
$$

Since the total energy of the gas is unaffected by collisions, and since also the energy of rotation about the axis of symmetry of the molecules is unaffected by collisions, it follows that $\mathrm{H}+\mathrm{K}$ is unaffected by collisions. Thus

$$
\frac{d \mathrm{H}}{d t}=-\frac{d \mathrm{~K}}{d t}=-\beta \nu \sqrt{\mathrm{K}}\left(\mathrm{H}-\frac{2}{3} \mathrm{~K}\right)
$$

116. From equations (252) and (253),

$$
\frac{d}{d t}\left(\mathrm{H}-\frac{2}{3} \mathrm{~K}\right)=-\frac{5}{3} \beta \nu \sqrt{\mathrm{K}}\left(\mathrm{H}-\frac{2}{3} \mathrm{~K}\right) .
$$

Since $\sqrt{\kappa}$ is always to be taken positively, it is clear that

$$
\frac{d}{d t} \log \left(\mathrm{H}-\frac{2}{3} \mathrm{~K}\right)
$$

is always negative, so that $\log \left(H-\frac{2}{3} K\right)$ continually decreases, until finally

$$
\mathrm{H}-\frac{2}{3} \mathrm{~K}=0 \text {. }
$$

This equation, then, expresses the relation between $\mathrm{H}$ and $\mathrm{K}$ in the steady state. Now $\mathrm{H}$ is the energy of two momentoids, of which the separate energies in the steady state must be equal from symmetry, and similarly $\mathrm{k}$ is the energy of three momentoids, of which the separate energies are equal in the steady state. Hence equation (254) expresses that, in the steady state, the energies of the five momentoids are equal, agreeing with the result predicted by the general dynamical theory.

The general result that the ratio of distribution of energy is independent of the structure of the molecule leads sometimes to striking results. In 
the case of the loaded sphere, let us suppose almost the whole mass concentrated at a point which must of course coincide very nearly but not quite with the geometrical centre, so that the weight of the remainder of the molecule is very small by comparison. Then $k$ is very small, and since $k^{2} \varpi_{1}^{2}$ is on the average equal to $\frac{1}{3} c^{2}$ independently of the value of $k$, it follows that $\varpi_{1}$ must be very great, becoming infinite in the limit when $k$ vanishes. In this case, then, the molecules will rotate with infinite angular velocity.

117. If the values of $H$ and $K$ are slightly disturbed from the value appropriate to the steady state, we have, from equation (254),

$$
\frac{d}{d t} \log \left(\mathrm{H}-\frac{2}{3} \mathrm{~K}\right)=-\frac{5}{3} \beta \nu \sqrt{\mathrm{K}}
$$

shewing that the disturbance will decrease exponentially with the time, and will fall to $\frac{1}{e}$ of its value in a time

$$
\frac{3}{5 \beta \nu \sqrt{K}}
$$

of which the value, on replacing $\beta$ from equation (251), and putting $K=\frac{3}{4 h}$ from equation (161), is found to be

$$
\frac{9 k^{2}}{20 r^{2}}\left(\frac{1}{2 \nu \sigma^{2}} \sqrt{\frac{h m}{2 \pi}}\right)
$$

The factor in brackets is the mean duration of a free path (cf. equation (48)), so that the time in question is

$$
\frac{9 k^{2}}{20 r^{2}} \times(\text { the average duration of a free path })
$$

This quantity naturally depends both upon the structure of the molecules and the state of the gas. Its reciprocal will, so to speak, measure the amount of "grip" which the translational motion is able to obtain over the rotational.

The problem of partition of energy in a system of loaded spheres was first investigated by Burnside*. His result was, however, erroneous. The correct result was subsequently obtained by Burbury + . In the same paper Burbury calculates the rate of subsidence of a disturbance in a gas consisting of two kinds of symmetrical molecules, the disturbance consisting of a small inequality in the mean translational energy of the two kinds of molecule. The corresponding calculation for loaded spheres was, so far as I know, first given in papers by myself.

* "On the Partition of Energy between the Translatory and Rotatory Motions of a set of nonhomogeneous Elastic Spheres," Trans. R. S. E. xxxin. Part ii. (1887).

+ "On the Collision of Elastic Bodier," Phil. Trans. cLxxxmr. p. 407 (1892).

‡ "The Distribution of Molccular Energy," Phil. Trans. cxcvi.p. 397 (1901): and "On the Partition of Energy in a system of Loaded Spheres," Quarterly Journal, xxxv. p. 224 (1904). 


\section{CHAPTER VI.}

\section{PHYSICAL PROPERTIES OF A GAS.}

\section{Pressure, Density and Temperature.}

118. In the preceding chapters the main dynamical problem of the motion of a gas has been solved with sufficient generality to justify us in hoping for some agreement with the results of experiment. We therefore attempt now to interpret the mathematical results which have been obtained, in terms of the physical properties of a gas. We shall begin by investigating the law which ought, as a consequence of our mathematical analysis, to connect the pressure, volume and temperature of a gas.

\section{Calculation of the Pressure of a Gas.}

119. Let us consider any small element $d S$ of the surface of the vessel containing the gas, this element being so small that it may, without appreciable error, be regarded as plane. The directions of the axes are at present perfectly arbitrary, so that we may choose them so that the outward normal to $d S$ is parallel to the axis of $x$.

If $p$ is the pressure per unit area at the element $d S$, the pressure on $d S$ is $p d S$, and therefore $p d S d t$ is equal to the sum of the normal components of the impulsive forces exerted upon $d S$ by the various molecules which enter into collision with it during the interval $d t$. If $A$ is any such molecule, the normal impulse exerted by $A$ on $d S$ may be divided into two parts $I_{a}$ and $I_{a}{ }^{\prime}, I_{a}$ being the total normal impulse up to the moment at which the velocity of the centre of gravity normal to the boundary $d S$ is nil, and $I_{a}^{\prime}$ the total normal impulse from this moment up to the time at which the molecule $A$ is again clear of the boundary. If the velocities of the centre of gravity of $A$ before and after collision are

we have

$$
u_{a}, v_{a}, w_{a} \text { and } u_{a}^{\prime}, v_{a}^{\prime}, w_{a}^{\prime} \text {, }
$$

$$
I_{a}=m_{a} u_{a}, \quad I_{a}^{\prime}=-m_{a} u_{a}^{\prime} \text {. }
$$


The total impulse due to $A$ is therefore $m_{a}\left(u_{a}-u_{a}^{\prime}\right)$, so that

$$
p d S d t=\sum_{\boldsymbol{A}} m_{a}\left(u_{a}-u_{a}{ }^{\prime}\right) \text {. }
$$

where the summation extends over all molecules which collide with $d S$ during the time $d t$. To calculate the value of this sum, we require to know the law of distribution of velocities.

120. In practice, what is observed is not the pressure at a particular instant, but the pressure averaged over a certain interval of time. In fact $p$, regarded as a function of the time, would be an infinitely rapidly varying quantity similar to the $H$ described in $\S 70$. The quantity which experiment enables us to determine is the value of $p$ averaged over a length of time sufficient for these rapid variations to be of no importance. In this way an experimental value of $p$ is obtained which is independent of the time.

Now the value of $p$ depends on the law of distribution of velocities $f$, and therefore on $H$. If we evaluate $H$ as a function of the time we shall find it to be an infinitely-rapidly varying function, but if we average its value over a sufficient interval of time, it has, as was shewn in Chapter III., a constant value; and this value is its minimum value, which corresponds to the normal state. Hence we see that the value of $p$ which is to be expected from experiment may legitimately be calculated on the supposition that the gas is in its normal state throughout.

\section{INFinitely small Molecules.}

121. The number of molecules which have velocities lying between $u$ and $u+d u$ etc., and which will impinge on $d S$ during the interval $d t$ is, as in $\$ 27$, equal to the number of molecules having velocities satisfying these conditions and lying within an element $u d S d t$ of volume at the beginning of the interval $d t$.

When the molecules are infinitely small, this number may be taken to be

$$
\nu f(u, v, w) d u d v d w u d S d t
$$

Hence we have, in equation (258),

$$
\sum_{A} m_{a} u_{a}=d S d t m \nu \iiint f(u, v, w) u^{2} d u d v d w
$$

where the integration extends over all values of $u, v$ and $w$ for which the molecules can collide with $d S$. In other words, the integration is to extend over all possible values of $v$ and $w$, and over all positive values of $u$.

In a similar way, we find that $-\sum_{\boldsymbol{A}} m u^{\prime}$ in equation (258) is equal to the quantity on the right-hand of equation (260), except that the integration must now be understood to extend over all values of $u, v$ and $w$ which are possible for molecules which are just rebounding from collision with the element $d S$. 
The integration is therefore over all values of $v$ and $w$, and over all negative values of $u$.

By addition of the two results just obtained, and comparison with equation (258) we obtain

$$
p=m \nu \iiint_{-\infty}^{+\infty} f(u, v, w) u^{2} d u d v d w
$$

the integration now extending over all possible values of the velocity components.

The integral is equal to the mean value of $u^{2}$ throughout the gas. If, as before, we denote this by $\overline{u^{2}}$, we have from equation (161),

$$
m \overline{u^{2}}=\frac{1}{2 h},
$$

in which we now introduce the supposition that the gas is in the normal state. Hence we have the pressure in the forms

$$
p=m \nu \overline{u^{2}}=\frac{\nu}{2 h}
$$

or again, if we use the relation $m \nu=\rho$, we have

$$
p=\rho \overline{u^{2}}=\frac{1}{3} \rho \overline{c^{2}} \text {. }
$$

This last result can be conveniently stated in words in the form :

The pressure per unit area of surface is equal to two-thirds of the kinetic energy of translational motion per unit volume of the gas.

\section{Boyle's Law.}

122. Since the heat of a gas, on the hypothesis of the Kinetic Theory, represents the energy of motion of the molecules of the gas, we must suppose that $\overline{c^{2}}$ remains the same so long as the temperature of the gas remains the same. Hence from equation (263) we can deduce Boyle's Law :

The pressure of a gas is proportional to its density so long as the temperature remains unaltered.

The exact relation between the temperature and the value of $\overline{c^{2}}$, must however be the subject of more careful discussion later.

\section{Pressure in a Mixture of Gases.}

123. If there are present in the gas molecules of different types $\alpha, \beta \ldots$, equation (261) must be replaced by

$$
p=\sum_{a} m_{a} \nu_{a} \iint_{-\infty}^{+\infty} \int_{a} f_{a}(u, v, w) u^{3} d u d v d w,
$$


where the notation is the same as in $\S 73$. This leads to the relation

or by equation (161),

$$
p=\sum_{a} \overline{m_{a} u^{2}} \nu_{a},
$$

$$
p=\sum_{a} \frac{\nu_{a}}{2 h}
$$

This again can be written

$$
p=\frac{\nu}{2 h},
$$

where $\nu$ is simply the number of molecules of all kinds, per unit volume of the gas.

This calculation shews that the pressure may be regarded as the sum of a number of contributions, one from each constituent gas. This is confirmed by Dalton's Law :

The pressure exerted by a mixture of gases is equal to the sum of the pressures exerted separately by its several components.

\section{Temperature.}

\section{Definition.}

124. The question which naturally presents itself at this stage is the relation of the pressure and density to the temperature. Before this can be discussed we must decide what is to be the Kinetic Theory interpretation of temperature. We have supposed that when the energy of internal motion of a gas is increased, its temperature is raised, but nothing has so far been said as to the quantitative relation between the energy and the temperature. The question of this relationship is complicated by the fact that there are a great number of different scales of temperature outside the Kinetic Theory: the scale of the mercury thermometer, those of the various possible gas thermometers, and the absolute thermodynamical scale may be mentioned as instances, and they all lead to essentially different definitions of temperature. It is true that in practice these scales are-except for additive and multiplicative constants-in substantial agreement, but this does not help in the least in a theoretical discussion. The simplest plan seems to be to introduce a new and independent Kinetic Theory scale. The selection of this scale is in a sense arbitrary; but if the hypotheses of the Kinetic Theory are true, it will be possible so to choose it that it will agree very approximately with all the existent scales. We shall define temperature on the Kinetic Theory scale as follows:

In the normal state of a gas, by equation (161),

$$
\overline{m u^{2}}=\overline{m v^{2}}=m w^{2}=\frac{1}{2 h} .
$$


We define the temperature $T$ of this gas to be such that each fraction is equal to $R T$, where $R$ is an absolute constant, as yet undetermined. We accordingly have the equations

$$
\overline{m u^{2}}=\overline{m v^{2}}=\overline{m w^{2}}=\frac{1}{2 h}=R T
$$

This temperature, as we shall see later, is an "absolute" temperature, the zero of the scale being at about $-273^{\circ} \mathrm{C}$.

\section{Equalisation of Temperature.}

125. If gases of types $\alpha, \beta \ldots$ are mixed, we have from equation (161),

$$
\overline{m_{a} u u^{2}}=\ldots=\overline{m_{\beta} u^{2}}=\ldots=\frac{1}{2 h},
$$

expressing of course that the average kinetic energy of a single molecule is the same for each kind of gas. In terms of the temperature, this becomes

$$
T_{a}=T_{\beta}=\ldots=\frac{1}{2 h R}
$$

-shewing that when two gases are mixed, they assume the same temperature.

In this connection an observation of Maxwell's is of importance. He says*:

"The theorem that the average kinetic energy of a single molecule is the same for molecules of different gases is not sufficient to establish the condition of equilibrium of temperature between gases of different kinds such as oxygen and nitrogen, because when the gases are mixed we have no means of ascertaining the temperature of the oxygen or of the nitrogen separately. We can only ascertain the temperature of the mixture by putting a thermometer into it.

"We cannot legitimately assert that the temperatures of the oxygen and of the nitrogen must be equal because they are in contact with each other, for the only way in which we can conceive the oxygen or the nitrogen as existing in the mixture is by picturing the medium as a system of molecules, and as soon as we begin to see the molecules distinctly, heat becomes resolved into motion.

"But since our investigation $\dagger$ is equally applicable to a system of any kind, provided only it satisfies the equations of dynamics, we may suppose it to consist of pure oxygen and pure nitrogen separated by a solid diaphragm, the solid diaphragm consisting of molecules capable of motion, but acting on each other with forces which are sufficient to prevent any molecule from

* Collected Works, ir. p. 727.

+ Maxwell here refers to the investigation which has been given in $\$ 88$ et seq. of the present book. 
getting far apart from its neighbours except under the action of disturbing forces greater than any which would occur in a system at the given temperature. In this system, though the oxygen and nitrogen cannot mix, each can make an exchange of molecular energy with the surface molecules of the diaphragm, and exchange of energy can go on within the solid diaphragm itself without any exchange of molecules between distant parts of the diaphragm.

"Hence in this system, the average kinetic energy of a molecule of oxygen will become equal to that of a molecule of nitrogen in the final state of the system, that is to say, when the temperatures of all parts of the system have become equal, and since in that final state we have pure oxygen on one side and pure nitrogen on the other, we can verify the equality of temperature by means of a thermometer. And we can now assert that the temperatures, not only of oxygen and nitrogen, but of all bodies, are equal when the average kinetic energy of a single molecule of each of these substances is the same."

Maxwell here rests his proof of the equalisation of temperatures upon his investigation which in turn rests upon the assumption of "contiuuity of path." This assumption, however, as we have seen, is not altogether satisfactory. Various attempts have been made to establish the theorem of the equalisation of temperatures without relying, in any form, upon this doubtful assumption. In particular, two may be mentioned, due respectively to Professors Bryan and Boltzmann and to Professor J. J. Thomson.

126. Bryan and Boltzmann* imagine a system in which gases of two different types $\alpha$ and $\beta$ occupy two distinct regions $A$ and $B$. These regions are separated by a layer $S$ of finite thickness which is occupied by a mixture of gases of types $\alpha$ and $\beta$. The gases inside $S$ are, however, acted upon by fields of force, such that no gas of type $\alpha$ can pass into the region $B$, and no gas of type $\beta$ can pass into the region $A$.

With reference to this system, the following propositions follow from Chapter V.:

(i) The mean translational energy of the molecules of type $\alpha$ in the region $A$ is equal to the mean translational energy of the molecules of type $\alpha$ in the region $S$.

(ii) The mean translational energy of the molecules of type $a$ in the region $S$ is equal to the mean translational energy of the molecules of type $\beta$ in the region $S$.

(iii) The mean translational energy of the molecules of type $\beta$ in the region $S$ is equal to the mean translational energy of the molecules of type $\beta$ in the region $B$.

\footnotetext{
*Wien. Sitzungsber., ur. Dec. 1894.
} 
Hence it follows that the mean translational energy per molecule in $A$ is equal to the mean translational energy per molecule in $B$, so that, in accordance with our definition of temperature, the temperature in $A$ is equal to the temperature in $B$.

127. J. J. Thomson* considers two gases in a tube separated by a piston of mass which is very great compared with that of a molecule of either gas. The condition that there shall be no transfer of energy from one gas to the other by means of the motion of the piston is found to be that the mean kinetic energy of a molecule is the same for the two gases.

Professor Thomson arrives at this result by a detailed calculation of the effect of individual collisions. The result is, however, obvious upon representing the whole system in a generalised space of the appropriate number of dimensions. For since the motion of the piston only represents one degree of freedom, the kinetic energy of its motion will only be an infinitesimal fraction of the whole energy. This energy may therefore be treated as the intermolecular potential energy $\Phi$ was treated in Chapter V., and we arrive at once at the conclusion that the mean energy of each type of molecule must be the same.

Maxwell's system of two gases separated by a diaphragm can be treated in the same way, if we assume that there are sufficient quantities of each of the two gases for their energies to be considered as infinite in comparison with the energy of the diaphragm.

\section{INFinitely S.Mald Molecules (continued).}

\section{Avogadro's Law.}

128. Replacing $\frac{1}{2 h}$ by $R T$ in equation (262), we have

$$
p=\frac{\nu}{2 h}=R \nu T
$$

Hence if two gases are at the same temperature and pressure, the molecular density is the same for both, namely, $p / R T$.

This is the well-known law of Avogadro:

Two different gases, when at the same temperature and pressure, contain equal numbers of molecules in equal volumes.

* Applications of Dynamics to Physics and Chemistry, p. 91. 


\section{Charles' Law.}

129. Again, replacing $\nu$ by $\frac{\rho}{m}$ in equation (267), we obtain the pressure in the form

$$
p=\frac{R}{m} \rho T
$$

or, since $N m=\rho \Omega$,

$$
p \Omega=R N T
$$

Equation (268) contains the combined laws of Boyle and Charles :

When the temperature of a gas is kept constant, the pressure varies as the density, and when the density is kept constant, the pressure varies as the temperature.

\section{Numerical estimate of velocities.}

130. From equation (263),

$$
p=\rho \overline{u^{2}},
$$

we can calculate the value of $\overline{u^{2}}$ for any gas with considerable accuracy. If we write $c^{2}=u^{2}+v^{2}+w^{2}$, and $C^{2}=\overline{c^{2}}$, then

$$
\overline{u^{2}}=\overline{v^{2}}=\overline{w^{2}}=\frac{1}{3}\left(\overline{u^{2}}+\overline{v^{2}}+\overline{w^{2}}\right)=\frac{1}{3} \bar{c}^{2}=\frac{1}{3} C^{2} \text {. }
$$

The quantity which is of the greatest physical interest, and which is usually tabulated, is $C$. The significance of this quantity is that the pressure is the same as if all the molecules were moving with a uniform velocity $C$. We have

$$
C^{2}=3 \overline{u^{2}}=\frac{3 R T}{m}
$$

hence $C$ is proportional to the square root of the absolute temperature, and inversely proportional to the square root of the molecular weight of the gas in question.

The equation connecting $C^{2}$ with the pressure and density (equation (263)) is

$$
p=\frac{1}{3} \rho C^{2}
$$

At normal pressure and temperature $0^{\circ} \mathrm{C}$. the weight of a cubic $\mathrm{cm}$. of oxygen reduced to Paris standard, is, according to Morley, $00142945 \mathrm{gms}$. The normal pressure is the weight of a column of mercury $76 \mathrm{~cm}$. in height. The specific gravity of mercury is 13.5953 , and at Paris the value of gravity is 980.939 . Hence for oxygen at $0^{\circ} \mathrm{C}$. equation (262) becomes

$$
76 \times 13.5953 \times 980.939=\frac{1}{3} \times 0014.2945 \times C^{2},
$$

whence we obtain $C=461 \cdot 18$ metres per sec.

and hence by equation (271),

$$
\frac{R}{m}=\frac{C^{2}}{3 T}=\frac{1}{3} C^{2} \div 273.04=259.65 \times 10^{4} .
$$


From these values in the standard case of oxygen at $0^{\circ} \mathrm{C}$, we can calculate the quantities $C$ and $R / m$ for other temperatures and substances. The following table is calculated from recent observations.

\begin{tabular}{|c|c|c|c|c|c|c|}
\hline Gas & $\begin{array}{l}\text { Density } \\
\left(\mathrm{O}_{2}=16\right)\end{array}$ & $\begin{array}{c}\text { Density at } \\
0^{\circ} \mathrm{C} . \\
(\text { Water }=1 \text { ) }\end{array}$ & $\begin{array}{c}\text { Molecular } \\
\text { Weight } \\
\left(\mathrm{H}_{2}=2\right)\end{array}$ & $\begin{array}{c}\frac{R}{m} \\
\text { (Centigrade) }\end{array}$ & $\begin{array}{l}\text { Temp. } \\
\text { (Cent.) }\end{array}$ & $\begin{array}{c}c \\
\text { (cms.) }\end{array}$ \\
\hline \multirow[t]{2}{*}{ Hydrogen ............. } & \multirow[t]{2}{*}{$1 \cdot 006$} & \multirow[t]{2}{*}{.000090} & \multirow[t]{2}{*}{2} & \multirow[t]{2}{*}{$4130 \times 10^{4}$} & $0^{\circ}$ & $1839 \times 10^{2}$ \\
\hline & & & & & $-253^{\circ}$ & $489 \times 10^{2}$ \\
\hline Helium ............... & 1.98 & & 3.93 & $2098 \times 10^{4}$ & $0^{\circ}$ & $1311 \times 10^{2}$ \\
\hline \multirow[t]{2}{*}{ Water-vapour ....... } & \multirow[t]{2}{*}{$8 \cdot 997$} & & \multirow[t]{2}{*}{$17 \cdot 88$} & \multirow[t]{2}{*}{$462 \times 10^{4}$} & $0^{\circ}$ & $615 \times 10^{2}$ \\
\hline & & & & & $100^{\circ}$ & $719 \times 10^{2}$ \\
\hline Carbon-monoxide.. & & .001234 & $27 \cdot 8$ & $296 \times 10^{4}$ & $0^{\circ}$ & $493 \times 10^{2}$ \\
\hline Nitrogen ... & $14 \cdot 071$ & .001257 & $27 \cdot 86$ & $295 \times 10^{4}$ & $0^{\circ}$ & $492 \times 10^{2}$ \\
\hline \multirow[t]{2}{*}{ Air..................... } & \multirow{2}{*}{$14 \cdot 479$} & \multirow[t]{2}{*}{.001293} & \multirow[t]{2}{*}{-} & \multirow[t]{2}{*}{$287 \times 10^{4}$} & $0^{\circ}$ & $485 \times 10^{2}$ \\
\hline & & & & & $15^{\circ}$ & $498 \times 10^{2}$ \\
\hline \multirow[t]{2}{*}{ Oxygen ................ } & \multirow[t]{2}{*}{16} & \multirow[t]{2}{*}{$\cdot 001429$} & \multirow[t]{2}{*}{$31 \cdot 76$} & \multirow[t]{2}{*}{$260 \times 10^{4}$} & $0^{\circ}$ & $461 \times 10^{2}$ \\
\hline & & & & & $-182 \cdot 5^{\circ}$ & $266 \times 10^{2}$ \\
\hline Argon .......... & \multirow[t]{4}{*}{$19 \cdot 9$} & \multirow{4}{*}{$\cdot 001974$} & $39 \cdot 6$ & $209 \times 10^{4}$ & $0^{\circ}$ & $413 \times 10^{2}$ \\
\hline Carbon-dioxide & & & $43 \cdot 7$ & $189 \times 10^{4}$ & $0^{\circ}$ & $393 \times 10^{2}$ \\
\hline Mercury .... & & & $198 \cdot 50$ & $416 \times 10^{3}$ & $0^{\circ}$ & $185 \times 10^{2}$ \\
\hline Iodine ......... & & & $251 \cdot 78$ & $328 \times 10^{3}$ & $0^{\circ}$ & $164 \times 10^{2}$ \\
\hline
\end{tabular}

Certain other quantities which are tabulated here will be found useful in later parts of the book.

The value of the constant $R$ can be found, although of course with less accuracy than the values of $R / m$, by assuming the value $4 \times 10^{10}$ for the number of molecules per cubic centimetre under normal conditions $(\$ 8)$. The weight of a cubic centimetre of hydrogen being 000090 grammes, and the number of molecules in this cubic centimetre being $4 \times 10^{19}$, we find that for hydrogen, $m=2.25 \times 10^{-24}$. Since $R / m$ for hydrogen is $413 \times 10^{5}$, this leads to the value

$$
R=9 \cdot 3 \times 10^{-17} .
$$$$
4.13 \times 10^{7}
$$

131. The quantity $C$ which has been tabulated, must be carefully distinguished from the mean velocity in the gas, of which the value is

$$
\begin{aligned}
& \bar{c}=4 \pi \sqrt{\frac{h^{3} m^{3}}{\pi^{3}}} \int_{0}^{\infty} e^{-h m c^{2} c^{3}} d c \\
& =\frac{2}{\sqrt{\pi h m}} \\
& =\sqrt{\frac{8}{3 \pi}} C
\end{aligned}
$$


It appears from this that the values of the mean velocities can be obtained by multiplying the last column in the above table by $\sqrt{\frac{8}{3 \pi}}$ or 921 .

At this stage, we obtain our first insight, as regards quantitative measurements, into the internal mechanism of a gas. The order of magnitude of the molecular-velocities could, however, have been predicted without actual detailed calculation.

For instance, if gas is allowed to stream out into a vacuum through a small hole in the containing vessel, the velocity of efflux is nothing else than the velocities of the individual molecules, which would have been simply molecular-velocities inside the vessel, had the hole not been present. Thus the mean molecular-velocity must be comparable with the velocity of efflux of the main stream of gas, and this velocity is known to be of the order of magnitude of the velocities tabulated in the last column of the table on the preceding page.

Or again, a disturbance at any point in a gas will produce an effect on the molecules in its immediate neighbourhood. - When these molecules collide with those in the next layer of gas, the effect of this disturbance is carried on into that layer, and so on indefinitely. Thus the molecules act as carriers of the effect of any disturbance, so that the disturbance is propagated, on the whole, with a velocity comparable with the mean velocity of motion of the molecules, just as, for instance, news which is carried by relays of messengers, spreads with a velocity comparable with the mean rate of travelling of the messengers. The propagation of a disturbance in the gas is, however, nothing but the passage of a wave of sound, and the velocity of sound is known to be comparable with the values of $C$ given in the table.

\section{Molecules of Finite Size.}

\section{Calculation of Pressure.}

132. When the size of the molecules may not be regarded as infinitesimal, the calculation of the pressure which has been given, in which the size of the molecules was neglected, requires correction.

A correction of this type was first given by Van der Waals, in his great essay On the Continuity of the Liquid and Gaseous States*. We shall first give an explanation of the corrections given by Van der Waals, which, it will be found, lead to an equation which expresses the deviations from Boyle's Law to a first approximation only, and we shall afterwards give a more general calculation of the pressure, which will not be restricted to small deviations from Boyle's Law.

* The original edition (1873) is in Dutch, published by Sigthoff, Leyden. There is a German translation by Roth (1881, Barth, Leipzig) and this bas been translated into English by Threlfall and Adair (1890, Physical Memoirs published under the direction of the Physical Society, Taylor and Francis, London). The references in the present book are to the English Translation. 


\section{Van der Waals' Equation.}

133. According to Van der Waals, equation (269),

$$
p v=R N T
$$

in which $v$ is now written for the volume instead of $\Omega$, to agree with the usual notation in this subject, must be corrected in two ways. The first correction is a correction to be applied to the term $v$ to represent the finite size of the molecules, and the second is a correction to be applied to the term $p$, to represent the influence upon the pressure of the forces of cohesion in the gas.

The argument of Van der Waals as to the first correction is as follows*. Let there be $N$ molecules each of diameter $\sigma$, and let us suppose the centre of each surrounded by a sphere of radius $\sigma$, and therefore of volume $\frac{4}{3} \pi \sigma^{3}$. In considering possible positions for the centre of molecule $A$, we know that it cannot lie within any of the $N-1$ spheres surrounding the $N-1$ other molecules, so that the space available for the centre of $A$ must not be taken to be $v$ but $v-(N-1) \frac{4}{3} \pi \sigma^{3}$. This expression, it is true, requires correction on account of the possibility of two or more of the $N-1$ spheres overlapping, but this correction will be of a higher order of small quantities than that already made, and may therefore be neglected.

Also the expression requires correction owing to the impossibility of the centre of a sphere being within a distance $\frac{\sigma}{2}$ of the boundary. This correction requires us further to reduce $v$ to the extent of the volume of a layer of thickness $\frac{\sigma}{2}$ taken round the boundary of the containing vessel, but clearly this correction may be neglected if $\sigma$ vanishes in comparison with the dimensions of the vessel. This condition is, of course, entirely different from the condition that the sums of the volumes of the molecules shall be small compared with the volume of the vessel. The former condition is satisfied if $\sigma v^{-\frac{1}{3}}$ may be neglected, the latter is satisfied if $N \sigma^{3} / v$ can be neglected. Using the figures given in $\S 8$, and taking the case of a gas at atmospheric pressure in a vessel of 1 litre capacity, we find

$$
\begin{aligned}
\sigma v^{-\frac{b}{3}} & =2 \times 10^{-9}, \\
N \sigma^{3} / v & =3.2 \times 10^{-4} .
\end{aligned}
$$

It is therefore rational to neglect the one correction, while taking the other into account.

* As regards method of presentation, I have followed Bolizmann (Gastheorie, ir. p. 7) more closely than the original work of Van der Wa,als, 
Hence in any element $d v$ which is known not to be within a distance $\frac{\sigma}{2}$ of the boundary, or to be included in any one of the spheres surrounding each molecule, the probability of finding the centre of a molecule is

$$
\frac{N d v}{v-(N-1) \frac{4}{3} \pi \sigma^{3}}
$$

If, however, the element is selected at random we must consider what is the probability that the conditions postulated as to its not lying inside a sphere, or within a distance $\frac{\sigma}{2}$ of the boundary, shall be satisfied.

The particular element of volume which is of importance for the calculation of the pressure is one of which the distance from the boundary is just greater than $\frac{\sigma}{2}$. The second condition, therefore, is satisfied as a matter of course. To calculate the probability of the other condition being satisfied, namely that the element $d v$ shall not lie inside any one of the $N-1$ spheres of radius $\sigma$, we notice that if it does lie in any one of these spheres, then the centre of the sphere, being at a distance not less than $\frac{\sigma}{2}$ from the boundary, must be at least as far away from the boundary as the element $d v$. In other words, if the sphere in question is divided into two hemispheres by a plane parallel to the boundary, the element $d v$ can only lie in that hemisphere which is the nearer of the two to the boundary.

Hence the probability that $d v$, selected at random, shall lie inside any particular sphere is

$$
\frac{\frac{2}{3} \pi \sigma^{3}}{v}
$$

so that the probability that it shall not lie in any of the $N-1$ spheres in question is, as far as the first order of small quantities

$$
1-\frac{(N-1) \frac{2}{3} \pi \sigma^{3}}{v}
$$

The product of this expression and expression (276) is

$$
\frac{N d v}{v} \frac{1-\frac{(N-1)}{v} \frac{2}{3} \pi \sigma^{3}}{1-\frac{(N-1)}{v} \frac{4}{3} \pi \sigma^{3}}
$$

This, then, is the probability that a molecule shall be found in the small element $d v$ of which the distance from the boundary is $\frac{\sigma}{2}$. As far as the first order of small quantities the expression is the same as

$$
\frac{N d v}{v} \frac{1}{1-\frac{(N-1)}{v} \frac{2}{3} \pi \sigma^{3}}
$$


or

where

$$
\begin{gathered}
\frac{N d v}{v-b}, \\
b=\frac{2}{3} N \pi \sigma^{3}
\end{gathered}
$$

in which the distinction between $N-1$ and $N$ is now ignored. The effect of allowing for the finite size of the molecules in the calculation of the pressure is therefore the same as that of reducing the volume from $v$ to $v-b$. To allow for this, then, we replace equation (275) by

$$
p(v-b)=R N T
$$

The value of $b$, it is of interest to notice, is four times the aggregate sum of the volumes of the molecules in the gas.

The use of the calculus of probabilities which is made in this argument is probably open to criticism. We shall not stop to discuss the validity or non-validity of the argument, as we shall subsequently arrive at exactly the same result by a method which does not rely on the calculus of probabilities for its justification.

134. The principle underlying Van der Waals' correction for cohesion is as follows. It is supposed that when the "spheres of molecular action" of two molecules do not intersect, the forces between the molecules, although small, are not negligible. Accordingly we suppose that a molecule in the gas is subjected to forces of cohesion acting between it and all the neighbouring molecules. The resultant of these forces varies continually both in direction and magnitude, with the position of the molecules. When the molecule is sufficiently far removed from the surface, all directions are equally likely for this resultant, and hence the aggregate force, averaged over a sufficient length of time, will be nil. When, however, the molecule is at or near the surface this is no longer true. Let the force from each molecule be resolved into tangential and normal components. Then all directions in the tangent plane are equally likely for the tangential components, but the normal component is in the majority of cases directed inwards. Averaged over a sufficient length of time the resultant force will therefore be a normal force always directed inwards.

We have here supposed the radii of curvature of the surface to be so large compared with molecular dimensions that the surface may at every point be regarded as plane. In this case the conditions will be the same at every point of the surface, and the normal force will depend only on the density of the gas, and the distance from the boundary of the point at which this force is estimated.

Thus the average effect of the forces of cohesion can be represented by a permanent field of force acting at and near the surface. It is this field of force which may be regarded as giving rise to the phenomena of capillarity and surface-tension in liquids. Now if we follow Van der Waals in supposing 
that the actual forces of cohesion are adequately represented by this permanent field of force, it will be easy to calculate the influence of this field of force upon the pressure.

For the field of force can be regarded as exerting an inward pressure, say $p_{1}$ per unit area, upon the outermost layers of molecules of the gas. Clearly this pressure must be supposed proportional jointly to the number of molecules per unit area in this layer, and to the intensity of the normal component of force. Each of these two factors is directly proportional to the density of the gas, so that $p_{1}$ will be proportional to the square of the density. Let us suppose, then, that

$$
p_{1}=c \rho^{2} \text {, }
$$

where $c$ is a constant depending only on the nature of the gas. The molecules are now deflected upon reaching the boundary, not by impact alone, but as the total result of their impact with the boundary and of the action of the supposed field of force. In other words they may be supposed acted on by a total pressure $p+p_{1}$ or $p+c p^{2}$, instead of by the simple pressure $p$.

Hence equation (279) must be further amended by writing it in the form

$$
\left(p+c \rho^{2}\right)(v-b)=R N T .
$$

or again, replacing $\rho$ by $N^{\top} m / v$,

$$
\left(p+\frac{a}{v^{2}}\right)(v-b)=R N T
$$

where

$$
a=c N^{2} m^{2} \text {. }
$$

This is Van der Waals' equation connecting $p, v$ and $T$. It will be noticed that $a$ and $b$ are constants for the same mass of gas, but depend on the amount of gas as well as on its nature, $a$ being proportional to the square and $b$ to the first power of the amount of gas.

135. One factor which is overlooked in the argument by which this equation is obtained, is that when cohesion forces exist, some molecules which would have reached the boundary had there been no cohesion forces, may never reach the boundary at all, being deflected by the cohesion forces before their paths meet the boundary. Actually, then, these molecules exert no pressure on the boundary, whereas Van der Waals' argument supposed them to exert a negative pressure. As a consequence, equation (281) admits of negative values for $p$, whereas an examination of the physical conditions shews that $p$ is necessarily positive.

This objection, however, is of no weight so long as it is clearly recognised that equation (281) is true only to the first order, as regards deviations from Boyle's Law. 
136. The equation of Van der Waals supplies the most convenient basis for discussing the behaviour of a gas over those ranges of pressure, volume and temperature for which the equation can be regarded as approximately true, i.e. over ranges in which the deviation from Boyle's Law is small. We shall return to a discussion of the equation later. In the meantime we shall investigate a general relation between pressure, volume and temperature, without the limitation that the deviations from Boyle's Law are to be such as may be regarded as small.

\section{General Calculation of Pressure.}

137. We return now to the calculation started in $\S 119$, but shall make no assumption as to the size of the molecules.

The number of molecules which have velocities lying between $u$ and $u+d u$, etc., and which will impinge on the element $d S$ of the boundary during the interval $d t$, is as before, equal to the number of molecules baving velocities satisfying these conditions, and lying within an element $u d S d t$ at the beginning of this interval. This number, however, is no longer given by expression (259).

If the molecules possess spheres of molecular action satisfying the conditions laid down in $\S 82$, the number required may be supposed to be

$$
\nu_{1} f(u, v, w) d u d v d w u d S d t .
$$

where $\nu_{1}$ is the "effective molecular density" (cf. $\S 66$ ) at the element in question-i.e. at a point distant $\frac{1}{2} \sigma$ from the boundary.

If the molecules do not possess spheres of molecular action, this expression must be replaced by

$$
d u d v d w u d S d t \iint \ldots \nu_{1} f\left(u, v, w, \xi_{1}, \xi_{2} \ldots\right) d \xi_{1} d \xi_{2} \ldots,
$$

since $\nu_{1}$ is no longer a function solely of $x, y, z$. This case is too complex for progress to be possible.

If we revert to expression (282), and if, as in the previous calculation of the pressure in a mixture of gases ( $\$ 123)$, we suppose the gas to consist of a mixture of molecules of types $\alpha, \beta \ldots$, then we have in equation (258)

$$
\sum_{A} m_{a} u_{a}=d S d t \sum_{a} m_{a}\left(\nu_{a}\right)_{1} \iiint f_{a}(u, v, w) u^{2} d u d v d w
$$

where, as before, the integration extends over all values of $u$, $v$, and $w$ for which the molecules can collide with $d S$, and therefore over all possible values of $v$ and $w$, and over all positive values of $u$.

Also, as before, the term $-\sum_{\boldsymbol{A}} m_{a} u_{a}^{\prime}$ in equation (258) is equal to the quantity on the right-hand of equation (283) except that the integration extends over all values of $u, v$, and $w$ which are possible for molecules which 
have just collided with $d S$, and therefore over all values of $v$ and $w$ and over all negative values of $u$.

Substituting these values in equation (258), we obtain

$$
p=\sum_{a} m_{\alpha}\left(\dot{\nu_{\alpha}}\right)_{1} \iiint_{-\infty}^{+\infty} \int_{\alpha}(u, v, w) u^{2} d u d v d w
$$

where the integration now extends over all values of $u, v$ and $v$. The integral is the mean value of $u^{2}$ for all molecules of type $\alpha$. If we denote this by $\overline{u_{a}^{2}}$ we have, from equation (161) as before,

so that

$$
m_{a} \overline{u_{a}^{2}}=\frac{1}{2 h}
$$

$$
\begin{aligned}
& p=\sum_{a} m_{\alpha}\left(\nu_{\alpha}\right)_{1} \iint_{-\infty}^{+\infty} \int_{\infty} f_{\alpha}(u, v, w) u^{2} d u d v d w \\
& =\Sigma\left(\nu_{1}\right)_{a} \overline{m_{a} u^{2}} \\
& =\frac{1}{2 h} \Sigma\left(\nu_{a}\right)_{1}
\end{aligned}
$$

and when there is only one kind of gas present,

$$
p=\frac{\nu_{1}}{2 h}
$$

Here $\nu_{1}$ is the "effective molecular density" at the nearest point to the boundary at which it is possible for the centre of a molecule to lie. To suggest the point at which $\nu_{1}$ is measured, let us denote its value for this point by $\nu_{b}$, so that

$$
p=\frac{\nu_{b}}{2 h}=R \nu_{b} T
$$

Deviations from the Laws of Dalton, Avogadro, Boyle, and Charles.

138. It will be clear both from Van der Waals' equation and the equation just obtained that the various simple laws deduced from the former equation (262), namely

$$
p=\frac{\nu}{2 h},
$$

all require correction when we abandon the simplifying hypotheses upon which this equation was deduced.

\section{Deviations from Avogadro's Law.}

139. If two gases are at the same temperature and pressure it follows from equation (287) that $\nu_{b}$ must be the same for both. The correction required for Avogadro's Law, therefore, consists simply in replacing $\nu$ by $\nu_{b}$. 


\section{Deviations from Dalton's Law.}

140. Equation (285) expresses the general law for the pressure in a mixture of gases. Dalton's law is no longer true, because $\left(\nu_{a}\right)_{b}$ depends not only on the number of molecules of type $\alpha$ which are present, but also on the numbers of molecules of other kinds.

The correction required, at any rate to a first approximation, can best be seen from an inspection of Van der Waals' equation (280), which, throughout the range for which it is true, namely as far as the first order of small quantities, may be written

$$
p=-c \rho^{2}+\frac{R N T}{v}\left(1+\frac{b}{v}\right)
$$

If there are two gases denoted by suffixes $\alpha$ and $\beta$, it will be found that in the simple case in which the molecules are supposed all of the same size, the total pressure is given by

$$
p=-\left(c_{a} \rho_{a}+c_{\beta} \rho_{\beta}\right)\left(\rho_{a}+\rho_{\beta}\right)+\frac{\left(N_{\alpha}+N_{\beta}\right) R T}{v}\left(1+\frac{b_{a}+b_{\beta}}{v}\right) .
$$

For $c_{a} \rho_{a}+c_{\beta} \rho_{\beta}$ replaces $c \rho$ as sthe intensity of the force of cohesion, and $b_{a}+b_{\beta}$ replaces $b$ as four times the sum of the molecular volumes.

The partial pressures, calculated on the supposition that only one kind of gas is present, are given by

$$
\begin{aligned}
& p_{a}=-c_{a} \rho_{a}{ }^{2}+\frac{N_{a} R T}{v}\left(1+\frac{b_{a}}{v}\right), \\
& p_{\beta}=-c_{\beta} \rho_{\beta}{ }^{2}+\frac{N_{\beta} R T}{v}\left(1+\frac{b_{\beta}}{v}\right)
\end{aligned}
$$

hence, instead of $p$ being equal to the sum of the partial pressures $p_{a}, p_{\beta}$, we have

$$
p=p_{\alpha}+p_{\beta}-\left(c_{\alpha}+c_{\beta}\right) \rho_{a} \rho_{\beta}+\frac{N_{\alpha} b_{\beta}+N_{\beta} b_{\alpha}}{v} .
$$

The correction to be applied to Dalton's law is too complex for further investigation to be profitable.

\section{Deviations from Boyle's Law and Charles' Law.}

141. It is obvious enough that the laws of Boyle and Charles will require correction.

If it were not for these corrections all gases would expand in the same proportion under an increase of temperature or a decrease of pressure, so that the scales of all gas thermometers would be the same, and the equation

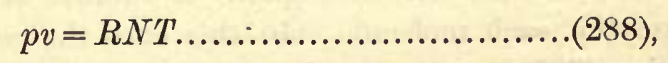

would identify the Kinetic Theory scale of temperature with that of any gas thermometer. 
It is convenient to classify gases into "ideal" and "real" according as they do or do not accurately satisfy the laws expressed by equation (288). No ideal gas is known to exist: all gases in nature shew deviations from equation (288) for certain temperatures and pressures. At the same time for some other temperatures and pressures, the deviation from equation (288) may be very slight, and in such cases we may say that for these temperatures and pressures the gas differs very little from an ideal gas. From equation (288) it is clear that the Kinetic Theory scale of temperature which we have introduced is the scale of an imaginary gas-thermometer in which an ideal gas is used as thermometric substance.

142. We proceed to discuss the divergencies from equation (288) which are to be looked for in a gas in which the molecules are not regarded as infinitely small. We shall do this first upon the assumption that it is permissible to use the conception of a "sphere of molecular action," defined in $\S 82$. Assuming this, we may use equation (287), calculating $\nu_{b}$ in the way already explained in Chapter IV. In the notation of $\$ 66$ (equation (124)) we have

$$
\nu_{1}=N \frac{I(b, c, d \ldots)}{I(a, b, c, d \ldots)}
$$

expressing the value of $\nu_{1}$ at the point $x_{a}, y_{a}, z_{a}$. Here $I(a, b, c, d \ldots)$ is the element of volume integrated throughoút the whole of the generalised space except those parts excluded by $\$ 33$.

The element of volume integrated through the whole of the generalised space is, as in $\S 65$, equal to $\Omega^{N}$. The excluded parts are of two types.

We have in the first place to exclude the region given by equation (53) in which $\phi\left(x_{a}, y_{a}, z_{a}\right)<\frac{1}{2} \sigma$. This exclusion can be represented fully by limiting the size of our vessel, and supposing it to have a layer of thickness $\frac{1}{2} \sigma$ removed from the interior. Let the remaining volume be supposed to be $\Omega^{\prime}$, then the corrected value of the integral $I(a, b, c \ldots)$ will obviously be $\Omega^{\prime N}$.

Secondly we must exclude from the generalised space regions of the type given by

$$
\left(x_{a}-x_{b}\right)^{2}+\left(y_{a}-y_{b}\right)^{2}+\left(z_{a}-z_{b}\right)^{2}<\sigma^{2}
$$

Let us consider the contribution to the whole integral $\Omega^{\prime N}$ which must be removed on account of this particular exclusion. The whole integral may be taken to represent all possible ways of distributing the centres of the molecules $A, B$... throughout a volume $\Omega^{\prime}$, each position being equally likely for each molecule. In this case the contribution which satisfies condition (290) represents all arrangements for which the centres of $A$ and $B$ lie within a distance $\sigma$ of one another. 
Now if $B$ is in a position such as I. in fig. 5 , in which its centre is at a distance greater than $\sigma$ from the boundary of the volume $\Omega^{\prime}$, the proportion of configurations represented in the generalised space in which condition (290) is satisfied-or, what is the same thing, in which the centre of $A$ lies within a sphere of radius $\sigma$ surrounding $B$-is equal to the ratio of the volume of this sphere, to the whole volume which is available for the centre of $A$, is therefore equal to $\frac{4}{3} \pi \sigma^{3} / \Omega^{\prime}$. If, however, $B$ is in a position in

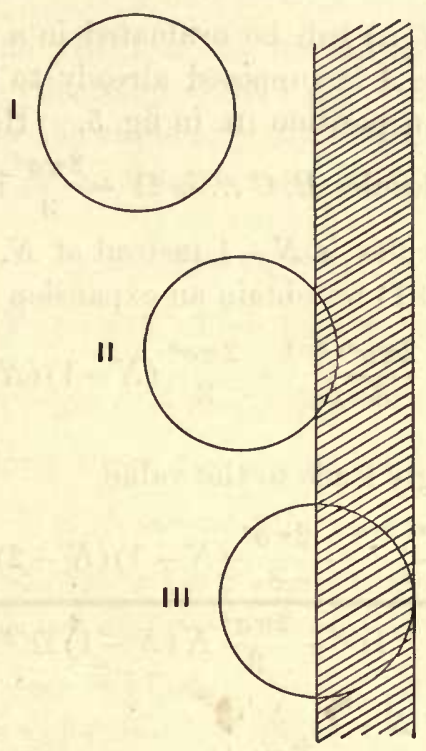

Fia. 5.

which its centre is at a distance less than $\sigma$ from the boundary, including being in a position such as II. in fig. 5 , in which its centre is at a distance less than $\frac{1}{2} \sigma$ from the boundary, the proportion in question is less than that just found, since it is only possible for $A$ to lie in that portion of a sphere of radius $\sigma$ surrounding $B$ which lies inside the volume $\Omega^{\prime}$. Ultimately, when $B$ is in position III., the proportion is only one-half of the above quantity, namely, $\frac{2}{3} \pi \sigma^{3} / \Omega^{\prime}$, for it is now only possible for $A$ to lie inside a single hemisphere of the sphere about $B$. On averaging over all positions of $B$ it is obviously legitimate to disregard the exceptional cases II. and III., and so we arrive at the result that the proportion of cases in which condition (290) is satisfied is $\frac{4}{3} \pi \sigma^{3} / \Omega^{\prime}$. Thus if condition (290) defined the only region to be excluded, the whole integral $\Omega^{\prime N}$ would have to be reduced by $\frac{4}{3} \pi \sigma^{3} / \Omega^{\prime N-1}$.

There are, however, $\frac{1}{2} N(N-1)$ such conditions, corresponding to all possible pairs of molecules. The total reduction is therefore $\frac{1}{2} N(N-1)$ times the foregoing amount. From this must be subtracted a quantity representing regions in which two of the conditions of the type of (290) are 
satisfied at once. This again must be corrected on account of the possibility of more than two of these conditions being satisfied at once, and so on, indefinitely.

In this way we obtain an expansion in descending powers of $\Omega^{\prime}$, of which the first two terms have been shewn to be

$$
I(a, b, c, d \ldots)=\Omega^{\prime N}-\frac{2 \pi \sigma^{3}}{3} N(N-1) \Omega^{\prime N-1}+\ldots
$$

The integral $I(b, c, d \ldots)$ can be evaluated in a similar way except that in this case the molecule $A$ is supposed already to be in collision with the boundary, and therefore in position III. in fig. 5. Hence the available space for the centres of the molecules $B, C \ldots$ is $\Omega^{\prime}-\frac{2 \pi \sigma^{3}}{3}$ instead of the former $\Omega^{\prime}$, and the number of molecules is $N-1$ instead of $N$. Making the necessary alterations in equation (291) we obtain an expansion in the form

$$
I(b, c, d \ldots)=\left(\Omega^{\prime}-\frac{2 \pi \sigma^{3}}{3}\right)^{N-1}-\frac{2 \pi \sigma^{3}}{3}(N-1)(N-2)\left(\Omega^{\prime}-\frac{2 \pi \sigma^{3}}{3}\right)^{N-2}
$$

Equation (289) accordingly leads to the value

$$
\begin{aligned}
\nu_{b} & =N \frac{\left(\Omega^{\prime}-\frac{2 \pi \sigma^{3}}{3}\right)^{N-1}-\frac{2 \pi \sigma^{3}}{3}(N-1)(N-2)\left(\Omega^{\prime}-\frac{2 \pi \sigma^{3}}{3}\right)^{N-2}+\ldots}{\Omega^{\prime} N-\frac{2 \pi \sigma^{3}}{3} N(N-1) \Omega^{\prime N-1}+\ldots} \\
& =\frac{N}{\Omega^{\prime}}\left(1+\frac{2 \pi \sigma^{3}}{3} \frac{N-1}{\Omega^{\prime}}+\ldots\right),
\end{aligned}
$$

on expanding as far as the first two terms; and since, in the limit, we may put

$$
\frac{N-1}{\Omega^{\prime}}=\frac{N}{\Omega^{\prime}}=\frac{N}{\Omega}=\nu
$$

we obtain $\nu_{b}$ in the form

$$
\nu_{b}=\nu+\frac{2 \pi \sigma^{3}}{3} \nu^{2}+\ldots
$$

Using this value for $\nu_{b}$ in equation (287) we obtain the equation

$$
p=\left(\nu+\frac{2 \pi \sigma^{3}}{3} \nu^{2}+\ldots\right) R T
$$

which agrees with equation (279) as far as the first two terms.

143. We shall now examine what correction is to be applied for the forces of cohesion. The principle underlying this correction has already been explained ( $\$ 134)$ in deducing Van der Waals' equation, so that we may start at once with the conception of a permanent field of force at and near the 
boundary of the gas. The effect of this field of foree upon the gas as a whole will be to alter the density of gas at points near the boundary. If $d$ is the distance from the boundary of a point near the boundary, we may assume a gas density of the form $\rho=f(d)$. If we calculate the potential of the supposed permanent field of force for this law of density we find a potential $\chi(d)$ at distance $d$ from the boundary, and we now have, by $\S 79$, a relation of the form

$$
f(d)=C e^{-2 h x(d)}
$$

from which to determine $f(d)$. If the intermolecular forces are small, we can calculate $\chi(d)$ upon the supposition that $\rho$ is constant throughout the gas, and we then have for the value of $\chi(d)$ an expression of the form $\rho \psi(d)$ where $\psi$ is independent of $\rho$. In this ease, we can write the density at the boundary in the form

$$
\rho_{0}=f(0)=C e^{-2 h \rho \psi(0)},
$$

and the mean density of the gas, which may be taken to be $f(\infty)$, will be given by

$$
\rho=f(\infty)=C e^{-2 h \rho \psi(\infty)} .
$$

The elimination of $C$ from these two equations gives

$$
\rho_{0}=\rho e^{-2 h \rho \psi}
$$

where $\psi$ is written for $\psi(0)-\psi(\infty)$, a quantity which is independent of $\rho$ and depends only on the law of force between the molecules.

In the more general case in which it is not legitimate to assume as a first approximation that $\rho$ is constant throughout the gas, we shall find it convenient to suppose that the value of $\rho_{0}$ is still given by equation (296), but we must in this case regard $\psi$ as a function of both $\rho$ and $h$.

The significance of the distinction between $\rho$ and $\rho_{0}$, expressed by equation (296), is as follows. We are supposing a density $\rho_{0}$ at the boundary, and have calculated $\nu_{b}$, and therefore $p$, on the supposition that $\rho_{0}$ is the density throughout the gas. Owing to cohesion, this density is $\rho$, so that the equation

$$
p=R \nu_{b} T
$$

expresses $p$ in the terms of the boundary density $\rho_{0}$, instead of the true density $\rho$.

Hence the relation between $p$ and $\rho$ must be obtained by the elimination. of $\rho_{0}$ between the equation

$$
p=\nu_{b}\left(\rho_{0}\right) R T
$$

in which $\nu_{b}\left(\rho_{0}\right)$ denotes the effective density at the boundary for a homogeneous gas of density $\rho_{0}$, and the equation

$$
\rho_{0}=\rho e^{-2 h \rho \psi}
$$


The result of this elimination can be expressed in the form

$$
p=\nu_{b}\left(\rho e^{-2 h \rho \psi}\right) R T
$$

This is the general relation between pressure and volume. Using the value of $\nu_{b}$ given by equation (293), the relation can be written in the form

$$
p=R T\left(\nu e^{-2 h \rho \psi}+\frac{2 \pi \sigma^{3}}{3} \nu^{2} e^{-4 h \rho \psi}+\ldots\right) .
$$

This again can be written

$$
p=R T e^{-2 h \rho \psi}\left(\nu+\frac{2 \pi \sigma^{3}}{3} \nu^{2} e^{-2 h \rho \psi}+\ldots\right) .
$$

The bracket on the right-hand side may be regarded as the product of $\nu$ and of a function expanded in powers of

$$
\nu \sigma^{3} e^{-2 h \rho \psi} \text {. }
$$

If we introduce $\sigma_{c}$ defined by

$$
\sigma_{c}=\sigma e^{-\frac{l}{3} h \rho \psi}
$$

the bracket becomes

$$
\nu+\frac{2 \pi \sigma_{c}^{3}}{3} \nu^{2}+\ldots
$$

and is now identical with the $\nu_{b}$ of equation (293), except that $\sigma_{c}$ replaces $\sigma$. Hence we can suppose the pressure given by

$$
p=R \nu_{b} T e^{-2 h \rho \psi}
$$

where $\nu_{b}$ is calculated upon the supposition that the diameters of the spheres are $\sigma_{c}$ instead of $\sigma$.

If we write $2 h=\frac{1}{R T}$ from equation (265), and $\rho_{b}=m \nu_{b}$, this assumes the form

$$
p=\frac{R T}{m} \rho_{b} e^{-\frac{\rho \psi}{R T}}
$$

in which $\rho_{b}$ is now given by

$$
\rho_{b}=\rho+\frac{2 \pi \sigma_{c}^{3}}{3 m} \rho^{2}+\ldots
$$

\section{ISOTHERMALS.}

144. The relation between $p, T$ and $\rho$ implied in equation (303) is best exhibited by drawing "isothermals" or graphs shewing the relation between $p$ and $\rho$ when $T$ is kept constant. Following the usual practice we shall assume that we are dealing with a unit mass of gas and therefore replace $\rho$ by $1 / v$. We now attempt to draw isothermals shewing the relation between $p$ and $v$ for constant values of $T$. 


\section{Isothermals of an ideal gas.}

145. When $\rho_{b}$ is put equal to $\rho$, and the cohesion factor $e^{-\rho \psi / R T}$ ignored, equation (303) becomes identical with the original equation (268)

$$
p=\frac{R}{m} \rho T^{\prime}=\frac{R T}{m v}
$$

and the isothermals are the family of curves

$$
p v=\text { constant }
$$

Plotted out with $p, v$ as rectangular coordinates, these curves are a family of similarly situated rectangular hyperbolas, having the axes for asymptotes. These, then, are the isothermals of an ideal gas.

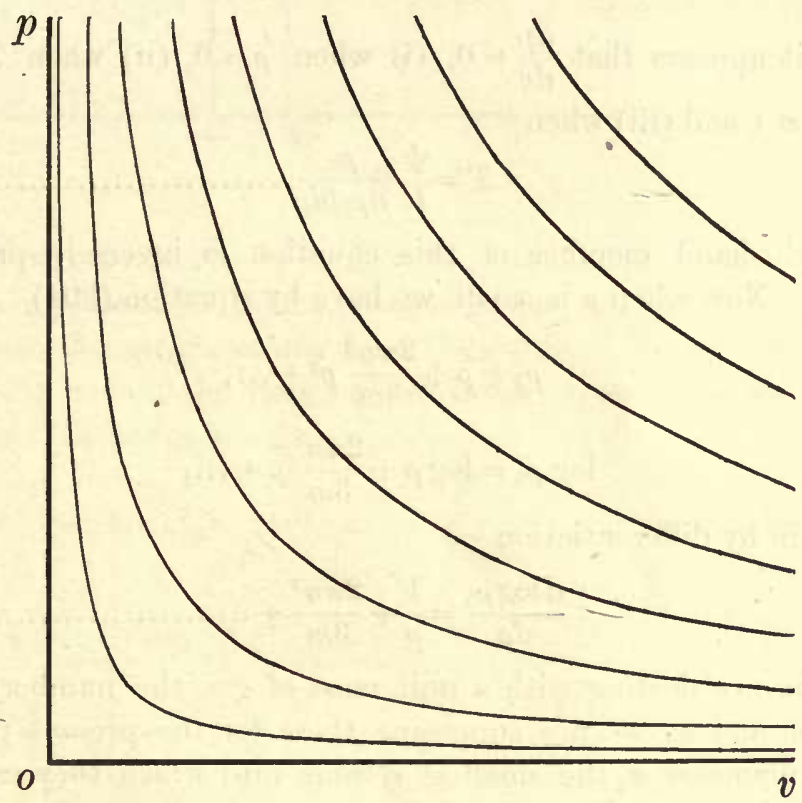

FIG. 6.

\section{Isothermals of a real gas.}

146. For extremely small values of $\rho$ it will still be legitinate to replace equation (303) by $(305)$, so that the isothermals represented by equation (303) will be similar to those of fig. 6 , at the extreme right-hand of the figure, but will require correction as we approach the region in which $v$ is small.

The nature of one correction is at once obvious. The molecules being of finite size cannot be compressed into a volume less than a certain minimum volume $v_{0}$. When $v$ is only just greater than $v_{0}$, the average free path of the 
molecules is vanishingly small. Thus the impacts upon the boundary, of the molecules nearest to the boundary, will be infinitely frequent, and the pressure will be infinite. The effective density of molecules in collision with the boundary is infinite, for all the molecules nearest to the boundary are in position III. of fig. 5. Hence when $v=v_{0}$, we have $p=\infty, \rho_{b}=\infty$. It follows that a system of values of $p, v, T$ for which $v<v_{0}$ is impossible; and that in the corrected form of fig. 6 the isothermals are limited to the region

$$
v>v_{0} \text {. }
$$

147. In general we have by differentiation of equation (303), treating $\psi$ and $\sigma_{c}$ as constants for the sake of simplicity,

$$
\frac{d p}{d v}=-\rho^{2} \frac{d p}{d \rho}=-\frac{R T}{m} \rho^{2} e^{-\frac{\rho \psi}{R T}}\left(\frac{d \rho_{b}}{d \rho}-\frac{\rho_{b} \psi}{R T}\right)
$$

Hence it appears that $\frac{d p}{d v}=0$, (i) when $\rho=0$, (ii) when $T=0$ (except when $\rho_{b}=\infty$ ), and (iii) when

$$
T=\frac{\psi}{R} \frac{\rho_{b}}{d \rho_{b} / d \rho}
$$

The right-hand member of this equation is inversely proportional to $d \log \rho_{b} / d \rho$. Now when $\rho$ is small, we have by equation (304)

$$
\rho_{b}=\rho+\frac{2 \pi \sigma^{3}}{3 m} \rho^{2}+\ldots
$$

whence

$$
\log \rho_{b}=\log \rho+\frac{2 \pi \sigma^{3}}{3 m} \rho+\ldots
$$

and therefore by differentiation

$$
\frac{d \log \rho_{b}}{d \rho}=\frac{1}{\rho}+\frac{2 \pi \sigma^{3}}{3 m}+
$$

Since we are dealing with a unit mass of gas, the number of molecules will be $1 / m$, and as we are supposing these for the present purpose to be spheres of diameter $\sigma$, the smallest volume into which they can be packed without overlapping will be given by

$$
v_{0}=\frac{1}{m} \frac{\sigma^{3}}{\sqrt{2}}
$$

whence

$$
\frac{2 \pi \sigma^{3}}{3 m}=\frac{2 \sqrt{2} \pi}{3} v_{0}
$$

The relation just found enables us to express equation (309) in the form

$$
\frac{d \log \rho_{b}}{d \rho}=v+\frac{2 \sqrt{2} \pi}{3} v_{0}+\text { terms in inverse powers of } v
$$

This equation enables us to determine the asymptotic value of $d \log \rho_{b} / d \rho$ when $v$ is great. We also know that $d \log \rho_{b} / d \rho$ becomes infinite and positive as $v$ approaches the value $v=v_{0}$ from the side $v>v_{0}$. 
The graph expressing $d \log \rho_{b} / d \rho$ as a function of $v$ is therefore that given in fig. 7 .

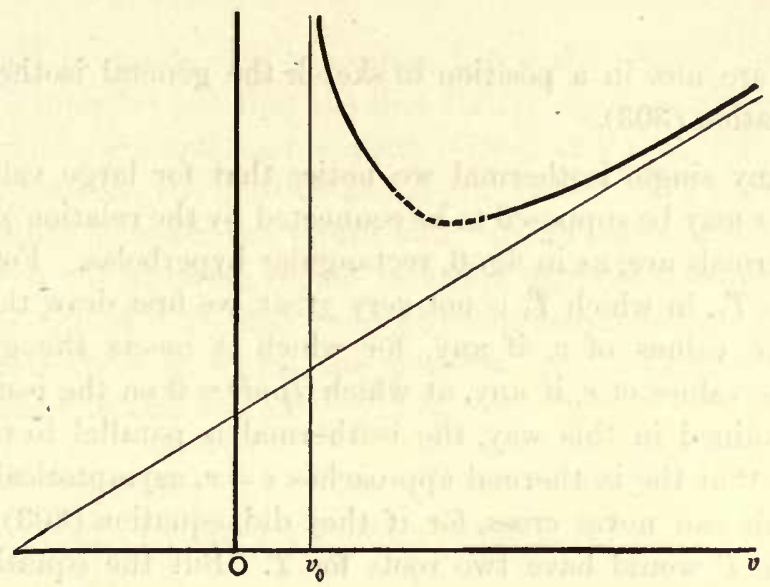

Fic. 7.

The dotted part is admittedly conjectural, but it is very unlikely that it is entirely misleading, for it is obvious enough that $\rho_{b}$ will increase steadily as $\rho$ increases. From this graph we can draw the graph of the values of $T$ given by equation (308), namely the values which cause $d p / d v$ to vanish. The latter graph is represented in fig. 8 .

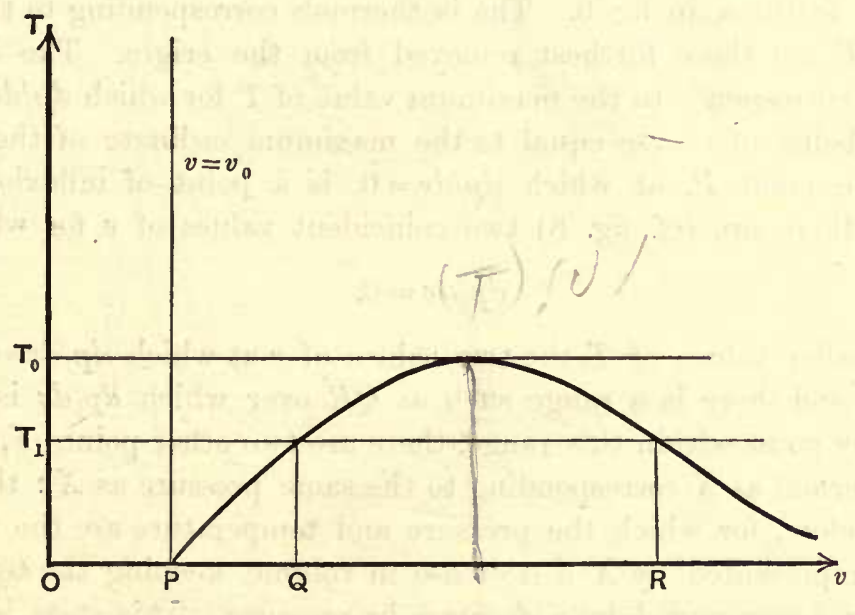

FIa. 8.

The only difficulty in drawing this figure lies in determining the form of the graph near the point $v=v_{0}$ at which $T=0$. It is however obvious, from the way in which $\rho_{b}$ is calculated, that near to $v=v_{0}, \rho_{b}$ must be proportional 
to some inverse power of $\left(v-v_{0}\right)$, and hence it follows that the value of $T$ given by equation (308) must for small values of $v-v_{0}$ be simply proportional to $\left(v-v_{0}\right)$.

148. We are now in a position to sketch the general isothermals represented by equation (303).

To draw any single isothermal we notice that for large values of $v$ the coordinates $p, v$ may be supposed to be connected by the relation $p v=$ constant, and the isothermals are, as in fig. 6, rectangular hyperbolas. For the general isothermal $T=T_{1}$, in which $T_{1}$ is not very great, we first draw the line $T=T_{1}$ in fig. 8. The values of $v$, if any, for which it meets the graph in this figure, give the values of $v$, if any, at which $d p / d v=0$ on the isothermal. At the points obtained in this way, the isothermal is parallel to the axis of $v$. Also we know that the isothermal approaches $v=v_{0}$ asymptotically. Finally, two isothermals can never cross, for if they did, equation (303) regarded as an equation in $T$ would have two roots for $T$. But the equation is of the well-known form

$$
e^{-a x}=b x,
$$

where $x$ stands for $1 / T$ and $a, b$ are positive constants, and it becomes obvious upon drawing the graphs of the two members of this equation that the equation can only have one root.

149. It will be seen, therefore, that these isothermals are represented, in their main features, in fig. 9. The isothermals corresponding to the highest values of $T$ are those furthest removed from the origin. The isothermal through $P$ corresponds to the maximum value of $T$ for which $d p / d v$ vanishes, this value being of course equal to the maximum ordinate of the graph of fig. 8. The point $P$, at which $d p / d v=0$, is a point of inflexion on this curve, for there are (cf. fig. 8) two coincident values of $v$ for which

$$
d p / d v=0 .
$$

For smaller values of $T$ the two values of $v$ at which $d p / d v=0$ are not coincident, and there is a range such as $Q R$ over which $d p / d v$ is negative. If $X$ is any point within this range, there are two other points $Y, Z$ on the same isothermal as $X$ corresponding to the same pressure as $X:$ three states in all, therefore, for which the pressure and temperature are the same. In the state represented by $X$ a decrease in volume, keeping the temperature constant, is accompanied by a decrease in pressure. This state is therefore unstable: any slight decrease in volume is attended by a tendency to a further decrease in the form of an unbalanced external pressure. Of the two stable states $Y, Z$, the state $Z$, corresponding to greater volume, must be the gaseous state, the state $Y$ corresponding to lesser volume is the liquid state. We see therefore that the temperature of the isothermal through $P$ is the 
"critical temperature" of the substance. So long as the temperature is kept above the critical temperature, no pressure, however great, can liquefy the substance.

It is usual to speak of a gas, when below the critical temperature, as a vapour. We therefore see that the line $P P_{2}$ in fig. 9 is the line of demarcation between the gaseous and vapour states, and that $P P_{1}$ is the line of demarcation between the gaseous and liquid states. We must now examine the demarcation between the liquid and vapour states, which is at present represented by the unstable region in which $d p / d v$ is positive. If $U$ is any point in this region it is clear from physical considerations that there must be some stable state in which the pressure and volume are those of the point $U$. What is this state?

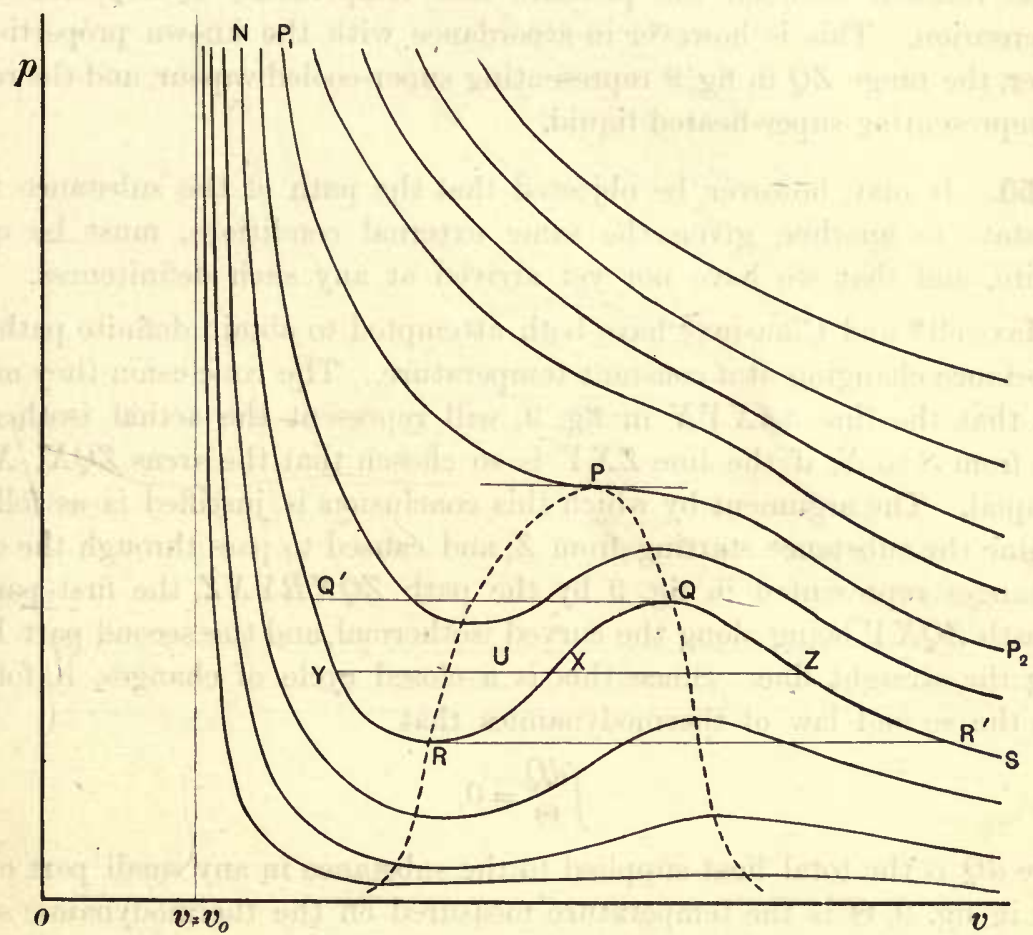

FIG. 9.

Through $U$ draw a line parallel to the axis of $v$. Let this cut any isothermal in the points $X, Y, Z$, the two latter representing stable statesliquid and vapour respectively. These states have the same pressure, so that a quantity of vapour in the state $Z$ can rest in equilibrium with a quantity of liquid in state $Y$. By choosing these quantities in a suitable ratio, the volume of the whole will be that represented by the point $U$. Here, then, we have an interpretation of the physical meaning of the point $U$. As the 
vapour is compressed at the temperature of the isothermal $S Z Q X R Y$, the substance remains a vapour until the point $Z$ is reached. At this point condensation sets in, and as the condensation proceeds the representative point moves along the straight line $Z X U Y$ until, by the time the point $Y$ is reached, the whole of the matter is in the liquid state. After this the substance, wholly in the liquid state, moves through the series of changes represented by the path $Y Q^{\prime} N$.

It will be seen that there is an element of arbitrariness in this, for instead of describing the path $S Z U Y N$ the substance might equally well be supposed to describe the path $S R^{\prime} R Y N$ keeping at the same temperature throughout; or any other path composed of two stable branches of an isothermal joined by a line of constant pressure. In other words there is no unique relation between the pressure and temperature of evaporation or condensation. This is however in accordance with the known properties of matter, the range $Z Q$ in fig. 9 representing super-cooled vapour, and the range $Y R$ representing super-heated liquid.

150. It may, however, be objected that the path of the substance from one state to another, given the same external conditions, must be quite definite, and that we have not yet arrived at any such definiteness.

Maxwell* and Clausius $\nmid$ have both attempted to obtain definite paths for a substance changing at a constant temperature. The conclusion they arrive at is that the line $S Z X Y N$ in fig. 9, will represent the actual isothermal path from $S$ to $N$, if the line $Z X Y$ is so chosen that the areas $Z Q X, X R Y$ are equal. The argument by which this conclusion is justified is as follows. Imagine the substance starting from $Z$, and caused to pass through the cycle of changes represented in fig. 9 by the path $Z Q X R Y X Z$, the first part of the path $Z Q X Y$ being along the curved isothermal, and the second part $Y X Z$ along the straight line. Since this is a closed cycle of changes, it follows from the second law of thermodynamics that

$$
\int \frac{d Q}{\Theta}=0
$$

where $d Q$ is the total heat supplied to the substance in any small part of its path in fig. $9, \Theta$ is the temperature measured on the thermodynamic scale, and the integral is taken round the whole closed path representing the cycle. Since the temperature is constant throughout the motion, this equation becomes $\int d Q=0$, so that the integral work done on the gas throughout the cycle is nil. This work is, however, equal to $\int p d v$ and therefore to the area, measured algebraically, of the curve in fig. 9 which represents the cycle.

* Nature, Vol. Ir. 1875 ; Collected Works, Ir. p. 425.

+ Wied. Ann. 1x. p. 337, 1880. 
Hence this area must vanish, which is the result already stated. Objections can be, and have been, urged against this argument, but to discuss the question any further would carry us too far into the domain of thermodynamics.

151. The figure which is obtained from figure 9 , upon replacing the curved parts of isothermals such as $Z Q X R Y$ by the straight line $Z X Y$, is represented in fig. 10. This figure then ought to represent the main features of the observed systems of isothermals of actual substances. There can of course be no attempt at anything of the nature of quantitative agreement.

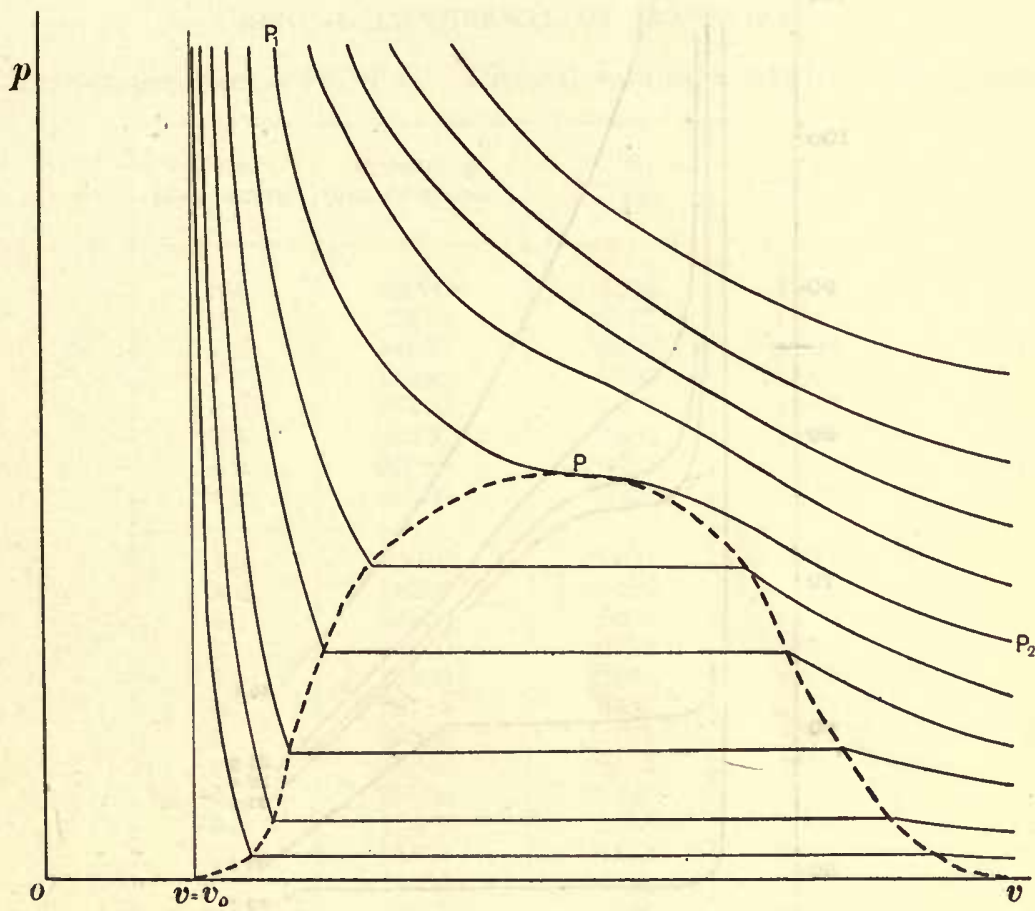

FIG. 10.

\section{Comparison with Experiment.}

152. It would in no way be consistent with the plan of this book to give a detailed account of the agreement or disagreement between the theory which has been arrived at and experiment. We shall therefore be content with mentioning two sets of experimental results, for the sake of illustration, rather than confirmation, of the theory.

153. In fig. 11, the curves are the isothermals of carbon dioxide as found experimentally by Andrews*. The figures on the left-hand denote

* Phil. Trans. clix. p. 575 (1869) and clxirir. p. 421 (1876). 
pressure measured in atmospheres, the isothermals only being shewn for pressures above 47 atmospheres. The figures on the right-hand denote the temperatures Centigrade of the corresponding isothermals.

The isothermal corresponding to the temperature $31 \cdot 1^{\circ}$ is of great interest, as being very near to the critical isothermal, the value of the critical temperature being given by Andrews as $30.92^{\circ}$. On this isothermal, as on all those above it, the substance remains gaseous, no matter how great the pressure.

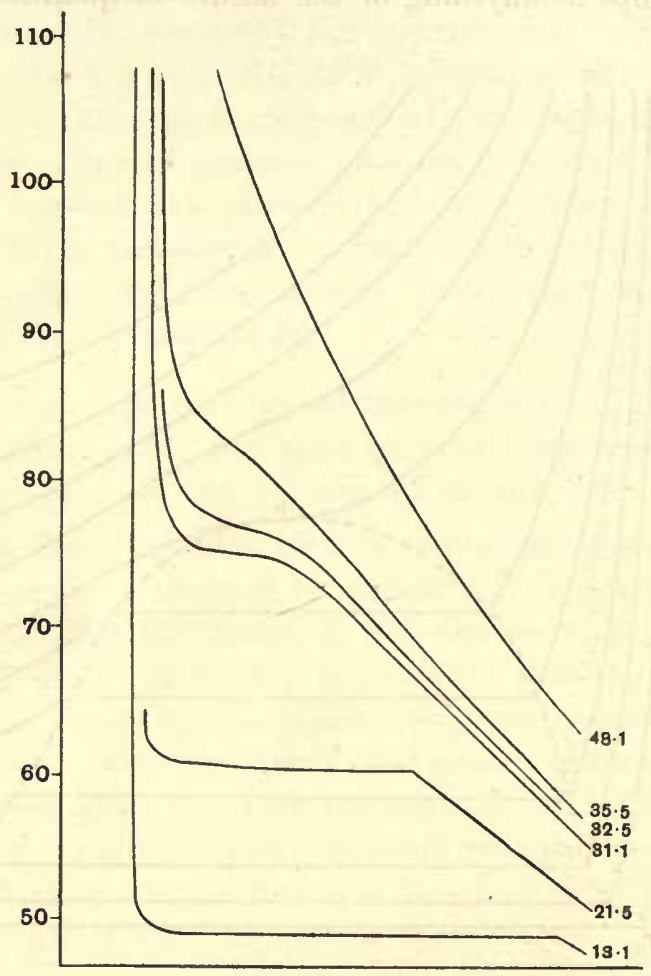

Fra. 11.

On the next lower isothermal, corresponding to temperature $21 \cdot 5^{\circ} \mathrm{C}$, we notice a horizontal range at a pressure of about 60 atmospheres. As the representative point moves over this range, boiling or condensation is taking place. Thus at a pressure of about 60 atmospheres the boiling point of carbon dioxide is about $21.5^{\circ} \mathrm{C}$. The ratio of volumes in the liquid and vapour states is equal to the ratio of the two values of $v$ at the extremities of the horizontal range-a ratio of about one to three.

The lowest isothermal of all corresponds to a temperature of $13 \cdot 1^{\circ} \mathrm{C}$. Here the inequality between the volumes of the liquid and the gas is greater than before. In fact an examination of the general theoretical diagram given in fig. 10 shews that as the temperature decreases the inequality 
becomes more and more marked, so that in all substances the distinction between the liquid and gaseous states becomes continually more pronounced as we recede from the critical temperature.

154. The following table gives corresponding values of $p$ and $v$ observed at the critical temperature of isopentane, by Young*. This temperature according to Young is $187.8^{\circ} \mathrm{C}$., the critical volume being 4.266 cubic centimetres for 1 gramme of the gas. The table is calculated by Dietericit from Young's observations.

\section{Critical Isothermal of Isopentane.}

Critical temperature $=187 \cdot 8^{\circ} \mathrm{C}$. Critical volume $=4 \cdot 266$ c.c. per gramme.

\begin{tabular}{|c|c|c|c|}
\hline $\begin{array}{l}\text { Volume, } v \\
\text { per gramme }\end{array}$ & $\begin{array}{l}\text { Pressure } p \\
\text { mm. of mercury }\end{array}$ & $\log \frac{R T}{p m v}$ & $v \log \frac{R T}{p m v}$ \\
\hline $2 \cdot 4$ & 49080 & •5296 & $1 \cdot 271$ \\
\hline $2 \cdot 5$ & 40560 & .5947 & $1 \cdot 486$ \\
\hline $2 \cdot 6$ & 34980 & .6419 & $1 \cdot 669$ \\
\hline $2 \cdot 8$ & 28940 & .6920 & $1 \cdot 938$ \\
\hline $3 \cdot 0$ & 26460 & $\cdot 7010$ & $2 \cdot 103$ \\
\hline $3 \cdot 2$ & 25490 & $\cdot 6893$ & $2 \cdot 205$ \\
\hline $3 \cdot 6$ & 25050 & $\cdot 6457$ & $2 \cdot 326$ \\
\hline $4 \cdot 0$ & 25020 & $\cdot 6004$ & $2 \cdot 402$ \\
\hline $4 \cdot 3$ & 25010 & 5691 & $2 \cdot 447$ \\
\hline $4 \cdot 6$ & 25000 & 5400 & $2 \cdot 483$ \\
\hline 5 & 24990 & $\cdot 5040$ & $2 \cdot 520$ \\
\hline 6 & 24840 & $\cdot 4273$ & $2 \cdot 564$ \\
\hline 7 & 24400 & $\cdot 3682$ & $2 \cdot 577$ \\
\hline 8 & 23710 & 3227 & $2 \cdot 582$ \\
\hline 9 & 22930 & $\cdot 2861$ & $2 \cdot 576$ \\
\hline 10 & 22040 & $\cdot 2575$ & 2.575 \\
\hline 12 & 20300 & .2140 & $2 \cdot 568$ \\
\hline 15 & 17980 & •1698 & $2 \cdot 548$ \\
\hline 20 & 14840 & $\cdot 1282$ & $2 \cdot 564$ \\
\hline 30 & 10950 & $\cdot 0842$ & $2 \cdot 526$ \\
\hline 40 & 8570 & .0656 & $2 \cdot 624$ \\
\hline 50 & 7068 & $\cdot 0525$ & $2 \cdot 625$ \\
\hline 60 & 6001 & $\cdot 0442$ & $2 \cdot 652$ \\
\hline 80 & 4614 & $\cdot 0335$ & $2 \cdot 680$ \\
\hline 90 & 4132 & .0293 & $2 \cdot 637$ \\
\hline 100 & 3750 & $\cdot 0268$ & $2 \cdot 680$ \\
\hline
\end{tabular}

To interpret this table, we revert to the general equation (303),

$$
p=\frac{R T}{m} \rho_{b} e^{-\frac{\rho \psi}{R T}}
$$

where (cf, equation (304))

$$
\rho_{b}=\rho+b_{1} \rho^{2}+b_{2} \rho^{3}+\ldots
$$

in which $b_{1}, b_{2} \ldots$ are constants.

* S. Young, Proc. Roy. Soc. xv. p. 126 and xvi. p. 11.

+ Annalen der Physik, v. p. 58, 1901. 
If $v$ is the volume per unit mass of gas, so that $v \rho=1$, we have, as far as squares of $\rho$,

$$
\begin{aligned}
e^{-\frac{\rho \psi}{R T}}=e^{-\frac{\psi}{R T v}} & =\frac{p m}{R T \rho_{b}} \\
& =\frac{p m}{R T \rho\left(1+b_{1} \rho\right)} \\
& =\frac{p m}{R T}\left(v-b_{1}\right) .
\end{aligned}
$$

Hence

$$
\begin{aligned}
\frac{\psi}{R T} & =-v \log \frac{p m}{R T}\left(v-b_{1}\right) \\
& =-v \log \frac{p m}{R T} v+b_{1}
\end{aligned}
$$

Now we have seen ( $§ 135)$ that so long as $\rho$ is approximately constant throughout the gas, $\psi$ may be regarded as constant in equation (312). This requires that $e^{-\frac{\rho \psi}{R T}}$ shall be approximately equal to unity throughout the gas, and therefore that $\rho$ shall be small. If this condition is satisfied, we may neglect terms beyond $b_{1} \rho^{2}$ in equation (313). Hence from equation (314) we may for sufficiently small values of $\rho$ suppose the equation of the isothermal to be

$$
v \log \frac{R T}{p m v}=\text { constant }
$$

The quantity on the left-hand is the function tabulated by Dieterici in the last column of the table on the last page. Inspection shews that although the function is by no means constant for all values of $v$, yet it is approximately constant for all values of $v$ which are greater than the critical volume. If Boyle's law were satisfied the entry in the third column would vanish throughout. It does not vanish, and our investigations on the deviations from Boyle's law have shewn that it ought approximately to vary as $1 / v$. The confirmation of our theory, then, lies in the fact that the variations of the entry in the last column are small compared with those of $v$.

\section{VAN DER WAaLS' Equation.}

155. As far as the first order of small quantities we have from equation (304),

$$
\begin{aligned}
\rho_{b} & =\rho+\frac{2 \pi \sigma^{3}}{3 m} \rho^{2}+\ldots \\
& =\frac{N m}{v-b}
\end{aligned}
$$


where

$$
\begin{aligned}
b & =\frac{2 \pi \sigma^{3}}{3 m} \\
& =\frac{2 \sqrt{2} \pi}{3} v_{0}
\end{aligned}
$$

upon substitution from equation (310).

Expanding $\rho_{b}$ and the cohesion factor as far as the first order of small quantities, we have as the equation giving the pressure,

$$
\begin{aligned}
p & =\frac{R T}{m} \rho_{b} e^{-\frac{\rho \psi}{R T}} \\
& =\frac{R T}{m} \frac{N m}{v-b}\left(1-\frac{\rho \psi}{R T}\right)
\end{aligned}
$$

so that

$$
p(v-b)=R N T-N \rho \psi
$$

or, again as far as the first order of small quantities,

$$
\left(p+\frac{a}{v^{2}}\right)(v-b)=R N T
$$

where

$$
a=N^{2} m \psi \text {, }
$$

agreeing with Van der Waals' equation (281).

We shall now use this equation to investigate the behaviour of gases when the deviations from Boyle's Law are slight.

\section{Changes at Constant Volume.}

156. Imagine the volume of a gas satisfying equation (317) to be kept constant, and let us suppose the temperature first to be $T_{0}$ and afterwards $T_{1}$.

We have, if $p_{0}, p_{1}$ are the corresponding pressures,

$$
\begin{aligned}
& \left(p_{0}+\frac{a}{v^{2}}\right)(v-b)=R N T_{0} \\
& \left(p_{1}+\frac{a}{v^{2}}\right)(v-b)=R N T_{1}
\end{aligned}
$$

By subtraction we get

$$
\left(p_{1}-p_{0}\right)(v-b)=R N\left(T_{1}-T_{0}\right)
$$

and dividing the members of this equation by the corresponding members of equation (318),

$$
\frac{p_{1}-p_{0}}{T_{1}-T_{0}} \frac{T_{0}}{p_{0}}=1+\frac{a}{p_{0} v^{2}}
$$

From this it is clear that if $T_{0}, p_{0}$ refer to a fixed temperature the increase in $T$ is proportional to that in $p$. Hence a gas kept at constant volume may be regarded as a thermometer giving readings on the kinetic theory scale, the reading being proportional to $p$. 
In the special case of hydrogen it is found that the ratio of the increase of temperature to that of pressure is very approximately independent of the volume. In other words, the value of $a$ is extremely small for hydrogen. For this reason the Comite internationale des poids et mesures decided on the constant-volume hydrogen thermometer as standard thermometer. The small value which can be found for $a$ is recognised in the stipulation of the committee that the volume at which the gas is used is to be such that the pressure at the melting-point of ice is to be the weight of $1000 \mathrm{~mm}$. of mercury at Paris. On this thermometer the readings are proportional to the pressure above that at the melting-point of ice. If $\theta$ is the observed temperature, we have

$$
T=T_{0}+\alpha \theta,
$$

where $\alpha$ is a constant and $T_{0}$ is the value of $T$ corresponding to the meltingpoint of ice. The relation between the two scales may obviously also be written

$$
T=\alpha\left(\theta_{0}+\theta\right) \ldots \ldots \ldots \ldots \ldots \ldots \ldots \ldots \ldots . . .(322) .
$$

If the thermometer scale is to be the Centigrade scale, the values of $\alpha$ must be such that at the boiling-point of water $\theta=100$. On this scale it is found that the point $T=0$ is given by

$$
\theta=-\theta_{0}=-273 \cdot 04^{\circ} \mathrm{C}
$$

This temperature is what is known as the absolute zero of temperature. It is the temperature at which matter is entirely devoid of internal motion.

If in equation (321) we take the temperature $T_{0}$ to be that of the meltingpoint of ice, and reduce the whole equation to the Centigrade scale, we put (equation (322)) $T_{0}=\alpha \theta_{0}, T_{1}-T_{0}=\alpha \theta$, and the equation becomes

$$
\frac{\left(p_{1}-p_{0}\right) \theta_{0}}{p_{0} \theta}=1+\frac{a}{p_{0} v^{2}}
$$

The quantity $\frac{p_{1}-p_{0}}{p_{0} \theta}$ is what is usually known as the "pressure coefficient," so that if we denote it by $\kappa_{p}$, the pressure at any temperature $\theta$ is given by

Equation (316) now becomes

$$
p_{1}=p_{0}\left(1+\kappa_{p} \theta\right) \text {. }
$$

$$
\kappa_{p}=\left(1+\frac{a}{p_{0} v^{2}}\right) \frac{1}{\theta_{0}}
$$

This equation depends on the density but not on the temperature; hence for a given density, the pressure coefficient is independent of the temperature. This law was verified by Regnault*, who found that gas thermometers filled with different gases gave identical readings over a great range of temperature. A single exception occurred in the case of sulphurous acid, of which the anomalous behaviour appears to have been due to evaporation $\dagger$.

* Mêm. de l'Acad. xxi. p. 180.

+ Van der Waals, Continuity, p. 392. 
The following table contains the values of the pressure coefficients of a number of gases, as given in Travers' Study of Gases.

$$
\begin{array}{ll}
\text { Hydrogen* } & -0036624, \\
\text { Nitrogen* } & 00367445, \\
\text { Oxygen }+ & 0036742, \\
\text { Air }+ & 0036700 .
\end{array}
$$

For an ideal gas the value would of course be $\frac{1}{273 \cdot 04}$ or $\cdot 0036635$.

\section{Changes at Constant Pressure.}

157. We next imagine the pressure kept constant. Experiments conducted under these conditions enable us to determine a "volume coefficient" $\kappa_{v}$ defined by

$$
\kappa_{v}=\frac{v_{1}-v_{0}}{v_{0} \theta}
$$

so that the volume at temperature $\theta$ is given by

$$
v_{1}=v_{0}\left(1+\kappa_{v} \theta\right) \text {. }
$$

By a method similar to that of the last section, we find

$$
\kappa_{v}=\left\{1+\frac{a}{p v_{0}}\left(\frac{1}{v_{0}}+\frac{1}{v}\right)-\frac{b}{v_{0}}\right\} \frac{1}{\theta_{0}}
$$

This is more complicated than the corresponding pressure-formula (325) in that it depends on both the pressure and volume.

\section{Determination of Size of Molecules.}

158. From a series of experiments in which the values of $\kappa_{p}$ and $\kappa_{v}$ are both determined, it will clearly be possible to calculate the values of both $a$ and $b$. Calculations of this type will be found in Van der Waals' essay.

The determination of $b$ is of special importance, because from it we can calculate the value of $\sigma$, the diameter of the sphere of molecular action.

The values for $b$ at which Van der Waals arrives from the discussion of a great number of experiments by Regnault are as follows :

$\begin{array}{ll}\text { Air } & \cdot 0026, \\ \text { Carbon dioxide } \| & 0030, \\ \text { Hydrogen } \uparrow & 00069 .\end{array}$

The numbers apply to unit volume of gas at a pressure of one metre of mercury.

* Obtained by Chappuis, the pressure at $0^{\circ} \mathrm{C}$. being $1000 \mathrm{~mm}$.

+ Von Jolly.

¥ Mendeleef, from observations of Regnault, Magnus and Jolly.

§ Continuity, p. 400 . $\quad \|$ ibid. p. 402 . See also p. 413.

T ibid.p. 401. 
Unfortunately the value of $b$ appears in the calculations as the difference of two larger quantities. The accuracy of the results, therefore, is not great. For instance in the case of hydrogen, Regnault's experiments lead to different values of $b$ of which the extreme values are $\cdot 0005$ and $\cdot 0008$. The number .00069 is, however, the mean of a very great number of experiments, so that the probable error is nothing like so great as the difference between the mean and either of the extremes. The agreement amongst themselves of the experiments in the case of the other two gases is much better. For instance, taking the value $b=0030$ for carbon dioxide, Van der Waals calculates for $a \times 10^{4}$ from Regnault's experiments the values 127, 114, 115, 107, 120,113, $116,111,116$. The first number according to Van der Waals is, from the experimental conditions, likely to be the least trustworthy: the remainder, as will be seen, agree to within a few per cent.

There are other ways of determining $b$, besides observations on the pressure and volume coefficients. Of these the most important is probably by measurement of the Joule-Thomson effect. From calculations by RoseInnes*, Callendar $\nmid$ deduces the following values for $b$ :

$$
\begin{array}{lr}
\text { Air } & \mathbf{1} \cdot 62, \\
\text { Nitrogen } & 2 \cdot 03, \\
\text { Hydrogen } & 10 \cdot 73 .
\end{array}
$$

These values are in cubic centimetres referred to unit mass of gas.

Reduced to a cubic cm. of gas at normal pressure, the two sets of values stand as follows :

VALUES FOR $b$.

\begin{tabular}{|c|c|c|}
\hline Gas & Van der Waals & Rose-Innes \\
\hline Hydrogen ............ & .00052 & .00096 \\
Nitrogen ............ & - & .00255 \\
Air...................... & .00198 & 00209 \\
Carbon dioxide ... & .00228 & - \\
\hline
\end{tabular}

As we have already seen, the value of $b$ is $\frac{2 \pi}{3} \nu \sigma^{3}$, and to give values of $b$ corresponding to those in the above table, $\nu$ múst be supposed to be the number of molecules per $\mathrm{cu}$. $\mathrm{cm}$. of the gas at normal temperature and pressure. 'I'hus $\nu$ is the same for all the gases, and if we take the value $\nu=4 \times 10^{19}$, from $\S 8$ we obtain the following series of values for $\frac{\sigma}{2}$, the radius of the molecules:

* Phil. Mag. Ir. p. 130.

† Phil. Mag. v. p. 48, or Proc. Phys. Soc. xviı. p. 282. 
VALUES FOR $\frac{1}{2} \sigma$.

\begin{tabular}{|c|c|c|c|}
\hline Gas & Van der Waals & Rose-Innes & Mean \\
\cline { 1 - 3 } Hydrogen ........... & $.92 \times 10^{-8}$ & $1.13 \times 10^{-8}$ & $1.02 \times 10^{-8}$ \\
Nitrogen ............ & - & $1.56 \times 10^{-8}$ & $1.56 \times 10^{-8}$ \\
Air................... & $1.44 \times 10^{-8}$ & $1.46 \times 10^{-8}$ & $1.45 \times 10^{-8}$ \\
Carbon dioxide ... & $1.50 \times 10^{-8}$ & - & $1.50 \times 10^{-8}$ \\
\hline
\end{tabular}

The Critical Point.

159. Referring to figure 9 on p. 131, we see that at a temperature below the critical temperature there are two points on every isothermal (e.g., $R, Q$ in the figure) at which $d p / d v=0$. At the critical point $P$ these two points coincide. It therefore follows that at the critical point

$$
\frac{d p}{d v}=0, \quad \frac{d^{2} p}{d v^{2}}=0
$$

Combining these equations with Van der Waals' equation, written in the form

$$
p+\frac{a}{v^{2}}=\frac{R N T}{v-b},
$$

we obtain, as the equations satisfied at the critical point,

$$
\begin{aligned}
& \frac{2 a}{v^{3}}=\frac{R N T}{(v-b)^{2}}, \\
& \frac{6 a}{v^{4}}=\frac{2 R N T}{(v-b)^{3}},
\end{aligned}
$$

from which we obtain

$$
v=3 b
$$$$
R N T=\frac{8 a}{27 b}
$$

$$
p=\frac{a}{27 b^{2}}
$$

giving the critical volume, temperature and pressure. From these we find for the value of $p v$ at the critical point

$$
p v=\frac{3}{8} R N T
$$

It appears from this that at this point the deviation of $p v$ from the value given by Boyle's Law is represented by a factor $\frac{8}{3}$, and therefore that this deviation cannot legitimately be treated as small. In spite of this, equations (328) to (331) lead to values which are in surprisingly good agreement with observation, when we consider the rough method by which the equations are arrived at. 
The following table, given by Berthelot*, gives the observed value for the ratio $\frac{R N T}{p v}$ at the critical point. It will be seen that argon is the only gas in which this value approximates with any reasonable accuracy to the value $\frac{8}{3}$ given by equation (331):

$\begin{array}{ll}\text { Marsh gas } & 3 \cdot 67, \\ \text { Carbon dioxide } & 3 \cdot 61, \\ \text { Nitrogen } & 3 \cdot 53, \\ \text { Oxygen } & 3 \cdot 49, \\ \text { Argon } & 2 \cdot 62 .\end{array}$

Other values of this quantity have been determined by Ramsay and Young $†$ ranging up to values as great as 5.00 for acetic acid. Young, however, is of opinion that for all substances which can attain the critical state without chemical change, the quantity is nearly constant, and has a value of about $3.7+$.

Before leaving this question, it is of interest to examine the numerical values at the critical point, given by the more general equation (303), namely,

$$
p=\frac{R T}{m} \rho_{b} e^{-\frac{\rho \psi}{R T}}
$$

Let us write $\rho=\frac{N m}{v}$ and $\rho_{b}=\frac{N m}{v-b}$, so that, as before, we neglect squares of $b / v$. Then the equation becomes

$$
p=\frac{R N T}{(v-b)} e^{-\frac{N m \psi}{R T v}} \text {. }
$$

The critical point is the point at which $d p / d v$ and $d^{2} p / d v^{2}$ both vanish. If $d p / d v=0$, we have by logarithmic differentiation,

$$
\frac{1}{v-b}-\frac{N m \psi}{R T v^{2}}=0
$$

and if also $d^{2} p / d v^{2}=0$, the equation just obtained, regarded as an equation for $v$, must have equal roots in $v$. The condition for this is that

$$
\frac{1}{(v-b)^{2}}-\frac{2 N m \psi}{R T v^{3}}=0
$$

From these two equations we obtain

$$
\frac{1}{v-b}=\frac{2}{v}
$$

so that

and

$$
b=\frac{1}{2} v \text {, }
$$

$$
\frac{N m \psi}{R T}=2 v \text {. }
$$

* Soc. Franç. Phys. Bull. clxvir. p. 4 (1901).

+ Phil. Mag. 1892, p. 503; 1894, p. 1 . See also Weinstein, Thermodynamik, 1. p. 469.

$\ddagger$ Physikalische Revue, r. p. 385 . 
Hence, at the critical point

$$
\begin{aligned}
p v & =\frac{R N T v}{(v-b)} e^{-\frac{N m \psi}{R T v}} \\
& =2 R N T e^{-2}
\end{aligned}
$$

The ratio of the value of $p v$ in the critical state as given by Boyle's Law to its actual value is therefore

$$
\frac{1}{2} e^{2} \text {, or } 3.6945 \text {. }
$$

This agrees almost exactly with Young's value $3 \cdot 7$. We must, however, not lay too much stress on the agreement. When, as in the present instance, we are dealing with values for which $\frac{b}{v}=\frac{1}{2}$, it is obvious that the error introduced by the neglect of $\left(\frac{b}{v}\right)^{2}$ must be very considerable.

\section{The Virial of Clausiús.}

160. Clausius attempted, in a way entirely different from that which we have followed, to determine the relation between the pressure, volume and temperature of a gas*.

Let $x, y, z$, be the coordinates of the centre of gravity of a molecule of mass $m$, and $X, Y, Z$, the components of force acting upon it. Then its motion is governed by the equations

$$
m \frac{d^{2} x}{d t^{2}}=X, \text { etc. }
$$

Since

$$
\left(\frac{d x}{d t}\right)^{2}+x \frac{d^{2} x}{d t^{2}}=\frac{1}{2} \frac{d^{2}}{d t^{2}}\left(x^{2}\right)
$$

we obviously have

$$
\begin{aligned}
\frac{1}{2} m\left(\frac{d x}{d t}\right)^{2} & =\frac{1}{4} m \frac{d^{2}}{d t^{2}}\left(x^{2}\right)-\frac{1}{2} m x \frac{d x^{2}}{d t^{2}} \\
& =\frac{1}{4} m \frac{d^{2}}{d t^{2}}\left(x^{2}\right)-\frac{1}{2} x X,
\end{aligned}
$$

by equation (332). If we sum this equation and the corresponding equations, for the two other axes and for all the molecules of the gas, we obtain

$$
\frac{1}{2} \Sigma m c^{2}=\frac{1}{4} \Sigma m \frac{d^{2}}{d t^{2}}\left(x^{2}+y^{2}+z^{2}\right)-\frac{1}{2} \Sigma(x X+y Y+z Z),
$$

where $\Sigma$ denotes summation over all the molecules of the gas. 
Averaged over all instants of time from $t=0$ to $t=\tau$, this equation becomes

$$
\begin{aligned}
\frac{1}{\tau} \int_{t=0}^{t=\tau} \frac{1}{2} \Sigma m c^{2} d t=\frac{1}{4 \tau} \Sigma m\left[\frac{d}{d t}\left(x^{2}+y^{2}+z^{2}\right)\right]_{t=0}^{t=\tau} \\
-\frac{1}{\tau} \int_{t=0}^{t=\tau} \frac{1}{2} \Sigma(x X+y Y+z Z) d t \ldots \ldots
\end{aligned}
$$

In the steady motion of a gas, the quantities

$$
\frac{1}{2} \Sigma m c^{2} d t, \quad \Sigma m \frac{d}{d t}\left(x^{2}+y^{2}+z^{2}\right) \text { and } \frac{1}{2} \Sigma(x X+y Y+z Z),
$$

are approximately constant throughout the motion. Hence as we increase $\tau$ indefinitely in equation (333), the first and last terms will remain approximately constant, while the middle term tends to vanish. Taking $\tau$ sufficiently large, equation (333) reduces to

$$
\frac{1}{2} \Sigma m c^{2}=-\frac{1}{2} \Sigma(x X+y Y+z Z)
$$

in which both sides, which are in any case constant except for the slight departures from the steady state which occur in the motion of the gas, are averaged over a time sufficient for them to be regarded as sensibly constant. The mean value of $-\frac{1}{2} \Sigma(x X+y Y+z Z)$ has been termed by Clausius the virial of the system. We have therefore shewn that when a gas moves, undisturbed from its steady state, the kinetic energy of its motion is equal to its virial.

161. The virial depends solely on the forces acting upon the molecules, and not upon the motion of the molecules. In the case of a gas these forces consist of the pressure exerted upon the gas by the walls of the containing vessel, and the forces exerted by the molecules upon one another.

If $d S$ is an element of the surface of the containing vessel, and $l, m, n$ the direction cosines of its outward normal, the pressure of the element $d S$ exerts upon the gas a, force of which the components are $-l p d S,-m p d S$, - npdS, so that the value of that part of $\Sigma x X$ which is contributed by the pressure will be $\iint-l p x d S$. The present treatment compels us to assume that the pressure is the same at all points of the containing vessel. If we make this assumption the quantity just obtained may be written $-p \iint l x d S$. Hence the contribution of the pressure to the virial is, in all,

by Green's Theorem,

$$
\begin{aligned}
& \frac{1}{2} p \iint(l x+m y+n z) d S \\
& \quad=\frac{1}{2} p \iiint\left(\frac{\partial x}{\partial x}+\frac{\partial y}{\partial y}+\frac{\partial z}{\partial z}\right) d x d y d z
\end{aligned}
$$

$$
=\frac{3}{2} p \Omega
$$

where $\Omega$ is the volume of the vessel. 
We suppose that the force between two molecules at distance $r$ is an attractive force $\phi(r)$, a function of the distance $r$ only. If the centres of the molecules are at $x, y, z, x^{\prime}, y^{\prime}, z^{\prime}$, and if $X, Y, Z, X^{\prime}, Y^{\prime}, Z^{\prime}$ are the components of the forces acting on them, then

$$
X=\phi(r) \frac{x-x^{\prime}}{r}, \quad X^{\prime}=\phi(r) \frac{x^{\prime}-x}{r} .
$$

The contribution to $\Sigma_{x} X$ made by the force between these two particles

$$
\begin{aligned}
& =x X+x^{\prime} X^{\prime} \\
& =\frac{\phi(r)}{r}\left(x-x^{\prime}\right)^{2} .
\end{aligned}
$$

The contribution to $\Sigma(x X+y Y+z Z)$ is therefore

$$
\frac{\phi(r)}{r}\left\{\left(x-x^{\prime}\right)^{2}+\left(y-y^{\prime}\right)^{2}+\left(z-z^{\prime}\right)^{2}\right\}=r \phi(r)
$$

so that the part of the virial which arises from intermolecular forces, is

$$
-\frac{1}{2} \Sigma \Sigma r \phi(r)
$$

where the summation extends over all pairs of molecules.

Equation (334) may now be replaced by

$$
\frac{1}{2} \Sigma m c^{2}=\frac{3}{2} p \Omega-\frac{1}{2} \Sigma \Sigma r \phi(r)
$$

so that the pressure is given by

$$
p \Omega=\frac{1}{3} \Sigma m c^{2}-\frac{1}{3} \Sigma \Sigma i \phi(r)
$$

162. Clerk Maxwell* makes an important observation on the subject of this equation. By obliterating one or other of the terms on the right-hand side, we notice that a pressure may be produced either wholly by molecular motion or wholly by intermolecular force. The latter is a hypothesis on which attempts have been made to account for the pressure in a gas. If this were the true account, then Boyle's Law that $p \Omega$ is constant could be satisfied only by making $\Sigma \Sigma r \phi(r)$ constant, and therefore by taking $\phi(r)=\frac{1}{r}$. In other words, two molecules would have to repel one another with a force proportional to the inverse distance. This is, however, an impossible law for a gas. It would make the action of the distant parts of the mass preponderate over that of the contiguous parts, and would not give a pressure which, for a given volume and temperature, would be constant as we passed from one vessel to another, or even from one part to another of the surface of the same vessel. We therefore conclude that the pressure of a gas cannot be explained by assuming repulsive forces between the molecules; it must arise, at any rate in part, from the motion of the molecules.

* “The Dynamical Evidence of the Molecular Constitution of Bodies." Collected Works, II. p. 422 . 
163. If we could calculate the term $\Sigma \Sigma r \phi(r)$ in equation (335) for any law of intermolecular force, we should have a complete knowledge of the corrections to be applied to Boyle's Law. Unfortunately this is not possible even in the simplest cases.

164. If there were no intermolecular forces, the probability that the centre of molecule $A$ should lie at a distance between $r$ and $r+d r$ from the centre of $B$ would be

$$
\frac{4 \pi r^{2} d r}{\Omega}
$$

In this expression $4 \pi r^{2} d r$ is the volume of a spherical shell of radius $r$, and we neglect the possibility of $B$ being within a distance $r$ of the boundary of the containing vessel, as we legitimately may if $r$ is sufficiently small. The probability of the centre of $A$ lying within a distance between $r$ and $r+d r$ from the centre of $C$ is also equal to expression (336), so that the probability of the centre of $A$ lying within this range of distance from the centre of some other molecule is $(N-1)$ times expression (336), except for a correction arising from the possibility of two of the $(N-1)$ shells of radius $r$ which surround these molecules intersecting. Ignoring this correction for the present, and replacing $N-1$ by $N$, it follows that the number of pairs of molecules of which the centres lie at a distance intermediate between $r$ and $r+d r$ apart, would, in the absence of intermolecular forces, be

$$
\frac{2 \pi N^{2}}{\Omega} r^{2} d r
$$

When, however, molecules at a distance $r$ attract one another with a force $\phi(r)$, it will be seen, upon examination of the results of $\S 85$, that expression (337) must be modified in two ways. The probability of finding two molecules at a distance $r$ apart is, by $\S 85$, less than the probability of finding the same two molecules at a distance $\infty$ apart $(\infty$ here denoting any distance great enough for the molecules to be out of range of each other's action) in the ratio

$$
e^{-2 h \int_{r}^{\infty} \phi(r) d r}
$$

And again the probability of finding two molecules at a distance $\infty$ apart is greater than it would be if there were no intermolecular forces, because some positions for the molecules, not at great distances apart, are less likely, on account of the intermolecular forces, than they would otherwise be. The former consideration requires us to multiply expression (337) by a factor

$$
e^{-2 h \int_{r}^{\infty} \phi(r) d r}
$$

the latter requires us to modify the factor $\frac{N^{2}}{\Omega}$ in expression (337), which is only accurate if all positions of the molecules are equally probable. 
If we write $N \nu^{\prime}$ for the modified value of $\frac{N^{2}}{\Omega}$, so that $\nu^{\prime}$ is a modified molecular density, the corrected form of expression (337) becomes

$$
2 \pi N \nu^{\prime} r^{2} e^{-2 h \int_{r}^{\infty} \phi(r) d r} d r
$$

This is the number of pairs of molecules each of which gives a contribution $r \phi(r)$ to $\Sigma \Sigma r \phi(r)$. Hence, multiplying the expression just obtained by $r \phi(r)$ and integrating over all values of $r$, we obtain

$$
\Sigma \Sigma r \phi(r)=2 \pi N \int_{0}^{\infty} \nu^{\prime} r^{3} \phi(r) e^{-2 h \int_{r}^{\infty} \phi(r) d r} d r
$$

165. Progress is for many reasons impossible in the general case. Let us fall back on the simplifying assumption of the existence of spheres of molecular action satisfying the conditions mentioned in $\$ 82$. If each sphere is of diameter $\sigma$, then in every term which supplies an appreciable contribution to $\Sigma \Sigma \phi(r)$, the value of $r$ is very nearly equal to $\sigma$. If we write

so that

$$
\int_{r}^{\infty} \phi(r) d r=x
$$

then equation (339) becomes

$$
\Sigma \Sigma r \phi(r)=-2 \pi N \int_{0}^{\infty} \nu^{2} r^{3} e^{-2 h x} d x
$$

In the integral on the right-hand side, $\nu^{\prime} r^{3}$ may be replaced by $\nu^{\prime} \sigma^{3}$ and taken outside the sign of integration. The value of the integral is accordingly $\frac{\nu^{\prime} \sigma^{3}}{2 h}$ and the equation becomes

$$
\Sigma \Sigma r \phi(r)=-\frac{\pi N \nu^{\prime} \sigma^{3}}{h}
$$

166. We next turn to the calculation of $\nu^{\prime}$. In virtue of the supposed existence of a sphere of molecular action, we can imagine each of $N^{T}-1$ molecules surrounded by spheres of radius $\sigma$, and then every position outside these spheres may be supposed equally probable as a possible position for the centre of the $N$ th molecule, while any position inside these spheres is impossible. Thus if $\Omega^{\prime}$ be the total volume excluded by these spheres, we shall have

$$
\nu^{\prime}=\frac{N}{\Omega-\Omega^{\prime}}
$$

instead of $\nu=\frac{N}{\Omega}$. Obviously if we neglect the distinction between $N^{r}-1$ and $N$,

$$
\Omega^{\prime}=\frac{4}{3} N \pi \sigma^{3}
$$

167. Equation (335) now becomes

$$
p \Omega=\frac{1}{3} \Sigma m c^{2}+\frac{1}{3} \frac{N \pi \sigma^{3}}{h}\left(\frac{N}{\Omega-\Omega^{\prime}}\right)
$$


by equations (340) and (341),

$$
=R N T+\frac{2}{3} R N T \frac{\pi N \sigma^{3}}{\Omega-\Omega^{\prime}}
$$

on introducing the temperature from equation (265),

$$
=R N T\left\{1+\frac{1}{2} \frac{\Omega^{\prime}}{\Omega-\Omega^{\prime}}\right\}
$$

Hence, as far as first powers of $\Omega^{\prime} / \Omega$,

$$
\begin{aligned}
p & =\frac{R N T}{\Omega}\left(1+\frac{1}{2} \frac{\Omega^{\prime}}{\Omega}\right) \\
& =\left(\nu+\frac{\dot{2} \pi \sigma^{3}}{3} \nu^{2}\right) R T .
\end{aligned}
$$

and this agrees with equation (294).

168. The approximation has been extended* to the second order of small quantities, by taking account of the further correction mentioned in $\S 164$, which arises from the possibility of two of the shells, which there are supposed to surround the molecules, intersecting one another. The corrected equation is found to be

$$
p=\left\{\nu+\frac{2 \pi \sigma^{3}}{3} \nu^{2}+\frac{5}{8}\left(\frac{2 \pi \sigma^{3}}{3}\right)^{2} \nu^{3}+\ldots\right\} R T
$$

169. If we now suppose that forces of cohesion act of the kind specified in $\S 135$, these forces will have a contribution to make to the virial. To the first order of small quantities, we may, in calculating $\Sigma \Sigma r \phi(r)$, ignore the effect of the forces of cohesion on the distribution of density of the gas. The value of $\Sigma \Sigma r \phi(r)$ is therefore obviously proportional simply to $\rho^{2}$. Allowing for this addition to the virial, equation (343) becomes

$$
p \Omega=R N T\left\{1+\frac{1}{2} \frac{\Omega^{\prime}}{\Omega-\Omega^{\prime}}\right\}-c \rho^{2} \Omega,
$$

where $c$ is the $c$ of $\S 134$, and is independent of the temperature. Or again this last equation may be written

$$
\left(p+\frac{a}{\Omega^{2}}\right)\left(\Omega-\frac{1}{2} \Omega^{\prime}\right)=R N T,
$$

agreeing with Van der Waals' equation (281), since the $b$ in that equation is the same as the present $\frac{1}{2} \Omega^{\prime}$.

170. It is obvious that the calculation of the effect of the forces of cohesion to a second approximation, would be extremely tedious, and I am not aware that it has ever been attempted. Indeed a comparison of the general equation of the virial (equation (335)), with the general equation giving the pressure (equation (303)) seems to suggest that the virial is hardly suited to the calculation of pressures to any accuracy other than that of a first approximation.

\footnotetext{
* Boltzmann, Vorlesungen über Gastheorie, II. § 51.
} 


\section{CHAPTER VII.}

\section{PHYSICAL PROPERTIES OF A GAS (CONTINUED).}

\section{Mass Motion and Calorimetry.}

\section{General hydrodynamical Equations of Motion.}

171. LET us consider the small element of volume $d x d y d z$ supposed entirely inside the gas, of which the centre is at $\xi, \eta, \zeta$ and which is bounded by the six planes, parallel to the coordinate planes, of which the equations are:

$$
x=\xi \pm \frac{d x}{2}, \quad y=\eta \pm \frac{d y}{2}, \quad z=\zeta \pm \frac{d z}{2}
$$

At any point let the molecular density be denoted by $\nu$. Strictly speaking, the quantity required is the "effective molecular density" $\nu_{1}$, defined in Chapter IV., but it will tend to clearness to suppose for the present that the molecules are vanishingly small, so that the distinction between $\nu$ and $\nu_{1}$ disappears. In either case $\nu$ (or $\nu_{1}$ ) is a function of $x, y, z$, and when it is necessary to specify the point at which $\nu$ (or $\nu_{1}$ ) is measured we shall write it in the form $\nu_{x, y, z}$.

Let the law of distribution at any point be $f(u, v, w)$, it no longer being supposed that the law of distribution is the same throughout the gas, so that $f(u, v, w)$ is a function of $x, y, z$ as well as of $u, v, w$. When it is necessary to express this we shall be able to replace

$$
f(u, v, w) \text { by } f(u, v, w, x, y, z) .
$$

As before, let us say that a molecule belongs to class $A$, when its velocity components lie within the limits

$$
u \text { and } u+d u, \quad v \text { and } v+d v, \quad w \text { and } w+d w \text {. }
$$


The number of molecules of class A which cross the plane $x=\xi-\frac{d x}{2}$ into the element of volume, in time $d t$, will be

$$
\int_{\eta-\frac{d y}{2}}^{\eta+\frac{d y}{2}} \int_{\zeta-\frac{d z}{2}}^{\zeta+\frac{d z}{2}} \nu_{\xi-\frac{d x}{2}, y, z} f\left(u, v, w, \xi-\frac{d x}{2}, y, z\right) d y d z d u d v d w u d t,
$$

and as far as squares of small quantities this may be written in the form

$$
\nu_{\xi-\frac{d x}{2}, \eta, \zeta} f\left(u, v, w, \xi-\frac{d x}{2}, \eta, \zeta\right) d y d z d u d v d w u d t
$$

Similarly the number of molecules of class $A$ which cross the plane $x=\xi+\frac{d x}{2}$ out of the element of volume is given by expression (346) if $\xi-\frac{d x}{2}$ is replaced by $\xi+\frac{d x}{2}$. By subtraction, the resulting loss to the element, of molecules of class A, caused by motion through the two faces perpendicular to the axis of $x$, is

$$
\frac{\partial}{\partial x}[v f(u, v, w)] d x d y d z d u d v d w u d t
$$

in which the differential coefficient is evaluated at $\xi, \eta, \zeta$. The net loss of molecules of class A caused by motion through all the faces is therefore

$$
\left(u \frac{\partial}{\partial x}+v \frac{\partial}{\partial y}+w \frac{\partial}{\partial z}\right)[v f(u, v, w)] d u d v d w d x d y d z d t \ldots \ldots .(348) .
$$

By integration over all values of $u, v$ and $w$, we obtain the total number of molecules which are lost to the element $d x d y d z$ in time $d t$. If we write

$$
\iiint u f(u, v, w) d u d v d w=u_{0}, \text { etc. }
$$

so that $u_{0}, v_{0}, w_{0}$ are the components of mass velocity of the gas at $x, y, z$, this number is seen to be

$$
\left(\frac{\partial}{\partial x}\left(\nu u_{0}\right)+\frac{\partial}{\partial y}\left(\nu v_{0}\right)+\frac{\partial}{\partial z}\left(\nu w_{0}\right)\right) d x d y d z d t
$$

Since, however, the number of molecules in the element at time $t$ is $\nu d x d y d z$, and at time $t+d t$ is $\left(\nu+\frac{d \nu}{d t} d t\right) d x d y d z$, the net loss is

$$
-\frac{d \nu}{d t} d t d x d y d z
$$

Equating expressions (350) and (351), we obtain

$$
\frac{d \nu}{d t}+\frac{\partial}{\partial x}\left(\nu u_{0}\right)+\frac{\partial}{\partial y}\left(\nu v_{0}\right)+\frac{\partial}{\partial z}\left(\nu w_{0}\right)=0
$$


This is the hydrodynamical equation of continuity, expressing the permanence of the molecules of the gas-in other words, the conservation of mass.

172. The loss of each molecule of class A to the element means a loss of momentum parallel to the axis of $x$ equal to $m u$. Hence the total loss of momentum parallel to the axis of $x$ arising from this cause in time $d t$, is, from expression (348),

$$
m d x d y d z d t \iiint\left(u^{2} \frac{\partial}{\partial x}+u v \frac{\partial}{\partial y}+u w \frac{\partial}{\partial z}\right)[\nu f(u, v, w)] d u d v d w \ldots . . .(353) .
$$

Let us write

$$
\begin{gathered}
\iiint u^{2} f(u, v, w) d u d v d w=\overline{u^{2}} \\
\iiint u v f(u, v, w) d u d v d w=\overline{u v} \\
\text { etc. }
\end{gathered}
$$

so that $\overline{u^{2}}, \overline{u v}$, etc. are the mean values of $u^{2}, u v$, etc. at the point $x, y, z$. Then expression (353) becomes

$$
m d x d y d z d t\left(\frac{\partial}{\partial x}\left(\overline{\nu u^{2}}\right)+\frac{\partial}{\partial y}(\nu \overline{u v})+\frac{\partial}{\partial z}(\nu \overline{u w})\right)
$$

With this must be compounded a gain of momentum caused by the action of forces on the nılecules. The gain of momentum parallel to the axis of $x$ to any single molecule is $X \partial t$, where $X$ is the component of force parallel to the axis of $x$, including of course intermolecular forces and forces at collision, acting upon the molecule in question. Combining the sum of these gains with the loss given by expression (356) we find for the net increase of momenturn inside the element $d x d y d z$ in time $d t$;

$$
\left[\Sigma X-m d x d y d z\left(\frac{\partial}{\partial x}\left(\nu \overline{u^{2}}\right)+\frac{\partial}{\partial y}(\nu \overline{u v})+\frac{\partial}{\partial z}(\nu \overline{u w})\right)\right] d t
$$

where $\Sigma$ denotes summation over all the molecules which were inside the element $d x d y d z$ at the beginning of the interval $d t$.

The total $x$-momentum inside the element $d x d y d z$ at time $t$ is, however, $m \nu u_{0} d x d y d z$. Hence the gain in time $d t$ may be expressed as

$$
\frac{d}{d t}\left(\nu u_{0}\right) m d x d y d z d t
$$

and equating this to expression (357), we obtain

$$
\left[\frac{d}{d t}\left(\nu u_{0}\right)+\frac{\partial}{\partial x}\left(\nu \overline{u^{2}}\right)+\frac{\partial}{\partial y}(\nu \overline{u v})+\frac{\partial}{\partial z}(\nu \overline{u w})\right] m d x d y d z=\Sigma X \ldots
$$

These and the similar equations in $y, z$ are the hydrodynamical equations of motion of the gas, expressing the conservation of momentum, except in so far as this momentum is changed by the action of external forces. 
173. If we write

$$
u=u_{0}+u \text {, etc., }
$$

as in $\S 26$, we have $\bar{u}=u_{0}$, and $\bar{U}=0$. We therefore have

$$
\begin{aligned}
\frac{\partial}{\partial y}(\nu \overline{u v}) & =\frac{\partial}{\partial y}\left[\nu \overline{\left(u_{0}+\mathrm{U}\right)\left(v_{0}+\mathrm{V}\right)}\right] \\
& =\frac{\partial}{\partial y}\left[\nu\left(u_{0} v_{0}+\overline{\mathrm{UV}}\right)\right] \\
& =\nu v_{0} \frac{\partial u_{0}}{\partial y}+u_{0} \frac{\partial}{\partial y}\left(\nu v_{0}\right)+\frac{\partial}{\partial y}(\nu \overline{\mathrm{UV}}),
\end{aligned}
$$

and from this and similar equations, by addition,

$$
\begin{aligned}
\frac{\partial}{\partial x}\left(\nu \overline{u^{2}}\right)+\frac{\partial}{\partial y}(\nu \overline{u v})+\frac{\partial}{\partial z}(\nu \overline{u w}) & \\
=\nu\left(u_{0} \frac{\partial}{\partial x}+v_{0} \frac{\partial}{\partial y}+w_{0} \frac{\partial}{\partial z}\right) u_{0} & +u_{0}\left(\frac{\partial}{\partial x}\left(\nu u_{0}\right)+\frac{\partial}{\partial y}\left(\nu v_{0}\right)+\frac{\partial}{\partial z}\left(\nu w_{0}\right)\right) \\
& +\frac{\partial}{\partial x}\left(\nu \overline{U^{2}}\right)+\frac{\partial}{\partial y}(\nu \overline{U V})+\frac{\partial}{\partial z}(\nu \overline{U W}) \ldots(35.9) .
\end{aligned}
$$

Also, by the equation of continuity (equation (352)),

$$
\begin{aligned}
\frac{d}{d t}\left(\nu u_{0}\right) & =\nu \frac{d u_{0}}{d t}+u_{0} \frac{d \nu}{d t} \\
& =\nu \frac{d u_{0}}{d t}-u_{0}\left(\frac{\partial}{\partial x}\left(\nu u_{0}\right)+\frac{\partial}{\partial y}\left(\nu v_{0}\right)+\frac{\partial}{\partial z}\left(\nu w_{0}\right)\right),
\end{aligned}
$$

so that on adding corresponding sides of this equation and (359), we obtain

$$
\begin{aligned}
\frac{d}{d t}\left(\nu u_{0}\right)+ & \frac{\partial}{\partial x}\left(\nu \overline{u^{2}}\right)+\frac{\partial}{\partial y}(\nu \overline{u v})+\frac{\partial}{\partial z}(\overline{\nu u w}) \\
= & \nu\left(\frac{d}{d t}+u_{0} \frac{\partial}{\partial x}+v_{0} \frac{\partial}{\partial y}+w_{0} \frac{\partial}{\partial z}\right) u_{0} \\
& +\frac{\partial}{\partial x}\left(\overline{\nu \bar{U}^{2}}\right)+\frac{\partial}{\partial y}(\nu \overline{U v})+\frac{\partial}{\partial z}(\nu \overline{u w}) .
\end{aligned}
$$

The left-hand member of the equation is, however, identical with the bracket in equation (358), so that this equation now becomes

$$
\begin{aligned}
\nu\left(\frac{d}{d t}+u_{0} \frac{\partial}{\partial x}\right. & \left.+v_{0} \frac{\partial}{\partial y}+w_{0} \frac{\partial}{\partial z}\right) u_{0} m d x d y d z \\
& =\Sigma X-\left[\frac{\partial}{\partial x}\left(\nu \overline{\mathrm{U}^{2}}\right)+\frac{\partial}{\partial y}(\nu \overline{\mathrm{uv}})+\frac{\partial}{\partial z}(\nu \overline{\mathrm{uw}})\right] m d x d y d z \ldots(360) .
\end{aligned}
$$

174. We now proceed to examine more closely the system of forces which act upon those molecules of which the centres are inside the element $d x d y d z$-the system of forces which we have so far been content to denote by $\Sigma X, \Sigma Y, \Sigma Z$. 
These forces may arise either from forces between pairs of molecules, or from the action upon the molecules of an external field of force. If there is an external field of force of amount $\Xi, \mathrm{H}, \mathrm{Z}$ per unit mass, the contribution made to $\Sigma X$ will of course be

$$
\Xi m \nu d x d y d z
$$

This part of the system of forces need not be discussed further: we now turn to the intermolecular forces. We are supposing those molecules to be inside $d x d y d z$ of which the centres are inside $d x d y d z$. To discuss the intermolecular forces, then, consistently with the suppositions already made, we must regard the molecules as point-centres of force, the position of the centre of force coinciding with the centre of the molecule. This supposition is, of course, perfectly general. For instance, if the molecules are elastic spheres of diameter $\sigma$, we take the law of force between the supposed point-centres to be some function of the distance which becomes infinite when the distance is less than $\sigma$, and zero when the distance is greater than $\sigma$-e.g. $\left(\frac{\sigma}{r}\right)^{n}$ where $n$ is infinitely great.

In general, it is not possible to represent the effect of a system of forces acting upon a material medium by a system of suitably-chosen pressures inside the medium. In the present case, however, this is possible, if we make the assumption that the range of molecular action is small compared with the linear scale of variation of density, etc., in the gas. For subject to this assumption we shall be able to choose the element $d x d y d z$ of such a size that the density, etc., may be regarded as constant throughout, while at the same time the intermolecular forces experienced by the molecules inside it are solely forces between pairs of molecules both of which are very near to one and the same face of the element: the element can, in fact, be too great for intermolecular action across it to be perceptible.

Choosing the element of volume in this way, the forces exerted across any one of the faces of the element may be regarded simply as a system of pressures acting upon the surface of the element. The components of pressure will obviously be continuous functions of position in space, so long as it is understood that the edges of the faces of the element are large compared with the distance between adjacent molecules.

If we denote the components of pressure per unit area acting across a small plane area perpendicular to the axis of $x$ by $\varpi_{x x}, \varpi_{x y}, \varpi_{x z}$, and adopt a similar notation as regards pressures across planes perpendicular to the axes of $y$ and $z$, we see that the $x$-component of all the molecular forces which act on the element $d x d y d z$ can be put in the form

$$
-\left(\frac{\partial \varpi_{x x}}{\partial x}+\frac{\partial \varpi_{y x}}{\partial y}+\frac{\partial \varpi_{z x}}{\partial z}\right) d x d y d z .
$$


Combining this contribution to $\Sigma X$ with that already found (expression (361)), we find as the whole value of $\Sigma X$,

$$
\Sigma X=\left[m \nu \Xi-\left(\frac{\partial \varpi_{x x}}{\partial x}-\frac{\partial \varpi_{y x}}{\partial y}-\frac{\partial \varpi_{z x}}{\partial z}\right)\right] d x d y d z
$$

and there are, of course, similar equations giving the values of $\Sigma Y$ and $\Sigma Z$.

Hence equation (360), on dividing throughout by $d x d y d z$ and replacing $m \nu$ by $\rho$, becomes

$$
\begin{aligned}
\rho\left(\frac{d}{d t}+\right. & \left.u_{0} \frac{\partial}{\partial x}+v_{0} \frac{\partial}{\partial y}+w_{0} \frac{\partial}{\partial z}\right) u_{0} \\
& =\rho \Xi-\frac{\partial}{\partial x}\left(\varpi_{x x}+\rho \overline{U^{2}}\right)-\frac{\partial}{\partial y}\left(\varpi_{y x}+\rho \overline{U V}\right)-\frac{\partial}{\partial z}\left(\varpi_{z x}+\rho \overline{U W}\right) \ldots . . .
\end{aligned}
$$

175. Let us write

$$
\left.\begin{array}{l}
P_{x x}=\varpi_{x x}+\rho \overline{\mathrm{U}}, \\
P_{y x}=\varpi_{y x}+\rho \overline{\mathrm{UV}}, \text { etc. }
\end{array}\right\}
$$

then equation (362) may be written

$$
\rho\left(\frac{d}{d t}+u_{0} \frac{\partial}{\partial x}+v_{0} \frac{\partial}{\partial y}+w_{0} \frac{\partial}{\partial z}\right) u_{0}=\rho \Xi-\frac{\partial P_{x x}}{\partial x}-\frac{\partial P_{y x}}{\partial y}-\frac{\partial P_{z x}}{\partial z}
$$

This equation is identical in form with the hydrodynamical equation of a fluid of density $\rho$ moving with a velocity $u_{0}, v_{0}, w_{0}$, the body forces being $\Xi, \mathrm{H}, \mathrm{Z}$ per unit mass, and the pressures being the system

$$
P_{x x}, P_{y x}, P_{z x}, P_{x y}
$$

We may, therefore, speak of this system of pressures as the total pressure at the point $x, y, z$. As is clear from equation (363), the pressures arise partly from the intermolecular forces and partly from the molecular agitation in the gas.

If we multiply equation ( 352 ) throughout by $m$, it becomes

$$
\frac{d \rho}{d t}+\frac{\partial}{\partial x}\left(\rho u_{0}\right)+\frac{\partial}{\partial y}\left(\rho v_{0}\right)+\frac{\partial}{\partial z}\left(\rho w_{0}\right)
$$

which is formally the same as the hydrodynamical equation of continuity, and the hydrodynamical analogy is now complete.

We have, therefore, seen that we may regard a gas as a continuous fluid, of which the motion is subject to hydrodynamical equations of motion and continuity of the usual type. This is the justification, in discussing the mass-motion of gases-the propagation of sound, for instance,-for treating the gas as if it was a continuous fluid of the kind contemplated in hydrodynamics. 


\section{Molecules of Finite Size.}

176. In order to separate the difficulties as much as possible, and so simplify the treatment of the subject, it has been found convenient to defer the difficulties introduced by the finite size of the molecules. The finite size can, as has already been explained, be supposed to have been allowed for in the field of intermolecular force, but if we wish to get results which admit of easy interpretation it is best to suppose the molecules to be of finite size, in addition to possessing fields of intermolecular force.

If $\nu_{x, y, z}$ is the "effective molecular density" at $x, y, z$, then the expectation of the number of molecules of which the centres lie within any element of volume is

$$
\iiint_{x, y, z} d x d y d z
$$

where the integration extends throughout the element of volume in question. It is, however, also equal, by the definition of $\nu$, to

$$
\nu \iiint d x d y d z
$$

so that $\nu_{x, y, z}$, averaged through a volume large enough to contain a great number of molecules, becomes equal to $\nu$.

Similarly the number of molecules crossing an element of surface $d S$ contains as a factor

$$
\iint v_{x, y, z} d S
$$

and if this element of surface is great compared with the size of a molecule, while at the same time $\nu$ is approximately constant while we pass in any direction over a distance comparable with the size of $d S$, then the factor may, we know, also be written

$$
\nu \iint d S
$$

so that $\nu$ is the average value of $\nu_{x, y, z}$ over the element of surface $d S$.

A complication occurs in the neighbourhood of the boundary, because here, as appeared in the last chapter, the value of $\nu$ may vary perceptibly over a distance comparable with the molecular diameter. If, then, $d S$ is an element of surface at a distance $\frac{1}{2} \sigma$ from the boundary and parallel to the boundary, expression (366) must be replaced, not by expression (368), but by

$$
\nu_{b} \iint d S
$$

where $\nu_{b}$ is the same as the $\nu_{b}$ of $\S 137$. 
It will now be clear that all the analysis of the present chapter will hold, even after allowing for the finite size of the molecules, if we take $\nu$ to be the density everywhere except at the boundary, provided that, in considering an element of surface at distance $\frac{\sigma}{2}$ from the boundary, we replace $\nu$ by $\nu_{b}$.

In calculating the pressure, it will be remembered, we assumed the element of volume so great that intermolecular forces could not act across it. At the same time we assume it so small that the density is approximately constant throughout. These assumptions become inconsistent at the boundary, because the density, as is clear from $\S 134$, varies over a range across which intermolecular forces can and do act. In this case, however, we can abandon the supposition that the density will be approximately constant throughout. All that is required is a knowledge of the mean density in the element of volume, and this, on account of the thinness of the layer near the surface in which the mean density differs perceptibly from $\nu$, may still be taken to be $\nu$.

\section{Gas in Equilibrium.}

177. We now examine the form assumed by our equations, when the gas is in equilibrium. In this case we can clearly take the law of distribution of velocities to be Maxwell's law at every point, so that

by equation (161), and

$$
\rho \overline{u^{2}}=\rho u^{\overline{2}}=\nu \overline{m u^{2}}=\frac{\nu}{2 h}
$$

$$
\overline{\mathrm{uV}}=\overline{\mathrm{vW}}=\overline{\mathrm{w} U}=0 .
$$

Thus of the system of pressures specified by equations (363), that part which arises from molecular agitation reduces to a simple hydrostatical pressure of amount $\frac{\nu}{2 h}$. Clearly also the $\approx$ system of pressures, arising from the intermolecular forces, will reduce to a simple hydrostatical pressure, and we shall therefore have

$$
\begin{aligned}
& P_{x x}=P_{y y}=P_{z z}=\varpi+\frac{\nu}{2 h}, \\
& P_{x y}=P_{x z}=P_{y z}=0 .
\end{aligned}
$$

The total pressure at the point $x, y, z$ is therefore a simple hydrostatical pressure of amount $P$ given by

$$
P=\varpi+\frac{\nu}{2 h}
$$

178. The equations of equilibrium become

$$
\rho \equiv=\frac{\partial P}{\partial x} \text { etc. }
$$


The conditions for equilibrium to be possible are, therefore, three of the type

$$
\frac{\partial}{\partial y}(\rho \Xi)=\frac{\partial}{\partial x}(\rho \mathrm{H})
$$

In general, the result of eliminating the unknown variable $\rho$ from these equations is *

$$
\Xi\left(\frac{\partial \mathrm{H}}{\partial z}-\frac{\partial Z}{\partial y}\right)+\mathrm{H}\left(\frac{\partial Z}{\partial x}-\frac{\partial \Xi}{\partial z}\right)+\mathrm{Z}\left(\frac{\partial \Xi}{\partial y}-\frac{\partial \mathrm{H}}{\partial x}\right)=0 \ldots \ldots
$$

If, however, the gas is at the same temperature throughout, the intermolecular pressure $\varpi$ will depend solely on $\rho$, so that we can regard $P$ as a function of $\rho$ only, and equation (371) can be written in the form

$$
\Xi=\frac{\partial}{\partial x} \int \frac{d P}{\rho} \text {. }
$$

There are of course two similar equations for $\mathrm{H}, \mathrm{Z}$, and the system of three equations taken together simply expresses that the forces must have a potential. This condition being satisfied, that expressed by equation (372) is satisfied also, but the two conditions are not identical. It is only in the case of a gas being at the same temperature throughout that the one can be deduced from the other.

If we suppose the potential to be $\psi$, the equations of equilibrium are

$$
-\frac{\partial \psi}{\partial x}=\frac{1}{\rho} \frac{\partial P}{\partial x}
$$

and two similar equations, and these have the common integral

$$
-\psi=\int \frac{d P}{\rho}
$$

Now $P=\varpi+\frac{\nu}{2 h}$ by equation (370). If we ignore intermolecular forces and put $\varpi=0$, we have $P=\frac{\nu}{2 h}=\frac{\rho}{2 h m}$, and equation (374) becomes

$$
\begin{gathered}
-\psi=C+\frac{\log \rho}{2 h m}, \\
\rho=D e^{-2 h m \psi} .
\end{gathered}
$$

or

In putting $\varpi=0$, we not only suppose the field of intermolecular force outside the molecules to be very small, but also that the pressures across any surface set up by collisions between molecules are very small. In other words we assume that the size of the molecules is negligible. Thus the equation obtained agrees, as it ought, with the equation

$$
\rho=D e^{-2 h x},
$$

obtained in Chapter V. (equation (165)), for the $\chi$ of that equation had the same meaning as the present $m \psi$.

\footnotetext{
* Besant and Ramsey, Hydromechanics, r. p. 12 (1904).
} 
179. When no external forces act, the equations of equilibrium (equations (371)) reduce to

$$
\frac{\partial P}{\partial x}=\frac{\partial P}{\partial y}=\frac{\partial P}{\partial z}=0
$$

so that $P$ is constant throughout the gas.

Obviously, in fact, since the gas is in equilibrium under the action of no forces the total pressure must be the same throughout. At the boundary this total pressure becomes identical with the pressure $p$ calculated in the last chapter. This, then, is the constant value of $P$.

Let us, however, examine carefully the form assumed at the boundary by the general equation (equation (370))

$$
P=\varpi+\frac{\nu}{2 h}
$$

If we calculate $P$ at a layer at distance just greater than $\frac{\sigma}{2}$ from the boundary, we put $\varpi=0$, for the number of molecules whose centres lie between this layer and the boundary is infinitesimal. Also, as explained in $\S 176$, we take $\nu_{b}$ as the value of $\nu$, and so obtain

$$
P=\frac{\nu_{b}}{2 h}
$$

If, on the other hand, we calculate $P$ at a layer just less than $\frac{\sigma}{2}$ from the boundary, we must not put $\varpi=0$, for we have now to take account of the stresses between the boundary and the molecules which are in collision with it. We accordingly put $\varpi=p$, and of course $\nu=0$, since no molecules can have their centres at a distance less than $\frac{\sigma}{2}$ from the boundary. Hence we obtain, as has already been foreseen,

$$
P=p
$$

Comparing the values of $P$ given by equations (376) and (377), we obtain

$$
p=\frac{\nu_{b}}{2 h}
$$

which is in agreement, as it ought to be, with the value of the pressure calculated in the last chapter (equation (287)).

If we substitute the value $\frac{\nu_{b}}{2 h}$ for $P$ in equation (375), we obtain

$$
\varpi=\frac{\nu_{b}-\nu}{2 h}
$$

giving a general expression for the intermolecular pressure, which will be found useful later. 


\section{Thermodynamics.}

\section{Equation of Energy.}

180. The total energy of the gas may be divided into internal and external. The former consists of the kinetic energy of molecular motion and the potential energy of intermolecular forces. The external energy is measured by the work which could be performed by the gas in expanding to a state of infinite rarity starting from its present configuration, this work of course being performed in opposition to the pressure of the gas. The external energy will be denoted by $\mathfrak{B}$, the internal energy by $\mathbb{E}$. As in equation (133) we have

$$
\xi=E_{a}+E_{b}+\ldots+\Phi=N \bar{E}+\Phi
$$

where $\bar{E}$ is the mean energy of the $N$ molecules, and $\Phi$ is the potential energy of the intermolecular forces.

181. Let us suppose that a quantity $d \mathbb{Q}$ of energy, either in the form of heat or otherwise, is absorbed by the gas from some external source, so that as a consequence the pressure, volume and temperature of the gas change. We shall suppose $d \mathbb{Q}$ measured in work-units; the corresponding quantity

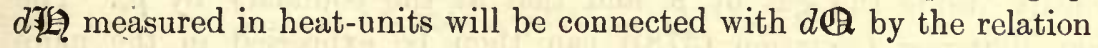

$$
d \mathbb{Q}=J d \underline{\mathrm{C}} \text { ? }
$$

where $J$ is the mechanical equivalent of heat.

Let us suppose that after this absorption of heat the gas again assumes a steady state, and that in the new state the values of $\mathscr{B}$ and $\mathbb{E}$ have become changed to $\mathfrak{W}+d \mathfrak{W}$ and $(\xi+d \mathfrak{E}$. The energy equation-obtained by equating the total energies before and after the change-is

$$
\begin{aligned}
d \mathbb{Q} & =d \mathfrak{W}+d \mathbb{E} \ldots \ldots . . \\
& =d \mathfrak{W}+N d \bar{E}+d \Phi .
\end{aligned}
$$

If we suppose the volume of the gas increased, by a small element $d S$ of the surface moving a small distance $d n$ parallel to itself normally outwards, then obviously the work done by the gas will be that of moving a force $p d S$ through a distance $d n$. We therefore have

$$
d \mathfrak{W}=p d S d n .
$$

The resulting increase in volume $(d v)$ is of course the sum of the contributions $d S d n$ from all the elements of surface which are moved, so that the equation becomes

$$
d \mathfrak{W}=p d v
$$


182. We know on experimental grounds $(\S 74)$ that $d \Phi$ is very small. If we take $d \Phi=0$, equation (382) assumes the form

$$
d \mathbb{Q}=N d \bar{E}+p d v
$$

and we have (equation (287))

$$
p=\frac{\nu_{b}}{2 h}=R \nu_{b} T,
$$

so that the equation becomes

$$
d \mathbb{Q}=N d \bar{E}+R v_{b} T d v
$$

183. If we do not neglect the term $d \Phi$, the calculation becomes considerably more difficult, for the intermolecular pressure $\varpi$ will do work when the gas expands.

Let us write

$$
\varpi=\varpi_{c}+\varpi_{i},
$$

where $\varpi_{c}$ is the part of $\varpi$ produced by collisions-i.e. encounters in which the molecules are so close that they cannot approach any nearer,-and $\varpi_{i}$ is the part of $\varpi$ produced by the outstanding intermolecular forces between pairs of molecules not in contact.

We have already calculated a value for $\varpi$; we must now try to calculate $\varpi_{i}$ and $\varpi_{c}$ separately.

As in the treatment of Van der Waals' cohesion $(\$ 141)$, we can denote the density inside the gas by $\rho$, and that at the boundary by $\rho_{0}$. The equation giving $\varpi$ (equation (378)) can then be expressed in the more complete form

$$
\varpi=\frac{\nu_{b}\left(\rho_{0}\right)-\nu}{2 h}
$$

expressing that $\nu_{b}$ is calculated for a density $\rho_{0}$.

Let us now imagine a rigid plane surface of area unity set up and held rigidly at rest at some point inside the gas. This alteration will not affect the distribution of density inside the gas, for the field of intermolecular force will exist on both sides of the area. The pressure produced by molecular impacts on either side of the area will of course be the same, say $p_{i}$. Treating this surface as we have already treated the surface which forms the true boundary of the gas, we obtain equations giving the constant $P$ in the form

$$
P=\varpi_{i}+\frac{\nu_{b}(\rho)}{2 h}
$$

by considering an area at a distance just greater than $\frac{\sigma}{2}$ from the fixed area; and

$$
P=\varpi_{i}+p_{i}
$$

by considering an area at a distance just less than $\frac{\sigma}{2}$ from the fixed area. 
Incidentally we notice that by comparing the two we obtain for the internal pressure $p_{i}$ the value

$$
p_{i}=\frac{\nu_{b}(\rho)}{2 h}=R \nu_{b}(\rho) T
$$

Returning to the values just found for $P$, and replacing $P$ by its known value

$$
P=\frac{\nu_{b}\left(\rho_{0}\right)}{2 h},
$$

we obtain

$$
\varpi_{i}=\frac{\nu_{b}\left(\rho_{0}\right)-\nu_{b}(\rho)}{2 h}
$$

We also have found that

hence, by subtraction,

$$
\varpi=\varpi_{i}+\varpi_{c}=\frac{\nu_{b}\left(\rho_{0}\right)-\nu}{2 h},
$$

$$
\varpi_{c}=\frac{\nu_{b}(\rho)-\nu}{2 h}
$$

These equations give $\varpi_{i}$ and $\varpi_{c}$ separately.

184. To obtain some insight into the meaning of these pressures, let us examine what they become when the deviations from Boyle's Law are small.

If we put $p=\frac{\nu_{b}\left(\rho_{0}\right)}{2 h}$ in Van der Waals' equation, we get

$$
\left(\frac{\nu_{b}\left(\rho_{0}\right)}{2 h}+\frac{a}{v^{2}}\right)(v-b)=R N T,
$$

or again, for the pressure on an area inside the gas, noticing that the cohesion term must be supposed to vanish in this case,

$$
\frac{\nu_{b}(\rho)}{2 h}(v-b)=R N T \text {. }
$$

From these equations, we have, on replacing $R T$ by $\frac{1}{2 h}$,

$$
\begin{aligned}
\nu_{b}\left(\rho_{0}\right)+\frac{2 h a}{\nu^{2}} & =\nu_{b}(\rho) \\
& =\frac{N}{v-b} \\
& =\nu\left(1+\frac{b}{v}\right),
\end{aligned}
$$

so that

$$
\varpi_{i}=\frac{\nu_{b}\left(\rho_{0}\right)-\nu_{b}(\rho)}{2 h}=-\frac{a}{v^{2}} .
$$

Thus $\varpi_{i}$, as we should expect, becomes identical, except as regards sign, with the cohesion pressure $p_{1}$ assumed in arriving at Van der Waals' equation, and

$$
\varpi_{c}=\frac{\nu_{b}(\rho)-\nu}{2 h}=\frac{\nu}{2 h}\left\{\frac{v}{v-b}-1\right\}=\frac{\nu b}{2 h v},
$$


so that $\varpi_{c}$, as we should expect, is proportional to the $b$ of Van der Waals, being in fact a fraction $\frac{b}{v}$ of the whole pressure. The internal pressure $p_{i}$ is therefore given by

$$
\begin{aligned}
p_{i} & =\frac{\nu_{b}(\rho)}{2 h} \\
& =p-\varpi_{i} \\
& =p+\frac{a}{v^{2}}
\end{aligned}
$$

in Van der Waals' equation.

185. To calculate $d \Phi$ we notice that when the gas expands or is compressed, the pressure $\varpi_{c}$ does not do any work, for the molecules themselves do not expand or contract with the gas. To a first approximation, we may suppose that every element of the gas expands in the same ratio, so that any element $d x d y d z$ expands to a volume $(1+\epsilon) d x d y d z$. The resulting contribution to $d \Phi$ is $-\varpi_{i} \epsilon d x d y d z$, the negative sign indicating that work is done by the pressure on the gas, and not as before by the gas against the pressure. The total value of $d \Phi$ is accordingly

$$
d \Phi=-\iiint \varpi_{i} \epsilon d x d y d z
$$

or, using the value of $\varpi_{i}$ given by equation (387),

$$
d \Phi=-\frac{\nu_{b}\left(\rho_{0}\right)-\nu_{b}(\rho)}{2 h}
$$

The energy equation is now

$$
\begin{aligned}
& d \Phi=N d \bar{E}+p d v+d \Phi \\
& =N d \bar{E}+\left[\frac{\nu_{b}\left(\rho_{0}\right)}{2 h}-\frac{\nu_{b}\left(\rho_{0}\right)-\nu_{b}(\rho)}{2 h}\right] d v \\
& =N d \bar{E}+\frac{\nu_{b}(\rho)}{2 h} d v \\
& =N d \bar{E}+R \nu_{b}(\rho) T d v \text {. }
\end{aligned}
$$

The equation is therefore of exactly the same form as equation (385), obtained on neglecting cohesion forces, but the value of $\nu_{0}$ must be calculated on the supposition of a uniform density $\rho$ equal to the mean density of the gas. This value of $\nu_{b}$ is of course a function only of the constants and volume of the gas (cf. equation (293)).

\section{The First Law of Thermodynamics.}

186. The law which is commonly termed the first law of thermodynamics is that contained in equation (380), and simply expresses that heat is energy, which is capable of transformation into the kinetic energy of motion of material masses. This, however, is all included in the hypothesis upon which the Kinetic Theory is based. Thus, in the Kinetic Theory, the first law of thermodynamics is reduced to a truism. 


\section{The Second Law of Thermodynamics.}

187. If equation (391) is divided throughout by $T$, it becomes

$$
\frac{d \mathbb{Q}}{T}=N \frac{d \bar{E}}{T}+R \nu_{b}(\rho) d v
$$

In this equation the gas remains of the same constitution throughout the change in its state. Thus $\bar{E}$ is a function of $T$ only and $\nu_{b}(\rho)$ of $v$ only. Hence the terms $\frac{d \bar{E}}{T}$ and $\nu_{b}(\rho) d v$ are each complete differentials.

It follows that the right-hand member of equation (392) is a complete differential, say $d \tilde{\delta}$, and we therefore have

$$
\frac{d Q}{T}=d \$
$$

This equation contains the second law of thermodynamics.

If we suppose that by the introduction of heat and by changes in pressure and volume, the gas is caused to pass through a series of states, such that finally it is brought back to the same volume and temperature from which it started, we have, since $\delta$ is the same at the beginning and end of the series of changes,

$$
\int \frac{d \mathbb{Q}}{T}=0
$$

Thus we have the second law in a different form :

The value of $\int \frac{d \Phi}{T}$, where the integral is taken through any closed cycle of thermal processes, is zero.

188. When equation (394) is reached from the thermodynamic standpoint, the temperature is not measured on the kinetic theory scale, which, as we have seen, is identical with the scale of a gas thermometer in which a perfect gas is used as thermometric substance, but is measured on the thermodynamical scale, which is independent of the working substance.

The result expressed by equation (394) does not in itself permit us to identify the kinetic theory temperature $T$, with the thermodynamic temperature $\Theta$. All that we have found so far is that $\frac{1}{\Theta}$ and $\frac{1}{T}$ are both of them

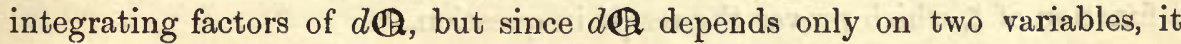
must in any case have an infinite number of integrating factors.

For in general, if

$$
d z=L d x+M d y
$$

the condition that $N$ shall be an integrating factor of $d z$ is that

$$
\frac{L}{N} d x+\frac{M}{N} d y
$$


shall be a complete differential, and is therefore

$$
\frac{d}{d y}\left(\frac{L}{N}\right)=\frac{d}{d z}\left(\frac{M}{N}\right)
$$

from which $N$ can always be found.

If $N$ is an integrating factor of (395), so that

$$
d z=N d \zeta
$$

where $d \zeta$ is a perfect differential, then we have also

$$
d z=N f(\zeta) \frac{d \zeta}{f(\zeta)}=N f(\zeta) d \int \frac{d \zeta}{f(\zeta)}
$$

so that if $N$ is an integrating factor, so also is $N f(\zeta)$-in fact $N$ multiplied by any function of $\zeta$ is an integrating factor. There are, therefore, an infinite number of integrating factors.

189. We can, moreover, shew that if $N$ is one integrating factor of (395), so that

$$
d z=N d \zeta
$$

then all the integrating factors are included under the form $N f(\zeta)$.

For let $N$ and $N^{\prime}$ be two separate integrating factors, so that

and also

$$
\frac{L}{N}=\frac{\partial \zeta}{\partial x}, \quad \frac{M}{N}=\frac{\partial \zeta}{\partial y}
$$

Then obviously

$$
\frac{L}{N^{\prime}}=\frac{\partial \zeta^{\prime}}{\partial x}, \frac{M}{N^{\prime}}=\frac{\partial \zeta^{\prime}}{\partial y}
$$

$$
\left|\begin{array}{ll}
\frac{\partial \zeta}{\partial x}, & \frac{\partial \zeta}{\partial y} \\
\frac{\partial \zeta^{\prime}}{\partial x}, & \frac{\partial \zeta^{\prime}}{\partial y}
\end{array}\right|=0
$$

from which it follows that $\zeta^{\prime}$ is a function of $\zeta$. Hence

$$
\frac{N^{\prime}}{N}=\frac{\frac{\partial \zeta^{\prime}}{\partial x}}{\frac{\partial \zeta}{\partial x}}=\frac{\partial \zeta^{\prime}}{\partial \zeta^{\prime}}
$$

a function of $\zeta$, which proves the result in question.

190. Applying this to the problem of thermodynamics before us, it appears that since $\frac{1}{T}$ and $\frac{1}{\Theta}$ are both of them integrating factors, there must be a relation of the form

$$
\frac{T}{\Theta}=f(\$)
$$


Now $T$ and $\Theta$, by definition, depend only on the heat-energy of the gas, whereas $\delta$ depends also upon the density of the gas. Hence equation (396) can only be satisfied by taking $f(\mathfrak{S})$ equal to a constant, $k$, so that

$$
T=k \Theta \text {, }
$$

identifying the absolute thermodynamical scale with the Kinetic Theory scale, except for a possible multiplicative constant.

\section{Entropy.}

191. The quantity $\delta$ introduced by equation (393) is defined to be the "Entropy" of the gas. It is, as we have seen, a function only of the quantities which determine the state of the gas. Since

$$
\delta=N \int \frac{d \bar{E}}{T}+R \int \nu_{b}(\rho) d v
$$

it is clear that $\delta$ is the sum of two terms, of which the first depends only on the motion of the molecules, and the second only on their positions.

The importance of the function $\delta$ is as follows: Let two systems, denoted by suffixes 1 and 2 , have initially entropies $\tilde{\Sigma}_{1}$ and $\boldsymbol{\Sigma}_{2}$, and let a quantity $d \mathbb{Q}$ of heat pass from the one to the other. The loss to $\Phi_{1}$ is $\frac{d \mathbb{Q}}{T_{1}}$, the gain to $\mathscr{\Xi}_{2}$ is $\frac{d \Phi}{T_{2}}$, so that

$$
d\left(\tilde{S}_{1}+\tilde{\Phi}_{2}\right)=d \mathbb{Q}\left(\frac{1}{T_{2}}-\frac{1}{T_{1}}\right)
$$

In nature, however, heat only passes from a hotter to a colder body, so that $T_{1}$ must be greater that $T_{2}$. Hence $d\left(\boldsymbol{\Sigma}_{1}+\boldsymbol{\Sigma}_{2}\right)$ must be positive.

It follows, then, that any natural transfer of heat tends to increase the entropy of the systems concerned, or, stated more concisely, the entropy tends to its maximum value.

\section{Apparent Irreversibility.}

192. We seem here to have arrived again at an irreversible phenomenon, although the equations of motion from which it ought to have been deduced were strictly reversible. In Chapter II. we had an experience with the

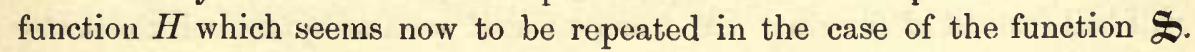
We apparently found in Chapter II. that $d H / d t$ was always negative or zero, although, as the whole calculation of $d H / d t$ had rested solely upon reversible equations of motion, it ought to have been found that $d H / d t$ was as likely to be positive as negative. In Chapters III. and IV. however, we were able to trace this apparent fallacy to the imperfections of the statistical method. We found that $H$ had a minimum value; if the initial value of $H$ 
were different from this minimum value, it was infinitely probable that $H$ would decrease, but on the other hand, it was infinitely probableassuming the basis of probability supplied by the generalised space,- that the initial value of $H$ would be equal to the minimum, in which case, as $H$ could not decrease further, the "expectation" was of a slight increase. Thus the large probability of a small increase in $H$ just balanced the small probability of a finite decrease in $H$, and on the whole the "expectation" of change in $H$ was nil.

A precisely similar explanation holds with reference to $\$$. Indeed it will be found that $\delta$ is very closely allied numerically, not with the simple function $H$ of Chapters II.-IV. but with the more general function $\mathfrak{S}$ of Chapter V. An increase in $\$$ presupposes an initial difference of temperature between the two component systems; and these initial conditions, looked at from the point of view of abstract dynamics, and judged with reference to the basis of probability supplied by a generalised space, are infinitely improbable. With reference to the same basis of probability, it is infinitely probable that the initial conditions will be those of equilibrium of temperature, in which case the only change possible in $\delta$ is a decrease. Or, physically, the only possible alteration in the state of the system is the production of inequalities of temperature. The production of such an inequality, although improbable when the motion is confined to a short time, is not impossible, and indeed becomes infinitely probable when the motion is continued for a sufficient time. Thus the increase of entropy, even granted the infinitely improbable (from the dynamical point of view) initial conditions which makes such an increase possible, is only a probability and not a certainty; and when the entropy starts initially at its maximum, it is infinitely probable that, granted sufficient time, the entropy will decrease.

193. Translated into physical language these results seem at first sight somewhat startling. To borrow an illustration from Lord Kelvin, if we have a bar of iron initially at uniform temperature, and subject neither to external disturbance nor to loss of energy, it is infinitely probable that, given sufficient time, the temperature of one half will at some time differ by a finite amount from that of the other half. Or again, if we place a vessel full of water over a fire, it is only probable, and not certain, that the water will boil instead of freezing. And moreover, if we attempt to boil the water a sufficient number of times, it is infinitely probable that the water will, on some occasions, freeze instead of boil. The freezing of the water, in this case, does not in any way imply a contravention of the laws of nature: the occurrence is merely what is commonly described as a "coincidence," exactly similar in kind to that which has taken place when the dealer in a game of whist finds that he has all the trumps in his hand. 
The analogy of the distribution of a pack of cards will help us to see further into the problem presented by the entropy of a gas. In dealing cards, it is just as likely that the dealer will have the thirteen trumps as that he will have any other thirteen cards that we like to specify. The occurrence of a hand composed of thirteen trumps might, however, be justly regarded as a "coincidence," whereas the occurrence of any specified hand in which the cards were more thoroughly mixed, could not reasonably be so regarded. The explanation is that there are comparatively few ways in which a hand which is all trumps can be dealt, but a great number in which a mixed hand can be dealt.

A similar remark applies to the result of putting cold water over a hot fire. There are comparatively few ways in which the fire can get hotter, and the water colder, but a great many ways in which the fire can impart heat to the water-a proposition which becomes obvious on looking at it from the dynamical point of view of the generalised space. Speaking loosely, it is just as likely that the water will freeze as that it will boil in any specified way. There are, however, so many ways in which the water can boil, all these ways being indistinguishable to us, that we can say that it is practically certain that the water will boil.

The increase of entropy, then, simply means the passage from a more easily distinguishable state to a less easily distinguishable state, or, in terms of the generalised space, from a less probable to a more probable configuration.

In reference to the generalised space, however, we saw that a decrease of $\mathfrak{S}$ denoted the passage of a system from a rarer to a more common, or, if we like, from a less probable to a more probable configuration. The theorem of increasing entropy is, therefore, identical with the principle of decreasing $\mathfrak{S c}$, by the help of which we investigated the motion of a gas towards its steady state.

194. A reference to equation (397) shews that the entropy consists of two parts, the former depending on the energy of the molecules of the gas, and the latter on their positions. So far we have considered only variations in the first term, resulting from inequalities in the temperature of the gas. Similar remarks could, however, be made about the variations of the second term, these denoting inequalities in the density of the gas. A single illustration, suggested by Willard Gibbs*, will, perhaps, make clear what is meant.

If we put red and blue ink together in a vessel, and stir them up, common experience tells us that, if we assume the inks initially to differ in nothing more than colour, the result of stirring is a uniform violet ink. Here we have the passage from a more easily distinguishable to a less easily

* Elementary Principles of Statistical Mechanics, p. 144. 
distinguishable arrangement of coloured inks. If, however, we start by stirring a uniform violet ink composed of a mixture of red and blue inks, then it is possible, although not probable, that the effect of the stirring will be to separate the inks of different colour, so that one half of the vessel is occupied solely by red, and the other solely by blue ink. And from the dynamical standpoint it is no less probable that this should occur, than that we should be able to start stirring inks which were separated initially as regards colour.

195. With reference to this subject, some well-known remarks of Maxwell* are of extreme interest. He says: "One of the best established facts in thermodynamics is that it is impossible in a system enclosed in an envelope which permits neither change of volume nor passage of heat, and in which both the temperature and the pressure are everywhere the same, to produce any inequality of temperature or of pressure without the expenditure of work. This is the second law of thermodynamics, and it is undoubtedly true so long as we can deal with bodies only in mass and have no power of perceiving or handling the separate molecules of which they are made up. But if we conceive a being whose faculties are so sharpened that he can follow every molecule in its course, such a being, whose attributes are still as essentially finite as our own, would be able to do what is at present impossible to us. For we have seen that the molecules in a vessel full of air at uniform temperature are moving with velocities by no means uniform though the mean velocity of any great number of them, arbitrarily selected, is almost exactly uniform. Now let us suppose that such a vessel is divided into two portions $A$ and $B$, by a division in which there is a small hole, and that a being, who can see the individual molecules, opens and closes this hole, so as to allow only the swifter molecules to pass from $A$ to $B$, and only the slower ones to pass from $B$ to $A$. He will thus, without expenditure of work, raise the temperature of $B$ and lower that of $A$, in contradiction to the second law of thermodynamics."

Thus Maxwell's sorting demon could effect in a very short time, what would probably take a very long time to come about if left to the play of chance. There would, however, be nothing contrary to natural laws in the one case any more than in the other.

The reader who wishes to study the question of irreversibility further is referred to the following works:

(i) "Report on the Present State of our knowledge of Thermodynamics, specially with regard to the Second Law," by J. Larmor and G. H. Bryan, British Association Report, 1891 (Cardiff), p. 85.

(ii) Elementary Principles of Statistical Mechanics, J. Willard Gibbs (Scribners, New York), 1902.

(iii) Vorlesungen über Gastheorie, Boltzmann.

* Theory of Heat, p. 328. 


\section{Calorimetry.}

\section{Specific Heats of a Perfect Gas.}

196. We now turn to an investigation of the specific heats of a gas, and shall begin by considering the simplest case, namely that of a perfect gas in which the relation between pressure, volume, and temperature is

$$
p=\frac{R N^{\prime} T}{v}
$$

The equation of energy in this case becomes

$$
\begin{aligned}
d \mathbb{Q} & =N d \bar{E}+p d v \\
& =N d \bar{E}+R N T \frac{d v}{v}
\end{aligned}
$$

and this may be regarded as the general equation of calorimetry.

197. Let us first suppose that a quantity $d \mathbb{Q}$ of energy in the form of heat is absorbed by the gas, while the volume of the gas is maintained constant. In this case all the heat goes towards raising the temperature of the gas, equation (400) assuming the form

$$
d \mathbb{Q}=N d \bar{E} .
$$

Let $C_{v}$ be the specific heat of the gas at constant volume, i.e., the amount of heat required to raise the temperature of a unit mass of gas by one degree, then

$$
d \mathbb{Q}=J d \underline{T}=J C_{v} N m d T
$$

and therefore equation (401) becomes

$$
C_{v}=\frac{1}{J m} \frac{d \bar{E}}{d T}
$$

198. Next, let us suppose that the absorption of heat takes place at constaut pressure. In this case both the volume and temperature will change, but from equation (399) they must change in such a manner that

$$
\frac{T}{v}=\text { constant }
$$

If we differentiate this equation logarithmically, we obtain

$$
\frac{d T}{T}=\frac{d v}{v}
$$

as the relation between $d T$ and $d v$ when the pressure is maintained constant, and using this relation, equation (400) becomes

$$
d \mathbb{Q}=N d \bar{E}+R N d T
$$

The value of $d \mathbb{Q}$ is now $J C_{p} N m d T$ where $C_{p}$ is the specific heat at constant pressure. Hence equation (403) leads to the relation

$$
C_{p}=\frac{1}{J m} \frac{d \bar{E}}{d T}+\frac{R}{J m}
$$


199. From equations (402) and (404) we obtain by subtraction

$$
C_{p}-C_{v}=\frac{R}{J_{m}}
$$

Since $m$ is proportional to the molecular weight of the particular gas we are discussing, this equation expresses Carnot's Law :

The difference of the two specific heats of a gas is inversely proportional to the molecular weight of the gas.

This law can be expressed in a different form. The specific heats referred to unit volume instead of to unit mass are of course $C_{p} \rho, C_{v} \rho$ and equation (405) may be written

$$
C_{p} \rho-C_{v \rho}=\frac{R \rho}{J m}=\frac{p}{J T}
$$

the last transformation depending on equation (399). Hence:

At a given temperature and pressure the difference of the two specific heats per unit is the same for all gases.

200. It is found by experiment that, at any rate for a large number of gases, $C_{p}$ and $C_{v}$ are approximately independent of the temperature. This, as is shewn by a reference to the formulae (402) and (404), must mean that $\frac{d \bar{E}}{d T}$ is a constant, and therefore that the mean energy of a molecule of the gas stands in a constant ratio to the translational energy. Let us denote this ratio by $(1+\beta)$, so that $\beta$ is the ratio of internal to translational energy. Then

$$
\begin{aligned}
\bar{E} & =(1+\beta) \frac{1}{2} m C^{2} \\
& =(1+\beta) \frac{3}{2} R T
\end{aligned}
$$

so that

$$
\frac{d \bar{E}}{d T}=\frac{3}{2} R(1+\beta) \text {. }
$$

Substituting this value for $\frac{d \bar{E}}{d T}$ in equations (402) and (404), we obtain

$$
\begin{aligned}
& C_{v}=\frac{3}{2}(1+\beta) \frac{R}{J m}, \\
& C_{p}=\left[1+\frac{3}{2}(1+\beta)\right] \frac{R}{J_{m}} .
\end{aligned}
$$

If we denote the ratio $C_{p} / C_{v}$ by $\gamma$, we obtain by division,

$$
\begin{aligned}
\gamma \equiv \frac{C_{p}}{C_{v}} & =\frac{1+\frac{3}{2}(1+\beta)}{\frac{3}{2}(1+\beta)} \\
& =1+\frac{2}{3(1+\beta)}
\end{aligned}
$$




\section{Adiabatic Motion.}

201. Let us suppose the pressure, volume and temperature to change in such a way that no heat enters or leaves the gas. Then since $d Q=0$, we have from equation (400),

$$
N d \bar{E}+R N T \frac{d v}{v}=0
$$

or, on substituting for $\bar{E}$ from equation (407), and dividing by $R N T$,

$$
\frac{3}{2}(1+\beta) \frac{d T}{T}+\frac{d v}{v}=0
$$

Hence upon integration

$$
T v^{\frac{2}{3(1+\beta)}}=\text { constant }
$$

or again, since $T$ is proportional to $p v$,

or

$$
\begin{aligned}
p v^{1+\frac{2}{3(1+\beta)}} & =\text { constant } \\
p v^{\gamma} & =\text { constant }
\end{aligned}
$$

This is the general relation between pressure and volume in a motion of the gas in which no heat enters or leaves the gas-a type of motion which is known as "adiabatic."

Since $\beta$ cannot be negative, we see from equation (409) that in adiabatic motion an increase in $v$ is accompanied by a decrease in $T$, and vice versâ.

\section{Calculation of $\gamma$.}

202. Let us now examine more closely the values of the two specific heats, abandoning the simplifying assumption that the mean internal energy of a molecule bears a constant ratio to the mean energy of translation.

As in equation (160) the energy of a molecule may be regarded as the sum of the potential energy $V$ and of the energies of $n$ momentoids. The mean energy of each momentoid is by equation (265) equal to $\frac{1}{2} R T$. The total mean energy of a molecule is accordingly given by

$$
\bar{E}=\bar{V}+\frac{1}{2} n R T
$$

This gives, upon differentiation with respect to $T$, .

$$
\frac{d \bar{E}}{d T}=\frac{d \bar{V}}{d T}+\frac{1}{2} n R
$$

and the substitution of this in equations (402) and (404) leads to

$$
\begin{aligned}
& C_{v}=\frac{1}{J m} \frac{d \bar{V}}{d T}+\frac{1}{2} n \frac{R}{J m} \ldots \ldots \\
& C_{p}=\frac{1}{J m} \frac{d \bar{V}}{d T}+\left(\frac{1}{2} n+1\right) \frac{R}{J m}
\end{aligned}
$$


By division we obtain

$$
\gamma=1+\frac{2}{n+\frac{2}{R} \frac{d \vec{V}}{d T}}
$$

the quantity $\gamma$ denoting, as before, the ratio of the two specific heats.

203. In the case of hard elastic spheres, we have $\bar{V}=0$ and $n=3$, since the only energy of the spheres is their energy of translation, which is represented by three momentoids. Hence for a gas composed of such spheres,

$$
\gamma=1 \frac{2}{3}
$$

This is (except for a possible small error) the value of $\gamma$ which is observed for mercury, argon, and as far as is known, for all monatomic gases. We must not, however, infer that the molecules of these gases are hard elastic spheres. Each of these gases, when raised to incandescence, exhibits a spectrum consisting of thousands of distinct lines, and therefore is presumably capable of executing vibrations of thousands of distinct periods. If, as we believe, all the molecules of the gas are exactly similar, each molecule must be capable separately of executing vibrations of all these periods. In other words the kinetic and potential energies of a molecule, specified as a dynamical system through coordinates $\phi_{1}, \phi_{2} \ldots \phi_{8}$, must contain thousands of terms of the forms

$$
\begin{aligned}
& 2 L=a_{1} \dot{\phi}_{1}{ }^{2}+a_{2} \dot{\phi}_{2}{ }^{2}+\ldots+a_{8} \dot{\phi}_{8}{ }^{2} \\
& 2 V=b_{1} \phi_{1}{ }^{2}+b_{2} \phi_{2}{ }^{2}+\ldots+b_{8} \phi_{8}{ }^{2}
\end{aligned}
$$

and since the evidence of the spectrum shews that the vibrations of any single type are (very approximately) isochronous, the coefficients $a_{1} \ldots a_{8}$, $b_{1} \ldots b_{8}$ must be constants. Averaged throughout the motion we find, as usual, that the mean kinetic energy of any vibration is equal to the mean potential energy, and hence, throughout the gas,

$$
\overline{b_{1} \phi_{1}{ }^{2}}=\overline{a_{1} \dot{\phi}_{1}{ }^{2}}=\frac{1}{2}\left(\overline{a_{1} \dot{\phi}_{1}{ }^{2}+b_{1} \dot{\phi}_{1}{ }^{2}}\right)
$$

The quantity $\overline{a_{1} \dot{\phi}_{1}^{2}}$ is, however, double the mean energy of a momentoid. Hence each line in the spectrum of a gas denotes a contribution to the mean energy of each molecule, equal to double the mean energy of a momentoid.

From this it appears that the value of $n$ in equation (415), must be taken to be some thousands, and therefore that the value of $\gamma$ given by equation (415) would, so far as experimental observation goes, be indistinguishable from unity.

It has, however, already been mentioned that the value observed for $\gamma$ in the case of the monatomic gases is very approximately equal to $1 \frac{2}{3}$. A table will be given later shewing the observed values of $\gamma$ for a large number of 
gases and in no case is $\gamma$ equal to unity within the limits of experimental error. Our theory has, then, led to a result which is in flagrant opposition to experiment.

\section{Dissipation of Energy.}

204. An explanation is not difficult to find. The whole discussion has rested upon the Law of Equipartition of Energy, expressed by equation (161). Now in arriving at equation (161) it was assumed that the molecules of the gas together formed a conservative dynamical system, whereas the very fact of our observing spectral lines shews that the molecules are radiating energy into the ether, and therefore do not form a conservative system. The application of the Law of Equipartition or of equation (161) to the energy represented by spectral lines is therefore illegitimate. In the next chapter we shall apply the general dynamical methods of Chapter V. to the motion of a gas in which the conservation of energy does not hold, and shall return later to a mathematical investigation of the question of the ratio of the specific heats of such a gas.

205. We may conclude the present chapter by giving a brief forecast of the results of this investigation. The energy of those degrees of freedom which are represented by the lines of the spectrum are, it will be seen, subject to losses from two causes and to gain from one. In the first place there is the continual radiation into the surrounding ether. This results in a loss which may be referred to as $\alpha$. Secondly at each collision the vibrations of the molecule are disturbed, so that a collision may either increase or decrease the energy of a particular vibration. The collisions which occur during a specified small interval of time may therefore be supposed to result in a loss $\beta$ and a gain $\gamma$ to any particular degree of freedom. The total decrease in the energy of this degree of freedom may therefore be written symbolically as

$$
\alpha+\beta-\gamma,
$$

and when the energy remains steady, we must have

$$
\alpha+\beta-\gamma=0 \text {. }
$$

In the previous investigations, there has been no radiation so that $\alpha=0$, and in the normal or steady state $\beta=\gamma$. Equation (420) was therefore satisfied in this case by the solutions

$$
\alpha=0, \beta=\gamma \text {. }
$$


As regards the degrees of freedom represented by the spectral lines, we shall find that equation (420) is satisfied very approximately by taking

$$
\beta=0, \alpha=\gamma
$$

That is to say, the vibrations set up by collisions are no longer balanced by the transfer of energy back from these degrees of freedom to the translational energy, but by radiation into the ether.

\section{Mechanical Illustration.}

206. A simple analogy will probably elucidate the meaning of these solutions better than pages of discussion. Suppose that we have two vessels $A, B$ filled with gas and connected by a passage $C$. Suppose the vessels and

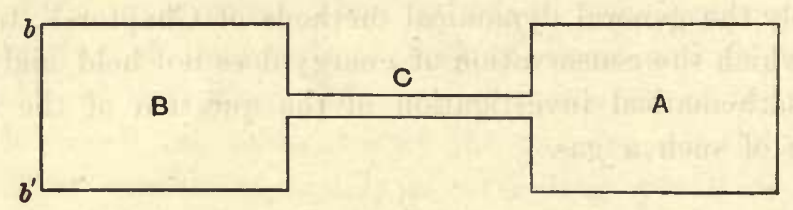

Fig. 12.

passage to be bounded by non-conducting walls, then the whole gas forms a conservative dynamical system, and in its steady or normal state, the mean energy of the molecules in $A$ is equal to the mean energy of the molecules in $B$-in other words the temperatures in $A, B$ are equal, being equalised by conduction through the passage $C$. The temperature in $A$ is analogous to the mean energy of one of the momentoids of translation, say $\frac{1}{2} m \overline{u^{2}}$, the temperature in $B$ is analogous to the mean-energy of a momentoid represented by one of the spectral lines, say $\frac{1}{2} \alpha \bar{\phi}^{2}$ in equation (417).

The equivalence of these two temperatures is the analogue of the solution $\beta=\gamma$ (equation (421)) in the case in which there is no dissipation of energy.

To represent the dissipation of energy, let us suppose that one of the walls $b b^{\prime}$ of the vessel $B$ is made a conductor of heat, the further face being kept at a zero temperature. The loss of heat which occurs by conduction through the wall $b b^{\prime}$ is now analogous to the loss of energy to which the momentoids represented by the spectral lines are subjected on account of the radiation into the ether. The analogy of the steady state represented by equation (422) is a state in which the gas in the chamber $B$ is almost entirely devoid of heat, so that the loss of energy by conduction through $b b^{\prime}$ is very small, and in which this loss is just counterbalanced by the supply of heat which reaches the gas in $B$ through the passage $C$. It is clear 
that if the passage $C$ is sufficiently narrow this last amount may be very small, and hence that the temperature of the gas in $A$ will remain perceptibly constant for a great time. It will be seen, then, reverting to the real gas, that the temperature of this gas can remain perceptibly constant for a great time if the analogy is a true one, and if the transfer of energy which has been represented by the transfer of heat through the passage can be shewn to be sufficiently slow. To examine this transfer will be the problem of Chapter IX. Meanwhile it is hoped that the analogy suggested in the present section, although of course not representing a step in the main argument, will be of service in making clearer the direction in which this main argument is tending. 


\section{CHAPTER VIII.}

\section{THE THEORY OF A NON-CONSERVATIVE GAS.}

\section{General Dynamics of Non-Conservative Systems.}

207. IT is proper to begin the study of the dynamics of a non-conservative gas by attempting to repeat, mutatis mutandis, the investigation which was carried out in Chapter V. for a conservative gas. In fact Chapter V. may be regarded as a study of the general equations of the dynamics of a conservative system, when the simplifying supposition is made that the dynamical system is an aggregate of a very great number of similar systems.

It might be thought at first sight that it would be an easy matter to repeat the former investigation, taking into account the dissipation of energy represented by an unknown general dissipation function. Unfortunately this is not so. In the former case the ultimate result happened to be entirely independent of the constitution of the molecules of the gas. In the present more general case in which the gas is not supposed to satisfy the conservation of energy, no ultimate general result of this kind can be obtained. It will therefore be found that no progress is possible which is at all comparable with that accomplished in the former case, but the fact that the possible progress is small would be no reason for neglecting the investigation.

\section{The Dissipation Function.}

208. As a preliminary to the general dynamical theory, it must be explained how it is that a gas in nature is adequately represented by a system of similar molecules and a dissipation function, and we must investigate the appropriate form for this dissipation function.

We shall assume that the complete dynamical system consists of the molecules of the gas and the ether by which these molecules are surrounded, and we shall therefore suppose that all energy lost to the molecules is energy gained to the ether. But in estimating this quantity there is a difficulty, for we do not know sufficient about the line of demarcation between ether and matter to be able to say at what stage precisely the energy is transferred from the one to the other. 
209. Happily there is no need to probe into this difficult question. The dynamics of those parts of the ether which do not contain any matter are, at any rate if we assume Maxwell's electrodynamical theory, sufficiently well understood. If $S_{1}, S_{2}$ are small surfaces surrounding the molecules, we know that at any point outside these surfaces we have, if $\mathbf{E ,} \mathbf{H}$ are the electric and magnetic forces,

$$
\operatorname{div} \mathbf{E}=0, \operatorname{div} \mathbf{H}=0
$$

The total energy, electrostatic and electromagnetic, inside any one of the surfaces, say $S_{1}$, is known to be

$$
\frac{1}{8 \pi} \iiint\left(P^{2}+Q^{2}+R^{2}+\alpha^{2}+\beta^{2}+\gamma^{2}\right) d x d y d z
$$

where $P, Q, R$ and $\alpha, \beta, \gamma$ are the components of electric and magnetic force respectively. The total flow of energy outwards across the surface $S_{1}$ is known, as shewn by Poynting*, to be

$$
\frac{1}{4 \pi} \iint[\mathbf{E H}] d S_{1}
$$

Now if $\xi_{1}, \xi_{2} \ldots$ are the generalised coordinates of position and velocity of the system inside $S_{1}$, we have at every point inside $S_{1}$

$$
\begin{aligned}
\operatorname{div} \mathbf{E} & =f\left(\xi_{1}, \xi_{2} \ldots\right) \ldots \ldots \ldots \ldots \ldots \ldots \ldots \ldots(426), \\
\operatorname{div} \mathbf{H} & =\phi\left(\xi_{1}, \xi_{2} \ldots\right) \ldots \ldots \ldots \ldots \ldots \ldots \ldots \ldots \ldots \ldots \ldots \ldots \ldots \ldots
\end{aligned}
$$

where $f$ and $\phi$ are functions of the coordinates which are theoretically calculable, the latter being almost certainly zero. From equations (423), (426) and (427) we can calculate $\mathbf{E}$ and $\mathbf{H}$ as functions of all the coordinates of the various systems, and from these we can calculate the Poynting flux given by expression (425), also as a function of those coordinates.

Let us, then, regard as the energy of the system inside $S_{1}$ the total material energy of this system, if any, plus the total electric energy, electrostatic and electromagnetic, associated with the ether inside $S_{1}$, this latter being given by expression (424). Then we may legitimately regard expression (425) as a dissipation function of the system inside $S_{1}$, and the motion of the system will be deducible from a knowledge of the forms of the energy function and of the dissipation function just defined. Taking this view of the system, the interaction between matter and ether is represented by the existence of the dissipation function, which may be interpreted physically as evidence of the radiation of electromagnetic waves and "pulses" into the ether; and the interaction of the various molecules inter se is represented by the fact that the energy and dissipation functions of system 1 depend not only on the coordinates of system 1 , but also on those of systems $2,3 \ldots$.

* J. H. Poynting, Phil. Trans. 1884, Ir. p. 343. J. J. Thomson, Recent Researches, p. 308. 
If, during the motion of the molecules, two or more of the surfaces $S_{1}, S_{2} \ldots$ intersect, an obvious slight modification must be made in the conventions just stated. Instead of integrating expression (425) over the surfaces separately to obtain the dissipation function, we must integrate only over the single closed surface formed by such as intersect. Similarly instead of integrating expression (424) throughout the volumes enclosed by the surfaces separately to obtain the energy, we integrate only throughout the single connected volume formed by such as intersect. In this way we fail to obtain the energy and dissipation functions as the sums of contributions from individual molecules, and obtain these functions only in the aggregate, a limitation which is of no importance as regards the main results to be obtained. If, on the other hand, we are willing to assume that there is a definite fixed line of demarcation between matter and ether, and that two portions of matter cannot occupy the same space, then these difficulties are obviated and we can associate definite terms of the energy and dissipation functions with each molecule.

\section{The General Dynamical Theory.}

210. We can now, in the main, follow the method of Chapter V. As that method was based on Liouville's Theorem $(\$ 72)$, we must begin the present discussion by investigating a theorem to replace this when the conservation of energy does not apply.

As in $\S 72$, we imagine a general dynamical system, specified by the $n$ coordinates $q_{1}, q_{2} \ldots q_{n}$ and the corresponding momenta $p_{1}, p_{2} \ldots p_{n}$, and we suppose all possible configurations of this system represented in a space of $2 n$ dimensions corresponding to the $2 n$ variables

$$
p_{1}, p_{2} \ldots p_{n}, q_{1}, q_{2} \ldots q_{n}
$$

If $E$ is the energy of the system expressed as a function of the $q$ 's and $\dot{q}^{\prime} \mathrm{s}$, we have

$$
p_{8}=\frac{\partial E}{\partial \dot{q}_{8}}
$$

and the equations of motion of the system are

$$
\begin{aligned}
& \frac{\partial q_{8}}{\partial t}=\frac{\partial E}{\partial p_{8}} \ldots \ldots \ldots . . . . \\
& \frac{\partial p_{8}}{\partial t}=-\frac{\partial E}{\partial q_{8}}-\frac{\partial F}{\partial \dot{q}_{8}}
\end{aligned}
$$

where $E$ is now expressed as a function of $p_{1}, p_{2} \ldots p_{n}, q_{1}, q_{2} \ldots q_{n}$, and $F$ is the dissipation function, expressed as a function of

$$
q_{1}, q_{2} \ldots q_{n}, \quad \dot{q}_{1}, \dot{q}_{2} \ldots \dot{q}_{n} .
$$

These, therefore, are the equations of motion of the fluid in the generalised space, and the elimination of the time from these equations will lead to the equations of the paths or stream lines along which the fluid moves. 
The hydrodynamical equation of continuity in the generalised space (equation $(130)$ ) is

$$
\frac{D \rho}{D t}+\rho \sum_{s=1}^{8=n}\left(\frac{\partial \dot{p}_{8}}{\partial p_{8}}+\frac{\partial \dot{q}_{8}}{\partial q_{8}}\right)
$$

where, as before, $D / D t$ denotes differentiation following an element of fluid in its motion.

From equations (4:30) and (431) we obtain

$$
\frac{\partial \dot{p}_{8}}{\partial p_{8}}+\frac{\partial \dot{q}_{8}}{\partial q_{8}}=-\frac{\partial^{2} F}{\partial p_{8} \partial \dot{q}_{8}}
$$

so that equation (4.32) becomes

$$
\frac{D \rho}{D t}=\rho \sum_{8=1}^{8=n} \frac{\partial^{2} F}{\partial p_{8} \partial \dot{q}_{8}}
$$

In the special case in which $F=0$, this reduces to the simple form $D \rho / D t=0$, which is, of course, the expression of Liouville's Theorem. Equation (433) therefore expresses the general theorem which must replace that of Liouville when the conservation of energy does not hold.

211. In the former case, in which we had $F=0$, the procedure was one of extreme simplicity. Since we had $D \rho / D t=0$, it was clear that a distribution of fluid which was of uniform density at one instant would remain of uniform density throughout all time. Hence a generalised space filled with fluid of uniform density supplied a basis of investigation which was independent of the time. It was therefore possible to prove, by taking a census of the different points in the generalised space, that all except an infinitesimal fraction of the space represented systems for which certain propositions of the Kinetic Theory-equipartition of energy, etc.-were true, and hence that these propositions would be true (except for a theoretical infinitesimal probability of error) for a gas selected at random.

In the present instance this method fails because the space filled initially with homogeneous fluid does not supply a basis of calculation which is independent of the time. It may be (and, as will be seen, actually is) the case that the fluid tends to crowd just to those parts of the space which represent systems in which the former propositions are not true. To investigate these questions, we commence by seeking for a basis which shall be independent of the time.

212. For the sake of brevity, let us write

$$
\sum_{s=1}^{s=n} \frac{\partial^{2} F}{\partial p_{8} \partial \dot{q}_{s}}=\Delta F
$$

so that we can write equation (433) in the form

$$
\frac{D \rho}{D t}=\rho \Delta F
$$


We now have

$$
\begin{aligned}
\Delta F & =\sum_{s=1}^{s=n} \frac{\partial^{2} F}{\partial p_{s} \partial \dot{q}_{s}} \\
& =\sum_{s=1}^{s=n} \sum_{t=1}^{=n} \frac{\partial^{2} F}{\partial p_{s} \partial p_{t}} \frac{\partial p_{t}}{\partial \dot{q}_{s}} \\
& =\sum_{s=1}^{s=n} \sum_{t=1}^{t=n} \frac{\partial^{2} F}{\partial p_{s} \partial p_{t}} \frac{\partial^{2} E}{\partial \dot{q}_{s} \partial \dot{q}_{t}}
\end{aligned}
$$

by equation (429), expressing $\Delta F$ as a function of the $q$ 's only. Since $E$ and $F$ are both positive for all values of the variables it follows that $\Delta F$ is positive throughout the space, and hence from equation (435) it is clear that as we follow the motion of any element, $\rho$ continually increases. Also, as we follow the motion $E$ decreases, so that the motion of the points in our imaginary space is one of concentration about minimum values of $E$ and, in particular, about $E=0$.

213. The rate of change in $E$ as we follow any element in its motion is of course

$$
\begin{aligned}
& \frac{D E}{D t}=\sum_{s=1}^{s=n}\left(\frac{\partial E}{\partial q_{s}} \frac{\partial q_{s}}{\partial t}+\frac{\partial E}{\partial p_{s}} \frac{\partial p_{s}}{\partial t}\right) \\
& =-\sum_{s=1}^{s=n} \frac{\partial E}{\partial p_{s}} \frac{\partial F}{\partial \dot{q}_{s}} \text {, by equations (430) and } \\
& =-\sum_{s=1}^{s=n} \frac{\partial E}{\partial \dot{q}_{s}} \frac{\partial F}{\partial p_{s}} \\
& =-\sum_{s=1}^{s=n} p_{\delta} \frac{\partial F}{\partial p_{8}} \\
& =-2 F
\end{aligned}
$$

since $F$ is a homogeneous quadratic function of the momenta $p_{1}, \ldots p_{n}$. From equations (435) and (437) it appears that as we follow the motion of any element

$$
\frac{D \rho}{D E}=-\rho \frac{\Delta F}{2 F}
$$

and $D$ may now be regarded as the symbol of differentiation along a stream line in the imaginary space. The equation just obtained has therefore the integral

$$
\rho=\rho_{0} e^{-\int \frac{\Delta F}{2 F} d E}
$$

where the integration is taken along any stream line, and $\rho_{0}$ is the value of $\rho$ at the lower limit of integration.

214. Equation (439) is an equation between $\rho$ and the coordinates in the imaginary space: it does not involve the time. Hence it follows that provided the initial distribution of points in the space is such as to satisfy 
equations (439) at every point, the motion of these points will be "steady motion" in the hydrodynamical sense. To obtain such a motion, then, we may assign arbitrary densities over any one of the loci $E=$ constant. The density of distribution of points is then uniquely determined throughout the whole space, and is given by equation (439). The space, filled with fluid in this way, supplies what we require, namely, a basis of investigation which is independent of the time.

By distributing the arbitrary densities in different ways, we shall obtain the various distributions of points in the imaginary space which are appropriate to the discussion of different problems, i.e., to the discussion of gases about which various data are given. For instance, suppose we know that a gas has cooled, undisturbed by external agencies, from some higher temperature at which its energy was $E_{0}$, and that nothing further as to its past history is known, then we may appropriately suppose the imaginary space filled with a distribution of points such that the density over $E=E_{0}$ is constant, say equal to unity. Let the present energy be $E_{1}$, then what we wish to know is the density of points over $E=E_{1}$, and this by equation (439) is given by

$$
\rho=e^{\int_{E_{1}}^{E_{0}} \frac{\Delta F}{2 F} d E}
$$

Application to a system with an infinite number of Degrees of Freedom.

215. We now introduce the simplifying assumption that the system consists of a very great number of parts (e.g., molecules) so that $n$, the number of momenta, is very great. Let $\overline{p^{2}}$ denote the mean value of

$$
p_{1}{ }^{2}, p_{2}{ }^{2} \ldots p_{n}{ }^{2}
$$

at any point in the imaginary space. Then in general $E$ is of the same order of magnitude as $n \overline{p^{2}}$ while $\Delta F / 2 F$ is obviously of the order of $1 / \overline{p^{2}}$. Hence we can write

$$
\frac{1}{n} \int_{E_{1}}^{E_{0}} \frac{\Delta F}{2 F} d E=\Phi
$$

and $\Phi$ will remain finite when $n$ becomes infinite, being, as we have seen, always positive.

The distribution of density in the region $E=E_{1}$ is now, by equation (440),

$$
\rho=e^{n \Phi}
$$

There is therefore a point (or series of points) of maximum density obtained by making $\Phi$ a maximum, and the density here is infinitely greater than that elsewhere. We have now to examine in what way the density falls off as we recede from such a point, so as to determine whether the whole aggregate of points for which $E=E_{1}$ may be regarded as being concentrated about this point (or series of points). 
216. Let $\Phi_{0}$ denote a maximum value of $\Phi$, and suppose that this value occurs at the point $p_{1}^{\prime}, p_{2}^{\prime} \ldots q_{n}{ }^{\prime}$; then in the immediate neighbuurhood of this point we can expand $\Phi$ in the form

$$
\begin{aligned}
\Phi= & \Phi_{0}+\left(p_{1}-p_{1}^{\prime}\right) \frac{\partial \Phi}{\partial p_{1}}+\ldots \\
& +\frac{1}{2}\left(p_{1}-p_{1}\right)^{2} \frac{\partial^{2} \Phi}{\partial p_{1}^{2}}+\ldots
\end{aligned}
$$

where the differential coefficients are all evaluated at the point at which $\Phi=\Phi_{0}$. Since $\Phi$ is, by hypothesis, a maximum so long as $E$ is kept constant, it follows that over the surface $E=E_{1}$,

$$
\left(p_{1}-p_{1}{ }^{\prime}\right) \frac{\partial \Phi}{\partial p_{1}}+\ldots+\left(q_{n}-q_{n}{ }^{\prime}\right) \frac{\partial \Phi}{\partial q_{n}}=0
$$

Hence the region for which $E=E_{1}$ and for which $\Phi$ lies between $\Phi_{0}$ and an adjacent value $\Phi_{1}$ is bounded by

$$
\left(p_{1}-p_{1}{ }^{\prime}\right)^{2} \frac{\partial^{2} \Phi}{\partial p_{1}^{2}}+2\left(p_{1}-p_{1}{ }^{\prime}\right)\left(p_{2}-p_{2}{ }^{\prime}\right) \frac{\partial^{2} \Phi}{\partial p_{1} \partial p_{2}}+\ldots=-2\left(\Phi_{0}-\Phi_{1}\right) \ldots
$$

When $p_{1}-p_{1}{ }^{\prime}, p_{2}-p_{2}{ }^{\prime}, \ldots$ are small enough, terms of degree higher than the second in these quantities may be neglected, so that the equation is that of a small closed boundary, an "ellipsoid" of $2 n$ dimensions. The volume of this ellipsoid is a Dirichlet integral of which the value is found to be

$$
\frac{(2 \pi)^{n}}{\Gamma(1+n)}\left(\Phi_{0}-\Phi_{1}\right)^{n} \Delta^{-\frac{1}{2}}
$$

where

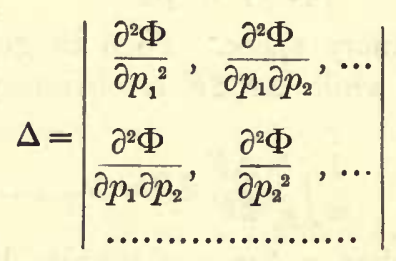

The equation of the plane $E=E_{1}$ is equation (444), and the intersection of this plane with the $2 n$ dimensional ellipsoid (445) determines a $2 n-1$ dimensional ellipsoid. This latter ellipsoid contains all points for which $E=E_{1}$ and $\Phi_{1}<\Phi<\Phi_{0}$. To find the volume of this $2 n-1$ dimensional ellipsoid, we must first find the lengths of its principal axes.

A $2 n$ dimensional sphere of radius $r$ intersects the $2 n$ dimensional ellipsoid (445) in a cone of which the equation is

$$
\left(p_{1}-p_{1}^{\prime}\right)^{2}\left(\frac{\partial^{2} \Phi}{\partial p_{1}^{2}}+\frac{2\left(\Phi_{0}-\Phi_{1}\right)}{r^{2}}\right)+2\left(p_{1}-p_{1}{ }^{\prime}\right)\left(p_{2}-p_{2}{ }^{\prime}\right) \frac{\partial^{2} \Phi}{\partial p_{1} \partial p_{2}}+\ldots=0
$$


If $r$ is the length of a principal axis of the section, the plane (444) must be a tangent plane to this cone, and the condition for this is the vanishing of the bordered determinant

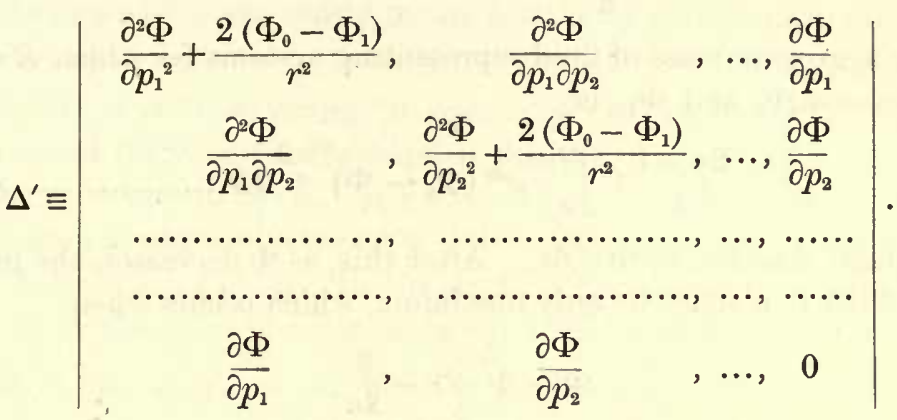

The equation $\Delta^{\prime}=0$ accordingly gives the lengths of the principal semiaxes.

In the expansion of $\Delta^{\prime}$, the coefficient of the highest power of $\frac{1}{r^{2}}$ is

$$
\left[2\left(\Phi_{0}-\Phi_{1}\right)\right]^{2 n-1} \Sigma\left(\frac{\partial \Phi}{\partial p_{1}}\right)^{2} \frac{\partial^{2} \Phi}{\partial p_{2}{ }^{2}} \frac{\partial^{2} \Phi}{\partial p_{3}{ }^{2}} \cdots \frac{\partial^{2} \Phi}{\partial q_{n}{ }^{2}},
$$

where the summation extends to all the variables. The term independent of $\frac{1}{r^{2}}$ is of course obtained from $\Delta^{\prime}$ by omitting terms containing $\frac{1}{r^{2}}$, leading to a new determinant, say $\Delta^{\prime \prime}$. Hence if we denote the product of the semiaxes by $\Pi(r)$, we have

$$
\left[2\left(\Phi_{0}-\Phi_{1}\right)\right]^{2 n-1} \Sigma\left(\frac{\partial \Phi}{\partial p_{1}}\right)^{2} \frac{\partial^{2} \Phi}{\partial p_{2}{ }^{2}} \frac{\partial^{2} \Phi}{\partial p_{3}{ }^{2}} \cdots \frac{\partial^{2} \Phi}{\partial q_{n}{ }^{2}}=\{\Pi(r)\}^{2} \Delta^{\prime \prime}
$$

The volume of the $2 n-1$ dimensional ellipsoid is now found (as in $\S 46$ ) to be

$$
\frac{\pi^{\frac{2 n-1}{2}}}{\Gamma\left(1+\frac{2 n-1}{2}\right)} \Pi(r) \text {. }
$$

If we denote this volume by $\Omega$, and substitute in the value just obtained for $\Omega$ the value of $\Pi(r)$ given by equation (448), we obtain an equation of the form

$$
\Omega=C\left(\Phi_{0}-\Phi_{1}\right)^{\frac{2 n-1}{2}},
$$

where $C$ does not involve $\Phi_{1}$, being in fact given by

$$
C=\frac{(2 \pi)^{\frac{2 n-1}{2}}}{\Gamma\left(\frac{2 n+1}{2}\right)}\left[\frac{\sum\left(\frac{\partial \Phi}{\partial p_{1}}\right)^{2} \frac{\partial^{2} \Phi}{\partial p_{2}{ }^{2}} \frac{\partial^{2} \Phi}{\partial p_{3}{ }^{2}} \cdots \frac{\partial^{2} \Phi}{\partial q_{n}{ }^{2}}}{\Delta^{\prime \prime}}\right]^{\frac{1}{2}}
$$


By differentiation, the volume for which $E=E_{1}$, while $\Phi$ lies between $\Phi_{1}$ and $\Phi_{1}+d \Phi$, will be

$$
\frac{2 n-1}{2} C\left(\Phi_{0}-\Phi_{1}\right)^{\frac{2 n-3}{2}} d \Phi \text {. }
$$

Hence the aggregate mass of fluid representing systems for which $E=E_{1}$ and $\Phi$ lies between $\Phi_{0}$ and $\Phi_{1}$, is

$$
\frac{2 n-1}{2} C \int_{\Phi_{1}}^{\Phi_{0}} e^{n \Phi}\left(\Phi_{0}-\Phi\right)^{\frac{2 n-3}{2}} d \Phi
$$

The integrand vanishes at $\Phi=\Phi_{0}$. After this, as $\Phi$ decreases, the integrand increases until it reaches its only maximum, which occurs when

$$
\Phi_{0}-\Phi=1-\frac{3}{2 n}
$$

and then decreases. In the limit when $n=\infty$, this maximum is given by

$$
\Phi=\Phi_{0}-1
$$

and if this value lies between $\Phi_{0}$ and $\Phi_{1}$, the whole value of the integral (450) is given by contributions from the immediate neighbourhood of this maximum, and is equal to

$$
\frac{C \Gamma\left(\frac{2 n-1}{2}\right)}{n^{\frac{2 n-1}{2}}} e^{-n \Phi_{0}}
$$

This result, however, only holds subject to the condition that we may neglect terms of order higher than the second in equation (443).

217. Assuming for the moment that this condition is satisfied, our result shews that all the fluid for which $E=E_{1}$, and which is in the neighbourhood of the point of maximum density $\Phi=\Phi_{0}$ may be supposed to lie within the boundary defined by equation (445). In other words the fluid is seen to flow into small clusters of stream lines, a cluster surrounding each maximum value of $\Phi$. All except an infinitesimally small proportion of the fluid for which $E^{\prime}=E_{1}$ will lie in one or other of these clusters.

The amount of fluid surrounding the maximum $\Phi=\Phi_{0}$ is given by expression (452). In comparing the amounts surrounding two different maxima $\Phi_{0}$ and $\Phi_{0}{ }^{\prime}$, the ratio of the two factors $e^{n \Phi_{0}}, e^{n \Phi_{0}{ }^{\prime}}$ will of course be infinite or zero according as $\Phi_{0}-\Phi_{0}^{\prime}$ is positive or negative, but it is unfortunately impossible to determine the ratio of the remaining factors, for the ratio of the two $C$ 's (equation (449)) may be infinite or zero. We therefore see that in general the ratio of two expressions of the form of (452) will be either infinite or zero, but it is impossible to determine which. In other words, we can see that all except an infinitesimally small amount of the fluid will be in a single cluster, but it is impossible to identify the particular cluster. 
218. An exception to the statement that all the fluid will be in a single cluster occurs when there are a number of clusters which are similar in every respect, so that expression (452) is identical for all. This will, for instance, occur in the case of a gas, owing to the possibility of interchanging the rôles of the different molecules.

219. We must now return to equations (443) and (445), and examine whether or not the condition is satisfied that we may neglect terms of degree higher than the second in $\left(p_{1}-p_{1}{ }^{\prime}\right)$, etc. Upon the assumption that we may do so, we have found the value of $\Phi-\Phi_{0}$ at the boundary of a cluster to be equal to 1 (equation (451)). Let us suppose that the corresponding value of $\left(p_{1}-p_{1}{ }^{\prime}\right)$ is of the order of magnitude $\delta p$, then the first term which has been neglected is $\left(p_{1}-p_{1}^{\prime}\right)^{3} \frac{\partial^{3} \Phi}{\partial p_{1}^{3}}$ or $(\delta p)^{3} \frac{\partial^{3} \Phi}{\partial p_{1}^{3}}$. Failing definite knowledge it is natural to suppose that $\frac{\partial^{3} \Phi}{\partial p^{3}}$ is of the order of magnitude of $\frac{1}{(\delta p)^{3}}$, and hence that the neglected term is of the same order of magnitude as 1 . In other words, the neglect of the remaining terms in equation (445) cannot be justified.

But this only affects the detail of the result we obtain : the main result remains. The value $\Phi_{0}$ being still a maximum for $\Phi$, the locus $\Phi=\Phi_{1}$ will surround the point $\Phi_{0}$, although it will only be ellipsoidal when $\Phi_{0}-\Phi_{1}$ is very small. The integral in expression (450) must be replaced by an integral of the form

$$
\int_{\Phi_{1}}^{\Phi_{0}} e^{n \Phi} f\left(\Phi_{0}-\Phi_{1}\right) d \Phi
$$

in which $f(x)$ is an unknown, but always positive, function of $x$, which becomes identical with $x^{\frac{2 n-3}{2}}$ in the neighbourhood of $x=0$. The integrand in this integral will, as before, have a maximum, and the integral will derive its whole value from the contributions supplied by values of $\Phi$ in the neighbourhood of this maximum. Also, as before, the value of the integral involves $\Phi_{0}$ merely through the factor $e^{n \Phi_{0}}$. Again, then, it appears that the fluid will flow into clusters surrounding maximum values of $\Phi$, and the strength of such a cluster will be proportional jointly to $e^{n \Phi}$ and to another factor. All except an infinitesimal fraction of the fluid will be in a single cluster, or, in cases such as that contemplated in $\S 218$, family of similar clusters, but as already explained, it is impossible to identify this cluster or family of clusters by general arguments of the kind which have so far been used.

220. Under the special assumption that we may neglect terms of degree higher than the second in equation (443), we have at any point inside a cluster (equation (445)),

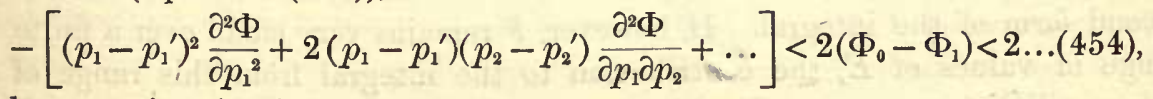
by equation (451). 
In the most general case it is obvious that the quadratic expression on the left hand is finite. The expression is, however, by hypothesis, positive for all values of $\left(p_{1}-p_{1}{ }^{\prime}\right) \ldots$ and as it contains the $2 n$ variables $p_{1}-p_{1}^{\prime}, \ldots q_{n}-q_{n}{ }^{\prime}$, it may be expressed as a sum of $2 n$ squares of linear functions of these variables. When $n$ is infinite, therefore, each square will be vanishingly small, so that $p_{1}-p_{1}^{\prime}, p_{2}-p_{2}^{\prime}, \ldots$ will be infinitesimal. We may say, then, that inside any cluster the coordinates $p_{1}, p_{2} \ldots$ of any point are indistinguishable from the coordinates $p_{1}^{\prime}, p_{2}^{\prime} \ldots$ of the corresponding point on the central line of the cluster.

221. The analysis has suppased the system to have started from an initial value of the energy $E=E_{0}$, and has, moreover, supposed all configurations for which $E=E_{0}$ to have been equally probable as the starting point. In nature it is not easy to find any value of $E_{0}$ which shall represent the conditions of the problem. When the system under consideration is a gas, we may probably assert with confidence that the gas has cooled from some higher temperature at which its energy has some value $E_{0}$, but we cannot say that at this temperature all configurations for which $E=E_{0}$ were equally likely. For obviously if it had previously cooled from some still higher temperature this statement would not be true. Again, the value $E_{0}=\infty$ does not supply a solution of the difficulty, for in taking this value we should not only have to suppose that the gas has cooled from a temperature in which it probably could not exist as a gas, but we should also have to suppose that it had cooled undisturbed throughout infinite time.

222. An escape from this difficulty is found as soon as we examine the way in which our work has depended on $E_{0}$. It will be seen that $E_{0}$ has entered only as a limit of integration in the definition of $\Phi$, namely (from equation (441)),

$$
\begin{aligned}
\Phi & =\frac{1}{n} \int_{E_{1}}^{E_{0}} \frac{\Delta F}{2 F} d E \\
& =-\frac{1}{n} \int_{E_{1}}^{E_{0}} \Delta F d t
\end{aligned}
$$

In this equation the integral has to be taken tentatively along all stream lines starting from $E=E_{0}$, and the course of the cluster or family is determined by the maximum values of $\Phi$. The integrand contains a factor $F$ in the denominator. From its nature $F$ cannot be negative, and in general will not vanish. If at a special point $F$ does vanish, then the integrand in the first integral becomes infinite, but the value of the integral taken over any part of the path which includes this point remains finite, because so long as $F=0$ we have also $d E=0$. The same result is of course obvious from the second form of the integral. If, however, $F$ remains very small over a finite range of values of $E$, the contribution to the integral from this range of values will be very great. In this case the definition of $\Phi$, for values of $E$ 
below a finite range of small values of $F$, becomes approximately independent of the upper limit of integration, so that the stream lines on which $\Phi$ is a maximum become identical for all such upper limits. Thus so soon as a value of the energy is reached so small that it is possible for $F$ to remain very small over a large range of values of $E$, we see that the positions of the clusters tend to become independent of their starting points. In other words, all clusters tend to combine into a single cluster; or again, all stream lines tend to combine into a single stream line.

The values of the energy at which the condition just stated is satisfied, are the only values which are of much importance in nature. They correspond to temperatures at which a substance can remain in appreciably the same state for a considerable interval of time; and in considering this question it must be borne in mind that the unit of time appropriate for comparison is the interval between successive collisions of the same molecules. We have already seen $(\S 8$, p. 8$)$ that under normal conditions this interval is comparable with $10^{-10}$ seconds. And for obvious reasons the state of a system which changes appreciably in $10^{-10}$ seconds is not of much interest to us.

When the condition in question is satisfied, the system may be said to be in an "approximately steady state."- It is then infinitely probable that it will be represented by a point on the single cluster (or family of clusters) just discussed. In this case it may be said to be in the "normal state," this state being analogous to the normal state previously found for a conservative gas.

223. It has already been assumed that the system possesses an infinite number of degrees of freedom. The application of the results must therefore be to "Statistical Mechanics," rather than to dynąmical problems of the ordinary type. Leaving out of mention the infinitesimal probability of error, it being understood, for reasons explained in $\S 104$, that this is inherent to any problem of statistical mechanies, it may be said that we have found that of our non-conservative systems, started from unknown configurations, all will, by the time a sufficiently low value of the energy has been reached, be represented in our generalised space by points on a single cluster or family of clusters.

In the case of a gas it is obvious that we must take the second alternative, and say "family of clusters," rather than "single cluster." For, as has already been pointed out in $\S 218$, by interchanging the rôles of any two molecules we get two distinct clusters which are identical in so far as the elements which are of importance in the dynamical problem are concerned. In other ways, too, it is clear that we shall get further distinct clusters which are identical as regards the important elements of the problem. Let $M$ be the number of clusters in any such family and $S$ the 
strength of each, given by an expression of the form of (453). Then the strength of the family of clusters is MS. Comparing two families of clusters we have already seen that the ratio of the two $S$ 's will be infinite, so that whether or not the ratio of the two $M$ 's is infinite, the ratio of the $M S$ 's is, in general, infinite. Thus all the fluid belongs to a single family. It is clear that systems in all the $M$ clusters have the same statistical specification.

224. Hence we may sum up as follows:

Out of a number of systems which have a given value for the energy, this value being sufficiently low, and have had their energy reduced from higher values by dissipation, the systems meanwhile being unaffected by external disturbance, all except an infinitesimal fraction will have the same statistical specification.

And as regards physical interpretation:

If nothing is known about a gas except that it has cooled undisturbed from some higher temperature, and is now at such a temperature that its changes of state are not very rapid (by "very rapid" being meant comparable with $10^{-10}$ seconds), then it is infinitely probable that it will have a definite statistical specification, which will depend solely on its present energy.

The way in which this specification has, in theory, been arrived at, shews how profoundly different it must be from that arrived at on the assumption of conservation of energy, namely the specification which leads to equipartition.

\section{Pripcipal and Subsidiary Temperatures. \\ Definition of Subsidiary Temperatures.}

225. The exact form of a law of distribution of coordinates possesses interest in itself from the point of view of abstract mathematics, but as regards utility it will be seen that its only importance is that it enables us to calculate the mean values of different functions of the coordinates, averaged throughout the gas. Of these functions of the coordinates, only one class of function is of much importance, namely the class which represents the whole or parts of the energy.

Let us suppose, as in $\S 77$, that $L$, the kinetic energy of a single molecule, expressed as a function of its coordinates, is reduced to a sum of squares in the form

$$
2 L=m u^{2}+m v^{2}+m w^{2}+c_{1} \eta_{1}{ }^{2}+c_{2} \eta_{2}{ }^{2}+\ldots \ldots \ldots \ldots \ldots . .(456),
$$

where $m$ is the mass of the molecule, $u, v, w$ are as usual the velocities of its centre of gravity in space, and $\eta_{1}, \eta_{2} \ldots$ are generalised momentoids. Equation (456) expresses the kinetic energy as the sum of contributions from 
momentoids. In the case of a conservative gas, it was shewn that if $\overline{m u^{2}}$, $\overline{c_{1} \eta_{1}^{2}} \ldots$ denoted the mean values of $m u^{2}, c_{1} \eta_{1}{ }^{2} \ldots$ averaged over all the molecules of the gas, then (equation (219))

$$
\overline{m u^{2}}=\overline{m v^{2}}=\overline{m w^{2}}=\overline{c_{1} \eta_{1}^{2}}=\ldots=R T .
$$

In the present case, we could, if our law of distribution were known, calculate the values of $\overline{m u^{2}}, \overline{c_{1} \eta_{1}{ }^{2}}$, etc. It is obvious that we should have $\overline{m u^{2}}=\overline{m v^{2}}=\overline{m w^{2}}$, and we may as before put each quantity equal to $R T$, these equations defining $T$. We shall also put $\overline{c_{1} \eta_{1}{ }^{2}}=R \tau_{1}, \overline{c_{2} \eta_{2}{ }^{2}}=R_{\tau_{2}} \ldots$, etc., these equations defining $\tau_{1}, \tau_{2} \ldots$ It will be convenient now to speak of $T$ as the principal temperature, and of $\tau_{1}, \tau_{2} \ldots$ as subsidiary temperatures. The temperatures are accordingly defined by

$$
\frac{\overline{m u^{2}}}{T}=\frac{\overline{m v^{2}}}{T}=\frac{\overline{m w^{2}}}{T}=\frac{\overline{c_{1} \eta_{1}^{2}}}{\tau_{1}}=\frac{\overline{c_{2} \eta_{2}{ }^{2}}}{\tau_{2}}=\ldots=R
$$

226. The information which has been obtained as to the law of distribution of coordinates admits of easy interpretation in terms of principal and subsidiary temperatures. The result is as follows. If we start an infinite number of gases, each with energy $E_{0}$, these gases being distributed with uniform density throughout the part of the generalised space which corresponds to energy $E_{0}$, then by the time the energy has been reduced to $E_{1}$, all except an infinitesimal fraction of the whole will have the same principal and subsidiary temperatures.

227. We can conveniently represent the temperatures of a gas in a generalised space, the coordinates in this space being $T, \tau_{1}, \tau_{2} \ldots$ Obviously only those parts of the space are needed in which every coordinate is positive. The motion of a gas can be represented by the motion of a point in this generalised space. Our theorem now shews that of an infinite number of representative points which start from the same point, all except an infinitesimal fraction pursue the same course.

Of the representative points for the system of gases, starting with any energy $E_{0}$, the systems being as we have assumed throughout equally distributed throughout the original generalised space, all except an infinitesimal fraction will, it is true, start from a point in the line

$$
T=\tau_{1}=\tau_{2}=\ldots
$$

on which there is equipartition of energy. It is however obvious that if we had considered only those systems of which the representative points started from the most general point $\left(T, \tau_{1}, \tau_{2} \ldots\right)$, the result of the last chapter could have been obtained in exactly the same way. It will therefore be seen that the whole space can be mapped out into stream lines, in such a way that when a representative point is once moving on a stream line, it is 
infinitely probable that it will remain on that same stream line throughout its motion. Obviously each stream line in this space represents a family of clusters in the former space. Unfortunately for the simplicity of treatment it is no longer true, as it was in the previous case, that two stream lines cannot intersect.

The result stated in the last section can now be expressed by the statement that when the energy is so small that there is very little dissipation of energy (or, more precisely, when a value of $E$ is reached so small that it is possible for $F$ to remain very small throughout a finite range of values for $E$ ), then the stream lines in the new generalised space tend to combine into a single stream line. And of the gases which have cooled from an unknown higher temperature, all except an infinitesimal fraction, will be represented by points on this stream line.

\section{The Normal State for a Non-Conservative Gas.}

228. When a gas is represented by a point on the single stream line just found we shall say that the gas is in the normal state. This state is obviously analogous to the normal state previously found for a conservative gas. For as we have just seen, if the conditions for a normal state to be possible are satisfied, it is infinitely probable that a gas selected at random from gases which have cooled from higher temperatures, will be in the normal state, and, in general, gases which are not in the normal state tend towards that state. And the conditions necessary for a normal state to be possible are that it shall be possible for $F^{\prime}$ to remain very small throughout a finite range of $E$ : in other words, that the rate of cooling shall be very slow.

In nature, this condition is fulfilled in the case of a gas in the ordinary state, which is not radiating energy to a perceptible extent, e.g., in the case of the air of the atmosphere. An investigation of the mechanism by which it comes about that the condition is fulfilled is given in the next chapter. At present we are content with the knowledge that a gas of this kind is in a definite normal state, which is entirely different from the normal state in a conservative gas, in which the energy of each momentoid is the same.

We conclude this part of the subject by discussing two mechanical illustrations of the theory which has been developed in this chapter.

\section{The example from $\S 206$.}

229. In the mechanical illustration suggested in $\S 206$, it was pointed out that the mean temperatures in the vessels $A$ and $B$ might be supposed to represent energies of two momentoids, of which the latter was affected by 
a dissipation function (represented by conduction of heat through the wall $b b^{\prime}$ ), while the former was not affected by a direct dissipation function but lost

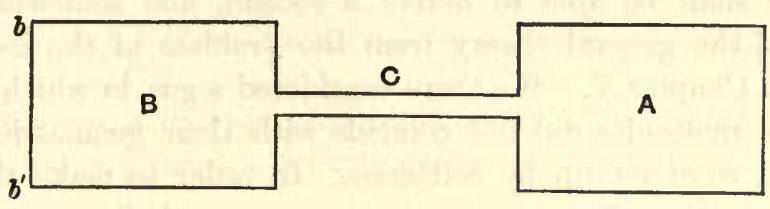

Fia. 13.

energy by transfer represented by conduction through the channel $C$. We may, therefore, suppose the actual physical temperatures of $A$ and $B$ to correspond to the principal and subsidiary temperatures, say $T$ and $\tau$, of a gas which has only one subsidiary temperature.

From a general knowledge of physics we can see what will ultimately happen to this system. According as $T$ is greater or less than $\tau$ at any instant, there will be conduction from $A$ to $B$ or from $B$ to $A$ through the channel $C$. If the conduction through the channel $C$ is good, the temperatures will rapidly equalise, and the system tends to approach a state defined by the equation $T=\tau$. There is, however, a constant loss of energy through the conducting wall $b b^{\prime}$ so that $\tau$ continually decreases. The value of $T$ also decreases, lagging behind $\tau$ by an amount which depends on the goodness of conduction through the channel $C$. The coefficient of conduction through the channel $C$ will however decrease as $T$ and $\tau$ decrease, for the coefficient of conduction in a uniform gas is proportional to some positive power of its absolute temperature. Hence, however good or bad the conducting power of the wall $b b^{\prime}$ may be, there will come a time, after the gas has cooled sufficiently, when the wall $b b^{\prime}$ will be a good conductor in comparison with the channel $C$. The conditions are now changed. A small quantity of heat escapes from $A$ through the channel $C$ but is immediately lost through the wall $b b^{\prime}$, and it is impossible for the value of $\tau$ to attain to any appreciable value. Here then we have the final (normal) state of the system. It is a state in which $T$ is finite but small and both $\tau$ and $d T / d t$ are, in the limit, vanishingly small.

This state is, moreover, analogous to that which observation shews to be the state of a real gas under normal conditions, namely a state in which all the energy is absorbed by a few degrees of freedom, corresponding to the principal temperature, and in which the rate of dissipation is extremely small. The illustration can be extended to apply to a gas with a large number of subsidiary temperatures by imagining a large number of chambers similar to $B$ opening out of $A$, each by a separate channel. 


\section{The example of the loaded spheres.}

230. We shall be able to derive a second, and somewhat important, illustration of the general theory from the problem of the loaded spheres, worked out in Chapter V. We there considered a gas in which the centre of gravity of the molecules did not coincide with their geometrical centres, so that rotations were set up by collisions. In order to make this system of molecules illustrative of a non-conservative gas, we shall suppose that energy is lost when these rotations take place. We may conveniently suppose that every rotation, by a mechanism which there is no need to specify with precision, is retarded by a force proportional to the velocity of rotation.

As before, we shall use $\mathrm{H}, \mathrm{K}$ to denote the mean energies of rotation and translation of a molecule. We previously found a value for $d \mathrm{k} / d t$ in terms of $\mathrm{K}$ and $\mathrm{H}$, independently of the law of distribution of the angular velocities, and as the change in $\mathrm{K}$ is still caused entirely by collisions, we can legitimately use this expression for the value of $d \mathrm{~K} / d t$ in the present instance. The value of $\mathbf{H}$ decreases for two reasons. There is first a decrease of $H$ caused by collisions, and the rate of this decrease must be $d \mathrm{k} / d t$, the rate of increase of $k$ caused by collisions, since the total energy remains unaltered by collisions. The second cause of decrease in $\mathrm{H}$ is the system of retarding forces. These may be supposed to cause a decrease in $\mathrm{H}$ of amount $2 \epsilon \mathrm{H}$ per unit time, where $\epsilon$ is a constant. Hence, on the whole, we shall have

$$
\frac{d \mathrm{H}}{d t}=-2 \epsilon \mathrm{H}-\frac{d \kappa}{d t} \text {. }
$$

Introducing the value of $\frac{d \kappa}{d t}$ from the former investigation (equation (253)), we have as the equations expressing the rates of change in $\mathrm{K}$ and $\mathrm{H}$,

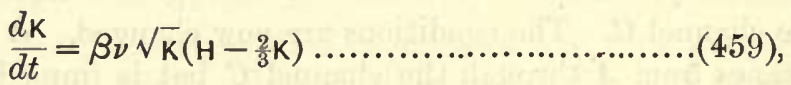

$$
\begin{aligned}
& \frac{d \mathrm{H}}{d t}=-2 \epsilon \mathrm{H}-\beta \nu \sqrt{\mathrm{K}}\left(\mathrm{H}-\frac{2}{3} \mathrm{~K}\right)
\end{aligned}
$$

231. Our aim is to obtain from these equations an illustration of the dynamical theory of a non-conservative gas, just as we found that the same equations, with the omission of the term $-2 \epsilon \mathrm{H}$, supplied an illustration of the much simpler theory of a conservative gas. We must begin by noticing that the present system possesses only one subsidiary temperature, and that $\mathrm{K}$ and $\mathrm{H}$ are respectively proportional to the principal and subsidiary temperatures.

Let us write

$$
u=2 \epsilon \mathrm{H}-\frac{2}{3} \beta \nu \mathrm{K}^{\frac{3}{2}}
$$


so that

$$
\begin{aligned}
\frac{d u}{d t} & =2 \epsilon \frac{d \mathrm{H}}{d t}-\beta \nu \sqrt{\mathrm{K}} \frac{d \mathrm{~K}}{d t} \\
& =-\frac{u}{2 \epsilon}\left(4 \epsilon^{2}+2 \epsilon \beta \nu \sqrt{\mathrm{K}}+\beta^{2} \nu^{2} \mathrm{~K}\right)-\frac{1}{3} \frac{\beta^{3} \nu^{3} \mathrm{~K}^{\frac{5}{2}}}{\epsilon}
\end{aligned}
$$

on substitution from equations (459) and (460). The general theory does not lead us to expect to find a definite steady state except for small values of $\mathrm{K}$.

Now as $k$ decreases, the relative importance of the three terms of the bracket $\left(4 \epsilon^{2}+2 \epsilon \beta \nu \sqrt{ } \mathrm{K}+\beta^{2} \nu^{2} \mathrm{~K}\right)$ changes, until ultimately the term $4 \epsilon^{2}$ is of preponderating importance. When these values of $k$ are reached, the last term in equation (462) becomes negligible, being of the same order of importance as the term $\beta^{2} \nu^{2} \mathrm{~K}$ of the bracket, so that the equation assumes the form

$$
\frac{d u}{d t}=-2 \epsilon u \text {. }
$$

It therefore appears that for these values of $\mathrm{k}$ the gas tends towards a state given by $u=0$, or

$$
\epsilon \mathrm{H}=\frac{1}{3} \beta \nu \mathrm{K}^{\frac{3}{2}}
$$

In this steady state, $\frac{H}{K}=0$ in the limit when $\mathrm{K}$ is very small, so that the energy tends to become wholly translational. It is therefore clear that in the appropriate generalised space of the kind defined in $\$ 227$, the stream lines will tend to form into a cluster, and that equation (463) is the equation of this cluster.

232. It will, however, be advantageous to examine the general arrangement of these stream lines.

If we eliminate the time between equations (459) and (460) we obtain

$$
\frac{d \mathrm{H}}{d \mathrm{~K}}=-1-\frac{2 \epsilon \mathrm{H}}{\beta \nu \sqrt{\mathrm{K}}\left(\mathrm{H}-\frac{2}{3} \mathrm{~K}\right)} .
$$

Since we may properly write

$$
\mathrm{k}=\frac{3}{2} R T, \quad \mathrm{H}=R_{\tau}
$$

where $T, \tau$ are principal and subsidiary temperatures, it is clear that equation (464) may be regarded as the differential equation of the stream lines in question. The case is one of extreme simplicity, partly because the necessary space is only two-dimensional, and partly because the stream lines are true hydrodynamical stream lines in the sense that no two lines intersect. We shall therefore proceed to draw this system of stream lines. It is not possible to solve the differential equation (464) in finite terms, but a graphical treatment will give all the information we require in a convenient form. 
It appears from the form of the differential equation that $\frac{d H}{d K}$ vanishes when

is infinite when

$$
\beta \nu \sqrt{K}\left(\mathrm{H}-\frac{2}{3} \mathrm{~K}\right)+2 \epsilon \mathrm{H}=0
$$

$$
\mathrm{H}=\frac{2}{3} \mathrm{~K}
$$

or $K=0$; and is negative, being equal to -1 , when $H=0$. If, then, we draw a two-dimensional space $(\mathrm{H}, \mathrm{K})$, the quadrant in which $\mathrm{H}$ and $\mathrm{K}$ are both positive will be divided into three sections by the axes and the two curves of which the equations are (466) and (467). Starting from the axis of $\mathrm{k}$, the signs of $\frac{d K}{d \mathrm{H}}$ in these three sections are respectively negative, positive and negative. The curves are therefore roughly as represented in fig. 1.4. It

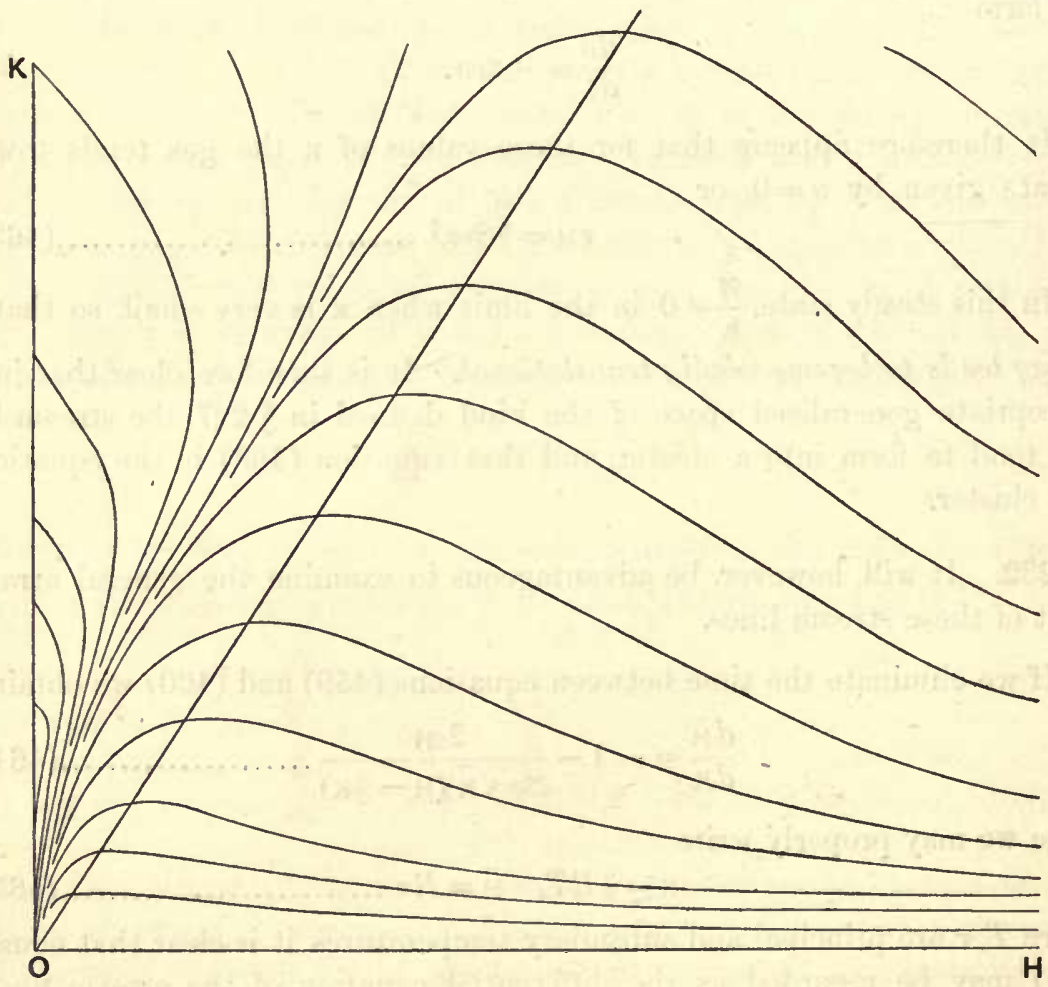

Fig. 14.

will be seen that they tend to converge for small values of $H$ and $K$ into a single stream line for which at the origin,

$$
\mathrm{Lt} \frac{\mathrm{H}}{\mathrm{K}}=0 \text {, and Lt } \frac{d \mathrm{H}}{d \mathrm{~K}}=0 \text {. }
$$


Combining these equations with the general differential equation (464) we obtain for the equation of this stream line near the origin

$$
\epsilon \mathrm{H}=\frac{1}{3} \beta \nu \mathrm{K}^{\frac{\pi}{2}}
$$

a semi-cubic parabola. Equation (468) accordingly defines the "normal state" for the imaginary gas now under consideration, and is in obvious agreement with equation (463).

If we replace $K$ and $H$ by their values in terms of the principal and subsidiary temperatures, as given by the equations (465),

$$
\mathrm{K}=\frac{3}{2} R T, \quad \mathrm{H}=R \tau,
$$

we obtain as the relation between $\tau$ and $T$ in the normal state,

$$
\tau=\frac{\beta}{\epsilon} \sqrt{\frac{3}{8} R} \nu T^{3}
$$

233. The corresponding normal state when $\epsilon=0$, is

$$
2 \mathrm{~K}=3 \mathrm{H}
$$

It is interesting and important to notice that this last equation cannot be deduced from equation (468) by putting $\epsilon=0$. In other words a conservative gas is a true special case: it is not legitimate to regard it as the limit of the general case. Hence also it is obviously futile to try to pass from the case of a conservative gas to the case of a non-conservative gas; the slightest amount of radiation of energy will entirely alter the circumstances of the case.

\section{Recapitulation.}

234. The present position is therefore as follows. We have (Chapters II.-V.) developed the dynamical theory of a conservative gas, and have found (Chapter VII.) that the results obtained do not agree with those of observation. We have now seen why this is, and have seen also that nothing less than an entirely new investigation, taking account of dissipation of energy from the outset, can be expected to lead to true results. An investigation of this kind has been attempted, but it has not been found to be possible to obtain results of the definite kind which had previously been obtained for a conservative gas. The impossibility of progress arose from a cause inherent in the subject itself: the results for a conservative gas are independent of the constitution of the molecule, whereas those for a nonconservative gas differ for different molecules. As we have no certain knowledge of the structure of molecules we have, so far, been unwilling to make any assumptions on this point. Now, however, that we have seen that further progress is only possible by the help of some definite assumption, we must be content to rest the remainder of our investigation upon hypothesis instead of upon comparative certainty. 
235. The phenomena which give the most definite and least questionable insight into the structure of matter are those of spectroscopy. The line spectrum of a gas can be most naturally interpreted as evidence of the existence of a number of isochronous free vibrations in the ultimate units of the gas.

It is true that other explanations of the line spectrum can be suggested; these will be reserved for discussion later. At present it is proposed to examine the one just mentioned. We shall therefore assume our gas to consist of molecules of which the internal structure is such that they possess a number of internal vibrations, of which the frequencies of free vibrations are equal to those of light.

The principal problem before us is of course to examine the rate of transfer of energy in this gas. Experiment suggests that the internal degrees of freedom do not receive anything like the amount of energy which would be assigned to them by the Law of Equipartition. The theory developed in the last two chapters indicates that this must occur if the internal degrees of freedom are capable of dissipating away their energy, and if also they receive energy with sufficient slowness from the translational motion. The mere fact that we are aware of the existence of these internal degrees of freedom shews that they are capable of dissipating their energy, for it is only their dissipated energy, in the form of light-waves, which tells us of their existence. As to the slowness of transfer of energy, the two illustrations of the theory discussed in the present chapter both suggest that for any type of structure the transfer of energy will, at sufficiently low temperatures, be "slow" in the sense required. The question before us is therefore as much to determine the actual limit of temperature within which this transfer may be regarded as "slow," as to determine whether such a limit of temperature exists. 


\section{CHAPTER IX.}

\section{THEORY OF A NON-CONSERVATIVE GAS (CONTINUED).}

\section{The Transfer and Dissipation of Energy. \\ The vibrations set up by collisions.}

236. WHEN a molecule is describing a free path, the changes in its coordinates will of course be deducible from its own energy function. Let us, on the grounds explained in the last chapter, suppose this energy function to contain terms of the form

$$
\begin{aligned}
& 2 L=a_{1} \dot{\phi}_{1}{ }^{2}+a_{2} \dot{\phi}_{2}{ }^{2}+\ldots \\
& 2 V=b_{1}{\phi_{1}}^{2}+b_{2}{\phi_{2}}^{2}+\ldots
\end{aligned}
$$

in which the $a$ 's and $b$ 's are constants, these terms representing the isochronous internal vibrations of the molecules. The quantities $\phi_{1}, \phi_{2} \ldots$ are principal coordinates of the system, and have the property that their equations of motion are independent of one another and of the other coordinates of the molecule. The variation of any coordinate $\phi$ is given by an equation of the form

$$
a \ddot{\phi}+b \phi=0
$$

The solution of this equation is

$$
\phi=A \cos p t+B \sin p t
$$

in which $A, B$ are arbitrary constants, and $p$ is given by

$$
a p^{2}-b=0 \text {. }
$$

Let us suppose that at time $t=0$ the motion of this moleçule is disturbed by an encounter with a second molecule, this encounter lasting until time $t=\tau$. The influence of this second molecule upon the first will be capable of being expressed by a force function of the form

$$
U_{1} \delta \phi_{1}+U_{2} \delta \phi_{2}+\ldots
$$

and from $t=0$ to $t=\tau$ the equation expressing the change of the coordinate $\phi$ will no longer be equation (473), but will be (dropping suffixes throughout)

$$
a \ddot{\phi}+p^{2} a \phi=U \ldots
$$


Before the time $t=0$, the coordinate $\phi$ is describing the free harmonic vibration expressed by equation (474). The impulse $U d t$ acting from $t=0$ to $t=d t$, sets up an additional free vibration of initial displacement zero and velocity $\frac{U d t}{a}$; the displacement of this additional vibration at any subsequent time is therefore $\frac{U d t}{a p} \sin p t$. Compounding all these vibrations with the original vibration expressed by equation (474), we obtain for the displacement $\phi$ at any instant subsequent to $t=\tau$, the well-known solution*

$$
\phi=A \cos p t+B \sin p t+\frac{1}{a p} \int_{t^{\prime}=0}^{t^{\prime}=\tau} U_{t=t^{\prime}} \sin p\left(t-t^{\prime}\right) d t^{\prime}
$$

237. Let is write

so that

$$
\left.\begin{array}{l}
X=\frac{1}{a p} \int_{t^{\prime}=0}^{t^{\prime}=\tau} U_{t=t^{\prime}} \cos p t^{\prime} d t^{\prime} \\
Y=\frac{1}{a p} \int_{t^{\prime}=0}^{t^{\prime}=\tau} U_{t=t^{\prime}} \sin p t^{\prime} d t^{\prime}
\end{array}\right\}
$$

$$
X+i Y=\frac{1}{a p} \int_{t^{\prime}=0}^{t^{\prime}=\tau} U_{t=t^{\prime}} e^{i p t^{\prime}} d t^{\prime}
$$

then equation (478) assumes the form

$$
\phi=(A-Y) \cos p t+(B+X) \sin p t .
$$

The energy of vibration is $\frac{1}{2}\left(a \dot{\phi}^{2}+b \phi^{2}\right)$, or

$$
\frac{1}{2} b\left[(A-Y)^{2}+(B+X)^{2}\right]
$$

If this is written in the form

$$
\frac{1}{2} b\left(A^{2}+B^{2}\right)+b(B X-A Y)+\frac{1}{2} b\left(X^{2}+Y^{2}\right)
$$

the first term represents what would have been the energy of vibration had the encounter not taken place; the two remaining terms represent the change effected by the encounter.

At the beginning of the encounter $\phi$ is given by putting $t=0$ in equation (474), so that

$$
\phi=A, \quad \dot{\phi}=p B .
$$

On averaging over all the molecules of the gas these quantities may be supposed to be equally likely to be positive as negative, so that on averaging over all encounters we find that the mean value of $B X-A \dot{Y}$ may be taken to be zero. Hence from expression (483), the increment in the energy of

* Rayleigh, Theory of Sound, I. $\S 66$. 
the $\phi$ coordinates which occurs throughout the whole gas, is the same as if the increase, in the case of each single molecule such as we have just been considering, were

$$
\frac{1}{2} b\left(X^{2}+Y^{2}\right)
$$

238. This quantity must be evaluated from equation (480). The form of the function $U$ is, however, almost entirely unknown. We are therefore compelled to examine the general properties of the function $X+i Y$ defined by equation (480), making as few assumptions about the function $U$ as we can.

A good deal can be learned from a graphical treatment. In fig. 15, let the thick line be the graph of the function $U$, regarded as a function of the time, then the dotted curve will be the graph of the function $U \cos p t$, and

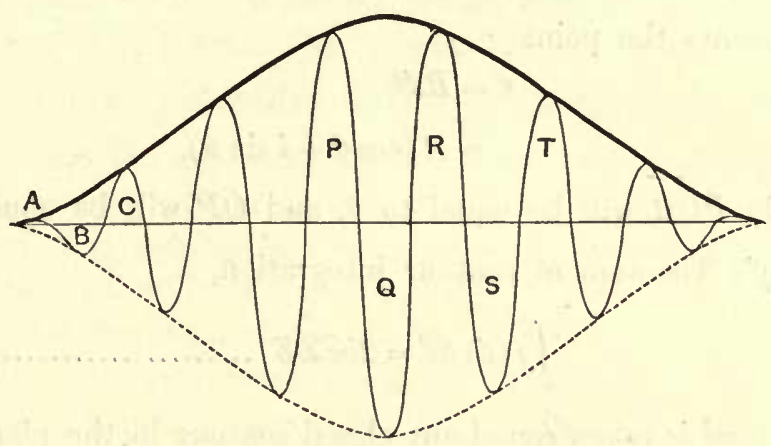

FIa. 15.

the value of $X$, given by equation (479), is equal to the area of this curve estimated algebraically, divided by ap. The area of this curve can, however, be regarded as made up of the areas $A, B \ldots P, Q, R, S, T \ldots$, and of these the alternate areas, say $B \ldots Q, S \ldots$ must be estimated negatively. Thus if we denote the numerical magnitudes of the areas by the corresponding letters, we have

$$
\begin{aligned}
\operatorname{apX}= & A-B+C+\ldots+P-Q+R-S+T-\ldots \\
= & \frac{1}{2}[A+(A-2 B+C)+(C-2 D+E)+\ldots \\
& +(P-2 Q+R)+(R-2 S+T)+\ldots]
\end{aligned}
$$

It is at once clear that each term in this sum is very small; $Q$ is nearly the arithmetic mean between $P$ and $R$, and so on, and the two isolated terms at the beginning and end are clearly seen to be small, on reference to the figure.

It is therefore obvious that $X$ is very small : to see clearly how small, we must have recourse to exact analysis. 
239. Let fig. 16 represent a plane in which all values of $t^{\prime}$, real and imaginary, are represented, and let $A O B$ be the axis along which $t^{\prime}$ is real, $O$ being the point $t^{\prime}=0$.

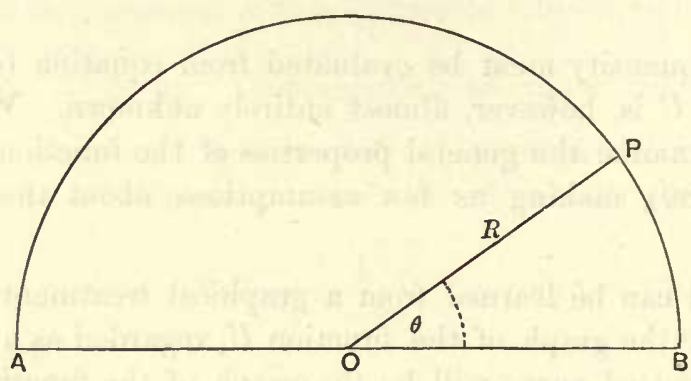

Fig. 16.

If $P$ represents the point

$$
\begin{aligned}
t^{\prime} & =R e^{i \theta} \\
& =R(\cos \theta+i \sin \theta),
\end{aligned}
$$

then the angle $P O B$ will be equal to $\theta$, and $O P$ will be equal to $R$.

By Cauchy's Theorem of contour integration,

$$
\int f\left(t^{\prime}\right) d t^{\prime}=2 i \pi \Sigma Z
$$

where the integral is taken round any closed contour in the plane of fig. 16, and $\Sigma Z$ is the sum of the residues of $f\left(t^{\prime}\right)$ inside this contour. Let us take $f\left(t^{\prime}\right)=U e^{i p t^{\prime}}$, and take the closed contour to consist of a semicircle BPA of radius $R$ described about the origin as centre, and the real axis $A O B$ which forms the bounding diameter of the semicircle. In the limit we shall take $R=\infty$.

We have supposed as a legitimate first approximation, that $U$ vanishes except between $t^{\prime}=0$ and $t^{\prime}=\tau$. But when we attempt to represent the conditions of nature by strict mathematical analysis, there can be no discontinuity of this kind and the function $U^{\prime}$ must be supposed to exist from $t=-\infty$ to $t=\infty$. This latter supposition, it is true, includes the former as a special case, but the special case is not only excluded on physical grounds, but is inconsistent with the supposition made in equation (485) that $U$ is an analytic function. When in the limit we make $R=\infty$, the real axis $A O B$ will extend from $-\infty$ to $+\infty$ so that the integral of $U e^{i p t^{\prime}}$ taken along this real axis may be supposed equal to the integral taken through the encounter, and therefore, by equation (480), to $a p(X+i Y)$.

The remainder of the left-hand member of equation (485), the integral being taken round the prescribed contour, is the integral taken round the 
semicircle $B P A$, over which $R$ is constant and infinite, while $\theta$ varies from 0 to $\pi$. On this part of the path we have, since $t^{\prime}=R e^{i \theta}$,

$$
\begin{aligned}
d t^{\prime} & =R e^{i \theta} i d \theta, \\
e^{i p t^{\prime}} & =e^{i p R(\cos \theta+i \sin \theta)},
\end{aligned}
$$

hence this part of the integral becomes

$$
I=\int_{\theta=0}^{\theta=\pi} U^{\prime} e^{i p R \cos \theta-p R \sin \theta} R e^{i \theta} i d \theta
$$

and the original equation (485) assumes the form

$$
a p(X+i Y)+I=2 i \pi \Sigma Z
$$

To evaluate $I$, we divide it into three parts $I_{1}, I_{2}$ and $I_{3}$, the first being the integral from $\theta=0$ to $\theta=\epsilon$, the second from $\theta=\epsilon$ to $\theta=\pi-\epsilon$, and the last from $\theta=\pi-\epsilon$ to $\theta=\pi$. The integrand can be written in the form

$$
U^{\prime}\{i \cos (\theta+p R \cos \theta)-\sin (\theta+p R \sin \theta)\} e^{-p R \sin \theta+\log R}
$$

Let us choose the quantity $\epsilon$ so that $\epsilon$ shall be vanishingly small, whilst $R \sin \epsilon$ shall be infinitely great in comparison with $\log R$, this being always possible when $R$ is infinite (e.g., we may take $\epsilon=R^{-\frac{1}{2}}$ so that $R \sin \epsilon=R^{\frac{1}{3}}$ ). Giving $\epsilon$ such a value, it is clear that $e^{-p R \sin \theta+\log R}$ will vanish from $\theta=\epsilon$ to $\theta=\pi-\epsilon$ and therefore, except in the special case in which $U^{\prime}$ becomes infinite within the range in question, we shall have $I_{2}=0$.

The cases in which $U^{\prime}$ becomes infinite within the range of integration must now be examined. It is clear that if, when $R=\infty, U$ becomes infinite less rapidly than an exponential $e^{\kappa R}$, then by taking $R$ sufficiently great, the integrand (487) will always vanish. When $U$ becomes infinite with the same rapidity as an exponential this is not in general true, and the same is the case if $U$ becomes infinite more rapidly than an exponential. The important case in which the result is not true is that in which $U$ contains a term of the form $\cos (q t+\epsilon)$, which gives rises upon the semicircle to terms containing the factors $e^{ \pm q R \sin \theta}$. If $q<p$, the result $I_{2}=0$ will be seen to be true; if $q>p$ the result $I_{2}=0$ is not true, but the main proposition can be proved by a slight modification of the present proof; if $q=p$ the main proposition is obviously not true. On general principles it will be seen that this is the only important case of exception. Physically, this possibility represents vibrations "forced" in one molecule, by vibrations of equal period in the second molecule. These vibrations, then, form an exception to the proposition which we are trying to prove, that the vibrations excited by collision are small in comparison with the energy of the exciting agency; but the physical deduction we wish to draw from this proposition will be in no way invalidated, for vibrations of the kind in question do not represent a transfer of energy between translational 
and vibratory degrees of freedom, but only between two degrees of freedom of the same type. The mean energies of the various degrees of freedom, therefore, are not affected.

Beyond this, however, it is impossible to discuss cases in detail : that the result is in general true there can be little doubt. We therefore suppose, for the present, that $I_{2}=0$ and proceed to the calculation of $I_{1}$ and $I_{3}$.

In evaluating $I_{1}, \theta<\epsilon$ and is therefore vanishingly small. We therefore have

$$
I_{1}=\int_{\theta=0}^{\theta=e} U^{\prime} e^{i(p R \cos \theta+\theta)} e^{-p R \theta} i d(R \theta) .
$$

In this the factor $e^{-p R \theta} d(R \theta)$ may be replaced by $-\frac{d u}{p}$ where $u=e^{-p R \theta}$ and the limits of integration, regarded as an integration with respect to $u$, are $u=1$ to $u=0$. Also, since $U^{\prime}=0$ when $\theta=0, U^{\prime}$ may in general be replaced by $\theta\left(\frac{d U^{\prime}}{d \theta}\right)_{\theta=0}$, and hence we have the equation

$$
I_{1}=\int_{u=0}^{u=1} \theta\left(\frac{d U^{\prime}}{d \theta}\right)_{\theta=0} e^{i(p R+\theta)} \frac{d u}{p},
$$

and this, in general, vanishes through the factor $\theta$. In a similar way we can shew that $I_{3}$ vanishes, and hence that $I=0$.

Equation (486) now becomes

$$
X+i Y=\frac{2 i \pi}{a p} \Sigma Z
$$

240. Let the residues of the function $U$ which occur inside the infinite semicircle which has formed the path of integration, be $\zeta_{1}$ at $\alpha_{1}+i \beta_{1}, \zeta_{2}$ at $\alpha_{2}+i \beta_{2}$, etc., in which $\alpha, \beta$ are in every case real, and in which, since the residues lie within the semicircle, $\beta$ must in every case be positive. Then the residues of the function $U e^{i p t^{\prime}}$ will be $\zeta_{1} e^{i p\left(\alpha_{1}+i \beta_{1}\right)}$ at $\alpha_{1}+i \beta_{1}$, etc. These are the quantities of which the sum has been denoted by $\Sigma Z$, so that equation (488) becomes

$$
X+i Y=\frac{2 i \pi}{a p} \Sigma \zeta_{1} e^{i p a_{1}} e^{-p \beta_{1}}
$$

We now proceed to study the variation of this quantity with $p$ when $p$ is very great. Let us imagine the molecule to change so that, although its configuration in equilibrium remains unaltered, the forces of restitution when it is disturbed are altered, and consequently the value of $p$ is altered. We shall suppose the purely geometrical coordinates $\phi_{1}, \phi_{2} \ldots$ to remain unaltered, and hence $a_{1}, a_{2} \ldots$ and the forces $U_{1}, U_{2} \ldots$ remain unaltered. Since $U_{1}, U_{2} \ldots$ remain unaltered with a change of $p$, it follows that $\zeta_{1}, \alpha_{1}$ and $\beta_{1}$ in equation (489) remain unaltered. If $\beta_{1}$ is the smallest of the quantities 
$\beta_{1}, \beta_{2} \ldots$ it appears that when $p$ is very great the term with suffix 1 will be the preponderating term in the sum on the right-hand of equation (489). We may accordingly write the equation in the form

$$
X+i Y=\frac{2 i \pi}{a p} \zeta_{1} e^{-i p \alpha_{1}} e^{-p \beta_{1}}
$$

and, since the modulus of a product is equal to the product of the moduli of its factors, it follows at once that

$$
X^{2}+Y^{2}=\frac{4 \pi^{2}}{a^{2} p^{2}}\left|\zeta_{1}\right|^{2} e^{-2 p \beta_{1}}
$$

Since, from equation (475) $a p^{2}=b$, it follows that expression (484), which gives the mean increase of the energy of vibration, becomes

$$
\frac{1}{2} b\left(X^{2}+Y^{2}\right)=\frac{2 \pi^{2}}{a}\left|\zeta_{1}\right|^{2} e^{-2 p \beta_{1}}
$$

and the right-hand member depends on $p$ only through the factor $e^{-2 p \beta_{1}}$. It therefore appears that, subject to the assumptions we have made, the energy of the vibrations set up in the molecules decreases exponentially with $p$. This immediately shews what an enormous range of values is possible for the rate at which the translational motion becomes dissipated through these vibrations. For instance, the two values of the expression given by equation (492) which correspond to $p \beta_{1}=200$ and $p \beta_{1}=100$ stand in the ratio $e^{-200}$, or about $10^{-88}$. It will, therefore, be seen that there is no difficulty in accounting for the slowness of the process of dissipation in a gas, provided we can attribute a sufficiently high value to $p \beta_{1}$.

241. The values of $p$ are known with great accuracy, being the frequencies of the light vibrations emitted by the molecule.

To determine the values of $\beta_{1}$ we should require to have full knowledge of the forces which come into play at an encounter of molecules. This is, of course, beyond our reach, but without it we can arrive at an estimate of the order of magnitude of $\beta_{1}$ which, although not exact, is sufficient for our purpose.

Let us begin by considering a collision between two elastic spheres. In this case the forces may be supposed to act.instantaneously. We do not require to know the variations in the magnitude of the force-function (476) which occur throughout the collision: all that is significant is its value integrated throughout the collision. We shall therefore get accurate results by assigning to this force-function any form which is such that the collision is instantaneous, and that its value integrated through the collision has a certain given value. Suppose, for instance, that $\int U d t$, where the integral 
is taken through a collision which occurs at time $t=0$, has to be equal to $I$. Then we may take

$$
U=\frac{I c}{\pi\left(c^{2}+t^{2}\right)}
$$

provided we put $c=0$ in the limit. For this value is such that $U$ vanishes except when $t=0$, and we have

$$
\int_{-\infty}^{+\infty} \frac{I c}{\pi\left(c^{2}+t^{2}\right)} d t=I
$$

In this case $U$, regarded as a function of the complex variable $t^{\prime}$, has two infinities occurring at $t=i c,-i c$, and each of residue $\pm \frac{I}{2 i \pi}$. Hence there is only one infinity $t=i c$, occurring within the contour of integration in fig. 16, and for this $\beta=c$. The right-hand member of equation (492) in this case is therefore

$$
\frac{I^{2}}{2 a} e^{-2 p c}
$$

Putting $c=0$ we notice that the expression becomes independent of $p$. This is as it should be; when the collision is instantaneous, the forces of restitution in the molecule do not have time to come into play, and therefore do not affect the result of the collision.

Taking $c$ small, but not quite zero, equation (493) can be supposed to represent the value of $U$ for an encounter which is not quite instantaneous. Here $U$ vanishes except through a small interval of time comparable with $c$ and occurring when $t=0$. The effect on expression (495) of taking $c$ small instead of zero, is to decrease it in the ratio $e^{-2 p c}$, and the forces of restitution now come into play. The quantity $\beta$ which in this case is equal to $c$ is, as we have seen, comparable with the time through which an encounter lasts. It will be seen that the interpretation of $\beta$ which has been obtained in this special case, will hold in every case as regards order of magnitude, and hence we conclude that the rate of dissipation of the energy of a gas may reasonably be expected to be slow, provided that the product of the time occupied by a collision and the frequency of vibration is small.

The physical principle upon which this result rests would of course have. been obvious enough without a mathematical discussion. What would not have been obvious is the extreme rapidity with which the rate of dissipation decreases as $p$ is increased.

242. Before we can determine the extent to which this principle applies to a gas, we must form an estimate of the duration of a collision. Let us at first suppose that the molecules are surrounded by spheres of molecular action, satisfying the conditions mentioned in $\S 82$. The figures of $\S 158$, as well as other experimental results to be mentioned later, shew that the 
radius of these spheres must be supposed comparable with $10^{-8} \mathrm{cms}$. The collisions which excite vibrations to the greatest extent will be "direct" collisions, and the vibrations most excited will be those of lowest frequency. For ultra-red light, which may for the present be supposed to represent the vibrations of lowest frequency, we may take $p=2 \times 10^{15}$. Let us, using the results of $\S 130$, take $C=5 \times 10^{4}$. If we suppose that at a direct collision the spheres penetrate one another to the extent of a hundredth part of their radius, we see that during collision the centre of a molecule would describe a path equal to $2 \times 10^{-10} \mathrm{~cm}$. at a mean velocity of $\frac{1}{2} \times 5 \times 10^{4}$. Hence the time of collision would be about $8 \times 10^{-15}$ secs. The product of this and the value given above for $p$ in the case of ultra-red light is 16 .

This is hardly a great quantity in the sense required; the value of $e^{-16}$ is about $10^{-7}$, and since the average nolecule undergoes more than $10^{7}$ collisions in a second, it is obvious that the dissipation of energy per second would be much too great to agree with observation.

243. On the other hand there is not the slightest evidence that it is permissible to assume the molecules to be surrounded by "spheres of molecular action" possessing the properties mentioned in $\S 82$. Or, what comes to the same thing, if we suppose the molecules to be surrounded by such spheres, we must also suppose the amount of interpretation to be comparable with the radii of the spheres. If we assume this to be one-half instead of one-hundredth part of the radius the time of collision previously calculated must be multiplied by 50 , and the product of this and $p$ will be 800 instead of 16 . The value of $e^{-800}$ is about $10^{-347}$ and it will be seen that this is amply small enough to account for the observed slowness of dissipation of energy. If we allow $10^{10}$ collisions per second, it follows that the translational energy will be reduced to $e^{-1}$ times its value in about $10^{337}$ seconds, or about $10^{330}$ years.

244. At the same time we must notice that even in a gas at an ordinary temperature, there will be some pairs of molecules which collide with enormously high relative velocities, and for which therefore the time of collision is much shorter than we have supposed it to be. Such a collision may obviously set up large vibrations in the molecules concerned.

In expression (45) we found the number of collisions of relative velocity between $V$ and $V+d V$ which occurred per unit time per unit volume to be

$$
\nu^{2} \sigma^{2} \sqrt{\frac{\pi h^{3} m^{3}}{2}} e^{-\frac{1}{2} h m V^{2}} V^{3} d V
$$

The total number of collisions, obtained by integrating this expression from $V=0$ to $V=\infty$, was also found to be

$$
\nu^{2} \sigma^{2} \sqrt{\frac{2 \pi}{h m}}
$$


so that the ratio to the total number of collisions, of the number of collisions for which $V$ is greater than $V_{0}$, is

$$
\begin{aligned}
\frac{h^{2} m^{2}}{2} \int_{V}^{V}=\nabla_{0} & e^{-\frac{1}{2} h m V^{2}} V^{3} d V \ldots \ldots . \\
& =\int_{x=\frac{1}{2} h m V_{0}^{2}}^{x=\infty} e^{-x} x d x \\
& =\left[e^{-x}(1+x)\right]_{x=\frac{1}{2} h m V_{0}^{2}} \\
& =e^{-\frac{1}{2} h m V_{0}^{2}}\left(1+\frac{1}{2} h m V_{0}^{2}\right)
\end{aligned}
$$

This quantity vanishes with extreme rapidity as $V_{0}$ increases, so that the number of collisions for which $V$ is great forms an extremely small fraction of the whole. If, as in $\S 242$, we take $C=5 \times 10^{4}$ we must put

$$
h m=\frac{3}{2 C^{2}}=6 \times 10^{-10} .
$$

Still using the figures of the last two sections, it appears that to obtain a collision of duration comparable with that of a vibration of light $\left(p=2 \times 10^{15}\right)$, the value of $V_{0}$ must be comparable with $2 \times 10^{7} \mathrm{~cm}$. per sec. Giving this value to $V_{0}$ and putting $h m=6 \times 10^{-10}$ the value of expression (498) becomes

$$
120,001 \times e^{-120,000} \text {. }
$$

Even if we give to $\nabla_{0}$ a value ten times as small as this, the value of expression (498) is

$$
1201 \times e^{-1200}
$$

which is equal to about $10^{-618}$, a quantity which is quite negligible for our present purpose.

245. To recapitulate, we have seen that there are two cases of transfer of energy to be considered, the first arising from the common collisions in which the duration of the collision is great in comparison with the periods of vibration of the molecule, and the second from the rare collisions in which the duration of the collision is comparable only with that of a vibration. We have seen that in the former case the transfer of energy is infinitesimal on account of the high frequency of the vibrations, and in the latter case the transfer is infinitesimal on account of the extreme rarity of these collisions.

Here, then, we have a sufficient explanation of the slow transfer, and consequently slow dissipation of energy, which is necessary in order that a normal state may exist. In this respect the analogy with the two mechanical illustrations of the preceding chapter is complete. Our hypothetical view of the structure of the atom having so far led to a satisfactory result, we shall proceed in the next chapter to investigate the calorimetry of a gas upon these hypotheses. 


\section{Physical Consequences of Hypotheses.}

246. We shall conclude the present chapter with the mention of a few physical considerations which arise from this hypothetical view of the structure of the molecule. They have very little bearing on the direct development of the Kinetic Theory, except in so far as they tend to strengthen the evidence for the hypotheses on which they rest.

\section{Calculation of Temperature of Incandescence.}

247. In $\S 242$, we assumed the value $C=5 \times 10^{4}$ corresponding roughly to air under normal conditions. If, instead of this we had taken $C=5 \times 10^{5}$ corresponding to air at a temperature of $28,000^{\circ} \mathrm{C}$., the value obtained for formula (498) in expression (499) would have to be replaced by

$$
13 e^{-12} \text {, }
$$

of which the value is about $\cdot 00008$, a quantity which is certainly not negligible for our present purpose. For if we take the number of collisions per second to be $10^{10}$ we find that the translational energy is reduced to $e^{-1}$ times its initial value in about a millionth of a second. We should therefore expect a gas at a temperature comparable with $28,000^{\circ}$ to lose energy with extreme rapidity. Our hypothesis would then lead to the prediction of a temperature of incandescence-a temperature, that is, at which the loss of energy by radiation becomes perceptible-lying somewhere between $0^{\circ} \mathrm{C}$. and $28,000^{\circ} \mathrm{C}$. Stated in this form, the result may seem at first sight to be almost worthless as a confirmation of the hypothesis on which it rests. It ought, however, to be noticed that the hypothesis enables us to predict the value of $C$ corresponding to the temperature of incandescence to within less than a power of 10-a result sufficiently remarkable when we consider the vagueness of the numerical data on which it rests.

\section{Experiments of Schuster and Hemsalech.}

248. The collisions between molecules moving with low velocities will give rise to but little radiation for reasons already explained: those between molecules moving with high velocities will be extremely rare. Hence the greater amount of the radiation will, when the radiation is perceptible, arise from the collisions of molecules moving with certain intermediate velocities; and, on account of the rapidity of change of the exponential expressions (cf. expressions (492) and (498)) on which the radiation depends, it is clear that the velocities will be closely grouped about a certain mean velocity. This mean velocity will, as is clear from an inspection of expression (492), 
depend on the frequency of the particular light-waves under consideration. Hence, speaking somewhat loosely, we may say that light of one frequency will be emitted mainly by molecules moving with a certain velocity, light of another frequency by molecules moving with another frequency, and so on. Obviously violet light will in general be emitted by molecules moving faster than those which emit red light. Something of this kind has been noticed by Schuster and Hemsalech* in the case of the light emitted by the electric arc. The conditions here are of course widely different from those contemplated by our simpler theory, but the cases are sufficiently parallel for a comparison to be of interest. The point which is of primary importance is that our hypothesis seems capable by itself of accounting for the observations of Schuster and Hemsalech: it is not necessary to introduce a further hypothesis to the effect that the light-radiating molecules are in a "special state."

\section{Light produced by heating.}

249. From the considerations just mentioned, it is clear that from our hypothesis we should expect that as the temperature of a gas is increased, the light first observed would be that of lowest frequency, provided of course that this light is the result solely of molecular collisions. This prediction cannot be verified by direct observation, for when a gas is heated until it emits light, it is not possible to decide whether this light is due to molecular collisions only or to chemical or electric action. But the analogous phenomenon in the case of a solid is one of everyday observation, and there is no reason to suppose that the behaviour of a gas would not be similar.

In connection with the radiation from heated gases, the experiments of Paschen $\dagger$ and others must be mentioned. It has been found that when certain gases (e.g. $\mathrm{H}_{2} \mathrm{O}, \mathrm{CO}_{2}$ ) are heated, there is an emission of rays of definite wave-length, this wave-length being many times greater than that of ultra-red light.

\section{Light produced by Electric Action.}

250. When a gas is radiating light the emission seems to be produced, in almost all cases which can be examined in the laboratory, by chemical or electrical action + .

* Phil. Trans. A. cxcrir., p. 189.

+ See a paper by Paschen (Wied. Ann. x. p. 409) for a summary of evidence on this question.

$\ddagger$ Pringsheim (Wied. Ann. xuv. p. 428) is of opinion that no light is ever produced in gases by collisions alone. This view is contested by Paschen (Wied. Ann. L. p. 409) and Smithells, Phil. Mag. [5], xxxvır. p. 244. See also Pringsheim, Rapports présentés au Congrès International de Physique, Paris (1900), p. 100. 
In the former case, if light can be caused at all by chemical action independently of electrical action, the direct cause of radiation is presumably the vibratory agitation of the atoms produced by the disruption of molecules.

Our knowledge of light emitted by a gas on account of electrical action, as for instance the well-known light of the vacuum tube, is at once more satisfactory and more definite. The cause of radiation seems to be the vibrations of the atoms caused by their being bombarded by free corpuscles.

Let us apply the considerations of this chapter to the collisions which cause this radiation. The mass of a corpuscle is, to within a few per cent., equal to about $\frac{1}{\gamma 00}$ of that of the hydrogen atom. Hence if we assume, as a preliminary hypothesis, that the corpuscles take up velocities in their motion equal to those which would be possessed by the molecules of a gas of masses equal to those of the free corpuscles, then the value of $C$, as will be seen from the table on p. 113, which corresponds to a temperature of $100^{\circ} \mathrm{C}$., will be about $7 \times 10^{6} \mathrm{~cm}$. per sec. The ratio of this velocity to the radius to an atom is $7 \times 10^{14}$, a ratio which is therefore comparable with, although not equal to, the frequency of light vibrations.

In many cases, however, the velocity of the corpuscles will be due to the action of an electric field. For instance in a vacuum tube in which the gas is hydrogen at a pressure of $2 \mathrm{~mm}$., the free path (cf. $\S 8$ ) is of the order of magnitude of $\frac{1}{16} \mathrm{~mm}$. If we assume a fall of potential of 40 volts per $\mathrm{cm}$., the fall on the free path is $\frac{1}{4}$ volt, or $25 \times 10^{6}$ c.G.S. (electromagnetic) units. The velocity $v$ at collision is therefore given by

$$
\frac{1}{2} m v^{2}=25 \times 10^{6} \times e,
$$

and on taking $e / m=10^{7}$, we obtain $v=2 \cdot 2 \times 10^{7}$. The ratio of this to the radius of the hydrogen atom is $2 \cdot 2 \times 10^{15}$, a number sufficiently large to be comparable with the frequency of even violet light.

In this way we see that the emission of light as the result of electric action is possible either when the free path is large, as in a vacuum tube, or when the electric intensity is great, as in the electric arc.

It therefore appears that our theory would almost enable us to predict the emission of light which is produced by electrical action : at any rate its consequences are fully in accordance with this observed emission. 


\section{CHAPTER X.}

THEORY OF A NON-CONSERVATIVE GAS (CONTINUED).

\section{Calorimetry.}

251. WE have seen that the hypothesis upon which we have been working leads to a possible explanation of the difficulties connected with the dissipation of energy. This hypothesis therefore puts us in a position to continue the investigation into the behaviour of a gas, at the point at which we had to abandon it before (Chapter VII.). We shall accordingly attempt to investigate the calorimetry of a gas with. the help of this hypothesis. Naturally the results obtained will give us further evidence as to the tenability or the reverse of the hypothesis in question.

\section{Calculation of subsidiary temperatures.}

252. We have found that so long as a gas is at a temperature comparable with the temperatures we find on this planet, or which we can reproduce in the laboratory, the transfer of energy from the principal degrees of freedom to the vibratory degrees of freedom is extremely slow. If, then, $\tau_{1}, \tau_{2} \ldots$ are the subsidiary temperatures corresponding to these vibrations, we shall have an equation for the change in $\tau_{1}$ of the form

$$
\frac{\partial \tau_{1}}{\partial t}=\theta_{1}-2 \epsilon_{1} \tau_{1}
$$

where $\theta_{2}$ is the rate of increase of $\tau_{1}$ caused by collisions.

The number of collisions of any specified kind which occur per unit of time is proportional to the square of the molecular density of the gas, so that the consequent increase in $\tau_{1}$ is proportional simply to the density. For the rest, since the subsidiary temperatures are small compared with the principal temperature, the value of $\theta_{1}$ may be supposed to depend solely on this principal temperature. We may therefore take $\theta_{1}$ to be of the form

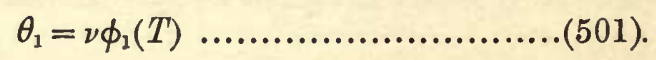


In the normal state $\frac{\partial \tau_{1}}{\partial t}, \frac{\partial \tau_{2}}{\partial t} \ldots$ vanish in comparison with $\tau_{1}, \tau_{2} \ldots$ so that from equations (500) and (501)

$$
2 \epsilon \tau_{1}=\theta_{1}=\nu \phi_{1}(T) \text { etc. }
$$

Or, writing $f_{1}(T)$ for $\frac{\phi_{1}(T)}{2 \epsilon_{1}}$

$$
\left.\begin{array}{c}
\tau_{1}=\nu f_{1}(T) \\
\tau_{2}=\nu f_{2}(T) \\
\text { etc. }
\end{array}\right\}
$$

These equations give the subsidiary temperatures as functions of the principal temperature and the molecular-density of the gas.

An illustration of these equations has already been obtained in equation (469).

253. There is, however, a second standpoint from which we may attack the problem of obtaining equations for the subsidiary temperatures. Up to the present we have taken full account of the dissipation of energy which is caused by radiation into the ether, but we have not followed this energy any further after it has once been transmitted to the ether. If we are dealing with a thin layer of gas isolated in space, this procedure will probably give an adequate representation of the facts of the case. The energy is merely radiated away from the gas, and since there is no way by which it can return, its existence need not further trouble us.

254. Suppose, on the other hand, that the gas is enclosed in an ideal vessel, of which the walls are perfect reflectors, and are therefore absolutely incapable either of transmitting or absorbing energy. The total energy inside this vessel must remain constant, so that the energy radiated out by the gas accumulates in the ether. It is now necessary to consider the possibility of a retransference of energy from the ether to the matter.

The energy lost to the gas by dissipation from a vibration of frequency $p$ will exist in the ether in the form of trains of waves of frequency $p$. Such a train of waves falling upon a second molecule will be represented by a force-function, simply-harmonic as regards the time and of frequency $p$, acting upon the coordinates of the molecule. From the analysis of the last chapter (p. 201) it is clear that this train of waves will "force" vibrations in the molecule of frequency $p$, if such vibrations are possible, but will have only an infinitesimal effect upon vibrations of other frequencies. Thus the only perceptible transfer of energy from the ether to the vibratory energy of the gas will be a transfer of energy represented by waves of one definite period to vibrations of the same period. This, of course, is fully in agreement with the results of spectroscopy, as shewn by absorption bands in the spectrum. 
In discussing the transfer of energy from the ether to the translational energy of a gas, we are dealing with the phenomenon known as the "pressure of radiation." From electrodynamical considerations, it can be shewn that a transfer of this kind will actually occur, and this again is fully in agreement with the results of observation-as, for instance, the fact that the air is warmed by sunlight.

To attempt to represent this mathematically, let us suppose that the aggregate energies in the gas represented by the temperatures $T, \tau_{1}, \tau_{2} \ldots$ are respectively $S T, s_{1} \tau_{1}, s_{2} \tau_{2} \ldots$ etc., so that $S, s_{1}, s_{2} \ldots$ are each proportional to $\nu$. Let us suppose that the wave energies in the ether of frequencies equal to those of the vibrations $\tau_{1}, \tau_{2} \ldots$ are $E_{1}, E_{2} \ldots$ Let us suppose further that the ether-energy $E_{1}$ increases the value of $\tau_{1}$ at a rate $\xi_{1} E_{1}$ and the value of $T$ at a rate $X_{1} E_{1}$, the quantities $\xi_{1}, X_{1}$ being of course independent of $\nu$. Then, if we neglect all the energy in the ether except that of definite trains of waves, we shall have equations of the forms

$$
\begin{gathered}
\frac{\partial \tau_{1}}{\partial t}=\theta_{1}-2 \epsilon_{1} \tau_{1}+\xi_{1} E_{1} \\
\frac{\partial \tau_{2}}{\partial t}=\theta_{2}-2 \epsilon_{2} \tau_{2}+\xi_{2} E_{2} \\
\text { etc. } \\
\frac{\partial T}{\partial t}=-\frac{1}{S}\left(s_{1} \theta_{1}+s_{2} \theta_{2}+\ldots\right)+\left(X_{1} E_{1}+X_{2} E_{2}+\ldots\right)
\end{gathered}
$$

for the changes of material energy, and for the changes of the energy of the ether, equations of the forms

$$
\frac{\partial E_{1}}{\partial t}=2 \epsilon_{1} s_{1} \tau_{1}-s_{1} \xi_{1} E_{1}-S X_{1} E_{1}, \text { etc. }
$$

From these equations we obtain at once

$$
\frac{\partial}{\partial t}\left(s_{1} \tau_{1}+s_{2} \tau_{2}+\ldots+S T+E_{1}+E_{2}+\ldots\right)=0
$$

expressing that the total energy remains unaltered.

255. There is now possible an absolutely steady state, the mathematical expression of which is obtained by equating to zero the right-hand side of every equation from (504) to (506). These equations, however, are not independent, in virtue of equation (507). We may therefore omit one equation, let us say (505). We are left with equations of the types

$$
\left.\begin{array}{r}
\theta_{1}-2 \epsilon_{1} \tau_{1}+\xi_{1} E_{1}=0 \\
2 \epsilon_{1} s_{1} \tau_{1}-s_{1} \xi_{1} E_{1}-S X_{1} E_{1}=0
\end{array}\right\}
$$

the remaining equations being obtained by replacing the suffix 1 by $2,3 \ldots$

Solving the pair of equations (508), we obtain

$$
\frac{E_{1}}{2 \epsilon_{1} s_{1}}=\frac{\tau_{1}}{s_{1} \xi_{1}+S_{1} X_{1}}=\frac{\theta_{1}}{2 \epsilon_{1} S_{1} X_{1}}
$$


and similar equations for the other suffixes. If we replace $\theta_{1}$ by its value as given by equation (501), we obtain

$$
2 \epsilon_{1} \tau_{1}=\theta_{1}\left(1+\frac{s_{1} \xi_{1}}{S_{1} X_{1}}\right)=\nu \phi_{1}(T)\left(1+\frac{s_{1} \xi_{1}}{S_{1} X_{1}}\right)
$$

and these equations lead to equations which are formally the same as equations (503), namely

$$
\tau_{1}=\nu f_{1}(T), \text { etc. }
$$

except that $f_{1}(T)$ no longer stands for

but for

$$
\frac{\phi_{1}(T)}{2 \epsilon_{1}},
$$

$$
\frac{\phi_{1}(T)}{2 \epsilon_{1}}\left(1+\frac{s_{1} \xi_{1}}{S_{1} X_{1}}\right)
$$

The value of $f_{1}(T)$, it will be noticed, is as before, independent of $\nu$, for $s_{1}, S_{1}$ are each directly proportional to $\nu$.

256. We have now seen that the values of the subsidiary temperatures are given by equations of precisely the same form, whether we suppose (i) that all the energy which passes into the ether is lost to the gas, or (ii) that all the energy is reabsorbed by the gas. It is therefore pretty obvious that the equations will also give a fair account of the state of things which will arise when part only of the radiated energy is reabsorbed by the gas. We shall, accordingly, in the present chapter, investigate the behaviour of a gas upon the supposition that the subsidiary temperatures are given by equations of the form of equations (503).

257. Before passing on it may be well to remark that there is a single case of failure of these equations. This occurs when $X_{1}=0$, and $\xi_{1}$ is not equal to zero, for then the value of $\tau_{1}$ given by equation (510) becomes infinite, suggesting that, in arriving at this equation, it is no longer legitimate to regard $\tau_{1}$ as small. The physical interpretation of this case is, however, obvious. The combined energies $E_{1}$ and $\tau_{1}$ can continue to gain energy through collision at the expense of $T$, but have no opportunity of losing energy, until $\tau_{1}$ becomes comparable with $T$. When this stage is reached energy can be repaid through the medium of collisions. The final stage is one in which the exchanges of energy produced by collisions are exactly balanced. This will be seen to be a state in which the material energy is equally divided between the different degrees of freedom, while the energy of the ether stands in a definite ratio to the material energy, the absorption exactly balancing the radiation.

This state, however, is not likely to occur in nature. It requires in the first place that the energy of the ether shall produce absolutely no effect on the translational energy of the molecules, and in the second place that the 
reabsorption of vibratory energy shall be perfect. Even if the first condition is satisfied, while only a definite fraction $\kappa$ of the radiated energy is reabsorbed, it will be seen that the equations of $\S 252$ are still true, except that $\epsilon$ must be replaced by $\epsilon(1-\kappa)$.

\section{The Total Energy of a Molecule.}

258. In the expression for the energy of a molecule of the gas, there will be momentoids other than those corresponding to the small vibrations we have been considering. The most obvious instance is that of the energy of rotation, which will be responsible for three momentoids.

If we consider the simplest case of molecules represented by perfectly symmetrical, smooth and elastic spheres, it is clear that these three momentoids do not enter at all into the dynamics of the gas. A collision cannot alter the rotation of the molecules, and the whole behaviour of the gas is exactly the same as if these rotations were of zero amount throughout, i.e., the same as if the three corresponding momentoids were ignored altogether. In nature, it is possible that we have to deal with very approximately spherical molecules, but we may be sure that the perfect conditions postulated for the ideal elastic spheres will not occur. If the molecules are approximately spherical, it is clear that only very small rotations will be set up by collisions. If, then, we suppose that rotations are accompanied by dissipation of energy, the momentoids which represent the energy of rotation may be supposed governed by a subsidiary temperature.

If the molecule is of a shape far removed from spherical symmetry, it is clear that the transfer of energy from translational to rotational energy at a collision will be comparable with the whole energy of the molecule. In this case the rotation will be governed by the principal temperature, if the radiation from the rotation is nil or is very small. If this radiation is not very small, the dissipation of energy from the gas will go on with great rapidity, a condition which need not be discussed since it obviously does not occur in nature.

The potential energy of the molecule must also be taken into account. The small vibrations will result in changes of potential energy, but these may be represented by terms of the second degree in the coordinates, and may therefore be regarded as momentoids. The potential energy of any small vibration, averaged over a sufficient time, is equal to the kinetic energy averaged over the same time, so that the potential energy of these vibrations will be governed by subsidiary temperatures, these being the same in every case as for the kinetic energy.

We therefore suppose the total energy of the molecule to consist of

(i) the energy of translation, proportional to the principal temperature; 
(ii) the energy of $n$ other momentoids (e.g. momentoids of rotation) also governed by the principal temperature; and

(iii) the energy of various degrees of freedom, governed by subsidiary temperatures $\tau_{1}, \tau_{2} \ldots$.

\section{General Equation of Calorimetry.}

259. We now examine the calorimetry of such a gas, without at present making the simplifying assumption that the gas obeys Boyle's Law.

The general equation of calorimetry is, as before (equation (391)),

$$
d \mathbb{Q}=R \nu_{b} T d v+N d \bar{E} \ldots \ldots \ldots \ldots \ldots \ldots \ldots(511),
$$

where $\nu_{b}$ is the molecular density at an imaginary boundary, and $\bar{E}$ is the mean value of the energy of the molecules. The value of the mean energy is, from equations ( 457 ),

$$
\bar{E}=\frac{1}{2} R\left\{(n+3) T+\tau_{1}+\tau_{2}+\ldots\right\}
$$

Instead of equation (511), the equation of calorimetry can now be written

$$
d \mathbb{Q}=R \nu_{b} T d v+\frac{1}{2} N R\left\{(n+3) d T+d \tau_{1}+d \tau_{2}+\ldots\right\} \ldots \ldots(513),
$$

and on division by $T$

$$
\frac{d \mathbb{Q}}{T}=R \nu_{b} d v+\frac{1}{2} N R(n+3) \frac{d T}{T}+\frac{1}{2} N R\left\{\frac{d \tau_{1}}{T}+\frac{d \tau_{2}}{T}+\ldots\right\} \ldots \ldots
$$

On the right-hand side the terms $\frac{d \tau_{1}}{T}, \frac{d \tau_{2}}{T}$ are not perfect differentials, so that there is no theorem corresponding to that of $\S 187$ (the Second Law of Thermodynamics) when the energy of subsidiary temperatures is taken into account.

\section{Specific Heat at Constant Volume.}

260. To find $C_{v}$, the specific heat at constant volume, we suppose that a quantity $d \mathbb{Q}$ of heat is absorbed by the gas, while the volume remains constant. In this case equation (513) assumes the form

$$
d \mathbb{Q}=\frac{1}{2} N R\left\{(n+3) d T+d \tau_{1}+d \tau_{2}+\ldots\right\}
$$

in which, since $\tau_{1}=\nu f_{1}(T)$ etc., we have

$$
d \tau_{1}=\nu f_{1}^{\prime}(T) d T
$$

Putting $d \mathbb{Q}=C_{v} J N m d T$ we obtain

$$
C_{v}=\frac{1}{2} \frac{R}{J m}\left\{n+3+\nu\left(f_{1}^{\prime}(T)+f_{2}^{\prime}(T)+\ldots\right)\right\} .
$$

It therefore appears that $C_{v}$ is, in general, a function of the temperature, but that it may be regarded as constant at temperatures at which the gas is not incandescent. The value of $C_{v}$, we notice, is in no way affected by deviations from Boyle's law. 


\section{Specific Heat at Constant Pressure.}

261. To find $C_{p}$, the specific heat at constant pressure, we suppose that the absorption of heat takes place while the pressure of the gas is maintained constant. The relation $d p=0$ will lead to a relation between $d T$ and $d v$, which must be substituted in equation (514).

If we assume for $p$ the form obtained in Chapter VI., namely

$$
p=\frac{R T}{m} \rho_{b} e^{-\frac{\rho \psi}{R T}}
$$

it is clear that the resulting equation will be one of extreme complexity, and the results will be so complicated as to be unintelligible. Let us, then, simplify the pressure equation by dropping the cohesion factor and assuming the relation

$$
p=\nu_{b} R T
$$

leaving aside for the present any discussion of the exact meaning of this procedure. The relation connecting volume and temperature when the pressure is kept constant is now $d\left(\nu_{b} T\right)=0$, and this may be written

$$
\frac{d T}{T}=-\frac{d \nu_{b}}{\nu_{b}}=-\frac{d v}{\nu_{b}\left(\frac{d v}{d \nu_{b}}\right)}
$$

Using this value for $d v$, and writing from equation (503)

$$
\begin{aligned}
d \tau_{1} & =f_{1}(T) d \nu+\nu f_{1}^{\prime}\left(T^{\prime}\right) d T, \\
& =-\frac{\nu^{2}}{N} f_{1}(T) d v+\nu f_{1}^{\prime}(T) d T,
\end{aligned}
$$

we find that equation (513) becomes

$$
\begin{aligned}
d \mathbb{Q}=d v\left\{R \nu_{b} T-\frac{1}{2} \frac{R \nu^{2}}{N}\right. & \left(f_{1}(T)+f_{2}(T)+\ldots\right) \\
& \left.+\frac{1}{2} N R d T\left\{n+3+\nu\left(f_{1}^{\prime}(T)+f_{2}^{\prime}(T)+\ldots\right)\right\}\right\} \\
=d T\left\{R \frac{d v}{d\left(1 / \nu_{b}\right)}+\right. & \frac{1}{2} \frac{R \nu^{2} \nu_{b}}{N T} \frac{d v}{d \nu_{b}}\left(f_{1}(T)+f_{2}(T)+\ldots\right) \\
& \left.+\frac{1}{2} N R\left\{n+3+\nu\left(f_{1}^{\prime}(T)+f_{2}^{\prime}(T)+\ldots\right)\right\}\right\}
\end{aligned}
$$

The value of $d \mathbb{Q}$ is now $C_{p} J N m d T$, so that

$$
\begin{aligned}
C_{p}=\frac{R}{J N m}\left\{\frac{d v}{d\left(1 / \nu_{b}\right)}+\frac{1}{2}\right. & \frac{\nu^{2} \nu_{b}}{N T} \frac{d v}{d \nu_{b}}\left(f_{1}(T)+\ldots\right) \\
& \left.+\frac{1}{2} N\left\{n+3+\nu\left(f_{1}^{\prime}(T)+\ldots\right)\right\}\right\}
\end{aligned}
$$

and it is clear from equations (516) and (518) that the quantities $C_{p}, C_{v}, C_{p}-C_{v}$ and $C_{p} / C_{v}(=\gamma)$ are no longer constants when the subsidiary temperatures are taken into account. 
Specific Heats when Subsidiary Temperatures are neglected.

262. If we neglect subsidiary temperatures, the values of the specific heats assume the simple forms.

$$
\begin{aligned}
& C_{v}=\frac{1}{2} \frac{R}{J m}(n+3) \ldots \ldots \ldots \ldots \ldots \ldots \ldots \ldots \ldots \\
& C_{p}=\frac{1}{2} \frac{R}{J m}\left\{(n+3)+\frac{2}{N} / \frac{d}{d v}\left(\frac{1}{\nu_{b}}\right)\right\}
\end{aligned}
$$

The quantities $C_{p}, C_{p}-C_{v}$ and $\gamma$ accordingly depend on the deviations from Boyle's law.

If we expand $\nu_{b}$ as far as the first order of small quantities in the form $\nu_{b}=\frac{N}{v-b}$, where $b$ is the $b$ of Van der Waals' equation,

$$
\frac{d}{d v}\left(\frac{1}{\nu_{b}}\right)=\frac{1}{N} \frac{d}{d v}(v-b)=\frac{1}{N},
$$

and equation (520) becomes

$$
C_{p}=\frac{1}{2} \frac{R}{J m}(n+5)
$$

so that as far as the first order of small quantities the quantities $C_{p}, C_{v}$, and of course also their difference and their ratio, become constants. The value of $\gamma$ is now

$$
\gamma=\frac{C_{p}}{C_{v}}=1+\frac{2}{n+3} .
$$

These results are exactly those which would be obtained if the molecules possessed no energy except that of the $(n+3)$ momentoids governed by the principal temperature.

\section{Experimental Values.}

$$
\text { Values of } C_{v} \text {. }
$$

263. With the help of the formula

$$
C_{v}=\frac{1}{2} \frac{R}{\sqrt{m}}(n+3)
$$

it is easy to deduce the value of $n$, from observations on the value of $C_{v}$. The value of $J$ is known, to within sufficient accuracy for the present purpose, to be

$$
J=4 \cdot 22 \times 10^{7} .
$$

The value of $R / m$ has already been tabulated for a number of gases (p. 113). Hence we obtain the following table: 
VALUES OF $C_{v}$ AT $0^{\circ} \mathrm{C}$.

\begin{tabular}{|c|c|c|c|c|c|}
\hline Gas & $C_{v}$ (observed) & Authority & Mean $C_{v}$ & $\frac{1}{2} \frac{R}{J m}$ & $n+3$ \\
\hline Hydrogen.......... & $2 \cdot 4269$ & Wüllner* & $2 \cdot 4269$ & $\cdot 489$ & $4 \cdot 96$ \\
\hline Nitrogen .......... & $0 \cdot 1738$ & Wüllner & $0 \cdot 1738$ & $\cdot 0349$ & $4 \cdot 98$ \\
\hline Ethylene ....... & $\begin{array}{l}0 \cdot 2782 \\
0 \cdot 2701\end{array}$ & $\begin{array}{c}\text { Wüllner } \\
\text { Wiedemannt }\end{array}$ & $0 \cdot 2741$ & .0349 & $7 \cdot 85$ \\
\hline Carbon monoxide & $\begin{array}{l}0 \cdot 1732 \\
0 \cdot 1729\end{array}$ & $\begin{array}{c}\text { Wiillner } \\
\text { Wiedemann }\end{array}$ & $0 \cdot 1730$ & .0350 & $4 \cdot 94$ \\
\hline Air....................... & $\begin{array}{l}0 \cdot 1701 \\
0 \cdot 1690\end{array}$ & $\begin{array}{c}\text { Wüllner } \\
\text { Wiedemann }\end{array}$ & $0 \cdot 1695$ & $\cdot 0340$ & 4.99 \\
\hline Nitric Oxide...... & $0 \cdot 1665$ & Wüllner & $0 \cdot 1665$ & .0329 & $5 \cdot 06$ \\
\hline Oxygen .............. & $0 \cdot 1563$ & Wüllner & $0 \cdot 1563$ & $\cdot 0308$ & $5 \cdot 08$ \\
\hline Carbon dioxide... & $\begin{array}{l}0 \cdot 1466 \\
0 \cdot 1489\end{array}$ & $\begin{array}{l}\text { Wüllner } \\
\text { Wiedemann }\end{array}$ & $0 \cdot 1477$ & $\cdot 0224$ & $6 \cdot 60$ \\
\hline Nitrous oxide ... & $\begin{array}{l}0 \cdot 1453 \\
0 \cdot 1513\end{array}$ & $\begin{array}{l}\text { Wüllner } \\
\text { Wiedemann }\end{array}$ & $0 \cdot 1483$ & .0224 & $6 \cdot 62$ \\
\hline
\end{tabular}

The values of $C_{v}$ given in the first column are not obtained by direct observations on $C_{v}$, but have been arrived at by using experimental values of $C_{p}$ in combination with the known value of either the ratio or difference of the two specific heats. There is only one method of determining $C_{v}$ directly, namely by the use of Joly's steam calorimeter, and the results of these experiments are hardly suitable for our present purpose.

\section{Values of $\dot{C}_{p}$.}

264. Since we have obtained the formula

$$
C_{p}=C_{v}+\frac{R}{J m}
$$

it appears that this table contains material for the calculation of $C_{p}$. It is of interest to calculate $C_{p}$ in this way, and to compare the results with those obtained by direct experiment. This is done in the following table, the experimental values being Regnault's values as quoted in a table in Travers' Study of Gases, p. 267.

* Meyer, Kinetic Theory, p. 295.

† ibid. p. 293. 
VALUES OF $C_{p}$ AT $0^{\circ} \mathrm{C}$.

\begin{tabular}{|c|c|c|c|c|}
\hline Gas & Mean $C_{v}$ & $\frac{R}{J m}$ & $C_{p}$ (calc.) & $C_{p}$ (obs.) \\
\hline Hydrogen ......... & $2 \cdot 4269$ & .978 & $3 \cdot 405$ & $3 \cdot 4046$ \\
\hline Nitrogen .......... & $0 \cdot 1738$ & .0698 & $0 \cdot 2436$ & $0 \cdot 2440$ \\
\hline Carbon monoxide & $0 \cdot 1730$ & .0700 & $0 \cdot 2430$ & $0 \cdot 2479$ \\
\hline Air................... & $0 \cdot 1695$ & .0680 & $0 \cdot 2375$ & $0 \cdot 2374$ \\
\hline Nitric oxide....... & $0 \cdot 1665$ & .0658 & $0 \cdot 2323$ & $0 \cdot 2315$ \\
\hline Oxygen ............. & $0 \cdot 1563$ & .0616 & $0 \cdot 2179$ & $0 \cdot 2182$ \\
\hline Carbon dioxide... & $0 \cdot 1477$ & .0448 & $0 \cdot 1925$ & 0.2164 \\
\hline Nitrous oxide ... & $0 \cdot 1483$ & .0448 & $0 \cdot 1931$ & $0 \cdot 2238$ \\
\hline
\end{tabular}

It will be seen that the agreement is good, except in the case of the last two gases. Since, however, $C_{v}$ was only arrived at by using an observed value of $C_{p}$, the agreement means little more than that various disturbing factors, such as the deviation from Boyle's Law, are of small importance. These deviations are allowed for in calculating the values of $C_{v}$ given in the former table, but are not allowed for by our simple theory.

\section{Values of $\gamma$.}

265. The last table suggests the possibility of calculating the ratio $\gamma$ $\left(=C_{p} / C_{v}\right)$ from the first and last columns. In the following table $\gamma$ is calculated in this way, and is compared with various observed values of $\gamma$, determined for the most part directly by methods depending on the velocity of sound or adiabatic expansion, and not on the separate values of $C_{p}$ and $C_{v}$. The observed values are largely taken from Meyer's Kinetic Theory, p. 125, and Travers' Study of Gases, p. 277. The values ascribed to Lummer and Pringsheim are from a paper in Wied. Annalen, LXIV. p. 555. 
VALUES OF $\gamma$.

\begin{tabular}{|c|c|c|c|c|}
\hline Gas & $\gamma$ (observed) & Authority & Mean & $\gamma$ (calc.) \\
\hline \multirow[t]{3}{*}{ Hydrogen .......... } & $1 \cdot 394$ & Dulong & & \\
\hline & $1 \cdot 405$ & Masson & $1 \cdot 402$ & $1 \cdot 403$ \\
\hline & $1 \cdot 408$ & Lummer \& Pringsheim & & \\
\hline \multirow{4}{*}{$\begin{array}{l}\text { Nitrogen ......... } \\
\text { Carbon monoxide }\end{array}$} & $1 \cdot 405$ & Masson & $1 \cdot 405$ & $1 \cdot 404$ \\
\hline & $1 \cdot 403$ & Wüllner & & \\
\hline & $1 \cdot 410$ & Dulong & $1 \cdot 409$ & $1 \cdot 433$ \\
\hline & $1 \cdot 413$ & Masson & & \\
\hline \multirow[t]{5}{*}{ Air................... } & $1 \cdot 4004$ & Lummer \& Pringsheim & & \\
\hline & $1 \cdot 4053$ & Röntgen & & \\
\hline & $1 \cdot 4053$ & Wüllner & $1 \cdot 4051$ & $1 \cdot 405$ \\
\hline & $1 \cdot 4106$ & Kaysir & & \\
\hline & $1 \cdot 4040$ & Leduc & & \\
\hline \multirow{5}{*}{$\begin{array}{l}\text { Nitric oxide....... } \\
\text { Oxygen ............. }\end{array}$} & $1 \cdot 394$ & Masson & $1 \cdot 394$ & $1 \cdot 390$ \\
\hline & 1.398 & Lummer \& Pringsheim & & \\
\hline & $1 \cdot 402$ & Dulong & & \\
\hline & $1 \cdot 403$ & Müller & $1 \cdot 402$ & $1: 396$ \\
\hline & $1 \cdot 405$ & Masson & & \\
\hline \multirow[t]{9}{*}{ Carbon dioxide... } & $1 \cdot 265$ & Muiller & & \\
\hline & $1 \cdot 277$ & Masson & & \\
\hline & $1 \cdot 292$ & Lucchi & & \\
\hline & $1 \cdot 300$ & Lummer \& Pringsheim & & \\
\hline & $1 \cdot 305$ & Röntgen & 1.300 & 1.466 \\
\hline & $1 \cdot 308$ & Capstick & 17 & 17.80 \\
\hline & $1 \cdot 311$ & Wiillner & 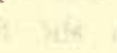 & \\
\hline & $1 \cdot 326$ & Dulong & & \\
\hline & $1 \cdot 319$ & Leduc & & $\cos 1$ \\
\hline \multirow[t]{3}{*}{ Nitrous oxide ... } & $1 \cdot 270$ & Masson & is & $x_{15}$ \\
\hline & $1 \cdot 311$ & Wüllner & $1 \cdot 304$ & $1 \cdot 509$ \\
\hline & $1 \cdot 331$ & Dulong & & \\
\hline
\end{tabular}

We again find that the agreement is excellent except in the case of the last two gases. 
The following table gives the values of $\gamma$ for some other gases, which are of interest :

VALUES OF $\gamma$.

\begin{tabular}{|c|c|c|c|}
\hline Gas & $\gamma$ (observed) & Authority & Mean \\
\hline Mercury ...... & $1 \cdot 666$ & Kundt \& Warburg & $1 \cdot 666$ \\
\hline Krypton & 1.666 & Rayleigh \& Ramsay & $1 \cdot 666$ \\
\hline Helium ...... & 1.652 & Travers & $1 \cdot 652$ \\
\hline Argon ... & $1 \cdot 64$ & Rayleigh & $1 \cdot 625$ \\
\hline & $1 \cdot 61$ & Ramsay & \\
\hline Hydrochloric acid ... & $1 \cdot 392$ & Masson & \\
\hline & $1 \cdot 394$ & Strecker & $1 \cdot 395$ \\
\hline & $1 \cdot 398$ & Müller & \\
\hline Chlorine........... & $1 \cdot 323$ & Strecker & 1.329 \\
\hline & $1 \cdot 336$ & Martini & \\
\hline Ethylene ................ & $1 \cdot 228$ & Dulong & \\
\hline & $1 \cdot 264$ & Capstick & \\
\hline & $1 \cdot 245$ & Wuillner & $1 \cdot 248$ \\
\hline & $\begin{array}{l}1 \cdot 243 \\
1 \cdot 260\end{array}$ & Müller & \\
\hline & 1200 & Hadsond & \\
\hline
\end{tabular}

Values of $n$.

266. When we know the value of $\gamma$, it is obviously possible to calculate the value of $n$ from the formula

$$
\gamma=1+\frac{2}{n+3}
$$

This has been done in the following table of values of $(n+3)$. The values of $(n+3)$ obtained from $C_{p}$ and $C_{v}$ separately (pp. 218, 219) are given for the sake of comparison. 
VALUES OF $n+3$.

\begin{tabular}{|c|c|c|c|c|}
\hline Gas & $\begin{array}{c}\text { Calculated } \\
\text { from mean } \gamma\end{array}$ & $\begin{array}{c}\text { Calculated } \\
\text { from mean } C_{v}\end{array}$ & $\begin{array}{l}\text { Calculated } \\
\text { from } C_{p}\end{array}$ & Mean \\
\hline Mercury.............. & 3.00 & - & - & 3.00 \\
\hline 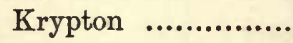 & 3.00 & - & - & 3.00 \\
\hline Helium ................... & $3 \cdot 07$ & - & - & 3.07 \\
\hline 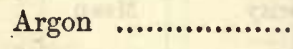 & $3 \cdot 20$ & - & - & $3 \cdot 20$ \\
\hline Hydrogen ................ & $4 \cdot 98$ & $4 \cdot 96$ & $4 \cdot 96$ & 4.97 \\
\hline Nitrogen ............. & $4 \cdot 94$ & $4 \cdot 98$ & $4 \cdot 99$ & 4.97 \\
\hline Carbon monoxide ... & $4 \cdot 89$ & $4 \cdot 94$ & $5 \cdot 01$ & 4.95 \\
\hline 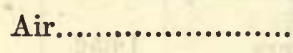 & $4 \cdot 95$ & $4: 99$ & 4.98 & 4.97 \\
\hline Nitric oxide ............. & $5 \cdot 08$ & $5 \cdot 06$ & $5 \cdot 04$ & 5.06 \\
\hline Oxygen .................. & $4: 98$ & $5 \cdot 08$ & $5: 08$ & $5 \cdot 05$ \\
\hline Hydrochloric acid ... & $5 \cdot 06$ & - & - & $5 \cdot 06$ \\
\hline Chlorine $\quad \ldots . . . . . . . . . . .$. & 6.08 & - & - & 6.08 \\
\hline Carbon dioxide & $6 \cdot 67$ & $6 \cdot 60$ & $7 \cdot 66$ & 6.98 \\
\hline Nitrous oxide .......... & $6 \cdot 58$ & $6 \cdot 62$ & $7 \cdot 69$ & 6.96 \\
\hline Ethylene ................... & $8 \cdot 06$ & $7 \cdot 85$ & - & $7 \cdot 95$ \\
\hline
\end{tabular}

\section{Discussion of RESULTS.}

267. If the experimental values were entirely trustworthy, and if our theory had taken a complete account of the physical conditions, the values obtained for $n$ by all the three methods ought to be the same, and these values ought to be exact integers in every case.

Approximately this is so, although the divergencies from the values indicated by theory are sufficiently marked to be noticeable. Undoubtedly this is partly due to the inaccuracies of experiment. For instance in the case of oxygen the values of $n+3$ calculated from the observed value of $C_{v}$ and $C_{p}$ are each 5.08, so that the discrepancy between this value and the value 4:98 obtained by calculation from direct observations of $y$ cannot be laid to the charge of the Kinetic Theory, but must be attributed to imperfections of observation. It is, in general, very much easier to obtain good experimental values of $\gamma$ from observations on the velocity of sound, than by finding $C_{v}$ and $C_{p}$ separately, so that the values of $n+3$ in the first column of the table ought to be given more weight than those in the other columns.

We shall find it convenient to discuss first the meaning of the values found for $n$ on the supposition that they are exact integers. 


\section{Monatomic Gases.}

268. There are four gases in the table, namely mercury, krypton, helium and argon, for which we may take $n+3=3$, corresponding to the value $\gamma=1 \frac{2}{3}$. For these gases, then, we have $n=0$; and since $n$ is the number of degrees of freedom, other than those of translation for which the energy is governed by the principal temperature, the result that $n=0$ must be interpreted to mean that the whole energy of the molecules of these gases is translational. This can only occur if the molecules of the gas are very approximately spherical in shape, and spherically symmetrical as regards internal structure. For if this were not so, an appreciable fraction of the translational energy of a molecule would be transformed into rotational energy at each collision. Now if on the one hand we assume the dissipation of rotational energy to be slight, this would be inconsistent with the value $\gamma=1 \frac{2}{3}$; while if, on the other hand, we do not assume the dissipation of rotational energy to be slight, it would be inconsistent with the observed slowness of loss of energy by the gas as a whole. We conclude then that the molecules of these substances are spherical. It follows that the molecules of the gases in question cannot be composed of two or more atoms, and we therefore conclude that the substances are monatomic. This is the view generally accepted by chemists. It rests primarily, at any rate in the case of krypton, helium and argon, upon the observed value of $\gamma$, but it accords well with the known chemical inertness of the substances. The Kinetic Theory, then, suggests the additional information that the atoms of these elements are spherical as regards both shape and internal structure.

269. After $n+3=3$, the next possible value for $n+3$ allowed by theory is $n+3=4$, corresponding to the value $\gamma=1 \frac{1}{2}$. In the table there is no gas for which $n+3=4$, and there is no gas known for which $\gamma=1 \frac{1}{2}$, even approximately. This, however, upon closer examination is seen to be additional confirmation of the truth of the theory. As a matter of geometry, a body, if not spherical, can either have one axis of symmetry, in which case its surface is a figure of revolution, or none at all. In the former case the energy of one of the three momentoids of rotation may be small; in the latter case none of these three energies can be small. It is impossible for the energy of two of the three momentoids of rotation to be small, while the third is not small. Thus a molecule for which $n=1$ is impossible, and therefore the value $\gamma=1 \frac{1}{2}$ is shewn by the Kinetic Theory to be an impossible one for any element.

\section{Diatomic Gases.}

270. After $n+3=3$, then, the next possible value is $n+3=5$, giving $\gamma=1 \frac{2}{5}$, and corresponding to a molecule possessing symmetry about an axis, as regards both shape and structure. From the table it appears that $n+3$ 
and $\gamma$ have very approximately these values in the case of air, of the elements hydrogen, nitrogen and oxygen, and of the gases $\mathrm{CO}, \mathrm{NO}$ and $\mathrm{HCl}$. These molecules are all diatomic, so that the structure which our theory indicates is fully in agreement with what we should expect. A molecule symmetrical about an axis as regards both shape and structure would obviously be formed by the conjunction of two spherical atoms. It might also conceivably be formed by two atoms which were themselves not spherical but each symmetrical about an axis, the axes coinciding with that of the molecule as a whole. The original shape of the atoms, whether spherical or not, will possibly be lost when the atoms are placed under the influence of each otber's forces, but there is nothing to destroy the symmetry about the single axis of figure of the whole molecule.

\section{More complex Gases.}

271. The next value possible is $n+3=6$, giving $\gamma=1 \frac{1}{3}$. This is obviously the value which ought to be found for a gas in which the molecules may be regarded as rigid bodies, having no axis of symmetry. We might reasonably have expected all triatomic molecules and molecules of higher atomicity to be of this type.

It is, however, quite clear from experiment that for many gases the value of $\gamma$ is very much lower than $1 \frac{1}{3}$. In other words, the molecules of many substances must not be regarded simply as rigid bodies, for the energy of internal motion is comparable with the energies of translation and rotation.

272. Various attempts have been made to connect the values of $n$ and $\gamma$ with the number of atoms in the molecule. Naumann*, for instance, suggested that $n$ is identical with the number of atoms in the molecule, while J. J. Thomson $\nmid$ suggests that in the special case of a symmetrically arranged molecule, $n+3$ may be found to be proportional to the number of atoms in the molecule.

The experiments of Capstick + have, however, shewn quite conclusively that no general law can be expected to relate $\gamma$ with the number of atoms, independently of the nature of the atoms. For instance, he finds the following values for the methane derivatives:

$\begin{array}{lccc} & & \gamma & n+3 \\ \text { Methane } & \mathrm{CH}_{4} & 1 \cdot 313 & 6 \cdot 4 \\ \text { Methyl Chloride } & \mathrm{CH}_{3} \mathrm{Cl} & 1 \cdot 279 & 7 \cdot 2 \\ \text { Methylene Chloride } & \mathrm{CH}_{2} \mathrm{Cl}_{2} & 1 \cdot 219 & 9 \cdot 0 \\ \text { Chloroform } & \mathrm{CHCl}_{3} & 1 \cdot 154 & 13 \cdot 0 \\ \text { Carbon tetrachloride } & \mathrm{CCl}_{4} & 1 \cdot 130 & 15 \cdot 4\end{array}$

* Annalen der Chemie, cxurrr. (1867) p. 284.

+ Watts' Dictionary of Chemistry, I. p. 89.

† Phil. Trans. cluxxvi. (1895) p. 564 ; clxxxv. (1894) p. 1. 
Thus the introduction of the series of chlorine atoms increases $n$ very perceptibly at every step.

A similar result was obtained by Strecker*, who shewed that hydrochloric, hydrobromic, hydriodic acids all have approximately the same values as hydrogen, namely

$$
\gamma=1 \cdot 4, \quad n+3=5,
$$

while for chlorine, bromine and iodine, the values are approximately

$$
\begin{array}{lll}
\text { Chlorine } & \gamma=1 \cdot 333 & n+3=6 \\
\text { Bromine, Iodine } \gamma=1 \cdot 293 & n+3=6.8 .
\end{array}
$$

Similarly for the iodides of bromine and chlorine,

$$
\begin{array}{lll}
\text { Bromine iodide } & \gamma=1.33 & n+3=6 \\
\text { Chlorine iodide } & \gamma=1.317 & n+3=6.3 .
\end{array}
$$

From these figures it appears that one halogen can be put in the place of hydrogen without producing any difference in the values of $\gamma$ and $n$, but that the substitution of the second halogen atom causes a marked increase in $n$. Capstick + finds a similar phenomenon in the case of the paraffin derivatives. In general the second chlorine atom introduced into the molecule causes a large change, although the first may or may not do so.

These facts will probably shew that the problem of determining a relation between the value of $n$ and the structure of the molecule is one of extreme complexity, and moreover that it is a problem for the chemist rather than for the mathematician.

\section{Non-integral values for $n$.}

273. A question which is still standing unanswered, is that of why the values found for $n+3$ are not exactly integral. Now in deducing the result that this value ought to be integral, we have throughout supposed the gas to consist of exactly similar molecules, whereas it is known that gases may be partially in a state of dissociation or molecular aggregation. A gas which is partially dissociated may be regarded as a mixture of the nondissociated gas, and another gas of simpler molecular structure. The observed value for $\gamma$ will in this case not be one of the series of values given by the simple theory, but will be intermediate between the values appropriate for the component gases, so that the value of $n$ deduced will not be integral. The case of molecular aggregation is similar. Obviously dissociation, being a tendency towards a simpler structure of molecule, tends to raise the value of $\gamma$, whereas aggregation, being a tendency towards a more complex structure, tends to decrease the value of $\gamma$. see Chapter $\bar{x}$

* Wied. Ann. xIIr. p. 20; xvir. p. 85.

+ l. c. ante. 
Since, then, the amount of dissociation in a gas increases with the temperature, the effect of dissociation ought to be to cause the value of $\gamma$ to decrease with the temperature. At any particular temperature we cannot say whether the effect of molecular aggregation or of dissociation is likely to be the greater. We cannot therefore say whether the actual value of $\gamma$ ought to be greater or less than that given by formula (522); all we can feel any confidence about is that the influence of both ought to be shewn in a decrease of $\gamma$ as the temperature increases. This decrease is casily observed in the case of large numbers of gases, and so far as I am aware, no gas is known in which $\gamma$ increases with the temperature.

The consideration of dissociation and aggregation, combined with the imperfection of experimental results, may be capable alone of giving a sufficient explanation of all discrepancies between theory and experiment.

274. There is, however, another consideration, which ought to be mentioned if only for the reason that it is impossible $\grave{a}$ priori to say how much weight ought to be assigned to it.

We have assumed that the subsidiary temperatures may be neglected altogether, and unfortunately the general argument by which this assumption was justified may break down in exactly those special cases which are of the greatest importance. The essential points of the question are all contained in the problem of the loaded spheres. We found, in equation (463), that the normal state at low temperatures is defined by the relation

$$
\beta \nu \mathrm{K}^{\frac{3}{2}}=3 \epsilon \mathrm{H} \text {. }
$$

Here $K$ is the energy of translation, and $H$ is the energy of rotation about an axis which is almost, but not quite, one of symmetry. The quantity $\beta$, which could be regarded as measuring the ease with which rotational energy was set up by collisions, was small. Hence for sufficiently small values of $\mathrm{K}, \epsilon \mathrm{H}$ could be small, and the dissipation of energy was small. In the case in nature to which this is analogous, $\beta$ is small on account of the approximate symmetry about the axis, so that $\mathrm{eH}$ can be small, in agreement with observation. But there is no evidence that $\epsilon$ is not very small: indeed the evidence points rather in the other direction, for we have already supposed $\epsilon$ to be very small as regards rotations about axes perpendicular to the axis of symmetry. Thus it is possible that instead of equation (523) being satisfied by the simultaneous smallness of $\beta$ and $H$ it may be satisfied by the simultaneous smallness of $\beta$ and $\epsilon_{0}$. This is quite consistent with the observed smallness of dissipation: it affects the argument of $\S 232$ in that instead of the equation for the normal state being equation (523) it will be

$$
\beta \nu K^{3}\left(1-\frac{3}{2} \frac{H}{K}\right)=3 \epsilon H
$$


and $H / K$ is no longer a small quantity. Hence we cannot safely neglect those particular subsidiary temperatures which measure energy of rotation about an axis of symmetry.

If we suppose $\mathrm{H}$ to be comparable with $\mathrm{K}$ in equation (524), the value of $\mathrm{H}$ in the normal state is given by

or

$$
\begin{aligned}
3 \epsilon \mathrm{H} & =\frac{\beta \nu \mathrm{K}^{\frac{3}{2}}}{1+\frac{\beta \nu \mathrm{K}^{\frac{1}{3}}}{2 \epsilon}}, \\
\mathrm{H} & =\frac{\frac{2}{3} \mathrm{~K}}{1+\frac{3 \epsilon}{\beta \nu \mathrm{K}^{\frac{1}{2}}}},
\end{aligned}
$$

so that $\mathrm{H}$ and $\mathrm{K}$ may be in any ratio.

In general when a subsidiary temperature cannot be neglected, it is clear that the value of $\gamma$ obtained after taking account of the influence of this subsidiary temperature must be expected to vary with $T$.

Neglecting the deviation from Boyle's Law, we may replace $\nu_{b}$ by $\nu$, and then obtain from equations (516) and (518),

$$
\gamma=1+\frac{2-\frac{\nu}{T}\left(f_{1}(T)+f_{2}(T)+\ldots\right)}{(n+3)+\nu\left(f_{1}^{\prime}(T)+f_{2}^{\prime}(T)+\ldots\right)}
$$

Since $f_{1}(T), f_{2}(T) \ldots$ and $f_{1}^{\prime}(T), f_{2}^{\prime}(T)$ are positive and increase with $T$, the value of $\gamma$ given by this equation will bo smaller than the corresponding value given by the formula

$$
\gamma=1+\frac{2}{n+3}
$$

and will decrease with the temperature.

275. We have now found two causes which ought to combine in producing a decrease in the value of $\gamma$ as the temperature increases. Also we can see that the amount of variation in the value of $\gamma$ ought to be greatest for gases in which the value of $\gamma$ is furthest removed from the values of the form

given by simple theory.

$$
1+\frac{2}{n+3}
$$

These conclusions are fully borne out by experiment. It is impossible to quote the whole experimental evidence. The two following results due to Leduc* may be taken as illustrations:

\begin{tabular}{lrc}
\multicolumn{1}{c}{ Gas } & $\gamma$ at $0^{\circ} \mathrm{C}$. & $\gamma$ at $100^{\circ} \mathrm{C}$. \\
Air & $1 \cdot 4040$ & $1 \cdot 4031$ \\
Carbon Dioxide & $1 \cdot 3190$ & $1 \cdot 2827$.
\end{tabular}

* Leduc, Comptes Rendus, cxxvIr. p. 661. 

Gases.

Further experimental data will be found in Meyer's Kinetic Theory of

276. There are two further complications which ought to be mentioned, although it seems probable that the magnitude of their effect must be considered small in comparison with those just discussed.

We have assumed that it is legitimate to neglect the cohesion-factor. This is certainly not negligible as regards its influence on the boundarypressure and ought therefore to be taken into account in the calculation of $C_{p}$. On the other hand, it seems improbable that it could affect the value of $\gamma$ to an extent comparable with the difference between the observed values of $\gamma$ and those given by assigning integral values to $n$.

277. In discussing the values obtained experimentally for $\gamma$, it onght to be remembered that most of these values have been obtained indirectly by observation of the velocity of propagation of sound through the gas in question. Now in arriving at Laplace's value for the velocity of propagation of sound in a gas*, it is assumed that the ratio of the two specific heats of a gas has a definite value, $\gamma$, which depends solely upon the nature of the gas. This of course amounts to assuming the hypothesis of $\$ 200$, namely that the mean internal energy of a molecule bears, at every instant, a constant ratio to the mean energy of translation.

After the lapse of infinite time, a gas tends to assume a steady state in which the two mean energies may be, as we have seen, supposed to bear to one another this constant ratio; but the case is different with a gas in which the value of one of the quantities in question is continually caused to vary owing to the passage of a wave of sound. For the mechanism by which the balance of energy is adjusted cannot be supposed to work with infinite rapidity, so that the ratio in question will never have the actual value which must be assigned to it in order to arrive at the Laplacean velocity of sound. The question as to whether or not this want of steadiness in the ratio of the two energies is sufficient to influence appreciably the transmission of sound, is therefore seen to be one requiring investigation.

A full investigation of this question is reserved for a later chapter. For the present, we may notice that since there are about $3 \times 10^{9}$ collisions per molecule per second in normal air, and $3 \times 10^{2}$ vibrations per second for sound of average pitch, therefore each molecule must undergo about $10^{7}$ collisions for each wave of sound. It therefore appears that there will be ample opportunity for an approximate adjustment of the ratio of different kinds of energy. We should accordingly expect the value of $\gamma$ to have very approximately the same values, whether calculated from the velocity of sound or. by direct methods.

\footnotetext{
* Lord Rayleigh, Theory of Sound, § 246.
} 


\section{CHAPTER XI.}

\section{FREE PATH PHENOMENA.}

\section{Calculation of the Free Path.}

278. In the problems which have so far been discussed, it has hardly been necessary to consider the dynamics of individual collisions at all. We now approach a class of problems of which the treatment must be made to depend mainly upon the occurrences at individual collisions. It is obvious that we cannot hope to get results of the same degree of accuracy as before, for the results will depend upon the details of occurrences at collisions, and of these we know almost nothing. The present chapter deals with some problems connected with collisions and free paths in a gas, the molecules being assumed for this purpose to be elastic spheres.

\section{Length of mean Free Path.}

279. In $\S 30$ we gave a calculation of the mean free path in a gas. We shall now give a more detailed investigation applicable to the free paths of molecules in a mixture of gases, the molecules of the different gases being of different sizes, and shall at the same time examine the correlation between the velocity of a molecule and its probable free path.

We shall suppose the constants of the molecules of different types to be distinguished by suffixes, those of the first type having a suffix unity $\left(\nu_{1}, m_{1}, \sigma_{1} \ldots\right)$, and so on.

We shall also require a system of symbols to denote the distance apart at collisions of the centres of two molecules of different kinds. Let these be $S_{11}, S_{12}, S_{23}$, etc., $S_{p q}$ being the distance of the centres of two molecules of types $p, q$ when in collision. Obviously, then,

$$
S_{12}=\frac{1}{2}\left(\sigma_{1}+\sigma_{2}\right), \quad S_{11}=\sigma_{1} .
$$

280. Consider a molecule of the first kind, having a velocity $c$ of which the components are $u, v, w$. The chance of collision per unit time with a molecule of the second kind having a specified velocity is equal to the 
probable number of molecules of this second kind in a cylinder of base $\pi S_{12}{ }^{2}$ and height $V$, where $V$ is the relative velocity of the two molecules. If $c$ and $c^{\prime}$ are the resultant velocities of the two molecules, and if these velocities are inclined at an angle $\phi$, the value of $V$ is given by

$$
V^{2}=c^{2}+c^{\prime 2}-2 c c^{\prime} \cos \phi
$$

The number per unit volume of molecules of the second kind moving with a resultant velocity intermediate between $c^{\prime}$ and $c^{\prime}+d c^{\prime}$ is

$$
4 \pi \nu_{2} \sqrt{\frac{h^{3} m_{2}^{3}}{\pi^{3}}} e^{-h m_{x} c^{\prime 2} c^{\prime 2}} d c^{\prime}
$$

These are moving in all directions equally, so that the proportion for which the direction makes an angle intermediate between $\phi$ and $\phi+d \phi$ with that of the first molecule is

$$
\frac{1}{2} \sin \phi d \phi \text {. }
$$

Multiplying expression (527) by this factor we obtain for the number per unit volume of molecules of the second kind for which the velocity is such that $c^{\prime}, \phi$ lie within limits $d c^{\prime}, d \phi$,

$$
2 \pi \nu_{2} \sqrt{\frac{h^{3} m_{2}^{3}}{\pi^{3}}} e^{-h m_{2} c^{\prime 2}} \sin \phi d \phi c^{\prime 2} d c^{\prime} .
$$

The number of molecules satisfying these conditions to be found inside a cylinder of volume $\pi S_{12}{ }^{2} V$ is therefore

$$
2 \nu_{2} S_{12}{ }^{2} \sqrt{\pi h^{3} m_{3}{ }^{3}} V e^{-h m_{2} c^{\prime 2}} \sin \phi d \phi c^{\prime 2} d c^{\prime} .
$$

From equation (526), we obtain by differentiation, keeping $c$ and $c^{\prime}$ constant,

$$
V d V=c c^{\prime} \sin \phi d \phi,
$$

so that expression (528) ean be replaced by

$$
2 \nu_{2} S_{12}{ }^{2} \sqrt{\pi h^{3} m_{2}{ }^{3}} e^{-h m_{2} c^{\prime 2}} \frac{c^{\prime}}{c} d c^{\prime} V^{2} d V .
$$

Keeping $c$ and $c^{\prime}$ constant, the value of $V$ changes as $\phi$ changes. Integrated with respect to this variation we have

$$
\int V^{2} d V=\frac{1}{3}\left[V^{3}\right]
$$

in which the limits for $V$ are $c+c^{\prime}$ and $c \sim c^{\prime}$.

Separating the two cases of $c^{\prime}$ being greater and less than $c$, we find

or

$$
\int V^{2} d V=\frac{2}{3} c\left(c^{2}+3 c^{\prime 2}\right) \text { when } c^{\prime}>c
$$

If, then, we integrate expression (529) with respect to $V$, and if, after substitution from the appropriate one of the equations (530) and (531), we 
again integrate with respect to $c^{\prime}$, we obtain for the aggregate chance per unit time of a collision between a given molecule of the first type moving with velocity $c$, and a molecule of the second type,

$2 v_{2} S_{12}{ }^{2} \sqrt{\pi l^{3} m_{2}{ }^{3}}\left[\int_{c}^{\infty} \frac{2}{3} c^{\prime}\left(c^{2}+3 c^{\prime \prime 2}\right) e^{-h m_{2} c^{\prime 2}} d c^{\prime}+\int_{0}^{c} \frac{2 c^{\prime 2}\left(c^{\prime 2}+3 c^{2}\right)}{3 c} e^{-h m_{2} c^{\prime 2}} d c^{\prime}\right]$

281. The former of the two integrals inside the square bracket can be evaluated directly, and is found to be equal to

$$
\left(\frac{4}{3} h m_{2} c^{2}+1\right) \frac{e^{-h m_{2} c^{2}}}{h^{2} m_{2}{ }^{2}} \text {. }
$$

The second integral cannot be evaluated in finite terms. If, however, we replace $h m_{2} c^{\prime 2}$ by $x^{2}$, the integral reduces to

$$
\frac{1}{c \sqrt{h^{5} m_{2}^{5}}} \int_{0}^{c \sqrt{h m_{2}}} \frac{2}{3} x^{2}\left(x^{2}+3 h m_{2} c^{2}\right) e^{-x^{2}} d x,
$$

and this again, after continued integration by parts with regard to $x^{2}$, is found to be equal to

$$
\frac{1}{c \sqrt{h^{5} m_{2}^{5}}}\left\{-\frac{1}{3} e^{-h m_{2} c^{2}} c \sqrt{h m_{2}}\left(4 h m_{2} c^{2}+\frac{3}{2}\right)+\frac{1}{2}\left(2 h m_{2} c^{2}+1\right) \int_{0}^{c \sqrt{h m_{2}}} e^{-x x^{2}} d x\right\} .
$$

The sum of the two integrals in expression (532) is accordingly

$$
\frac{1}{c \sqrt{h^{5} m_{2}^{5}}}\left[\frac{1}{2} c \sqrt{h m_{2}} e^{-h m_{2} c^{2}}+\frac{1}{2}\left(2 h m_{2} c^{2}+1\right) \int_{0}^{c \sqrt{h m_{2}}} e^{-x^{2}} d x\right] \ldots \ldots
$$

If we introduce a function* $\psi(x)$ defined by

$$
\psi(x)=x e^{-x^{2}}+\left(2 x^{2}+1\right) \int_{0}^{x} e^{-x^{2}} d x
$$

expression (533) may be expressed in the form

$$
\frac{1}{2 c \sqrt{h^{5} m_{2}^{5}}} \psi\left(c \sqrt{h m_{2}}\right)
$$

and hence if we denote expression (532) by $\Theta_{12}$, its value is found to be

$$
\Theta_{12}=\frac{\sqrt{\pi \nu_{2} S_{12}{ }^{2}}}{h m_{2} c} \psi\left(c \sqrt{h m_{2}}\right)
$$

With this definition of $\Theta_{12}$ we see that when a molecule of the first kind is moving with a velocity $c$, the chance that it collides with a molecule of the second kind in time $d t$ is $\Theta_{12} d t$.

* The value of $\int_{0}^{x} e^{-x^{2}} d x$ cannot be expressed in simpler terms, so that $\psi(x)$ as defined by equation (534) is already in its simplest form. Tables for the evaluation of $\psi(x)$ are given in an appendix. 
282. If we change the suffix 2 into 1 wherever it occurs, we obtain an expression $\Theta_{11}$ for the chance per unit time that a molecule of the first kind moving with velocity $c$ shall collide with another molecule of the same kind.

By addition, the total chance per unit time that a molecule of the first kind moving with velocity $c$ shall collide at all with a molecule of any kind is

$$
\Sigma \Theta_{18}=\Theta_{11}+\Theta_{12}+\Theta_{13}+\ldots
$$

In unit time the molecule we are considering describes a distance $c$, hence the chance of collision per unit length of path, for a molecule moving with velocity $c$, is

$$
B_{c}=\frac{1}{c} \Sigma \Theta_{18}
$$

and the mean free path $\lambda_{c}$, for molecules of the first kind moving with velocity $c$, is accordingly

$$
\lambda_{c}=\frac{1}{B_{c}}=\frac{c}{\sum \Theta_{18}}
$$

\section{Tait's Free Path.}

283. When there is only one kind of gas, this assumes the form

$$
\lambda_{c}=\frac{c}{\Theta}=\frac{h m c^{2}}{\sqrt{\pi \nu} \nu \sigma^{2} \psi(c \sqrt{h m})}
$$

and from this formula we can without difficulty calculate Tait's expression for the mean free path defined as explained in $\$ 30$. For, in a single gas, there is at any instant a fraction

$$
\sqrt{\frac{h^{3} m^{3}}{\pi^{3}}} 4 \pi e^{-h m c^{2} c^{2} d c}
$$

of the whole number of molecules moving with velocity $c$, and therefore, on the average, starting to describe distances $\frac{c}{\Theta}$ each before collision. Hence Tait's mean free path $\left(\lambda_{T}\right)$ is given by

$$
\begin{aligned}
\lambda_{T} & =\int_{0}^{\infty} \frac{c}{\Theta} \sqrt{\frac{h^{3} m^{3}}{\pi^{3}}} 4 \pi e^{-h m c^{2}} c^{2} d c \\
& =\frac{1}{\pi \nu \sigma^{2}} \int_{0}^{\infty} \frac{4 x^{4} e^{-x^{2}} d x}{\psi(x)} \ldots \ldots \ldots .
\end{aligned}
$$

This integral can only be evaluated by quadrature. The evaluation has been performed by Tait* and Boltzmannt, who agree in assigning to it the value 0.677 , leading to the value for $\lambda_{T}$ which has already been given in $\S 30$.

* Edin. Trans. xxxır. p. 74 (1886).

+Wiener Sitz. xcvr. p. 305 (1887) ; Gastheorie, r. p. 73. 


\section{Maxwell's Free Path.}

284. From the formula just obtained we can also deduce Maxwell's expression for the free path given in $\S 30$, and can extend it so that it applies to a mixture of gases.

For, from expression (536), the chance of a collision per unit time for a molecule of the first kind moving with velocity $c$ is $\Sigma \Theta_{18}$, and out of all the molecules of the first kind, a fraction

$$
\sqrt{\frac{h^{3} m_{1}^{3}}{\pi^{3}}} 4 \pi e^{-h m_{1} c^{2}} c^{2} d c
$$

will be moving with velocity $c$. Hence the average chance of collision per unit time, for all molecules of the first kind, is

$$
\sqrt{\frac{h^{3} m_{1}^{3}}{\pi^{3}}} \int_{0}^{\infty} 4 \pi e^{-h m_{1} c^{23}} c^{2}\left(\Sigma \Theta_{18}\right) d c,
$$

and this again, by equation (535), is equal to

where as before

$$
\sum_{s} \frac{4 \nu_{s} S_{18}^{2} \sqrt{h^{3} m_{1}^{3}}}{h m_{s}} \int_{0}^{\infty} e^{-h m_{1} c^{2}} c \psi\left(c \sqrt{h m_{s}}\right) d c
$$

If we put

$$
\Psi(x)=x e^{-x^{2}}+\left(2 x^{2}+1\right) \int_{0}^{x} e^{-y^{2}} d y
$$

expression (541) becomes

$$
c \sqrt{h m_{s}}=x,
$$

$$
\sum_{\delta} \frac{4 \nu_{\delta} S_{18}^{2} \sqrt{h^{3} m_{1}^{3}}}{h^{2} m_{s}^{2}} \int_{0}^{\infty} e^{-\frac{m_{1}}{m_{s}} x^{2}} x \psi(x) d x
$$

The integral, upon substitution for $\psi(x)$, becomes the sum of the two integrals

$$
\int_{0}^{\infty} e^{-x^{2}\left(1+\frac{m_{1}}{m_{s}}\right)} x^{2} d x+\int_{0}^{\infty} \int_{0}^{x} x\left(2 x^{2}+1\right) e^{-y^{2}-\frac{m_{1}}{m_{s}} x^{2}} d x d y
$$

The first integral has for its value

$$
\frac{1}{4} \sqrt{\frac{\pi}{\left(1+\frac{m_{1}}{m_{8}}\right)^{3}}}
$$

To evaluate the second we write $y=K x$, so that $d x d y=x d x d K$, and the integral

$$
\begin{aligned}
& =\int_{0}^{\infty} \int_{0}^{1} x^{2}\left(2 x^{2}+1\right) e^{-x^{2}\left(K^{2}+\frac{m_{1}}{m_{s}}\right)} d x d K \\
& =\int_{0}^{1}\left[\frac{3}{4} \sqrt{\frac{\pi}{\left(K^{2}+\frac{m_{1}}{m_{s}}\right)^{5}}}+\frac{1}{4} \sqrt{\frac{\pi}{\left(K^{2}+\frac{m_{1}}{m_{s}}\right)^{3}}}\right] d K \\
& =\frac{3 \sqrt{ } \pi}{4}\left\{\frac{m_{8}{ }^{2}}{m_{1}{ }^{2} \sqrt{1+\frac{m_{1}}{m_{s}}}}-\frac{1}{3} \frac{m_{s}{ }^{2}}{m_{1}{ }^{2}\left(1+\frac{m_{1}}{m_{o}}\right)^{\frac{3}{2}}}\right\}+\frac{\sqrt{ } \pi}{4} \frac{m_{s}}{m_{1} \sqrt{1+\frac{m_{1}}{m_{s}}}} .
\end{aligned}
$$


Adding this to expression (544), we obtain as the value of expression (543),

$$
\frac{\sqrt{ } \pi}{4}\left[\frac{3 \frac{m_{8}{ }^{2}}{m_{1}{ }^{2}}+\frac{m_{8}}{m_{1}}}{\left(1+\frac{m_{1}}{m_{8}}\right)^{\frac{1}{2}}}+\frac{1-\frac{m_{s}{ }^{2}}{m_{1}^{2}}}{\left(1+\frac{m_{1}}{m_{8}}\right)^{\frac{3}{2}}}\right],
$$

of which the value, after simplification, is found to be

$$
\frac{\sqrt{ } \pi}{2} \frac{m_{8}^{2}}{m_{1}^{2}} \sqrt{1+\frac{m_{1}}{m_{s}}} \text {. }
$$

This is the value of the integral which occurs in expression (542). The whole expression is therefore equal to

$$
\sum_{s} 2 \nu_{8} S_{18}{ }^{2} \sqrt{\frac{\pi}{h}\left(\frac{1}{m_{1}}+\frac{1}{m_{8}}\right)}
$$

This is the mean chance of collision per unit time for a single molecule of the first kind, and every collision terminates a free path of this molecule. The total number of free paths described by all molecules of the first kind per unit time per unit volume is therefore

$$
n_{1}=\Sigma 2 \nu_{1} \nu_{8} S_{18}{ }^{2} \sqrt{\frac{\pi}{h}\left(\frac{1}{m_{1}}+\frac{1}{m_{8}}\right)}
$$

This agrees with expression (47), when there is only one kind of gas. The general expression (546) also could have been arrived at by the shorter method of $\S 30$.

The distance described per unit time by the $\nu_{1}$ molecules of the first kind in a unit volume is, as in $\$ 30$, equal to

$$
\frac{2 \nu_{1}}{\sqrt{\pi / h m_{1}}} \text {. }
$$

Hence the mean free path, $\lambda_{1}$, of all molecules of the first kind is

$$
\begin{aligned}
\lambda_{1} & =\frac{2 \nu_{1}}{\mathfrak{u}_{1} \sqrt{\pi h m_{1}}} \\
& =\frac{1}{\pi \Sigma \nu_{s} S_{18}{ }^{2} \sqrt{1+\frac{m_{1}}{m_{s}}}}
\end{aligned}
$$

These formulae are due to Maxwell.

\section{Dependence of Free Path on Velocity.}

285. The way in which $\lambda_{c}$ depends on the value of $c$ is of some interest. The formula expressing $\lambda_{c}$ as a function of $c$ is, however, too complex to convey much definite meaning to the mind, and we are therefore compelled to fall back on numerical values. The following table, which is taken from 
Meyer's Kinetic Theory of Gases (p. 429), gives the ratio of $\lambda_{c}$ to Maxwell's mean free path $\lambda$ for different values of $c$, from $c=0$ to $c=\infty$.

\begin{tabular}{|c|c|c|c|}
\hline$c / \bar{c}$ & $h m c^{2}$ & $\lambda_{c} / \lambda$ & $\lambda / \lambda_{c}$ \\
\hline 0 & & 0 & $\infty$ \\
\hline $0 \cdot 25$ & & $0 \cdot 3445$ & $2 \cdot 9112$ \\
\hline 0.5 & $t_{1}$ & $0 \cdot 6411$ & $1 \cdot 5604$ \\
\hline 0.627 & $\frac{1}{2}$ & 0.7647 & $1 \cdot 3111$ \\
\hline 0.886 & 1 & 0.9611 & 1.0407 \\
\hline $1 \cdot 0$ & & 1.0257 & $\cdot 9749$ \\
\hline $1 \cdot 253$ & 2 & $1 \cdot 1340$ & 8819 \\
\hline 1.535 & 3 & $1 \cdot 2127$ & $\cdot 8247$ \\
\hline 1.772 & 4 & $1 \cdot 2572$ & $\cdot 7954$ \\
\hline 2 & & $1 \cdot 2878$ & $\cdot 7765$ \\
\hline 3 & & $1 \cdot 3551$ & $\cdot 7380$ \\
\hline 4 & & $1 \cdot 3803$ & $\cdot 7244$ \\
\hline 5 & & $1 \cdot 3923$ & $\cdot 7182$ \\
\hline 6 & & $1 \cdot 3989$ & $\cdot 7149$ \\
\hline$\infty$ & & $1 \cdot 4142$ & ·7071 \\
\hline
\end{tabular}

\section{Probability of a given Free Path.}

286. A further problem which is of importance is the probability that a molecule shall describe a free path of given length.

If $f(l)$ is the probability that a molecule shall describe a path at least equal to $l$, then $f(l+d l)$, the probability that a molecule has already described a path $l$ and shall describe a further distance $d l$, will be the product of $f(l)$ and a second factor, this second factor expressing the probability of no collision occurring within the length $d l$. This factor is, however, known to be $1-B_{c} d l$, where $B_{c}$ is given by equation (537). Hence we have the equation

$$
f(l+d l)=f(l)\left(1-B_{c} d l\right),
$$

or, what is the same thing, $\frac{\partial f(l)}{\partial l}=-B_{c} f(l)$,

of which the solution is $\quad f(l)=e^{-B_{c} l}$

the arbitrary constant of the integration being determined by the condition that $f(0)=1$.

The probability of a molecule moving with velocity $c$ describing a free path $\lambda$, of length intermediate between $l$ and $l+d l$, is therefore

$$
B_{c} e^{-B_{c} l} d l
$$


287. It is clear from the form of this expression that free paths which are many times greater than the mean free path will be extremely rare. Since $B_{c}$ is the reciprocal of $\lambda_{c}$ (equation (538)), the probability that a molecule moving with velocity $c$ shall describe a path greater than $n$ times $\lambda_{c}$ is $f\left(n \lambda_{c}\right)$ or, by equation (548), is $e^{-n}$. Thus only onc in 148 describes a path as great as $5 \lambda_{c}$, only one in 22027 a path as great as $10 \lambda_{c}$, only one in $9 \times 10^{43}$ a path as great as $100 \lambda_{c}$, and so on.

This result applies of course only to molecules moving with a given velocity $c$.

At any given instant the fraction of the whole number of molecules which have described a distance greater than $l$ since their last collision, is

$$
\sqrt{\frac{\pi^{3}}{h^{3} m^{3}}} \int_{0}^{\infty} 4 \pi c^{2} e^{-h m c^{2}-B_{c} l} d c
$$

This function is not easy to calculate in any way. As the result of a rough calculation by quadrature, I have found that through the range of values for $l$ in which its value is appreciable, it does not ever differ by more than about 1 per cent. from $e^{-1 \cdot 0 t l / \lambda}$, the value for molecules moving with velocity $1 / \sqrt{h m}$.

\section{Persistence of Velocities after Collision.}

288. We have next to consider what is the average effect of a collision, as regards reversal of path. We shall find that on the average a collision does not reverse the velocity in the original direction of motion, or even reduce it to rest, but that there is a tendency for the original velocity to persist after collision. Obviously it is of importance to form a numerical estimate of the extent to which this occurs.

289. Let us begin by considering two equal molecules colliding with velocities $a, b$, the magnitude of these velocities but not their directions being known. In fig. 17 let $O P$ and $O Q$ represent these velocities, and let $R$ be the middle point of $P Q$. Then we can resolve the motion of the

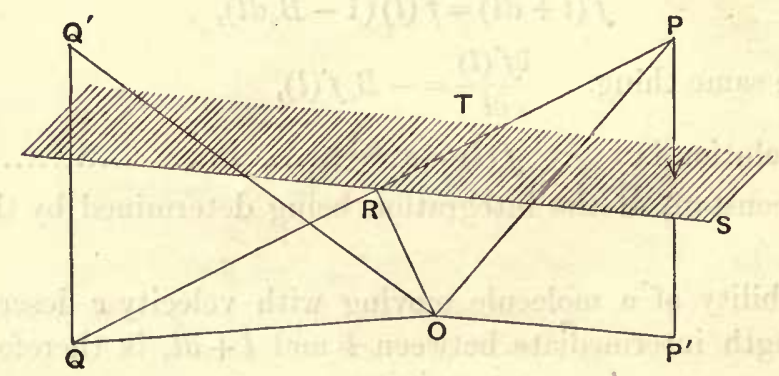

FIG. 17. 
two molecules into (i) a motion of the centre of mass of the two, the velocity of this motion being represented by $O R$, and (ii) two equal and opposite velocities relative to the centre of mass, these being represented by $R P$ and $R Q$.

Imagine a plane RTS drawn throngh $R$ parallel to the common tangent to the spheres at the moment of impact, and let $P^{\prime}, Q^{\prime}$ be the images of $P, Q$ in this plane. Then clearly $R P^{\prime}$ and $R Q^{\prime}$ represent the velocities relatively to the centre of gravity after impact, so that $O P^{\prime}$ and $O Q^{\prime}$ represent the actual velocities in space.

In fig. 18, let the directions of motion relatively to the centre of gravity before impact be $A B, D E$, and let those after impact be $B C, E F$. Then the line of centres bisects each of the angles $A B C, D E F$. Let us call each of

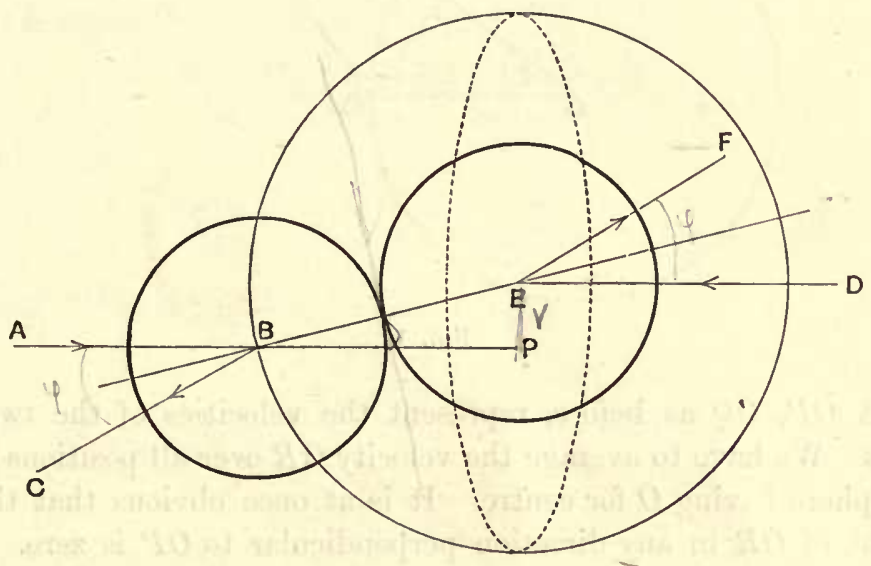

FIG. 18.

these angles $\phi$, measured so as to be acute in the figure. Imagine the point $E$ surrounded by a circle of radius $\sigma$ (the diameter of a molecule) of which the plane is perpendicular to the direction $A B$. Then in order that a collision may take place, the line $A B$ produced must cut the plane of this circle at some point $P$ inside the circle. Also, all positions of $P$ inside this circle are equally probable, so that the probability that the distance $E P$ shall lie between $r$ and $r+d r$ is $\frac{2 r d r}{\sigma^{2}}$. Since $r=\sigma \sin \frac{\phi}{2}$, this may be written $\sin \frac{\phi}{2} \cos \frac{\phi}{2} d \phi$. This, then, is the probability that $\phi$ shall lie between $\phi$ and $\phi+d \phi$, and therefore that the angle which $E F$ makes with $D E$ shall be between $\phi$ and $\phi+d \phi$. The expression found is, however, equal to $\frac{1}{2} \sin \phi d \phi$ and therefore to that part of the area of a unit sphere for which the radius makes an angle between $\phi$ and $\phi+d \phi$ with a given line. Hence 
all directions for $E F$ are equally probable*. Hence in fig. 17 all directions of $R Q^{\prime}, R P^{\prime}$ are equally probable so that the "expectation " of the component of velocity of either molecule after impact in any direction is equal to the component of $O R$ in that direction.

290. Let us now average over all possible directions for the velocity of the second molecule, keeping the magnitude of this velocity constant. In

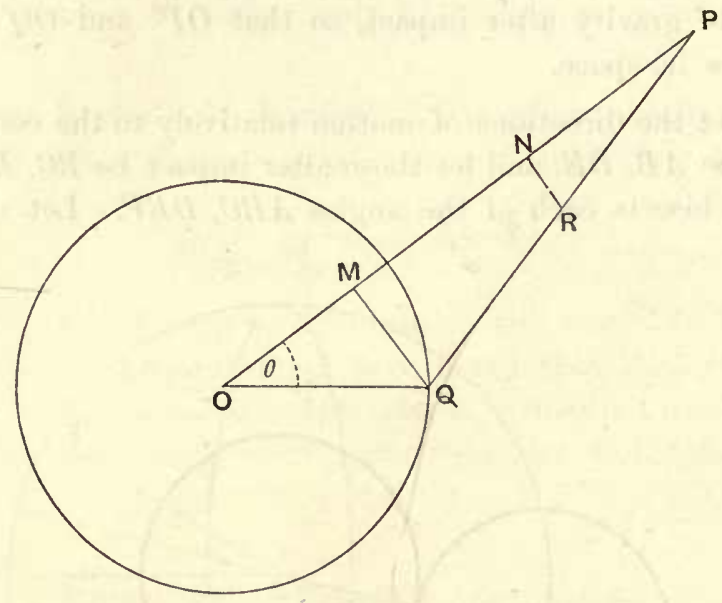

Fia. 19.

fig. 19 let $O P, O Q$ as before, represent the velocities of the two colliding molecules. We have to average the velocity $O R$ over all positions of $Q$ which lie on a sphere having $O$ for centre. It is at once obvious that the average component of $O R$ in any direction perpendicular to $O P$ is zero. We have, therefore, only to find the component in the direction $O P$. We must not suppose all directions for $O Q$ to be equally likely, for (cf. Chapter II.) the probability of collision with any two velocities is proportional to the relative velocity. Thus the probability of the angle $P O Q$ lying between $\theta$ and $\theta+d \theta$ is not simply proportional to $\sin \theta d \theta$, but is proportional to $P Q \sin \theta d \theta$, for $P Q$ represents the relative velocity. The average value of the component $O N$ is therefore

$$
\overline{O N}=\frac{\int_{0}^{\pi} O N \cdot P Q \sin \theta d \theta}{\int_{0}^{\pi} P Q \sin \theta d \theta}
$$

To evaluate this fraction, let us write

$$
O P=a, \quad O Q=b, \quad P Q=r,
$$

so that

$$
r^{2}=a^{2}+b^{2}-2 a b \cos \theta
$$

\footnotetext{
* This theorem was first given by Maxwell in 1859, Collected Works, r. p. 378.
} 
and

$$
\begin{aligned}
O N & =\frac{1}{2}(O P+O M) \\
& =\frac{1}{2}(a+b \cos \theta) \\
& =\frac{1}{4 a}\left(3 a^{2}+b^{2}-r^{2}\right)
\end{aligned}
$$

By differentiation of the relation (552), we have

$$
r d r=a b \sin \theta d \theta .
$$

Hence the fraction (551) becomes

$$
\overline{O N}=\frac{\int\left(3 a^{2}+b^{2}-r^{2}\right) r^{2} d r}{\int 4 a r^{2} d r}
$$

the limits of integration now being from $r=a+b$ to $r=a-b$. When $a>b$, the fraction is equal to

$$
\frac{3 a^{2}+b^{2}}{4 a}-\frac{3}{20 a}\left(\frac{5 a^{4}+10 a^{2} b^{2}+b^{4}}{3 a^{2}+b^{2}}\right)
$$

and when $a<b$, to

$$
\frac{3 a^{2}+b^{2}}{4 a}-\frac{3}{20 a}\left(\frac{5 b^{4}+10 a^{2} b^{2}+a^{4}}{3 b^{2}+a^{2}}\right)
$$

Expression (553) is equal to

$$
\frac{15 a^{4}+b^{4}}{10 a\left(3 a^{2}+b^{2}\right)}
$$

Expression (554) is equal to

$$
\frac{a\left(5 b^{2}+3 a^{2}\right)}{5\left(3 b^{2}+a^{2}\right)}
$$

The essential point is that these expressions are necessarily positive for all values of $a$ and $b$, so that whatever the velocity of the second molecule may be, the "expectation" of the velocity of the first molecule after collision is definitely in the same direction as its velocity before collision. Expressions (555) and (556) are too complex to convey much meaning to the mind. The following table gives $\alpha$, the expectation of velocity in the same direction as $\alpha$, corresponding to different values of the velocity $b$ of the second molecule.

$$
\left.\begin{array}{lcccc}
\frac{b}{a}=0 & \frac{1}{4} & \frac{1}{2} & \frac{3}{4} & 1 \\
\frac{\alpha}{a}=\cdot 500 & \cdot 492 & \cdot 473 & \cdot 437 & \cdot 400
\end{array}\right\}
$$


We have now proved the statement made in $\S 287$, to the effect that there is a tendency for the original velocity to persist after collision. It will be convenient to refer to the ratio $\frac{\alpha}{a}$ as the "persistence" of the velocity $a$. The persistence, then, is measured, as we have found, by a quantity which varies from $33 \frac{1}{3}$ to 50 per cent. of the original velocity, according to the velocity of the colliding molecule.

291. By averaging over all possible velocities we can obtain the mean value of this persistence averaged over all collisions.

From expression (529), we find as the chance per unit time that a molecule moving with velocity $a$ shall collide with a second molecule moving with velocity $b$, in such a direction that their relative velocity is $V$,

$$
2 \nu \sigma^{2} \sqrt{\pi h^{3} m^{3}} e^{-h m b^{2}} \frac{b}{a} d b V^{2} d \dot{V}
$$

and hence for the total number of collisions of this type in the gas, per unit time,

$$
8 \nu^{2} \sigma^{2} h^{3} m^{3} e^{-h m\left(a^{2}+b^{2}\right)} a b d a d b V^{2} d V
$$

Let us suppose that of the two velocities $a$ and $b, a$ is the greater. Then the limits for $V$ are $a+b$ and $a-b$. Integrating expression (558) with respect to $V$ we find as the total number of collisions per unit time in which the velocities of the two colliding molecules are $a, b$,

$$
\frac{16}{3} \nu^{2} \sigma^{2} h^{3} m^{3} e^{-h m\left(a^{2}+b^{2}\right)} a b^{2}\left(b^{2}+3 a^{2}\right) d a d b .
$$

If we put $a=\kappa b$, so that $\kappa$ is greater than unity, then since

this becomes

$$
\frac{\partial(a, b)}{\partial(\kappa, b)}=b
$$

$$
\frac{16}{3} \nu^{2} \sigma^{2} h^{3} m^{3} e^{-h m b^{2}\left(1+\kappa^{2}\right)} \kappa b^{6}\left(3 \kappa^{2}+1\right) d \kappa d b
$$

Integrating with respect to $b$ from $b=0$ to $b=\infty$, we find, for the total number of collisions per unit time in which $\kappa$, the ratio of the greater to the less velocity, lies between $\kappa$ and $\kappa+d \kappa$,

$$
5 \sqrt{\frac{\pi}{h m}} \nu^{2} \sigma^{2} \frac{\kappa\left(3 \kappa^{2}+1\right)}{\left(1+\kappa^{2}\right)^{7 / 2}} d \kappa
$$

Since the total number of collisions, by formula (46), is

$$
\sqrt{\frac{2 \pi}{h m}} \nu^{2} \sigma^{2}
$$

the fraction of the whole number of collisions, for which $\kappa$ lies between $\kappa$ and $\kappa+d \kappa$, is

$$
\frac{5 \kappa\left(3 \kappa^{2}+1\right)}{\sqrt{2}\left(1+\kappa^{2}\right)^{7 / 2}} d \kappa
$$


of which the value, when integrated from $\kappa=1$ to $\kappa=\infty$, is unity, as it ought to be.

From expressions (555) and (556) we find that when two molecules collide, having velocities in the ratio $\kappa: 1$, the "persistences" of the two velocities are

$$
\frac{15 \kappa^{4}+1}{10 \kappa^{2}\left(3 \kappa^{2}+1\right)} \text { and } \frac{5 \kappa^{2}+3}{5\left(3 \kappa^{2}+1\right)}
$$

respectively. Hence the mean "persistence" for the two molecules of the collision, the mean of the two expressions just written down, is

$$
\frac{25 \kappa^{4}+6 \kappa^{2}+1}{20 \kappa^{2}\left(3 \kappa^{2}+1\right)} \text {. }
$$

Multiplying this by expression (561) and integrating from $\kappa=1$ to $\kappa=\infty$, we find, for the mean persistence of all velocities after collision

$$
\int_{1}^{\infty} \frac{\left(25 \kappa^{4}+6 \kappa^{2}+1\right)}{4 \sqrt{2} \kappa\left(1+\kappa^{2}\right)^{7 / 2}} d \kappa
$$

of which the value is found to be

$$
\frac{1}{4}\left(1+\frac{1}{\sqrt{2}} \log _{e}(\sqrt{2}+1)\right),
$$

or $\cdot 406$. Thus the average value of the persistence is very nearly equal to $\frac{2}{5}$, the value when two molecules collide with equal velocities.

292. For other laws of force between molecules the persistence will clearly be different from what it is for elastic spheres. Everything will, however, depend on our definition of a collision. If we suppose that a very small interaction is sufficient to constitute a collision, then the mean free path will be very short while the persistence will be nearly equal to unity. If, on the other hand, we require large forces to come into play before calling a meeting of two molecules a collision, then the free path will be long, but the persistence very small, or perhaps negative. The variation in the value of the persistence of velocities just.balances the arbitrariness of the standard we set up in defining a collision. 


\section{CHAPTER XII.}

\section{FREE PATH PHENOMENA (CONTINUED).}

\section{VISCOSITY.}

293. WE have so far discussed only gases in which the molecular motion at every point has been symmetrical with respect to every direction in space. We now approach a much more complex class of problems for which this property is not true. If we refer to the expression obtained for the law of distribution of velocities at any point of a gas in the normal state, namely,

$$
A e^{-h m\left[\left(u-u_{0}\right)^{2}+\left(v-v_{0}\right)^{2}+\left(w-w_{0}\right)^{2}+2 x\right]},
$$

we notice that there are five independent constants $u_{0}, v_{0}, w_{0}, h$ and $A$. The constancy of $u_{0}, v_{0}, w_{0}$ indicates that the mass motion of the gas is the same throughout the gas: if this mass motion varies from point to point in the gas, the layers of gas move relatively to one another, and we have the problem of determining the viscosity of the gas. Similarly the constancy of $h$ indicates the equality of temperature throughout the gas: if this varies from point to point we have the problem of conduction of heat. Finally the constancy of $A$ indicates the mass-equilibrium of the gas: if this equilibrium does not exist we have the problem of diffusion. These three problems of viscosity, conduction, and diffusion have now to be dealt with.

294. It may be said at once that there is no general method of the kind which was available for the determination of the steady state. The method which will be followed first in every instance is of the most primitive kind, and the results obtained cannot claim any high degree of accuracy. On the other hand the method in question possesses the great advantage of depending only in a slight degree upon the actual structure of the molecules of the gas. And as the exact nature of this structure is very largely a matter of speculation, the comparative inaccuracy of the result obtained will be less serious than it might have been if we had had fuller knowledge of the molecular structure.

We shall afterwards be able to correct the first general result so as to obtain results of considerable accuracy upon the special supposition that the molecules are elastic spheres. 
There is also an entirely different method of attacking the problem, due to Maxwell, which will be presented in Chapter XV. This method is an exact method, but unfortunately only leads to a solution in very special cases. We shall give the solution obtained by Maxwell on the supposition that molecules are point-centres of force repelling one another with a force proportional to the inverse fifth power of the distance. This exact solution will be of interest in that it will enable us to estimate the amount of inaccuracy in the results obtained by the general method of the present chapter.

\section{General Equations of Viscosity.}

295. We shall now discuss the motion in a gas in which the massvelocity varies from point to point. At the particular point considered, let us choose our axes so that the mass-velocity is parallel to the axis of $x$, while the surfaces of equal velocity are parallel to the plane of $x y$, so that in the neighbourbood of the point in question the mass-velocity is a function of $z$ only.

Let us write $\mu$ for $m u$, the momentum of any single molecule in the direction of the $x$-axis, so that the total momentum of any element of the gas is obtained by summing $\mu$ over all the molecules inside the element. The mean value of $\mu$ at any point will be denoted by $\bar{\mu}$, and of course $\bar{\mu}$ varies from point to point in the gas. At the particular point considered we have chosen the direction of our axes so that the gas is arranged, as regards the distribution of $\bar{\mu}$, in a series of layers parallel to the plane of $x y$. We proceed to attempt to calculate the amount of $\mu$ which is transferred by the molecular motion across any one of the planes $z=$ constant.

296. The physical principle underlying the calculation can easily be explained. Let us, to fix our ideas, suppose that the average value of $\mu$ increases as $z$ increases, that the planes $z=$ constant are horizontal and that $z$ increases as we move upwards. The molecules will cross the planes $z=$ constant in both directions. Those which cross any plane, say $z=z_{0}$, in the downward direction will, however, be coming from regions in which the average value of $\mu$ per molecule is greater than it is over the plane $z=z_{0}$, and will therefore, on the average, possess a value of $\mu$ in excess of that appropriate to the plane $z=z_{0}$. In the same way, those molecules which cross this plane in the upward direction will, on the average, possess a value of $\mu$ smaller than that appropriate to the plane $z=z_{0}$. Since, however, there is no mass motion parallel to the axis of $z$, the number of molecules which cross the plane $z=z_{0}$ in one direction is exactly equal to the number which cross it in opposite directions. There is, therefore, more momentum carried through the plane $z=z_{0}$ in the downward direction than in the upward direction. In other words, there is a downward transfer of momentum. 
297. As regards any single molecule which meets the plane $z=z_{0}$ at any point $P$, the amount of $\mu$ carried across the plane $z=z_{0}$ will of course depend, in actual fact, upon the whole past history of the molecule before reaching the point $P$. We are going to conduct our preliminary calculations upon the supposition that the history of the molecule previous to the last collision before meeting $P$, say at $Q$, is immaterial. This would be justifiable if, on the average, all past histories previous to the point $Q$ were equally probable. This, unfortunately, is not so when the molecules are elastic spheres. From the persistence of velocities investigated in the last chapter, it follows that a molecule which is known to have arrived at $P$ from $Q$ has probably started originally from some point further away from $P$ than the point $Q$. Since, however, the amount of the persistence depends in general on the particular molecular structure assumed, it will be simplest to neglect it altogether at first, and subsequently correct our results for it as far as possible.

298. Consider a molecule meeting $z=z_{0}$ in $P$, having previously come from a collision at $Q$. Let the velocity components of the molecule be $u, v, w$, and let the velocity be regarded as consisting of two parts:

(i) a velocity $u_{0}$, of components $u_{0}, 0,0$, equal to the mass-velocity of the gas at $P$;

(ii) a velocity $c$, of components $u-u_{0}, v, w$, the molecular-velocity of the molecule relatively to the gas at $P$.

Let $Q P$ be the actual path described, and let this make an angle $\chi$ with the axis of $z$. Let the length of the path described relatively to the motion of the gas at $P$ be $\lambda_{r}$, and let this make an angle $\theta$ with the axis of $z$. The direction-cosines of the direction of $\lambda_{r}$ are of course proportional to the components of the relative velocity $c$, and therefore to $u-u_{0}, v, w$. Since all directions of this molecular velocity may be regarded as equally probable, the probability of $\theta$ lying between $\theta$ and $\theta+d \theta$ is proportional to $\sin \theta d \theta$.

Also since the projection of the relative path on the axis of $z$ is equal to that of the absolute path,

$$
P Q \cos \chi=\lambda_{r} \cos \theta
$$

The $z$ coordinate of $Q$ is therefore

$$
z_{0}-\lambda_{r} \cos \theta
$$

We are working upon the hypothesis that the expectation of $\mu$ for the molecule in question is that appropriate to the point $Q$. We shall therefore take it to be the mean value of $\mu$ at the point $Q$.

Since $\bar{\mu}$, the mean value of $\mu$, is a function of $z$ only, we can denote the value of $\bar{\mu}$ over the plane $z=\zeta$ by $\bar{\mu}(\zeta)$, and the value of $\bar{\mu}$ at $Q$ will now be

$$
\bar{\mu}\left(z_{0}-\lambda_{r} \cos \theta\right) \text {. }
$$


Since $\lambda$ is small compared with the scale of variation in $\bar{\mu}$, this expression may be written as

$$
\bar{\mu}\left(z_{0}\right)-\lambda_{r} \cos \theta\left(\frac{\partial \bar{\mu}}{\partial z}\right)_{z_{0}=z}
$$

This is the expectation of $\mu$ for any molecule which crosses the plane $z=z_{0}$, having a relative molecular-velocity $c$ inclined at an angle $\theta$ to the axis of $z$.

The number of molecules per unit volume which have relative molecularvelocities for which $c$ and $\theta$ lie within specified small ranges $d c, d \theta$ may be taken to be

$$
\frac{1}{2} \nu f(c) \sin \theta d \theta d c
$$

where of course $\int_{0}^{\infty} f(c) d c=1$.

The number of molecules having a velocity satisfying these conditions, which cross a unit area of the plane $z=z_{0}$ in time $d t$, is equal to the number which at any instant occupy a cylinder of base unity in the plane $z=z_{0}$ and of height $c \cos \theta d t$, and is therefore

$$
\frac{1}{2} \nu c f(c) \cos \theta \sin \theta d \theta d c d t \text {. }
$$

Each molecule, on the average, carries with it the amount of momentum given by expression ( 564 ). The total momentum transferred across unit area of the plane by the molecules now under discussion is therefore

$$
\frac{1}{2} \nu c f(c)\left\{\bar{\mu}\left(z_{0}\right)-\lambda \cos \theta\left(\frac{\partial \bar{\mu}}{\partial z}\right)_{z=z_{0}}\right\} \cos \theta \sin \theta d \theta d c d t,
$$

where $\lambda$ is the mean value of $\lambda_{r}$ for all such molecules. "If we integrate this expression with respect to $\theta$, the limits being 0 to $\pi / 2$, so as to include all directions in which the plane can be crossed from below, we obtain

$$
\frac{1}{2} \nu c f(c)\left\{\frac{1}{2} \bar{\mu}\left(z_{0}\right)-\frac{1}{3} \lambda\left(\frac{\partial \mu}{\partial z}\right)_{z=z_{0}}\right\} d c d t,
$$

and if we now integrate this with respect to $c$, remembering that $\int_{0}^{\infty} f(c) d c=1$, we obtain

$$
\left\{\frac{1}{4} \nu \bar{c} \bar{\mu}\left(z_{0}\right)-\frac{1}{6} \nu \overline{\lambda c}\left(\frac{\partial \bar{\mu}}{\partial z}\right)_{z=z_{0}}\right\} d t
$$

where $\bar{c}$ is the mean value of $c$, calculated as explained in $\S 131$, and $\overline{\lambda c}$ is the mean value of $\lambda c$. We have seen that in general $\lambda$ is a function of $c$, and have calculated the dependence of $\lambda$ upon $c$ when the molecules are supposed to be elastic spheres. At present we are not making any assumption as to the structure of the molecules, and so cannot carry the calculation of the term $\overline{\lambda c}$ any further. 
299. The calculation of the transport of momentum across the plane $z=z_{0}$ in the reverse direction is exactly similar, and we obtain for this quantity the expression

$$
\left\{\frac{1}{4} \nu \bar{c} \bar{\mu}\left(z_{0}\right)+\frac{1}{6} \nu \overline{\lambda c}\left(\frac{\partial \bar{\mu}}{\partial z}\right)_{z=z_{0}}\right\} d t
$$

On the whole, then, the net excess transfer from above to below, which is the difference of the two expressions (565) and (566), is found to be

$$
\frac{1}{3} \nu \overline{\lambda c}\left(\frac{\partial \bar{\mu}}{\partial z}\right)_{z_{0}} d t
$$

We can conveniently suppose that

$$
\lambda c=\bar{c} l
$$

where $l$ is a new quantity, which is of course the mean free path of a molecule, this mean being taken in a certain way. The way in which the mean has to be taken is not the same as the way in which it was taken in $\S 284$, so that we do not obtain an accurate result, in the case of elastic spheres, by replacing $l$ by the $\lambda$ of equation (547). At the same time the mean values calculated in different ways will not greatly differ from one another, and as our present calculation is at best one of approximation, we shall be content for the moment to suppose $l$ to be identical with the mean free path, however calculated. The extent of the error involved in this procedure will be examined later.

300. It might be thought that $l$ ought to be replaced by $\frac{1}{2} \lambda$ instead of by $\lambda$. For if $O Q$ is the whole free path, there is no reason why $P O$ should be less than $P Q$, so that the probable value of $P Q$ might be thought to be $\frac{1}{2} \lambda$.

The fallacy in this reasoning becomes obvious when we consider that in selecting free paths at random by choosing points on these free paths, the longer free paths have a greater chance of being chosen than the shorter ones, the chance of any path being chosen being in fact exactly proportional to the length of the path. The average path chosen in this way, accordingly, will be much longer than that calculated in $\S 284$. To see that $\lambda$ is the right value to assign to $l$, we notice that after a molecule has left $O$, its chances of collision are exactly the same as if it had just undergone collision at $P$. Hence $\overline{P O}=\lambda$, and therefore, by a similar argument, $\overline{P Q}=\lambda$.

A simple example taken from Boltzmann's Vorlesungen* will perhaps elucidate the point further. In a series of throws with a six-faced die the average interval between two throws of unity is of course five throws. But starting from any instant the average number of throws since a unit throw last occurred will be five, and similarly, working back from any instant, the average number of throws since a unit throw occurred is also five.

$$
\text { * I. p. } 72 \text {. }
$$


301. If $\Gamma$ is the aggregate transfer of momentum per unit of time across a unit area of a plane parallel to the plane of $x y$, we have shewn that

$$
\Gamma=\frac{1}{3} \nu \bar{c} l \frac{\partial \bar{\mu}}{\partial z}
$$

Across the plane $z+d z$ the similar transfer is

$$
\frac{1}{3} \nu \bar{c} l\left(\frac{\partial \bar{\mu}}{\partial z}+d z \frac{\partial^{2} \bar{\mu}}{\partial z^{2}}\right)
$$

hence the gain of momentum to the layer between the planes $z$ and $z+d z$ is

$$
\frac{1}{3} \nu \bar{c} l \frac{\partial^{2} \bar{\mu}}{\partial z^{2}} d z
$$

Also if we have a viscous fluid of coefficient of viscosity $\kappa$ moving with the mass velocity of the gas, of which the components are $u_{0}, 0,0$, the force per unit area of the $z$ plane in the direction of the axes of $x$, acting upon the layer of fluid enclosed by the planes $z$ and $z+d z$ is

$$
\kappa \frac{\partial u_{0}}{\partial z}
$$

Similarly that on the plane $z+d z$, acting in the other direction, is

$$
\kappa\left(\frac{\partial u_{0}}{\partial z}+d z \frac{\partial^{2} u_{0}}{\partial z^{2}}\right)
$$

Hence, acting upon this layer per unit area there is a resultant force

$$
\kappa \frac{\partial^{2} u_{0}}{\partial z^{2}} d z
$$

This force increases the momentum per unit area of the layer per unit time at a rate

$$
\kappa \frac{\partial^{2} u_{0}}{\partial z^{2}} d z
$$

In expression (570), we can replace $\bar{\mu}$ by $m u_{0}$, where $u_{0}$ is the massvelocity of the gas, and expression (571) now becomes identical with (570) if

$$
\kappa=\frac{1}{3} \nu \bar{c} l m
$$

We have therefore found that our gas will behave exactly like a viscous fluid, of which the coefficient of viscosity is given by equation (572). If we replace $m \nu$ by $\rho$, this takes the simple form

$$
\kappa=\frac{1}{3} \rho \bar{c} l
$$

302. From the results of our analysis we can now obtain an insight into the molecular mechanics of viscosity in the case of a gas. Let us imagine two molecules, with velocities $u, v, w$ and $-u, v, w$ penetrating from a layer at which the mass velocity is $0,0,0$ to one at which it is $u, 0,0$. By the 
time the molecules have reached this second layer we must suppose that their velocities are divided into two parts, namely,

for the first, and

$$
u-u_{0}, v, w \text { and } u_{0}, 0,0
$$

$$
-u-u_{0}, v, w \text { and } u_{0}, 0,0
$$

for the second. The first part in each case will represent molecular-motion, and the second part will represent mass-motion. Now in equation (32), we saw that the total energy of the gas could be regarded as the sum of the energies of the molecular and mass-motions. The sum of the energies of the molecular-motions of the two molecules now under discussion is, however,

$$
\frac{1}{2} m\left[\left(u-u_{0}\right)^{2}+v^{2}+w^{2}\right]+\frac{1}{2} m\left[\left(-u-u_{0}\right)^{2}+v^{2}+w^{2}\right]
$$

which can be written

$$
m\left(u^{2}+v^{2}+w^{2}\right)+m u_{0}^{2} .
$$

The first term is equal to the energy of the molecular-motion of the two molecules at the start; the second term represents an increase which must be regarded as gained at the expense of the mass-motion of the gas. Thus the phenomenon of viscosity in gases consists essentially in the degradation of the energy of mass-motion into energy of molecular-motion; and is therefore accompanied by a rise of temperature in the gas.

The phenomena of viscosity in liquids and of friction in solids (as explained in §3) can of course be accounted for in a similar way.

\section{Corrections when Molecules are assumed to be Elastic Spheres.}

303. For want of definite knowledge of the molecular structure we have introduced two errors into our calculations. In the first place, we have neglected the persistence of velocities after collision, and in the second place we have ignored the difference between two different ways of estimating the mean free path. If we assume the molecules to be elastic spheres, it is possible to calculate the amount of error introduced by both these simplifications. This we now proceed to do. Both of the necessary corrections are small, so that we shall calculate them as independent corrections, and sum the results.

304. We can begin by an exact calculation of $\overline{\lambda c}$, to replace the assumption of equation (568). The required value of $\overline{\lambda c}$ is

$$
\overline{\lambda c}=\int_{0}^{\infty} f(c) \lambda_{c} c d c
$$

where $\lambda_{c}$ is the same as the $\lambda_{c}$ of $\S 282$. Substituting the value given for $\lambda_{c}$ by equation (539), and putting

$$
f(c)=\sqrt{\frac{h^{3} m^{3}}{\pi^{3}}} 4 \pi c^{2} e^{-h m c^{2}},
$$


we find

$$
\begin{aligned}
\overline{\lambda c} & =\int_{0}^{\infty} \frac{4(h m)^{\frac{5}{5}} c^{5} e^{-h m c^{2}}}{\pi \nu \sigma^{2} \psi(c \sqrt{h m})} d c \\
& =\frac{4}{\pi \sqrt{h m} \nu \sigma^{2}} \int_{0}^{\infty} \frac{x^{5} e^{-x^{2}} d x}{\psi(x)}
\end{aligned}
$$

and if $l$ is defined by equation (568), we have

$$
\overline{\lambda c}=l \bar{c}=\frac{l}{\sqrt{2 h m}},
$$

so that in formula (573), we must take

$$
\begin{aligned}
l & =\overline{\lambda c} \sqrt{2 h m} \\
& =\frac{8}{\sqrt{2} \pi \nu \sigma^{2}} \int_{0}^{\infty} \frac{x^{5} e^{-x^{2}} d x}{\psi(x)} .
\end{aligned}
$$

The integral has to be evaluated by quadrature. Tables for its evaluation are given by Tait*. The integral has also been evaluated by Boltzmannt, whose result agrees to three significant figures with that obtained by Tait.

Using this value for the integral, we find that

$$
l=1.051 \frac{1}{\sqrt{2} \pi \nu \sigma^{2}}
$$

The value of $l$, calculated accurately for our present purpose, accordingly differs by about 5 per cent. from the mean free path,

calculated in $\S 30$.

$$
\frac{1}{\sqrt{2} \pi \nu \sigma^{2}}
$$

305. We turn now to the error which has been introduced by ignoring the persistence of velocities. We have found that this persistence is measured, in a gas of which the molecules are elastic spheres, by a numerical factor which is always intermediate between $\frac{1}{2}$ and $\frac{1}{3}$, and of which the mean value, averaged over all collisions, is $\bullet 406$.

If, on the average, each particle has described a path of which the projection on the axis of $z$ is $\zeta$, with a velocity of which the component parallel to the direction of the axis of $z$ is $w$, then, on tracing back the motion, we know that as regards the previous path of each molecule the expectation of average velocity parallel to the axis of $z$ is $\theta w$, where $\theta$ measures the persistence, and therefore, on the average, the expectation of the projection of this path on the axis of $z$ may be taken to be $\theta \zeta$. Similarly,. the expectation of the projection of each of the paths previous to these may be taken to be $\theta^{2} \zeta$, and so on.

* Collected Works, II. pp. 152 and 178.

† Wiener Sitzungsber. LxxxIv. p. 45 (1881). 
It follows that if we trace the motion a sufficient distance back, each molecule must be supposed to have come, not from a distance $\zeta$ measured along the axis of $z$, but from a distance

$$
\zeta+\theta \zeta+\theta^{2} \zeta+\ldots
$$

of which the value is

$$
\frac{\zeta}{1-\theta}
$$

If we take $\theta=\frac{2}{5}$, this becomes

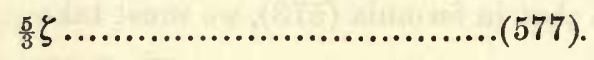

It would not, however, be legitimate to assume that each molecule on arriving at the plane $z=z_{0}$ has, on the average, a value of $\mu$ appropriate to the plane $z=z_{0}-\frac{\hbar}{3} \zeta$. For the molecule has not travelled a distance $\frac{\hbar}{3} \zeta$ undisturbed, and at each collision a certain amount of its excess of momentum will have been shared with the colliding molecule. Of the various simple assumptions possible, the most obvious one to make is that at each collision, the excess of momentum above that appropriate to the point at which the collision takes place is halved, half going to the colliding molecule and half remaining with the original molecule. Making this assumption, it is clear that the excess of momentum to be expected is not that due to having travelled undisturbed a distance equal to that given by expression (576), but a distance

$$
\zeta+\frac{1}{2}\left(\theta \zeta+\frac{1}{2}\left(\theta^{2} \zeta+\frac{1}{2}\left(\theta^{3} \zeta+\ldots\right)\right)\right)
$$

of which the value is

$$
\frac{\zeta}{1-\frac{1}{2} \theta} .
$$

Again taking $\theta=\frac{2}{5}$, this becomes

$$
\frac{5}{4} \zeta \text {. }
$$

It follows that the persistence of velocities, when the molecules are elastic spheres, can roughly be allowed for by supposing the free path in the viscosity formula to be the mean free path multiplied by a factor 1-25. Combining this with expression (539), we find that the free path in the viscosity formula must be taken to be

$$
l=\frac{1 \cdot 31}{\sqrt{2} \pi \nu \sigma^{2}}
$$

Hence for a gas, in which the molecules are elastic spheres, the viscosity coefficient (equation (573)) is given by

$$
\kappa=\frac{1}{3} \rho \bar{c} l=\cdot 44 \frac{\rho \bar{c}}{\sqrt{2} \pi \nu \sigma^{2}}
$$




\section{COMPARISON WITH EXPERIMFNT.}

Determination of Size of Molecules.

306. Since the value of $\kappa$ can be determined experimentally, it is clear that, upon the supposition that the molecules may be regarded as elastic spheres, equation (581) will give valuable information as to the magnitude of $\sigma$. The agreement or disagreement of the value of $\sigma$ obtained in this way with that obtained in other ways, can be regarded as evidence as to the tenability or the reverse, of the hypotheses on which we have been working.

In the following table are given the coefficients of viscosity of various gases, and the calculated values of $\frac{1}{2} \sigma$, the radius, obtained by taking $\nu=4 \times 10^{10}$ in accordance with $\S 8$.

\begin{tabular}{|c|c|c|c|c|c|}
\hline Gas & & $\begin{array}{c}\kappa \text { (observed) } \\
0^{\circ} \mathrm{C} .\end{array}$ & Authority & Mean & $\begin{array}{l}\text { Calculated value } \\
\text { of } \frac{1}{2} \sigma \times 10^{8}\end{array}$ \\
\hline Hydrogen ........... & $\mathrm{H}_{2}$ & $\begin{array}{r}.000084 \\
086 \\
088\end{array}$ & $\begin{array}{l}1 \\
2 \\
3\end{array}$ & $0000864^{*}$ & $1 \cdot 024$ \\
\hline Helium ............... & $\mathrm{He}$ & 165 & 4 & 165 & 0.905 \\
\hline Water vapour ...... & $\mathrm{H}_{2} \mathrm{O}$ & 093 & 3 & 093 & $1 \cdot 695$ \\
\hline Carbon monoxide... & $\mathrm{CO}$ & $\begin{array}{l}167 \\
162\end{array}$ & $\begin{array}{l}1 \\
2\end{array}$ & $1628^{*}$ & $1 \cdot 448$ \\
\hline Ethylene ... & $\mathrm{C}_{2} \mathrm{H}_{4}$ & 099 & 2 & 0944 & 1.883 \\
\hline Nitrogen .... & $\mathrm{N}_{2}$ & $\begin{array}{l}167 \\
166\end{array}$ & $\begin{array}{l}1 \\
2\end{array}$ & 1647 & $1 \cdot 448$ \\
\hline Air....... & - & - & 5 & $1714^{*}$ & $1 \cdot 430$ \\
\hline Nitric oxide.... & NO & 168 & 2 & 168 & $1 \cdot 412$ \\
\hline Oxygen .......... & $\mathrm{O}_{2}$ & $\begin{array}{l}191 \\
189\end{array}$ & $\begin{array}{l}1 \\
2\end{array}$ & $1873^{*}$ & $1 \cdot 405$ \\
\hline Argon & A & 208 & 4 & 208 & $1 \cdot 393$ \\
\hline Carbon dioxide & $\mathrm{CO}_{2}$ & $\begin{array}{l}145 \\
138 \\
144\end{array}$ & $\begin{array}{l}1 \\
2 \\
3\end{array}$ & $1431^{*}$ & $1 \cdot 736$ \\
\hline Nitrous oxide ...... & $\mathrm{N}_{2} \mathrm{O}$ & 144 & 2 & $1381^{*}$ & $1 \cdot 770$ \\
\hline Ethyl Chloride...... & $\mathrm{C}_{2} \mathrm{H}_{6} \mathrm{CI}$ & 094 & 2 & 094 & $2 \cdot 340$ \\
\hline Chlorine & $\mathrm{Cl}_{2}$ & 128 & 2 & 128 & $2 \cdot 055$ \\
\hline
\end{tabular}

The meaning of the figures in the third column is as follows:

* These values are taken from Landolt and Börnstein's tables. 
1. Calculated by Meyer from Graham's Transpiration Experiments. Meyer, Kinetic Theory, p. 192.

2. From transpiration experiments by von Obermayer. Meyer, Kinetic Theory, p. 192, or Weinstein, Thermodynamik und Kinetik der Körper, I. p. 332 .

3. From oscillation experiments by Kundt and Warburg. The experiments were conducted at $15^{\circ} \mathrm{C}$., but the coefficients of viscosity are corrected, according to the experimentally observed variation with the temperature, to $0^{\circ}$ C. Meyer, Kinetic Theory, p. 192.

4. Lord Rayleigh, Proc. Roy. Soc. LIX. p. 108.

5. The value for air is the mean value of a great number of experiments, taken from Landolt and Börnstein's tableș. Meyer, also considering a great number of experiments, gives the value 000172 (Kinetic Theory, p. 197).

The mean values in the fourth column are not always the mean of the observations recorded in the second column. Where possible I have used the mean values given in Landolt and Börnstein's tables, which are derived from the consideration of a greater number of experimental data than those recorded in the second column. These values are marked by an asterisk.

The agreement of the values of $\frac{1}{2} \sigma$ given in the last column, with those already calculated from the deviations from Boyle's law (p. 141), is seen to be tolerably good.

\section{Variation of $\kappa$ with Density.}

307. Since $\rho=m \nu$, equation (581) can be put in the form

$$
\kappa=\cdot 44 \frac{m \bar{c}}{\sqrt{2} \pi \sigma^{2}}
$$

shewing that theoretically $\kappa$ is independent of the density of the gas.

Indeed, whatever structure we assume for the molecules of the gas, it is clear that $l$ will, to a first approximation, vary inversely as the number of molecules per unit volume of the gas. Hence equation (573) gives a value of $\kappa$ which is independent of $\nu$, and we obtain Maxwell's law:

The coefficient of viscosity of a gas is independent of its density.

In spite of its apparent improbability, this law was predicted by Maxwell on purely theoretical grounds, and its subsequent experimental confirmation has constituted one of the most striking triumphs of the Kinetic Theory. 
At the same time, it must be mentioned that the experimental confirmation of this law is by no means complete. Meyer* gives a detailed account of experimental work which has been performed to test this law. He comes to the conclusion that the divergencies from the law exhibited by experimental results are not so great as to be inconsistent with the truth of the law, at any rate within the limits of pressure from 1 to $\frac{1}{60}$ atmosphere. That the divergencies are very considerable outside these limits will be shewn by the two following tables, taken from Weinstein ${ }^{\prime}$.

\section{Viscosity of Carbon dioxide.}

I. Two sets of experiments at pressures less than one atmosphere :

\begin{tabular}{|c|rr|}
\hline$p(\mathrm{~mm} . \mathrm{Hg})$ & \multicolumn{2}{|c|}{$\kappa$} \\
\hline 750 & 0001493, & 0001524 \\
380 & 1493, & 1511 \\
$20 \cdot 5$ & 1483, & \\
$8 \cdot 8$ & & 1506 \\
$2 \cdot 4$ & 1467, & 1498 \\
$1 \cdot 5$ & 1442, & 1473 \\
$0 \cdot 6$ & 1381, & 1433 \\
\hline
\end{tabular}

II. Experiments at pressures greater than one atmosphere:

\begin{tabular}{|c|c|c|}
\hline Pressure (atmos.) & Density & $\kappa$ \\
\hline $43 \cdot 1$ & $0 \cdot 100$ & $\cdot 000177$ \\
\hline $60 \cdot 7$ & 170 & 189 \\
\hline $70 \cdot 3$ & 240 & 212 \\
\hline $75 \cdot 0$ & 310 & 240 \\
\hline $77 \cdot 1$ & 380 & 277 \\
\hline 77.9 & 450 & 323 \\
\hline $78 \cdot 4$ & 520 & 370 \\
\hline - $\quad 79 \cdot 3$ & 590 & 429 \\
\hline $82 \cdot 3$ & 660 & 499 \\
\hline . $90 \cdot 7$ & 730 & 579 \\
\hline 111.5 & 800 & 683 \\
\hline
\end{tabular}

* Kinetic Theory of Gases, p. 181, § 77.

† Thermodynamik und Kinetik der Körper, I. pp. 325 and 326. 


\section{Variation of $\kappa$ with Temperature.}

308. Since $\bar{c}$ is proportional to the square root of the absolute temperature, it is seen that if the molecules were true elastic spheres, the value of $\kappa$ would be proportional to the square root of the temperature.

As a matter of fact, it is found that $\kappa$ varies a good deal more rapidly than this, as the temperature increases. The divergence between experiment and the theoretical value obtained for elastic spheres is, however, one that could have been predicted. The assumption that the molecules are elastic spheres is, at best, only an approximation, and we must continually examine what deviations are to be expected from the results obtained on this assumption.

The peculiarity of a system of elastic spheres is that the motion remains geometrically the same if the velocity of each sphere is increased in the same ratio. Thus in order to determine the motion of two spheres after collision it is only necessary to know the directions of motion before collision, and the ratio of their velocities; we are not concerned with the actual values of these velocities. When, on the other hand, we suppose the molecules to be surrounded by fields of force, this ceases to be true: the paths after collision do not depend solely on the ratio of the velocities, but on the absolute magnitudes of these velocities. For instance, in fig. 20 let $O P Q R$, $O^{\prime} P^{\prime} Q^{\prime} R^{\prime}$ be the paths described when two molecules surrounded by fields of force meet one another, the figure being drawn for the sake of simplicity, for the case in which the two velocities are equal and opposite. If now we suppose the molecules moving along the same lines before encounter, but each with double its former velocity, the paths will be different. For obviously the higher velocity will carry each molecule further into the other's field of force before the centres of the two molecules reach their shortest distance apart, so that the path described, instead of being $O P Q R$ will be, let us say, OPST.

We can, however, by an obvious geometrical construction find the size of two spheres which would describe paths having the same deflections as $O P Q R$ or OPST. If we perform the construction for the two paths of fig. 20 we find that the size of the sphere for the former path described with small velocity is greater than for the latter path described with large velocity. We can therefore see that, if we attempt to represent our molecules by spheres, the size of these spheres must be supposed to decrease as the mean molecular velocity increases, and therefore as the temperature rises. 


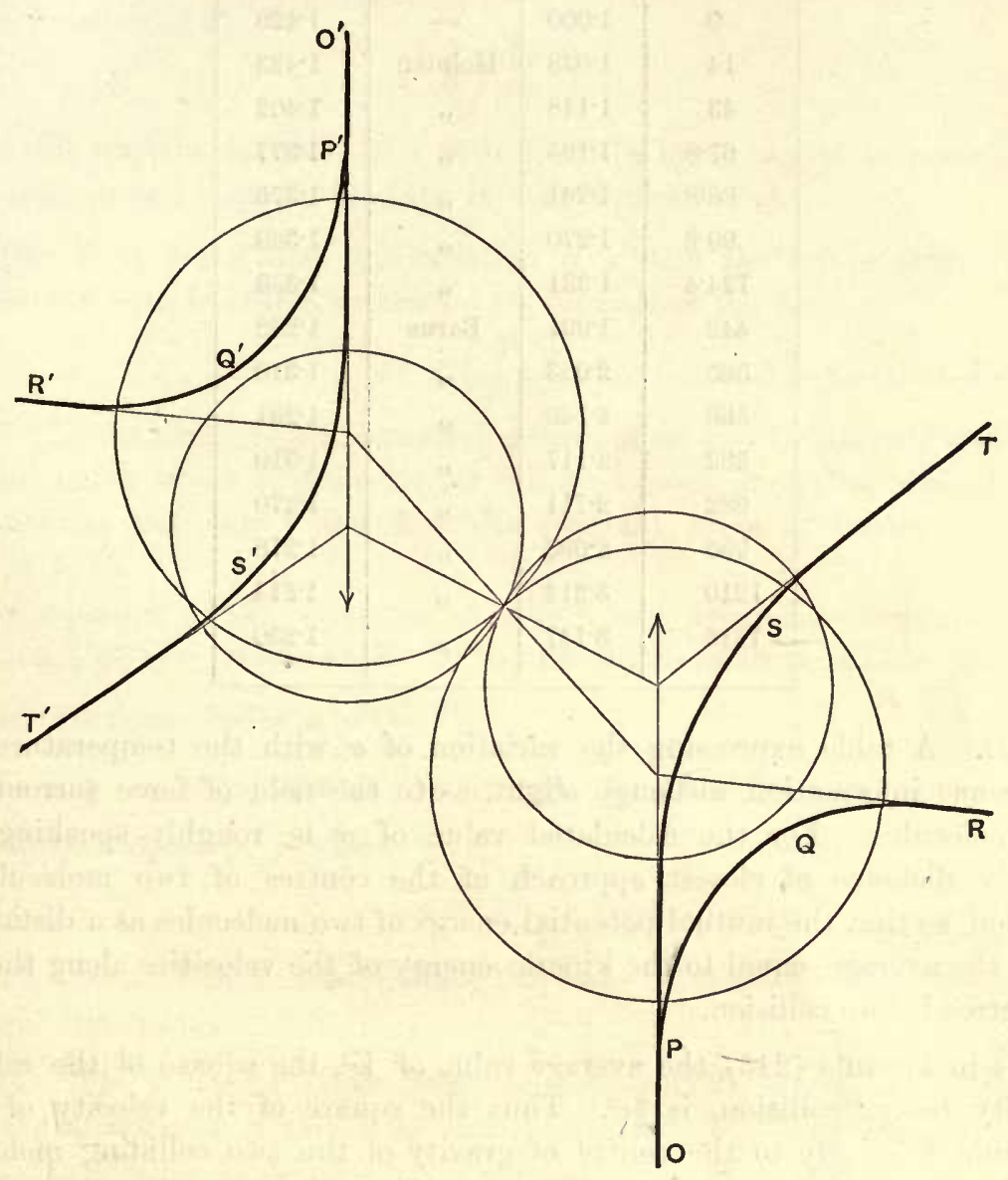

Fig. 20.

These considerations, then, fully account for the apparent variation of $\sigma$ with the temperature. Full accounts of experiments to determine the variation of $\kappa$ with the temperature will be found in Meyer's Kinetic Theory of Gases*. From three sets of observations I have calculated the following table of values for $\frac{1}{2} \sigma$, the mean effective radius of a molecule of air. The first column gives the temperature in degrees centigrade, the second gives $\kappa$ in terms of $\kappa_{0}$, the value of $\kappa$ at $0^{\circ} \mathrm{C}$. 


\begin{tabular}{|c|c|c|c|}
\hline Temp. & $\kappa / \kappa_{0}$ & Observer & $\frac{1}{2} \sigma \times 10^{-8}$ \\
\hline 0 & $1 \cdot 000$ & - & $1 \cdot 428$ \\
\hline 14 & $1 \cdot 038$ & Holman & $1 \cdot 423$ \\
\hline 43 & $1 \cdot 118$ & " & $1 \cdot 402$ \\
\hline $67 \cdot 8$ & $1 \cdot 185$ & " & $1 \cdot 377$ \\
\hline $88 \cdot 8$ & $1 \cdot 241$ & , & $1 \cdot 375$ \\
\hline $99 \cdot 2$ & $1 \cdot 270$ & $"$ & $1 \cdot 369$ \\
\hline $124 \cdot 4$ & $1 \cdot 331$ & $"$ & $1 \cdot 359$ \\
\hline 442 & 1.991 & Barus & $1 \cdot 288$ \\
\hline 565 & $2 \cdot 083$ & " & $1 \cdot 310$ \\
\hline 569 & $2 \cdot 149$ & $"$ & $1 \cdot 291$ \\
\hline 592 & $2 \cdot 117$ & $"$ & $1 \cdot 310$ \\
\hline 982 & $2 \cdot 711$ & $"$ & $1 \cdot 270$ \\
\hline 995 & $2 \cdot 693$ & $"$ & 1.278 \\
\hline 1210 & $3 \cdot 214$ & $"$ & $1 \cdot 214$ \\
\hline 1216 & $3 \cdot 147$ & $"$ & $1 \cdot 230$ \\
\hline
\end{tabular}

309. A table expressing the variation of $\sigma$ with the temperature will give some information, although slight, as to the field of force surrounding the molecules. For the calculated value of $\sigma$ is, roughly speaking, the average distance of closest approach of the centres of two molecules in collision, so that the mutual potential energy of two molecules at a distance $\sigma$ is, on the average, equal to the kinetic energy of the velocities along the line of centres before collision.

As in formula (245), the average value of $V^{2}$, the square of the relative velocity before collision, is $\frac{8}{3} c^{2}$. Thus the square of the velocity of each molecule relatively to the centre of gravity of the two colliding molecules will be, on the average, $\frac{2}{3} c^{2}$. The probability that the direction of this velocity makes an angle with the line of centres, which lies between $\theta$ and $\theta+d \theta$, is $2 \sin \theta \cos \theta d \theta$, so that the average square of the relative velocity along the line of centres is

$$
\frac{2}{3} c^{3} \int_{0}^{\pi / 2} 2 \sin \theta \cos ^{3} \theta d \theta=\frac{1}{3} c^{2} .
$$

The kinetic energy which has been destroyed by the intermolecular field of force when the molecules are, on the average, at their point of closest approach at distance $\sigma$ apart is therefore

$$
\frac{1}{3} m c^{2}=R T .
$$

Thus we may say that the mutual potential energy of two molecules at a distance $\sigma$ apart will be $R T$, where $T$ is the temperature corresponding to the 
value of $\sigma$ in question. The force of repulsion between two molecules at distance $\sigma$ is accordingly $R \frac{d T}{d \sigma}$, or more conveniently, since we are regarding $\sigma$ as a function of $T$,

$$
R / \frac{d \sigma}{d T}
$$

In this way the variation of $\sigma$ with $T$ can be made to give us some idea of the variation and magnitude of the intermolecular forces.

310. It is found that the variation of $\kappa$ with the temperature can be represented with tolerable accuracy by a formula of the form

$$
\kappa=\kappa_{0}\left(\frac{T}{273}\right)^{n}
$$

where $T$ is the absolute temperature, measured on the Centigrade scale, and $n$ is an index which is different for different gases, its value always being intermediate between $\frac{1}{2}$ and 1 . The constant $\kappa_{0}$ is of course the value of $\kappa$ at $0^{\circ} \mathrm{C}$.

By equation (582), $\kappa$ varies as $T^{\frac{1}{2}} \sigma^{-2}$, and hence by comparison with equation (583), $\sigma$ varies as $T^{-\frac{1}{2}\left(n-\frac{1}{2}\right)}$. By differentiation, $d T / d \sigma$ varies as $\frac{T}{\sigma}$, and this again varies as $\sigma^{-\frac{2}{n-\frac{1}{2}}-1}$.

We may say, then, that on the average, the force between two molecules at distance $r$ apart is $\frac{\mu}{r^{s}}$, where

$$
s=\frac{2}{n-\frac{1}{2}}+1
$$

In a later section we shall deduce this same equation from a discussion of physical dimensions.

\begin{tabular}{|c|c|c|c|c|}
\hline Gas & Formula & Atomicity & $n$ & 8 \\
\hline Helium .......... & $\mathrm{He}$ & 1 & 681 & $12 \cdot 0$ \\
\hline Hydrogen ....... & $\mathrm{H}_{2}$ & 2 & $\cdot 70$ & $11 \cdot 0$ \\
\hline Nitrogen ............ & $\mathrm{N}_{2}$ & 2 & $\cdot 74$ & $9 \cdot 3$ \\
\hline Carbon monoxide... & $\mathrm{CO}$ & 2 & $\cdot 74$ & $9 \cdot 3$ \\
\hline 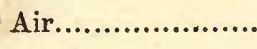 & - & 2 & $\cdot 76$ & $8 \cdot 7$ \\
\hline Oxygen ................ & $\mathrm{O}_{2}$ & 2 & $\cdot 80$ & $7 \cdot 7$ \\
\hline Nitrous oxide ...... & $\mathrm{N}_{2} \mathrm{O}$ & 3 & $\cdot 93$ & $5 \cdot 6$ \\
\hline Carbon dioxide & $\mathrm{CO}_{2}$ & 3 & $\cdot 94$ & $5 \cdot 5$ \\
\hline Ethylene ............. & $\mathrm{C}_{2} \mathrm{H}_{4}^{\prime \prime}$ & 6 & $\cdot 96$ & $5 \cdot 3$ \\
\hline Ethyl chloride...... & $\mathrm{C}_{2} \mathrm{H}_{5} \mathrm{Cl}$ & 8 & $\cdot 98$ & $5 \cdot 2$ \\
\hline
\end{tabular}

311. The following table gives various observed values for $n$, together with the corresponding values for $s$ calculated from equation (584).

J. 
The table is taken from Weinstein's Thermodynamik (I. p. 329) with the exception of the entry Helium, for which the value of $n$ is due to Lord Rayleigh*.

A similar table, in which the numbers are in almost complete agreement with those given above, will be found in Landolt and Börnstein's tables.

We notice at once that $s$ is greatest for the molecules of which the structure is simplest. This is what we should expect. In the case of a complex molecule the atoms will not fill the whole of the circumscribing sphere, so that it will be possible for the circumscribing spheres of two molecules in certain positions to intersect, while the force between them remains comparatively small. A glance at fig. 21 will probably make this clearer than a long explanation. The effect of including forces such as these in the average of forces at a given distance apart is not only to lessen the average force between two molecules at a given distance apart, but also to lessen the rapidity with which the average force falls off, or, in other words, to decrease $s$.

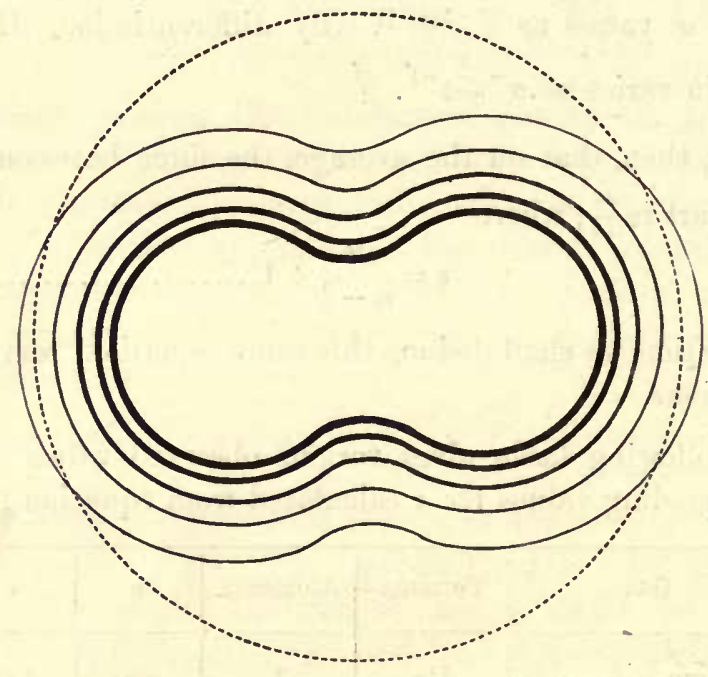

Fig. 21.

* See also Schultze, Annalen der Physik, 11. p. 302. 


\section{CHAPTER XIII.}

\section{FREE PATH PHENOMENA (CONTINUED)}

\section{CONDUCTION OF HeAT.}

\section{Elementary Theory.}

312. AN elementary theory of conduction, similar to the elementary theory of viscosity arrived at in the last chapter, can be arrived at without trouble. The physical principle underlying the phenomenon of conduction of heat is exactly similar to that underlying the phenomenon of viscosity; the difference of detail being that instead of an excess of momentum being carried in one direction, we have in the present instance, an excess of energy. In equation (569), we arrived at the relation

$$
\Gamma=\frac{1}{3} \nu \bar{c} l \frac{\partial \bar{\mu}}{\partial z}
$$

and this will apply to the present problem if we now suppose $\bar{\mu}$ to be the mean energy at the plane $z=z_{0}$, and $\Gamma$ the transfer of energy across this plane. If $g$ is the coefficient of conduction of heat, the flow of heat is $9 \frac{\partial T}{\partial z}$, so that the flow of energy is $J 9 \frac{\partial T}{\partial z}$, where $J$ is the mechanical equivalent of heat.

Also $\bar{\mu}$, the mean energy of a molecule, is identical with the quantity which has previously been denoted by $\bar{E}$, so that equation (585) can now be written

$$
\begin{aligned}
J 9 \frac{\partial T}{\partial z} & =\frac{1}{3} \nu \bar{c} l \frac{\partial \bar{E}}{\partial z} \\
& =\frac{1}{3} \nu \bar{c} l \frac{d \bar{E}}{d T} \frac{\partial T}{\partial z}
\end{aligned}
$$

from which it follows that the value of $\mathrm{g}$ is

$$
\vartheta=\frac{1}{3} \frac{\nu \bar{c} l}{J} \frac{d \bar{E}}{d T}
$$


From equation (402) we have the relation

$$
C_{v}=\frac{1}{J m} \frac{d \bar{E}}{d T}
$$

where $C_{v}$ is the specific heat at constant volume, and again, from equation (572), if $\kappa$ is the coefficient of viscosity,

$$
\kappa=\frac{1}{3} \nu \bar{c} l m
$$

Hence, by comparison of equations (586), (587) and (588),

$$
9=\kappa C_{v}
$$

\section{Correction when Molecules are Elastic Spheres.}

313. We found that the first formula obtained for the coefficient of viscosity was true as regards order of magnitude, but required correction by multiplication by a numerical factor substantially different from unity. So also here, we shall find that strict analysis leads to a value of 9 which differs very appreciably from that given by equation (589), although the only difference lies in the occurrence of a numerical multiplier. We proceed to apply analysis as rigorously as possible, to the case of conduction of heat in a gas of which the molecules are elastic spheres. The solution which follows is substantially that given in Meyer's Kinetic Theory of Gases. The main difference is that Meyer neglects certain terms expressing the variation of collision-frequency, although these terms are of the same order of magnitude as terms retained. I have found it possible to give the more complete investigation in which these terms are taken into account, but find that their ultimate effect on the result obtained by Meyer is nil.

314. We consider any element $d x d y$ of the plane $z=z_{0}$, and with the centre of this element as origin, we take spherical polar coordinates $r, \theta, \phi$, the line $\theta=0$ being parallel to the axis of $z$.

The curvilinear element of volume for which $r, \theta, \phi$ lie between $r$ and $r+d r$, $\theta$ and $\theta+d \theta, \phi$ and $\phi+d \phi$ is the volume $d v=r^{2} \sin \theta d \theta d \phi d x$.

We begin by considering the possibility of a molecule undergoing collision in the element $d v$, leaving it with a velocity $c$ in such a direction as to pass through the small area $d x d y$, and describing a free path

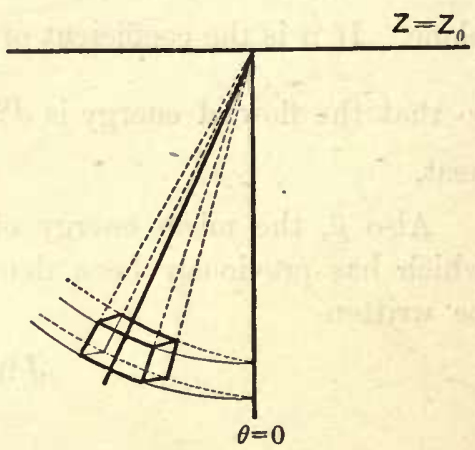

FIG. 22. which reaches at least as far as the element $d x d y$ without collision.

Since the whole motion is reversible, the number of collisions in which one of the molecules has a velocity between $c$ and $c+d c$ after collision in the 
element $d v$ is exactly equal to that of the collisions in which one of the molecules before collision has velocities within these limits.

The number of molecules which at any instant are moving with a velocity between $c$ and $c+d c$ in the element $d v$ is

$$
\nu r^{2} \sin \theta d \theta d \phi d r \sqrt{\frac{h^{3} m^{3}}{\pi^{3}}} 4 \pi c^{2} e^{-h m c^{2}} d c
$$

so that the number of collisions experienced by these molecules in time $d t$ is, by $\S 251$, equal to

where

$$
\Theta d t \times(\text { expression (590)) }
$$

$$
\Theta=\frac{\sqrt{\pi} \nu \sigma^{2}}{h m c} \psi(c \sqrt{h m})
$$

This, then, is the number of molecules which, in time $d t$, experience a collision in the element $d v$, and leave it with a velocity between $c$ and $c+d c$. All directions are equally likely. Now the element $d x d y$ subtends a solid angle

$$
d x d y \frac{\cos \theta}{r^{2}}
$$

at the element $d v$, so that the chance that, if a molecule escapes collision, it will pass through the element $d x d y$, is

$$
d x d y \frac{\cos \theta}{4 \pi r^{2}} .
$$

Multiplying expressions (591) and (593) together, we obtain

$$
d x d y d t \sin \theta \cos \theta d \theta d \phi d r \nu \sqrt{\frac{h^{3} m^{3}}{\pi^{3}}} \Theta c^{2} e^{-h m c^{2}} d c
$$

This is the number of molecules which leave the element $d v$ in time $d t$ with a velocity between limits $c$ and $c+d c$, in a direction suitable for passing through the element of area $d x d y$.

315. We have next to calculate the probability of a molecule describing the free path $r$ from $d v$ to $d x d y$ without collision.

We cannot use the analysis of $\S 286$ to determine this probability, for in the present problem the state of the gas varies from point to point as we proceed along the path of the molecule. If, however, we denote by $f(l)$ the fraction of the whole which travel a distance at least equal to $l$ without collision, we obtain, just as in $\S 286$, the differential equation

$$
\frac{\partial f(l)}{\partial l}=-B_{c} f(l)
$$

in which $B_{c}$ is now evaluated at a point at distance $l$ along the path. The solution of this equation is

$$
f(l)=e^{-\int_{0}^{l} B_{c} d l}
$$


If the path is small compared with the scale of variation of structure in the gas, we may to a sufficient approximation write the value of $B_{c}$ at a distance $l$ along the path in the form

so that

$$
\left(B_{c}\right)_{l}=\left(B_{c}\right)_{0}+l\left(\frac{\partial B_{c}}{\partial l}\right)_{0}
$$

$$
\begin{aligned}
\int_{0}^{l} B_{c} d l & =l\left(B_{c}\right)_{0}+\frac{1}{2} l^{2}\left(\frac{\partial B_{c}}{\partial l}\right)_{0} \\
& =l\left(B_{c}\right)_{l / 2} .
\end{aligned}
$$

Hence the fraction of the whole number of molecules which describe the distance $r$ from $d v$ to $d x d y$ without collision is

$$
e^{-r B_{c}}
$$

where $B_{c}$ is evaluated at the middle point of the path.

316. By multiplication of expressions (594) and (598) we find, as the number of particles which cross the plane $z=z_{0}$ per unit area per unit time with velocities between $c$ and $c+d c$, having started from the element $d v$,

$$
d \mathfrak{n}=d r \sin \theta \cos \theta d \theta d \phi \nu \sqrt{\frac{h^{3} m^{3}}{\pi^{3}}} e^{-h m c^{2} \Theta} e^{-r B_{c}} c^{2} d c \ldots . .
$$

In this expression, $\Theta, \nu$ and $h$ are evaluated at the point $(r, \theta, \phi)$ while $B_{c}$ is evaluated at the point $\left(\frac{r}{2}, \theta, \phi\right)$. Since, however, $\nu, h$ and $B_{c}$ are functions of $z$ only and not of $x$ and $y$, we can write the values of $\nu, h$ and $B_{c}$ required for expression (599) in the forms

$$
\begin{gathered}
\Theta=\Theta-r \cos \theta \frac{\partial \Theta}{\partial z}, \\
\nu=\nu-r \cos \theta \frac{\partial \nu}{\partial z}, \\
h=h-r \cos \theta \frac{\partial h}{\partial z}, \\
B_{c}=B_{c}-\frac{1}{2} r \cos \theta \frac{\partial B_{c}}{\partial z},
\end{gathered}
$$

in which all the quantities on the right-hand side are evaluated in the plane $z=z_{0}$.

Substituting these values, we obtain as the value of $d \mathfrak{n}$

$$
\begin{aligned}
& d \mathfrak{n}=d r \sin \theta \cos \theta d \theta d \phi \nu \sqrt{\frac{h^{3} m^{3}}{\pi^{3}}} e^{-h m c^{2} \Theta e^{-r B_{c}} c^{2} d c} \\
& {\left[1-r \cos \theta \frac{1}{\Theta} \frac{d \Theta}{d z}-r \cos \theta \frac{1}{\nu} \frac{d \nu}{d z}\right.} \\
&\left.-\frac{3}{2} r \cos \theta \frac{1}{h} \frac{d h}{d z}+r \cos \theta m c^{2} \frac{d h}{d z}+\frac{1}{2} r^{2} \cos \theta \frac{d B_{c}}{d z}\right],
\end{aligned}
$$

in which all the quantities are evaluated in the plane $z=z_{0}$. 
We next integrate this expression with respect to $\theta$ and $\phi$ so as to obtain the total number of molecules which cross the plane $z=z_{0}$ from the side $z<z_{0}$ with velocities between $c$ and $c+d c$, coming from a distance intermediate between $r$ and $r+d r$. In this integration, the limits for $\phi$ are from 0 to $2 \pi$, those for $\theta$ are from 0 to $\frac{\pi}{2}$ only. If we denote the number in question by $d n_{r, c}$, then, since

we have

$$
\begin{aligned}
& \int_{\theta=0}^{\theta=\pi / 2} \int_{\phi=0}^{\phi=2 \pi} \sin \theta \cos \theta d \theta d \phi=\frac{1}{2}, \\
& \int_{\theta=0}^{\theta=\pi / 2} \int_{\phi=0}^{\phi=2 \pi} \sin \theta \cos ^{2} \theta d \theta d \phi=\frac{1}{3},
\end{aligned}
$$

$$
\begin{array}{r}
d n_{r, c}=\sum_{\theta=0}^{\theta=\pi / 2} \sum_{\phi=0}^{\phi=2 \pi} d n \\
=2 \pi \nu \sqrt{\frac{h^{3} m^{3}}{\pi^{3}}} e^{-h m c^{2} \Theta} e^{-r B_{c}} c^{2} d c d r \\
{\left[\frac{1}{2}-\frac{r}{3 \Theta} \frac{d \Theta}{d z}-\frac{r}{3 \nu} \frac{d \nu}{d z}-\frac{r}{2 h} \frac{d h}{d z}\right.} \\
\left.+\frac{r m c^{2}}{3} \frac{d h}{d z}+\frac{r^{2}}{6} \frac{d B_{c}}{d z}\right]
\end{array}
$$

We can now sum this number over all possible values of $r$. The limits are of course $r=0$ and $r=\infty$. The number obtained, which we can denote by $d n_{c}$, will be the total number of molecules crossing the plane $z=z_{0}$ per unit area from the direction $z<z_{0}$, with velocities between $c$ and $c+d c$. Since

$$
\begin{aligned}
& \int_{0}^{\infty} e^{-r B_{c}} d r=\frac{1}{B_{c}}, \\
& \int_{0}^{\infty} e^{-r B_{c}} r d r=\frac{1}{B_{c}{ }^{2}}, \\
& \int_{0}^{\infty} e^{-r B_{c}} r^{2} d r=\frac{2}{B_{c}{ }^{3}},
\end{aligned}
$$

we obtain, on integration,

$$
\begin{aligned}
& d n_{c}=\sum_{r=0}^{r=\infty} d n_{r, c} \\
& =2 \pi \nu \sqrt{\frac{h^{3} m^{3}}{\pi^{3}}} e^{-h m c^{2} \Theta c^{2} d c} \\
& {\left[\frac{1}{2 B_{c}}-\frac{1}{3 \Theta B_{c}{ }^{2}} \frac{d \Theta}{d z}-\frac{1}{3 \nu \overline{B_{c}}} \frac{d \nu}{d z}-\frac{1}{2 h B_{c}{ }^{2}} \frac{d h}{d z}\right.} \\
& \left.+\frac{m c^{2}}{3 B_{c}{ }^{2}} \frac{d h}{d z}+\frac{1}{3 B_{c}^{3}} \frac{d B_{c}}{d z}\right]
\end{aligned}
$$


We have, however, since there is only one kind of gas, by equations (537) and (535)

so that

$$
B_{c}=\frac{\Theta}{c}=\frac{\sqrt{\pi} \nu \sigma^{2}}{h m c^{2}} \psi(c \sqrt{h m}),
$$

$$
\frac{1}{\Theta} \frac{\partial \Theta}{\partial z}=\frac{1}{B_{c}} \frac{\partial B_{c}}{\partial z},
$$

and hence the terms in $\frac{\partial \Theta}{\partial z}$ and $\frac{\partial B_{c}}{\partial z}$ destroy one another in equation (601). Also

$$
\frac{\Theta}{B_{c}^{2}}=\frac{h m c^{3}}{\sqrt{\pi} \nu \sigma^{2} \psi(c \sqrt{h m})},
$$

so that the equation can be written in the simplified form

$$
d n_{c}=\frac{2 \sqrt{h^{5} m^{5}}}{\pi \sigma^{2} \Psi(c \sqrt{h m})}\left[\frac{1}{2} B_{c}-\frac{1}{3 \nu} \frac{d \nu}{d z}-\frac{1}{2 h} \frac{d h}{d z}+\frac{m c^{2}}{3} \frac{d h}{d z}\right] e^{-h m c^{2} c^{5} d c .}
$$

317. Clearly the corresponding number which cross the plane in the opposite direction, say $d n_{c}{ }^{\prime}$, is given by an expression which is of the same form except that the signs of all differential coefficients with respect to $z$ are changed. Hence $\delta n_{c}$, the excess flow of molecules with velocities intermediate between $c$ and $c+d c$, is given by

$$
\begin{aligned}
\delta n_{c} & =d n_{c}^{\prime}-d n_{c} \\
& =\frac{2 \sqrt{h^{5} m^{5}}}{\pi \sigma^{2} \psi(c \sqrt{h m})}\left[\frac{2}{3 \nu} \frac{d \nu}{d z}+\left(1-\frac{2}{3} h m c^{2}\right) \frac{1}{h} \frac{d h}{d z}\right] e^{-h m c} c^{5} d c .
\end{aligned}
$$

If we write $x$ for $c \sqrt{h m}$, this becomes

$$
\delta n_{c}=\frac{2}{\pi \sigma^{2} \sqrt{h m} \psi(x)}\left[\frac{2}{3 \nu} \frac{d \nu}{d z}+\left(1-\frac{2}{3} x^{2}\right) \frac{1}{h} \frac{d h}{d z}\right] x^{5} e^{-x^{2}} d x \ldots \ldots(602) .
$$

318. The total transfer of mass across the plane $z=\bar{z}_{0}$ is

$$
\sum_{x=0}^{x=\infty} \delta n_{c}
$$

while the total transfer of translational energy is

$$
\Gamma_{t}=\sum_{x=0}^{x=\infty} \frac{1}{2} m c^{2} \delta n_{c}=\frac{1}{2 h} \sum_{x=0}^{x=\infty} x^{2} \delta n_{c}
$$

Heat can, as we know, be transferred either by conduction, or by convection. If we are dealing, as in the present case, with conduction only, we must introduce the condition that there is to be no convection. This simply requires that there shall be no transfer of mass, and therefore that expression 
(602) shall vanish. This condition will of course lead to a relation between $\frac{\partial \nu}{\partial z}$ and $\frac{\partial h}{\partial z}$. Substituting for $\delta n_{c}$ in expression (602), the relation in question is seen to be

$$
\frac{1}{\nu} \frac{\partial \nu}{\partial z} \int_{0}^{\infty} \frac{x^{5} e^{-x^{2}}}{\psi(x)} d x=\frac{1}{h} \frac{\partial h}{\partial z} \int_{0}^{\infty} \frac{\left(x^{2}-\frac{3}{2}\right) x^{5} e^{-x^{2}}}{\psi(x)} d x
$$

The integrals have been calculated by W. Conrau, and the result is given in Meyer's Kinetic Theory (p. 464). It appears that equation (605) can be written in the form

$$
\frac{1}{\nu} \frac{\partial \nu}{\partial z}=0 \cdot 71066 \frac{1}{h} \frac{\partial h}{\partial z}
$$

so that, instead of equation (602), we have

$$
\delta n_{c}=\frac{2}{\pi \sigma^{2} \sqrt{h m} \psi(x)} \frac{2}{3} \frac{d h}{h d z}\left(2 \cdot 21066-x^{2}\right) x^{5} e^{-x^{2}} d x
$$

Hence, upon substitution for $\delta n_{c}$ in equation (604), we obtain

where

$$
\Gamma_{t}=\frac{2}{3 \pi \sigma^{2} \sqrt{h m}} \frac{1}{h^{2}} \frac{d h}{d z} I
$$

$$
I=\int_{0}^{\infty} \frac{\left(2 \cdot 21066-x^{2}\right) x^{7} e^{-x^{2}}}{\psi(x)} d x
$$

319. The mean total energy carried over the plane $z=z_{0}$ by the molecules crossing it, no matter where they come from, will always bear the same constant ratio to the mean translational energy, this ratio being $\bar{E} / \frac{1}{2} m C^{2}$, or

$$
\frac{4 h \bar{E}}{3}
$$

Hence the total transfer of energy $\Gamma$ stands to $\Gamma_{t}$ in this same ratio, and is therefore given by

$$
\begin{aligned}
\Gamma & =\frac{4 h \bar{E}}{3} \Gamma_{t} \\
& =\frac{8}{9 \pi \sigma^{2} \sqrt{h m}} \frac{\bar{E}}{h} \frac{d h}{d z} I
\end{aligned}
$$

Also, since the ratio $(610)$ is constant in space, we have

$$
\bar{E} \frac{d h}{d z}+h \frac{d \bar{E}}{d z}=0
$$

so that $\Gamma$ can be put in the form

$$
\Gamma=-\frac{8 I}{9 \pi \sigma^{2} \sqrt{h m}} \frac{d \bar{E}}{d z}
$$


As in $\S 312$, the flow of energy is $J \curvearrowright \frac{\partial T}{\partial z}$, so that by comparison we find

$$
\stackrel{\vartheta}{=\frac{8 I}{9 \pi \sigma^{2} \sqrt{h m}}} \frac{1}{J} \frac{\partial \bar{E}}{\partial T}
$$

By equations (273) and (402), we have

$$
\begin{aligned}
\bar{c} & =\frac{2}{\sqrt{\pi h m}}, \\
C_{v} & =\frac{1}{J m} \frac{\partial \bar{E}}{\partial T},
\end{aligned}
$$

so that equation (613) becomes

$$
\vartheta=\frac{4 I m}{9 \sqrt{\pi} \sigma^{2}} \bar{c} C_{v}
$$

320. We have so far made no correction for the persistence of velocities. We are, however, trying to correct equation (589), namely,

$$
\vartheta=\kappa C_{v},
$$

by determining the numerical multiplier. We are therefore trying to evaluate the ratio $\Phi / \kappa$. Now $\Phi$ and $\kappa$ are each proportional to the mean free path, and are therefore both affected in the same way by the persistence of velocities. It is therefore clear that the fraction $\$ / \kappa$ will be approximately unaffected by this persistence.

Hence, if we use the value of $\kappa$ which was obtained before correction, we can use the value of $\mathcal{A}$ given by equation (614) without further correction, and shall obtain approximately accurate results.

The value of $\kappa$ obtained before correction (equations (537) and (539)) was

$$
\begin{aligned}
\kappa & =\frac{1}{3} \rho \bar{c} l \\
& =\frac{1}{3} \rho \bar{c}\left[\frac{1 \cdot 051}{\sqrt{2} \pi \nu \sigma^{2}}\right]
\end{aligned}
$$

By comparison with equation (614),

$$
\vartheta=\frac{4}{3} \frac{I \sqrt{2 \pi}}{1 \cdot 051} \kappa C_{v}
$$

giving the multiplying factor, by which equation (589) must be corrected.

The value of the integral $I$ has been calculated by P. Neugebauer, and from this value Meyer reduces equation (616) to the form

$$
\vartheta=1 \cdot 6027 \kappa C_{v}
$$


Comparison with Experiment.

321. This relation between 9 and $\kappa$ is found to be in excellent agreement with experiment. This is shewn in the following table:

RAtio of $\kappa C_{v}$ TO 9.

\begin{tabular}{|c|c|c|c|c|c|}
\hline Gas & Mean $\kappa($ p. 251) & $1.6027 \kappa C_{v}$ & 9 & Authority* & $\frac{\text { Mean } 9}{\kappa C_{v}}$ \\
\hline Hydrogen.......... & .0000864 & .0003346 & $\begin{array}{r}0003872 \\
3190\end{array}$ & $\begin{array}{l}1 \\
2\end{array}$ & $1 \cdot 70$ \\
\hline Nitrogen ............ & .0001647 & 0464 & 0512 & 1 & 旺 \\
\hline Ethylene ........ & $\cdot 0000944$ & 0434 & 0395 & 1,3 & 4.57 \\
\hline Carbon monoxide... & .0001628 & 0456 & $\begin{array}{l}0498 \\
0499\end{array}$ & $\begin{array}{l}1 \\
3\end{array}$ & $\begin{array}{l}176 \\
1.43 \\
\end{array}$ \\
\hline Air................... & $\cdot 0001714$ & 0468 & $\begin{array}{l}0561 \\
0483 \\
0513\end{array}$ & $\begin{array}{l}1 \\
2 \\
3\end{array}$ & $1 \cdot 77$ \\
\hline Nitric oxide ......... & .000168 & 0448 & 0451 & 1 & $1 \cdot 61$ \\
\hline Oxygen ............... & .0001873 & 0475 & 0551 & 1 & 1.80 \\
\hline Carbon dioxide & $\cdot 0001431$ & 0337 & $\begin{array}{l}0335 \\
0309 \\
0305\end{array}$ & $\begin{array}{l}1 \\
2 \\
3\end{array}$ & $\begin{array}{r}1.50 \\
1.51\end{array}$ \\
\hline Nitrous oxide ...... & .0001381 & 0342 & $\begin{array}{l}0353 \\
0350\end{array}$ & $\begin{array}{l}1 \\
3\end{array}$ & 1.65 \\
\hline
\end{tabular}

From these figures it will be seen that the agreement between theory and observation is in every way as good as could be expected. The mean of the entries in the last column is 1.65 , which is in excellent agreement with the theoretical number 1.6027. And we shall see later that the discrepancy, small though it is, is such as could reasonably be attributed to the intermolecular forces in the gas.

* Authorities:-1 Winkelman, 2 Graetz (Meyer, Kinetic Theory, p. 295), 3 Wüllner (Meyer, p. 293). 


\title{
CHAPTER XIV.
}

\author{
FREE PATH PHENOMENA (CONTINUED).
}

\section{Diffusion.}

\section{Elementary Theory.}

322. THE difficulties in the way of an exact mathematical treatment of diffusion are similar to those which occurred in the problems of viscosity and heat conduction. Following the method adopted in discussing these earlier problems, we shall begin by giving a simple, but mathematically inaccurate, treatment of the question.

We imagine two gases diffusing through one another in a direction parallel to the axis of $z$, the motion being the same at all points in a plane perpendicular to the axis of $z$. The arrangement of the gases is accordingly in layers perpendicular to the axis of $z$. Let us denote the mass velocity of the whole gas in the direction of $z$ increasing by $w_{0}$, and the molecular deusities of the two gases by $\nu_{1}, \nu_{2}$. Then $\nu_{1}, \nu_{2}$ and $w_{0}$ are functions of $z$ only.

We assume that as far as the order of approximation required in the problem, the mass-velocity of the gas is small compared with its molecularvelocity, and we also assume that the linear scale of variation of either gas is great compared with the average mean free path of a molecule.

We may, therefore, to a first approximation, assume that Maxwell's law of distribution of velocities obtains at every point, and that $h$ is the same for the two gases.

Since the mass velocities are small quantities of the first order, and their squares are therefore small quantities of the second order, it follows that, as far as the first order of small quantities, the pressure $p$ is constant throughout the gas. 
Since, by equation (264),

$$
p=\frac{\nu_{1}+\nu_{2}}{2 h}
$$

and since $p$ and $h$ are constant throughout the gas, it follows that

$$
\nu_{1}+\nu_{2}=\text { constant }
$$

This, in fact, is nothing more than a re-statement of Avogadro's Law (§ 128).

323. The number of molecules of the first kind, which cross the plane $z=z_{0}$ per unit area per unit time in the direction of $z$ increasing, is

$$
\sqrt{\frac{h^{3} m_{1}^{3}}{\pi^{3}}} \iiint \nu_{1} e^{-h m_{1}\left[u^{2}+v^{2}+\left(w-w_{0}\right)^{2}\right]} w d u d v d w
$$

in which the limits are from $-\infty$ to $+\infty$ as regards $u$ and $v$, and from 0 to $\infty$ as regards $w$.

These, however, do not all come from the same point, and $\nu_{1}$ must, in accordance with the principles already explained, be evaluated at the point from which they started after their last collision. Those which move so as to make an angle $\theta$ with the axis of $z$ may be supposed, on the average, to come from a point of which the $z$ coordinate is $z_{0}-\lambda \cos \theta$, and at this point the value of $\nu_{1}$ may be taken to be

$$
\nu_{1}=\nu_{1}\left(z_{0}\right)-\lambda \cos \theta\left(\frac{\partial \nu_{1}}{\partial z}\right)_{z_{0}}
$$

If, then, we wish to go as far as the first order of small quantities we divide the integral (620) into two integrals corresponding to the two terms of the right-hand of equation (621).

We obtain as the value of the first

$$
\begin{aligned}
& \sqrt{\frac{h^{3} m_{1}^{3}}{\pi^{3}}} \iiint \nu_{1}\left(z_{0}\right) e^{-h m_{1}\left[u^{2}+v^{2}+\left(w-w_{0}\right)^{2}\right]} w d u d v d w \\
& =\nu_{1}\left(z_{0}\right) \sqrt{\frac{h^{3} m_{1}^{3}}{\pi^{3}}} \iiint e^{-h m_{1}\left(u^{2}+v^{2}+\mathrm{w}^{2}\right)}\left(\mathrm{w}+w_{0}\right) d u d v d \mathrm{w} \\
& \quad \text { where } \mathrm{w}=w-w_{0} \\
& =\nu_{1}\left(z_{0}\right) \sqrt{\frac{h^{3} m_{1}^{3}}{\pi^{3}}} \int_{-\infty}^{+\infty} e^{-h m_{1} u^{2}} d u \int_{-\infty}^{+\infty} e^{-h m_{1} v^{2}} d v \\
& \int_{-w_{0}}^{\infty} e^{-h m_{1} \mathrm{w}^{2}}\left(\mathrm{w}+w_{0}\right) d \mathrm{w} \ldots \ldots \ldots . . .(622)
\end{aligned}
$$

Now

$$
\int_{-\infty}^{+\infty} e^{-h m_{1} u^{2}} d u=\int_{-\infty}^{+\infty} e^{-h m_{1} v^{2}} d v=\sqrt{\frac{\pi}{h m}}
$$


and as far as the first order of small quantities,

$$
\begin{gathered}
\int_{-w_{0}}^{\infty} e^{-h m_{1} \mathrm{~W}_{2}} \mathrm{~W} d \mathrm{~W}=\int_{0}^{\infty} e^{-h m_{1} \mathrm{~W}^{2}} \mathrm{~W} d \mathrm{~W}=\frac{1}{2 h m}, \\
\int_{-w_{0}}^{\infty} e^{-h m_{1} \mathrm{~W}_{2}} w_{0} d \mathrm{~W}=w_{0} \int_{0}^{\infty} e^{-h m_{1} \mathrm{~W}^{2}} d \mathrm{~W}=\frac{1}{2} w_{0} \sqrt{\frac{\pi}{h m}} .
\end{gathered}
$$

Hence the value of expression (622) is

$$
\frac{1}{2} \nu_{1}\left(z_{0}\right)\left(\frac{1}{\sqrt{\pi h m}}+w_{0}\right)
$$

The second integral required for the evaluation of expression (620) is

$$
\lambda\left(\frac{\partial \nu_{1}}{\partial z}\right)_{z_{0}} \sqrt{\frac{h^{3} m_{1}^{3}}{\pi^{3}}} \iiint e^{-h m_{1}\left[u^{2}+v^{2}+\left(w-w_{0}\right)^{2}\right]} \cos \theta w d u d v d w
$$

Since $\lambda$ is a small quantity of the first order, we may put $w_{0}=0$ under the sign of integration, and if we now use the transformation

the expression

$$
\begin{aligned}
u & =c \sin \theta \cos \phi, \\
v & =c \sin \theta \sin \phi, \\
w & =c \cos \theta,
\end{aligned}
$$

$$
\begin{aligned}
& c=\infty, \theta=\frac{\pi}{2}, \phi=2 \pi
\end{aligned}
$$

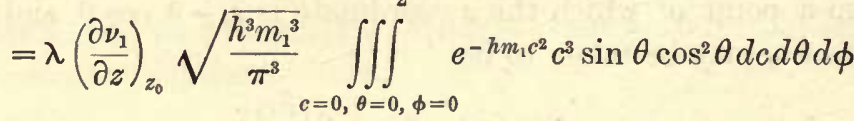

$$
\begin{aligned}
& =\lambda\left(\frac{\partial \nu_{1}}{\partial z}\right)_{z_{0}} \sqrt{\frac{h^{3} m_{1}^{3}}{\pi^{3}}} \cdot \frac{1}{2 h^{2} m^{2}} \cdot \frac{1}{3} \cdot 2 \pi \\
& =\lambda\left(\frac{\partial \nu_{1}}{\partial z}\right)_{z_{0}} \frac{1}{3 \sqrt{\pi h m_{1}}}
\end{aligned}
$$

By subtraction of expressions (625) and (623), we find as the total number of molecules of the first kind which cross the plane $z=z_{0}$ per unit area per unit time in thë direction of $z$ increasing (expression (620)),

$$
\frac{1}{\sqrt{\pi h m}}\left\{\frac{1}{2} \nu_{1}\left(z_{0}\right)-\frac{\lambda}{3}\left(\frac{\partial \nu_{1}}{\partial z}\right)_{z_{0}}\right\}+\frac{1}{2} w_{0} \nu_{1}\left(z_{0}\right)
$$

Similarly the number crossing in the reverse direction is

$$
\frac{1}{\sqrt{\pi h m}}\left\{\frac{1}{2} \nu_{1}\left(z_{0}\right)+\frac{\lambda}{3}\left(\frac{\partial \nu_{1}}{\partial z}\right)_{z_{0}}\right\}-\frac{1}{2} w_{0} \nu_{1}\left(z_{0}\right)
$$

The rate of increase of molecules of the first kind on the positive side of the plane $z=z_{0}$ per unit time per unit area is the difference of these two expressions, and therefore denoting this quantity by $\Gamma_{1}$,

$$
\Gamma_{1}=w_{0} \nu_{1}\left(z_{0}\right)-\frac{2}{3} \frac{\lambda_{1}}{\sqrt{\pi h m_{1}}}\left(\frac{\partial \nu_{1}}{\partial z}\right)_{z_{0}}
$$


If the similar quantity for the second kind of gas is denoted by $\Gamma_{2}$,

$$
\Gamma_{2}=w_{0} \nu_{2}\left(z_{0}\right)-\frac{2}{3} \frac{\lambda_{2}}{\sqrt{\pi h m_{2}}}\left(\frac{\partial \nu_{2}}{\partial z}\right)_{z_{0}}
$$

324. The increase per unit time of molecules of the first kind per unit volume at $z=z_{0}$ is clearly $\frac{d \Gamma_{1}}{d z}$, so that

$$
\begin{aligned}
& \frac{d \Gamma_{1}}{d z}=\frac{d \nu_{1}}{d t} \\
& \frac{d \Gamma_{2}}{d z}=\frac{d \nu_{2}}{d t}
\end{aligned}
$$

Hence

$$
\frac{d}{d z}\left(\Gamma_{1}+\Gamma_{2}\right)=\frac{d}{d t}\left(\nu_{1}+\nu_{2}\right)
$$

This quantity is in any case independent of $z$, since $\nu_{1}+\nu_{2}$ is constant throughout the gas, and vanishes in the steady state. Hence in the steady state $\Gamma_{1}+\Gamma_{2}$ is constant throughout the gas. Clearly $\Gamma_{1}+\Gamma_{2}$ measures the net flow of gas per unit area across any plane perpendicular to the axis of $z$. If there is no steady current of this kind,

$$
\Gamma_{1}+\Gamma_{2}=0
$$

We have also the relation (equation (619))

$$
\nu_{1}+\nu_{2}=\text { constant }
$$

whence, by differentiation,

$$
\left(\frac{\partial \nu_{1}}{\partial z}\right)+\left(\frac{\partial \nu_{2}}{\partial z}\right)=0
$$

If we add together equations (628) and (629), simplifying by means of the equation just obtained, we get

$$
\Gamma_{1}+\Gamma_{2}=w_{0}\left(\nu_{1}+\nu_{2}\right)-\frac{2}{3}\left(\frac{\partial \nu_{1}}{\partial z}\right)\left(\frac{\lambda_{1}}{\sqrt{\pi h m_{1}}}-\frac{\lambda_{2}}{\sqrt{\pi h m_{2}}}\right)
$$

and since $\Gamma_{1}+\Gamma_{2}=0$, this gives the value of $w_{0}$ in the form

$$
w_{0}=\frac{\frac{2}{3} \frac{\partial \nu_{1}}{\partial z}}{\nu_{1}+\nu_{2}}\left(\frac{\lambda_{1}}{\sqrt{\pi h m_{1}}}-\frac{\lambda_{2}}{\sqrt{\pi h m_{2}}}\right) .
$$

Substituting this value in equations (628) and (629) we obtain

$$
-\Gamma_{1}=\Gamma_{2}=\frac{2}{3} \frac{\frac{\partial \nu_{1}}{\partial z}}{\nu_{1}+\nu_{2}}\left(\frac{\nu_{2} \lambda_{1}}{\sqrt{\pi h m_{1}}}+\frac{\nu_{1} \lambda_{2}}{\sqrt{\pi h m_{2}}}\right)
$$


The coefficient of $\frac{\partial \nu_{1}}{\partial z}$ on the right-hand side is the coefficient of diffusion (D) for the two gases in question. If we write

$$
\begin{aligned}
& \frac{2}{\sqrt{\pi h m_{1}}}=\bar{c}_{1}, \\
& \frac{2}{\sqrt{\pi h m_{2}}}=\bar{c}_{2},
\end{aligned}
$$

so that $\bar{c}_{1}, \bar{c}_{2}$ are the mean velocities in the gases in question, this assumes the form

$$
\mathfrak{D}=\frac{1}{3} \frac{\lambda_{1} \bar{c}_{1} \nu_{2}+\lambda_{2} \bar{c}_{2} \nu_{1}}{\nu_{1}+\nu_{2}}
$$

325. If we consider diffusion between two gases in which the molecules are approximately of equal size and weight, and agree to neglect the differences in size and weight, we may take $\lambda$ and $c$ to be the same for each gas, and so obtain

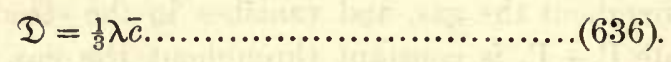

Comparing this with the value of the coefficient of viscosity (equation (573))

$$
\kappa=\frac{1}{3} \lambda \bar{c} \rho
$$

we obtain the relation

$$
\mathfrak{D}=\frac{\kappa}{\rho}
$$

\section{Correction when Molecules are Elastic Spheres.}

326. This equation of course requires correction by multiplication by a numerical factor, and this we shall now try to estimate. As in the case of viscosity, there are two sources of error, the first arising from our inaccurate evaluation of $\lambda$, and the second from neglect of the persistence of velocities.

As regards the first, it is clear that in expression (624), $\lambda$ must be replaced by $\lambda_{c}$ and taken under the sign of integration. Hence instead of $\lambda$ in the final result, we must have $l$, where

$$
l=\frac{\int_{0}^{\infty} \lambda_{c} e^{-h m c^{2}} c^{3} d c}{\int_{0}^{\infty} e^{-h m c^{2}} c^{2} d c}=\frac{\overline{\lambda_{c} c}}{\bar{c}}
$$

This however is exactly the same as the $l$ of the viscosity formula, of which the value was found in $\S 304$ to be

$$
\frac{1 \cdot 051}{\sqrt{2} \pi \nu \sigma^{2}}
$$

Hence this correction affects $\mathfrak{D}$ and $\kappa$ exactly similarly, multiplying each by 1.051, but does not affect equation (638). 
327. We now examine the effect of the persistence of velocities. When the molecule arrives in a given direction we found that the expectation of the distance it had described in that direction was not $\lambda$, but $\frac{\lambda}{1-\theta}$ where $\theta=406$. Hence the expectation of its belonging to one gas or the other is not that appropriate to a distance $\lambda$ back, but to a distance $\frac{\lambda}{1-\theta}$. The effect of "persistence" is therefore to multiply the value of $\mathfrak{D}$ given by equation (636), by a factor $\frac{1}{1-\theta}$. Also, as we have already seen, the effect on equation (637), is to multiply the value of $\kappa$ there found by $\frac{1}{1-\frac{1}{2} \theta}$.

The two equations, both corrected for persistence, accordingly become

$$
\begin{aligned}
\mathfrak{D} & =\frac{1}{3(1-\theta)} \lambda \bar{c}, \\
\kappa & =\frac{1}{3\left(1-\frac{1}{2} \theta\right)} \lambda \bar{c} \rho,
\end{aligned}
$$

so that the corrected form of equation (638) must be

$$
D=\frac{1-\frac{1}{2} \theta}{1-\theta} \frac{\kappa}{\rho}
$$

Putting $\theta=\cdot 406$, the value found in $§ 291$, this becomes

$$
D=1 \cdot 34 \frac{\kappa}{\rho}
$$

It is of interest to examine the difference between the effect of persistence of velocities on diffusion on the one hand, and on viscosity and conduction of heat on the other. Diffusion, it will be seen, is a transport of a quality, while viscosity and heat-conduction are transports of quantities. The difference rests ultimately upon the circumstance that qualities remain unaltered by collisions, whereas quantities do not.

\section{Comparison with Experiment.}

328. The last equation gives a quantity which it is impossible to measure experimentally, the interdiffusivity of a gas into itself.

If, however, we take any three gases, and measure the coefficients of diffusion $\mathfrak{D}_{12}, \mathfrak{D}_{23}, \mathfrak{D}_{31}$ of the three pairs of gases separately, we can calculate $\mathfrak{D}_{11}, \mathfrak{D}_{22}$ and $\mathfrak{D}_{33}$. For our knowledge of $\mathfrak{D}_{12}, \mathfrak{D}_{23}$ and $\mathfrak{D}_{31}$ will, by the use of equation (635), lead to three equations between $\sigma_{1}, \sigma_{2}$ and $\sigma_{3}$. If we solve these equations, we can use the values of $\sigma_{1}, \sigma_{2}$ and $\sigma_{3}$ to calculate $\mathfrak{D}_{11}$, $\mathfrak{D}_{22}$ and $\mathfrak{D}_{33}$. 
From some experiments of Loschmidt, treated in this way, the values of coefficients of interdiffusivity of four gases can be calculated. The following table is taken from Kelvin's Baltimore Lectures (p. 295).

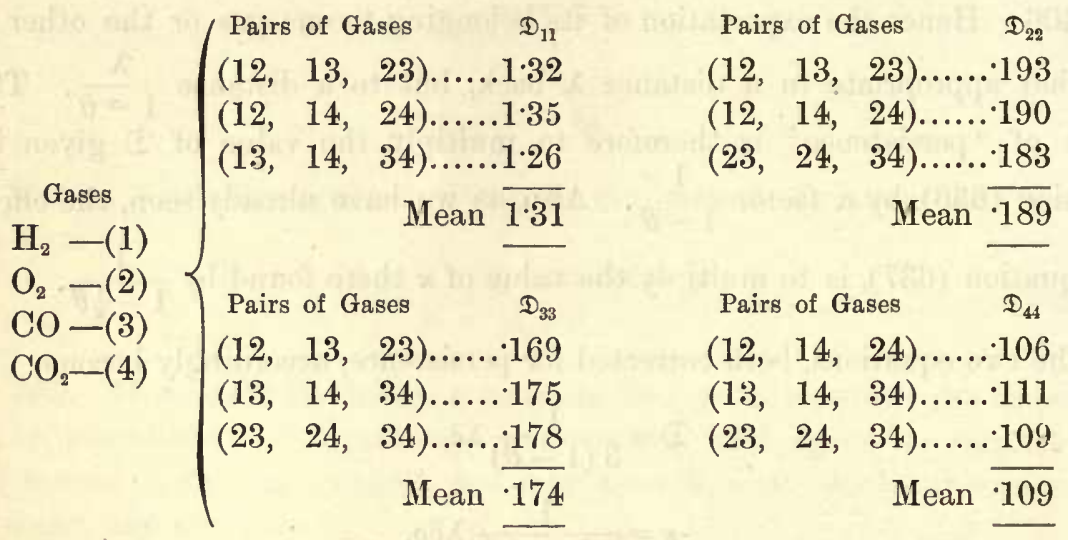

'The agreement inter se of the values obtained by a set of three gases gives in itself a striking confirmation of the theory.

Let us, however, carry our test further by comparing the values of the coefficients of interdiffusivity just found with those given by equation (640). In the following table, the first column gives $\kappa$, the mean of the values given in the table of p. 251, the second column gives $\rho$, the third gives the value of $D$ calculated from equation (640), and the fourth gives the value of $\mathcal{D}$ calculated from Loschmidt's experiments.

\begin{tabular}{|c|c|c|c|c|c|}
\hline Gas & $\kappa(\mathrm{p} .251)$ & $\rho($ p. 113) & $\frac{1 \cdot 34 \kappa}{\rho}$ & $D$ (exper.) & $\frac{D \rho}{\kappa}$ \\
\hline Hydrogen............ & $\cdot 0000864$ & .0000900 & $1 \cdot 28$ & $1 \cdot 31$ & $1 \cdot 37$ \\
\hline Oxygen............... & $\cdot 0001873$ & $\cdot 001429$ & $\cdot 175$ & - $\cdot 189$ & $1 \cdot 44$ \\
\hline Carbon monoxide... & $\cdot 0001628$ & $\cdot 001234$ & $\cdot 177$ & $\cdot 174$ & $1 \cdot 32$ \\
\hline Carbon dioxide...... & .0001431 & .001974 & .097 & $\cdot 109$ & 1.50 \\
\hline & & & & Mean & 1.41 \\
\hline
\end{tabular}

The mean of the entries in the last column is 1.41 which is in excellent agreement with the theoretical 1.34 ; and, as previously occurred in the similar case of heat-conduction, we shall see that the small discrepancy is such as could be attributed to intermolecular forces. 


\section{CHAP'TER XV.}

\section{FREE PATH PHENOMENA (CONTINUED).}

\section{MAXWell's Theory.}

329. THE subject of the present chapter is Maxwell's theory of the behaviour of a gas in which the molecules are supposed to be point centres of force, repelling according to the inverse fifth power of the distance*. Maxwell's original theory has been greatly improved and elaborated by Kirchhoff and Boltzmann. In the present chapter we shall follow Kirchhoff's discussion of the problem more closely than the original investigation of Maxwell.

We have already seen, from the observed variation of the coefficient of viscosity with the temperature, that molecules may not legitimately be regarded as elastic spheres. If, as an alternative hypothesis, we suppose them to be point centres, repelling with a force $\mu r^{-8}$, the observed relation between viscosity and temperature gives us information as to the value of $s$.

For $\kappa$, the coefficient of viscosity, can only depend on the following quantities: $m$ and $C$ which measure the mass and mean velocity of the molecules, $\mu$ which measures the distance at which their action on one another reaches a certain intensity (replacing the "size" of the molecules, which has now become meaningless), and $\nu$ the number of molecules per cubic centimetre. It is clear as in $\S 307$, that $\kappa$ must be independent of $\nu$; so that $\kappa$ must be expressible as a function of $m, C$ and $\mu$.

The physical dimensions of $\kappa, m, C$ and $\mu$ are as follows:

$\kappa$ is of dimensions $M L^{-1} T^{-1}$,

$\begin{array}{llll}m & , & & M, \\ C & " & " & L T^{-1}, \\ \mu & " & , & M L^{8+1} T^{-2}\end{array}$

Hence we must have that $\kappa$ is proportional to

$$
\left(m^{s+1} C^{s+3} \mu^{-2}\right)^{\frac{1}{s-1}},
$$

* "On the Dynamical Theory of Gases," Collected Works, Ir. p. 26. 
this being the only way of combining $m, C$ and $\mu$ so as to get a quantity of the same physical dimensions as $\kappa$. If $\kappa$ is observed to vary as the $n$th power of the absolute temperature, and therefore as the $2 n$th power of $C$, we have the relation

$$
2 n=\frac{s+3}{s-1}
$$

the same relation as is given by equation (584).

From experimental evidence we found values for $s$ ranging from $5 \cdot 2$ to 12 . Maxwell* believed the value of $n$ for air and some other gases to be $n=1$, and hence supposed that $s=5$ gave the true law of force for all gases. It is now known that this is not true, but nevertheless the law $s=5$ gives an interesting mechanical illustration of the theory of gases, and is moreover the only law for which the theory has been worked out with strict mathematical accuracy.

If we make the assumption of a repulsion varying as some inverse power of the distance, the value of $s$ for all the gases tabulated on p. 257, lies between $s=5$ and $s=\infty$. Now the law of force for elastic spheres is one in which the force is zero for $r>\sigma$, and is infinite for $r<\sigma$. This may suitably be represented by the function $\mu_{0}\left(\frac{\sigma}{r}\right)^{s}$ where $s=\infty$. Thus $s=\infty$ (the law worked out in previous chapters) and $s=5$ (the law worked out in the present chapter), may be regarded as limits between which all gases lie.

\section{General Equation of Transfer.}

330. Let $Q$ be any function of the velocity components of a single molecule, e.g. momentum, energy. We proceed to form general equations expressing the transfer of $Q$.

At any point $x, y, z$ let $\bar{Q}$ be the mean value of $Q$, so that

$$
\bar{Q}=\iiint f(u, v, w) Q d u d v d v
$$

If we consider a fixed rectangular parallelepiped $d x d y d z$ at this point, the number of molecules inside it is $\nu d x d y d z$, and hence $\Sigma Q$, the aggregate amount of $Q$ inside the element of volume, is given by

$$
\Sigma Q=\nu \bar{Q} d x d y d z
$$

We now examine the various causes of change in $\Sigma Q$. In the first place some molecules will leave the element $d x d y d z$, taking of course a certain amount of $Q$ with them. It has been already found in expression (348), that the total number of molecules of class $\mathbf{A}$ lost to the element $d x d y d z$ in time $d t$ is

$$
d x d y d z d t\left(u \frac{\partial}{\partial x}+v \frac{\partial}{\partial y}+w \frac{\partial}{\partial z}\right)(\nu f) d u d v d w,
$$

* "On the Viscosity of Internal Friction of Air and other Gases," Collected Works, Ir. p. 1. 
and hence the total amount of $Q$ lost by motion into and out of the element is

$$
d x d y d z d t \iiint\left[\left(u \frac{\partial}{\partial x}+v \frac{\partial}{\partial y}+w \frac{\partial}{\partial z}\right)(\nu f)\right] Q d u d v d w \ldots . . .
$$

If we write

$$
\iiint u Q f(u, v, w) d u d v d w=\overline{u Q}, \text { etc., }
$$

expression (644) can be put in the form

$$
d x d y d z d t\left[\frac{\partial}{\partial x}(\nu \overline{u Q})+\frac{\partial}{\partial y}(\nu \overline{v Q})+\frac{\partial}{\partial z}(\overline{\nu w Q})\right]
$$

Another cause of change in $\Sigma Q$ is supplied by the action of external forces on the molecules. For any single molecule, we have

$$
\frac{\partial Q}{\partial t}=\frac{\partial Q}{\partial u} \frac{d u}{d t}+\frac{\partial Q}{\partial v} \frac{d v}{d t}+\frac{\partial Q}{\partial w} \frac{d w}{d t}=\frac{1}{m}\left(X \frac{\partial Q}{\partial u}+Y \frac{\partial Q}{\partial v}+Z \frac{\partial Q}{\partial w}\right)
$$

where $X, Y, Z$ are the components of external force, so that in time $d t$ the total value of $\Sigma Q$ experiences an increase

$$
d x d y d z d t \frac{\nu}{m}\left[X\left(\frac{\partial \bar{Q}}{\partial u}\right)+Y\left(\frac{\partial \vec{Q}}{\partial v}\right)+Z\left(\frac{\overrightarrow{\partial Q}}{\partial w}\right)\right]
$$

Lastly, $\Sigma Q$ may be changed by collisions between molecules. If $Q$ is any one of the quantities which have previously been denoted by $\chi_{1}, \chi_{2} \ldots \chi_{5}$, namely the mass, energy, and the three components of momentum of a molecule, there is no such change, but if $Q$ is any other function of the velocities such changes will occur. In general let us denote the increase in $\Sigma Q$ which is caused in the element $d x d y d z$ by collisions in time $d t$ by

$$
d x d y d z d t \Delta Q
$$

Expressions (645), (646) and (647) now contain between them, the effect of all possible changes in $\Sigma Q$. The value of $\Sigma Q$ is however given by expression (64.3), so that the change in $\Sigma Q$ in time $d t$ will be

$$
\frac{d}{d t}(\nu \bar{Q}) d x d y d z d t
$$

Comparing the two different values which have been obtained for this change, we have

$$
\begin{aligned}
\frac{d}{d t}(\nu \bar{Q})=-\left[\frac{\partial}{\partial x}(\nu \overline{u Q})\right. & \left.+\frac{\partial}{\partial y}(\nu \overline{v Q})+\frac{\partial}{\partial z}(\nu \overline{w Q})\right] \\
& +\frac{\nu}{m}\left[X\left(\frac{\partial \bar{Q}}{\partial u}\right)+Y\left(\frac{\partial \bar{Q}}{\partial v}\right)+Z\left(\frac{\partial Q}{\partial w}\right)\right]+\Delta Q
\end{aligned}
$$


331. From this general equation, we can obtain the equations of Chapter VII. as special cases. If we put $Q=1$, so that $\bar{Q}=1$, the equation becomes

$$
\begin{aligned}
\frac{d \nu}{d t} & =-\left[\frac{\partial}{\partial x}(\nu \bar{u})+\frac{\partial}{\partial y}(\nu \bar{v})+\frac{\partial}{\partial z}(\nu \bar{w})\right] \\
& =-\left[\frac{\partial}{\partial x}\left(\nu u_{0}\right)+\frac{\partial}{\partial y}\left(\nu v_{0}\right)+\frac{\partial}{\partial z}\left(\nu w_{0}\right)\right]
\end{aligned}
$$

the equation of continuity already obtained in equation (352).

Similarly, on putting $Q=m u$, we obtain

$$
m \frac{d}{d t}\left(\nu u_{0}\right)=-m\left[\frac{\partial}{\partial x}\left(\nu \overline{u^{2}}\right)+\frac{\partial}{\partial y}(\nu \overline{u v})+\frac{\partial}{\partial z}(\nu \overline{u w})\right]+\nu X+\Delta Q \ldots(650) .
$$

When the molecules collide only with other molecules of the same kind, $\Delta Q$ of course vanishes, and equation (650) now becomes identical with equation (358).

332. If we multiply equation (649) by $\bar{Q}$, and subtract from equation (648), we obtain as a new form for the general equation,

$$
\nu \frac{d \bar{Q}}{d t}=\Sigma\left[\bar{Q} \frac{\partial}{\partial x}(\nu \bar{u})-\frac{\partial}{\partial x}(\nu \overline{u Q})+\frac{\nu}{m} X\left(\frac{\partial \bar{Q}}{\partial u}\right)\right]+\Delta Q
$$

where $\Sigma$ denotes summation with respect to the three coordinates $x, y$ and $z$.

Let us now write

$$
u=u_{0}+\overrightarrow{\mathrm{U}} \text {, etc. }
$$

so that $\mathrm{u}, \mathrm{v}, \mathrm{W}$ are components of molecular velocity. Then

Hence

$$
\bar{u}=u_{0}, \quad \overline{\mathbf{U}}=0, \quad \overline{u Q}=\overline{u_{0} Q+\mathrm{U} Q}=u_{0} \bar{Q}+\overline{\mathrm{U} Q} .
$$

$$
\begin{aligned}
\bar{Q} \frac{\partial}{\partial x}(\nu \bar{u})-\frac{\partial}{\partial x}(\nu \bar{\psi} \bar{Q}) & =\bar{Q} \frac{\partial}{\partial x}\left(\nu u_{0}\right)-\frac{\partial}{\partial x}\left(\nu u_{0} \bar{Q}\right)-\frac{\partial}{\partial x}(\nu \overline{\mathrm{U} Q}) \\
u & =-\nu u_{0} \frac{\partial \bar{Q}}{\partial x}-\frac{\partial}{\partial x}(\nu \overline{\mathrm{U} Q}) .
\end{aligned}
$$

We further have

$$
\frac{\partial Q}{\partial u}=\frac{\partial Q}{\partial\left(u_{0}+U\right)}=\frac{\partial Q}{\partial u_{0}}
$$

where $Q$ is now expressed as a function of $u_{0}, u$, etc., so that

$$
\left(\frac{\partial \bar{Q}}{\partial u}\right)=\left(\frac{\partial \bar{Q}}{\partial u_{0}}\right)=\frac{\partial \bar{Q}}{\partial u_{0}} \text {. }
$$

Making these substitutions, equation (651) becomes

$$
\nu \frac{d \bar{Q}}{d t}=\Sigma\left[-\nu u_{0} \frac{\partial \bar{Q}}{\partial x}-\frac{\partial}{\partial x}(\nu \overline{\mathrm{UQ}})+\frac{\nu}{m} X \frac{\partial \bar{Q}}{\partial u_{0}}\right]+\Delta Q
$$


or, again, if we write

$$
\frac{D}{D t}=\frac{d}{d t}+u_{0} \frac{\partial}{\partial x}+v_{0} \frac{\partial}{\partial y}+w_{0} \frac{\partial}{\partial z}
$$

so that $\frac{D}{D t}$ denotes differentiation following an element of gas in its motion, the equation becomes

$$
\nu \frac{D \bar{Q}}{D t}=\Sigma\left[-\frac{\partial}{\partial x}(\nu \overline{\cup Q})+\frac{\nu}{m} X \frac{\partial \bar{Q}}{\partial u_{0}}\right]+\Delta Q
$$

As before if we put $Q=u$ in this equation, we have $\Delta Q=0$. Also $\bar{Q}=u_{0}$, so that

and also

$$
\begin{array}{r}
\frac{\partial \bar{Q}}{\partial u_{0}}=1, \\
\frac{\partial \bar{Q}}{\partial v_{0}}=\frac{\partial \bar{Q}}{\partial w_{0}}=0
\end{array}
$$

$$
\begin{aligned}
& \overline{u Q}=\overline{U\left(U+u_{0}\right)}=\overline{U^{2}}, \\
& \overline{v Q}=\overline{v\left(u+u_{0}\right)}=\overline{u v}, \text { etc. }
\end{aligned}
$$

Hence the equation becomes

$$
\nu \frac{D u_{0}}{D t}=-\frac{\partial}{\partial x}\left(\nu \overline{\mathrm{U}^{z}}\right)-\frac{\partial}{\partial y}(\nu \overline{\mathrm{UV}})-\frac{\partial}{\partial z}(\nu \overline{\mathrm{UW}})+\frac{\nu}{m} X
$$

an equation of motion which is identical with our previous equation (360).

There are of course two similar equations obtained from the general equation by putting $Q=v$ and $Q=w$ respectively. When these equations were obtained before in $\S 173$, they were regarded as expressing the motion of the gas in terms of the forces acting upon the gas. On the present occasion it is convenient to regard them as equations expressing $X, Y$ and $Z$ in terms of the motion of the gas.

From these three equations and equation (652) we can obtain an equation which does not contain $X, Y$ or $Z$, and which is therefore true for a gas independently of the action of external forces. Effecting the elimination of $X, Y$ and $Z$, this equation is found to be

$$
\nu \frac{D \bar{Q}}{D t}=\Sigma\left[-\frac{\partial}{\partial x}(\nu \overline{\cup Q})+\frac{\partial \bar{Q}}{\partial u_{0}}\left\{\nu \frac{D u_{0}}{D t}+\frac{\partial}{\partial x}\left(\nu \overline{\mathrm{U}^{2}}\right)+\frac{\partial}{\partial y}(\nu \overline{\mathrm{UV}})+\frac{\partial}{\partial z}(\nu \overline{\mathrm{UW}})\right\}\right]+\Delta Q
$$

or, arranging the terms in a form more convenient for use,

$$
\begin{aligned}
& \nu\left[\frac{D \bar{Q}}{D t}-\frac{\partial \bar{Q}}{\partial u_{0}} \frac{D u_{0}}{D t}-\frac{\partial \bar{Q}}{\partial v_{0}} \frac{D v_{0}}{D t}-\frac{\partial \bar{Q}}{\partial w_{0}} \frac{D w_{0}}{D t}\right] \\
& =\Sigma\left[-\frac{\partial}{\partial x}(\nu \bar{U} \bar{Q})+\frac{\partial \bar{Q}}{\partial u_{0}}\left\{\frac{\partial}{\partial x}\left(\nu \overline{\mathrm{U}^{2}}\right)+\frac{\partial}{\partial y}(\nu \overline{\mathrm{UV}})+\frac{\partial}{\partial z}(\nu \overline{\mathrm{UW}})\right\}\right]+\Delta Q
\end{aligned}
$$


Since $\bar{Q}$ is in general a function of $u_{0}, v_{0}, w_{0}$ and the mean values $\overline{U^{2}}, \overline{U V}$, etc., etc., the bracket on the left-hand side is seen to be the differential coefficient of $\bar{Q}$ with respect to the time, regarding the mean values as functions of the time, but $u_{0}, v_{0}, w_{0}$ as constants.

\section{Special Forms assumed by Equation of Transfer.}

333. The equation just found is the general equation expressing the transfer of $Q$. Further progress is only possible by calculating $\Delta Q$, the term which arises from collisions, directly from the dynamics of a collision. This, however, is only possible when the value of $Q$ is known, and when we have made a special assumption as to the law of force between molecules.

Maxwell uses the same equation (654) for the investigation of the three phenomena of viscosity, conduction of heat and diffusion, regarding viscosity as a transfer of momentum, conduction of heat as a transfer of energy, and diffusion as a transfer of mass.

Before calculating the form assumed by $\Delta Q$ on giving the requisite forms to $Q$ and making special assumptions as to the law of force, we shall find it convenient to examine the simplifications introduced into equation (654) on giving these forms to $Q$.

$$
\text { I. } Q=u^{2}+v^{2}+w^{2} \text {. }
$$

334. Let us first put

$$
\begin{aligned}
Q & =u^{2}+v^{2}+w^{2} \\
& =u_{0}{ }^{2}+v_{0}{ }^{2}+w_{0}{ }^{2}+u^{2}+v^{2}+w^{2}+2 u_{0} u+2 v_{0} v+2 w_{0} w .
\end{aligned}
$$

Thus $\bar{Q}=u_{0}^{2}+v_{0}^{2}+w_{0}^{2}+\overline{U^{2}}+\overline{\mathrm{V}}^{2}+\overline{\mathrm{W}}^{2}$,

$$
\overline{\mathrm{uQ}}=\overline{\mathrm{u}\left(\mathrm{u}^{2}+\mathrm{V}^{2}+\mathrm{W}^{2}\right)}+2 u_{0} \overline{\mathrm{U}^{2}}+2 v_{0} \overline{\mathrm{UV}}+2 w_{0} \overline{\mathrm{uW}} \text {. }
$$

We are going to assume the molecules to be point centres of force, so that their only kinetic energy is energy of translation. Hence, by the conservation of energy, we may take $\Delta Q=0$. The equation now becomes

$$
\begin{aligned}
& \nu \frac{D}{D t} \overline{\left(\mathbf{u}^{2}+\mathrm{v}^{2}+\mathrm{w}^{2}\right)} \\
& =\Sigma\left[-\frac{\partial}{\partial x}\left\{\nu \overline{\mathrm{U}\left(\mathrm{U}^{2}+\mathrm{V}^{2}+\mathrm{W}^{2}\right)}+2 \nu u_{0} \overline{\mathrm{U}^{2}}+2 \nu v_{0} \overline{\mathrm{UV}}+2 \nu w_{0} \overline{\mathrm{UW}}\right\}\right. \\
& \left.+2 u_{0}\left\{\frac{\partial}{\partial x}\left(\nu \overline{\mathrm{U}^{2}}\right)+\frac{\partial}{\partial y}(\nu \overline{\mathrm{UV}})+\frac{\partial}{\partial z}(\nu \overline{\mathrm{UW}})\right\}\right] \\
& \left.=\Sigma\left[-\frac{\partial}{\partial x}\left\{\nu \overline{U\left(U^{2}+\mathrm{V}^{2}+\mathrm{W}^{2}\right)}\right\}-2 \nu\left(\overline{\mathrm{U}^{2}} \frac{\partial u_{0}}{\partial x}+\overline{\mathrm{UV}} \frac{\partial u_{0}}{\partial y}+\overline{\mathrm{UW}} \frac{\partial u_{0}}{\partial z}\right)\right]\right)
\end{aligned}
$$


335. We shall require to give other values to $Q$ in equation (654), but in calculating these it will, for reasons which will be seen afterwards, be sufficient to neglect deviations from Maxwell's Law of distribution of velocities. We accordingly take

$$
\begin{array}{r}
\overline{\mathrm{U}^{2}}=\overline{\mathrm{V}^{2}}=\overline{\mathrm{W}^{2}}=q, \\
\overline{\mathrm{UV}}=\overline{\mathrm{VW}}=\overline{\mathrm{WU}}=0,
\end{array}
$$

and equation (654) becomes

$$
\begin{aligned}
\nu\left[\frac{D \bar{Q}}{D t}-\frac{\partial \bar{Q}}{\partial u_{0}} \frac{D u_{0}}{D t}-\frac{\partial \bar{Q}}{\partial v_{0}} \frac{D v_{0}}{D t}\right. & \left.-\frac{\partial \bar{Q}}{\partial w_{0}} \frac{D w_{0}}{D t}\right] \\
& =\Sigma\left[-\frac{\partial}{\partial x}(\nu \overline{\cup Q})+\frac{\partial \bar{Q}}{\partial u_{0}} \frac{\partial}{\partial x}(\nu q)\right]+\Delta Q
\end{aligned}
$$

$$
\text { II. } Q=u^{2} \text {. }
$$

336. We first use this equation by putting

so that

$$
\begin{aligned}
& Q=u^{2}=u_{0}^{2}+2 u_{0} \mathrm{u}+\mathrm{u}^{2}, \\
& \bar{Q}=u_{0}^{2}+q, \\
& \frac{\partial \bar{Q}}{\partial u_{0}}=2 u_{0}, \quad \frac{\partial \bar{Q}}{\partial v_{0}}=\frac{\partial \bar{Q}}{\partial w_{0}}=0, \\
& \overline{\mathrm{u} Q}=2 u_{0} q, \quad \overline{\mathrm{v} Q}=\overline{\mathrm{w} Q}=0 .
\end{aligned}
$$

Then the equation becomes

$$
\begin{aligned}
\nu \frac{D q}{D t} & =-\frac{\partial}{\partial x}\left(2 \nu u_{0} q\right)+2 u_{0} \frac{\partial}{\partial x}(\nu q)+\Delta u^{2} \\
& =-2 \nu q \frac{\partial u_{0}}{\partial x}+\Delta u^{2} \ldots \ldots \ldots \ldots \ldots \ldots
\end{aligned}
$$

There are two similar equations for $v^{2}$ and $w^{2}$. If we add the system of three together, we obtain

$$
3 \nu \frac{D q}{D t}=-2 \nu q\left(\frac{\partial u_{0}}{\partial x}+\frac{\partial v_{0}}{\partial y}+\frac{\partial w_{0}}{\partial z}\right)+\Delta u^{2}+\Delta v^{2}+\Delta w^{2}
$$

We have, however,

$$
\Delta u^{2}+\Delta v^{2}+\Delta w^{2}=\Delta\left(u^{2}+v^{2}+w^{2}\right)=0
$$

since, as already explained, $\Sigma\left(u^{2}+v^{2}+w^{2}\right)$ remains unchanged by collisions. Hence equation (657) becomes

$$
\frac{D q}{D t}+\frac{2}{3} q\left(\frac{\partial u_{0}}{\partial x}+\frac{\partial v_{0}}{\partial y}+\frac{\partial w_{0}}{\partial z}\right)
$$

This equation may be regarded as giving $\frac{D q}{D t}$. Substituting this value in equation (657), we obtain

$$
-\frac{2}{3} \nu q\left(\frac{\partial u_{0}}{\partial x}+\frac{\partial v_{0}}{\partial y}+\frac{\partial w_{0}}{\partial z}\right)+2 \nu q \frac{\partial u_{0}}{\partial x}=\Delta u^{2}
$$




\section{Adiabatic Motion.}

337. The equation of continuity (equation (649)) can be expressed in the form

$$
\frac{D \nu}{D t}+\nu\left(\frac{\partial u_{0}}{\partial x}+\frac{\partial v_{0}}{\partial y}+\frac{\partial w_{0}}{\partial z}\right)=0
$$

and with the help of this, equation (660) becomes

or

$$
\begin{gathered}
\frac{1}{q} \frac{D q}{D t}=\frac{2}{3} \frac{1}{\nu} \frac{D \nu}{D t} \\
\frac{D}{D t}\left(q \nu^{-\frac{9}{3}}\right)=0
\end{gathered}
$$

Thus as we follow an element of the gas in its motion $q \nu^{-\frac{8}{8}}$ remains constant. Since

$$
q=\overline{\mathrm{U}^{2}}=\frac{1}{3} C^{2}=\frac{p}{\rho}
$$

this equation simply expresses that $p \rho^{-\frac{6}{8}}$ remains constant, as an element is followed in its motion. This is the particular case of the adiabatic law obtained in $\S 201$, in which $\gamma=\frac{5}{3}$. This result we can understand. We have neglected the conduction of heat in making the suppositions of equation (662), and we have supposed the whole energy to be translational on putting $\Delta\left(u^{2}+v^{2}+w^{2}\right)=0$ in equation (659). We therefore get $\gamma=\frac{5}{3}$, as in $\S 203$.

\section{III. $Q=u v$.}

338. We next put $Q=u v$ in equation (656). We have

$$
\begin{aligned}
& \bar{Q}=\overline{\left(u_{0}+u\right)\left(v_{0}+\mathrm{v}\right)}=u_{0} v_{0}, \\
& \overline{\mathrm{u} Q}=v_{0} q, \quad \overline{\mathrm{v} Q}=u_{0} q, \quad \overline{\mathrm{w} Q}=0, \\
& \frac{d \bar{Q}}{d u_{0}}=v_{0}, \quad \frac{d \overline{\mathbf{Q}}}{d v_{0}}=u_{0}, \quad \frac{d \overline{\mathbf{Q}}}{d w_{0}}=0,
\end{aligned}
$$

so that equation (656) becomes

$$
0=-\left[\frac{\partial}{\partial x}\left(\nu v_{0} q\right)+\frac{\partial}{\partial y}\left(\nu u_{0} q\right)\right]+v_{0} \frac{\partial}{\partial x}(\nu q)+u_{0} \frac{\partial}{\partial y}(\nu q)+\Delta(u v),
$$

giving, upon simplification,

$$
\nu q\left(\frac{\partial v_{0}}{\partial x}+\frac{\partial u_{0}}{\partial y}\right)-\Delta(u v)=0
$$




$$
\text { IV. } Q=u\left(u^{2}+v^{2}+w^{2}\right) \text {. }
$$

339. Lastly, in equation (656) we put

$$
\begin{aligned}
Q & =u\left(u^{2}+v^{2}+w^{2}\right) \\
& =\left(u_{0}+\mathrm{U}\right)\left(u_{0}{ }^{2}+v_{0}{ }^{2}+w_{0}{ }^{2}+2 u_{0} \mathrm{U}+2 v_{0} \mathrm{v}+2 w_{0} \mathrm{~W}+\mathrm{u}^{2}+\mathrm{v}^{2}+\mathrm{W}^{2}\right),
\end{aligned}
$$

so that

$$
\begin{aligned}
& \bar{Q}=u_{0}\left(u_{0}^{2}+v_{0}{ }^{2}+w_{0}^{2}\right)+5 u_{0} q, \\
& \frac{\partial \bar{Q}}{\partial u_{0}}=3 u_{0}^{2}+v_{0}^{2}+w_{0}^{2}+5 q, \quad \frac{\partial \bar{Q}}{\partial v_{0}}=2 u_{0} v_{0}, \quad \frac{\partial \bar{Q}}{\partial w_{0}}=2 u_{0} w_{0}, \\
& \overline{\mathrm{U} Q}=\left(3 u_{0}^{2}+v_{0}{ }^{2}+w_{0}^{2}\right) q+\overline{\mathrm{U}^{2}\left(\mathrm{U}^{2}+\mathrm{v}^{2}+\mathrm{W}^{2}\right),} \\
& \overline{\mathrm{v} Q}=2 u_{0} v_{0} q, \quad \overline{\mathrm{W} Q}=2 u_{0} w_{0} q .
\end{aligned}
$$

Since we are supposing the gas to be in a steady state, we may suppose $\mathrm{u}, \mathrm{v}, \mathrm{w}$ to be distributed according to Maxwell's Law. Assuming this law we have

$$
q=\overline{\mathrm{U}^{2}}=\frac{1}{3} C^{2}=\frac{1}{2 h m},
$$

so that

$$
\begin{gathered}
\overline{\mathrm{U}^{4}}=\frac{\int_{0}^{\infty} \mathrm{U}^{4} e^{-h m \mathrm{U}^{2}} d \mathrm{U}}{\int_{0}^{\infty} e^{-h m \mathrm{U}^{2}} d \mathrm{U}}=\frac{3}{4 h^{2} m^{2}}=3 q^{2}, \\
\overline{\mathrm{U}^{2} \mathrm{~V}^{2}}=\overline{\mathrm{U}^{2} \mathrm{~W}^{2}}=q^{2},
\end{gathered}
$$

and hence

$$
\overline{u^{2}\left(u^{2}+v^{2}+w^{2}\right)}=5 q^{2} .
$$

Hence on putting $Q=u\left(u^{2}+v^{2}+w^{2}\right)$ in equation (6こ6), we obtain

$$
\begin{aligned}
5 u_{0} \nu \frac{D q}{D t}= & -\frac{\partial}{\partial x}\left\{\nu\left(3 u_{0}{ }^{2}+v_{0}{ }^{2}+w_{0}{ }^{2}+5 q\right) q\right\} \\
& -\frac{\partial}{\partial y}\left(2 u_{0} v_{0} \nu q\right)-\frac{\partial}{\partial z}\left(2 u_{0} w_{0} \nu q\right) \\
& +\left(3 u_{0}{ }^{2}+v_{0}{ }^{2}+w_{0}{ }^{2}+5 q\right) \frac{\partial}{\partial x}(\nu q) \\
& +2 u_{0} v_{0} \frac{\partial}{\partial y}(\nu q)+2 u_{0} w_{0} \frac{\partial}{\partial z}(\nu q) \\
& +\Delta u\left(u^{2}+v^{2}+w^{2}\right) \\
= & -q\left[\frac{\partial}{\partial x}\left(3 u_{0}{ }^{2}+v_{0}{ }^{2}+w_{0}{ }^{2}+5 q\right)+\frac{\partial}{\partial y}\left(2 u_{0} v_{0}\right)+\frac{\partial}{\partial z}\left(2 u_{0} w_{0}\right)\right] \\
& +\Delta u\left(u^{2}+v^{2}+w^{2}\right) .
\end{aligned}
$$


If we substitute for $\frac{D q}{D t}$ from equation (660) and further simplify, we find

$$
\begin{aligned}
\Delta u\left(u^{2}+v^{2}+w^{2}\right) & =4 q u_{0}\left[\frac{\partial u_{0}}{\partial x}-\frac{1}{3}\left(\frac{\partial u_{0}}{\partial x}+\frac{\partial v_{0}}{\partial y}+\frac{\partial w_{0}}{\partial z}\right)\right] \\
& +2 q v_{0}\left(\frac{\partial v_{0}}{\partial x}+\frac{\partial u_{0}}{\partial y}\right)+2 q w_{0}\left(\frac{\partial w_{0}}{\partial x}+\frac{\partial u_{0}}{\partial z}\right)+5 q \frac{\partial q}{\partial x} \ldots \ldots
\end{aligned}
$$

\section{The Dynamics of Collisions.}

340. We now proceed to calculate the values of $\Delta Q$ which are required, directly from the dynamics of collisions, assuming the law of force between molecules to be that of the inverse fifth power of the distance.

With a view to a subsequent investigation of diffusion, we shall suppose there to be gases of two kinds, the masses of the molecules being $m_{1}$ and $m_{2}$ respectively.

Let us consider a collision between two molecules of different kinds. Their mutual potential energy at distance $r$ is

$$
\Phi=\int_{\infty}^{r} m_{1} m_{2} \frac{K}{r^{5}} d r,
$$

where $K m_{1}$ replaces our former $\mu$ for the first kind of molecule, and $K m_{2}$ replaces $\mu$ for the second. Integrating, we get

$$
\Phi=-m_{1} m_{2} \frac{K}{4 r^{4}}
$$

If at any instant $x_{1}, y_{1}, z_{1}$ are the coordinates of the first molecule, and $x_{2}, y_{2}, z_{2}$ those of the second, the equations of motion of the first molecule are

$$
m_{1} \ddot{x}_{1}=\frac{\partial \Phi}{\partial x_{1}}+m_{1} X, \text { etc. }
$$

while those of the second are

$$
m_{2} \ddot{x}_{2}=\frac{\partial \Phi}{\partial x_{2}}+m_{2} X, \text { etc. }
$$

From these we obtain

$$
m_{1} m_{2}\left(\ddot{x}_{1}-\ddot{x}_{2}\right)=m_{1} \frac{\partial \Phi}{\partial x_{1}}-m_{2} \frac{\partial \Phi}{\partial x_{2}}
$$

Let us write $x_{2}-x_{1}=x$, etc., so that $x, y, z$ are the coordinates of the second molecule relatively to the first. Then

$$
r^{2}=x^{2}+y^{2}+z^{2}
$$

so that since $\Phi$ is a function of $r$,

$$
\frac{\partial \Phi}{\partial x_{1}}=-\frac{\partial \Phi}{\partial x_{2}}=-\frac{\partial \Phi}{\partial x}
$$


and equation (666) becomes

$$
m_{1} m_{2} \ddot{x}=\left(m_{1}+m_{2}\right) \frac{\partial \Phi}{\partial x} .
$$

Thus the equations of motion can be written in the form

$$
\ddot{x}=\frac{\partial \Phi^{\prime}}{\partial x}, \text { etc. }
$$

where

$$
\Phi^{\prime}=\frac{m_{1}+m_{2}}{m_{1} m_{2}} \Phi=-\left(m_{1}+m_{2}\right) \frac{K}{r^{4}}
$$

341. With the centre of the first molecule as origin, let us take new coordinates $r, \theta$ in the plane of motion of the second. The motion of the second particle in this plane is, by equations (667), an orbit described by a particle of unit mass under the influence of a central force of potential $\Phi^{\prime}$.

Hence we have the usual two first integrals

$$
\begin{aligned}
r^{2} \dot{\theta} & =h \quad \ldots . \\
\frac{1}{2}\left(\dot{r}^{2}+r^{2} \dot{\theta}^{2}\right) & =\Phi^{\prime}+C
\end{aligned}
$$

where $C$ and $h$ are constants. Eliminating the time we obtain as the equation of the orbit,

or

$$
\frac{1}{2}\left\{\left(\frac{\partial r}{\partial \theta}\right)^{2}+r^{2}\right\} \frac{h^{2}}{r^{4}}=\Phi^{\prime}+C
$$

$$
\left(\frac{\partial r}{\partial \theta}\right)^{2}+r^{2}=\frac{2 r^{4}}{h^{2}} C-\frac{2}{h^{2}}\left(m_{1}+m_{2}\right) K
$$

If we take the direction of one asymptote for initial line this has the integral

$$
\theta=\int_{\infty}^{r} \frac{d r}{\sqrt{\frac{2 C}{h^{2}} r^{4}-r^{2}-\frac{2}{h^{2}}\left(m_{1}+m_{2}\right) K}}
$$

342. If $V$ is the velocity at infinity (i.e. the relative velocity before collision), and $p$ the perpendicular from the centre on to the asymptote described with velocity $V$, we have from equations (669) and (670),

$$
h=p V, \quad C=\frac{1}{2} V^{2}
$$

so that equation (672) can be written in the form

$$
\theta=\int_{\infty}^{r} \frac{d r}{\sqrt{\frac{r^{4}}{p^{2}}-r^{2}-\frac{2}{p^{2} V^{2}}\left(m_{1}+m_{2}\right) K}}
$$

If we now write $\eta$ for $\frac{p}{r}$, this becomes

$$
\theta=\int_{0}^{\eta} \frac{d \eta}{\sqrt{1-\eta^{2}-\frac{2 \eta^{4}}{a^{4}}}}
$$


where

$$
\alpha^{4}=\frac{p^{4} V^{2}}{\left(m_{1}+m_{2}\right) K}
$$

The apse is of course given by the vanishing of the denominator in the integral of equation (674). If the corresponding positive root for $\eta$ is $\eta_{0}$, the angle between the asymptotes, being twice the angle between either asymptote and the apsidal line, is given by

$$
\theta^{\prime}=2 \int_{0}^{\eta_{0}} \frac{d \eta}{\sqrt{1-\eta^{2}-\lambda^{4} \eta^{4}}}
$$

After collision the velocities parallel and perpendicular to the initial line are of course

$-V \cos \theta^{\prime}$ and $V \sin \theta^{\prime}$.

343. There is naturally a doubly infinite series of possible orbits corresponding to different values of $p$ and $V$. Except for a difference of linear scale, however, these may be reduced to a singly infinite system corresponding to the variation of $p^{2} V$. In fig. 23, which is taken from Maxwell's original paper*, some members of this singly infinite system are shewn.

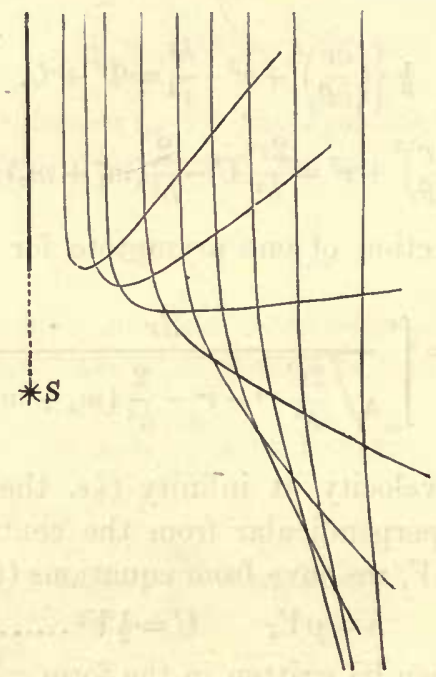

Fio. 23.

344. Let us consider a collision between two molecules, the velocities before collision being $u, v, w$ and $u^{\prime}, v^{\prime}, w^{\prime}$. The relative velocity $V$ is given by

$$
V^{2}=\left(u^{\prime}-u\right)^{2}+\left(v^{\prime}-v\right)^{2}+\left(w^{\prime}-w\right)^{2}
$$

and $V$ is here the same as the $V$ of $\S 342$.

* Collected Works, Ir. p. 42. I am indebted to the University Press for the use of the original block. 
Let us imagine the first molecule to be at rest, and the second to approach it with a velocity of components $u^{\prime}-u, v^{\prime}-v, w^{\prime}-w$. Let the direction of this velocity meet a plane perpendicular to it through the centre of the second molecule in a point of which the polar coordinates referred to the

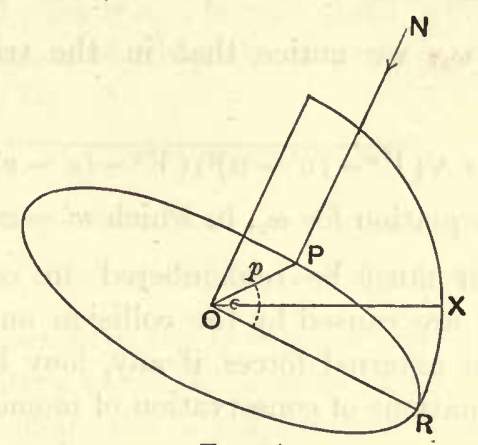

Fra. 24.

second molecule as origin are $p, \epsilon$. Then $p$ is the same as the $p$ of $\$ 342$. Let $\epsilon$ be measured from $O R$, the intersection of the plane normal to $N P$ with a plane through $O$ containing the directions of $P N$ and of the axis of $x$.

In fig. 25, every point except $O$ is on a unit sphere having $O$ as centre.

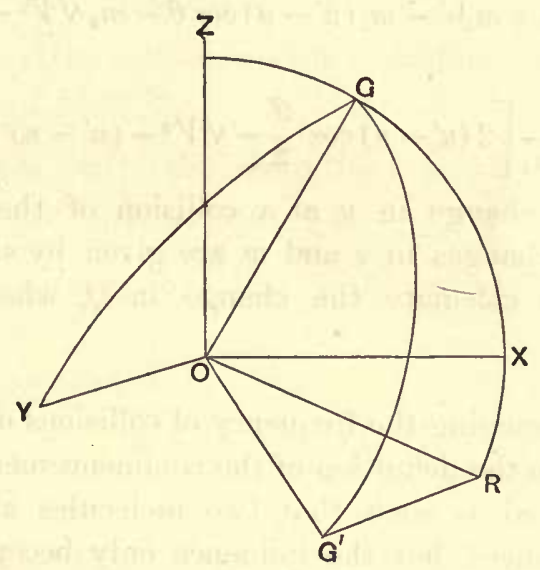

Fra. 25.

Thus if $O G, O G^{\prime}$ are directions of motion before and after collision,

$$
\widehat{R G G^{\prime}}=\epsilon, \quad G G^{\prime}=\theta^{\prime}
$$

therefore $\quad \cos G^{\prime} X=\cos G X \cos G G^{\prime}+\sin G X \sin G G^{\prime} \cos \epsilon$.

$$
\text { But } \quad \cos G^{\prime} X=\frac{\bar{u}^{\prime}-\bar{u}}{V}, \quad \cos G X=\frac{u^{\prime}-u}{V},
$$

where $\bar{u}, \bar{u}^{\prime}$, etc. are components of velocity after collision, so that

$$
\bar{u}^{\prime}-\bar{u}=\left(u^{\prime}-u\right) \cos \theta^{\prime}+\sqrt{V^{2}-\left(u^{\prime}-u\right)^{2}} \sin \theta^{\prime} \cos \epsilon
$$


If we suppose the angle $X G Y$ to be $\omega_{2}$ and $X G Z$ to be $\omega_{3}$, we shall have in a similar way,

$$
\begin{aligned}
\bar{v}^{\prime}-\bar{v} & =\left(v^{\prime}-v\right) \cos \theta^{\prime}+\sqrt{V^{2}-\left(v^{\prime}-v\right)^{2}} \sin \theta^{\prime} \cos \left(\epsilon-\omega_{2}\right) \ldots \ldots \\
\bar{w}^{\prime}-\bar{w} & =\left(w^{\prime}-w\right) \cos \theta^{\prime}+\sqrt{V^{2}-\left(w^{\prime}-w\right)^{2}} \sin \theta^{\prime} \cos \left(\epsilon-\omega_{3}\right) \ldots
\end{aligned}
$$

To determine $\omega_{2}, \omega_{3}$, we notice that in the triangle $G X Y, X Y=\frac{\pi}{2}$, $X G Y=\omega_{2}$, hence

$$
\left(u^{\prime}-u\right)\left(v^{\prime}-v\right)+\sqrt{\left(V^{2}-\left(u^{\prime}-u\right)^{2}\right)\left(V^{2}-\left(v^{\prime}-v\right)^{2}\right)} \cos \omega_{2}=0
$$

and there is a similar equation for $\omega_{3}$, in which $w^{\prime}-w$ replaces $v^{\prime}-v$.

We are engaged, it must be remembered, in calculating the changes in the velocities which are caused by the collision only, so that the changes caused by the action of external forces, if any, may be neglected. We may accordingly use the equations of conservation of momentum. Thus

$$
m_{1} \bar{u}+m_{2} \bar{u}^{\prime}=m_{1} u+m_{2} u^{\prime}
$$

an equation which can of course be obtained directly, by the integration of the equations of $\S 340$.

Multiplying equation (678) by $m_{2}$ and subtracting from this the equation of momentum, we obtain

$$
\left(m_{1}+m_{2}\right) \vec{u}=m_{1} u+m_{2} u^{\prime}-m_{2}\left(u^{\prime}-u\right) \cos \theta-m_{2} \sqrt{V^{2}-\left(u^{\prime}-u\right)^{2}} \sin \theta^{\prime} \cos \epsilon,
$$

so that finally

$$
\bar{u}-u=\frac{m_{2}}{m_{1}+m_{2}}\left[2\left(u^{\prime}-u\right) \cos ^{2} \frac{\theta^{\prime}}{2}-\sqrt{V^{2}-\left(u^{\prime}-u\right)^{2}} \sin \theta^{\prime} \cos \epsilon\right] \ldots(683) \text {. }
$$

This gives the change in $u$ at a collision of the type we have been considering. The changes in $v$ and $w$ are given by similar equations, and from these we can calculate the change in $Q$, when $Q$ is any function of $u, v$, and $w$.

345. Before discussing the frequency of collisions of given type, we must make a remark as to the definition of the commencement of a collision. The law of force assumed is such that two molecules are influenced by one another at all distances, but the influence only becomes perceptible when the molecules are near to one another. If we suppose the influence to be perceptible when the molecules come within a distance $\sigma$ of one another, we may regard $\sigma$ as the diameter of a sphere of molecular action, and may define the commencement of a collision as the instant at which either molecule crosses a sphere of radius $\sigma$ surrounding the other. This gives an approximation which is the better, the greater the value of $\sigma$. In the limit we shall find it possible to pass to the limit and take $\sigma=\infty$, in this way disposing entirely of the error involved in assuming the existence of a sphere of molecular action. 
With this definition, the probability that a molecule of mass $m_{1}$ and of class $A$ undergoes collision with a second molecule of mass $m_{2}$ and of class $B$ in time $d t$, the relative position of the molecules being such that $p, \epsilon$ lie within certain limits $d p, d \epsilon$, is seen to be equal to the probability of finding a molecule of mass $m_{2}$ and class $B$ in a certain small cylinder of height $V d t$ and of base $p d p d \epsilon$. The number of molecules of mass $m_{2}$ and of class $B$ per unit volume may be taken to be

$$
\nu_{2} f_{2}\left(u^{\prime}, v^{\prime}, w^{\prime}\right) d u^{\prime} d v^{\prime} d v^{\prime}
$$

so that the probability in question is

$$
\nu_{2} f_{2}\left(u^{\prime}, v^{\prime}, w^{\prime}\right) d u^{\prime} d v^{\prime} d w^{\prime} V d t p d p d \epsilon
$$

Let $[Q]$ denote the change in the $Q$ of a molecule of mass $m_{1}$ and of class $A$ caused by collision with a molecule of mass $m_{2}$ and of class $B$. Then the average change in $Q$ for molecules of mass $m_{1}$ and of class $A$ caused in time $d t$ by collisions with molecules of mass $m_{2}$ and of class $B$, is obtained by multiplying the value of $[Q]$ obtained from equations of the type of (683) by expression (684) and integrating over all values of $p$ and $\epsilon$.

346. We can conveniently divide $\Delta Q$ into two parts, so that

$$
\Delta Q=\Delta_{11} Q+\Delta_{12} Q
$$

the former being caused by collisions with molecules of mass $m_{1}$, and the latter with molecules of mass $m_{2}$.

Then in accordance with the definition of $\Delta Q$ already given (expression (647)),

$\Delta_{12} Q=\iiint \iiint \int[Q] \nu_{1} \nu_{2} f_{1}(u, v, w) f_{2}\left(u^{\prime}, v^{\prime}, w^{\prime}\right) d u d v d w d u^{\prime} d v^{\prime} d w^{\prime} V p d p d \epsilon$

Since, however, (equation (675)),

$$
\alpha^{4}=\frac{p^{4} V^{2}}{\left(m_{1}+m_{2}\right) K}
$$

we have

$$
V p d p=\sqrt{\left(m_{1}+m_{2}\right) K} \alpha d \alpha
$$

so that

$$
\begin{array}{r}
\Delta_{12} Q=\sqrt{\left(m_{1}+m_{2}\right) K} \iiint \iiint \iint[Q] \nu_{1} \nu_{2} f_{1}(u, v, w) f_{2}\left(u^{\prime}, v^{\prime}, w^{\prime}\right) \\
d u d v d w d u^{\prime} d v^{\prime} d w^{\prime} \alpha d \alpha d \epsilon .
\end{array}
$$

If we write $|\phi|$ for the mean value of any quantity $\phi$ averaged over all molecules of type $\mathbf{1}$ and also over all molecules of type 2 , so that

$$
|\phi|=\iiint \iiint_{-\infty}^{+\infty} \int_{-\infty}^{+\infty} \phi f_{1}(u, v, w) f_{2}\left(u^{\prime}, v^{\prime}, w^{\prime}\right) d u d v d w d u^{\prime} d v^{\prime} d w^{\prime}
$$

J. 
then this equation becomes

$$
\Delta_{12} Q=\nu_{1} \nu_{2} \sqrt{\left(m_{1}+m_{2}\right) K} \mid \int_{\epsilon=0}^{\epsilon=2 \pi} \int_{\alpha=0}^{\alpha=\infty}[Q] \alpha d \alpha d \epsilon
$$

\section{Calculations of $\Delta Q$.}

I. $Q=u$.

347. We begin by putting $Q=u$. The value of $[Q]$, or of $\bar{u}-u$, is now given at once by equation (683), and we have, by equation (686)

$$
\begin{aligned}
\Delta_{12} u & =\nu_{1} \nu_{2} m_{2} \sqrt{\frac{K}{m_{1}+m_{2}}} \\
& \left|\int_{\epsilon=0}^{\epsilon=2 \pi} \int_{a=0}^{\alpha=\infty}\left\{2\left(u^{\prime}-u\right) \cos ^{2} \frac{\theta^{\prime}}{2}-\sqrt{V^{2}-\left(u^{\prime}-u\right)^{2}} \sin \theta^{\prime} \cos \epsilon\right\} \alpha d \alpha d \epsilon\right| .
\end{aligned}
$$

The integration with respect to $\epsilon$ can be effected at once, resulting in the disappearance of the last term, and leaving

$$
\begin{aligned}
\Delta_{12} u & =4 \pi \nu_{1} \nu_{2} m_{2} \sqrt{\frac{K}{m_{1}+m_{2}}}\left|\int_{\alpha=0}^{\alpha=\infty}\left(u^{\prime}-u\right) \cos ^{2} \frac{\theta^{\prime}}{2} \alpha d \alpha\right| \\
& =4 \pi \nu_{1} \nu_{2} m_{2} \sqrt{\frac{K}{m_{1}+m_{2}}}\left(u_{0}^{\prime}-u_{0}\right) \int_{\alpha=0}^{\alpha=\infty} \cos ^{2} \frac{\theta^{\prime}}{2} \alpha d \alpha .
\end{aligned}
$$

Maxwell writes

$$
4 \pi \int_{\alpha=0}^{\alpha=\infty} \cos ^{2} \frac{\theta^{\prime}}{2} a d \alpha=A_{1}
$$

Tables for the calculation of $A_{1}$ by quadrature are given in Maxwell's original paper. He finds for its value

$$
A_{1}=2 \cdot 6595
$$

and we accordingly have

$$
\Delta_{12} u=\nu_{1} \nu_{2} m_{2} \sqrt{\frac{K}{m_{1}+m_{2}}} A_{1}\left(u_{0}^{\prime}-u_{0}\right)
$$

To calculate $\Delta_{11}$, we notice that $u_{0}^{\prime}$ and $u_{0}$ now become identical, so that $\Delta_{11} u=0$. This could have been foreseen: the value of $u$ averaged through a system of equal molecules cannot be changed by collisions with one another.

Thus the expression on the right-hand of equation (689) contains the whole value of $\Delta u$, so that

$$
\Delta u=\nu_{1} \nu_{2} m_{2} \sqrt{\frac{K}{m_{1}+m_{2}}} A_{1}\left(u_{0}^{\prime}-u_{0}\right)
$$


348. We require also to calculate $\Delta Q$ where $Q$ is replaced by certain functions of the second and third degree, but these quantities will be required for a single gas only. In this case equation (686) becomes

$$
\Delta Q=\nu^{2} \sqrt{2 m K}\left|\int_{\epsilon=0}^{\epsilon=2 \pi} \int_{\alpha=0}^{\alpha=\infty}[Q] \alpha d \alpha d \epsilon\right|
$$

\section{II. $Q=u^{2}$.}

349. We begin by putting $Q=u^{2}$. The value of $\bar{u}^{2}-u^{2}$ for a single collision, from equation (683), is given by

$$
\bar{u}^{\varepsilon}-u^{2}=\left\{u+\left(u^{\prime}-u\right) \cos ^{2} \frac{\theta^{\prime}}{2}-\frac{1}{2} \sqrt{V^{2}-\left(u^{\prime}-u\right)^{2}} \sin \theta^{\prime} \cos \epsilon\right\}^{2}-u^{2} .
$$

If we substitute this value for $[Q]$ in equation (691), and perform the integration with respect to $\epsilon$, we obtain

$\Delta u^{2}=2 \pi \nu^{2} \sqrt{2 m K}$

$$
\left|\int_{a=0}^{\alpha=\infty}\left(2 u\left(u^{\prime}-u\right) \cos ^{2} \frac{\theta^{\prime}}{2}+\left(u^{\prime}-u\right)^{2} \cos ^{4} \frac{\theta^{\prime}}{2}+\frac{1}{8}\left(V^{2}-\left(u^{\prime}-u\right)^{2}\right) \sin ^{2} \theta^{\prime}\right) \alpha d \alpha\right|
$$

Since

$$
\begin{aligned}
& V^{2}-\left(u^{\prime}-u\right)^{2}=\left(v^{\prime}-v\right)^{2}+\left(w^{\prime}-w\right)^{2} \text {, } \\
& \text { and } \quad \cos ^{4} \frac{\theta^{\prime}}{2}=\cos ^{2} \frac{\theta^{\prime}}{2}-\frac{1}{4} \sin ^{2} \theta^{\prime}
\end{aligned}
$$

the bracket in the integrand becomes

$$
\left(u^{\prime 2}-u^{2}\right) \cos ^{2} \frac{\theta^{\prime}}{2}+\frac{1}{8}\left\{-2\left(u^{\prime}-u\right)^{2}+\left(v^{\prime}-v\right)^{2}+\left(w^{\prime}-w\right)^{2}\right\} \sin ^{2} \theta^{\prime}
$$

Now

$$
\left|u^{\prime 2}-u^{2}\right|=0
$$

$$
\begin{aligned}
\mid-2\left(u^{\prime}-u\right)^{2}+\left(v^{\prime}-v\right)^{2} & +\left(w^{\prime}-w\right)^{2} \mid \\
= & \left|-2\left(\mathrm{u}^{\prime}-\mathrm{u}\right)^{2}+\left(\mathrm{v}^{\prime}-\mathrm{v}\right)^{2}+\left(\mathrm{w}^{\prime}-\mathrm{w}\right)^{2}\right| \\
= & 2\left(-2 \overline{\mathrm{u}^{2}}+\overline{\mathrm{v}^{2}}+\overline{\mathrm{W}^{2}}\right) .
\end{aligned}
$$

Hence equation (692) becomes

$$
\Delta u^{2}=2 \pi \nu^{2} \sqrt{2 m K} \cdot \frac{1}{4}\left(-2 \overline{U^{2}}+\overline{V^{2}}+\overline{W^{2}}\right) \int_{0}^{\infty} \sin ^{2} \theta^{\prime} \alpha d \alpha
$$

Maxwell writes

$$
\pi \int_{0}^{\infty} \sin ^{2} \theta^{\prime} \alpha d \alpha=A_{2}
$$


and calculates for $A_{2}$ the value

$$
A_{2}=1 \cdot 3682
$$

Hence

$$
\Delta u^{2}=\frac{1}{2} \nu^{2} \sqrt{2 m K} A_{2}\left(-2 \overline{\mathrm{U}^{2}}+\overline{\mathrm{V}^{2}}+\overline{\mathrm{W}^{2}}\right)
$$

\section{III. $Q=u v$.}

350. We also require $\Delta u v$, but this is more easily found by transformation of axes than by direct calculation.

Let us write $l x+m y+n z$ instead of $x$, so that we write $l u+m v+n w$ instead of $u$. The left-hand member of equation (696) becomes

$$
l^{2} \Delta u^{2}+2 l m \Delta u v+\ldots .
$$

The bracket on the right-hand may be written

$$
\overline{u^{2}+v^{2}+w^{2}}-3 \overline{u^{2}}
$$

and therefore transforms into

$$
\left(l^{2}+m^{2}+n^{2}\right)\left\{\overline{\mathbf{U}^{2}+\mathbf{v}^{2}+\mathbf{W}^{2}}-3(\overline{l \mathbf{u}+m \mathbf{v}+n \mathbf{W}})^{2}\right\} .
$$

Equating coefficients of $2 l m$, we have at once

$$
\Delta u v=-\frac{3}{2} \nu^{2} \sqrt{2 m K} A_{2} \overline{U V} .
$$

$$
\text { IV. } Q=u\left(u^{2}+v^{2}+w^{2}\right) \text {. }
$$

351. To evaluate $\Delta u\left(u^{2}+v^{2}+w^{2}\right)$, we need the complete system of three equations of the type of (683).

On putting $m_{1}=m_{2}$, equation (683) becomes

in which

$$
\bar{u}=u+a-a^{\prime} \cos \left(\epsilon-\omega_{1}\right) \text {. }
$$

$$
a=\left(u^{\prime}-u\right) \cos ^{2} \frac{\theta^{\prime}}{2}, \quad a^{\prime}=\frac{1}{2} \sqrt{V^{2}-\left(u^{\prime}-u\right)^{2}} \sin \theta^{\prime},
$$

and $\omega_{1}=0$. The angle $\omega_{1}$ has been introduced for the sake of symmetry, for we have now, from equations (679) and (680),

$$
\begin{aligned}
& \bar{v}=v+b-b^{\prime} \cos \left(\epsilon-\omega_{2}\right) \ldots \ldots \ldots \ldots \ldots \ldots . . .(699) \text {, } \\
& \bar{w}=w+c-c^{\prime} \cos \left(\epsilon-\omega_{3}\right) \ldots \ldots \ldots \ldots \ldots \ldots \ldots(700),
\end{aligned}
$$

where $b, b^{\prime}$ and $c, c^{\prime}$ are obtained from $a, a^{\prime}$ by replacing $u$ by $v$ and $w$ respectively, and, by equation (681),

$$
\begin{aligned}
& \cos \omega_{2}=\frac{\left(u^{\prime}-u\right)\left(v^{\prime}-v\right)}{4 a^{\prime} b^{\prime}} \sin ^{2} \theta^{\prime}, \\
& \cos \omega_{3}=\frac{\left(u^{\prime}-u\right)\left(w^{\prime}-w\right)}{4 a^{\prime} c^{\prime}} \sin ^{2} \theta^{\prime} .
\end{aligned}
$$


Squaring the system of three equations (698), (699) and (700), and adding,

$$
\bar{u}^{2}+\bar{v}^{2}+\bar{w}^{2}=\Sigma(u+a)^{2}-2 \Sigma(u+a) a^{\prime} \cos \left(\epsilon-\omega_{1}\right)+\Sigma a^{\prime 2} \cos ^{2}\left(\epsilon-\omega_{1}\right),
$$

so that

$$
\begin{gathered}
\bar{u}\left(\bar{u}^{2}+\bar{v}^{2}+\bar{w}^{2}\right)=(u+a) \Sigma(u+a)^{2}-2(u+a) \Sigma a^{\prime} \cos \left(\epsilon-\omega_{1}\right) \\
+(u+a) \Sigma a^{\prime 2} \cos ^{2}\left(\epsilon-\omega_{1}\right) \\
+a^{\prime} \cos \epsilon \Sigma(u+a)^{3}-2 a^{\prime} \cos \epsilon \Sigma a^{\prime 2} \cos \left(\epsilon-\omega_{1}\right) \\
+a^{\prime} \cos \epsilon \Sigma a^{\prime 2} \cos ^{2}\left(\epsilon-\omega_{1}\right) .
\end{gathered}
$$

Hence, on integration with respect to $\epsilon$,

$$
\begin{aligned}
\frac{1}{2 \pi} \int_{0}^{\epsilon} & {\left[u\left(u^{2}+v^{2}+w^{2}\right)\right] d \epsilon } \\
& =-u \Sigma u^{2}+(u+a)\left(\Sigma(u+a)^{2}+\frac{1}{2} \Sigma a^{\prime 2}\right)-2 \alpha^{\prime} \Sigma a^{\prime 2} \cos \omega_{1}
\end{aligned}
$$

To simplify this, we notice that

$a^{\prime} \Sigma a^{\prime} \cos \omega_{1}=\frac{1}{4} \sin ^{2} \theta^{\prime}\left\{V^{2}-\left(u^{\prime}-u\right)^{2}+\left(u^{\prime}-u\right)\left(v^{\prime}-v\right)+\left(u^{\prime}-u\right)\left(w^{\prime}-w\right)\right\}$,

$$
\begin{aligned}
\Sigma(u+a)^{2} & =\Sigma u^{2}+2 \Sigma u\left(u^{\prime}-u\right) \cos ^{2} \frac{1}{2} \theta^{\prime}+V^{2} \cos ^{4} \frac{1}{2} \theta^{\prime}, \\
\frac{1}{2} \Sigma \alpha^{\prime 2} & =\frac{1}{8} \sin ^{2} \theta^{\prime} \Sigma\left(V^{2}-\left(u^{\prime}-u\right)^{2}\right)=V^{2} \sin ^{2} \frac{1}{2} \theta^{\prime} \cos ^{2} \frac{1}{2} \theta^{\prime} ;
\end{aligned}
$$

so that we also have

$$
\Sigma(u+a)^{2}+\frac{1}{2} \Sigma a^{\prime 2}=\Sigma u^{2}+\Sigma\left(u^{\prime 2}-u^{2}\right) \cos ^{2} \frac{1}{2} \theta^{\prime} .
$$

It is clear therefore that the right-hand side of equation (701) can be expressed as the sum of two terms multiplied by $\cos ^{2} \theta^{\prime} / 2$ and $\sin ^{2} \theta^{\prime}$ respectively. Simplified as far as possible, we find for this expression the value

$$
\begin{aligned}
\left(u^{\prime} \Sigma u^{\prime 2}\right. & \left.-u \Sigma u^{2}\right) \cos ^{2} \frac{1}{2} \theta^{\prime} \\
& +\frac{1}{4} \sin ^{2} \theta^{\prime}\left[u\left(2 \Sigma u^{\prime 2}-\Sigma u^{2}-\Sigma u u^{\prime}\right)+u^{\prime}\left(2 \Sigma u^{2}-\Sigma u^{\prime 2}-\Sigma u u^{\prime}\right)\right] .
\end{aligned}
$$

After integrating with respect to $\alpha$, and averaging over all values of the velocities, it is obvious that the first line vanishes, while from the second we obtain, by the use of equation (691),

$$
\begin{aligned}
\Delta Q= & \nu^{2} \sqrt{2 m K}\left|\int_{\epsilon=0}^{\epsilon=2 \pi} \int_{a=0}^{a=\infty}\left[u\left(u^{2}+v^{2}+w^{2}\right)\right] \alpha d \alpha d \epsilon\right| \\
= & 2 \pi \nu^{2} \sqrt{2 m K} \int_{a=0}^{\alpha=\infty} \frac{1}{4} \sin ^{2} \theta^{\prime} \alpha d \alpha \\
& \left|u\left(2 \Sigma u^{\prime 2}-\Sigma u^{2}-\Sigma u u^{\prime}\right)+u^{\prime}\left(2 \Sigma u^{2}-\Sigma u^{\prime 2}-\Sigma u u^{\prime}\right)\right| \\
= & \frac{1}{2} A_{2} \nu^{2} \sqrt{2 m K} \\
& \left.\left\{4 u_{0}\left(\overline{u^{2}+v^{2}+w^{2}}\right)-2 \overline{\left(u^{2}+v^{2}+w^{2}\right.}\right)-2\left(u_{0} \overline{u^{2}}+v_{0} \overline{u v}+w_{0} \overline{u w}\right)\right\}
\end{aligned}
$$


This, then, is the value of $\Delta u\left(u^{2}+v^{2}+w^{2}\right)$. In terms of $\mathrm{u}, \mathrm{v}$ and $\mathrm{W}$, it becomes

$$
\begin{aligned}
& \Delta u\left(u^{2}+v^{2}+w^{2}\right)=2 A_{2} \nu^{2} \sqrt{2 m K} \\
& \quad\left\{u_{0}\left(\overline{\mathrm{U}^{2}}+\overline{\mathrm{V}^{2}}+\overline{\mathrm{W}^{2}}\right)-3\left(u_{0} \overline{\mathrm{U}^{2}}+v_{0} \overline{\mathrm{UV}}+w_{0} \overline{\mathrm{UW}}\right)+3 \overline{\mathrm{U}\left(\mathrm{U}^{2}+\mathrm{V}^{2}+\mathrm{W}^{2}\right)}\right\} \ldots
\end{aligned}
$$

352. Substituting the value which has been obtained for $\Delta u^{2}$ from equation (696) into equation (661), we have

$$
\frac{1}{2} \nu \sqrt{2 m K} A_{2}\left(\overline{\mathrm{V}^{2}}+\overline{\mathrm{W}^{2}}-2 \overline{U^{2}}\right)=q\left(2 \frac{\partial u_{0}}{\partial x}-\frac{2}{3}\left(\frac{\partial u_{0}}{\partial x}+\frac{\partial v_{0}}{\partial y}+\frac{\partial w_{0}}{\partial z}\right)\right) \ldots . .(704) .
$$

Similarly substituting the value obtained for $\Delta u v$ from equation (697) into equation (663)

$$
\frac{3}{2} \nu \sqrt{2 m K} A_{2} \overline{U V}=-q\left(\frac{\partial v_{0}}{\partial x}+\frac{\partial u_{0}}{\partial y}\right)
$$

and lastly, substituting the value of $\Delta u\left(u^{2}+v^{2}+w^{2}\right)$ just obtained into equation (664) we have, after simplification from equations (704) and (705),

$$
\nu \sqrt{2 m K} A_{2} \overline{u\left(U^{2}+v^{2}+\mathrm{W}^{2}\right)}=-5 q \frac{\partial q}{\partial x}
$$

\section{Time of relaxation.}

353. We have now obtained a sufficient amount of mathematical working material, and proceed to the discussion of physical phenomena.

Let us in the first place consider a gas in which the law of distribution is initially some law other than that of Maxwell. Our equations enable us to determine the rate at which the gas approaches the steady state. We take the simplest case, and suppose that there is no mass motion, so that $u_{0}=v_{0}=w_{0}=0$; we also suppose that the law of distribution is the same throughout the gas, so that $\overline{U^{2}}, \overline{U V}$, etc. are constants in space. With these suppositions equation (654) becomes

$$
\nu \frac{\partial \bar{Q}}{\partial t}=\Delta Q
$$

expressing that the whole change in $\bar{Q}$ is caused by collisions. If we put

$$
Q=u^{2}-v^{2}=u^{2}-v^{2}
$$

we have from equation (696),

$$
\Delta\left(u^{2}-v^{2}\right)=-\frac{3}{2} \nu^{2} \sqrt{2 m K} A_{2}\left(\overline{u^{2}}-\overline{v^{2}}\right)
$$

giving, upon substitution in equation (707),

$$
\begin{aligned}
\frac{\partial}{\partial t}\left(\overline{u^{2}}-\overline{v^{2}}\right) & =-\frac{3}{2} \nu \sqrt{2 m K} A_{2}\left(\overline{u^{2}}-\overline{v^{2}}\right) \\
& =-\eta \nu\left(\overline{u^{2}}-\overline{v^{2}}\right) \ldots \ldots \ldots \ldots \ldots \\
\eta & =\frac{3}{2} \sqrt{2 m K} A_{2} \ldots \ldots \ldots \ldots \ldots \ldots
\end{aligned}
$$

where 
The solution of equation (708) is

$$
\overline{u^{2}}-\overline{v^{2}}=\left[\overline{u^{2}}-\overline{v^{2}}\right]_{t=0} e^{-\eta \nu t},
$$

shewing that $\overline{u^{2}}-\overline{v^{2}}$ decreases exponentially with the time. The value of $\overline{u^{2}}-\overline{v^{2}}$ is reduced to $1 / e$ of its original value in a time $\frac{1}{\eta \nu}$. This time is called by Maxwell, the "time of relaxation."

Analysis of a similar kind enables us to find the rate at which any other deviation from Maxwell's law subsides. For instance, if in equation (707) we put $Q=u v$, and use the value of $\Delta u v$ given by equation (697), we obtain

$$
\nu \frac{\partial \overline{u v}}{\partial t}=-\frac{3}{2} \nu^{2} \sqrt{2 m K} A_{2} \overline{u v}
$$

or

$$
\frac{\partial \overline{u v}}{\partial t}=-\eta \nu \overline{u v}
$$

Thus the value of $\overline{u v}$ also decreases to $1 / e$ of its amount in a time $\frac{1}{\eta \nu}$.

It is not possible to find the absolute value of this "time of relaxation" from equation (709). We shall, however, be able to compare it with the known values of coefficients of viscosity, and shall find that it is extremely small.

\section{Viscosity.}

354. We have already seen $(\$ 175)$, that the system of pressures at any point in the gas is given by the equations

$$
\left.\begin{array}{l}
P_{x x}=\rho \overline{\mathrm{U}^{2}} \\
P_{y x}=\rho \overline{\mathrm{UV}}, \text { etc. }
\end{array}\right\}
$$

To arrive at these formulae we have taken $\varpi_{x x}=\varpi_{x y}=\ldots=0$ in equations (363). This does not mean that we neglect the intermolecular forces which vary inversely as the fifth power of the distance, for we have already taken full account of these forces in supposing that two molecules are in collision as soon as these forces become appreciable; in neglecting the system of pressures $\varpi_{x x}, \varpi_{x y}$, etc. we are merely assuming that no forces exist other than those which vary inversely as the fifth power of the distance.

When the gas is in its normal state, the quantities $P_{x x}, P_{y y}, P_{z z}$ each become identical with $p$, the pressure. When the gas is not in its normal state, let us define $p$ by the relation

$$
\dot{p}=\frac{1}{3} \rho\left(\overline{\mathrm{u}^{2}}+\overline{\mathrm{v}^{2}}+\overline{\mathrm{w}^{2}}\right),
$$

so that $p$ is still two-thirds of the energy per unit volume, and becomes identical with the hydrostatic pressure in the normal state. 
In calculations in which we ignored deviations from the normal state, we took (equation (662)),

$$
q=\overline{u^{2}}=\overline{\mathrm{v}^{2}}=\overline{\mathrm{w}^{2}} .
$$

Hence we may now, to the same order of approximation, take

$$
q=\frac{p}{\rho} \text {. }
$$

Introducing $\eta$, defined by equation (709), we can write equations (704) and (705) in the form

$$
\begin{aligned}
\overline{\mathrm{V}^{2}}+\overline{\mathrm{W}^{2}}-2 \overline{\mathrm{U}^{2}} & =\frac{3 q}{\eta \nu}\left(2 \frac{\partial u_{0}}{\partial x}-\frac{2}{3}\left(\frac{\partial u_{0}}{\partial x}+\frac{\partial v_{0}}{\partial y}+\frac{\partial w_{0}}{\partial z}\right)\right) \\
\overline{\mathrm{UV}} & =-\frac{q}{\eta \nu}\left(\frac{\partial v_{0}}{\partial x}+\frac{\partial u_{0}}{\partial y}\right) \ldots \ldots \ldots \ldots \ldots \ldots \ldots \ldots \ldots \ldots \ldots \ldots \ldots
\end{aligned}
$$

Hence

$$
\begin{aligned}
\overline{\mathrm{U}^{2}} & =\frac{1}{3}\left(\overline{\mathrm{U}^{2}}+\overline{\mathrm{V}^{2}}+\overline{\mathrm{W}^{2}}\right)-\frac{1}{3}\left(\overline{\mathrm{V}^{2}}+\overline{\mathrm{W}^{2}}-2 \overline{\mathrm{U}^{2}}\right) \\
& =\frac{p}{\rho}-\frac{q}{\eta \nu}\left(2 \frac{\partial u_{0}}{\partial x}-\frac{2}{3}\left(\frac{\partial u_{0}}{\partial x}+\frac{\partial v_{0}}{\partial y}+\frac{\partial w_{0}}{\partial z}\right)\right)
\end{aligned}
$$

so that $\quad P_{x x}=\rho \overline{\mathrm{U}^{2}}$

$$
=p-\frac{p}{\eta \nu}\left(2 \frac{\partial u_{0}}{\partial x}-\frac{2}{3}\left(\frac{\partial u_{0}}{\partial x}+\frac{\partial v_{0}}{\partial y}+\frac{\partial w_{0}}{\partial z}\right)\right)
$$

Similarly

$$
\begin{aligned}
P_{x y} & =\rho \overline{\mathrm{UV}} \\
& =-\frac{p}{\eta \nu}\left(\frac{\partial v_{0}}{\partial x}+\frac{\partial u_{0}}{\partial y}\right)
\end{aligned}
$$

Now in a viscous fluid of coefficient of viscosity $\kappa$, the system of pressures is given by equations of the form*

$$
\begin{aligned}
& P_{x x}=p-2 \kappa \frac{\partial u}{\partial x}+\frac{2}{3} \kappa\left(\frac{\partial u}{\partial x}+\frac{\partial v}{\partial y}+\frac{\partial w}{\partial z}\right), \\
& P_{x y}=-\kappa\left(\frac{\partial v}{\partial x}+\frac{\partial u}{\partial y}\right) .
\end{aligned}
$$

These pressures agree exactly with those found for the gas, if we take

$$
\kappa=\frac{p}{\eta \nu}
$$

Putting $p=\frac{1}{3} \nu m C^{2}$, this reduces to

$$
\kappa=\frac{1}{3} \frac{m C^{2}}{\eta}=\frac{R T}{\eta},
$$

so that $\kappa$ is found to be independent of the density, and directly proportional to the temperature, in accordance with the results already obtained.

* See, for instance, Lamb's Hydrodynamics, p. 512. 
355. We cannot use this equation for numerical calculation of $\kappa$ because it involves the unknown quantity $\eta$. We can, however, use the equation to obtain a value for $\eta$, by utilising an observed value for $\kappa$.

Let us take the case of air at $0^{\circ} \mathrm{C}$. We have $\kappa=000172$, and the general formula is

$$
\kappa=\frac{R T}{\eta} .
$$

Taking $T=273$, and using the value $R=9.3 \times 10^{-17}$ from p. 113 , we obtain

$$
\eta=1.5 \times 10^{-10} .
$$

The value of $\eta \nu$ is accordingly $6 \times 10^{9}$, so that the time of relaxation is

$$
\frac{1}{6 \times 10^{9}} \text { seconds. }
$$

It is, as we should expect, comparable with the time of describing a free path.

\section{Conduction of Heat.}

356. Multiplying equation (655) throughout by $m$, we obtain the relation

$$
\begin{aligned}
& \rho \frac{D}{D t}\left(\overline{\mathrm{U}^{2}}+\overline{\mathrm{V}^{2}}+\overline{\mathrm{W}^{2}}\right) \\
= & \Sigma\left[-\frac{\partial}{\partial x}\left\{\rho \overline{\mathrm{U}\left(\mathrm{U}^{2}+\mathrm{V}^{2}+\mathrm{W}^{2}\right)}\right\}-2 \rho\left(\overline{\mathrm{U}^{2}} \frac{\partial u_{0}}{\partial x}+\overline{\mathrm{UV}} \frac{\partial u_{0}}{\partial y}+\overline{\mathrm{UW}} \frac{\partial u_{0}}{\partial z}\right)\right]
\end{aligned}
$$

This is the equation of transfer of $Q$, when $Q$ is taken to be $\overline{U^{2}}+\overline{V^{2}}+\overline{W^{2}}$. It is therefore the equation of transfer of energy, and this, in the Kinetic Theory, is the transfer of heat.

Writing $\overline{\mathrm{U}^{2}}+\overline{\mathrm{V}^{2}}+\overline{\mathrm{W}^{2}}=3 q$, the left-hand member becomes $3 \rho \frac{D q}{D t}$. As regards the first term on the right-hand side, we have from equation (706),

$$
\begin{aligned}
\rho \overline{\mathrm{U}\left(\mathrm{U}^{2}+\mathrm{V}^{2}+\mathrm{W}^{2}\right)} & =-\frac{5 q \rho \frac{\partial q}{\partial x}}{\nu \sqrt{2 m \bar{K}} A_{2}} \\
& =-\frac{15}{2} \frac{q \rho \frac{\partial q}{\partial x}}{\eta \nu}
\end{aligned}
$$

by equation (709). The remaining terms on the right hand, containing 
$\overline{U^{2}}, \overline{U V}$, etc., are given by equations (712) and (713). On substituting these values in equation (717), we obtain

$$
\begin{aligned}
& 3 \rho \frac{D q}{D t}=\Sigma \frac{\partial}{\partial x}\left\{\frac{15 q \rho}{2 \eta \nu} \frac{\partial q}{\partial x}\right\} \\
& -2 q \rho\left(\frac{\partial u_{0}}{\partial x}+\frac{\partial v_{0}}{\partial y}+\frac{\partial w_{0}}{\partial z}\right) \\
& +\frac{4 q \rho}{\eta \nu}\left\{\left(\frac{\partial u_{0}}{\partial x}\right)^{2}+\left(\frac{\partial v_{0}}{\partial y}\right)^{2}+\left(\frac{\partial w_{0}}{\partial z}\right)^{2}\right\} \\
& -\frac{4}{3} \frac{q \rho}{\eta \nu}\left(\frac{\partial u_{0}}{\partial x}+\frac{\partial v_{0}}{\partial y}+\frac{\partial w_{0}}{\partial z}\right)^{2} \\
& +\frac{2 q \rho}{\eta \nu} \Sigma\left(\frac{\partial w_{0}}{\partial y}+\frac{\partial v_{0}}{\partial z}\right)^{2}
\end{aligned}
$$

If there is no mass motion all the terms disappear after the first line, and the equation reduces to

$$
3 \rho \frac{d q}{d t}=\Sigma \frac{\partial}{\partial x}\left\{\frac{15 q \rho}{2 \eta \nu} \frac{\partial q}{\partial x}\right\}
$$

the equation of conduction of heat in a gas at rest. Comparing this equation with Fourier's equation

we obtain

$$
C_{v} \rho \frac{d T}{d t}=\Sigma \frac{\partial}{\partial x}\left(\vartheta \frac{\partial T}{\partial x}\right)
$$

$$
9=\frac{5 q \rho C_{v}}{2 \eta \nu}
$$

or, introducing $\kappa$ from equation (716),

$$
9=\frac{5}{2} \kappa C_{v}^{*}
$$

In Chapter XIII, assuming the molecules to be elastic spheres, we obtained the value

$$
9=1 \cdot 6027 \kappa C_{v}
$$

which is only equal to about two-thirds of that just found. Regarding the law of force for elastic spheres $(n=\infty)$ and for the present point centres $(n=5)$ as extremes between which the actual law of force must lie, we may say that theory predicts for the ratio $9: \kappa C_{v}$, a value intermediate between the limits $1 \cdot 6027$ and $2 \cdot 5$.

\section{Diffusion.}

357. In equation (651) we obtained the general relation

$$
\nu \frac{d \bar{Q}}{d t}=\sum_{x, y, z}\left[\bar{Q} \frac{\partial}{\partial x}(\nu \bar{u})-\frac{\partial}{\partial x}(\nu \overline{u Q})+\frac{\nu}{m} X\left(\frac{\partial \bar{Q}}{\partial u}\right)\right]+\Delta Q
$$

* Maxwell, as the result of an arithmetical mistake, gives the factor as $\frac{5}{3}$ instead of $\frac{5}{2}$. The error has been pointed out by Boltzmann and Poincaré. 
We can use this equation to determine the value of the coefficient of diffusion. There must be two kinds of gas, which we shall denote by suffixes 1,2 , and which we shall suppose to be diffusing into one another in a direction parallel to that of the axis of $x$. Equation (720) will of course apply only to one kind of gas, let us say the first, but there will be an exactly similar equation for the other kind of gas.

In this equation we put $Q=u$, so that $\bar{Q}=\left(u_{0}\right)_{1}$, the mass velocity of the first gas. We assume the motion of diffusion to be so slow that the deviations from Maxwell's law and squares of mass velocities may be neglected to a first approximation.

Then

$$
\overline{u Q}=\overline{u^{2}}=\overline{u^{2}}=\frac{p_{1}}{\rho_{1}},
$$

where $p_{1}$ is the partial pressure due to the first gas.

The equation accordingly becomes

$$
\nu \frac{d\left(u_{0}\right)_{1}}{d t}=\left(u_{0}\right)_{2} \frac{\partial}{\partial x}\left(\nu_{1}\left(u_{0}\right)_{1}\right)-\frac{\partial}{\partial x}\left(\nu_{1} \frac{p_{1}}{\rho_{1}}\right)+\frac{\nu_{1}}{m_{1}} X_{1}+\Delta Q
$$

or, simplifying by the neglect of $\left(u_{0}\right)_{1}{ }^{2}$, and multiplying throughout by $m_{1}$,

$$
m_{1} \nu_{1} \frac{d\left(u_{0}\right)_{1}}{d t}=-\frac{\partial p_{1}}{\partial x}+\nu_{1} X_{1}+m_{1} \Delta(u)_{1}
$$

The value of $\Delta\left(u_{1}\right)$ is of course not zero, because collisions with molecules of the second kind change the momentum of the first gas. In equation (690) we obtained the value of $\Delta(u)_{1}$ in the form

$$
\Delta(u)_{1}=m_{2} \nu_{1} \nu_{2} \sqrt{\frac{K}{m_{1}+m_{2}}} A_{1}\left(\left(u_{0}\right)_{2}-\left(u_{0}\right)_{1}\right) .
$$

If the motion of the gas has become "steady motion," the left-hand member of equation (721), namely $m_{1} \nu_{1} \frac{d}{d t}\left(u_{0}\right)_{1}$, must vanish, so that the equation becomes

$$
\frac{\partial p_{1}}{\partial x}=\nu_{1} X_{1}+m_{1} m_{2} \nu_{1} \nu_{2} \sqrt{\frac{K}{m_{1}+m_{2}}} A_{1}\left(\left(u_{0}\right)_{2}-\left(u_{0}\right)_{1}\right) .
$$

Similarly, of course

$$
\frac{\partial p_{2}}{\partial x}=\nu_{2} X_{2}-m_{1} m_{2} \nu_{1} \nu_{2} \sqrt{\frac{K}{m_{1}+m_{2}}} A_{1}\left(\left(u_{0}\right)_{2}-\left(u_{0}\right)_{1}\right) .
$$

By addition we obtain

$$
\frac{\partial}{\partial x}\left(p_{1}+p_{2}\right)=\nu_{1} X_{1}+\nu_{2} X_{2}
$$

the hydrostatic equation of equilibrium. 
If there are no external forces, we can put $X_{1}=X_{2}=0$, and obtain

$$
\frac{\partial p_{1}}{\partial x}=-\frac{\partial p_{2}}{\partial x}=m_{1} m_{2} \nu_{1} \nu_{2} \sqrt{\frac{K}{m_{1}+m_{2}}} A_{1}\left(u_{2}-u_{1}\right)
$$

where $u_{2}, u_{1}$ are now mass-velocities. Since the temperature is constant throughout the gas, we may take

$$
\begin{aligned}
& \frac{\partial p_{1}}{\partial x}=\frac{\partial}{\partial x}\left(\frac{\nu_{1}}{2 h}\right)=\frac{1}{2 h} \frac{\partial \nu_{1}}{\partial x}, \\
& \frac{\partial p_{2}}{\partial x}=\frac{\partial}{\partial x}\left(\frac{\nu_{2}}{2 h}\right)=\frac{1}{2 h} \frac{\partial \nu_{2}}{\partial x} .
\end{aligned}
$$

Hence we have

$$
\frac{\partial \nu_{1}}{\partial x}=-\frac{\partial \nu_{2}}{\partial x}=2 h m_{1} m_{2} \nu_{1} \nu_{2} \sqrt{\frac{K}{m_{1}+m_{2}}} A_{1}\left(u_{2}-u_{1}\right)
$$

The total flow of molecules of the first kind per unit area per unit time is clearly $\nu_{1} u_{1}$, and is also $\mathfrak{D}_{12} \frac{\partial \nu_{1}}{\partial x}$, if $\mathfrak{D}_{12}$ is the coefficient of diffusion. Hence we have

$$
\begin{aligned}
& \nu_{1} u_{1}=\mathfrak{D}_{12} \frac{\partial \nu_{1}}{\partial x} \\
& \nu_{2} u_{2}=\mathfrak{D}_{12} \frac{\partial \nu_{2}}{\partial x}=-\mathfrak{D}_{12} \frac{\partial \nu_{1}}{\partial x},
\end{aligned}
$$

so that equation (722) becomes

$$
\frac{\partial \nu_{1}}{\partial x}=2 h m_{1} m_{2} \sqrt{\frac{K}{m_{1}+m_{2}}} A_{1}\left(\nu_{1}+\nu_{2}\right) \mathfrak{D}_{12} \frac{\partial \nu_{1}}{\partial x} .
$$

From this we obtain as the value of $\mathfrak{D}_{12}$,

$$
\mathfrak{D}_{12}=\frac{1}{2 h m_{1} m_{2} A_{1}\left(\nu_{1}+\nu_{2}\right)} \sqrt{\frac{m_{1}+m_{2}}{K}} .
$$

For diffusion of a single gas into itself, this becomes

$$
\mathfrak{D}=\frac{1}{2 h m^{2} A_{1} \nu} \sqrt{\frac{2 m}{K}}=\frac{1}{h \rho A_{1} \sqrt{2 m K}},
$$

and if from equation (709) we introduce $\eta$, given by

this value of $\mathfrak{D}$ becomes

$$
\eta=\frac{3}{2} \sqrt{2 m K} A_{2}
$$

$$
\mathfrak{D}=\frac{\frac{3}{2} A_{2}}{h \rho A_{1} \eta}
$$

Introducing the value of the coefficient of viscosity,

$$
\kappa=\frac{p}{\eta \nu}=\frac{1}{2 h \eta}
$$


we obtain equation (723) in the form

$$
\mathfrak{D}=\frac{3 A_{2}}{A_{1}} \frac{\kappa}{\rho}
$$

This equation is of the same form as the equation

$$
D=1 \cdot 34 \frac{\kappa}{\rho}
$$

obtained for elastic spheres in Chapter XIV. Using Maxwell's values

$$
A_{1}=2 \cdot 6595, \quad A_{2}=1 \cdot 3682
$$

we find that

$$
\frac{3 A_{2}}{A_{1}}=1.543
$$

This numerical factor does not differ widely from the factor 1.34 previously found. The values for the ratio of $\mathfrak{D}$ to $\kappa / \rho$ found on $p .274$, are all intermediate between the two values 1.34 and 1.543 , with the exception of Carbon monoxide for which the value is 1.32 .

\section{Energy.}

358. On substituting the values which have been found for 9 , $\kappa$, etc., we find that equation (718) assumes the form

$$
\begin{aligned}
. \rho C_{v} \frac{D T}{D t}=\Sigma \frac{\partial}{\partial x}\left\{9 \frac{\partial T}{\partial x}\right\} & -\frac{2}{3} \rho C_{v}\left(\frac{\partial u_{0}}{\partial x}+\frac{\partial v_{0}}{\partial y}+\frac{\partial w_{0}}{\partial z}\right) \\
& +\frac{\kappa}{J}\left[2 \Sigma\left(\frac{\partial u_{0}}{\partial x}\right)^{2}+\Sigma\left(\frac{\partial w_{0}}{\partial y}+\frac{\partial v_{0}}{\partial z}\right)^{2}-\frac{2}{3}\left(\frac{\partial u_{0}}{\partial x}+\frac{\partial v_{0}}{\partial y}+\frac{\partial w_{0}}{\partial z}\right)^{2}\right] .
\end{aligned}
$$

Obviously the term on the left-hand side is the increase of heat-energy of an element of the gas. On the right-hand side, the first term is the increase of heat which ordinary physics regards as due to conduction, the second term is that due to adiabatic expansion or compression, and the third term is that which ordinary physics attributes to the action of viscosity, being in fact twice the "dissipation function" of the viscous motion*.

To the Kinetic Theory, however, conduction of heat, change of temperature resulting from adiabatic motion and "heat generated by viscosity" are all equally resolved into the transfer of energy by molecules, so that to the Kinetic Theory the equation just obtained expresses nothing more than the conservation of this energy. 


\section{CHAPTER XVI.}

\section{FREE PATH PHENOMENA (CONTINUED).}

\section{The Propagation of Sound.}

359. AT the end of Chapter X. (p. 228), it was pointed out that the problem of the propagation of sound requires special investigation from the point of view of the Kinetic Theory. In any gas for which $\gamma$, the ratio of the two specific heats, is less than $1 \frac{2}{3}$, the propagation of sound is dependent on a transfer of internal energy through collisions, and if this energy is not transferred with sufficient rapidity to keep pace with the transfer of translational energy, complications will arise which are not contemplated by a simple theory of the kind which is given in books on Sound or Hydrodynamics. This simple theory deals only with the mass motion of a gas on the assumption that it may be regarded as a homogeneous fluid: it is the province of the Kinetic Theory to investigate what modifications, if any, are required when the molecular structure of the gas is taken into account.

We shall work ont the problem in detail in the special case in which the molecules of the gas are loaded spheres. We shall be able to infer the nature of the general solution from the special solution obtained in this way.

\section{The molecules are loaded spheres.}

360. At any point in the gas we suppose as usual that the mass motion is so small that squares of its components may be neglected. Also the mass motion is in one dimension, so that if we choose this direction for the axis of $x$, the components of mass velocity may be taken to be $u_{0}, 0,0$, and $u_{0}^{2}$ may be neglected.

Along the path of the wave, the quantities $u_{0}, \nu, \mathrm{H}$ and $\mathrm{K}$ will vary, differing only slightly from their values in the undisturbed state. The 
equations from which the propagation of sound is to be deduced will accordingly be the equations of transfer of the four quantities $u_{0}, \nu, \mathrm{H}$ and $\mathrm{K}$, corresponding respectively to momentum, mass, and rotational and translational energy.

From equation (648), the general equation of transfer of any quantity $Q$, simplified by the suppositions that there are no external forces, that squares of the mass-motion may be neglected, and that the whole motion is parallel to the axis of $x$, is found to be

$$
\frac{d}{d t}(\nu \bar{Q})=-\frac{\partial}{\partial x}(\nu \overline{u Q})+\Delta Q
$$

To find the transfer of momentum we put $Q=u$, so that $\bar{Q}=u_{0}$ and $\Delta Q=0$. We obtain

$$
\frac{d}{d t}\left(\nu u_{0}\right)=-\frac{\partial}{\partial x}\left(\nu \overline{u^{2}}\right)=-\frac{\partial}{\partial x}\left(\frac{1}{3} \nu \overline{c^{2}}\right)=-\frac{2}{3 m} \frac{\partial}{\partial x}(\nu \mathrm{K})
$$

the equation of motion in the simpler theory.

To find the transfer of mass, we put $Q=1$, so that $\bar{Q}=1$ and $\Delta Q=0$. We obtain

$$
\frac{d \nu}{d t}=-\frac{\partial}{\partial x}\left(\nu u_{0}\right)
$$

the equation of continuity.

To obtain the equation of transfer of $\mathrm{H}$, we put $Q=\frac{1}{2} m k^{2} \varpi^{2}$, so that $\bar{Q}=\mathrm{H}$, $\overline{u Q}=u_{0} \mathrm{H}$, and $\Delta Q$ is the same as the value of $\nu \frac{d H}{d t}$ given by equation (253), namely

$$
\nu \frac{d \mathrm{H}}{d t}=-\beta \nu^{2} \sqrt{\mathrm{K}}\left(\mathrm{H}-\frac{2}{3} \mathrm{~K}\right) .
$$

The equation of transfer is accordingly

$$
\frac{d}{d t}(\nu \mathrm{H})=-\frac{\partial}{\partial x}\left(\nu u_{0} \mathrm{H}\right)-\beta \nu^{2} \sqrt{\mathrm{K}}\left(\mathrm{H}-\frac{2}{3} \mathrm{~K}\right)
$$

Similarly the rate of transfer of $K$ is obtained by putting $Q=\frac{1}{2} m c^{2}$, so that $\bar{Q}=k$, and

$$
\begin{aligned}
\overline{u Q} & =\frac{1}{2} m \overline{u\left(u^{2}+v^{2}+w^{2}\right)} \\
& =\frac{1}{2} m \overline{\left(u_{0}+\mathrm{U}\right)\left(u_{0}^{2}+2 u_{0} \mathrm{U}+\mathrm{U}^{2}+\mathrm{V}^{2}+\mathrm{W}^{2}\right)} \\
& =\frac{1}{2} m u_{0}\left(3 \mathrm{U}^{2}+\mathrm{V}^{2}+\mathrm{W}^{2}\right) \\
& =\frac{1}{2} m u_{0}\left(\frac{5}{3} \bar{c}^{2}\right) \\
& =\frac{5}{3} u_{0} \mathrm{~K} .
\end{aligned}
$$

The equation is accordingly

$$
\frac{d}{d t}(\nu \mathrm{K})=-\frac{\partial}{\partial x}\left(\frac{5}{3} u_{0} \mathrm{~K}\right)+\beta \nu^{2} \sqrt{\mathrm{K}}\left(\mathrm{H}-\frac{2}{3} \mathrm{~K}\right)
$$


Since $x$ and $t$ enter only through the differential operators $\frac{\partial}{\partial t}$ and $\frac{\partial}{\partial x}$, there will be a solution in which each variable differs from its value in the stearly state by a small term proportional to $e^{i(p t-q x)}$, and the solution represents wave motion parallel to the axis of $x$. Let us then assume a solution of the form

$$
\begin{aligned}
u_{0} & =u_{0}^{\prime} e^{i(p t-q x)} \\
\nu & =\nu+\nu^{\prime} e^{i(p t-q x)} \\
\mathrm{H} & =\mathrm{H}+\mathrm{H}^{\prime} e^{i(p t-q x)} \\
\mathrm{K} & =\mathrm{K}+\mathrm{K}^{\prime} e^{i(p t-q x)},
\end{aligned}
$$

in which the unaccented $\nu, H$ and $K$ on the right-hand side refer to equilibrium values.

The forms assumed by equations (725) to (729), on substitution of these solutions and neglect of small quantities of the second order, are as follows :

$$
\begin{array}{r}
p \nu u_{0}^{\prime}-\frac{2 q}{3 m}\left(\nu \mathrm{K}^{\prime}+\nu^{\prime} \mathrm{K}\right)=0 \ldots \ldots \ldots . . \\
p \nu^{\prime}-q \nu u_{0}^{\prime}=0 \ldots \ldots \ldots \ldots . . \\
p\left(\nu \mathrm{K}^{\prime}+\nu^{\prime} \mathrm{K}\right)-\frac{5}{3} q \mathrm{~K} \nu u_{0}^{\prime}+i \beta \nu^{2} \sqrt{\mathrm{K}}\left(\mathrm{H}^{\prime}-\frac{2}{3} \mathrm{~K}^{\prime}\right)=0 . \\
p \nu \mathrm{H}^{\prime}-i \beta \nu^{2} \sqrt{\mathrm{K}}\left(\mathrm{H}^{\prime}-\frac{2}{3} \mathrm{~K}^{\prime}\right)=0 \ldots \ldots \ldots . .
\end{array}
$$

Eliminating the accented letters, we arrive at the relation

$$
\frac{i \beta \nu \sqrt{\mathrm{K}}}{p}=\frac{\frac{5}{3} q^{2} \mathrm{~K}-\frac{3 m}{2} p^{2}}{\frac{7}{3} q^{2} \mathrm{~K}-\frac{5 m}{2} p^{2}}
$$

361. If the left-hand member is very great, the relation between $q$ and $p$ is expressed by the vanishing of the denominator on the righthand, so that

$$
q^{2}=\frac{15 m}{14 \mathrm{~K}} p^{2}
$$

Since

$$
\mathrm{K}=\frac{3}{4 h}=\frac{3}{2 \nu} p_{0}
$$

where $p_{0}$ is the pressure in the undisturbed gas, the foregoing equation can be written as

$$
q^{2}=\frac{5}{7} \frac{\rho}{p_{0}} p^{2}
$$

so that the velocity of propagation is

$$
\frac{p}{q}=\sqrt{\frac{7}{5} \frac{p_{0}}{\rho}}
$$


This is simply Laplace's formula, since $\gamma=\frac{7}{5}$ for loaded spheres. In fact in supposing the left-hand member of equation (734) to be very great, we assume that the ratio of the energies adjusts itself with a rapidity which is great compared with the rate of wave motion-an assumption which can be fulfilled either by the greatness of $\beta \nu \sqrt{\mathrm{K}}$ or the smallness of $p$.

362. If the left-hand member of equation (734) is very small, the equation reduces to

$$
\frac{p}{q}=\sqrt{\frac{5}{3} \frac{p_{0}}{\rho}}
$$

and this becomes identical with Laplace's formula if $\gamma=1 \frac{2}{3}$. The smallness of the term $\frac{i \beta \nu \sqrt{K}}{p}$ can be effected either by the smallness of $\beta \nu \sqrt{\mathrm{K}}$ or by the greatness of $p$. The energy here adjusts itself slowly in comparison with the rate of passage of the sound, so that the variations in the translational energy are too rapid to affect the rotational energy at all. Here, then, we have a gas of which the molecules have five degrees of freedom, and yet from experiments on the velocity of sound we should deduce the value $\gamma=1 \frac{2}{3}$. This is the possibility to which it was necessary to refer in Chapter X., although a brief calculation shewed that it was not likely to occur in nature.

363. Let us write $V$ for Laplace's value of the velocity of sound, so that

$$
V^{2}=\frac{14 \mathrm{~K}}{15 m}
$$

then equation (734) becomes

so that

$$
\frac{i \beta \nu \sqrt{\mathrm{K}}}{p}=\frac{3}{5} \frac{p^{2}-\frac{25}{2} q^{2} V^{2}}{p^{2}-q^{2} V^{2}},
$$

$$
q^{2}=\frac{p^{2}}{V^{2}} \frac{1+\frac{3}{5} \frac{i p}{\beta \nu \sqrt{\mathrm{K}}}}{1+\frac{5}{7} \frac{i p}{\beta \nu \sqrt{\mathrm{K}}}}
$$

If $\frac{i p}{\beta \nu \sqrt{K}}$ is small, we have as far as the first order of small quantities

$$
q=\frac{p}{V}\left(1-\frac{2}{3 \hbar} \frac{i p}{\beta \nu \sqrt{\mathrm{K}}}\right)
$$

so that the exponential

$$
e^{i(p t-q x)}
$$

may be replaced by

$$
e^{-\frac{2 p^{2}}{35 \beta \nu \sqrt{\kappa} V} x} e^{i p\left(t-\frac{x}{V}\right)}
$$

J. 
This corresponds physically to propagation with velocity $V$, the whole wave motion being damped with a modulus of decay

per unit length.

$$
\frac{2 p^{2}}{35 \beta \nu \sqrt{\mathrm{K}} V}
$$

Thus the first effect produced by the slowness of adjustment between internal and translational velocities will not be a change in the velocity of propagation, but a damping of the sound.

364. There is a second cause which tends to diminish the amplitude of a wave of sound propagated in free air, namely the viscosity of the gas. This has of course been neglected in the present investigation because we have assumed Maxwell's law to give a sufficiently good approximation--an assumption which, as is evident from the last chapter, includes the neglect of viscosity. The effect of viscosity* is to introduce a linear modulus of decay

$$
\frac{2 p^{2}}{3 m \nu \bar{V}^{3}} \kappa
$$

proportional to the first power of the coefficient of viscosity $\kappa$, when $\kappa$ is small, and to alter the velocity of propagation only by terms depending on $\kappa^{2}$.

It will be noticed that expressions (738) and (739) are both proportional to $p^{2}$, so that their ratio depends only on the gas, and not on the frequency of the sound. The effect of the lag in rotatory energy can accordingly be fully allowed for by supposing $\kappa$ increased to

$$
\kappa+\frac{3 m V^{2}}{35 \beta \sqrt{\bar{K}}}
$$

Since, by equation $(737), V^{2}=\frac{14 k}{15 m}$, this expression can be written in the form

$$
\kappa+\frac{2 \sqrt{k}}{25 \beta}
$$

The last term is independent of the density and proportional to the square root of the temperature, as also is $\kappa$. Hence formula (741) is the mathematical expression of an increase in $\kappa$ which depends only on the structure of the molecules and not on the state of the gas. To determine the amount of this increase, we use the formulae

$$
\begin{array}{rlrl}
\kappa & =\frac{1}{3} m \nu \bar{c} l & (\$ 301), \\
\frac{1}{\beta \nu \sqrt{\mathrm{K}}}=\frac{3 k^{2}}{4 r^{2}}\left(\frac{l}{\bar{c}}\right) & (\$ 117), \\
\mathrm{K}=\frac{1}{2} m C^{2} & =\frac{3 \pi m}{16}(\bar{c})^{2} & (\$ 131),
\end{array}
$$

* See Lord Rayleigh's Theory of Sound, Ir. $\S 346$. 
from which we find that

$$
\kappa+\frac{2 \sqrt{K}}{25 \beta}=\kappa\left(1+\frac{27 \pi}{800} \frac{k^{2}}{r^{2}}\right)
$$

so that the apparent viscosity would be equal to the true viscosity increased in the ratio

$$
1+\frac{27 \pi}{800} \frac{k^{2}}{r^{2}}
$$

\section{Molecules of more general type.}

365. This completes the actual calculation for loaded spheres. Clearly the result for any other type of molecule would be similar. To illustrate, let us consider an ideal diatomic molecule formed by placing two homogeneous spheres each of radius $b$, in contact. The value of $k^{2}$, the square of the radius of gyration, is now $\frac{7}{5} b^{2}$, while $r^{2}$, the square of the distance from the centre of gravity to the point at which a normal to the surface meets the axis, is $b^{2}$. Substituting these values, expression (742) becomes

$$
1+\frac{7}{5} \frac{27 \pi}{800}
$$

We ought, however, to correct the multiplying factor $\frac{27 \pi}{800}$, this correction being necessitated by the considerations explained in $\S 109$. To obtain an estimate of the order of magnitude of the effect under discussion, we may neglect this correction altogether, so that expression (742) must simply be replaced by the quantity already found, of which the value is $1 \cdot 146$.

The damping of sound due to the "lag" in the adjustment of sound is therefore about one-seventh as great as that caused by viscosity.

366. There is less difficulty in forming an estimate of the effect of this "lag" upon the velocity of propagation of sound.

As far as squares of $\frac{i p}{\beta \nu \sqrt{\mathrm{K}}}$, equation (738) gives the value

$$
q=\frac{p}{V}\left(1-\frac{2}{35} \frac{i p}{\beta \nu \sqrt{\mathrm{K}}}-\frac{48}{1225} \frac{p^{2}}{\beta^{2} \nu^{2} \kappa}+\ldots\right)
$$

so that the exponential $e^{i(p t-q x)}$ becomes

$$
e^{-\frac{2 p^{2}}{35 \beta \sqrt{\mathrm{K} V}} x} e^{i p\left[t-\frac{x}{V}\left(1-\frac{48}{1225} \frac{p^{2}}{\beta^{2} \nu^{2} \mathrm{~K}}\right)\right]} .
$$

The velocity of propagation, corrected as far as squares of the small quantity, is therefore

$$
V\left(1+\frac{48}{1225} \frac{p^{2}}{\beta^{2} \nu^{2} \mathrm{~K}}\right)
$$


Using the three formulae of $\S 364$, this reduces to

$$
V\left[1+\frac{48}{1225}\left(\frac{3 k^{2}}{4 r^{2}}\right)^{2}\left(\frac{p l}{\bar{c}}\right)^{2}\right]
$$

Now $\frac{l}{\bar{c}}$ is, roughly, the time of describing a free path, and is therefore of the order of magnitude of $3 \times 10^{-10}$ seconds. For a sound of moderate pitch $p$ is of the order of 3000 , so that $\frac{p l}{\bar{c}}$ is of the order of $10^{-7}$. For the sounds of highest pitch which are audible, we may take $p=10^{\text {s }}$, so that $\frac{p l}{\bar{c}}$ still remains of the order of $3 \times 10^{-5}$. It is therefore clear that for all sound, the difference between expression (743) and the limiting velocity $V$ is in every way quite imperceptible, at any rate upon the supposition that the factor $\frac{3 k^{2}}{4 r^{2}}$ (or the corresponding factor for molecules of different structure), is comparable with unity. 


\section{CHAPTER XVII.}

\section{PLANETARY ATMOSPHERES.}

367. IN the last six chapters we worked out certain problems connected with the free path in gases and molecular transport. In the present chapter we shall apply the results obtained to the discussion of problems connected with the atmosphere. These problems will consist of various problems of aerostatics, and an investigation into the question of the dissipation of planetary atmospheres.

\section{Aerostatics.}

Atmosphere in Isothermal (Conductive) Equilibrium.

368. In $\$ 79$ we obtained the equation

$$
\nu_{a}=D_{a} e^{-2 h x}
$$

connecting the molecular density of gas of type $a$ with the potential of a permanent field of force. To apply this result to a planetary atmosphere, we replace $\chi$ by $m_{a} g z$, where $g$ is the value of gravity, and $z$ is the height measured vertically upwards. The equation accordingly becomes

$$
\nu_{a}=D_{a} e^{-2 h m_{a} g z}
$$

In this equation we neglect variations in the value of $g$, and also the rotation of the planet.

If we measure $z$ from the surface of the planet, $D_{a}$ must be the molecular density of gas of type $\alpha$ at the surface, say $\left(\nu_{a}\right)_{0}$, so that we obtain the equations

$$
\left.\begin{array}{l}
\nu_{a}=\left(\nu_{a}\right)_{0} e^{-2 h m_{a} g z}, \\
\nu_{\beta}=\left(\nu_{\beta}\right)_{0} e^{-2 h m_{\beta} g z}, \text { etc. }
\end{array}\right\}
$$

for the different constituents of the atmosphere. 
These equations are independent in the sense that each is concerned with one and only one of the different constituents of the atmosphere. The equations therefore contain the mathematical expression of the law formulated by Dalton for an atmosphere in isothermal equilibrium:

An atmosphere in isothermal equilibrium may be regarded as the aggregate of a number of atmospheres, one for each constituent gas, the law of density in each atmosphere being the sume as if it alone was present.

369. Considering for the sake of simplicity an atmosphere which consists only of two constituents $\alpha, \beta$, the ratio $\nu_{a} / \nu_{\beta}$ will measure the proportion in which the gases are mixed at any point. From equations (746),

$$
\frac{\nu_{a}}{\nu_{\beta}}=\left(\frac{\nu_{a}}{\nu_{\beta}}\right)_{0} e^{-2 h g z\left(m_{\alpha}-m_{\beta}\right)}
$$

If the gas of type $\alpha$ is the heavier of the two, $m_{\alpha}-m_{\beta}$ is positive, and therefore $\nu_{a} / \nu_{\beta}$ has its maximum value at $z=0$. In other words, the heavier gas tends to sink to the bottom.

To obtain some idea of the magnitude of this imperfection of diffusion, let us make use of the numerical values given in $\$ 130$. Since $2 h m=\frac{3}{C^{2}}$ for any gas, we may write the index of the exponential in equation (747) in the form

$$
2 h g z\left(m_{\alpha}-m_{\beta}\right)=\frac{g z}{3}\left(\frac{1}{C_{\alpha}^{2}}-\frac{1}{C_{\beta}^{2}}\right) .
$$

If $\alpha$ refers to oxygen and $\beta$ to nitrogen, the value of this quantity at $0^{\circ} \mathrm{C}$. is, from the table given on p. 113, found to be

$$
2 g z \times 10^{-11} \text { C. G. S. units. }
$$

If we put $g=981$, and $z=5 \times 10^{5}$, corresponding to a height of five kilometres, the value of this quantity is found to be $\cdot 01$. Thus the rate at which the proportions of oxygen to nitrogen would change is very small, amounting only to about 1 per cent. in five kilometres.

\section{Atmosphere in Adiabatic (Convective) Équilibrium.}

370. The atmospheric distribution which has just been investigated, a distribution in which the temperature is constant throughout, while the density of each component gas falls off exponentially with the height above the earth's surface, is the law which would undoubtedly become established if the earth's atmosphere were left at rest for a sufficient time.

As a matter of fact, however, the earth's atmosphere is incessantly being agitated by currents and storms, so that there is a continual mechanical transference of air from one part of the earth's atmosphere to another. From this consideration, coupled with the fact that the conduction of heat in 
gases is very slow, it follows that the atmosphere is never permitted to assume the equilibrium distribution which has just been discussed. As the density of an element is changed by its enforced motion from one layer of the atmosphere to another, the temperature also tends to change, but before the temperature has adjusted itself by conduction to the temperature of its new surroundings the element finds itself again moved away. Hence it happens that the factor which determines the distribution of the atmosphere is not the equalisation of temperature necessary to a permanent state, but is the condition that an element of gas, on being moved from one place to another, shall take up the requisite pressure and volume in its new position without any loss or gain of heat by conduction taking place. The law connecting the pressure and volume in the atmosphere must accordingly be the adiabatic law found in $\$ 230$, namely,

$$
p v^{\gamma}=\text { constant }
$$

371. The equation of equilibrium of the atmosphere is

$$
\frac{\partial p}{\partial z}=-g \rho,
$$

and if, in accordance with the adiabatic law, we write

$$
p=k \rho^{\gamma}
$$

we obtain

$$
k \gamma \rho^{\gamma-1} \frac{\partial \rho}{\partial z}=-g \rho,
$$

of which the integral is

$$
\frac{k \gamma}{\gamma-1}\left(\rho_{0}^{\gamma-1}-\rho^{\gamma-1}\right)=g z
$$

where $\rho_{0}$ is the density at $z=0$. This is the law according to which the density falls off with the height. Since by equation (749), $T$ is proportional to $\rho^{\gamma-1}$, it follows that equation (750) can be expressed in the form

$$
T_{0}-T=c z
$$

where $c$ is a constant, and $T_{0}$ is the temperature at $z=0$. Thus the temperature decreases as we move upwards in the atmosphere, the amount of decrease being proportional to the height.

The process of diffusion, as well as that of conduction, is very slow in gases, and from this it follows that the constituent gases of an atmosphere in convective equilibrium, will be found to occur in approximately the same proportion at all heights. This is found by experiment to be true of the atmosphere of the earth. Frankland* has found that the proportions of nitrogen and oxygen in our atmosphere are the same for all altitudes up to 14,000 feet. As has already been remarked, there would be a variation of about one per cent. at this height in an atmosphere in conductive equilibrium.

* Journ. Chem. Soc. xrIr. 22. 
372. On putting $\rho=0$ in equation (750) we obtain

$$
z=\frac{k \gamma \rho_{0}^{\gamma-1}}{g(\gamma-1)}
$$

from which it appears that there is a superior limit to the height of an atmosphere in convective equilibrium. Since, by equation (749), $p=k \rho^{\gamma}$, this limiting height may be written in the form

$$
\frac{p_{0} \gamma}{g \rho_{0}(\gamma-1)}
$$

where $p_{0}, \rho_{0}$ are the pressure and density at the earth's surface. This again can be put in the form

$$
\frac{R}{m} \frac{\gamma}{g(\gamma-1)} T
$$

Substituting numerical values, this height is found to be about 29 kilometres.

According to Dewar* the greatest height at which clouds have been observed is 17 miles, or 27.37 kilometres.

Our atmosphere, then, if supposed to be in convective equilibrium throughout, must be regarded as a layer of gas of uniform composition throughout, having a height of about 29 kilometres, the temperature decreasing uniformly as we ascend.

The rate at which the temperature decreases must be about $10^{\circ} \mathrm{C}$. per kilometre, or in English units, about $54^{\circ} \mathrm{F}$. per $100 \mathrm{ft}$. This theoretical estimate of the temperature gradient is approximately confirmed by observation. In practice, however, the problem is one of extreme complexity, owing mainly to the irregularities of the earth's surface which prevent the surfaces of equal temperature from being strictly spherical. Detailed accounts of observations on this point will be found in Meteorological Reports $\dagger$.

\section{Outer Atmosphere.}

\section{Outer Atmosphere in Conductive Equilibrium.}

373. When, however, we examine in detail the molecular mechanism by which the adiabatic law is maintained in an atmosphere we find that there must be a limit beyond which adiabatic equilibrium is impossible. For at the free surface which is predicted from the supposition that the adiabatic law obtains throughout, the density would be zero, and therefore the mean free path infinite. Hence there would be molecules arriving at this surface from layers of gas inside it, with finite velocities and infinitesimal probabilities

* Royal Institution Proc. xvir. 1, p. 223.

+ See for example a general discussion in the Report of the Chief of the U.S. Weather Bureau, 1900-1, Vol. Ir. p. 25. 
of collision. The majority of these molecules would of course pass outside the free surface predicted by the simpler theory, in a manner somewhat similar to that in which molecules escape from the free surface of a liquid and form a vapour.

These molecules form what may be described as an "outer" atmosphere. In this atmosphere, the density is very small, so that collisions are rare, and the majority of molecules will simply describe orbits under the earth's gravitation, undisturbed by collisions, and will finally fall back again into the "inner" or adiabatic atmosphere. This at any rate is true of those molecules wbich start with velocities such that they describe elliptic orbits under the earth's attraction. Others, starting with greater velocities, will describe parabolic or hyperbolic orbits, and these may be regarded as lost altogether to the earth's atmosphere. We shall return to the consideration of these losses later.

374. In nature it is obvious that there must be a gradual, and not a sudden, transition from the state of things which we have supposed to exist in the inner atmosphere, to that which has been supposed to exist in the outer. There must, in fact, be an intermediate region, in which neither set of conditions is satisfied with any accuracy. Passing through this layer from inside outwards, we pass from a region at which the massagitation is sufficient for the adiabatic law to be obeyed, to a region at which the frequency of collisions is so small that a mass-agitation is impossible. At this outer region conductive equilibrium must clearly obtain. And outside this region, the law of density for gases of type $\alpha$ must be

$$
\nu_{a}=A e^{-2 h \chi_{a}}
$$

just as if the whole of the atmosphere were in conductive equilibrium, a result which can be deduced either as in equation (165), or as in $\S 178$, without making any assumption as to the frequency of collisions in the outer atmosphere.

375. There is no superior limit to the height of the outer atmosphere which is in conductive equilibrium, since, in equation (752), $\nu_{a}$ does not vanish at any finite height. It must, however, be remembered that in arriving at equation (752) no account has been taken of the rotation of the planet, and when there is no limit to the height of the atmosphere, the neglect of rotation is inadmissible.

For a planet rotating about the axis of $z$ with angular velocity $\omega$, the law of density of the atmosphere is found, after the manner of $\S 80$, to be

$$
\nu_{\alpha}=A e^{-2 h \chi_{\alpha}+h m_{a^{\omega^{2}}\left(x^{2}+y^{2}\right)}}
$$

and clearly $\nu_{a}$ not only does not vanish at any distance, but becomes infinite at infinity. 
The pressure in the atmosphere is given by

$$
p=\sum_{a} \frac{\nu_{a}}{2 h}
$$

and therefore does not vanish anywhere, and becomes infinite at infinity. It is therefore clear that no steady state is possible for the atmosphere of a rotating planet, for there is no physical agency capable of supplying the requisite pressure at the boundary. This simply means that the atmosphere is continually flowing away to infinity, and so rendering a steady state an impossibility.

376. Let us, however, examine the atmospherie distribution expressed by equation (753), without at present considering how this distribution is to be maintained. The surfaces of equal density for any constituent gas are given by the equation

$$
m_{a} \omega^{2}\left(x^{2}+y^{2}\right)-2 \chi_{\alpha}=\text { constant. }
$$

If $g$ is the value of gravity at the planet's surface, the value of gravity at a distance $r$ from the planet's centre is $\frac{g a^{2}}{r^{2}}$, so that $\chi_{a}=-m_{a} g a^{2} / r$, and the equation of the surfaces of equal density may be taken to be

$$
\omega^{2}\left(x^{2}+y^{2}\right)+\frac{2 g a^{2}}{r}=\text { constant }
$$

The form of these surfaces was first studied by Edward Roche*; a discussion of his results is given in a paper by Prof. G. H. Bryan $\dagger$. Sufficient information for our present purpose will be obtained by examining the distribution of density in the equatorial plane of the planet. Replacing $x^{2}+y^{2}$ by $r^{2}$, we have in the equatorial plane

$$
\nu_{\alpha}=A e^{-h m_{\alpha}\left[\omega^{2} r^{2}+\frac{2 g a^{2}}{r}\right]}
$$

Differentiating, we find that $d \nu_{\alpha} / d r$ vanishes when

$$
\omega^{2} r=\frac{g a^{2}}{r^{2}}
$$

There is therefore a single minimum of density, and the position of this minimum is the same for each eonstituent atmosphere, being in fact the series of points at which the apparent centrifugal force exactly balances the gravitational attraction of the planet. As we pass outwards the density deereases until this minimum is reached, and afterwards increases continually.

In the ease of our earth, $\frac{\omega^{2} a}{g}$, the ratio of the apparent eentrifugal force to gravity at the equator, is $\frac{1}{293}$, so that the minimum of density occurs at a distance from the earth's centre given by

$$
r=\sqrt[3]{293} a=6 \cdot 642 a
$$

* Mémoires Acad. Sci. Montpellier.

+ "The Kinetic Theory of Planetary Atmospheres," Phil. Trans. cxcvi. A, p. 12. 


\section{Constitution of the Outer Atmosphere.}

377. Throughout the whole of the outer atmosphere the law of distribution is that expressed by equation (753). We can now carry out calculations similar to those of $\S 369$, but having reference to the outer atmosphere:

We shall not carry these calculations to a height above the earth's surface so great that the rotational term becomes of any importance. We can therefore replace equation (753) by the simpler equation

$$
\nu_{a}=A e^{-2 h_{\chi \alpha}} \text {. }
$$

At a height $z$ above the surface of the planet, the value of $\chi_{a}$ is

$$
-\frac{m_{a} g a^{2}}{a+z}
$$

so that the equation becomes

$$
\nu_{a}=A e^{2 h m_{a j a}\left(\frac{a}{a+z}\right)},
$$

and $\left(\nu_{a}\right)_{0}$, the value of $\nu_{a}$ at the lower boundary of the outer atmosphere, is given by

$$
\left(\nu_{a}\right)_{0}=A e^{2 h m_{a} g a},
$$

since we inay neglect the thickness of the inner (convective) atmosphere in comparison with the earth's radius.

The elimination of $A$ leads to

$$
\nu_{a}=\left(\nu_{a}\right)_{0} e^{-2 h m_{a} g a}\left(\frac{z}{a+z}\right)
$$

Also at the inner surface the proportion in which the different con-

\begin{tabular}{|c|c|c|}
\hline \multicolumn{3}{|c|}{ Hydrogen*..... less than 1 in 100,000} \\
\hline Helium $\nmid \ldots$ & 1 to 2 & „1,000,000 \\
\hline Neont ... & 1 to 2 & \#100,000 \\
\hline Argon $_{+}^{+}$ & 3 to 0.95 & $\because 100$ \\
\hline Krypton§ & 1 & $" 20,000,000$ \\
\hline Xenon§ & 1 & , $170,000,000$ \\
\hline Oxygen ... & $21 \cdot 0$ & „100 \\
\hline Nitrogen & $78 \cdot 0$ & „100 \\
\hline
\end{tabular}
stituents occur is the same as throughout the inner atmosphere, and therefore the same as at the earth's surface.

378. The following table gives the constitution of the atmosphere at the earth's surface :

Traces of Carbon dioxide and Ammonia are also present in the atmosphere.

* Dewar, l.c. ante.

$\mp$ Moissan, Comptes Rendus, cxxxvir. p. 600.

§ W. Ramsay, Royal Soc. Proc. Lxxi. p. 421. 
379. To study the way in which these proportions change as we pass upwards, we use formula (757).

If $T$ is the temperature (absolute) of the outer atmosphere, the index of the exponential may be written in the form

$$
-2 h m_{a} g a\left(\frac{z}{a+z}\right)=\frac{m_{a} g}{R T}\left(\frac{a z}{a+z}\right) .
$$

The value of $T$ being unknown, we shall calculate the function in terms of $\frac{1}{T}\left(\frac{a z}{a+z}\right)$ and can then calculate values of $z$ corresponding to different values for $T$. The result of the calculations, putting $g=981$, and taking the values of $R / m$ from p. 113, is given in the following table:

\begin{tabular}{|c|c|c|c|c|c|}
\hline Temp. & \multicolumn{5}{|c|}{$z$ in kilometres measured from outside of adiabatic atmosphere } \\
\hline $273^{\circ}=0^{\circ} \mathrm{C}$. & 0 & 25 & 100 & 200 & 1000 \\
\hline $100^{\circ}=-173^{\circ} \mathrm{C}$. & 0 & $9 \cdot 13$ & $36 \cdot 27$ & 80 & $333: 3$ \\
\hline $10^{\circ}=-263^{\circ} \mathrm{C}$. & 0 & .912 & $3 \cdot 61$ & $7 \cdot 11$ & $31 \cdot 83$ \\
\hline $1^{\circ}=-272^{\circ} \mathrm{C}$. & 0 & .091 & $\cdot 36$ & $\cdot 71$ & $3 \cdot 17^{\circ}$ \\
\hline$\frac{1}{T}\left(\frac{a z}{a+z}\right)$ in cms. & 0 & 9120 & 36070 & 71000 & 316800 \\
\hline Gas & \multicolumn{5}{|c|}{ Proportion measured by volume } \\
\hline Hydrogen .. & .00001 & .00018 & $\cdot 485$ & $\cdot 983$ & •99998 \\
\hline Helium ... & .000001 & .00002 & $\cdot 021$ & .017 & .00002 \\
\hline Nitrogen ......... & $\cdot 781$ & 850 & $\cdot 473$ & .00001 & {$\left[10^{-43}\right]$} \\
\hline Oxygen ....... & .210 & $\cdot 147$ & $\cdot 021$ & $\cdot 000000$ & {$\left[10^{-60}\right]$} \\
\hline Argon .. & $\cdot 009$ & .003 & .000000 & $\cdot 000000$ & {$\left[10^{-65}\right]$} \\
\hline
\end{tabular}

From these tigures it appears that as soon as we get beyond a certain distance from the earth's surface, the atmosphere may be supposed to consist solely of hydrogen. It is difficult to say exactly how far we have to go to reach this region, on account of our ignorance of the temperature of the upper air. If we regard clouds at a height of $\mathbf{1 7}$ miles as evidence of an adiabatic atmosphere extending to at least this height above the earth's surface, then, since the temperature at a height of 17 miles would be about 
$17^{\circ}$ absolute $\left(-256^{\circ} \mathrm{C}\right.$.), we may say that the temperature of the outer atmosphere would be less than $17^{\circ}$ absolute. In this case an atmosphere of hydrogen only would be reached at a height of about 53 kilometres from the adiabatic atmosphere-say 80 kilometres from the earth's surface. Any numerical estimate must, however, be very uncertain.

\section{Collisions in the Outer Atmosphere.}

380. It is not possible to form an exact estimate of the frequency of collisions in the outer atmosphere, for the obvious reason that we know hardly anything as to the actual value of the absolute density of the gas at this height. 'We can, however, easily obtain an upper limit to the collisionfrequency. As we shall find that this leads, at a certain height, to a mean free path many times greater than the earth's diameter, the information obtained will be sufficient for our purpose.

Throughout that part of the atmosphere which is in adiabatic equilibrium, the density falls off more rapidly than it would if the equilibrium were isothermal throughout, and hence the density at any point is less, for the same value at the earth's surface, than it would be if the atmosphere were in isothermal equilibrium. On this latter hypothesis, the density at height $z$ would be

$$
\nu=\nu_{0} e^{-2 h x},
$$

where $\chi$ is the difference of potential of a molecule between a height $z$ and the earth's surface. We accordingly have

$$
\begin{aligned}
\chi & =m \int_{r=a}^{r=a+z} \frac{g a^{2}}{r^{2}} d r \\
& =m g a^{2}\left(\frac{1}{a}-\frac{1}{a+z}\right) \\
& =\frac{m g a z}{a+z},
\end{aligned}
$$

as in $§ 377$ and hence the density at a height $z$ is

$$
\nu=\nu_{0} e^{-2 h m g}\left(\frac{a z}{a+z}\right)
$$

in which the variations in gravity are now taken into account, but not the planetary rotation.

We shall consider a height $z=a$, at which, it must be noticed, the apparent centrifugal force is still small compared with gravity, the ratio being about 1 to 37 . At such a height, even assuming a temperature as 
high as $0^{\circ} \mathrm{C}$., the atmosphere may be regarded as consisting entirely of hydrogen. If we take

we have, at height $z=a$,

$$
\begin{aligned}
& \nu_{0}=4 \times 10^{14}, \\
& C=18.4 \times 10^{4},
\end{aligned}
$$

$$
\begin{aligned}
\nu & =\nu_{0} e^{-h m g a} \\
& =340, \text { roughly } ;
\end{aligned}
$$

so that, taking $\sigma=2 \times 10^{-8}$, the mean free path is

$$
\frac{1}{\sqrt{2} \pi \nu \sigma^{2}}=1.6 \times 10^{12} \mathrm{cms} \text {., roughly, }
$$

so that even in this least favourable case the free path is many times greater than $a$.

We have assumed a temperature of $0^{\circ} \mathrm{C}$., although the actual temperature of the outer atmosphere is probably much lower than this. A lower temperature means a greater value for $h$, and hence a smaller value for $\nu$ and a greater mean free path. The result is therefore $\grave{a}$ fortiori true at temperatures lower than that which we have assumed.

Thus even on the hypothesis of isothermal equilibrium throughout, the calculated free path at a height $\alpha$ above the earth's surface is many thousands of times as great as $a$ : $\grave{a}$ fortiori the result is true when the density is diminished by the fact of part of the atmosphere being in adiabatic instead of isothermal equilibrium.

381. It follows that outside a sphere of radius equal to twice the earth's radius, we have an atmosphere in which practically no collisions occur. The molecules are simply describing orbits about the earth uninfluenced by one another, the velocities and directions of these orbits being determined by their last collision, which may be supposed to have taken place inside the sphere $r=2 a$. Of these orbits, some will be elliptic, some parabolic, and some hyperbolic. As has already been remarked, those molecules which describe parabolic or hyperbolic orbits must be regarded as permanently lost to the earth's atmosphere.

\section{Dissipation of Planetary Atmospheres.}

\section{The Earth.}

382. The number of molecules which cross the sphere $r=2 a$ and describe parabolic or hyperbolic orbits is identical with the number which cross with a velocity equal to or greater than that necessary to carry them to infinity against the earth's attraction. At the surface of a sphere of radius $2 a$ the gravitational potential is $\frac{1}{2} g a$, so that a molecule with a velocity $c$, such that $\frac{1}{2} c^{2}=\frac{1}{2} g a$, will just pass to infinity, describing a parabolic 
orbit. The molecules which leave the earth's atmosphere are therefore those which cross the sphere $r=2 a$ with a velocity equal to or greater than $\sqrt{g a}$.

At a point on the sphere $r=2 a$, of which the latitude is $\theta$, the gas has a mass velocity due to the earth's rotation, equal to $2 a \omega \cos \theta$. This, however, as we have seen, is small compared with $\sqrt{g a}$, so that the effect of the rotation of the earth in determining the dissipation of its atmosphere is slight. We shall neglect it altogether, as our calculations are necessarily of the roughest kind.

383. There has, however, been an arbitrary element introduced in choosing the radius of the sphere to be $2 a$. Let us consider the more general sphere of unknown radius $R$ drawn in the earth's atmosphere. The gravitational potential at the surface of this sphere is $\frac{g a^{2}}{R}$, so that the critical velocity is $c=\sqrt{\frac{2 g a^{2}}{R}}$, let us say $c_{0}$.

Maxwell's Law must of course hold throughout the outer atmosphere, which is in conductive equilibrium, and we shall suppose the sphere of radius $R$ to lie within this part of the atmosphere. If at any point of this sphere we take axes in such a direction that the axis of $z$ coincides with the outward normal, we find that the number of molecules of any type and of class A (i.e. having velocity components with a given range $d u d v d v$ ) which cross an element $d S$ of the sphere in time $d t$ is

$$
v \sqrt{\frac{h^{3} m^{3}}{\pi^{3}}} e^{-h m\left(u^{2}+v^{2}+w^{2}\right)} w d u d v d w d S d t \ldots \ldots \ldots \ldots \ldots . . .(759),
$$

where $\nu$ is the molecular density of molecules of this type at the sphere $r=R$, and is therefore, by equation (758), given by .

$$
\nu=\nu_{0} e^{-2 h m g \frac{a(R-a)}{R}}
$$

Integrating expression (75.9), we find for the total number of molecules crossing the sphere $r=R$ in the outward direction in time $d t$

$$
4 \pi R^{2} v \sqrt{\frac{h^{3} m^{3}}{\pi^{3}}} d t \iint e^{-l m\left(u^{2}+v^{2}+w^{2}\right)} w d u d v d w
$$

where the integration extends over all values of $u, v, w$ for which $w$ is positive and

$$
u^{2}+v^{2}+w^{2}>\overleftarrow{c_{0}^{2}} \text { ? }
$$

384. It will facilitate integration to divide the integral

into two parts, so that

$$
I=\iiint e^{-h m\left(u^{2}+v^{2}+w^{2}\right)} w d u d v d w
$$

$$
I=I_{1}-I_{2}
$$


where $I_{1}, I_{2}$ are formally the same as $I$, but in $I_{1}$ the variables are subject only to the condition that $w$ is positive, while in $I_{2}$ they are subject to this condition and also to the condition that

$$
u^{2}+v^{2}+w^{2}<c_{0}{ }^{2} \ldots \ldots \ldots \ldots \ldots \ldots \ldots \ldots \ldots \ldots . .(762) .
$$

We begin by performing the integration with respect to $w$ in $I_{2}$. The upper limit for $w^{2}$ is $c_{0}{ }^{2}-u^{2}-v^{2}$, the lower limit is zero. Hence

$$
\begin{aligned}
I_{2} & =-\frac{1}{2 h m} \iint e^{-h m\left(u^{2}+v^{2}\right)}\left[e^{-h m w^{2}}\right] d u d v \\
& =\frac{1}{2 h m} \iint\left(e^{-h m\left(u^{2}+v^{2}\right)}-e^{\left.-h m c_{0}^{2}\right)} d u d v,\right.
\end{aligned}
$$

in which $u$ and $v$ are now subject to

$$
u^{2}+v^{2}<c_{0}^{2} .
$$

Writing $u=\rho \cos \theta, v=\rho \sin \theta$, the first integral in the above expression for $I_{2}$ becomes

$$
\int_{\theta=0}^{\theta=2 \pi \rho=c_{0}} \int_{\rho=0}^{\rho=c_{0}} e^{-h m \rho^{2}} \rho d \rho d \theta=\frac{\pi}{h m}\left(1-e^{-h m c_{0}^{2}}\right),
$$

while the value of the second integral is

$$
-\pi c_{0}^{2} e^{-h m c_{0}^{2}} \text {. }
$$

Hence the value of $I_{2}$ is

$$
I_{2}=\frac{\pi}{2 h^{2} m^{2}}\left\{1-e^{-h m c_{0}^{2}}\left(1+h m c_{0}^{2}\right)\right\} .
$$

Putting $c_{0}=\infty$, we obtain the value of $I_{1}$, which is therefore

$$
I_{1}=\frac{\pi}{2 h^{2} m^{2}}
$$

and hence by subtraction,

$$
\begin{aligned}
I & =I_{1}-I_{2} \\
& =\frac{\pi}{2 h^{2} m^{2}} e^{-h m c_{0}^{2}}\left(1+h m c_{0}^{2}\right) .
\end{aligned}
$$

385. The value of expression (761), the number of molecules which cross the sphere $r=R$ outwards in time $d t$, is accordingly

$$
4 \pi R^{2} \nu \sqrt{\frac{h^{3} m^{3}}{\pi^{3}}} I d t=2 R^{2} \nu \sqrt{\frac{\pi}{h m}} e^{-h m c_{0}^{2}}\left(1+h m c_{0}^{2}\right) d t .
$$

Replacing $c_{0}{ }^{2}$ by its value $\frac{2 g a^{2}}{R}$ and replacing $\nu$ from equation (760) this expression becomes

$$
\begin{array}{r}
2 R^{2} \nu_{0} e^{-2 h m g} \frac{a(R-a)}{R} \sqrt{\frac{\pi}{h m}} e^{-2 h m g \frac{a^{2}}{R}}\left(1+2 h m g \frac{a^{2}}{R}\right) d t \\
=2 R^{2} \nu_{0} e^{-2 h m g a} \sqrt{\frac{\pi}{h m}}\left(1+2 h m g \frac{a^{2}}{R}\right) d t
\end{array}
$$


386. This expression is not, however, independent of $R$, as we might at first have expected it to be. The reason for this is as follows. In the complete atmosphere, supposing it to be constituted according to Maxwell's Law throughout, there will be a number of molecules describing orbits which never pass within a sufficiently small distance from the earth's centre for the chance of collision to be appreciable. Some of these describe hyperbolic or parabolic orbits, passing from infinity past the earth to infinity again without collision. Now if $p$ is the distance of the apse of any orbit from the earth's centre, it is clear that a molecule describing this orbit will be counted in expression (763) as escaping from the earth's atmosphere if $R>p$, but not if $R<p$. We should therefore expect expression (763) to increase with $R$ as is in fact seen to be the case.

It is questionable whether molecules of the kind just described ought to be supposed to exist in the actual atmosphere. The analysis by which the specification of the steady state is arrived at, takes no account of the length of time required for the establishment of this steady state. In the present instance the steady state implies the arrival of molecules which have described hyperbolic and parabolic orbits from infinity. It is therefore obvious that it will require infinite time to establish the steady state.

On the other hand, molecules which are supposed to describe orbits in the regions in which no collisions occur have no influence on the rest of the atmosphere and may therefore be removed without disturbing the equilibrium of the remainder of the atmosphere. In nature these molecules cannot be supposed to exist. They would be counted in our estimate of the escape of molecules from the atmosphere by taking $R$ great. We therefore obtain the most accurate results by taking $R$ as small as possible, and the error vanishes altogether when we reduce the sphere of radius $R$ to such a size that collisions may be regarded as frequent everywhere inside it.

387. In the case of a rotating atmosphere, we found that there must be supposed to be a complete atmosphere extending to infinity, lying outside the region in which practically no collisions occur. This atmosphere can be treated in the same way in which individual molecules coming from infinity have been treated. It can be supposed to be removed bodily without disturbing the equilibrium of the remainder of the atmosphere.

It must, nevertheless, be noticed that the order of magnitude of expression (763) is determined solely by the exponential $e^{-2 h m g a}$, so that the value of 2hmga determines whether the escape of molecules is appreciable or not. This criterion, as we should expect, is independent of $R$.

388. The total number of molecules of any specified kind in the planet's outer atmosphere, supposed limited by spheres of radii $a$ and $R$, is

$$
\int_{a}^{R} 4 \pi R^{2} \nu_{0} e^{-2 h m g a \frac{R-a}{R}} d R
$$


This integral cannot be evaluated in finite terms. Since, however, we are ultimately going to take $R$ nearly equal to $a$, we can obtain a sufficiently accurate value of the integral as follows.

The integral may be replaced by

$$
\int_{a}^{R} 4 \pi \nu_{0} \frac{R^{4}}{a^{2}} e^{-2 h m g a} \frac{a^{2}}{R^{2}} e^{2 h m g} \frac{a^{2}}{R} d R
$$

and this again is equal to

$$
4 \pi \nu_{0} \frac{a^{4}}{a^{2}} e^{-2 h m g a} \int_{a}^{R} e^{2 h m g} \frac{a^{2}}{R} \frac{a^{2}}{R^{2}} d R
$$

where $a^{\prime}$ is some quantity intermediate between $a$ and $R$. The value of the integral in this expression is

$$
\frac{1}{2 h m g}\left(e^{2 h m g a}-e^{2 h m g \frac{a^{2}}{R}}\right)
$$

so that the whole expression is equal to

$$
\frac{2 \pi \nu_{0}}{h m g} \frac{a^{\prime}}{a^{2}}\left(1-e^{-2 h m g a \frac{R-a}{R}}\right)
$$

389. The loss per second, from formula (763), is

$$
2 R^{2} \nu_{0} e^{-2 h m g a} \sqrt{\frac{\pi}{h m}}\left(1+2 h m g \frac{a^{2}}{R}\right)
$$

so that the fraction of the whole which is lost per second-the ratio of expression (765) to expression (764) -is

$$
g \sqrt{\frac{h m}{\pi}} \frac{R^{2} a^{2}}{a^{\prime 4}}\left(1+2 h m g \frac{a^{2}}{R}\right) \frac{e^{-2 h m g a}}{1-e^{-2 h m g a\left(\frac{R-a}{R}\right)}}
$$

As regards order of magnitude, we may replace $\frac{R^{2} a^{2}}{a^{\prime 4}}$ by unity, and $1+2 h m g \frac{a^{2}}{R}$ by $1+2 h m g a$. If for brevity we replace $2 \dot{h} m g a$ by $x$, expression (766) becomes

$$
g \sqrt{\frac{h m}{\pi}}(1+x) \frac{e^{-x}}{1-e^{-x\left(\frac{R-a}{R}\right)}}
$$

For the earth and all the other planets $g$ is of the order of magnitude of $10^{3}$. The value of $\sqrt{\frac{h m}{\pi}}$ is $\sqrt{\frac{3}{2 \pi}} \frac{1}{C}$, and may therefore be supposed to be of the order of $10^{-5}$. Hence, roughly, expression (767) may be replaced by

$$
\frac{10^{-2}(1+x) e^{-x}}{1-e^{-x\left(\frac{R-a}{R}\right)}}
$$


This may fairly be said to be negligible when $x=30$, in which case it has the value $3 \times 10^{-14}$, and to be appreciable (for our present purpose) when $x=15$, in which case it has the value $5 \times 10^{-8}$. In the former instance, half of the upper atmosphere is lost in about two million years; in the latter, in about one year.

We can therefore say that an atmosphere is certainly lost if $x<15$, and is certainly retained through millions of years if $x>30$.

\section{Numerical Values.}

390. The final part of the problem consists of the calculation of numerical values. We shall find the values of $C$ for which $x=15$ and $x=30$ respectively. Since $x=\frac{3 g a}{C^{2}}$, these values are

$$
C^{2}=\frac{g a}{5}, \frac{g a}{10}
$$

\section{The Earth.}

For the earth,

$$
g=981, \quad a=6.4 \times 10^{8},
$$

so that the critical values of $C$ are

$$
3.5 \times 10^{5}, 2.5 \times 10^{5} \text {. }
$$

For hydrogen, the value $C=255 \times 10^{5}$ corresponds to a temperature of $230^{\circ} \mathrm{C}$. (say $500^{\circ}$ absolute), the value $C=3.5 \times 10^{5}$ to a temperature of about $740^{\circ} \mathrm{C}$. Since the temperature of the present outer atmosphere of the earth is certainly less than $230^{\circ} \mathrm{C}$., it is clear that there can be no perceptible escape of gas from our present atmosphere. But if at any time in the history of our earth the temperature of the outer atmosphere has been greater than about $740^{\circ} \mathrm{C}$., the hydrogen atmosphere will have been lost, and the hydrogen in the present atmosphere must be explained as an addition, probably as the result of chemical action at and near the earth's surface, since that time.

\section{Sun, Moon, and Planets.}

391. Other atmospheres are best treated by reference to the earth as the standard case. The structure of the planet is involved through the term $g a$, and since $g$ is proportional to $\frac{M}{a^{2}}$ where $M$ is the mass, and $a$ the radius of the planet, it follows that ga will be proportional to $\frac{M}{a}$. The following table 
gives the values of $M, a$ and $g a$ in terms of the corresponding quantities for the earth :

\begin{tabular}{|c|c|c|c|}
\hline & Mass & Radius & $g a$ \\
\cline { 1 - 4 } Earth & 1 & 1 & 1 \\
\cline { 2 - 4 } Sun ........ & 333,432 & 109 & 3060 \\
Moon ........ & $\cdot 013$ & $\cdot 27$ & 05 \\
Mercury ... & $\cdot 066$ & $\cdot 38$ & $\cdot 17$ \\
Venus ...... & $\cdot 8$ & $\cdot 97$ & $\cdot 8$ \\
Mars ........ & $\cdot 11$ & $\cdot 53$ & $\cdot 2$ \\
Jupiter ...... & 300 & $11 \cdot 0$ & 27 \\
Saturn ...... & $90 \cdot 0$ & $9 \cdot 0$ & 10 \\
Uranus ...... & $14 \cdot 0$ & $4 \cdot 0$ & $3 \cdot 5$ \\
Neptune ... & $16 \cdot 0$ & $4 \cdot 4$ & $3 \cdot 6$ \\
\hline
\end{tabular}

Superior Planets. From this table it appears that for all the superior planets except Mars, $g a$ is greater than the case of the earth, so that we may conclude that all constituents of the atmosphere would be retained at temperatures up to $230^{\circ} \mathrm{C}$. Indeed, the lowest value of $g a$ is 3.5 times that of the earth, so that the absolute temperature of the outer atmosphere would have to be more than 3.5 times as great as $230^{\circ} \mathrm{C}$., say $1500^{\circ} \mathrm{C}$., before the loss even of hydrogen would be perceptible. A temperature as high as this is highly improbable, so that we may say that, so far as the Kinetic Theory is concerned, the superior planets except Mars ought to retain all constituents of their atmospheres.

There is general agreement amongst astronomers as to the existence of atmospheres on Jupiter and Saturn. As regards Uranus, spectroscopic evidence (absorption bands) suggests an atmosphere. The spectrum of Neptune is too faint for any such inference to be possible, but it has been suggested that its green appearance is due to absorption bands at the red end of the spectrum, similar to those in the spectrum of Uranus.

The Sun. For the Sun, ga is 3060 times as great as for the earth. The temperature of the outer atmosphere could therefore be about $3060 \times 500$ absolute, say $1,530,000^{\circ} \mathrm{C}$., before any appreciable loss, even of hydrogen, would occur from the causes now contemplated.

Venus. For Venus the value of $g a$ is 8 times as great as for the earth, so that the critical values of $C$ are $\sqrt{\cdot 8}$ times as great as the critical values for the earth, and are therefore 
For hydrogen, the value $C=2 \cdot 24 \times 10^{5}$ corresponds to a temperature of about $130^{\circ} \mathrm{C}$. It therefore seems probable that Venus retains the whole of her atmosphere. There is conclusive astronomical evidence of the existence of an atmosphere on Venus.

Mars. For Mars, $g a$ is only one-fifth as great as for the earth. The critical velocities are accordingly

$$
1.6 \times 10^{5} \text { and } 1.1 \times 10^{5} \text {. }
$$

From the table on page 113 it appears that at a moderate temperature hydrogen and helium would escape from the planet, while water vapour, air, and heavier gases would be retained.

Mercury. The case of Mercury is exactly similar to that of Mars, as regards the evaluation of the critical velocities, but as Mercury is much nearer the Sun, the temperature of the outer atmosphere would probably be much higher, and therefore the chance of an atmosphere of any kind being found on Mercury would be much smaller. The evidence of astronomy leaves little doubt that Mercury is entirely devoid of atmosphere.

The Moon. For the moon the critical velocities are found to be about

$$
7 \cdot 8 \times 10^{4} \text { and } 5.6 \times 10^{4} \text {, }
$$

so that if the moon ever possessed an atmosphere at a temperature much above zero, all constituents, except possibly carbon-dioxide and denser gases, would rapidly escape.

The bearing of the Kinetic Theory on the question of the dissipation of planetary atmospheres was first brought into prominence by the writings of Dr Johnstone Stoney*. An adequate mathematical treatment was first given by Professor G. H. Bryan in the paper to which reference has already been made. In the present chapter I have borrowed largely from Professor Bryan's paper, although not following his treatment in detail. The results arrived at are practically identical.

Dr Stoney now maintains that the Kinetic Theory is not competent to decide on the question, being unable to take full account of the physical conditions of the problem, and that the conclusions of the Kinetic Theory are inconsistent with the facts. His present position is stated in a recent letter "Escape of Gases from Atmospheresf."

* "Atmospheres upon Planets and Satellites," Trans. Roy. Soc. Dublin, vi. p. 305, and earlier papers.

† Phil. Mag. vir. p. 690 (June, 1904). 


\title{
CHAPTER XVIII.
}

\author{
MOLECULAR AGGREGATION AND DISSOCIATION.
}

392. Through all our investigations we have relied upon the supposition that a gas can be regarded as a collection of separate dynamical systems, namely molecules, each of which retains its identity through all time. We must now discuss what changes are required when this supposition is regarded only as an approximation to the truth, and not as expressing the complete truth.

\section{Molecular aggregation.}

393. We have already seen that there must be a small attractive force between those molecules in a gas which are sufficiently near to one another, or, more precisely, that the potential energy of the total intermolecular forces in a gas is negative.

This result, it is worth noticing, is intelligible without assuming that there is any definitely attractive force inherent in a single molecule. In $§ 85$ we obtained as the laws of distribution for those molecules which were free from intermolecular force and for those molecules which were under the influence of intermolecular force, equations of the forms

$$
\left.\begin{array}{rl}
\phi_{\alpha} & =A e^{-2 h E_{\alpha}} \\
\phi_{a \alpha} & =A^{2} e^{-2 h\left(E_{\alpha}+E_{\alpha}^{\prime}+W_{\alpha a}\right)} \\
& \text { etc. }
\end{array}\right\}
$$

it being sufficient for our present purpose to consider a gas in which only one kind of molecule is present, i.e. a gas which is chemically pure. In the above equations $W_{a a}$ is the potential of the intermolecular forces between the two molecules. If we denote the potential of the intermolecular forces between three molecules by $W_{\text {aaa }}$, and so on, we obtain as the total intermolecular potential energy of the gas,

$$
\begin{aligned}
\Phi & =A^{2} \iint \ldots W_{\alpha \alpha} e^{-2 h\left(E_{\alpha}+E_{\alpha}^{\prime}+W_{\alpha \alpha}\right)} d \xi_{1} d \xi_{2} \ldots \\
& +A^{3} \iint \ldots W_{\alpha a \alpha} e^{-2 h\left(E_{\alpha}+E_{\alpha}^{\prime \prime}+E^{\prime \prime}{ }_{\alpha}+W_{\alpha a \alpha}\right)} d \xi_{1} d \xi_{2} \ldots+\text { etc. }
\end{aligned}
$$


where the integrations extend over all configurations in which the intermolecular forces are appreciable. Now if, when the configuration of two molecules is selected at random from all possible configurations, $W_{a a}$ is as likely to be positive as negative, then the whole of the first integral can be expressed as a sum of terms of the form

$$
W_{\alpha a} e^{-2 h W_{\alpha a}}-W_{a \alpha} e^{2 h W_{\alpha a}},
$$

this term being obtained by combining two configurations in which the values of $W_{a a}$ are equal in magnitude but opposite in sign. This expression is, however, negative for all values of $W_{a a}$. The second integral can be similarly treated, so that we arrive at the final result that $\Phi$ is negative.

If now $\eta_{1}, \eta_{2} \ldots$ are any of the $\xi$ s which determine orientation (or any other coordinates which enter into the intermolecular potential, but not into the internal energy of a single molecule), then the first integral in expression (769) can be written in the form

$$
A^{2} \iint \ldots e^{-2 h\left(E_{\alpha}+E_{\alpha}^{\prime}\right)} d \xi_{1} \cdot d \xi_{2} \ldots \iint \ldots W_{\alpha a} e^{-2 h W_{\alpha \alpha} d \eta_{1}} d \eta_{2} \ldots,
$$

and this again can be written as

$$
A^{2} \iint \ldots \Psi_{\alpha a} e^{-2 h\left(E_{\alpha}+E_{\alpha}^{\prime}+\Psi_{\alpha \alpha}\right)} d \xi_{1} d \xi_{2} \ldots
$$

where $\Psi_{a a}$ is an average intermolecular potential for pairs of molecules of which all the coordinates except $\eta_{1}, \eta_{2} \ldots$ are specified, and is negative.

\section{Degree of Aggregation.}

394. Consistently with what has been said, we may now simplify the problem by regarding molecules as point-centres of force, acting on one another with a force depending only on their distance apart. The chance of finding a free molecule of class $A$ inside an element of volume $d x d y d z$ is now, by equation (768)

$$
A e^{-h m c^{2}} d u d v d w d x d y d z
$$

while the chance of finding two molecules of classes $A$ and $B$ in adjacent elements $d x d y d z$ and $d x^{\prime} d y^{\prime} d z^{\prime}$ is

$$
A^{2} e^{-h m\left(c^{2}+c^{\prime 2}\right)-2 h \Psi} d u d v d w d x d y d z d u^{\prime} d v^{\prime} d w^{\prime} d x^{\prime} d y^{\prime} d z^{\prime} .
$$

If we take the element $d x^{\prime} d y^{\prime} d z^{\prime}$ to be a spherical shell of radii $r$ and $r+d r$ surrounding the centre of the first molecule, this last expression becomes

$$
A^{2} e^{-h m\left(c^{2}+c^{\prime 2}\right)-2 h \Psi} d u d v d w d u^{\prime} d v^{\prime} d w^{\prime} 4 \pi r^{2} d r d x d y d z,
$$

$\Psi$ being a function of $r$. If, as in $\S 30$, we use the transformations

$$
\mathbf{u}=\frac{1}{2}\left(u+u^{\prime}\right) \text { etc., } \quad \alpha=u^{\prime}-u, \text { etc., }
$$


and write

$$
\mathbf{u}^{2}+\mathbf{v}^{2}+\mathbf{w}^{2}=\mathbf{c}^{2}, \quad \alpha^{2}+\beta^{2}+\gamma^{2}=V^{2},
$$

we can transform the foregoing expression into

$$
A^{2} e^{-2 h m \mathrm{c}^{2}} d \mathbf{u} d \mathbf{v} d \mathbf{w} d x d y d z e^{-\frac{1}{2} h m V^{2}-2 h \Psi} d \alpha d \beta d \gamma 4 \pi r^{2} d r \ldots . .(771) .
$$

The first factor after the $A^{2}$ expresses the law of distribution of translational velocities for a double molecule. It is exactly the same as if each double molecule were a permanent structure of mass $2 \mathrm{~m}$. The remaining factors express the distribution of those coordinates which may be regarded as internal to the double molecule.

395. Throughout the motion of a double molecule, so long as it is undisturbed by collisions, $\mathbf{c}^{2}$ will remain constant, so that from the energy equation it follows that $\frac{1}{2} m V^{2}+2 \Psi$ remains constant. The possible orbits which the component molecules can describe about their common centre of gravity fall into two classes, according as they pass to infinity or not. Analytically these two classes are differentiated by the sign of $\frac{1}{2} m V^{2}+2 \Psi$. Double molecules for which $\frac{1}{2} m V^{2}+2 \Psi$ is positive consist of two molecules which have approached one another from outside each other's sphere of action, and which after passing once within a certain minimum distance of each other, will again recede out of each other's sphere of influence. On the other hand, double molecules, for which $\frac{1}{2} m V^{2}+2 \Psi$ is negative, consist of two molecules describing orbits about one another, these orbits being entirely within the two spheres of action, and this motion continues except in so far as it is interrupted by collisions with other molecules. It is clear that double molecules of the first kind are simply pairs of molecules in collision. In discussing molecular aggregation we must confine our attention to double molecules of the second kind, i.e. those for which $\frac{1}{2} m V^{2}+2 \Psi$ is negative. It is to be noticed that double molecules of this kind cannot be produced solely by the meeting of two single molecules. It is necessary that while the single molecules are in collision something should happen to change the motion-in fact to change the sign of $\frac{1}{2} m V^{2}+2 \Psi$. This might be effected by collision with a third molecule, or possibly if $\frac{1}{2} m V^{2}+2 \Psi$ were very small at the beginning of an encounter, sufficient energy might be dissipated into the ether for $\frac{1}{2} m V^{2}+2 \Psi$ to become negative before the termination of the encounter. We shall return to the consideration of this second possibility later. If this were the primary cause of aggregation, we should no longer be able to use the equations with which we have been working, since they rest upon the assumption of conservation of energy.

396. Integrating expression (770) over all values of $u, v$ and $w$, we find for $\nu_{1}$, the molecular density of uncombined molecules,

$$
\nu_{1}=A \sqrt{\frac{\pi^{3}}{h^{3} m^{3}}} .
$$


Similarly, if $\nu_{2}$ is the molecular density of double molecules, we obtain by integration of expression (771),

$$
\begin{aligned}
\nu_{2} & =A^{2} \sqrt{\frac{\pi^{3}}{8 h^{3} m^{3}}} \iiint \int e^{-h\left[\frac{1}{[3} m V^{2}+2 \Psi\right]} d \alpha d \beta d \gamma 4 \pi r^{2} d r \\
& =A^{2} \sqrt{\frac{\pi^{3}}{8 h^{3} m^{3}}} \iint e^{-h\left[l_{3} m V^{2}+2 \psi\right]} 16 \pi^{2} V^{2} r^{2} d V d r,
\end{aligned}
$$

in which the integration extends over all values of $V$ and $r$ for which $\frac{1}{2} m V^{2}+2 \Psi$ is negative.

The total number of constituent molecules per unit volume $(\nu)$ is

$$
\begin{aligned}
& \nu=\nu_{1}+2 \nu_{2}+3 \nu_{3}+\ldots \\
& =\nu_{1}\left(1+\frac{A}{\sqrt{2}} \iint e^{\left.-h[1] m V^{2}+2 \psi\right]} 16 \pi^{2} V^{2} r^{2} d V d r+\frac{A^{2}}{\sqrt{3}} \iint \ldots+\ldots\right) \ldots \ldots
\end{aligned}
$$

so that if we denote the fraction of the whole mass which is free by $q$, we have

$$
q=\frac{\nu_{1}}{\nu}=\frac{1}{1+\frac{A}{\sqrt{2}} \iint e^{-h\left[\frac{1}{2} m V^{2}+2 \Psi\right]} 16 \pi^{2} V^{2} r^{2} d V d r+\ldots}
$$

\section{Continuity of Liquid and Gaseous States.}

397. At very high temperatures $h$ is very small, so that $A$, or

$$
\nu_{1} \sqrt{\frac{h^{3} m^{3}}{\pi^{3}}}
$$

is very small. Also $e^{-h\left[t m \nu^{2}+2 \Psi\right]}$ is very nearly equal to unity. Thus at these temperatures the series (772) reduces to its first term, and $q=1$. There are therefore no molecules in permanent combination, as indeed we should expect.

At lower temperatures $h$ is greater, so that not only is $A$ greater, but the exponential $e^{-h\left[\mathrm{~s} m V^{2}+2 \psi\right]}$, in which it will be remembered that the index is always positive, is also greater. The relative importance of the later terms of the series (772) is therefore greater. Ultimately we reach low values of the temperature for which $h$ has so great a value that the series (772) becomes divergent. At this point the molecules tend, according to our analysis, to form into clusters, each containing an infinitely great number of molecules. By the time this point is reached, however, the analysis ceases to apply, as the assumption that the molecular clusters are small, made in $\S 83$, is now invalidated. It is, however, easy to give a physical interpretation of the point now reached : obviously it is the point at which liquefaction begins, and the collection of molecular clusters is a "saturated vapour." 
398. Regarded as a series in terms of $A$, the series (772) is a power series, and so has a single radius of convergence. Thus for a given value of $h$, say $h_{0}$, there is a single value of $A$, say $A_{0}$, such that the series is convergent for all values of $A$ less than $A_{0}$ and is divergent for all values of $A$ greater than $A_{0}$. In other words, corresponding to a given temperature, there is a definite density at which the substance liquefies. This of course is the vapour-density corresponding to this temperature. Clearly as $h$ increases, $A$ decreases, and conversely, so that an increase of pressure is accompanied by a rise in the boiling point of the substance.

399. For very small values of $h$, as we have seen, $\nu$ and $\nu_{1}$ become almost identical, so that we can replace $A$ by $\nu \sqrt{\frac{h^{3} m^{3}}{\pi^{3}}}$, and the series (772) becomes very approximately,

$$
\nu_{1}\left(1+\nu \sqrt{\frac{h^{3} m^{3}}{2 \pi^{3}}} \iint 16 \pi^{2} V^{2} r^{2} d V d r+\ldots\right),
$$

the exponential factor, which is very nearly equal to unity, being omitted.

The integrals are now all independent of $h$, and we can suppose the integration with respect to $V$ in the first integral and similar variables in the other integrals to be performed, so that we are left with a series of integrals each extending over a sphere of molecular action-integrals, then, which depend solely on the structure of the molecules and which may therefore be treated as constants. The series is now a power-series in ascending powers of $\nu h^{\frac{3}{2}}$, and therefore becomes divergent when $\nu h^{\frac{\pi}{2}}$ exceeds a certain value.

We are considering only the limit in which $h$ vanishes, so that for the series to become divergent, $\nu$ must, in the limit, be infinite. This is, however, inconsistent with the supposed finite extension of the spheres of molecular action. Thus when $h$ is very small there is no possibility of the series which we have been discussing becoming divergent. In other words, at very high temperatures, a substance will not liquefy no matter how great the pressure.

\section{The Critical Point.}

400. We have already found that corresponding to every value of $h$ there is a definite value of $A$ at which a substance liquefies. This leads to a functional relation between $\nu$ and $T$, say

$$
f(\nu, T)=0
$$

expressing the relation between $\nu$ and $T$, at the boiling point of a liquid. We have now seen that corresponding to $T=\infty$, there is only a single root of equation (773), namely $\nu=\infty$. This value of $\nu$ is, however, prohibited by the molecular structure of the gas, from exceeding a certain critical 
maximum value, say $\nu_{\text {crit. }}$, consistently with the suppositions we have made as to the smallness of the clusters of molecules. Hence there is a certain critical value of $T$, say $T_{\text {crit. }}$, such that for values of $T$ greater than $T_{\text {crit., }}$, equation (773) cannot be satisfied by any physically possible value of $\nu$. In other words, at temperatures greater than $T_{\text {erit. }}$, liquefaction cannot set in, no matter how great $\nu$ is; at temperature $T_{\text {crit., }}$, liquefaction sets in when the molecular density is $\nu_{\text {erit.; }}$; and at temperatures below $T_{\text {crit. }}$, liquefaction sets in as soon as the molecular density reaches some value which is below $\nu_{\text {erit. }}$.

It is therefore obvious that $T_{\text {erit. }}$ is simply the "critical temperature" in the ordinary sense, and that $\nu_{\text {crit. }}$ is the "critical molecular-density." At temperatures above $T_{\text {erit., }}$, the molecules can be brought closer together by pressure, and the density can be increased to values greater than $\nu_{\text {crit., }}$, but the suppositions on which we have been working are now violated-there are no longer small and separate molecular clusters, but the clusters are crowded into one another by the pressure. Thus at densities above the critical density, the liquid and gaseous states are continuous. For values of $T, \nu$ below the critical values, it is clear that equation (773) represents that branch of the chain line in fig. 10 (p. 133), which is to the right of the critical point $P$.

\section{Pressure, Density and Temperature.}

401. From what has been said, it is clear that when a gas or vapour is at a temperature which is only slightly greater than its boiling point at the pressure in question, it cannot be regarded as consisting of single molecules, but must be supposed to consist partly of single molecules and partly of clusters of two, three or more molecules. If $m$ is the mass of a single molecule, and if $\nu_{1}, \nu_{2}, \nu_{3} \ldots$ have the same meaning as before, the density is given by

$$
\rho=m\left(\nu_{1}+2 \nu_{2}+3 \nu_{3}+\ldots\right)
$$

In calculating the pressure, we must treat each type of cluster as a separate kind of gas, exerting its own partial pressure. We accordingly obtain for the pressure, as in $\S 123$,

$$
\begin{aligned}
p & =\frac{1}{2 h}\left(\nu_{1}+\nu_{2}+\nu_{3}+\ldots\right) \\
& =R T\left(\nu_{1}+\nu_{2}+\nu_{3}+\ldots\right)
\end{aligned}
$$

From a comparison of equations (774) and (775), remembering that $\nu_{1}, \nu_{2} \ldots$ are functions of $T$ and $\rho$, it is clear that neither Boyle's Law, Charles' Law nor Avogadro's Law will be satisfied with any accuracy. 
402. The observed deviations from the laws obeyed by a perfect gas must of course be attributed partly to aggregation, as has just been explained, and partly to the causes which have already been discussed in Chapter VI. The two sets of causes are not, however, independent; so that it is not sufficient to consider the effects separately, and then add. The state of the question is, perhaps, best regarded as follows.

The effect of the forces of cohesion is too complex for an exact mathematical treatment to be possible. We have therefore, in Chapter VI. and the present chapter, examined their effect with the help of two separate simplifying assumptions. In Chapter VI., following Van der Waals, we regarded the gas as a single molecular cluster containing an infinite number of molecules, and in replacing the whole system of the forces of cohesion by a permanent average force, we virtually neglected the effect of any formations of small clusters inside the large cluster. In the present chapter, on the other hand, we have been concerned solely with the formation of small clusters, and have disregarded the large cluster altogether. As a consequence of the omission of the former treatment to take account of the formation of small clusters, this treatment led to the erroneous result (equation (386)), that the internal pressure is exactly proportional to the temperature, whereas as a consequence of the omission of the present treatment to consider the clustering of the gas as a whole, we are led in the present chapter to the erroneous conclusion that the internal pressure is identical with the boundary pressure. The situation may then be summed up by saying that the treatment of Chapter VI. considers only the tendency to mass-clustering, while that of the present chapter considers only the tendency to molecular-clustering.

So long as the deviations from the behaviour of a perfect gas are small, the two tendencies may be considered separately, and the total deviation regarded as the sum of the deviations caused by the two tendencies separately. On the other hand, as we approach the critical point the phenomena of massclustering and molecular-clustering merge into one another and ultimately become identical at the critical point. The two effects are no longer additive, for each has become identical with the whole effect.

By the side of these considerations must be placed the consideration that we have only found an exact mathematical treatment of either effect to be possible by making the assumption that the effect itself is small. In other words, so far as our results apply, the effects are additive. It may be noticed that the deviations from the laws of a perfect gas which were discussed in Chapter VI., fell off proportionally to $\frac{1}{T}$ and $\frac{1}{T^{2}}$, whereas the deviations discussed in the present chapter fall off much more rapidly as the temperature increases. 
As a conspicuous instance of a gas in which these laws are not satisfied we may take chlorine, of which the boiling point at normal pressure is $-33.4^{\circ} \mathrm{C}$. According to Stas's determination of the atomic weight of chlorine its density relatively to that of air would be 2.4501 at all temperatures if Avogadro's Law were satisfied. The observed densities are as follows:

$\begin{array}{rr}\text { Temp. C. } & \text { Density } \\ 20^{\circ} & 2 \cdot 4807 \\ 50^{\circ} & 2 \cdot 4783 \\ 100^{\circ} & 2 \cdot 4685 \\ 150^{\circ} & 2 \cdot 4609 \\ 200^{\circ} & 2 \cdot 4502\end{array}$

On inspection of these figures, we see that the deviation at $150^{\circ} \mathrm{C}$. is about 100 times as great as that at $200^{\circ} \mathrm{C}$. This rapid diminution as the temperature increases, suggests that aggregation plays the preponderating part in the production of these deviations.

\section{Calorimetry.}

403. The total translational energy per unit volume is

$$
3 R T\left(\nu_{1}+\nu_{2}+\ldots\right) \text {. }
$$

We must also regard each compound molecule as possessing internal energy of amount $\left[\frac{1}{2} m V^{2}+2 \Psi\right]$. This is negative, the explanation of course being that it is referred to the energy of two molecules at rest and clear of each other's sphere of influence as zero. Let us write $-I_{2},-I_{3}, \ldots$ for the average internal energy of double molecules, triple molecules, etc., so that the total internal energy of the clusters of molecules, referred to the standard already explained, is

$$
-\left(\nu_{2} I_{2}+\nu_{3} I_{3}+\ldots\right)
$$

The general equation of calorimetry is now

$$
d \mathbb{Q}=d\left[3 R T\left(\nu_{1}+\nu_{2}+\ldots\right) v-\left(\nu_{2} I_{2}+\nu_{3} I_{3}+\ldots\right) v\right]+p d v
$$

At constant volume, this becomes

$$
d \mathbb{Q}=v d\left[3 R T\left(\nu_{1}+\nu_{2}+\ldots\right)-\left(\nu_{2} I_{2}+\nu_{3} I_{3}+\ldots\right)\right]
$$

and since the value of $d \mathbb{Q}$ is $J \rho v C_{v} d \not$, we have by comparison,

$$
C_{v}=\frac{1}{J_{\rho}} \frac{d}{d T}\left[3 R T\left(\nu_{1}+\nu_{2}+\ldots\right)-\left(\nu_{2} I_{2}+\nu_{3} I_{3}+\ldots\right)\right]
$$

If there were no aggregation, the value of $C_{v}$ would be

$$
C_{v}^{\prime}=\frac{1}{J_{\rho}} \frac{d}{d T}\left[3 R T\left(\nu_{1}+2 \nu_{2}+3 \nu_{3}+\ldots\right)\right]
$$

so that by subtraction,

$$
C_{v}^{\prime}-C_{v}=\frac{1}{J_{\rho}} \frac{d}{d T}\left[3 R T\left(\nu_{2}+2 \nu_{3}+\ldots\right)+\left(\nu_{2} I_{2}+\nu_{3} I_{3}+\ldots\right)\right]
$$


It would be fruitless to try to evaluate this expression further; we cannot even determine its sign with certainty. We shall simply remark that it depends on both the temperature and the density, and this to an extent which increases as we approach low temperatures and high densities.

The case is similar as regards the quantities $C_{p}-C_{v}, C_{v}$ and $\gamma$. We should expect them all to depend on the pressure and density to an extent of which it is hardly possible to form any estimate.

404. As an illustration we may take the case of steam. Wet steam is steam in which large molecular clusters occur, dry steam is steam in which the molecules are all separate, and our quantity $q$ measures what engineers speak of as the dryness of wet steam. For the value of $\gamma$ for wet (saturated) steam, Rankine and Zeuner give respectively the values 1.0625, 1.0646. For dry steam ("steam gas") the recognised value is 1:30. If we used the formula

$$
\gamma=1+\frac{2}{n+3},
$$

for the calculation of $n$, we should come to the conclusion that $n+3$ had the value 32 for wet steam, and 6.6 for dry steam. From what has been said, it will be seen that we may with some confidence take the value $n+3=6$ for the molecule, this being the value we should naturally expect for a triatomic molecule (cf. $§ 271$ ) and regard the difference between this and the apparent value 32 for wet steam, as caused by molecular aggregation.

405. A further instance is supplied by carbon-dioxide for which we have already (p. 252) noticed the large influence of pressure on the coefficient of viscosity. The following values of $C_{p}$ are given by Lussana*.

\begin{tabular}{|c|c|c|}
\hline Temp. C. & Press. in atmos. & $C_{p}$ \\
\cline { 2 - 3 } $13 \cdot 2^{\circ}$ & $85 \cdot 40$ & $2 \cdot 1096$ \\
$"$ & $61 \cdot 70$ & $0 \cdot 8900$ \\
$"$ & $54 \cdot 10$ & $0 \cdot 7301$ \\
$114 \cdot 9^{\circ}$ & $85 \cdot 40$ & 0.5324 \\
$"$ & $61 \cdot 70$ & $0 \cdot 3133$ \\
\hline
\end{tabular}

At normal temperature and pressure, the value of $C_{p}$ is $0 \cdot 2164$. The variations of $C_{p}$ with temperature and pressure are therefore enormous.

From the value $C_{p}=2 \cdot 1096$, we obtain, by the use of the formula (521)

$$
C_{p}=\frac{1}{2} \frac{R}{J m}(n+5),
$$

* Nuovo Cim. 1894, xxxvr., 1895, r. 
the value $n+3=92$; from the values of $C_{p}, C_{v}$ and $\gamma$ at normal temperature and pressure, we have (p. 222) found the values

$$
n+3=7 \cdot 66, \quad 6 \cdot 60, \quad 6 \cdot 67 \text {. }
$$

We may therefore again, with some show of reason, suppose that if the influence of aggregation were removed the value of $n+3$ would be six, so that the $\mathrm{CO}_{2}$ molecule would be a rigid body without an axis of symmetry.

406. A further instance, as has already been mentioned, is chlorine. The value of $n+3$ calculated for chlorine in the table on p. 222 , was 6.08 , but it is quite conceivable that if we could dispose of molecular aggregation we should find the value $n+3=5$, so that chlorine would fall into line with the other diatomic gases.

407. There is no reason why we should not go further. On the evidence which has been obtained, the supposition that we have

$$
\left\{\begin{array}{l}
n+3=3 \text { for all monatomic gases, } \\
n+3=5 \text { for all diatomic gases, } \\
n+3=6 \text { for all more complex gases, }
\end{array}\right.
$$

seems at any rate to be worth serious consideration. It disposes of the tremendous difficulty of explaining how it is possible for the molecules of a gas to keep up large internal motions without loss of energy, a difficulty which would have to be faced if it were not for the loophole suggested. This difficulty, as we shall see later $(\$ 411)$, seems almost insuperable in the present state of our knowledge of molecular structure.

\section{Dissociation.}

408. So far as the mathematical analysis goes, there is nothing in the preceding treatment to prevent it being applied to dissociation. The former molecules must be replaced by atoms, and the former clusters of molecules by single molecules. Dissociation has been treated on these lines by Boltzmann*, whose analysis is almost identical with that which we have just applied to the question of aggregation. The question has also been treated by Willard Gibbs $†$ and others by a method which, although at first sight appearing very different from that of Boltzmann, will be found, as Boltzmann remarks+, to rest ultimately upon exactly the same physical basis. On the other hand the question has been treated by Prof. J. J. Thomson $\S$ by a method which rests upon a physical basis entirely different from that of Boltzmann and Gibbs.

* Vorlesungen über Gastheorie, II. p. 177.

+ Trans. Connecticut Acad. Irr. (1876), p. 108.

$\ddagger$ Vorlesungen, II. p. 211.

§ Phil. Mag. [5] xvin. p. 233. 
409. Let us consider a gas in which the complete molecules are each composed of two atoms, of types $\alpha, \beta$ respectively.

As in equations (768) the laws of distribution of dissociated atoms and complete molecules are

$$
\begin{aligned}
\phi_{a} & =A e^{-2 h E_{\alpha}}, \\
\phi_{\beta} & =B e^{-2 h E_{\beta}}, \\
\phi_{a \beta} & =A B e^{-2 h\left(E_{\alpha}+E_{\beta}+\Psi\right),}
\end{aligned}
$$

where $\Psi$ is the potential energy of the two atoms forming the molecule. The analysis will be simplified, and the theory sufficiently illustrated, by regarding the atoms as point centres of force, and supposing the masses of the two atoms to be equal, say $m$. Making these simplifications, we find as the laws of distribution of dissociated atoms

$$
\left.\begin{array}{l}
A e^{-h m c^{2}} d u d v d w \\
B e^{-h m c^{2}} d u d v d w
\end{array}\right\}
$$$$
\text { a }
$$

and as the law of distribution of complete molecules

$$
A B e^{-2 h m c^{2}} d u d v d w e^{-\frac{1}{2} h m V^{2}-2 h \Psi} d \alpha d \beta d \gamma 4 \pi r^{2} d r
$$

the law being arrived at in the same way as the law (771).

410. Although the mathematical analysis is similar to that of aggregation there is an important difference in the physical conditions. The law of distribution (777) is limited to values of the variable such that $\frac{1}{2} m V^{2}+2 \Psi$ is negative; as soon as $\frac{1}{2} m V^{2}+2 \Psi$ becomes positive the molecule splits up into its component atoms. Now in the case of molecular aggregation, the attraction between complete molecules is not great, so that $\Psi$ is a small negative quantity, and the range of values for $V$ is correspondingly small. In the case of chemical dissociation $\Psi$ is a large negative quantity, and the range for $V$ is practically unlimited.

An estimate of the value of $\Psi$ can be formed by considering the amount of heat evolved when chemical combination takes place. For instance when 16 grammes of hydrogen combine with 2 grammes of oxygen to form 18 grammes of water the amount of heat developed according to Thomsen's determination, is 68,376 units, - sufficient to raise the temperature of the whole mass of water by $3,600^{\circ} \mathrm{C}$. The value of $V$ necessary for dissociation to occur is therefore comparable with the mean value of $V$ at $3,600^{\circ} \mathrm{C}$, and these high values of $V$ will be very rare in a gas at ordinary temperatures. The exclusion from the law of distribution (777) of high values of $V$ will therefore have but little effect either on the law of distribution or on the energy represented by the internal degrees of freedom, and we may, without serious error, regard the law of distribution as holding for all values of $V$. 
This being so, it appears that the molecule has six degrees of freedomthe three translational degrees represented by the differentials $d u d v d w$, and the three internal degrees represented by the differentials $d a d \beta d \gamma$.

We notice first that $\alpha, \beta, \gamma$, the components of $V$, enter similarly into the law of distribution, so that for the velocity of a single atom relatively to the centre of gravity of a molecule, all directions are equally probable. There is no tendency for the atoms to fall into circular orbits.

We notice next that as there are six degrees of freedom, the value of $\gamma$, even if we neglect potential energy, must be $1 \frac{1}{3}$, and will be less if potential energy is taken into account. As we have seen that for diatomic molecules $\gamma$ is fairly uniformly equal to $1 \frac{2}{5}$, this suggests that we are on the wrong track as regards underlying physical hypotheses.

411. We have made practically no assumptions except that of conservation of energy, so that we are again led to examine what modifications have to be made if dissipation of energy is taken into account.

Since the atoms are known, from the evidence of spectroscopy, to be capable of internal vibration, it seems extremely improbable that two atoms could describe orbits about one another, and therefore exert varying forces on one another, without "forcing" some at least of these vibrations. A rough numerical calculation suggests that these forced vibrations would be of considerable amplitude. The internal kinetic energy of the molecule, $\frac{1}{4} m V^{2}$, can be written in the form

$$
m\left(\dot{r}^{2}+r^{2} \dot{\theta}^{2}+r^{2} \sin ^{2} \theta \dot{\phi}^{2}\right),
$$

where $r, \theta, \phi$ are the polar coordinates of either atom referred to the centre of gravity of the molecule. If there were no dissipation of energy, we should have, from the law of equipartition,

$$
\overline{m \dot{r}^{2}}=R T=\frac{1}{3} m C^{2},
$$

so that $\dot{r}$ is, on the average, comparable with $C$. The value of $r$, however, increases and decreases alternately as the apses of the orbit are described, and for a considerable number of the orbits the range of variation of $r$ will be small compared with the molecular diameter. It follows that the time of oscillation of the value of $r$ is small compared with the time which we took in Chapter IX as the most probable duration of a collision, and hence, by the analysis of Chapter IX, that the interral vibrations forced in the atoms will be large. Thus any initial tendency to oscillations in the value of $r$ will be damped, and the atoms will describe circular orbits about one another.

There are now only five degrees of freedom for a diatomic molecule, and the molecule is dynamically identical with the single rotating body 
of $\S 270$. Indeed the molecule is in every way identical so long as we treat the atoms as points, each having only three degrees of freedom; it is merely a difference of point of view whether we regard the molecule as a single body of which the two components are slightly separated by centrifugal force, or as two bodies describing circular orbits about one another. When we regard the atoms as something more complex than mere points, a distinction has to be drawn between rotation of the atoms as part of the molecule and rotation of the atoms as separate systems. This distinction can hardly be discussed without formulating definite views as to the internal structure of the atom.

Similar remarks apply to molecules which consist of more than two atoms. The condition for no dissipation from the vibrations forced by the mass motion of the atoms is that the relative distance of the atoms shall remain the same, so that the molecule must rotate as a rigid body. If this argument is sound, the molecule must have six degrees of freedom altogether, so that if it were not for the influence of aggregation, etc., we should have to have $\gamma=1 \frac{1}{3}$ for all gases other than monatomic and diatomic, as suggested in $\S 403$.

As soon, however, as we accept the view that dissipation of energy plays a part of the kind explained, in the phenomenon of dissociation and recombination, we have to give up all hope of calculating the degree of dissociation from the principles of the Kinetic Theory. For the factors which are of greatest importance in determining the degree of dissociation will be just those which measure the dissipation of energy, and of these we know nothing. We can only proceed further by introducing some simplifying supposition which is altogether extraneous to the Kinetic Theory, as for instance that of J. J. Thomson (l.c. ante, p. 335) that there is a definite average time during which two or more atoms of known kinds remain in combination. 


\section{CHAPTER XIX.}

\section{THE EVIDENCE OF THE KINETIC THEORY AS TO THE SIZE OF MOLECULES.}

412. WE may appropriately devote a final chapter to collecting and discussing the evidence which has been obtained as to the size of the molecular structure.

Throughout the book we have examined to what extent observations are in agreement with theoretical predictions, calculated on the supposition that the molecules may be treated as hard elastic spheres. We have also had another picture of a molecule before us-namely, a point-centre of force, the law of force between two molecules being that of the inverse fifth power of the distance. Neither of these two representations were supposed to reproduce all the properties of the molecule; we found it necessary continually to bear in mind that they were at best only approximations, and to examine how the imperfections in our representation of a molecule would affect the results arrived at with the help of this representation.

413. The question which we must attempt to answer first is as to which of these two representations gives the better picture of a molecule. In deciding this question the following table will be of use :

\begin{tabular}{|c|l|l|}
\hline Ratio & $\frac{9}{\kappa C_{v}}$ & $\frac{D}{\kappa}$ \\
\hline CALCULATED : & & \\
General Theory ...... & 1 & 1 \\
Elastic Spheres ...... & 1.60 & 1.34 \\
Inverse fifth power... & 2.50 & 1.54 \\
OBSERVED : & & \\
Mean .................... & 1.68 & 1.41 \\
\hline
\end{tabular}

As regards each ratio it will be seen that the best approximations to the observed values are given by the hypothesis of elastic spheres. 
This representation of a molecule has the further advantage that the conceptions of "the diameter of a molecule," "a collision," "a free path," and so on, have definite meanings when associated with hard spherical molecules, but none at all when associated with point-centres of force.

\section{The Size of Molecules, regarded as Elastic Spheres.}

414. We accordingly agree for the present to regard molecules as elastic spheres. Two series of calculations of the radii of these spheres have already been given, the first (p. 141) from observed deviations from Boyle's Law, the second (p. 251) from the observed coefficients of viscosity. To these can of course be added calculations from the observed coefficients of conduction of heat and diffusion. Collected together, the four sets of values are as follows :

VALUES OF $\frac{1}{2} \sigma \times 10^{8}$.

\begin{tabular}{|c|c|c|c|c|c|}
\hline Gas & Boyle's Law & Viscosity & Heat & Diffusion & Mean \\
\hline Hydrogen ............. & 1.025 & 1.024 & 0.995 & $1 \cdot 01$ & 1.01 \\
\hline Helium ................ & - & 0.905 & - & - & $0 \cdot 90$ \\
\hline Water vapour ...... & - & $1 \cdot 695$ & - & - & $1 \cdot 69$ \\
\hline Carbon monoxide... & - & $1 \cdot 448$ & $1 \cdot 37$ & $1 \cdot 46$ & $1 \cdot 43$ \\
\hline Ethylene ............ & - & $1 \cdot 883$ & 1.94 & - & $1 \cdot 91$ \\
\hline Nitrogen ............ & $1 \cdot 56$ & $1 \cdot 448$ & $1 \cdot 37$ & - & $1 \cdot 46$ \\
\hline Air..................... & $1 \cdot 45$ & $1 \cdot 430$ & $1 \cdot 36$ & - & $1 \cdot 41$ \\
\hline Nitric oxide .......... & - & $1 \cdot 412$ & $1 \cdot 41$ & - & $1 \cdot 41$ \\
\hline Oxygen ................ & - & $1 \cdot 405$ & $1 \cdot 29$ & $1 \cdot 35$ & $1 \cdot 35$ \\
\hline Argon ................ & - & $1 \cdot 393$ & - & - & $1 \cdot 39$ \\
\hline Carbon dioxide...... & 1.50 & $1 \cdot 736$ & $1 \cdot 79$ & $1 \cdot 64$ & $1 \cdot 66$ \\
\hline Nitrous oxide ...... & - & $1 \cdot 770$ & $1 \cdot 74$ & - & $1 \cdot 75$ \\
\hline Ethyl chloride ...... & - & $2 \cdot 340$ & - & - & $2 \cdot 34$ \\
\hline Chlorine ............. & - & $2 \cdot 055$ & - & - & $2 \cdot 05$ \\
\hline Mercury vapour*... & - & $1 \cdot 74$ & $1 \cdot 24$ & - & $1 \cdot 49$ \\
\hline
\end{tabular}

415. A further way of forming an estimate of the molecular radius is by considering the density of the substance in the solid state. This way, however, only enables us to calculate an upper limit to the molecular radius.

* The values for mercury vapour refer to $203^{\circ} \mathrm{C}$., this being the lowest temperature at which $\kappa$ has been determined. I have used Koch's value for $\kappa$ at $203^{\circ}(\kappa=\cdot 000395$, Wied. Ann. xIx. p. 857) and Schleiermacher's value for 9 , also at $203^{\circ}(9=0000185$, Wied. Ann. xxxvi. p. 346). Meyer, from a consideration of the experimental difficulties, douits the accuracy of the value of $\kappa$ (Kinetic Theory, pp. 197, 296), and this doubt is confirmed by the facts that the ratio $9 / \kappa C_{v}$ is found to be as great as $3 \cdot 15$, and that the calculated values of $\frac{1}{2} \sigma$ are in disagreement with the upper limit calculated in $\S 415$. 
For instance, Dewar* has found the density of solid hydrogen at $13 \cdot 1^{\circ}$ absolute to be 0763 . The mass of a cubic centimetre of solid hydrogen is accordingly 0763 grammes, while the mass of each molecule (assuming $4 \times 10^{19}$ for Avogadro's number) is known to be $2.25 \times 10^{-24}$ grammes. The number of molecules in a cubic centimetre is accordingly $3.39 \times 10^{22}$. If the molecules are spheres of diameter $\sigma$, these molecules, if packed as closely as possible, would occupy a volume

$$
3.39 \times 10^{22} \times \frac{\sigma^{3}}{\sqrt{2}} .
$$

This volume, then, is certainly less than a cubic centimetre. Or, what comes to the same thing, the value of the molecular radius of hydrogen is certainly less than the value of $\frac{1}{2} \sigma$ which makes the above expression equal to a cubic centimetre. This value is

$$
\frac{1}{2} \sigma=1 \cdot 734 \times 10^{-8} \text {. }
$$

In this way we obtain a superior limit to the value of $\frac{1}{2} \sigma$ for hydrogen. The similar limits for other gases can be obtained in the same way, and we

\begin{tabular}{|c|c|c|c|c|}
\hline Substance & Temp. (C.) & Density & $\begin{array}{l}\text { Upper limit of } \\
\frac{1}{2} \sigma \times 10^{8}\end{array}$ & $\begin{array}{c}\text { Value of } \\
\frac{1}{2} \sigma \times 10^{8}(\mathrm{p} .340)\end{array}$ \\
\hline Hydrogen * ................... & $-259 \cdot 9^{\circ}$ & $0 \cdot 0763$ & $1 \cdot 73$ & $1 \cdot 01$ \\
\hline Water $($ liquid $)+\ldots \ldots \ldots \ldots . . . .$. & $4 \cdot 0$ & $1 \cdot 0000$ & $1 \cdot 52$ & $1 \cdot 69$ \\
\hline Ethylene (liquid) $\ddagger \ldots . . . . . . . .$. & $-21 \cdot 0$ & $0 \cdot 414$ & $2 \cdot 36$ & $1 \cdot 91$ \\
\hline Nitrogen $* \ldots \ldots \ldots \ldots \ldots \ldots \ldots$ & $-253 \cdot 0$ & $1 \cdot 0265$ & $1 \cdot 75$ & $1 \cdot 46$ \\
\hline Oxygen ${ }^{*} . . . \ldots \ldots \ldots \ldots \ldots \ldots$ & $-253 \cdot 0$ & $1 \cdot 4256$ & $1 \cdot 64$ & $1 \cdot 35$ \\
\hline Argon $\S \ldots \ldots \ldots \ldots \ldots \ldots \ldots \ldots$ & $-187 \cdot 0$ & $1 \cdot 396$ & $1 \cdot 78$ & $1 \cdot 39$ \\
\hline Carbon dioxide $($ liquid $) \ddagger \ldots$ & $-34 \cdot 0$ & $1 \cdot 057$ & $2 \cdot 00$ & $1 \cdot 66$ \\
\hline Nitrous oxide $($ liquid $) \ddagger \ldots . .$. & $-20 \cdot 6$ & $1 \cdot 003$ & $2 \cdot 04$ & $1 \cdot 75$ \\
\hline Ethyl chloride $($ liquid) $\| \quad \ldots$ & 0.0 & 0.9253 & $2 \cdot 34$ & $2 \cdot 34$ \\
\hline Chlorine (liquid) $\Psi$............ & $-80 \cdot 0$ & $1 \cdot 6602$ & $2 \cdot 03$ & $2 \cdot 05$ \\
\hline Mercury** ...................... & $-40 \cdot 0$ & $14: 39$ & $1 \cdot 39$ & $1 \cdot 49+t$ \\
\hline
\end{tabular}
arrive at the following table:

416. Considering the general crudeness of the supposition upon which we have been working, the comparative agreement of these two sets of figures cannot be regarded as otherwise than satisfactory. In regarding all

* Dewar, Royal Soc. Proc., Lxxir. p. 251.

+ The density is taken at the point of maximum density in the liquid state, this being greater than the density in the solid state.

‡ Cailletet and Mathias, Jour. de Phys. 1886, v. p. 555.

§ Ramsay, see Lord Kelvin in Phil. Mag. Iv. p. 197.

\|l Meyer, Kinetic Theory, p. 319.

T. Knietsch, Ann. Chem. 1890, ccuIx. p. 100.

** Mendeleef, Chemistry, p. 47 (English Trans.). t† At $203^{\circ} \mathrm{C}$. 
molecules, regardless of their true shape, as spherical, we have run the risk of an error in $\frac{1}{2} \sigma$ comparable with the value of the quantity itself. That discrepancies of this magnitude occur in the two sets of figures cannot, therefore, be regarded as a matter for surprise. At the same time it is worth trying whether the errors themselves cannot be reduced to some kind of order.

417. A first possible cause, which has probably already occurred to the reader, is the variation of $\sigma$ with the temperature. We have already seen that $\sigma$ must be supposed to depend on the temperature of the gas. Now the last column of the above table gives the value of $\frac{1}{2} \sigma$ at $0^{\circ} \mathrm{C}$., while the preceding column gives the value at temperatures which vary from $-260^{\circ} \mathrm{C}$. to $4^{\circ} \mathrm{C}$.

If we assume the empirical formula by which $\kappa$ (the coefficient of viscosity) varies as $T^{n}$, we have seen that $\sigma$ must be supposed to vary as $T^{-\frac{1}{2}\left(n-\frac{1}{2}\right)}$. Values of $n$ for a number of gases are given on p. 257, and with the help of these we can reduce the values of $\frac{1}{2} \sigma$ for the corresponding gases to the uniform temperature of $0^{\circ} \mathrm{C}$. Corrected in this way, the numbers stand as follows:

\begin{tabular}{|c|c|c|}
\hline Substance & $\begin{array}{c}\text { Upper limit } \\
\text { at } 0^{\circ} \mathrm{C} .\end{array}$ & $\begin{array}{l}\text { Mean value } \\
\text { at } 0^{\circ} \mathrm{C} \text {. }\end{array}$ \\
\hline Hydrogen ....... & $1 \cdot 29$ & 1.01 \\
\hline Water*.......... & $1 \cdot 52$ & $1 \cdot 69$ \\
\hline Ethylene ........ & $2 \cdot 32$ & 1.91 \\
\hline Nitrogen ....... & $1 \cdot 31$ & $1 \cdot 46$ \\
\hline Oxygen .......... & $1 \cdot 23$ & $1 \cdot 35$ \\
\hline Carbon dioxide & $1 \cdot 95$ & $1 \cdot 66$ \\
\hline Nitrous oxide & $2 \cdot 02$ & $1 \cdot 75$. \\
\hline Ethyl chloride. & $2 \cdot 34$ & $2 \cdot 34$ \\
\hline Mercury †..... & $1 \cdot 29$ & $1.49 \ddagger$ \\
\hline
\end{tabular}

From these figures it is clear that the agreement is greatly improved by the temperature-correction, all gases except mercury now shewing almost as good an agreement as could be expected. It must, however, be remarked that the temperature-corrections have been based on an empirical formula obtained from the variation of $\kappa$ at temperatures above $0^{\circ} \mathrm{C}$. We are therefore on doubtful ground in extending it to temperatures as low as $-260^{\circ} \mathrm{C}$.

418. A large number of the corrected upper limits, it will be noticed, are below the corresponding mean value. This, however, is not so incomprehensible as might at first be thought. Owing to the forces of cohesion, the

* The difference between $4^{\circ} \mathrm{C}$. and $0^{\circ} \mathrm{C}$. is neglected.

$\dagger$ Assuming the somewhat doubtful value $n=1 \cdot 5$.

$\ddagger$ At $203^{\circ} \mathrm{C}$. 
molecules inside a solid or liquid are subjected to a pressure of which the amount, measured by ordinary standards, seems enormous. The molecules, then, may be said to be pressed together by one another's attraction, and so to occupy less space in the solid or liquid state than they otherwise would.

To form a numerical estimate of the amount of this compression, let us consider the process of changing water initially at $0^{\circ}$ into steam, the pressure being atmospheric pressure throughout. To raise the water to boiling point, 100 units of heat are required per gramme. This heat does not change the density of the water to any great extent, so that only a small fraction of the 100 units will be spent in doing work against the intermolecular forces. To change the water at $100^{\circ}$ into steam at $100^{\circ}$, we now require 537 units of heat per gramme. Of this heat, part is spent in doing external work, and part in overcoming the attractive forces between the molecules. The former part can easily be calculated. A gramme of steam at $100^{\circ}$ occupies about $1647 \mathrm{cu}$. cms., so that the external work done per gramme of steam is equal to the product of the atmospheric pressure by 1646. In C.G.S. work-units this is found to be

$$
1 \cdot 668 \times 10^{9} \text { C.G.S. work-units. }
$$

Dividing by $4 \cdot 22 \times 10^{7}$ to reduce to heat-units, we obtain

\section{$39 \cdot 52$ c.G.s. heat-units.}

Hence of the 537 heat-units per gramme required, about 40 are spent in external work, and therefore about 497 in overcoming intermolecular attractions. This is equal to $2.1 \times 10^{10}$ work-units, and therefore if spent in increasing the translational energy of the molecules, would be equivalent to a rise of temperature of

$$
\frac{2}{3} \frac{2 \cdot 1 \times 10^{10}}{R / m}
$$

or about $3000^{\circ} \mathrm{C}$. It must, however, be borne in mind that in a liquid each molecule is "compressed" at more than one point of its surface. If, as a rough guess, we assume contact at ten points, the rise of temperature just calculated must be divided by ten. The "compression" of molecules in wate $i$ is therefore about equal to that of molecules in collision at a temperature of about $300^{\circ} \mathrm{C}$.

Other substances might be similarly treated. It would be absurd to apply the empirical temperature-correction over so large a range of temperature, and under such different physical conditions, but it is obvious that the difference between the two values for $\frac{1}{2} \sigma$ is sufficiently explained. As regards order of magnitude of the correction, an examination of the table on p. 256 will be of use.

419. There is a further consideration which is capable of reconciling to some extent the discrepancies between the two sets of figures given on p. 341. If we arrange the substances there given in the order of the 
differences, measured algebraically, between the two sets of figures, we find that the order is roughly that of the boiling points of the substances.

\begin{tabular}{|c|c|c|}
\hline Substance & $\begin{array}{c}\text { Difference } \\
\text { per cent. }\end{array}$ & $\begin{array}{c}\text { Boiling Point } \\
\text { abs. }\end{array}$ \\
\cline { 2 - 3 } Hydrogen ........... & +71 & 20 \\
Argon ............. & +27 & 87 \\
Carbon dioxide...... & +26 & 193 \\
Ethylene ........... & +24 & $169 \cdot 5$ \\
Oxygen ............. & +21 & $90 \cdot 5$ \\
Nitrogen ........... & +20 & $77 \cdot 5$ \\
Nitrous oxide ...... & +17 & $183 \cdot 2$ \\
Ethyl chloride...... & 0 & $285 \cdot 5$ \\
Chlorine ............ & -1 & $239 \cdot 6$ \\
Water .............. & -10 & 373 \\
Mercury ........... & - & 630 \\
\hline
\end{tabular}

This circumstance suggests that as regards the gases which occur near the bottom of the table, a considerable error may have been introduced into the determination of $\frac{1}{2} \sigma$, by the neglect of molecular aggregation.

This consideration is of course by no means independent of that of the last section. The two considerations together seem to suggest that the determination of $\frac{1}{2} \sigma$ for some of the vapours tabulated, and for mercury in particular, is almost worthless except as regards order of magnitude.

420. Finally mention must be made of an interesting determination of the size of molecules which does not depend upon the Kinetic Theory at all. Regarding molecules as spheres which are perfect conductors of electricity*, the dielectric capacity $K$ of a medium containing $N$ molecules per unit volume is found to be given by

$$
\frac{K-1}{K+2}=\frac{4}{3} \pi N\left(\frac{\sigma}{2}\right)^{3}
$$

For a gas $K$ will be very nearly equal to unity, so that the relation becomes

$$
K-1=\frac{1}{2} \pi N \sigma^{3} \text {. }
$$

It is therefore possible to calculate $\sigma$ from values of $K$. On Maxwell's Theory $K$ is equal to $\mu^{2}$ where $\mu$ is the refractive index for light of infinite wave-length, so that the foregoing equation becomes

$$
\mu-1=\frac{1}{4} \pi N \sigma^{3} \text {. }
$$

It is difficult to calculate either $\mu$ or $K$ directly, but values of $\mu$ for different substances can be compared with great accuracy by an interferencemethod. In the following table, many of the values are calculated in this

\footnotetext{
* Mossotti's Hypothesis: see Maxwell, Elect. and Mag. p. 70 (Third Edn.).
} 
way, assuming for $(\mu-1)_{\text {arr }}$ the value 1.000294. These ratios of values of $(\mu-1)$ are taken from Travers' Study of Gases, p. 296. The direct values used are taken from Preston's Light, p. 137.

\begin{tabular}{|c|c|c|}
\hline Gas & $(\mu-1) \times 10^{6}$ & $\frac{1}{2} \sigma \times 10^{8}$ (p. 340) \\
\hline Helium ................ & 35 & 0.90 \\
\hline Hydrogen............ & 139 & $1 \cdot 01$ \\
\hline Oxygen ................. & 273 & $1 \cdot 35$ \\
\hline 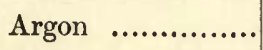 & 284 & $1 \cdot 39$ \\
\hline Air.................... & 294 & $1 \cdot 41$ \\
\hline Nitrogen ........... & 298 & $1 \cdot 46$ \\
\hline Nitric oxide ......... & 303 & $1 \cdot 41$ \\
\hline Carbon monoxide... & 340 & $1 \cdot 43$ \\
\hline Carbon dioxide...... & 449 & $1 \cdot 66$ \\
\hline Nitrous oxide ...... & 503 & $1 \cdot 75$ \\
\hline Ethylene ............ & 678 & $1 \cdot 91$ \\
\hline Chlorine ............ & 772 & $2 \cdot 05$ \\
\hline Ethyl chloride...... & 1095 & $2 \cdot 34$ \\
\hline
\end{tabular}

The arrangement, it will be seen, is that of order of magnitude of $(\mu-1)$. The order is also, with one slight exception, that of order of magnitude of $\frac{1}{2} \sigma$. The agreement of these two orders gives a sufficient indication of the general accuracy of the values obtained for $\frac{1}{2} \sigma$.

The absolute values obtained are not so satisfactory, but this is hardly surprising. For instance, from the value $(\mu-1)_{\text {air }}=000294$ we obtain the absolute value

$$
\frac{1}{2} \sigma=1.054 \times 10^{-8}
$$

of which the agreement with the value

$$
\frac{1}{2} \sigma=1 \cdot 41 \times 10^{-8}
$$

could not be considered satisfactory if it were not for the large number of inaccuracies which have necessarily been introduced in regarding the molecules as elastic spheres. It should be noticed that this is the first occasion on which we have been dealing with the actual matter of the molecule and not with its field of force.

Perhaps, then, we may see in the difference between the two values of $\frac{1}{2} \sigma$ some indication of the extension of the field of force surrounding the molecules. If so, regarding $\sigma$ as variable with the temperature, the values of $\sigma$ calculated from $\mu-1$ must be the limiting values of $\sigma$ when $T=\infty$. Looked at from this point of view, the value $\frac{1}{2} \sigma=1.054 \times 10^{-8}$ is in tolerably good agreement with the figures tabulated on p. 256 . 


\section{APPENDIX A.}

\section{INTEGRALS INVOLVING EXPONENTIALS.}

The type of integral which occurs most frequently in the mathematics necessary to the Kinetic Theory is

$$
\int u^{n} e^{-\lambda u^{2}} d u
$$

where $n$ is integral. This can be evaluated in finite terms when $n$ is odd, and can be made to depend on the integral

$$
\int_{0}^{u} e^{-\lambda u^{2}} d u
$$

when $n$ is even. In each case the reduction is most quickly performed by successive integrations by parts with respect to $u^{2}$. Tables for the evaluation of the integral (ii) will be found in Appendix B.

When, as is generally the case, the limits of integration are from $u=0$ to $u=\infty$, the results of integration are expressed by the formulae

$$
\begin{gathered}
\int_{0}^{\infty} u^{2 \kappa} e^{-\lambda u^{2}} d u=\frac{1.3 \ldots(2 \kappa-1)}{2^{\kappa+1}} \sqrt{\frac{\pi}{\lambda^{2 \kappa+1}}}, \\
\int_{0}^{\infty} u^{2 \kappa+1} e^{-\lambda u^{2}} d u=\frac{\kappa !}{2 \lambda^{\kappa+1}} .
\end{gathered}
$$

The following cases of the general formulae are of such frequent occurrence that it may be useful to give the results separately:

$$
\begin{aligned}
& \int_{0}^{\infty} e^{-h m u^{2}} d u=\frac{1}{2} \sqrt{\frac{\pi}{h m}}, \\
& \int_{0}^{\infty} e^{-h m u^{2}} u d u=\frac{1}{2 h m}, \\
& \int_{0}^{\infty} e^{-h m u^{2}} u^{2} d u=\frac{1}{4} \sqrt{\frac{\pi}{h^{3} m^{3}}}, \\
& \int_{0}^{\infty} e^{-h m u^{2}} u^{3} d u=\frac{1}{2 h^{2} m^{2}}, \\
& \int_{0}^{\infty} e^{-h m u^{2}} u^{4} d u=\frac{3}{8} \sqrt{\frac{\pi}{h^{5} m^{5}}}, \\
& \int_{0}^{\infty} e^{-h m u^{2}} u^{5} d u=\frac{1}{h^{3} m^{3}}, \\
& \int_{0}^{\infty} e^{-h m u^{2}} u^{6} d u=\frac{15}{16} \sqrt{\frac{\pi}{h^{7} m^{7}}} .
\end{aligned}
$$

Each integral can be obtained by differentiating that next but one before it with respect to $\mathrm{hm}$. In this way the system can be extended indefinitely. 


\section{APPENDIX B.}

THE following tables will be found of use for numerical calculations in connection with the Kinetic Theory. The values of $\psi(x)$ are from a table by Tait in the paper already referred to (p. 232).

\begin{tabular}{|c|c|c|c|c|}
\hline$x$ & $x^{2}$ & $e^{-x^{2}}$ & $\frac{2}{\sqrt{\pi}} \int_{0}^{x} e^{-x^{2}} d x$ & $\begin{array}{c}\psi(x) \\
\text { Defined by equation } \\
(534), \text { p. } 231\end{array}$ \\
\hline $\begin{array}{l}\cdot 1 \\
\cdot 2 \\
\cdot 3 \\
\cdot 4 \\
\cdot 5\end{array}$ & $\begin{array}{l}\cdot 01 \\
\cdot 04 \\
\cdot 09 \\
\cdot 16 \\
\cdot 25\end{array}$ & $\begin{array}{l}.99905 \\
.96080 \\
.91393 \\
.85214 \\
\cdot 77880\end{array}$ & $\begin{array}{l}\cdot 11246 \\
\cdot 22270 \\
\cdot 32863 \\
\cdot 42839 \\
\cdot 52050\end{array}$ & $\begin{array}{r}.20066 \\
.40531 \\
.61784 \\
.84200 \\
1.08132\end{array}$ \\
\hline $\begin{array}{r}\cdot 6 \\
\cdot 7 \\
\cdot 8 \\
\cdot 9 \\
1 \cdot 0\end{array}$ & $\begin{array}{r}\cdot 36 \\
\cdot 49 \\
\cdot 64 \\
\cdot 81 \\
1 \cdot 00\end{array}$ & $\begin{array}{l}\cdot 69768 \\
\cdot 61263 \\
\cdot 52729 \\
\cdot 44486 \\
\cdot 36788\end{array}$ & $\begin{array}{l}\cdot 60386 \\
.67780 \\
.74210 \\
.79691 \\
.84270\end{array}$ & $\begin{array}{l}1 \cdot 33907 \\
1 \cdot 61819 \\
1 \cdot 92132 \\
2 \cdot 25072 \\
2 \cdot 60835\end{array}$ \\
\hline $\begin{array}{l}1 \cdot 1 \\
1 \cdot 2 \\
1 \cdot 3 \\
1 \cdot 4 \\
1 \cdot 5\end{array}$ & $\begin{array}{l}1 \cdot 21 \\
1 \cdot 44 \\
1 \cdot 69 \\
1 \cdot 96 \\
2 \cdot 25\end{array}$ & $\begin{array}{l}\cdot 29820 \\
\cdot 23693 \\
\cdot 18452 \\
\cdot 14086 \\
\cdot 10540\end{array}$ & $\begin{array}{l}\cdot 88021 \\
\cdot 91031 \\
.93401 \\
.95229 \\
\cdot 96611\end{array}$ & $\begin{array}{l}2 \cdot 99582 \\
3 \cdot 41448 \\
3 \cdot 86538 \\
4 \cdot 34939 \\
4 \cdot 86713\end{array}$ \\
\hline $\begin{array}{l}1 \cdot 6 \\
1 \cdot 7 \\
1 \cdot 8 \\
1 \cdot 9 \\
2 \cdot 0\end{array}$ & $\begin{array}{l}2 \cdot 56 \\
2 \cdot 89 \\
3 \cdot 24 \\
3 \cdot 61 \\
4 \cdot 00\end{array}$ & $\begin{array}{l}\cdot 07730 \\
\cdot 05558 \\
.03916 \\
\cdot 02705 \\
\cdot 01832\end{array}$ & $\begin{array}{l}.97635 \\
.98379 \\
\cdot 98909 \\
.99279 \\
.99532\end{array}$ & $\begin{array}{l}\mathbf{5 \cdot 4 1 9 1 1} \\
6 \cdot 00570 \\
6 \cdot 62715 \\
7 \cdot 28366 \\
7 \cdot 97536\end{array}$ \\
\hline $\begin{array}{l}2 \cdot 1 \\
2 \cdot 2 \\
2 \cdot 3 \\
2 \cdot 4 \\
2 \cdot 5\end{array}$ & $\begin{array}{l}4 \cdot 41 \\
4 \cdot 84 \\
5 \cdot 29 \\
5 \cdot 76 \\
6 \cdot 25\end{array}$ & $\begin{array}{l}\cdot 01215 \\
\cdot 00791 \\
\cdot 00504 \\
\cdot 00315 \\
\cdot 00197\end{array}$ & $\begin{array}{l}\cdot 99702 \\
\cdot 99814 \\
\cdot 99886 \\
\cdot 99931 \\
\cdot 99959\end{array}$ & $\begin{array}{r}8 \cdot 70234 \\
9 \cdot 46467 \\
10 \cdot 26236 \\
11 \cdot 09547 \\
11 \cdot 96402\end{array}$ \\
\hline $\begin{array}{l}2 \cdot 6 \\
2 \cdot 7 \\
2 \cdot 8 \\
2 \cdot 9 \\
3 \cdot 0\end{array}$ & $\begin{array}{l}6 \cdot 76 \\
7 \cdot 29 \\
7 \cdot 84 \\
8 \cdot 41 \\
9 \cdot 00\end{array}$ & $\begin{array}{l}\cdot 00116 \\
.00068 \\
.00039 \\
\cdot 00022 \\
\cdot 00012\end{array}$ & $\begin{array}{l}\cdot 99976 \\
.99987 \\
.99992 \\
\cdot 99996 \\
.99998\end{array}$ & $\begin{array}{l}12 \cdot 86798 \\
13 \cdot 80734 \\
14 \cdot 78225 \\
15 \cdot 79255 \\
16 \cdot 83830\end{array}$ \\
\hline
\end{tabular}




\title{
INDEX OF SUBJECTS.
}

\author{
The numbers refer to pages.
}

A. Assumption A (of Burbury), see Molecular chaos

Adiabatic motion of a gas, 171, 282

" equilibrium of atmosphere, 310

Aerostatics, 309

Aggregation of molecules, 326

Arc, emission of light by electric, 208, 209

Assumption A (of Burbury), see Molecular chaos

, of molecular chaos, see Molecular chaos

Atmosphere, constitution of earth's, 315

, dissipation of earth's, 318

, general theory of planetary, 309

Avogadro, constant of, 6

" law of, 111,120 ; deviations from law, 120,331

Boiling point, theory of, 132,330

, numerical values, 344

Boundary, effect of boundary on law of distribution, 25

, pressure at boundary influenced by cohesion, 117, 125

Boyle's Law, 107, 112, 145

" , deviations from 121, 331

Calorimetry of a conservative gas, 169

$" \quad$ of a non-conservative gas, 210

" of a gas in a state of aggregation, 333

Carbon-dioxide Isothermals, 134

" Specific heats, 334

Carnot's Law $\left(C_{p}-C_{v}\right), 170$

Chaos, molecular, see Molecular chaos

Charles' Law, 112

Chemical affinity, magnitude of, 336

Chlorine, variation of density of, 333

Cohesion in gases, 64, 326, 332

, Van der Waals' treatment of, 117, 124, 332

, in water, magnitude of forces of, 343
Collisions between elastic spheres, 15

" between loaded spheres, 96

, between diatomic molecules, 307

" between molecules with law $\mu / r^{5}, 284$

" total number of, 31

, number with given relative velocity, 31

", frequency of, in mixture of gases, 229

" arbitrary standard in definition of, 61,288

" arbitrariness corrected by "persist. ence of velocities," 241

Conduction of Heat, see Heat

Conductive equilibrium in atmosphere, 309, 312

Continuity of path. Maxwell's assumption, 82 ; relation to assumption of molecular chaos, 91

, of state, $114,131,329$

" hydrodynamical equation of, 151

Convection of Heat, see Heat

Convective equilibrium of atmosphere, 310

Correlation between velocity and positional coordinates:

" Absence of correlation assumed in statistical method, 52

" Result disputed by Burbury, 52

" Proof of result, 58

" between components of velocity:

" Absence of correlation assumed by Maxwell, 51

" Proof of result, 28 period of light emitted, 207

Critical point, 141,330 .

, temperature, 131, 141, 331

Dalton's Law-Pressure in a mixture of gases, 108

deviations from, 121

", -Planetary atmospheres, 310 
Degrees of freedom of a molecule, 172, 221, 335,337

Demon, sorting demon of Maxwell, 168

Density, definition, 11, 46

, distribution in field of force, 70, 157

" influenced by aggregation, 331

" effective molecular, see Effective

Diameter of molecules:

, ,, apparent variation with temperature, $254,275,342$

, , determination of numerical values, 339

Diatomic gases, 223, 307, 337

Diffusion of gases, $5,268,298$

, experimental values, 273

Dissipation of énergy, 173, 176, 197, 337

, function for a gas, 176

, of planetary atmospheres, 309

Dissociation, 335

Earth's atmosphere, constitution of, 315, 316

$" \quad$ " equilibrium of, 310

$" \quad$ " dissipation of, 318

Effective molecular density, 58

" , with reference to intermolecular forces, 72

, , calculation of, 122

Electric arc, light of, 208, 209

" vacuum tube, light of, 209

Energy, equipartition of, see Equipartition

, internal, of a molecule, $65,172,337$

" transfer of, by collisions, 197

,. transfer of, by molecular motion, 301

Entropy, 165

Equilibrium of atmosphere, 309

, of gas in field of force, 156

, of vapour in contact with liquid,

$$
3,131,330
$$

Equipartition of energy, 67

" , enunciated by Waterston, 79

, , Maxwell's treatment of, 79

" ", modified by interaction with ether, 173,176

Escape of gases from the atmosphere, 318

Expansive property of a gas, 3, 145

Force, intermolecular, see Intermolecular

Free-paths, total number described, 31

, average duration of, 31

" mean length of, 31, 232, 233

Friction, Kinetic Theory interpretation of, 3, 248

Generalised space, 33
H-theorem, 19, 32 ; second proof, 88

" analysis of theorem, 59

, relation to theorem of increasing entropy, 165

Heat, conduction of, 259, 297; experimental values, 267

" convection of, 264

Historical notes:

General Theory, 9

Proofs of Law of Distribution, 51

Proofs of Law of Equipartition, 79

Partition of Energy in Loaded Spheres, 104

Planetary Atmospheres, 325

Hydrodynamical equations of a gas, 149

Impact of masses, interpreted by Kinetic Theory, 2,29

Incandescence, temperature of, 207 ; of gases, 208

Integrating factors of energy-equation, 163

Intermolecular force in gases, 64, 73, 153, 326, 345

, $\quad$, in liquids and solids, 343

" " law of force, 256, 275, 326

Intermolecular pressure, $160,162,343$

Internal pressure in a gas, $160,162,332$

" energy of a molecule, $65,172,337$

Irreversibility of motion, apparent, 32, 59, 165

Isopentane, critical isothermal of, 135

Isothermals, 127

Isothermal equilibrium of atmosphere, 309, 312

Latent heat, 343

Law of distribution, definition, 13

", , statistical method, 12

", method of general dynamics, 33

" " comparison of the two methods, 53

" , extension to complex molecules, 61

", "Maxwell's original proof, 51

Light, emitted by heated gases, 208 ; by electrio action, 208

Liouville's Theorem, 62; modified for nonconservative system, 178

Liquid in contact with vapour, $3,131,330$

Liquid state, continuity with gaseous state, 131, 329

Loaded spheres, see Sphere

Mass and Molecular motion, 27, 247

Mass motion of a gas, equations of, 149

Masses of molecules, 7 
Matter, three states of, 2

Maxwell's Law of Distribution, see Law

Mean free path, see Free-paths

Mechanical llustrations:

General Theory, 4

Equipartition of energy, 92

Equalisation of temperature, 4, 110, 111

Dissipation of Energy, 174, 190

Partition of energy in non-conservative gas, 192

Molecular action, sphere of, see Sphere

Molecular chaos, assumption of, 14

, , analysis of assumption, 54

" " justification when molecules are infinitely small, 57

" " modification when molecnles are of finite size, 58

" " relation of assumption to "continuity of path," 91

Molecules, mass of, 7

, number per c.c., 6

" size of, see Size

" velocity of, 113

Momentolds, definition of, 68

, partition of energy between, 69

, number of, 172

Monatomic gases, 172, 223

Non-conservative gas, general theory, 176

Normal state, 49 ; for non-conservative gas, 190, 211

Number of molecules per c.c., 6

Persistence of velocities after collision, 236

, , effect on coefficients of viscosity, 249

, " effect on conduction of heat, 267

," , effect on diffusion, 273

Planetary atmospheres, 309

Pressure in a gas, 105, 114, 119, 158, 331, 332

, calculated by the Virial, 143

, in a mixture of gases, 107

, of vapour in equilibrium with liquid,

$$
3,131,330
$$

, of radiation, 212

Pressure-coefflcient of a gas, 138

Probability, use of calculus of, 13,53

Propagation of sound, see Sound

Radiation of gases, 208

" caused by electrical or chemical action, 209

" pressure of, 212

Rate of dissipation of translational energy, 197

, " of planetary atmospheres, 318
Ratio of Specific Heats, of a conservative gas, 171

, , , $\quad$ of a non-conservative gas, 219

experimental values, 220,221

gas in state of aggregation, 334

Refractive index in gases, 345

" $\quad$ size of molecules determined by, 345

Relaxation, time of, 294, 297

Rotating gas, 67, 71

Rotational energy of a molecule, 92, 192, 226

$$
\text { , } \quad \text {, transfer of, } 303
$$

Size of molecules, 339

" , determined from deviations from Boyle's Law, 139

, , determined from viscosity, 251

" , determined from coefficients of conduction and diffusion, 340

, , determined from densities of liquids and solids, 341

", determined from specific inductive capacity, 344

" , apparent variation with temperature, 254,342

Sorting demon of Maxwell, 168

Sound, propagation of, 228, 302

"Special state" of Schuster and Hemsalech, 207

Specific Heats of conservative gas, 169

, " of non-conservative gas, 215

" $\quad$ of gas in state of aggregation, 333

" $\quad$ experimental values, 217, 218, 334

Specific Inductive Capacity of gas, 344

Spectroscopy, 172

sphere of molecular action, 72

" loaded, as illustrative type of molecule:

" $\quad$ " equipartition, conservative gas, 92

" " partition of energy, non-conservative gas, 192

, " propagation of sound, 302

state, change of, 2

, continuity of, 114, 131, 329

Statistical mechanics, 91, 187

Statistical method, 11

" , imperfections of, 12, 91, 165

Steam, wet and dry, 334 
Subsidlary temperatures, definition of, 188

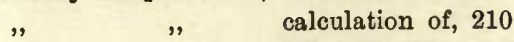

" , mechanical illustra-

Super-cooled vapour, 132

Super-heated liquid, 132

Temperature, definition of, 108

,$\quad$ equalisation of, $5,109,166$

" thermodynamic scale of, 165

" principal and subsidiary, 188 (see also Subsidiary temperatures)

, gradient in atmosphere, 312

Thermodynamics, 162

Thermometry' (gas), 138

Transfer of energy by collisions, 197

, of energy by molecular motion, 301

, general equations of, $276,280,301$

Transition from liquid to gaseous states, 114, 131, 329
Vacuum-tube, free path in, 8

$$
\text { , light emitted by, } 209
$$

Van der Waals' equation, 115, 136, 148

, " treatment of cohesion, 117, 124, 332

Vapour, 3, 131, 329

Velocity of molecules, numerical values, 113 , of sound, 307

Vibrational energy of molecule, 172,337

$$
\text { , } \quad \text { rate of increase caused by }
$$

Virial, 143

Viscosity, 29, 242, 295; experimental values, 251

" variation with density, 252, 334; with temperature, 254, 275

Volume-coefficient of a gas, 139

Wave-length of light, correlation with velocity of molecule, 207

\section{INDEX OF NAMES.}

\section{Andrews, 133}

Barus, 256

Bernoulli, Daniel, 10

Berthelot, 142

Boltzmann, 10, 11, 51, 110, 115, 168, 246, 249, 275,335

Bryan, 84, 110, 168, 314, 325

Burbury, 10, 12, 52, 104

Burnside, 104

Cailletet, 341

Callendar, 140

Capstick, 220, 221, 224, 225

Clausius, 10, 132, 143

Conrau, 265

Dalton, 108, 310

Democritus, 9

Dewar, 312, 315, 341

Dulong, 220, 221

Epicurus, 9

Frankland, 311
Gassendi, 9

Gibbs, J. Willard, 167, 168, 335

Graetz, 267

Graham, 252

Hemsalech, 207

Herapath, 10

Holman, 256

Hooke, 10

Johnstone stoney, 325

Joule, 10, 64

Kaysir, 220

Kelvin, Lord, 6, 64, 166, 274

Kirchhoff, 10, 52, 275

Knietsch, 341

Koch, 340

Krönig, 10

Kundt, 221, 252

Larmor, 168

Lasswitz, 9

Leduc, 220, 227

Leucippus, 9 
Liouville, 62

Lorentz, 10, 51

Loschmidt, 274

Lucchi, 220

Lucretius, 9

Lummer, 219

Lussana, 334

Martini, 221

Masson, 220, 221

Mathias, 341

Maxwell, 10, 11, 51, 79, 109, 132, 145, 168, $233,238,252,275,276$

Mendeleef, 139, 341

Meyer, 52, 219, 235, 252, 253, 255, 269

Moissan, 315

Müller, 220, 221

Naumann, 224

Neugebauer, 266

Obermeyer, 252

Paschen, 208

Pirogoff, 52

Pringsheim, 208, 219

Ramsay, w., 142, 221, 315, 341
Rayleigh, Lord, 10, 79, 82, 84, 221, 252, 258

Regnault, 138, 139

Roche, 314

Röntgen, 220

Rose-Innes, 140

Schleiermacher, 340

Schultze, 258

Schuster, 207

Smithells, 208

stoney, 325

Strecker, 221, 225

Tait, 10, 32, 232, 249

Thomson, J. J., 6, 110, 224, 335

Townsend, 6

Van der Waals, 10, 114, 140

warburg, 221, 252

Waterston, 10, 79

Wiedemann, 218

Wilson, H. A., 6

Winkelmann, 267

Wüllner, 218, 220, 221, 267

Young, s., 135, 142 



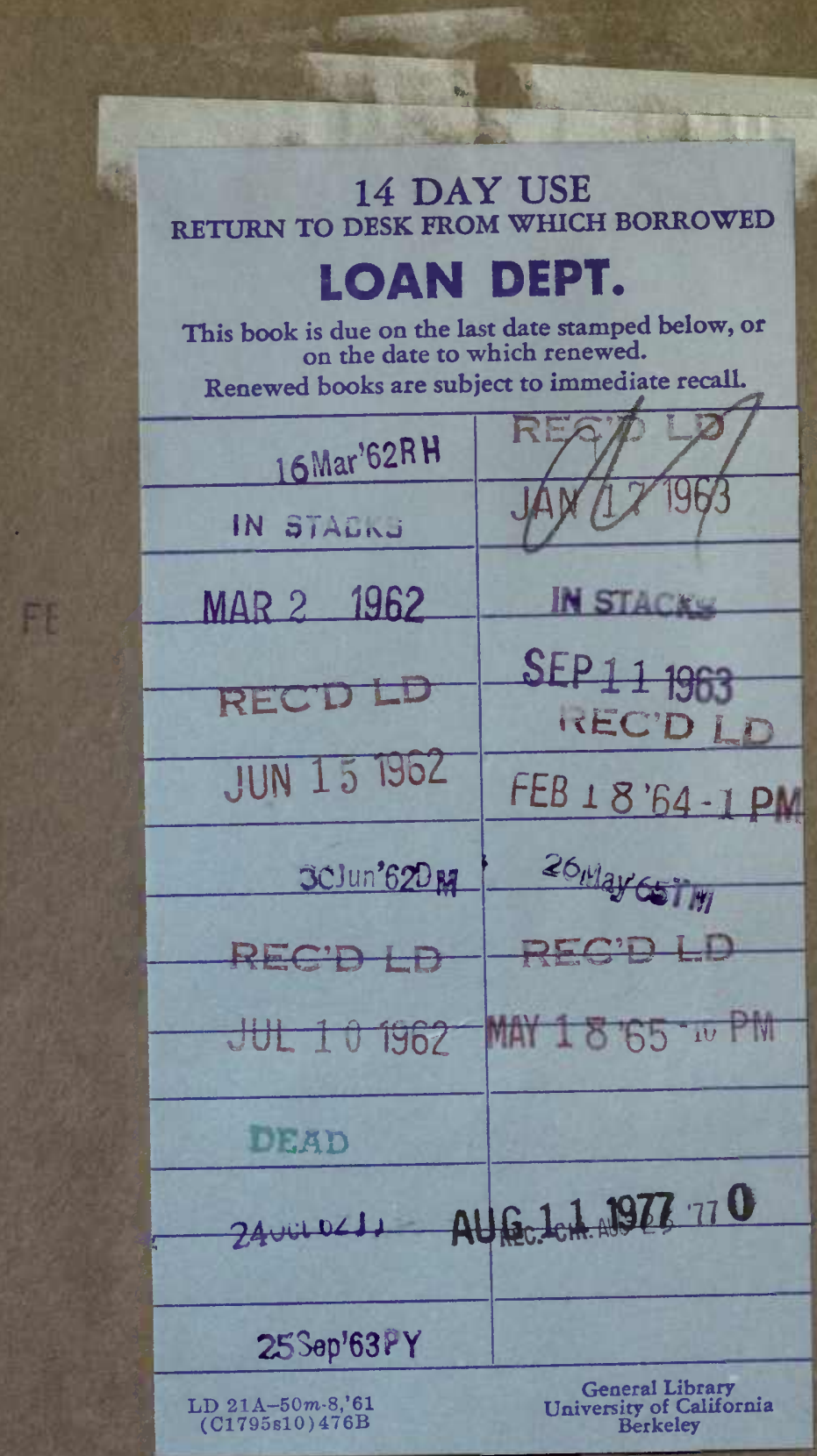



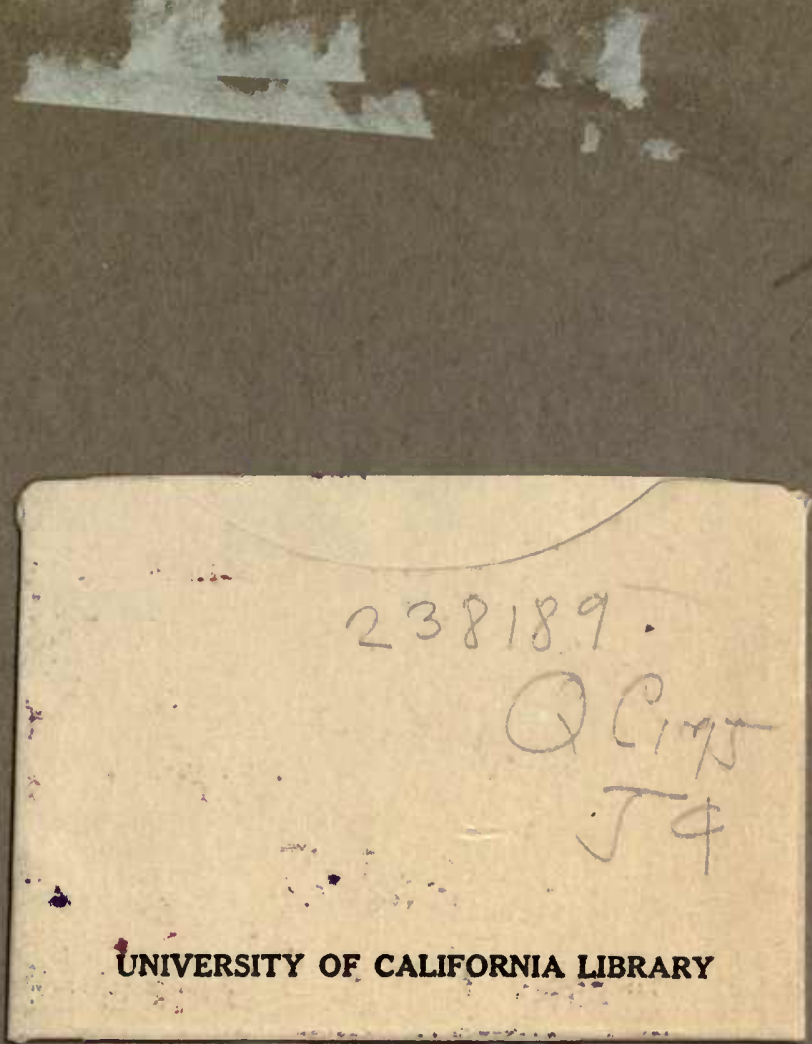


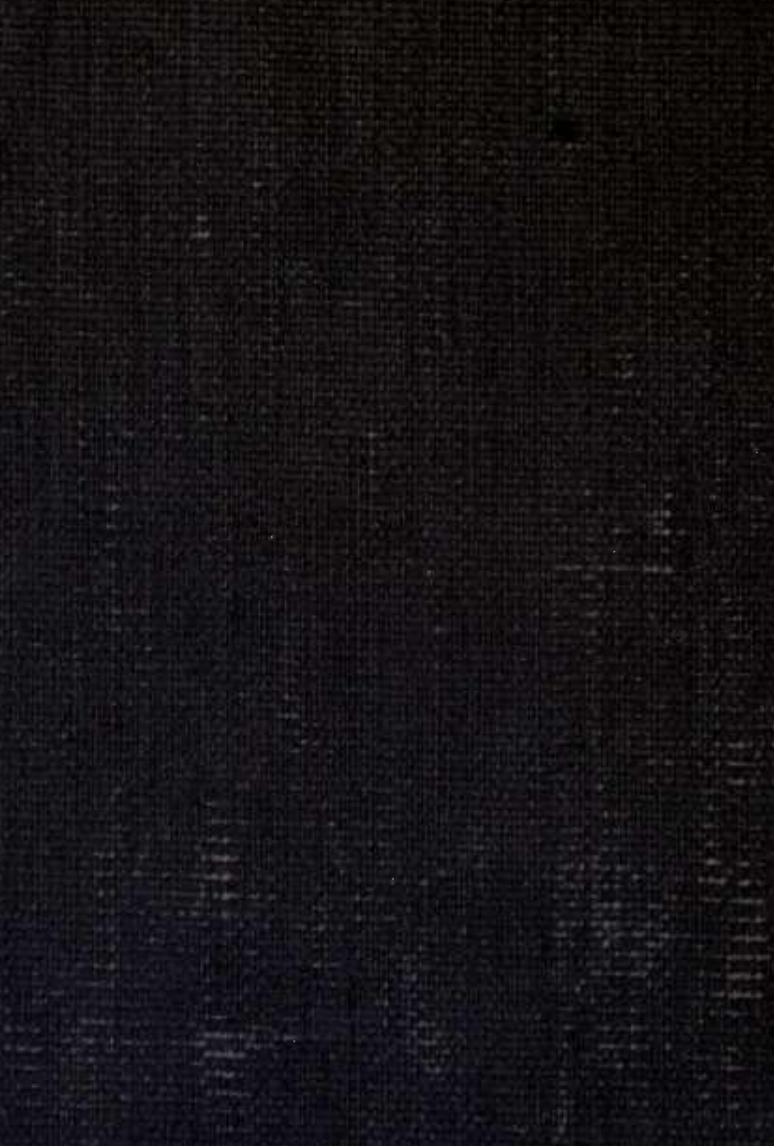

